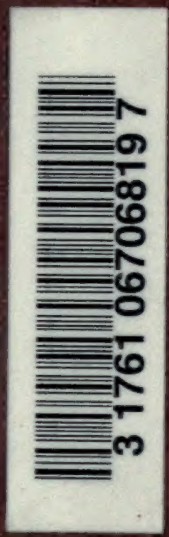




Digitized by the Internet Archive in 2007 with funding from Microsoft Corporation

http://www.archive.org/details/elementarycourse00parkuoft 
AN ELEMENTARY COURSE

$$
\text { OF }
$$

PRACTICAL ZOOLOGY 


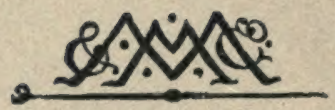

\section{MACMILLAN AND CO., LIMITED}

IONDON - BOMBAY . CALCUTTA - MADRAS MELBOURNE

\section{THE MACMILIAAN COMPANY}

NEW YORK - BOSTON - CHICAGO

DALLAS . SAN FRANCISCO

THE MACMILLAN CO. OF CANADA, LTD. TORONTO 


\section{AN ELEMENTARY COURSE}

OF

\section{PRACTICAL ZOOLOGY}

BY THE LATE

T. JEFFERY PARKER, D.Sc., F.R.S.

PROFESSOR OF BIOLOGY IN THE UNIVERSTTY OF OTAGO DUNEDIN, NEW. ZEALAND

\section{AND}

W. N. PARKER, PH.D.

RROFESSOR OF ZOOLOGY AT THE UNIVERSITY COLLEGE OF SOUTH WALES AND MONMOUTHSHIRE, IN THE UNIVERSITY OF WALES

\section{FIFTH EDITION}

With One Hundred and Sixly-eight Illustrations

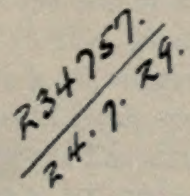

MACMILLAN AND CO., LIMITED ST. MARTIN'S STREET, LONDON 


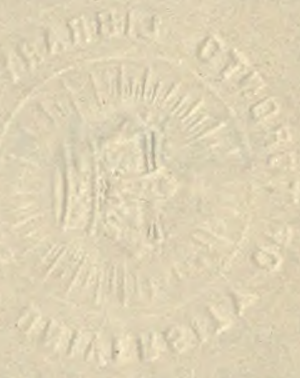

COPYKIGHT.

First Eaition 1899. Second Edition rgos. -Third Edition I9r. Fourth Edition rgi6. Fifth Edition, r92a. 


\section{PREFACE}

IN the early part of I897, my brother and I had arranged to collaborate in writing a practical text-book of Elementary Zoology, adapted more particularly to the requirements of Students pursuing courses in the subject as laid down by various examining bodies. We had, however, only reached the stage of deciding on a general plan at the time of my brother's death in November of the same year.

The following are the chief points on which we had agreed :

I. To adopt the method pursued in Huxley and Martin's Elementary Biology of giving a connected account of each example. 2. To give brief practical directions which should serve mainly as a guide, the student being able to refer, in case of difficulty, to the descriptive accounts preceding them. 3. In the larger animals, to arrange for as much work as possible to be done on one specimen: there is much to be said in favour of this plan apart from the fact that the average student cannot give sufficient time to the subject to dissect a fresh specimen for each system of organs. 4. To begin the course of instruction by an introductory study of one of the higher animals; to include in this introduction the elements of Histology and Physiology ; and to select the Frog for the purpose: after trying various methods, I 
have found this plan to be the most satisfactory in practice. 5. To give drawings and diagrams of difficult dissections, and of details which the beginner cannot as a rule make out satisfactorily for himself ; but otherwise to limit the number of illustrations so as not to tempt the student to neglect observing the things themselves. 6. To include a short account of methods and technique, limited to the barest essential outlines, sufficient for a student working by himself to make out the things described, but not going into such details as would naturally be learnt in a properly organised laboratory.

In the meantime, my brother had in preparation a Biology for Beginners, in which he intended to carry out the plan, suggested in the preface to his Elementary Biology, of giving a simple account, with practical directions, of one of the higher animals and one of the higher plants, as an introduction to the study of Biology. The animal he selected was the Frog, and the manuscript of the greater part of this section of the book was already finished and the rest in rough draft. He had previously suggested that some of this work might be utilised for our proposed Practical Zoology; and I found that, with certain additions and with modifications in the arrangement, the whole of it was exactly the kind of introduction I had in view for the first part of our book. Some of the illustrations that my brother had intended to insert in the Biology for Beginners I have found it advisable to omit, and even now the figures in the introductory part are purposely nearly as numerous as those in the rest of the book. But apart from these various minor modifications, Chapters I-XII in Part I are almost entirely taken from my brother's manuscript.

We felt that there could be no object in entirely rewriting the descriptions of several familiar animals 
already given in my brother's published works; and, in Part II, I have intentionally made use of these descriptions, borrowing very freely from the Elementary Biology, as well as (with Professor Haswell's permission) from the Textbook of Zoology; and to a less extent, from the Zootomy.

The practical directions are mainly based on a series of Laboratory-instructions I drew up some years ago for the use of my junior classes, which consist principally of students preparing for the Intermediate Science examination of the University of Wales, the Preliminary Scientific examination of the London University, and the first examination of the Conjoint Board of the Royal Colleges of Surgeons and Physicians. The time such students can devote to an elementary course in the subject is limited; and throughout the book I have borne in mind that the main object of teaching Zoology " as a part of a liberal education is to familiarise the student not so much with the facts as with the ideas of the science," but at the same time that he should be provided with a sound basis of facts so arranged, selected, and compared as to carry out this principle.

Our original intention was to include one or more cxamples of each of the larger phyla, and also to ald a practical exercise after each type, giving general directions for the examination of an allied form for comparison. But I found that this would be impossible within the space of a single volume, and it was therefore necessary to limit the descriptions mainly to those animals to which the students for whom the book is chiefly intended have to give special attention. This has resulted in rather a heavy balance on the side of Vertebrates; but on the whole, I think that if sufficient work is done on the lower animals to illustrate certain main facts and generalisations, a comparative studv of 
several Vertebrates forms as good a training as any for beginners-more especially in the case of medical students.

I am indebted to Mr. H. Spencer Harrison, B.Sc., Demonstrator of Biology in this College, for much assistance in testing and improving the practical instructions, as well as for various suggestions and for help while the work was passing through the press. The new figures were redrawn from the originals by Mr. M. P. Parker.

\section{W. N. PARKER.}

University College, Cardiff,

November, 1899.

\section{PREFACE TO THE FOURTH EDITION}

I. addition to a careful revision and to numerous minor modifications, the last chapter of previous Editions has been subdivided and largely re-written, and the embryology of the chick and rabbit treated in somewhat further detail. Certain of the illustrations have been replaced, and several additional figures inserted. A change has also been made as regards the type throughout, larger type being used for the Practical 1)irections.

I am indebted to my colleague, Mr. T. H. Burlend, for many useful criticisms and suggestions.

Feloruary, т9เ6.

$$
\text { W. N. PARKER. }
$$




\section{CONTENTS}

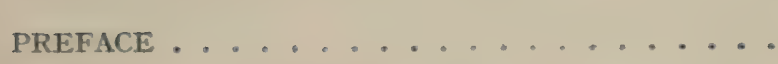

\section{PART I}

CHAPTER I

SCOPE OF THE SCIETCE OF BIOLOGY - THE FROG : PRELIMINARY SKETCH OF ITS STRLCTURE, LIFE-HISTORY, AND VTTAL FUNCTIONS ................. . . I HINTS ON DISSECTION AND DRAWING , . . . . . . . I2

\section{CHAPTER II}

THE FROG (contimued): GENERAL INTERNAL STRUCTURE . . I 6 PRACTICAL DIRECTIONS . . . . . . . . . . . . 3 I

\section{CHAPTER III}

THE FROG (continued): THE SKELETON

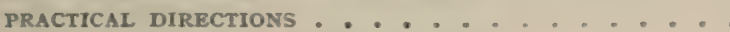

\section{CHAPTER IV}

THE FROG (continued): THE JOTNTS AND MUSClES . . . . 55 PRACTICAL DIRECTIONS . . . . . . . . . . 64

\section{CHAPTER V}

THE FROG (continued): WASTE AND REPAIR OF SUBSTANCE 一THE DIGESTIVE ORGAXS-NUTRITION . . . . . . 66 PRACTICAL DIRECTIONS .............. 76 


\section{CHAPTER VI}

THE FROG (COntinued): THE VASCULAR SYSTEM-THE CIRCULATION OF THE BLOOD ........... . 78 PRACTICAL DIRECTIONS . . . . . . . . . . 98

\section{CHAPTER VII}

THE FROG (continued): THE MICROSCOPICAL EXAMINATION OF THE SIMPLE TISSUES ........... 104 PRACTICAL DIRECTIONS ............ II9

\section{CHAPTER VIII}

THE FROG (continued) : THE MICROSCOPICAL EXAMINATION OF THE COMPOUND TISSUES-GLANDS-SECRETION AND ABSORPTION . . . . . . . . . . . . I 126 PRACTICAL DIRECTIONS ........... I 36

CHAPTER IX

THE FROG (continued): RESPIRATION AND EXCRETION . I4I PRACTICAL DIRECTIONS .......... I 152

\section{CHAPTER $\mathbf{X}$}

THE FROG (continued): THE NERVOUS SYSTEM . . . . I54 PRACTICAL DIRECTIONS . . . . . . . . . . I75

\section{CHAPTER XI}

THE FROG (continued): THE ORGANS OF SPECIAL SENSE - I79 PRACTICAL DIRECTIONS ................. IgI

\section{CHAPTER XII}

I HE FROG (continued): REPRODUCTION AND DEVELOPMENT .

\section{CHAPTER XIII}

THE FROG (continued): MEANING OF THE TERM SPECIES-THE PRINCIPLES OF CLASSIFICATION-EVOLUTION-ONTOGENY AND PHYLOGENY-HEREDITY AND VARIATIONSTRUGGLE FOR EXISTENCE-NATURAL AND ARTIFICIAL 


\section{PART II}

\section{CHAPTER I}

AMGBA-UNICELLULAR AND MULTICELLELAR ANIMALS • 229 PRACTICAL DIRECTIONS . . . . . . . . . . 238

\section{CHAPTER II}

SPHFRELLA AND EUGLENA-MONADS AND BACTERIA-DIFFERENCES BETWEEN ANIMALS AND PLANTS-SAPROPHYTES

PRACTICAL DIRECTIONS

\section{CHAPTER III}

PARAMCECIUM : VORTICELLA AND ITS ALLIES-COLONIAL ORGANISMS

\section{CHAPTER IV}

OPALINA : MONOCYSTIS-PARASITISM-BIOGENESIS AND ABIOGENESIS-CLASSIFICATION OF THE UNICELLULAR ORGANISMS EXAMINED ............... 280 PRACTICAL DIRECTIONS . . . . ........ . . 293

\section{$\sigma_{\text {CHAPTER V }}$}

HYDRA : OBELIA-SYMBIOSIS-ALTERNATION OF GENERATIONS-CHARACTERS OF THE PHYLLM COELNTERATA . 294 PRACTICAL DIRECTIONS ............... 322

\section{CHAPTER VI}

THE EARTHWORM : NEREIS-CHARACTERS OF THE PHYLUM ANNULATA .. . . . . . . . . . . . . . 326 PRACTICAL DIRECTIONS . . . . . . . . . 354

\section{CHAPTER VII}

THE CRAYFISH CHARACTERS OF THE PHYLUM ARTHROPUDA 360 PRACTICAL DIRECTIONS .............. 386 
xii

\section{$\int_{\text {CHAPTER VIII }}^{\text {CONTENTS }}$}

THE FRESH-WATER MUSSEL-CHARACTERS OF THE PHYLUM MOLLUSCA-ENUMERATION OF THE CHIEF PHYLA OF THE ANIMAL KINGDOM . . . . . . . . . . . 396 PRACTICAL DIRECTIONS . . . . . . . . . . . 412

\section{CHAPTER IX}

CHARACTERS OF THE PHYLUM VERTEBRATA-THE LANCELET 418

PRACTICAL DIRECTIONS ............. 426

CHAPTER $\mathrm{X}$

CHARACTERS OF THE CLASS PISCES-THE DOGFISH . . . 430

PRACTICAL DIRECTIONS . . , . . . . . . . . 472

\section{CHAPTER XI}

CHARACTERS OF THE CLASS MAMMALIA-THE RABBIT • . 482

PRACTICAL DIRECTIONS . . . . . . . . . . . 542

\section{CHAPTER XII}

THE MINUTE STRUCTURE OF CELLS-CELL-DIVISION-STRUCTURE OF THE OVUM-GAMETOGENESIS- -(SPERMATOGENESIS AND OOGENESIS) - MATURATION AND FERTILISATION OF THE OVUM -DIFFERENT TYPES OF OVA AND OF SEGMENTATION-EFFECT OF FOOD-YOLK ON DEVELOPMENT +. . . . . . . . . . . . . . 558 PRACTICAL DIRECTIONS . . . . . . . . . . 575

\section{CHAPTER XIII}

LIEVELOPMENT OF THE LANCELET : EARLY DEVELOPMENT OF OTHER TYPES, INCLUDING THE CHICK AND RABBITFORMATION OF THE CHIEF ORGANS OF VERTEBRATES,

AND OF THE AMNION, ALLANTOIS, AND PLACENTA . . 577 PRACTICAL DIRECTIONS .............. 6 I5 


\title{
AN ELEMENTARY COURSE OF PRACTICAL ZOOLOGY
}

\author{
PART I
}

\section{CHAPTER I}

SCOPE OF THE SCIENCE OF BIOLOGY-THE FROG : PRELIMINARY SKETCH OF ITS STRCCTURE, IIFE-HISTORY, A.ND VITAL FUNCTIONS-HINTS ON DISSECTION AND DRAWING

Biology, Zoology, and Botany:- There is a good deal of misconception as to the scope of the science of Biology. One often meets with students who think that while the study of animals as a whole is Zoology and the study of plants as a whole Botany, Biology is the study of a limited number of animals and plants, treated as if they had no connection with anything else,--even with one another.

This is quite wrong. Biology is the master-science which deals with all living things, whether animals or plants, under whatever aspect they may be studied. Physiology, treated for practical purposes as a separate subject, is a branch of biology ; so is anatomy, to which the medical student devotes so much time ; so are botany 
and zoology, in the ordinary sense of the words, i.e., the study of the structure, the mutual relations, and the arrangement or classification of plants and animals. But biology may also be pursued, and very profitably pursued too, quite independently of teachers, classrooms, and examinations. The country boy who knows the song of every bird, its nesting place, the number of its eggs, the nature of its food, the lurking place of the trout in the stream or the frogs in the marsh; who has watched the ants with their burden of grain, or the bees with their loads of honey or pollen; has began the study of biology in one of its most important branches. The intelligent gardener who observes the habits of plants, their individual tastes as to the soil, moisture, sunshine and the like, is also something of a biologist without knowing it. So also is the collector of eggs, shells, or insects, provided he honestly tries to learn all he can about the objects he collects, and does not consider them merely as a hoard or as objects for barter. Indeed, all that is often spoken of as natural history, so far as it deals with living things-plants and animalsand not with lifeless natural objects, such as rocks and minerals, is included under the head of biology.

What then is the connection between biology in this wide sense and the kind of thing you are expected to learn in a limited number of lessons? Simply this :- In the class-room nature cannot be studied under her broader aspects : indeed, much out-door natural history cannot be taught at all, but must be picked up by those who have a love of the subject, a keen eye and patience. But there is one thing we can do within the narrow limits of the class-room: we can confine ourselves to some department of biology small enough to be manageable: we can take, for instance, one or more familiar animals and plants, and, by studying them in some 
detail, get some kind of conception of animals and plants as a whole. This book deals with the zoological side of biology only; and what we have now to do is, in fact, what you have often done in the study of English : you take a single verse of a poem at a time, analyse it, parse it, criticise its construction, try to get at its exact meaning. If you have any real love of literature this detailed study of the part will not blind you to the beauty of the whole. And so if you have any real love of nature, the somewhat dry and detailed study we have now to enter upon should serve to awaken your interests in the broader aspects of biology by showing you, in a few instances, what wonderful and complex things animals are.

One word of warning before we begin work. You must at the outset disabuse your mind of the fatal error that zoology or any other branch of natural science can be learnt from books alone. In the study of languages the subject-matter is furnished by the words, phrases, and sentences of the language; in mathematics, by the figures or other symbols. All these are found in books, and, as languages and mathematics are commonly the chief subjects studied at school, they tend to produce the habit of looking upon books as authorities to which a final appeal may be made in disputed questions. But in natural science the subject-matter is furnished by the facts and phenomena of nature; and the chief educational benefit of the study of science is that it sends the student direct to nature, and teaches him that a statement is to be tested, not by an appeal to the authority of a teacher or of a book, but by careful and repeated observation and experiment.

The object of this book, therefore, is not only to give you some idea of what animals are, but also to induce you to verify the statements contained in it for your- 
CHAP.

self. The description of each animal you should follow with the animal before you ; and if you find the account in the book does not agree with what you see, you must conclude, not that there is something wrong with your subject, but either that the description is imperfect or erroneous, or that your observation is at fault and that the matter must be looked into again. In a word, zoology must be learnt by the personal examination of animals : a text-book is merely a guide-post, and all doubtful points must be decided by an appeal to the facts of nature.

It matters very little what animal we choose as a starting-point-a rabbit, a sparrow, or an earthwormone will serve almost as well as another to bring out the essential nature of an animal, how it grows, how it is nourished, how it multiplies. On the whole, one of the best subjects to begin with is a frog : partly because it is casily obtained, partly because its examination presents no difficulties which an intelligent student may not be expected to surmount by due exercise of patience.

Let us therefore begin our studies by catching a frog and placing it in a convenient position for examination, as, for instance, under an inverted glass bell-jar or even a large tumbler.

External Characters:-Notice, first of all, the short, broad trunk, passing insensibly in front into the flattened head - there being no trace of a neck-and ending behind without the least vestige of a tail : these constitute the axial parts of the animal. In the ordinary squatting position the back has a bend near the middle, producing a peculiar humped appearance. The head ends in front in a nearly semicircular snout, round the whole edge of which extends the huge slit-like mouth. On the top of fore-end of the snout are the two small nostrils, one on 
each side of the middle line; and some distance behind them, the large, bright, prominent eves, in which we can distinguish, as in our own eyes, a coloured ring or iris, surrounding a roundish black space or pupil. The cyelids, however, are rather different from our own : the upper is fairly well developed, but the lower is a mere fold of skin, incapable itself of covering the eye, but produced into a thin transparent skin, the nictitating membrane, which can be drawn upwards over the eye. The entire absence of eyebrows and eyelashes is a point worthy of notice.

Extending backwards from the eve is a large dark patch, in the middle part of which is a circular area of tightly stretched skin. reminding one of the parchment of a tambourine; this is the drum-membrane or tympanic membrane, a part of the ear. Here again we see a striking difference from our own organs : in ourselves the drummembrane, instead of being flush with the surface of the head, is placed at the inner end of a deep passage or tunnel, the entrance to which is guarded by the large external ear. Of the latter there is no trace in the frog.

Attached to the trunk are two pairs of offshoots or appendages, the arms and legs, or fore- and hind-limbs, in which the resemblance to our own limbs will be at once obvious. The arms are very short : each consists of an upper-arm, a fore-arm, and a hand, the latter provided with four fingers, which are slender and tapering and have no nails. The legs, on the other hand, are very long : each consists of a stout thigh, a long shank, with a well-marked "calf," and a very curious foot. The ankle-region is long-almost like a second shank-and has no heel : it is followed by five toes, the first or innermost short, the second of moderate length, the third longer, the fourth longer still, and the fifth of about the 
same length as the third. All the toes are joined together by thin transparent webs, and, like the fingers, have no nails. The name digit is conveniently applied both to fingers and toes. Between the bases of the thighs, at the hinder end of the trunk, is a small aperture, the vent or anus.

In the squatting posture the body is raised upon the arms, which are kept slightly bent at the elbows, with the fingers spread out and directed forwards. In this position the innermost of the four fingers corresponds with our own index-finger, the frog having no thumb. The hind-limb, under similar circumstances, is bent into a sort of $Z$, the knee being directed forwards and the ankle-joint backwards. The toes are turned forwards, and the inner one, which is the smallest of all, corresponds with our own great toe.

Owing to the bent position of the limbs, we cannot very well, as in our own arms and legs, speak of their upper and lower ends. It is therefore customary to call the end of a limb, or of any division of a limb, which is nearest to the trunk, the proximal end, that which is furthest away the distal end. Thus the proximal end of the fore-arm is the elbow region, the distal end of a digit is its tip.

The whole body, including head, trunk, and limbs, is covered with a soft, slimy skin, of a brown colour, irregularly spotted with brown or black on the upper or dorsal surface, and whitish on the under or ventral surface. The colouring is, however, not constant; in a frog kept in the dark the black spots increase to such an extent that the whole animal becomes almost black, while if kept in full daylight a corresponding brightening of the tints takes place. Moreover, the spots and patches of brighter colour are very variable: if you examine a dozen specimens you will see at once that no two are 
alike in this respect. The large dark patch situated behind the eye and containing the tympanic membrane. is, however, always present, and is one of the chief distinguishing marks of the common British frog as compared with other kinds, such as the "edible frog" of the Continent.

Sexual Characters. - As in so many of the more familiar animals there are two sexes of frogs, easily distinguished from one another. If you examine several of them you will find that a certain number have on the palm of the hand, towards the inner side, a large swelling, rather like the ball of our own thumb, but much more prominent and of a black colour. Frogs having this structure are males; it is not present in the females.

Actions performed by the Living Frog.-Kept under suitable conditions a frog very soon shows evidences of life. If touched or otherwise alarmed it attempts to escape by making a series of vigorous leaps-suddenly extending the hind legs and jumping to a considerable height. Thrown into water it swims by powerful strokes of the hind-limbs. It has thus, like so many living things with which we are familiar, the power of voluntary movement.

If kept under observation for a sufficient time-weeks or months-it will be found that frogs grow until they reach a certain limit of size. Growth, in the case of the frog, is an increase in size and weight affecting all parts of the body, so that the proportions remain practically unaltered, and no new parts are added.

Careful observation shows that the throat is constantly rising and falling, and the nostrils opening and shutting. These movements, like the expansion and contraction of the human chest, are respiratory or breathing movements, and serve to pump air into and out of the lungs.

It requires frequent watching and sharp observation to see a frog feed. It lives upon insects, worms, slugs, and 
the like. Opening its mouth it suddenly darts out a tolerably long, nearly colourless, and very sticky tongue ; if the prey is a small insect, such as a fly, it adheres to the end, and the tongue is quickly drawn back into the mouth, the whole operation being performed with almost inconceivable rapidity.

Like other animals the frog discharges waste matters from its body. Its droppings or faces, discharged from the vent, are black and semi-solid. From the same aperture, it expels periodically a quantity of fluid, the urine, which is perfectly clear and colourless, and contains little besides water.

Sometimes a frog will escape from confinement, leaving its damp box or vivarium for the warm, dry atmosphere of an ordinary room. When this happens the animal is usually found next morning dead and shrunken, and with its naturally moist skin dry and hard. From this it may be inferred that there is a constant evaporation of water from the skin, which, under ordinary circumstances, is checked by a damp atmosphere or by occasional immersion in water.

Hibernation.--In winter frogs bury themselves in damp places, and become sluggish, manifestations of life becoming hardly apparent until the following spring, when they emerge from their holes. In this way they escape the dangers of frost which would otherwise be fatal to them. This suspension of activity during winter is known as hibernation, or the winter-sleep.

Reproduction and Development.-If you examine a number of frogs towards the end of winter-about February-you will find that the full-grown females are distinguished from the males, not only by the absence of the pad on the hand, but by the swollen condition of the trunk, due to the interior being distended with eggs. After a time the eggs are laid, being passed out of the 
vent by hundreds; each is a little globular body about $\frac{1}{1}$ th inch in diameter, half black and half white, and surrounded by a sphere of clear jelly, by means of which the eggs adhere together in large irregular masses, the well-known "frog-spawn." As the eggs are laid, the male passes out of his body, also by the vent, a milky spermatic substance or milt, which gets access to the eggs and impregnates or fertilises them. Without impregnation they are incapable of developing.

Neither male nor female takes the slightest care of the eggs when once they are deposited and fertilised. They are simply left in the water unprotected in any way; and, naturally enough, the mortality among them during the course of development is very great, the majority being eaten or otherwise destroyed, and only a very small percentage coming to maturity.

The first noticeable change in the spawn is that the sphere of jelly surrounding each egg swells up so as to acquire several times the diameter of the enclosed egg. The egg itself, or cmbryo, as it must now be called, gradually becomes entirely black, then elongates, and takes on the form of a little creature (Fig. I, I) with a large head, a short tail, and no limbs; which after wriggling about for a time, escapes from the jelly and fixes itself, by means of a sucker on the underside of its head, to a water-weed. Great numbers of these tadpoles, as the free-living immature young or larice of the frog are called, may be seen attached in this way. At first they are sluggish and do not feed, but, before long, they begin to swim actively by lashing movements of their tails, and to browse on the weeds. They are thus in the main vegetable-feeders, not carnivorous, like the adult frog. On each side of the head appear three little branched tufts or gills, which serve as respiratory organs $\left(2,2^{\circ}\right)$, the tadpole, like a fish, breathing air which is dissolved 
in the water. After a while the gills begin to shrivel up $(3,4)$, and the tadpole then comes periodically to the surface to breathe, lungs having in the meantime made

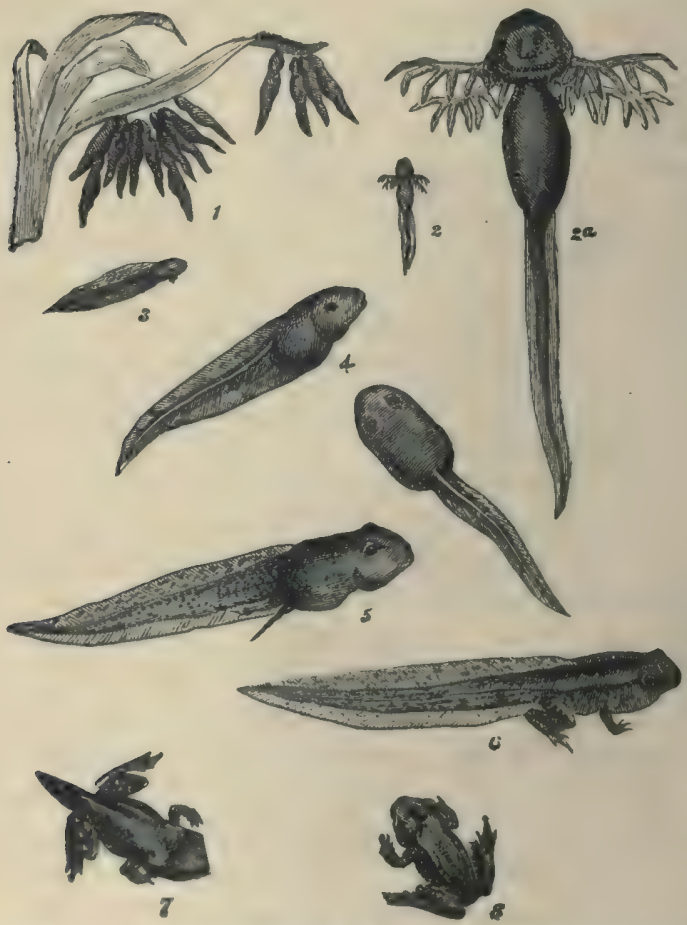

Fic. x.-Stages in the life-history of a Frog, from the newly-hatched tadpoles ( $\mathrm{x}$ ) to the young frog ( 8 ). Natural size. $-2 a$ is a magnified view of 2 . (After Mivart.)

their appearance. A pair of little hind-limbs appears at the root of the tail, and a pair of fore-limbs behind the head $(5,6)$. As these increase in size the tail slowly dwindles, the head and trunk assume the characteristic frog-form, and the animal now comes on land and hops 
about as a small tailed frog (7). As growth goes on, the tail further diminishes and finally disappears altogether, the transformation or metamorphosis being thus completed (8).

Death and Decomposition:-Frogs may live for many years, but, sooner or later, either in the ordinary course of nature or by accident, they die. The heart stops beating, the flesh undergoes what is called " death-stiffening," becoming hard and rigid, and all vital manifestations cease. Before long the process of decomposition ensues, the flesh, viscera, etc., soften and emit a bad smell, and in course of time rot away completely, leaving only the bones.

Summary of Chapter.-The very brief and cursory study we have made so far shows us (I) that a frog has certain definite parts arranged in a particular way; (2) that it performs characteristic movements, some of them, such as leaping and swimming, voluntary ; others, such as breathing, involuntary ; (3) that it takes in solid food, consisting mainly of vegetable matter in the tadpole, of living animals in the adult; (4) that it gives off waste matters; (5) that it reproduces its kind by laying eggs, which develop only if impregnated ; (6) that it undergoes transformation or metamorphosis, the egg giving rise to a larva, the tadpole, which, after living for a time the life of a fish, gradually changes into a frog. 


\section{HINTS ON DISSECTION AND DRAWING}

Instruments and other Requisites for Dissection. ${ }^{1--}$ In order to carry out the dissection of the frog and other animals successfully it is necessary to be provided with proper tools. The most important are--

I. Three or four sharp dissecting knives, or scalpels, of different sizes.

2. A large and a small pair of straight dissecting-forceps; the small pair should have a peg on one leg fitting into a hole on the other, to prevent the points crossing; the points should be roughened.

3. A large and a small, fine-pointed pair of dissecting scissors; the small pair for the more delicate work, and the large pair for coarser work and for cutting through bones. For the latter purpose a pair of bone-forceps is useful, but is not necessary in the case of such a small animal as the frog.

4. A seeker, i.e., a blunt needle mounted in a handle.

5. Three or four probes; a seeker or knitting needle, or a thin slip of whalebone will answer for some purposes, but the most generally useful form of probe is made by sticking the end of a hog's bristle into melted sealing wax, and immerliately withdrawing it so as to affix a little knob or guard.

6. An anatomical blowpipe, or, failing this, a piece of glass-tubing, 6 or 8 inches long, with one end drawn out in the flame until it is not more than ${ }_{20}^{1}$ th to $\frac{1}{20}$ th of an inch in diameter.

7. An ordinary "medicine-dropper," or "feeder" of a self-feeding pen (see Fig. 25), made of a piece of glass-tubing about three inches long, drawn out in the flame at one end, and thickened at the other so as to form a collar, over which an india-rubber cap-an ordinary non-perforated teat--is fixerl. This is useful for washing fine dissections, as well as for injecting.

8. A dissecting-dish. Get a common pie-dish, about 6 or 8 inches long, with rather low sides. Cut out a piece of cork-carpet or thick linoleum the size of the bottom of the dish, and a piece of sheet-lead of the same size, and fasten the two together by three or four ties of copper wire or

1 A suitable box of clissecting instruments can be bought from most scientific instrument makers for about $\mathrm{r} 3 \mathrm{~s}$. $-\mathrm{f}-\mathrm{I}$. (Fior further apparatus required in connection with injection and microscopical work, see pp. 99, XI9, and I35.) 
strong thread. Place this in the dish with the lead (which is simply to keep the cork-board from floating in water) downwards. Or, place a few strips of sheet lead in the bottom of the dish, and then pour in some melted paraffinwax into which a little lamp-black has been stirred, so as to make a layer half an inch or more in thickness.

For larger animals than the frog, in addition to a larger dish, a dissecting-board will also be required. Get a piece of soft deal or pine about Is inches $X$ I I inches and 3 inch thick, and nail round its edge a strip of wood about $\frac{3}{4}$ inch $\times \frac{1}{4}$ inch, so as to form a projecting rim.

9. A magnifying glass. Any good pocket-lens or a common watchmaker's glass will answer the purpose. As it is often desirable to have both hands free while using the lens, a stand of some kind is useful. One of the simplest is made by fixing a piece of 1 -inch brass-tubing, about 6 inches long, into a heavy block of wood, about 3 inches in diameter, and coiling round it, in a close spiral, one end of a piece of thick wire, which can be raised and lowered on the tube : leave 6 or 7 inches of the wire standing out at right angles, and bend the free end into a loop to carry the lens. Or, get a piece of narrow clock-spring, about 13 inches long, and rivet one end to the outside of the rim of a watchmaker's glass, and the other to a small piece of zinc or brass; on passing the spring round the head, the lens is kept in place at the eye without exertion.

I0. Medium and small-sized pins. Large blanket pins are useful for fixing down larger animals.

II. A small sponge and a duster.

12. One or more wide-mouthed bottles or jars, containing a preservative in which to place your subjects after each day's work. The most convenient preservative for the purpose in most cases is the fluid sold as formaline, ${ }^{1}$ which can be diluted as it is wanted. For preserving your dissections from day to day, a I per cent. solution of formaline is strong enough in many cases-i.e., I cubic centimetre of formaline to 99 c.c. of water, or three-quarters of a dram of formaline to half a pint of water. For permanent preservation, a stronger solution-2 to 5 per cent., according to circumstances-should be used, or methylated spirit. If formaline is not available, use strong methylated spirit (i.e., about 90 per cent.) diluted with one-third of its bulk of water.

I3. A plentiful supply of clean water.

If. An ounce or two of chloroform.

1 A 40 per cent. solution of the gas formic aldehyde. 
Rules to be Observed in Dissection.-Many of the parts and organs of animals are bound together by means of a substance known as " connective-tissue," and the main object of dissection is to tear away and remove this substance so as to separate the parts from one another.

The subject should be firmly fixed down in the dissectingdish or on the dissecting-board by means of pins, inserted obliquely, so that they do not interfere with the dissection. The dissecting-dish must always be used for finer dissections, which should be done under water; only just enough water being put into the dish to cover the dissection, which should be washed under the tap from time to time.

When dissecting a part keep it on the stretch, and avoid fingering it or damaging it with the forceps.

Never remove anything until you know what you are removing. Dissect along and not across such structures as blood-vessels and nerves.

See that your instruments are kept clean and sharp, and never use the smaller scissors and scalpels for coarse work.

Drawing.-You should make a point of drawing as many of your preparations, as well as of the living animals, as possible: an accurate sketch, taken from nature, no matter how rough, is of more value in teaching observation and in impressing the facts on your memory than the examination and copying of more perfert drawings, made by others. Anyone can soon learn to make sketches of "this kind, even without having any previous knowledge of drawing.

Fach sketch should be made to scale, and small objects should be enlarged several times ; it is much easier to insert details in a large drawing than in a small one. Mark the scale against each drawing-e.g., $\times 2, \times \frac{1}{2}$.

Using a rule and compasses, first sketch in an outline of the principal parts with a hard pencil; if your object is bilaterally symmetrical, draw a faint line down the middle of the paper and then sketch in one side first. When vou have sketched in all the outlines correctly, go over them again with a softer pencil, so as to make them clear and distinct. Do not at temnt any shacling unless you have some knowledge of drawing.

Then tint the various parts in clifferent colours, using very light tints except for such structures as vessels and nerves. You should keep to the same colours for the corresponding organs or tissues in all the animals you examine : thus you 
- might in all cases colour the alimentary canal yellow, the arteries red, the veins blue, glands brown, cartilage green, and so on.

Wake your drawings on one side of the page only; the opposite side can then be used for explanations of the figures.

Nerer insert on your original sketches anything you have not actually seen; you can copy as many other figures as you like from various sources, but these should be kept apart from your own original drawings.

Directions for the examination of the external characters of the adult frog, as described in this chapter, are given at the end of Chapter II., p. 31 ; and of the eggs and tadpoles in Chapter XII., p. 212. 


\section{CHAPTER II}

THE FROG (continued): GENERAL INTERNAL STRUCTURE

You have now seen that a frog can perform a number of very complicated actions; and, if you have any curiosity in these matters, you will probably want to know something of the mechanism by which these actions are brought about. Now, the best way to understand the construction of a machine, such as a clock or a steam-engine, is to begin by taking it to parts of which the living machine we call a frog is made, and the way they are related to one another, only by
taking it to pieces, or dissecting it.

First notice, in addition to the external characters described in the last chapter, that the various parts of the body are strengthened or stiffened, as in ourselves, by a number of bones, which together form the greater part of the skcleton. It is quite easy to ascertain by feeling that the head contains a hard skull; the lower jaw, a lower-jaw-bone or mandible; that running through the back is a jointed backbone or vertebral column; that the region of the chest is protected by a breastbone or bone or bones.

The Mouth-Cavity. - There are also 
be observed in the interior of the mouth. All round the edge of the upper jaw is a row of small conical teeth (Fig. 7). There are no teeth in the lower jaw ; but on the roof of the mouth, a short distance behind the snout, are two little patches of teeth, called the vomerine teeth ( $\left.\tau^{\circ} \circ . t\right)$, and nearer the jaw are two apertures, called the internal nostrils $(p, n a)$ : a guarded bristle passed into one of the external nostrils and pushed gently backwards and downwards will be found to enter the mouth by the corresponding internal nostril.

Behind the internal nostrils are two large hemispherical projections, due to the roof of the mouth being bulged out by the huge eyes, as can be readily made out by pushing the eyes from outside.

On the floor of the mouth is the large, flat tongue $(\operatorname{lng})$, remarkable for the fact that it is attached at its front end, its hinder end being free and double-pointed. When the frog uses it to catch insects it is suddenly thrown forwards, almost like a released spring. Just behind the backwardly-turned tips of the tongue is an oval elevation, having on its surface a longitudinal slit, called the glottis $(g l)$, which leads, as we shall see afterwards, into the lungs.

The back of the mouth narrows considerably, and the soft skin or mucous membrane lining it is here thrown into folds. A probe gently pushed backwards passes, as we shall see, into the stomach. The narrowed region of the mouth is the throat, or pharynx. On its upper wall, near the angles of the mouth, are two pits: a guarded bristle passed into one of these will be found to come into contact with the corresponding tympanic membrane, which will be pierced if sufficient force is used. The pits are known as the Eustachian recesses or tubes (eus. $t$ ).

Dissection of the Frog: Skin and Museles.-If a slit is made in the skin of the belly, and a probe pushed in 
under it, it will be seen that the skin, instead of being firmly attached to the underlying flesh, as in a rabbit or a sheep, is for the most part quite loose, a spacious cavity lying between it and the flesh. Not, however, a single continuous cavity for the whole body: the probe, gently pushed in various directions, is stopped, in front, at about the level of the arms; behind, at the junction of the thighs with the trunk; and at each side, along an oblique line joining the armpit with the thigh. Moreover, by opening the skin of the back, throat, and limbs, and inserting the probe as before, similar cavities will be found in these regions, all separated from one another by partitions, along which the skin is firmly united to the underlying flesh. It will be noticed also that the probe, when withdrawn from any of these cavities, is wet. The cavities contain a watery fluid, called lymph, and are hence known as sub-cutaneous lymph-sinuses (Fig. $7, d . l y . s, v . l y . s$ ).

When the skin is removed it will be seen that under the skin and separated from it by the lymph-sinuses is a nearly. colourless, semi-transparent, fibrous substance, the flesh. At first this appears to be continuous over the whole body, but, by careful dissection with a sharp scalpel, a very delicate, transparent membrane, called the fascia, can be separated from the flesh, which is then seen to consist of a number of separate bands (Fig. 2, my. hy, $p c t, r c t$. $a b d$; see also Fig. I6), covered as aforesaid by the fascia, and separated from one another by a kind of packing substance, also very delicate and transparent and known as connective-tissue. These bands or sheets are the muscles, and the whole of the flesh is made up of distinct muscles, readily separated from one another when once the requisite anatomical skill is attained. Here and there-for instance on the top of the head and the front of the shanks-there are no muscles, 
and the bones are covered only by skin and connectivetissue.

Passing along the middle line of the belly is a dark longitudinal streak (Fig. 2,abd. v): this is a blood-ressel, the abdominal vein. On each side of the body another

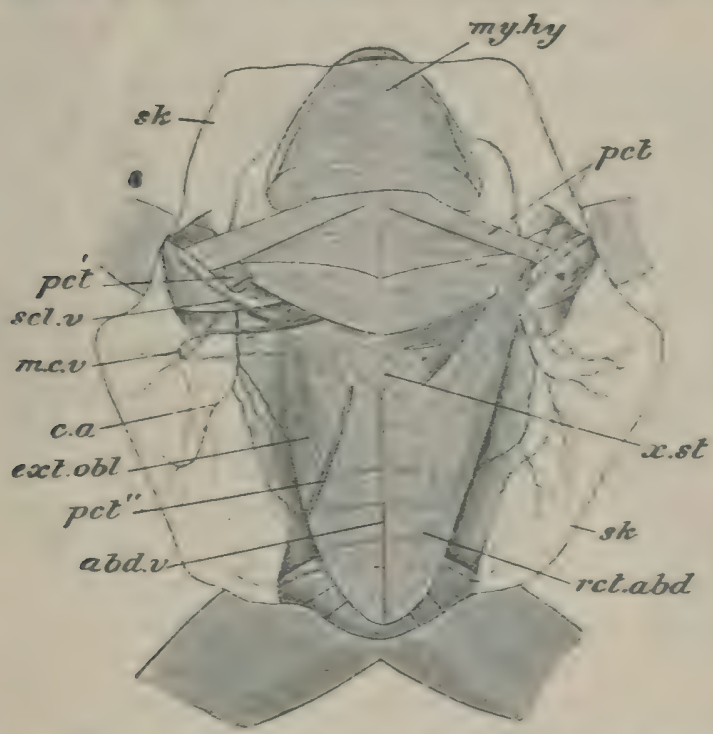

Fic. 3.-A Froz with the skin (sk) of the ventral surface cut through and turned back rizht and left, sn as to expose the muscles. Of these the mylohyoid ( $m y \cdot h y$ ), pectorailis (pef). external ntigue (evt. obi), and restus abiominis (rot. abd)

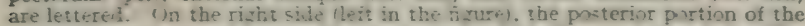

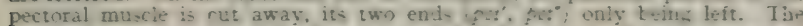
cartilazinmus extreruity of the breast-hone ixiphisternum, $x$. st) is shown, as well

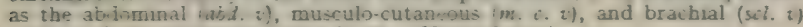
veins, and the cutaneous artery ( $c . a)$. (Natural sze.)

vein (m. c.v) is seen forming a loop, one limb of which is on the turned-back flap of skin, while the other passes between the muscles not far from the armpit : this vessel is the musculo-cutaneous rein. Both these veins, and many others which will be seen in the course of the 
dissection, are thin-walled tubes full of blood, as will be proved if you should happen to cut one of them, when the blood will escape in considerable quantity.

Between the right and the left fore-limbs the ventral region of the trunk is protected by certain bones which form part of the shoulder-girdle: projecting backwards from this in the middle line is a flat, heart-shaped plate of a softer, gristle-like substance, known as cartilage (compare Fig. I2). Immediately between the thighs a cartilage called the pubis, part of the hip-girdle (Fig. I4), can be felt. Between the shoulder-and hip-girdles the ventral body-wall is soft, being formed only of muscle and connective-tissue.

The Abdomen and its Contents.-By cutting through the muscles of the belly or abdomen, a large cavity, the body-cavity or calome, is exposed, in which are contained numerous structures presently to be described. In order, however, to open the whole of the cavity the ventral part of the shoulder-girdle must be removed.

In the middle line, between the fore-limbs, and therefore covered in the entire animal by the shoulder-girdle, is a pink conical body (Figs. 3 and $4, v$ ) connected in front with a thin-walled bag $(r . a u, l$. au), of a purplish colour. The whole thing is the heart: the pink posterior portion is called the ventricle: the purple anterior part consists of two chambers, the auricles. The heart is enclosed in a transparent, membranous bag, the pericardium $(p c d)$, which contains a lymphatic fluid.

Just behind or posterior to the heart are two large masses $(l r)$ which have a dark reddish-brown colour; these are the right and left lobes of the liver. They extend forwards, one on either side of the heart : between them is a globular bag of a greenish colour (Fig. $3, g l . b l$ ), the gall-bladder. In front of the liver and left and right 


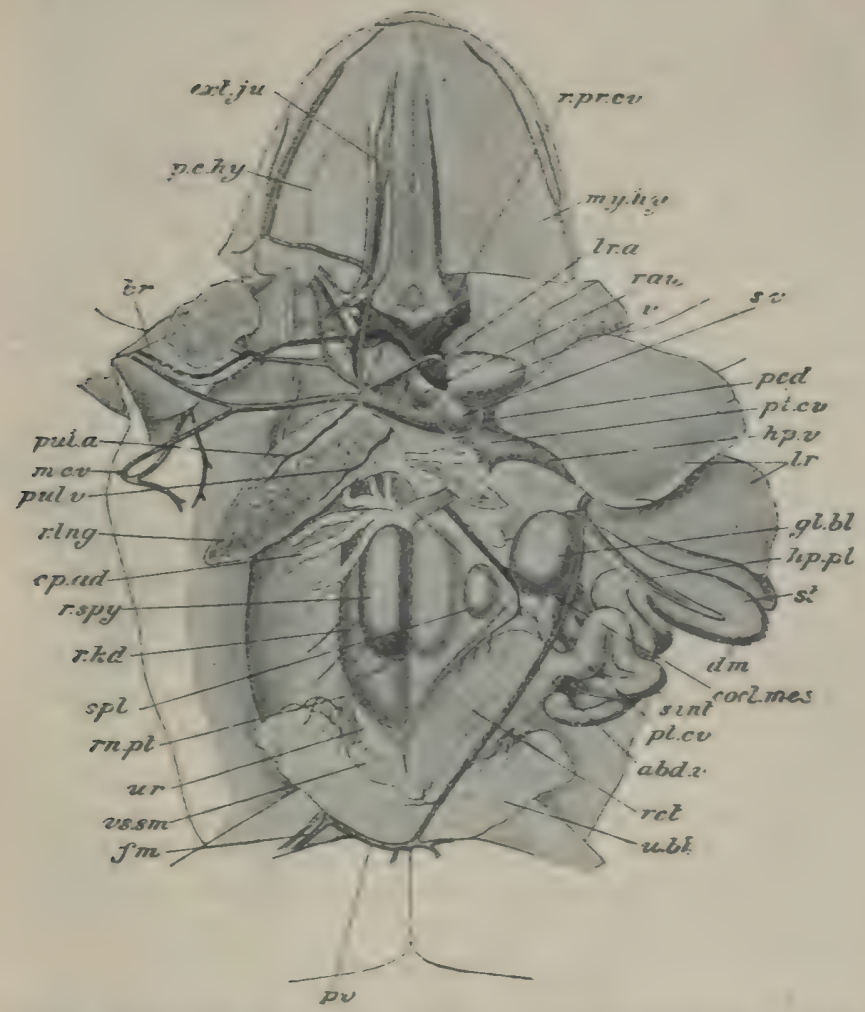

Fig. 3--Dissection of a Male Frog. The enteric canal and liver are displaced to the animal s left. ani part of the Inve is cut away. Some of the muscles are cut away and certain nerves and blond-vessels traced into the head and limbs ( $x$ I

abd. $v$ abdominal vein : br. brachial artery, vein, and nerve ; cal. mes. splanchnic or colizcomesenteric artery: op. ah. right fat-body; dm. dwotutum; ext. ju. external jurular vein: fn. iemoral vein: gl. bi. Eall-bladiet: Fp. pr.

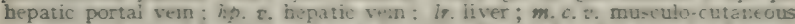
vein: wy. hy. mylohvoid musle: pod. perivardiun; ; p. c. ky. anterion bern of hyoid; $p$ t. on. postcaval vein: sul. a. pulmenary artery; pwi. $v$. puimonary vein; pe. pelvic vein: $\%$. as. tiaht auricle: res. rectum: $\%$ ka. riaht kidnev; \%. Ing. right lung: $\%$ pr. 2 . rizht pretaval vein: "n. pt. right renal fortal vein: r. spy. right spermary: s. : w. aleum: stl spleen: st. stemach: s. $v$. sinus venesus ; tr. a. comes arienosus: w. bi. urinary bladder; wr. ureter; ะ. ventricle; : s. sm. seminal vesicle. 
of the heart are two thin-walled transparent sacs $(r$. $\operatorname{lng}, l$. $\operatorname{lng}$ ) with a honeycombed surface, the lungs. Their appearance varies very much according to their state of distension. When full of air they are about two inches in length in a full-sized frog, and protrude freely as soon as the abdomen is opened: when empty they hardly show unless the liver is turned aside.

Emerging from beneath the left lobe of the liver (beneath in the present position of the animal, actually above) is a wide whitish tube (Fig. $3, s t$ ), which almost immediately turns to the right (the frog's right, not yours), so as to form a U-shaped bend $(s t, d m)$. This is the stomach, which is connected with the pharynx by a short tube called the gullet or cesophagus (compare Fig. 7 , $g u l, s t)$, and which varies considerably in size according to whether it is empty or distended with food. The stomach becomes continuous with a narrower tube, the first part of which $(\tilde{d} m)$ passes forwards parallel with the stomach, thus forming the narrow limb of the $U$, while the rest of it $(s . i n t)$ is thrown into a rather complex coil. This tube is the small intestine; the part in immediate connection with the stomach $(d m)$ is distinguished as the duodenum and the coiled part as the ileum. Between the stomach and duodenum, in the bend of the $\mathrm{U}$, is a small yellowish-white body of irregular form, the pancreas (Figs. 7, pn, and I8, $P$ ).

The stomach and intestine are kept in place and suspended to the dorsal wall of the body-cavity by a delicate membrane, the mesentery (Fig. 5, mes), which is folded in correspondence with the various coils. As we shall see, the mesentery is really a portion of a thin, moist membrane, the peritoneum, with which the bodycavity is lined.

The small intestine becomes continuous, posteriorly, with a much wider tube (Figs. 3 and $7, r c t$ ), lying against 
the dorsal wall of the abdomen, and called the large intestine or rectum. It is continued into a short tube, the cloaca $(\mathrm{cl})$, which passes backwards, between the backbone above and the pubis below, to open externally by the vent. Thus the mouth-cavity, pharynx, gullet, stomach, small intestine, rectum, and cloaca form a continuous tube, opening externally at each end, by mouth and vent respectively, and, for the greater part of its extent, contained within the body-cavity. The whole tube is known as the enteric or alimentary canal.

Attached to the mesentery, close to the anterior end of the rectum, is a rounded body of a deep-red colour, the spleen (Figs. 3 and $7, s p l$ ). Quite at the posterior end of the abdominal cavity a very thin-walled and transparent $\operatorname{sac}(u . b l)$ will be seen, connected with the ventral side of the cloaca, and varying much in size according to its state of distension. This is the urinary bladder, which communicates by an aperture (Fig. $7, b l$ ) with the cloaca, and when distended will be seen to be bilobed and of considerable size.

If your specimen should be an adult female, and the time of year approaching the breeding season, you will already have observed, as the most prominent organs in the body, two large, lobed structures of a dark colour, protruding one on either side, and partly obscuring the view of the other organs. Each (Fig. 4,l. ovy) contains a great number of small globular bodies, half black and half white, and is suspended to the roof of the bodycavity by a sheet of peritoneum. These bodies are the ovaries, or organs for the manufacture of the eggs; the rounded bodies of which they are largely composed are the eggs themselves. To each ovary is attached a vellow structure, produced into a number of streamerlike lobes $(c p . a d)$; this is the fat-body, which serves as a storehouse of reserve nutriment. 


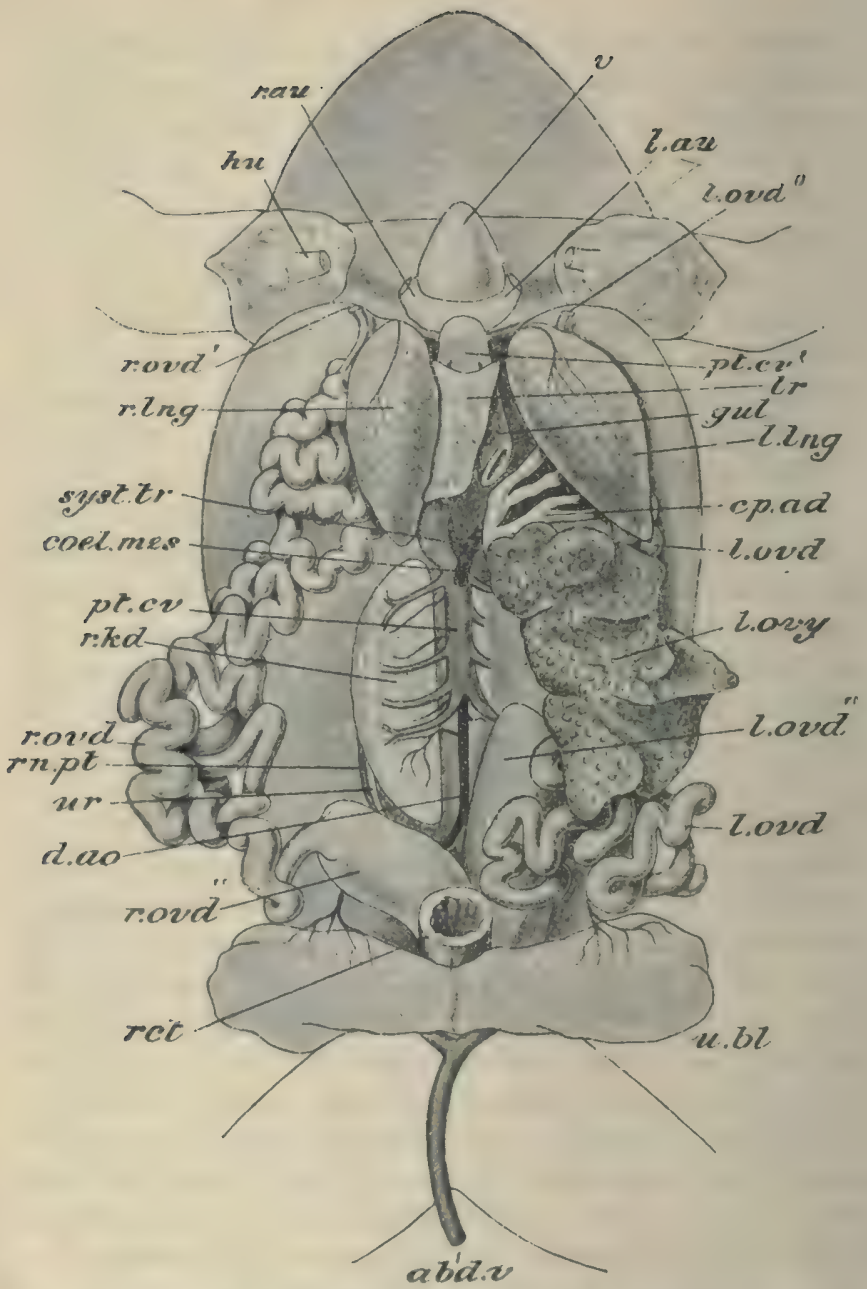

Fis. 4. Dissection of a young Female Frog. The gullet (gul) and rectum (ret) have been cut through, and the enteric canal remover between these two points. lise lives is removel, with the exception of a small portion (lr) surrounding the postcaval vein $\left(p^{t}, c v\right)$. The ventricle of the heart $(v)$ is turneclforwards, and 
the abdominal vein $(a b d .:)$ is severed and turned backwards. The right ovary and fat-tody are removed, and the right oviduct $(r$.ovd) is slightly displaced outwards. ( $x \quad x$.

abd. $y$. abdominal vein: cxl. mes. splanchnic or coliaco-mesenteric artery; $c p$. $a d$. corpus adiposum, or fat-body ; $d$. ao. dorsal aorta : gul. gullet; hi. cut end of humerus or upper-arm bone; $l$. aw. left auricle; $l$. Ing. left lung : l. otd. Ifefe oviduct; $I$. mod'. its opening into the body-cavity; $l$. ovd". its pus-

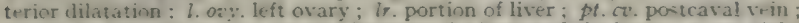
pi. $c^{\prime}$. its anterior portion passing between the liver and the heart; 7 , au. right auriel. ; rct. rectum : r. kd. -ight kikney; $r$. logg. right lung; $r n$. pt. renal porzal vein; $r$. end. right ovjduct; $r$. ond'. its openine inte the beuly-eavity:

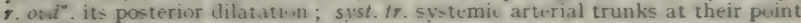
of union; $u . b l$. urinary bladder; wr. ureter; v. ventricle.

By lifting up either of the ovaries there is seen beneath it -in the natural position of the parts, above or dorsal to it-a greatly convoluted colourless tube (l.ovd, r. ovd) of about the same diameter as the intestine. This is the oviduct, through which the eggs pass from the ovary to the cloaca. If the specimen is allowed to remain long in water the oviducts will be found to swell and finally to become disintegrated; this is due to the fact that in them is formed the jelly in which the laid eggs are enclosed, and which, as already mentioned (p. 9), swells in water.

In the male there is seen, on turning the intestines aside, a pair of yellow ovoidal bodies (Fig. 3, r. spy) about half an inch long, attached by peritoneum to the dorsal wall of the body-cavity. These are the spermaries or testes; they manufacture the spermatic substance or milt by which the eggs are impregnated. To the anterior end of each is attached a fat-body $(c p . a d)$, like that of the female. In young specimens of both sexes the reproductive organs-spermaries, ovaries, and oviducts-are very small.

When the intestine is turned aside there will also be seen, in both sexes, a pair of flattened, irregularly-oval bodies (Figs. 3 and $4, r . k d$ ) lying in the posterior part of the abdominal carity just above or dorsal to the ovaries or spermaries. These are the kidneys. With the outer edge of each is connected a tube, the ureter 
(ur), by which the urine, formed in the kidneys, is carried to the cloaca (Fig. 7).

It has been pointed out that the abdomen is lined by peritoneum, and that the various organs are suspended by folds of the same membrane, called, in the case of the enteric canal, the mesentery. The relations of this membrane are best seen in a diagrammatic transverse section of the body (Fig. 5), though many

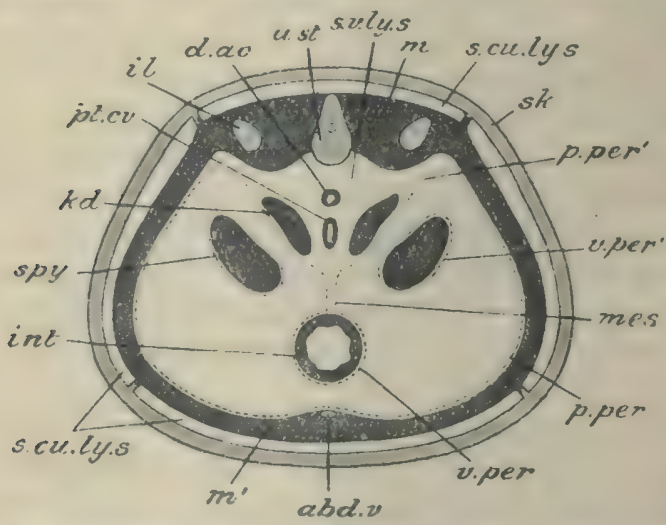

Fic. 5.- Diagrammatic transverse section through the trunk of a Frog, to show the relations of the peritoneum. ( $x 2 \frac{1}{2}$.)

$a b d . v$. abdominal vein ; $d$. ao. dorsal aorta ; $i l$. ilium; inl. intestine; $k d$. kidney; $m$. museles of back; $m$. muscles of abdomen; mes. mesentery ; $p$. per. parietal layer of peritoneum; $p$. per'. the same, turning down to cover the kidney; $p i . c v$. postcaval vein; sk. skin; s. cu. $l y . s$. sub-cutaneous lymph-sinuses; spy. spermary; s.v.ly.s. sub-vertebrallymph-sinus; u. st. urostyle (part of the vertetural column); v. per. visceral layer of peritoneum, investing intestine; $v$. por. the same, investing spermary.

points can be perfectly well made out from the actual specimen. The body-cavity is lined by what is called the parietal layer of the peritoneum $(p \cdot p e r)$, which adheres closely to the body-wall except in the middle dorsal region, where it becomes closely applied to the ventral surface of the kidneys and reproductive organs. L.eaving these, the peritoneum of the right side 
approaches that of the left, and the two, coming into contact, form a double vertical sheet, the mesentery (mes), which extends ventrally towards the enteric canal. On reaching the latter, the two layers diverge again and surround the canal, forming the visceral layer of the peritoneum ( $\tau$. per). The liver, oviducts, etc., are suspended and covered in the same way. Thus the lining of the body-cavity, the investment of the various organs contained in it, and the folds by which they are suspended, are all parts of one continuous membrane. The space left between the two diverging layers of peritoneum, in the mid-dorsal region, contains lymph, and is known as the sub-vertebral lymph sinus (s. v.ly.s).

We have already noticed the abdominal and musculocutaneous veins. Other veins of greater or less size will be seen everywhere: passing, for instance, to the head and limbs (Fig. 3), and in the mesentery. Running parallel with many of the veins are smaller vessels, many of which have pigment in their walls, and which are of distinctly stouter texture. These are the arteries. They contain little blood in the dead animal, and, owing to the stoutness and elasticity of their walls, do not collapse when empty. Hence they are quite easy to see in a frog from which all the blood has been drained, while the thin-walled veins are almost invisible under like circumstances. Finally, there will be seen in many parts of the body, often lying parallel to an artery and a vein, white cords, the nerves.

\section{The Neural Cavity and its Contents.-By turning} the frog with its back upwards and cutting through the muscles of the back and the arches of the vertebræ as well as, in front, the roof of the skull (see Fig. 6), you will see that the backbone contains a distinct cavity, the neural cawal, in which lies a white rod, made of the same soft kind of substance as the nerves, and called the spinal 
cord $(s p . c d)$, which ends behind in a thread-like prolongation $(f . t)$, some distance in front of the thighs. It will also be found that the neural canal is continued, with a slightly increased diameter, into the skull, and that the spinal cord becomes continuous with the brain (br), a

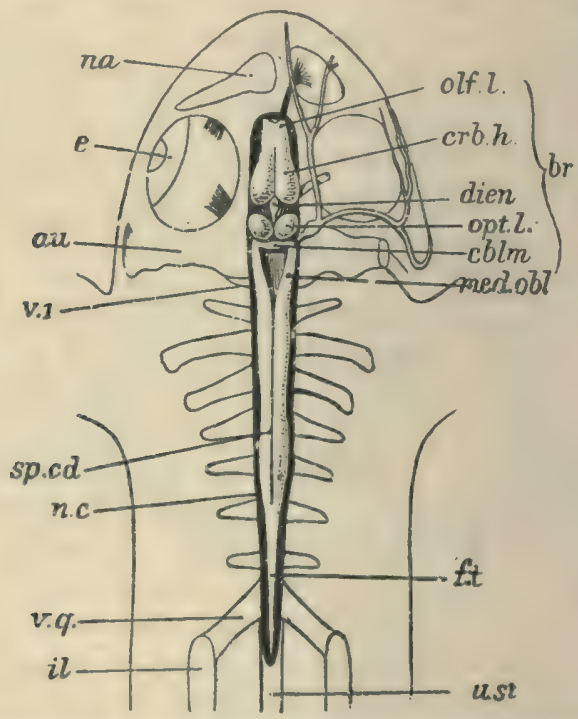

Fig. 6.-Dissection of a Frog in which the entire neural canal ( $n . c)$ has been opened from above, and the brain (br) and the spinal cord (sp.cd) laid bare. The brain consists of olfactory lobes (olf. $l$ ), cerebral hemispheres $(c r b . h)$, diencephalon $\left(d_{l e n}\right)$, optic lobes (opl. l), cerebellum $(\mathrm{cblm})$, and medulla oblongrata (med. $\left.a b l\right)$. wheh will be referred to in Chapter $X$. The spinal cord ends in a delicate proJormation, the filum terminale $(f, t)$. The nasal bones $(n a)$, eyes $(c)$, auditory regien of the skul! $(a u)$, transverse processes of the nine vertebra $(v, z-v . q)$, urostyle $(u, s l)$ and ilia (il) are indicated in outline, and serve as landmarks. ( $x$ I1.) (After Howes, slightly altered.)

complex organ formed of several parts, which will be referred to hereafter.

General structure of the Limbs.-A transverse section cut across one of the legs, at about the middle of the thigh, will show in the middle of the cut surface the 
thigh-bone, around it the flesh or muscle, and around this again the skin. Similar cuts through various parts of both fore- and hind-limbs show that these appendages of the body are solid, containing no cavities, except the sub-cutaneous lymph-sinuses previously observed.

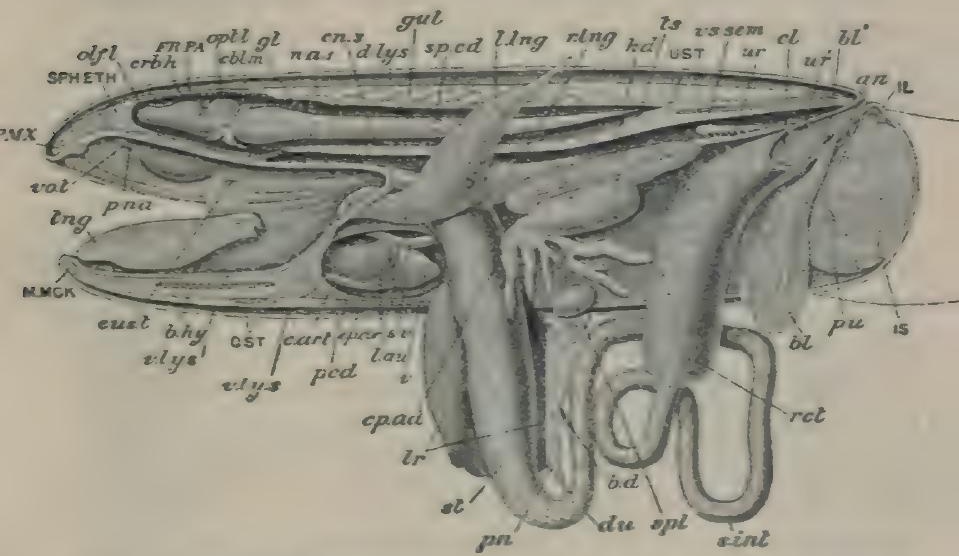

FrG. 7.-Dissection of a Male Froz from the left side. The left fore- and hind-limbs and the left sides of the head and trunk have been cut away, the enteric canal and liver are displaced dowmwards, and the mouth, pharynx, and cloaca laid open. ( $x$ It $)$.)

an. anus : $b$. $d$. bile-duct; $b$. ky. body of hyoid; $b l$. urinazy bladder; $b l$. jts opening inte the clnaca ; c. ent. conus arteriosus; colm. cerebellum ; cl. cloaca ; cr. 3 , centrum of third vertebra : $c p$. ad. fat body : $c v b$. $h$. cerebral hemisphere; d. ly. s. dorsal lymph-sinus; dis. duodenum; ep. cor. epiecracoid : cus. t. Eustarhian recess ; FK. PA. fronto-pariotal; gl. glottis : gui gullet : II. ilium; IS. schum. kd. Kilney: $l$. au. leit auricle; $l$. ing. left lung: lr. liver; M.MCK. mento-meckithan bone; $r$. a. $I$, arth of first vertebra ; olf.l. olfactory lobe: op: l. opuc lobe: O. ST. omonternum ; pui perteardium : PMX. pre-

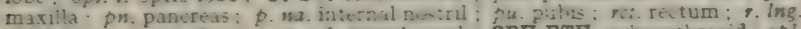
right lung: s. wo leum : sp. $c d$. spinal cord: SPE.ETE. sphenthmoid : spl.

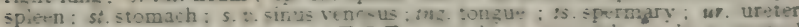

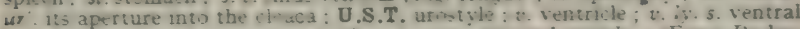
lymph-sas: \% t. vomerine teeth; *s. scm. siminal vesicle. (From Parker and Haswell's Zoology:)

Summary.- We thus get a notion of the general plan of construction of a frog as follows. It consists of a central or axial portion, the head and trunk, and of two pairs of lateral offshoots or appendages, the fore- and hind-limbs. 
The trunk is hollowed out into two cavities: the $a b$ dominal or body-cavity (ccelome) below, and the neural canal above ; of these the neural cavity alone is continued into the head. The abdominal cavity contains the greater part of the enteric canal, the liver, gall-bladder, pancreas, spleen, lungs, heart, kidneys, urinary bladder, and reproductive organs. The neural canal contains the brain and spinal cord. The anterior end of the enteric canal is continued forwards into the head, forming the mouth-cavity, and opens externally by the aperture of the mouth; its posterior end opens externally by the vent. The enteric canal passes through the containing body-cavity, having no communication with it. The lungs open into the pharynx, and thus communicate with the exterior not only by the mouth but also by the nostrils. The kidneys, bladder, and oviducts communicate with the cloaca, and thus with the exterior through the vent. Neither the neural nor the abdominal cavity has any communication with the exterior. The walls of the head and trunk consist largely of muscles and bones covered with skin. The limbs are solid outgrowths of the trunk, formed mainly of muscle, with bony supports and a covering of skin.

Organs.-Notice that the body consists of various definite structures, or organs as they are technically termed, which have various purposes or functions to perform. The enteric canal, together with the liver and pancreas, are organs of digestion; the lungs and skin, organs of respiration or breathing; the heart and bloodvessels organs of circulation, serving as they do to propel and conduct the blood through the body; the kidneys, aided by the skin, organs of excretion, for getting rid of waste matters; the ovaries and spermaries, organs of reproduction; the muscles, organs of movement; the brain and spinal cord, together with the nerves, organs 
of control, serving to direct or control the actions of the body: the skin, nose, eye, and ear, sensory organs, by which communications are kept up with the external world.

Tissues.-Notice also that the various organs of the body are built up of different materials, or tissues as they are called. We have already distinguished muscle, bone, cartilage, connective-tissue and nervous tissue. Other tissues we shall meet with in the course of a more careful examination.

\section{PRACTICAL DIRECTIONS}

To Kill a Frog for Dissection.-Place a frog en a plate, and cover it with a tumbler, or put it into a stopnered bottle. Soak a little bit of cotton-wool or sponge in chloroform, and push it under the edge of the tumbler, or drop it into the bottle. In a few minutes the vapour will make the animal quite insensible, and a somewhat longer exposure will kill it painlessly.

External Characters.-Observe the voluntary and the involuntary respiratory movements of the living animal, and compare with a dead frog when making out the external characters (pp. 4-8) and the position of the various parts of the skeleton (p. I6).

Sketch the entire animal from the side or from above.

The Cavity of the Mouth.-Gently open the mouth of a dead frog as wide as possible, and make out the points described on p. I7. Sketch.

The Body-wall.-Lay the frog on its back in the dissecting-dish, and fix it firmly by sticking pins through the skin of the arms and legs. With the forceps, held in the left hand, pinch up the skin of the abdomen near the middle line between the thighs, and make a nick in it with the point of the scissors. Then, holding the edge of the hole thus made with the forceps, pass in a probe and push it forwards as far as it will go without opposition.

Note :-

The sub-cutaneous lymph-sinuses, and the underlying muscle. 
With the scissors extend the incision made in the skin of the belly forwards, in a straight line, to the lower jaw. Holding up the edge of the skin with the forceps, cut through, with a scalpel, the partitions between adjacent lymphsinuses, so as to separate the whole of the skin of the ventral surface from the muscle ; and, having done so, pin back the flaps, right and left (see Fig. 2). Similar cuts should be made in the skin of the limbs and back. Observe-

The fascia, the muscles of the body-wall, the abdominal and musculo-cutaneous veins, and the position of the hyoid (p. 4o), sternum (p. 47), shoulder-girdle, and pubic region of the hip-givdle (pp. 46 and 50 ).

The Abdomen and its Contents.-Pinch up the muscles on one side of the abdominal vein with the forceps, and make an incision in them by a single snip of the scissors. Then, holding the edge of the wound with the forceps, extend the cut forwards to the shoulder-girdle and backwards to the pubis. Keep the cut parallel to the abdominal vein, and be careful not to wound the latter. You will find that the incision thus made opens a large body-cavity or colome, in which a number of structures, the abdominal viscera, are contained. Note that the body-wall consists of three layers: (I) skin, (2) muscles, with their fascia, and (3) peritoneum.

So far, however, the cavity is not thoroughly opened. I.ift up the side of the abdominal wall to which the abdominal vein is attached, and very carefully separate the vein by tearing through, with a needle or the point of a scalpel, the connective-tissue by which it is attached to the inner face of the muscles: or, in order to prevent the possibility of injuring the vein, cut through the muscles of the body-wall longitudinally on the other side of the abdominal vein, so as to leave a narrow strip of muscle attached to it. Then make two cross-cuts, starting from the anterior end of the longitudinal incision, and extending outwards towards the fore-limbs: take care not to injure the musculo-cutaneous veins, and pin back the two flaps into which the soft abdominal wall is now divided (Figs. 3 and 4 ). Next dissect away the muscles covering the shoulder-girdle, so as to expose the bones: irlentify the bones called coracoid and clavicle (compare a skeleton and fig. 12). With the strong scissors cut through these bones on either side as near as possible to the shoulder-joint: then lift up the stermum and middle portions of the shoulder-girdle, and carefully clissect them away from the underlying parts. 
Having thus exposed the whole of the abdominal cavity, pour just enough water into the dissecting-dish to cover the animal, furst washing away any blood which may have escaped from cut vessels. If your specimen is female, dissolve a little common salt in the proportion of I per cent. in the water (I gramme to 100 c.c.), or mix it with about one-third of its bulk of methylated spirit, in order to prevent the excessive swelling of the oviducts. (If, however, you wish to make out the blood-vessels in this specimen without injecting them, it is as well to defer putting water into the dish until a later stage of dissection.)

\section{Note-}

I. The peritoneum-parietal and visceral layers.

2. The pericardium, containing the heart. If not already opened, the pericardium should be slit through, so that the auricles and ientricle can be plainly scen. If the frog has been killed quite recently, you will be able to observe the pulsation of the heart.

3. The right and left lobes of the liver, and the gallbladder.

4. The two lungs : if contracted, inflate with a blowpipe through the glottis.

5. The enteric or alimentary canal, consisting of gullet or osophagus, stomach, smali intestine (duodenum and ileum), and large intestine or rectum communicating with the cloaca, which will be scen at a later stage, and which opens to the exterior by the vent.

\section{The mesentery.}

7. The pancreas.

8. The spleen.

9. The urinary b!adder. If collapsed, insert a blowpipe into the vent and inflate. (Tou will very likely find some small parasitic flat-worms, called Polystomum integerrinum, in the bladder; each worm has a ring of suckers round the hinder end.)

10. In the male the spermaries and fat-bodies, and in the female the oiaries, fat-bodies and oitulucts.

II. The kidney's and ureters.

12. The mode of suspension of all these organs (p. 26), and the position of the sub-iertetral lymph-simus. In order to understand clearly the relations of these parts, a thick transverse section should be made through another frog in 
the region of the kidneys and examined under water (compare Fig. 5).

Sketch the contents of the abdomen in situ.

The Neural Cavity and its Contents.-Now turn the frog with its back upwards, and pin it firmly to the bottom of the dissecting-dish or to the dissecting-board as before. Pinch up the skin, make a longitudinal cut through it from the snout to within a short distance of the vent, and turn the flaps right and left. The muscles of the back will be exposed, and, in front, the roof of the skull, which lies just beneath the skin. Carefully dissect away the muscles along the middle line of the back until the vertebral column is seen. Compare a prepared skeleton and Fig. 8, and make out the arches of the vertebra. Between the first of these and the back of the skull is a slight space: insert one blade of the strong scissors into this, directing the point backwards, and cut through the arch of the first vertebra, first on one, then on the other side, and finally detach and remove the little piece of bone. The neural canal will then be exposed, in which lies the spinal cord, ending behind in a thread-like prolongation (compare Fig. 6) : work backwards, cutting away the arches of the remaining vertebræ.

Next, using the scissors in the same manner, cut away, bit by bit, the roof of the skull : two large bones - the frontoparietals (see Figs. 8 and 9), forming a considerable part of the roof, can be more easily removed by raising them up with the edge of a scalpel. Note the cavity of the skull and its contained brain.

General Structure of the Limbs.-With a strong knife, cut across one of the legs at about the middle of the thigh. Compare p. 28 , and notice the thigh-bone, muscles, and skin. Sketch.

Now preserve your specimen in formaline (about 2 per cent.) or spirit (70 per cent.). 


\section{CHAPTER III}

THE FROG (continued): THE SKELETON

If you have followed the description given in the preceding chapter with a frog beiore you, testing every statement as you proceeded by reference to the specimen, you will now have a very fair notion of the general build of the animal. The next thing to do is to study its various parts in somewhat greater detail.

As the bones and cartilages form the framework on which all the other parts are supported, it is convenient to begin with them. You may study them on a prepared skeleton, but a far better plan is to make a skeleton for yourself as directed on p. 53 .

Parts of the Skeleton.-The skeleton consists of the following regions:-

I. The skull (Figs. 8 and 9): a complex mass of mingled bone and cartilage, enclosing the brain and the organs of smell and hearing, and supporting the upper jaw. Connected loosely with the skull, but really forming part of it, are the lower jaw and a cartilage in the floor of the mouth known as the hyoid.

2. The vertebral column or backbone, consisting of nine movably united pieces, the vertebre (Fig. 8 v.I-v.9), and of a long bony rod, the urostyle (UST), representing a number of fused vertebræ belonging to the tail-region of the tadpole. 
3. The shoulder-girdle or pectoral arch (Figs. 8 and I2), an inverted arch of bone and cartilage nearly encircling the anterior part of the trunk and giving attachment to-

4. The bones of the fore-limbs.

5. The hip-girdle or pelvic arch (Figs. 8 and I4), an apparatus shaped somewhat like a bird's "merrythought ": it is attached in front to the ninth vertebra and behind gives attachment to-

6. The bones of the hind-limbs.

The Vertebral Column.-The essential structure of a vertebra may be best studied by examining any of the nine from the second to the seventh: the first, eighth, and ninth present certain peculiarities, and so may be left till last.

The whole vertebra (Fig. 8, B) has something the form of a signet-ring with its sides produced into two outstanding projections. The part comparable to the stone of the ring is ventral in position, and is called the body or centrum (cn), the form of which is procclous, i.e., its anterior face is concave, its posterior face convex, and both faces are covered with a thin layer of cartilage. The part corresponding with the circle of the ring is the neural arch $(p d, l m)$ : it arches over the spinal cord and is produced in the middle line above into a blunt projection, the neural spine $(n . s p)$. From the arch is given off, on either side, the large outstanding projection already referred to, the transverse process (tr. pr), which is tipped with cartilage in the second, third, and fourth vertebræ.

The neural arch gives off from its anterior face, just above the origin of the transverse processes, a pair of small shelf-like projections, the articular processes or zygapophyses (a. zyg). Each has its upper surface flat and smooth, and covered with a thin layer of cartilage. A similar pair of processes spring from the posterior 


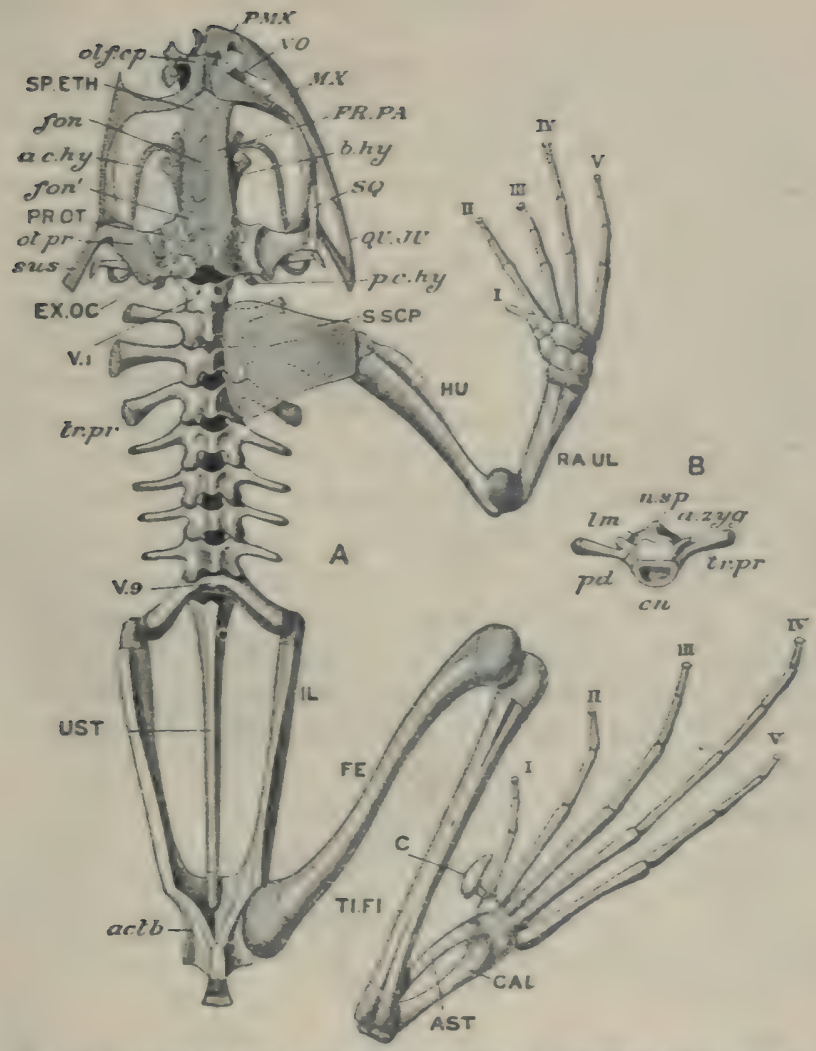

Fig. 8.-A, skeleton of Frog from the dorsal aspect; F, anterior face of the fourth vertebra. $\left(\mathrm{r}_{\mathrm{r}} \mathrm{s}^{3}\right)$ In $\mathrm{A}$ the left $\mathrm{h}$.lf of the shoulder-girdle and the left foreand hind-limbs are removed, as als, are the inves:ing honis of the left side of the skull. Cartilazinous parts ar distinguished ty dotting. The names of replacing bones are in thick capital letiers, those of investing bones in italic capitals, other references in smal! italics.

a.c.hy. anterior bern of hynid! avt. acetabulum; AST. astrazalus; a. =yg. anterior articular prosesses. or zyeapephysis: b. h: basi-h:al: C. calcar; CAL. calcaneum; cn. centrum : EX. OC exccciptial: FE. timur; fon, fon'. fontanelies: FR. P.A. frento-parietal : HU. humerus: IL. ilum ; im. roof of neural arch: MX. maxilla: ns sp. neurai spine: $\theta^{\prime} f . c p$. cliactory capsule ; of. pr. ofic process: p.c. h. posterior hom of hvoid: ful base of neural arch; P.M. premaxilla: PR. OT. preotic: $Q t^{\circ}, J l^{\circ}$. guadrato-jugal : RA. UL. radio-ulna : SP. ETE. spherethmeld: SQ. squamcsat: S. SCP. supra-scapula; sus. suspensorium: TI. FI. :ibio-îbula : $r$. pr. transverse process : UST. Urostyle; $\nabla .1$. cervical vertebra: $\nabla .9$, sacral vertebra: 10 . vomer; $I-V$ digits. (From Parker and Haswell's Zon'ogy, after Howes, slightly altered.) 
face of the arch, but have the smooth, cartilage-covered surface or facet looking downwards.

When two vertebræ are placed in position, the convex posterior face of the foremost centrum fits into the concave anterior face of its successor, like a cup and ball, and at the same time the posterior articular processes of the first fit over the anterior articular processes of the second. All the touching surface is, as we have seen, capped with cartilage, and the vertebræ can be moved upon one another, either up and down or from side to side.

The centra and articular processes are the only parts of the vertebræ which are in contact when the bones are in their natural positions. Large gaps or notches are left between the dorsal portions of the arches (see Fig. 8, A) to allow of the requisite amount of up and down movement, and there are similar gaps between the sides of the arches, bounded by the articular processes above and the centra below. These are called the intervertebral foramina: through them the nerves pass from the spinal cord.

The only differences of importance between the vertebræ now under consideration is in the form and direction of their transverse processes, which are specially large in the third, short and devoid of cartilaginous tips in the fifth, sixth, and seventh.

The first vertebra has no transverse processes, and on either side of the very small " body" its anterior face bears, instead of the ordinary articular processes, a pair of obliquely placed, oval, slightly concave surfaces or facets, covered with cartilage, and serving for the articulation of the condyles of the skull, presently to be described. The transverse processes of the ninth or sacral vertebra are very long and strong, directed obliquely backwards, and tipped with cartilage: to them the arms of the hip girdle are articulated. 
The eighth vertebra differs from its predecessors in having its body concave behind as well as in front. Corresponding with this, the ninth (v. 9) has its centrum convex in front, while behind it presents two little rounded elevations placed side by side.

It will be seen that the vertebræ are all corresponding structures, following one another in a regular series from before backwards. A correspondence of this kind, in which there is a repetition of similar parts along the body, is termed a serial homology, and thus not only the vertebræ as a whole, but also their various parts are serially homologous, each to each, the correspondence being disturbed only by the first vertebra, in which the transverse processes are absent and the anterior face is modified for articulation with the skull.

The urostyle (UST) is a long bone with a gradually diminishing ridge along its dorsal surface (see p. 35). Its anterior face has somewhat the appearance of a small vertebra with no transverse processes, and has a double concavity for articulation with the double convexity on the ninth vertebra. Near its anterior end there is on each side a small aperture, representing an intervertebral foramen, for the last spinal nerve.

The skull is a very complex structure, consisting partly of bone, partly of cartilage. It is divided into the following regions:-

I. The brain-case or cranium, a sort of oblong box containing the brain (Figs. 8 and 9): it forms the middle portion of the skull and is a direct forward continuation of the vertebral column.

2. The auditory capsules (aud. $c p$ ), a pair of outstanding masses arising, right and left, from the posterior end of the brain-case. They lodge the organs of hearing.

3. The olfactory capsules (olf. $c p$ ), smaller masses proceeding from the anterior part of the brain-case and 
united with one another in the middle line. They lodge the organs of smell.

4. The suspensoria (sus), a pair of projections springing from the outer and upper portions of the auditory capsules, and directed downwards, outwards and backwards. To them the ends of the lower jaw are attached.

5. The upper jaw, a half-circle of bone and cartilage, united in front to the olfactory capsules and behind to the auditory capsules and suspensoria. On either side of the skull, between the cranium and upper jaw, is a large space, the orbit, in which the eye is contained.

6. The lower jaw, a roughly semicircular bar of bone and cartilage, articulated at its ends with the suspensoria.

7. The hyoid (b.hy), a shield-shaped cartilage connected by delicate curved rods $(a, c$. $h y$ ) with the auditory capsules.

On the posterior surface of the brain-case is a large hole, the foramen magnum (Fig. 9, c, for. mag), on either side of the lower edge of which is a large oval elevation covered with cartilage, the occipital condyle (oc. cn). The foramen magnum leads into the cavity in which the brain is contained. If the first vertebra is placed in its natural position with regard to the skull it will be seen that the foramen magnum corresponds with the neural canal of the vertebra, and that the condyles fit into its articular surfaces. Thus the skull readily moves up and down upon the vertebra, the condyles acting as rockers; a space between the neural arch and the dorsal edge of the foramen magnum covered by membrane in the fresh state allows of the requisite amount of play.

The discrimination of the separate bones and detailed structure of the skull is rather difficult, and may very well be omitted by the beginner at the present stage.

The occipital condyles are borne on a pair of irregular 
bones (EX.OC) which bound the sides of the foramen magnum, nearly uniting above and below it, and extending over a considerable portion of the posterior surface of the auditory capsule. These bones are the exoccipilals. In

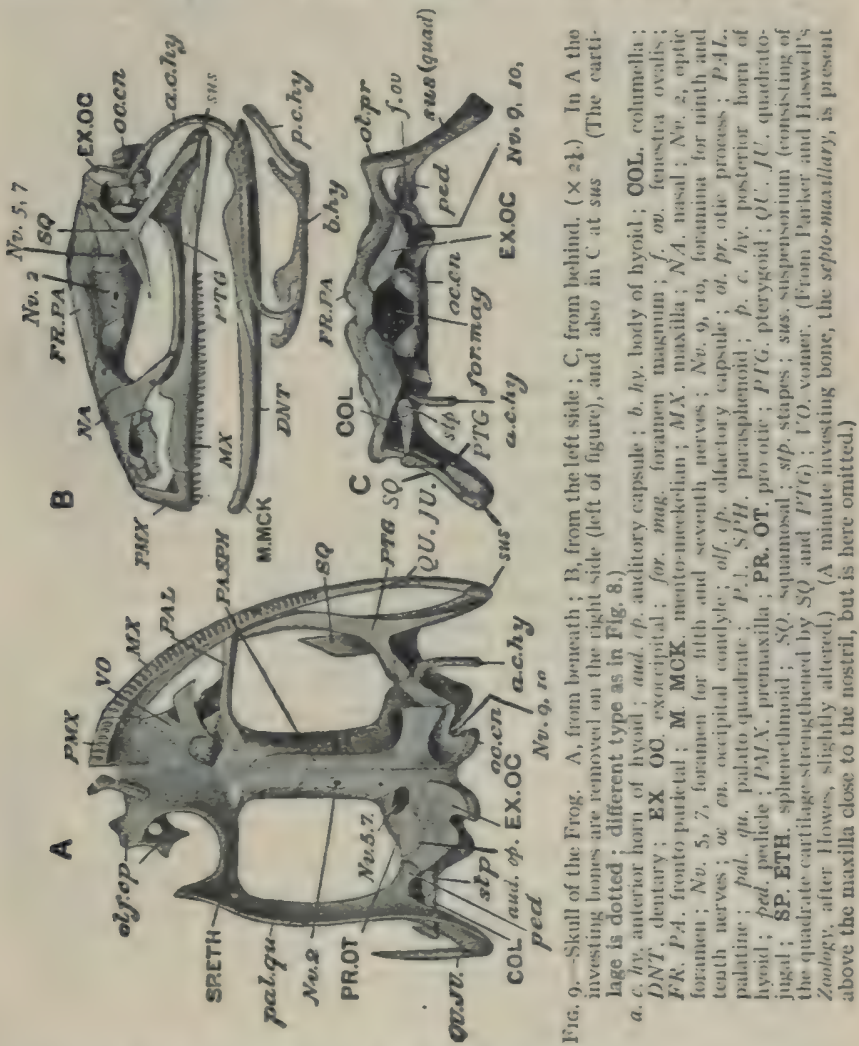

front of each exoccipital is another irregular bone (PR.OT) forming the front part of the auditory capsule, and called the pro-otic. Each pro-otic is separated from the corresponding exoccipital, in young frogs, by a band of cartilage, but in old specimens the two bones are more or less completely united. 
In the disarticulated skull it can be made out that the exoccipital and pro-otic of either side enclose a cavity ; in this the organ of hearing is contained. The exoccipital is perforated, just in front of the condyle, by a double aperture $(N v .9$, Io), through which two nerves, the glossopharyngeal and the vagus, pass on their way from the brain (see Chapter X.). The pro-otic is similarly perforated or notched for the trigeminal and facial nerves $(N v, 5,7)$.

The dorsal surface of the brain-case is covered by two longish, flat bones $(F R . P A)$. In the young condition each of these consists of two distinct bones, the front one the frontal, the hinder the parietal. As the young frog grows the frontal and parietal of either side become completely fused, forming a single fronto-parietal. On the upper surface of each olfactory capsule is a roughly triangular bone, the nasal $(N A)$, in front of which is the corresponding nostril.

The ventral surface of the brain-case is covered by a single bone $(P A . S P H)$ having the shape of a $T$. The stem extends forwards in the middle line as far as the olfactory capsules, while the arms stretch outwards beneath the auditory capsules. This very characteristic bone is the parasphenoid. On the under surface of the olfactory capsules, corresponding to the nasals above, are a pair of irregular bones, the vomers ( $V O$ ). Their outer edges are notched and help to bound the internal nostrils: their posterior ends bear the vomerine teeth.

The anterior end of the brain-case is surrounded by a bone (SP.ETH) which extends forwards into the region of the olfactory capsules, and is partly covered by the frontoparietals and nasals above and by the parasphenoid below. This is the girdle-bone or sphenethmoid. In the disarticulated skull it is seen to have a very peculiar shape. Its posterior half encloses a single cavity in which the fore-end of the brain (Fig. 6, olf. $l$ ) is lodged. Its anterior half encloses two cavities, right and left, separated from one another by a vertical partition, and serving to lodge the posterior ends of the olfactory sacs or organs of smell. Each of these cavities communicates with the single posterior cavity by a small hole through which the nerve of smell passes.

Between the girdle-bone in front and the pro-otic behind, the side-walls of the skull are formed of cartilage perforated by a rouncled aperture, the optic foramen $(N v, 2)$, for the nerve of sight.

Forming the outer part of the suspensorium is a hammershaped bone, the squamosal $(S Q)$; its head is applied to the auditory capsule and projects forwards into the orbit. 
The upper jaw is formed of three bones on either side. In front is the premaxilla $(P . M X)$, a short bone, sending off an upward process towards the nostril. Next follows the maxilla $(M X)$, a long, curved bone, forming the greater part of the upper jaw, and joined at its posterior end to a small, slender bone, the quadrato-jugal $(Q U . J C)$, which is firmly connected with the lower end of the suspensorium. Both premaxilla and maxilla are produced below into a prominent edge from which spring a number of small conical teeth, arranged in a single row.

Besides these three bones there are two others which seem, as it were, to brace the upper jaw to the brain-case and suspensorium. The palatine $(P A L)$ is a narrow, rodlike bone, placed transversely behind the olfactory capsule. The pterygoid ( $P T G$ ) is a large three-rayed bone; one ray is directed forward and connected with the outer end of the palatine and with the inner face of the maxilla; another passes backwards and inwards and is connected with the auditory capsule; the third extends backwards and outwards and forms the inner and ventral portion of the suspensorium. The main mass or core of the suspensorium, between the squamosal outside and the pterygoid within, is a rod of cartilage (sus), which is continued forwards by a bar (pal. qu) supporting the pterygoid and palatine.

There is an important distinction to be drawn between the bones of the skull which can be made out only by the exercise of a good deal of care and patience. By suftening the connective-tissue which binds the bones together, it is possible to remove the majority of them without injuring the underlying cartilage (compare the right and left sides of the skull in Figs. 8 and 9, $A$ and C), provided, of course, that the operation is skilfully performed : these bones are the nasals, vomers, fronto-parietals, parasphenoid, premaxillæ, maxillæ, quadrato-jugals, palatines, pterygoids, and squamosals. A sort of foundation or groundwork (left side of figure in Figs. 8 and $9, \mathrm{~A}$; right side in Fig. 9, C) is then left behind, consisting mainly of cartilage, but containing the exoccipitals, pro-otics, and girdle-bone. These five bones cannot be removed without pulling the cartilaginous groundwork or chondrocranium to pieces. We thus get a distinc tion between replacing bones ("cartilage bones") which are actually continuous with the cartilage and form part of the chondrocranium, and investing bones ("membrane bones") which lie outside the chondrocranium, united to it only by connective-tissue.

The chondrocranium has a cartilaginous roof, underlying the fronto-parietals ; it is pierced by one large (Fig. 8, fon) 
and two small ( $f \circ n^{\prime}$ ) spaces, called fontanelles, covered by membrane. It has also a cartilaginous floor (Fig. 9, A) underlaid by the parasphenoid. The olfactory capsules (olf. $c p$ ) also have a cartilaginous roof and floor of irregular form, with the posterior end of which is united the cartilaginous palato-quadrate bar (pal. qu), with which the palatine and pterygoid bones are connected. Posteriorly this bar is continuous with the cartilaginous groundwork (quadrate) or core of the suspensorium (sus), which unites above with the auditory capsule by two processes (Fig. 9, C, ot. $p r, p e d)$ and below furnishes an articular surface for the lower jaw.

Notice that in describing the vertebral column no distinction was drawn between replacing and investing bones. As a matter of fact the vertebræ and the urostyle are all replacing bones; each consists, in the tadpole, of cartilage which subsequently undergoes ossification, i.e., is replaced by bone in which a deposition of lime salts takes place.

The lower jaw (Fig. 9, B) consists of two halves, or rami, united with one another in front by ligament. At its posterior end each half bears on its upper surface a shallow pit, by which it articulates with the suspensorium, and a little in advance of this pit is an elevation of the dorsal edge of the jaw, called the coronary process.

Each half of the lower jaw consists of a cartilaginous core, the mandibular or Meckel's cartilage, which furnishes the articular surface referred to, and in front is ossified as a small replacing bone, the mento-meckelian (M.MCK). Outside the cartilage are two investing bones. One, the angulo-splenial, extends along the inner surface and lower edge of the jaw and forms the coronary process, while the dentary (DNT) forms the outer surface of the anterior half of the jaw.

The hyoid is a thin, shield-shaped plate of cartilage (Figs. 8 and 9, b. hy) produced, both in front and behind, into a pair of processes or horns, as well as into less important offshoots. The anterior horns (Fig. 9, a.c.liy) are long, delicate, cartilaginous rods which curve backwards and then upwards, finally joining with the auditory capsules. The posterior horns (p.c.hy) are short, 
bony rods which pass backwards, diverging as they go, one on either side of the glottis.

Two apparently insignificant structures connected with the skull must be described because of their connection with the organ of hearing. Behind the suspensorium is a recess, roofed over by the squamosal, and, in the entire frog, converted by muscle and other tissues

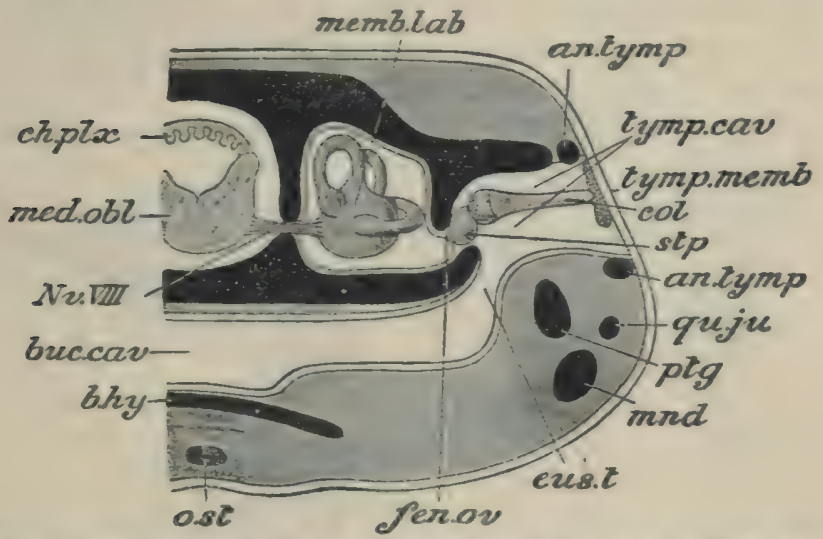

Frc. I0.- Transverse section (diagrammatic) through the head of a Frog at the level of the tympanic cavity. The various parts of the skull shown in section are black, the muscles, \&c., grey, and the skin and mucous membrane white. (; 5.)

an. tymp. tympanic ring : b. hr. boly of hyoid; buc. cat. cavity of pharynx; ch. plx. choroid plexus; col. columella : eus. t. Eustachian tube: fens. ov. fenestra ovalis; med.obl. medulla oblongata; memb.lab. membranous labyrinth; mnd. mandible; Nv. IIII. auditory nerve; o. st. omosternum; p:g. pterygoid; qu. ju. quadrato-jugal; stp. stapes; tymp. car. tympanic cavity; tymp. memb. tympanic membrane. (From Parker and Haswell's Zoology.)

into a chamber, the tympanic cavity (Fig. 1o, tymp. cav), bounded externally by the tympanic membrane, and communicating with the mouth by the Eustachian tube. Supporting the tympanic membrane, as the frame of a tambourine supports the parchment, is a cartilaginous ring, the tympanic ring (an. tymp, shown in section). Stretching across the tympanic cavity from the outer wall of the auditory capsule to the tympanic 
membrane is a small, hammer-shaped rod, the columella (Figs. 9 and Io, col), having a bony handle and a cartilaginous head, the latter firmly fixed to the inner face of the tympanic membrane. The inner end of the handle is tipped with cartilage, and is attached to a small cartilaginous nodule, the stapes (st), which is inserted into an aperture in the auditory capsule known as the fenestra ovalis (fen. ov). With care the columella, in a wet skull, may easily be removed with small forceps, and examined under a magnifying glass.

The shoulder-girdle has the form of an inverted

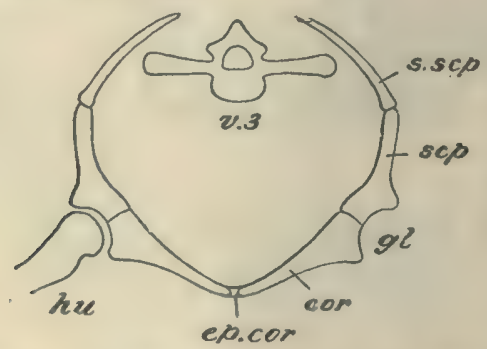
FIG. 11.-Diagrammatic transverse section through the shoulder-girdle of a
Frog. $\left(x_{2 .}\right)$

cor. coracoid ; ep. cor. epicoracoid; gl. glenoid cavity; hu. humerus ; scp. scapula ; s. scp. supra-scapula; $v .3$, third vertebra.

arch encircling the anterior region of the trunk, and having its dorsal ends turned inwards so as partly to cover the second to the fourth vertebræ (Figs. 8 and I I). The dorsal region, on either side, is formed by a broad plate, the supra-scapula (s. scp), or upper blade-bone. It is mostly formed of bone, but its free edge consists of cartilage which, when dried, is seen to be impregnated with a granular deposit of lime-salts. This rough, brittle tissue is called calcified cartilage, and is distinguishable from true bone, which has usually a smooth, enamelled surface. 
Connected with the ventral end of the supra-scapula and passing vertically downwards is a flat bone, broadened at each end, the scapula or blade-bone (Fig. II, scp: Fig. 12, sc). From its lower end two bones (Fig. I2, cl, co: Fig. II, cor) pass directly inwards, parallel with one another, to end in a plate of cartilage which meets with its fellow of the opposite side in the middle line of the chest. The more anterior of these $(c l)$

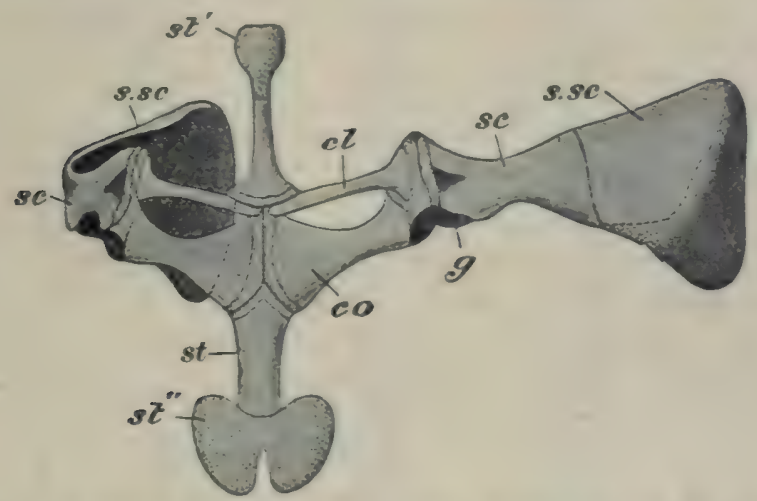

Fig. I2.-The shoulder-girdle of the Frog from the ventral aspect, the left scapula being straightmed out. The cartilage is dotted. ( $x 3$.

cl. clavicle; co. coracnid; $g$. glenoid cavity; sc. scapula; s. sc. supra-scapula : st strmum: st'. ommsternum: st". I. phisternum. (From Howes's Atlas of Practical Elementary Zootomy.).

is a narrow bone called the clavicle, or collar-bone, the posterior one is broader and is known as the coracoid (co). Between the scapula on the one hand and the clavicle and coracoid on the other, there is a cartilaginous interval, the posterior edge of which is scooped out into a depression, the glenoid cavity (Fig. I2, g; Fig. II, gl) for the articulation of the upper-arm bone.

Connected with the median ventral portion of the shoulder-girdle is the stermum, or breast-bone, which 
consists of two separate parts, one extending forwards, the other backwards, in the middle line, and each formed of a flattened bony rod $\left(s t^{\prime}, s t^{\prime \prime}\right)$, tipped with a flat plate of cartilage.

The anterior bony rod with its terminal cartilage is called the omosternum $\left(s t^{\prime}\right)$, the posterior bony rod the sternum $(s t)$, and the bilobed cartilage at its end the xiphisternum $\left(s t^{\prime \prime}\right)$. The cartilages uniting the inner or ventral ends of the clavicles and coracoids are distinguished as the epicoracoids (Fig. II, ep. cor.).

All the bones of the shoulder-girdle and sternum are replacing bones except the clavicle. This can be removed, and is seen partly to surround a bar of cartilage, the procoracoid, which stretches between the scapula and the epicoracoid and is ordinarily completely concealed by the clavicle.

The Fore-limb.-The upper arm is supported by a single bone, the humerus (Fig. 8, HU), the first example we have had of what is conveniently called a long bone. It consists of a roughly cylindrical shaft, formed of dense bone, and of two extremities - the proximal of partially calcified cartilage, the distal of spongy or cancellated bone. The proximal extremity or head is convex, and fits into the glenoid cavity of the shoulder girdle (Fig. II) ; the distal extremity or condyle is almost globular, and is articulated with the bone of the forearm.

In a longitudinal section of the humerus which has not been allowed to dry you will see that the shaft (Fig. I3, A, sh) is not a solid rod, but a tube, containing a cavity, the marrow-cavity. In this way the weight of the bone is diminished without its strength being impaired. The marrow-cavity contains a substance called bonemarrow, composed chiefly of connective-tissue and fat, with blood-vessels. The proximal end of the hollow shaft is, as it were, plugged by the cartilaginous extremity. 
The fore-arm is also supported by a single bone, the radio-ulna (Fig. 8, RA.UL ; Fig. I3, B). Its proximal end is concave and articulates with the almost globular condyle of the humerus: the outer or posterior edge of the concavity is produced into a short process, the
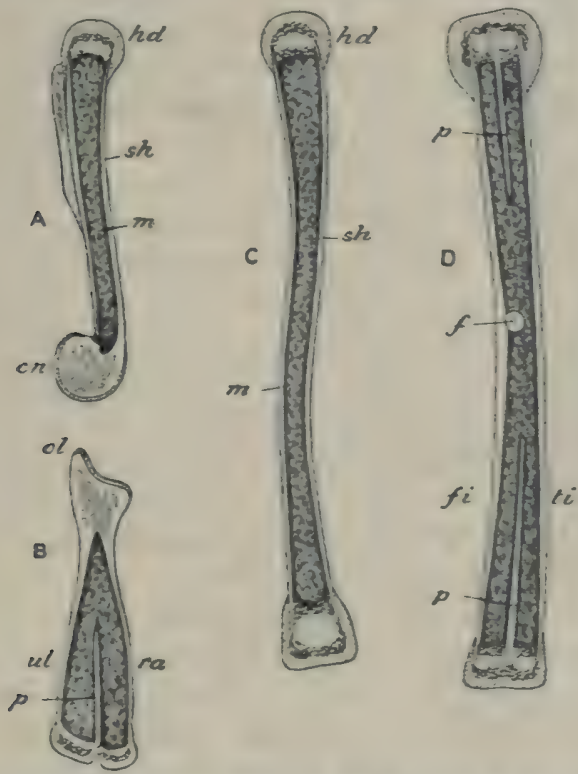

Frc. 13.-Longitudinal sections of the principal long bones of the Frog. ( $X 21$.

A, humerus; B, radio-ulna ; C, femur; D, tibio-fibula ; $c h$. condyle; $f$. foramen for artery; fi. fibula; $h d$. head; $m$. marrow : ol. olecranon process; $p$. bony partition; ra. radius; sh. shaft ; ti. tibia; wl. ulna.

olecranon or elbow. The distal end is incompletely divided into two articular surfaces, and between these is a groove passing for some distance towards the proximal end of the bone. A section shows that at this end there are two distinct marrow-cavities, indicating that the bone is really double. That this is the case is 
proved by the examination of a very young frog, in which the single fore-arm bone is represented by two distinct cartilages, the radius on the inner or thumb side, and the ulna on the outer or little-finger side. The olecranon is a process of the ulna.

The skeleton of the hand is divisible into three regions : the carpus or wrist, the metacarpus or mid-hand, and the phalanges or finger-bones. The carpus consists of six small irregular bones, arranged in two rows (Fig. 8). The proximal row articulates with the radioulna, while to the distal row are attached the metacarpals, which together constitute. the metacarpus. Four of these are long, rod-like bones and support the bases of the four fingers or digits: to them are attached the phalanges, of which the first or innermost digit (II) has two, the next two, and the remaining two digits three apiece. A very small metacarpal, with a single phalanx (I), occurs on the radial side and is concealed by the skin in the entire frog: it corresponds with our own thumb, so that the apparent first digit of the frog is really the second or index finger.

The Hip-girdle. - This, as we have seen, has somewhat the form of a bird's merrythought. It consists of two long arms (Fig. 8, IL; Fig. I4, Il), which are articulated with the transverse processes of the ninth vertebra, and sweeping backwards, unite in a discshaped mass, having on either side of it a deep, hemispherical cavity, the acetabulum (Fig. 8, actb ; Fig. I4, G), for the articulation of the thigh-bone.

Two sutures, or lines of separation, nearly at right angles to one another, divide the disc-shaped portion into three parts. One of these, dorsal and anterior in position, is continued into one of the arms of the hipgirdle and forms half of the acetabulum; this is the ilium (Fig. I $4, I l, P$ ). The second, posterior in position, 
is the sschium (Is); like the ilium it is made of true bone. The third, or pubis $(K n)$, is ventral, and is formed of calcified cartilage. Originally each of these elements is paired, i.e., there is an ilium, an ischium, and a pubis on either side, the three together forming the innominate; but in the adult the right and left ischia and pubes become united in the median plane, the ilia only remaining free.

The Hind-limb.-The thigh, like the upper arm, is supported by a single long bone, the femur (Fig. 8, FE; Fig. I3, C), having a gently curved shaft and extremities of calcified cartilage. Its rounded proximal extremity, or head, fits into the acetabulum: its distal end articulates with the single bone of the shank, the tibio-fibula (TI. FI). This, the longest bone in the body, also has a shaft and extremities, and is further distinguished by

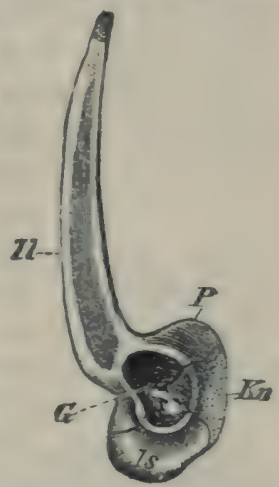

FIG. If - The hip-girdle of a Frog seen from the right side. $(0$ 2.)

G. acetabulum; $K n$. pubis ; Il., P. ilium; Is. ischium. (From Wiedersheim's Comparative Anatomy.)

grooves running from each end towards the middle of the shaft. Sections show that the grooved portions of the bone contain a double marrow-cavity (Fig. I3, D), and in the young animal there are found two shank-bones which afterwards unite, the tibia on the inner side, the fibula on the outer side.

The foot, like the hand, is divisible into three regions: the tarsus or ankle, the metatarsus or mid-foot, and the phalanges or toe-bones. The tarsus, like the carpus, consists of two rows, but with only two bones in each: Those of the proximal row (astragalus and calcaneum) are greatly elongated (AST., CAL.), and furnish an additional segment to the limb, thus increasing the 
frog's leaping powers: those of the distal row are very small.

The metatarsals are five in number : those of the first and second digits (I, II) bear two phalanges each, those of the third and fifth, three each, and that of the - fourth, four. Attached to the inner side of the tarsus is a little claw-like structure (C) composed of two or three bones and called the calcar or spur.

Notice the striking correspondence in structure between the fore- and hind-limbs, a correspondence which extends also, though less obviously, to the limb-girdles. The humerus corresponds or is serially homologous (p. 39) with the femur, the radius with the tibia, the ulna with the fibula, the carpals with the tarsals, the metacarpals with the metatarsals, and the phalanges of the fingers with those of the toes. Then in the limb-girdles the glenoid cavity corresponds with the acetabulum, the scapula and supra-scapula (being above the articular cavity) with the ilium, the precoracoid and clavicle (being ventral and anterior in position) with the pubis, and the coracoid with the ischium. Thus not only are the limbs and limb-girdles serially homologous structures, but their several parts are also serially homologous, each to each.

Nature of Bone.-It is a mistake to suppose that bones are made exclusively of hard mineral matter, like rocks or stones. If one of the long bones, for example, is put into weak acid, bubbles of gas will rise from the bone, showing that the phosphate and carbonate of lime, of which it is partly composed, is being decomposed with the liberation of carbonic acid gas. When the liberation of bubbles is over, the bone will be found to be unaltered in form, but to be quite flexible instead of hard and rigid. It can be bent in any direction, and a bone of sufficient length, such as a sheep's rib, can be 
tied in a knot. This shows that the bone contains a large amount of organic or animal matter. On the other hand, a bone may be completely calcined by heating to redness in a closed vessel, when its animal matter is completely consumed and its mineral matter left. Under these circumstances it becomes very brittle, falling to pieces at a touch, and its appearance is far more altered than by the removal of the mineral matter.

\section{PRACTICAL DIRECTIONS}

Preparation of the Skeleton.-Kill a frog with chloroform (p. 3I), open the abdomen as directed on p. 32, but without cutting the shoulder-girdle, and remove the contained organs. Then, the frog being firmly pinned down, remove the skin and gradually cut away the flesh from the bones. In the case of the long bones of the limbs, it is best to cut through the muscles near one end of the bone and then gradually to strip them back towards the other end until the bone is exposed. The process is facilitated by dipping the frog occasionally into boiling water (maceration in cold water requires a considerable time): this softens the connective-tissue by which the bones and muscles are bound together, and thus allows them to be more readily separated. While at work keep Fig. 8 before you, and be Farticularly careful not to injure those parts of the skeleton which are made of cartilage (dotted in the figure), and are therefore easily cut : the most important of these parts are the hyoid or tongue-cartilage lying in the floor of the mouth, the omosternum, the xiphisternum, and the supra-scapula (Fig. I2). Great care will also be required in cleaning the bones of the hands and feet, since the fine cords or tendons which pass to them from the muscles are very strong, and if pulled upon with much force are sure to bring away the small toe-bones with them : they should be separated as far as possible and then cut off, close to the bones, with scissors.

Kieep all the parts of the skeleton together, avoiding separation of the various bones, until the general characteristics of the entire skeleton have been made out: the only part which cannot be kept in connection with the rest is the shoulder-girdle, together with the fore-limbs.

Examination of the Skeleton.-You should have two skeletons to examine-one dried, after it has been thoroughly 
cleaned, and one which has been kept from the first in spirit or formaline: the latter is the more instructive (see also the paraffin method, p. 473). An additional skull should be carefully cleaned, and then boiled until the numerous bones become separated from one another or disarticulated.

After observing the form and relations of different parts of the skeleton as described on pp. 35 and 36 (Fig. 8, A), they may be separated from one another for more detailed examination. The individual vertebræ should be strung on a piece of wire or string so as to prevent them from being lost or misplaced.

With the specimen before you, work through the characters of the axial skeleton (pp. 36-46) : if you omit the details given in small type at the present stage, do not forget to examine them subsequently. Make sketches of $a$. any one of the vertebrx from the Ist to the 7 th, from the side and from the front or back ; $b$. the Ist vertebra ; $c$. the urostyle; $d$. the skull from above and from below; and $e$. the hyoid.

It requires considerable skill to make a satisfactory preparation of the chondrocranium, and it is advisable to examine that of a Dogfish first; but if you wish to attempt it, procure a large skull which has not been dried, and boil it in water. Carefully separate, by means of a scalpel, most of the investing bones (p. 43) ; the palatines, pterygoids and quadratojugals, and the dentaries and angulosplenials cannot well be disarticulated without destroying the soft cartilaginous parts beneath them.

Make out-I. The brain-case and its fontanelles and nerveapertures. 2. The olfactory capsules. 3. The auditory capsules. 4. The palatoquadrate bar (to which the palatine, pterygoid, and quadratojugal bones have been left attached). 5. The mandibula; or Mleckel's cartilage (to which the angulosplenial and dentary have been left attached). 6 . The replacing-bones (exoccipitals, pro-otics, sphenethmoid, and mento-meckelians). 7. The columella, stapes, and fenestra ovalis.

Sketch from above and from below.

Now proceed to examine the appendicular skeleton (pp. $46-52$ ), and sketch the shoulder-girdle and fore-limb, and the hip-girdle and hind-limb.

Split some of the longer limb-bones longitudinally with a knife, and note the marrow-cavity in the shaft (Fig. 13). Place another of the long bones in io per cent. hydrochloric acid for an hour or two; wash thoroughly in water and examine. 


\section{CHAPTER IV}

THE FROG (continued): THE JOINTS AND MUSCLES

IN the previous chapter the bones-more than 150 in number-which together constitute the greater part of the skeleton of a frog have been considered as so many separate parts, fitting into or against one another in certain ways. We must now see how they are joined together in the entire animal so as to afford the requisite support, and, at the same time, to allow of free movement.

The Hip-joint.-Let us begin by a study of the hipjoint (Fig. I5).

The acetabulum ( $a c t b)$, as you have already seen (p. 50), is a hemispherical depression on the outer surface of the hip-girdle. It is formed of cartilage, continued into a projecting rim round the edge of the cavity. The head of the femur ( $h d$ ) is also formed of cartilage, and fits accurately but rather loosely in the acetabulum.

The acetabulum is lined, and the head of the femur is covered, by a thin layer of connective-tissue, the perichondrium ( $p$. chd), which, in both cases, is continued on to the adjacent bone, where it receives the name of periosteum (p.ost).

Attached all round the rim of the acetabulum is a strong sheet of connective-tissue called the capsular liga- 
ment (cps. lg), forming a short, fibrous tube. The other end of this tube is fixed to the femur, just below the head, the ligament being continuous, in each case, with the perichondrium. There is thus a space between the head of the thigh bone and the acetabulum, closed all round by the capsular ligament. This space is filled with a delicate, fibrous, closed bag, the synovial capsule (sy. $c p s$ ), one side of which fits closely into the acetabulum, while the other as closely invests the head of the femur.

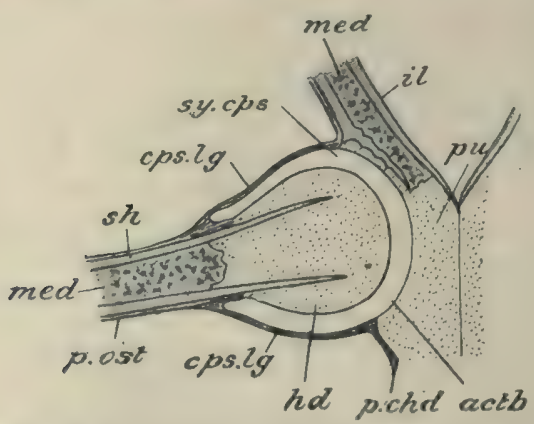

FIG. 15.--Horizontal section of the Frog's hip-joint. ( $x$ 5.) $a c t b$. acetabulum; cps. lg. capsular ligament; hd. head of femur; il. ilium; med. marrow; $p$.chd. perichondrium; $p$. ost. periosteum; $p u$. pubis; sh. shaft of femur; sy. cps. synovial capsule.

The capsule is filled with a watery fluid, the synovia, and thus forms a buffer or water-cushion between the adjacent parts of the skeleton. Thus the synovial capsule keeps the two parts slightly apart and prevents friction, while the capsular ligament keeps them together and prevents dislocation.

It is obvious that, in such a joint as this, movement is possible in all directions. The femur can be inclined either upwards, downwards, or sideways, and is capable of a certain amount of rotation. The joint is, in fact, a cup-and-ball joint, and is capable of movement in any 
plane. A similar but less perfect cup-and-ball joint is that of the shoulder, in which the cup is furnished by the glenoid cavity, the ball by the head of the humerus.

other Joints.-The elbow- and knee-joints are constructed on the same general plan, but, owing partly to the form of the adjacent surfaces, partly to the mode of attachment of the ligaments, they are capable of movement in one plane only, i.e., up and down, but not from side to side. They are therefore distinguished as hinge-joints.

The vertebræ are connected with one another in a similar way. Between the convex hinder face of one centrum and the concave front face of its' successor is a synovial capsule, and the two centra are bound together by ligament, a shallow cup-and-ball joint, with a very limited range of movement, being produced. There are also synovial capsules between the articular processes, which, being in contact with one another by flat surfaces and working mainly from side to side, form glidingjoints. Strong ligaments connect the neural arches with one another and join the first vertebra to the skull.

In all cases where free movement is necessary the joints are formed in the same way ; the bones are bound together by ligaments, and a synovial capsule is interposed between their adjacent cartilage-covered surfaces. Where little or no movement is required, as between the bones of the shoulder- and hip-girdles, the union is effected by cartilage or ligament only, and there is no synovial capsule. Such joints are therefore distinguished as immovable or imperfect joints.

The Muscles.-We see then that the bones of the skeleton are attached to or articulated with one another by means of ligaments, so arranged, in most cases, as to allow of more or less free movement between the bones. 


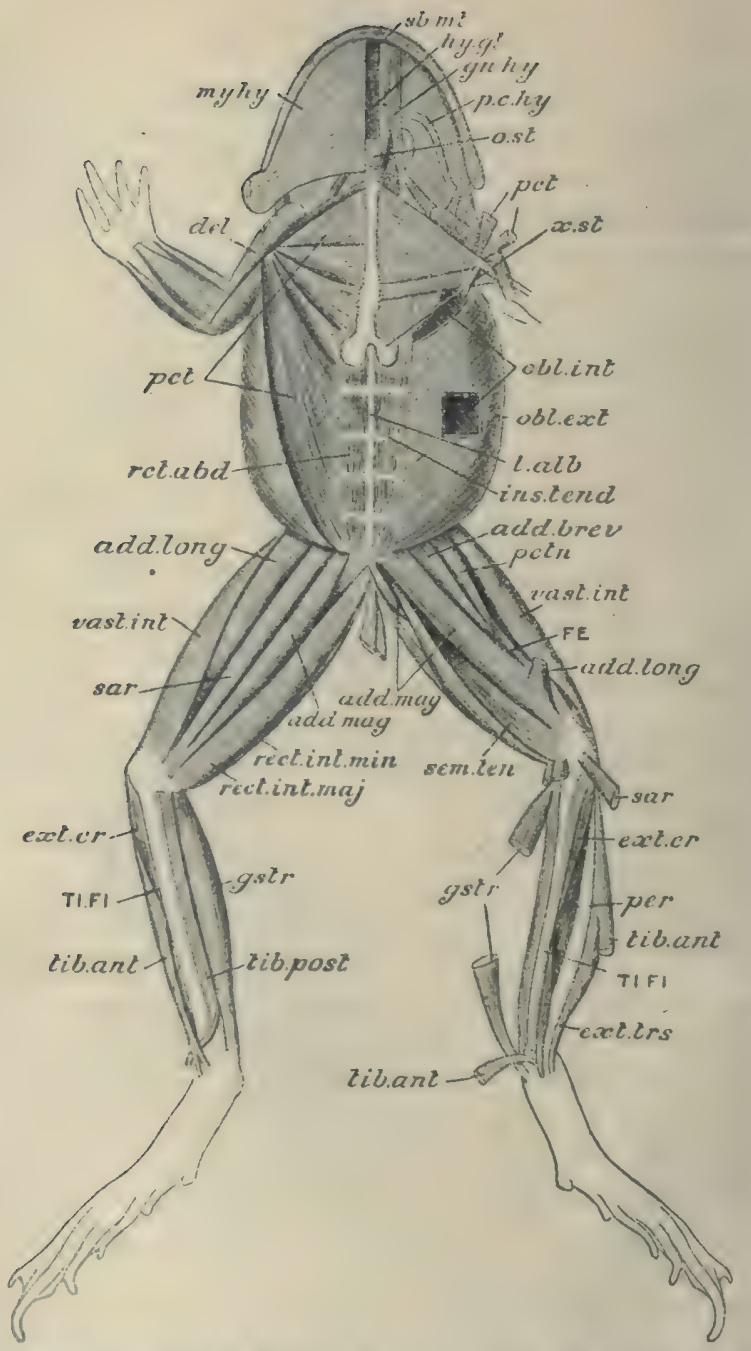

Fic. 16. - The muscles of a Frog from the ventral aspect. On the left side (right of figure) many of the superficial muscles have been cut and reflected to show the deep layer. (Natural size.)

add. brev. adductor brevis; add. long. adductor longus; add. mag. adductor mag. nus; del. deltoid : ext. cr. extensor cruris; ext. Irs. extensor tarsi ; FE. femur.; 
gr. hy. genio-hyoid; gstr. gastrocnemius; $h y$. gl. hyoglossus; ins. ten. tendinous inscription; $l$. alb. linea alba ; $m y . h y$. mylo-hyoid; $a b l$. int. obliquus in ternus; obl. ext. obliquus externus; $0 . s t$. omosternum ; p.c. ky. posterior horn of hyoid ; pct. pectoralis; petn. pectineus; per. peronæus; rct. abd. rectus abdominis; rect. int. maj. rectus internus major; rect. int. min. rectus internus uninor; sar. sartorius; $s b$. mt. submentalis; sem. ten. semitendinosus; $t u b$. $a n t$. tibialis anticus; tib. post. tibialis posticus; TI. FI. tibio-fibula; tast. int. vastus internus; $x$. st. xiphisternum. (From Parker and Haswell's Zoology.)

We must now try to find out how the movements are effected in the living frog.

It was pointed out in the second chapter that the flesh is made up of distinct bands or sheets, the muscles, some of which came under your notice in your first dissection. It is quite easy to convince yourself that the whole of the flesh has this character by skinning a frog and carefully removing the fascia (p. I8) which covers the muscles and the more delicate web of connective-tissue which forms a sort of packing substance between them. After noticing some of the muscles shown in Fig. I6, especially those of the leg, give your attention to the muscle marked gstr, a prominent spindleshaped mass of flesh forming the calf of the leg, and known as the gastrocnemius (gstr).

The spindle-shaped, fleshy mass or belly of this muscle is continued at either end into a band of strong, tough connective-tissue, the tendon (Fig. I7). The tendon at the proximal end is flat, and is attached to the distal end of the femur and to the proximal end of the tibio-fibula, in each case becoming continuous with the periosteum of the bone. The tendon at the distal end has the form of a stout cord, and is distinguished as the tendo Achillis; it corresponds with the strong tendon just above the heel of the human foot. At its distal end it is continued into a broad sheet of connective-tissue, the plantar fascia, which spreads over the whole sole or plantar surface of the foot.

If the foot is bent upon the shank as in the ordinary sitting position of the frog, and the gastrocnemius pulled 
upwards or towards the thigh, the foot will instantly be bent backwards, so as to come into a straight line with the shank, the action being one of those performed by the living frog when leaping. It will be seen that the proximal tendon is attached to a relatively fixed point : it is distinguished as the tendon of origin, or the muscle is said to arise from the femur and tibio-fibula. The distal tendon is attached to a relatively movable part, the foot, and is called the tendon of insertion, the muscle being said to be inserted into the plantar fascia.

Muscular Contraction.--Obviously, however, there is nothing to pull upon the muscle from outside in the living frog. We must, therefore, try to form some idea as to how the action of bending the foot, roughly imitated in the dead subject, is performed during life. If the gastrocnemius be exposed in a recently killed frog, the foot bent up as before, and a smart pinch be given to the belly of the gastrocnemius, the foot will be bent back, although no pull has been exerted on the muscle. The same thing will happen if you drop on the gastrocnemius a single drop of weak acid or of a strong solution of common salt, or if you touch it with a hot wire, or if you apply to it the electrodes from an induction coil so as to pass an electric current through it.

Careful observation shows that what happens under either of these circumstances is that the belly of the muscle decreases in length and at the same time increases in breadth, so as to become shorter and thicker (Fig. I7). The result of this must necessarily be to cause its two ends to approach one another. As the tendon of origin is attached to the femur, which we suppose to be fixed, it is unable to move, and the insertion is therefore drawn upwards, bringing with it the movably articulated foot. In fact exactly the same thing takes place as 
when we raise our own fore-arm. This action is performed by means of the biceps muscle which arises from the scapula and is inserted into the fore-arm. When the latter is raised we feel a lump rise on the front of the upper arm due to the thickening of the biceps.

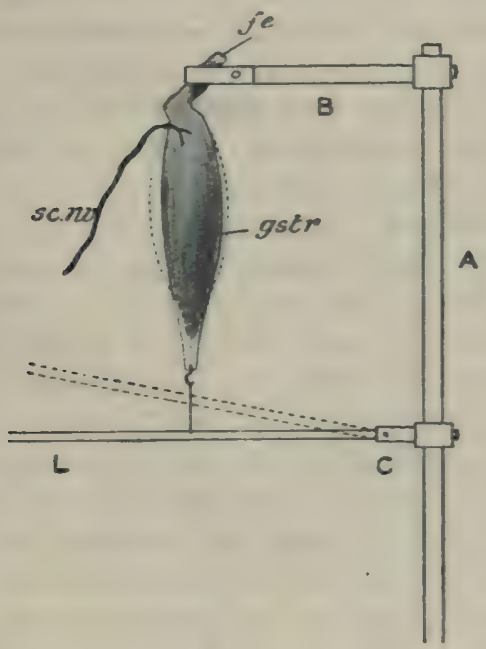

Fic. 17.-Diagram of apparatus for demonstrating the contraction of the gastrocnemius muscle.

$A$, cpright, bearing two adjustable horizontal arms. To the upper of these $(B)$ is fixed by a clamp the fernur ( $f e$ ), having the gastrocnemius (gstr) in connection with it. To the lower arm (C) is fixed a light lever (L) movable in a vertical plane, and having the tendon of insertion of the muscle attached to it by a thread. The dotted lines show the form of the gastrncnemius and the position of the lever during contraction of the muscle. sc. we. the sciatic nerve.

This shortening and thickening of the muscle is termed a contraction. Do not fail to notice that this word is used in a special sense. When we say that a red-hot bar of iron contracts on cooling, we mean that it becomes smaller in all dimensions-undergoes an actual decrease in bulk. But in muscular contraction there is no alteration in bulk: the decrease in length is 
balanced by an increase in thickness, as when a stretched piece of india-rubber is relaxed.

The external influence by which a contraction is induced is called a stimulus. As we have seen, a stimulus may be produced by actual contact of some external object (mechanical stimulus), or by chemical action (chemical stimulus), or by heat (thermal stimulus), or by an electrical current (electrical stimulus).

Relation of Muscle and Nerve.--Evidently, however, we have by no means got to the bottom of the matter. In the living frog movements are always going on, and all are due to the contraction of muscles, and yet no stimuli of the kind enumerated are applied to any of them. As the muscles retain the power of contraction for some little time after the death of the animal, it is easy to make such experiments as that described in the next paragraph.

Running longitudinally between the muscles on the dorsal side of the thigh is a shining, white cord, the sciatic nerve (Fig. I7, sc.nv), accompanied by an artery: it gives off branches to the muscles and skin, and, amongst others, one to the gastrocnemius. If, when quite fresh, this nerve be carefully separated as it traverses the thigh and pinched with the forceps, the gastrocnemius will contract just as if the stimulus had been applied to it directly, and the same will happen if a chemical, thermal, or electrical stimulus be applied.

Thus a stimulus applied to the nerve of a muscle has the same effect as if applied to the muscle directly : it gives rise to a nervous impulse, which, travelling along the nerve, induces contraction of the muscle.

Once more, however, external stimuli are not applied to the frog's nerves during life, and it is obvious that we must carry our inquiry a little further. The sciatic nerve if traced upwards will be found to pass into the 
trunk (Fig. 5I, sci), and finally to join the spinal cord, which, as we have seen, is in connection with the brain. In the living frog nervous impulses originate in the brain, without the direct intervention of an external stimulus, and are conducted along the cord and nerves to the muscles. But further consideration of this subject must be deferred until we have made a special study of the nervous system.

The Museular System in General.-All over the body the muscles, though varying greatly in form-some being elongated and band-like (Fig. I6, sar), others spindle-shaped (gastr), others in the form of broad, flat sheets $(m y . h y, o b l . e x t)$-have the same general relation to the skeleton as in the case of the gastrocnemius. Each muscle arises or has its origin in a relatively fixed part, and is inserted into a relatively movable part. As each muscle contracts in one direction only, it follows that the more complex the movements any part is capable of performing, the more numerous must be its muscles. For instance, the femur, which, as we have seen, is capable of universal movement, has no fewer than nine muscles, arising from various parts of the hipgirdle, inserted into it. Even the minute phalanges of the fingers and toes all have their little slips of muscle by which the various movements of grasping and relaxing, approximating and separating the digits, are effected.

There are certain terms applied to muscles which it is useful to know. A muscle which raises a part, e.g., the lower jaw, is called a leiator, one which lowers a part a depressor. A muscle which serves to straighten one part upon another, e.g., to bring the shank into line with the thigh, is an extensor, one which bends one part on another is a flexor. A muscle which draws, e.g., a limb towards the trunk is an adductor, one which draws it away an abductor. one which rotates one part upon another (e.g., the femur on the pelvis), a rotator 
Thus all the complex and accurately adjusted movements of the frog are performed by the contraction of its numerous muscles, acting either singly or in concert. The contractions of these muscles are brought about by nervous impulses sent from the brain or spinal cord along nerves which branch out and are distributed to the muscles, thus bringing the whole of the complex machinery which affects the movements of the animal under the direct control of its will.

\section{PRACTICAL DIRECTIONS}

\section{The Joints and Ligaments.}

I. The hip-joint. Strip off most of the muscles from the thigh and the adjacent parts of the pelvis ; decalcify in weak acid and then wash thoroughly. Cut the femur through lengthwise and continue the section through the pelvic girdle (Fig. 15). The details are more easily made out if the femur of a larger animal (e.g., rabbit) be used.

Note : $a$. The cartilage of the acetabulum and head of the femur; the perichondrium and periosteum. (b) The capsular ligament and its relations to the synovial capsule. Observe that the hip-joint is a cup-and-ball joint. Sketch.

In a prepared wet skeleton-

2. Examine and compare a hinge-joint (e.g., elbow or knee).

3. Examine the cartilaginous union between the bones of the shoulder- or hip-girdle (immovable or imperfect joints).

4. Examine the joints and ligaments of the vertebral column.

\section{The Muscles.}

x. Remove the skin from part of the body and legs of a preserved frog (the one you have already dissected will do). Then clear away the fascia here and there and separate some of the muscles by dissecting away the connective-tissue which binds them together. Notice the different forms of the muscles in different parts (Fig. I6 and p. 63).

2. In the hind-leg carefully dissect away the connectivetissue investing the gastrocnemius muscle (Figs. I6 and 17), and trace it upwards towards the thigh and downwards towards the foot, cutting away any of the other muscles 
which obscure the view. Notice the belly, and the tendons of origin and insertion (p. 6o). Sketch.

Bend the foot upon the shank as in the ordinary sitting position of the frog. Hold the thigh firmly with one hand, and with the other take hold of the gastrocnemius and pull it upwards or towards the thigh. Note the result.

3. In a quite freshly killed frog (see p. 3I) expose the gastrocnemius as directed above, and with the small forceps give a sharp pinch to the belly of the muscle. Note the contraction following the stimuius.

Then remove the skin on the dorsal side of the thigh, and separate the muscles in this region so as to expose the sciatic nerie (Figs. $x-$ and $5 \mathrm{I}$ ). Trace this towards the shank and notice its branch going to the gastrocnemius. Carefully separate the nerve as it traverses the thigh and pinch it with the forceps, noting again the contraction following the stimulus. 


\section{CHAPTER V}

THE FROG (continued) : WASTE AND REPAIR OF SUBS? -THE DIGESTIVE ORGANS-NUTRITION

Waste and Repair.-The effects of prolonged cular exertion are familiar to everyone. Sooner on sensations of fatigue, hunger, and thirst are proc accompanied by a loss of weight. Indeed, ho little exertion we make and however often we feer weight always goes down between meals and rises when we take iood. The loss of substance, of the diminution in weight is the index, takes place l: in the form of perspiration, a fluid consisting of with certain organic and inorganic matters in sol A further loss is due to the air breathed out frol lungs; this is always moist, i.e., contains a good d water, and is further distinguished by containing siderable volume of carbonic acid gas or carbon a $\left(\mathrm{CO}_{2}\right)$. Besides these two constant sources of there is an intermittent loss in the urine, which co of water containing certain matters in solution most characteristic of which are two complex subst called urea $\left(\mathrm{CON}_{2} \mathrm{H}_{4}\right)$ and uric acid $\left(\mathrm{C}_{5} \mathrm{H}_{4} \mathrm{~N}_{4} \mathrm{O}_{3}\right)$. of these, as well as carbon dioxide, act as pois allowed to remain in the system. Lastly there intermittent source of loss in the waste matte freces which are passed out from the intestine. 
These losses are made good in two ways. Firstly, by breathing, in which process we constantly inhale pure air and replace the poisonous carbon dioxide by oxygen. Secondly, by eating and drinking, by which, at intervals, we make good the loss of solids and liquids. Just as a clock is constantly running down and has to be wound up in order to keep it going, so our bodies run down by loss of substance between meals, and require to be wound up by the repair of substance which results from food and drink.

The same thing is true of the frog. Every one of its numerous and often vigorous movements is done at the expense of a certain waste of substance. The various tissues of the body are constantly undergoing a process of wear and tear, expressed not as in machines of human construction, by a wearing away of surfaces and a loosening of bolts and screws, but by a slow and almost imperceptible dwindling, the lost material being carried off principally in the form of water, carbon dioxide, and urea or some allied compound containing nitrogen.

\section{Food of the Frog: general characteristies of the Diges-} tive Process.-As we have seen, the food of the frog consists of worms, slugs, insects, and the like. These it catches and swallows whole, the stomach often becoming immensely distended with numbers of captured animals. After remaining for some time in the stomach the carcases are found to have undergone a marked change. Their soft parts become softer and slimy and finally semi-fluid, and in this way. the food undergoes gradual disintegration. The quantity of food in the stomach decreases, some of it is passed into the intestine, which it traverses from ducdenum to rectum, and certain portions of it are finally ejected from the vent in the form of freces. 
It is not difficult to assure one's self that the weight of the fæces passed during a certain time is very much less than that of the food swallowed during the same time. Obviously some constituents of the food have disappeared during its progress through the enteric canal. The character of the fæcal matter, moreover, is very different from that of the food; the only portions of the swallowed animals discoverable in the rectum are bits of their hard parts; for the rest, the fæces form a pulpy, black mass. That this change is due to certain definite chemical processes taking place in the enteric canal may be inferred from the fact that the contents of the stomach, as well as the walls of that organ, have an acid reaction, and turn blue litmus paper red. On the other hand, the contents of the small intestine are, to a greater or less extent, alkaline, restoring reddened litmus paper to its original blue colour.

It is also obvious that there must be some definite mechanism for propelling the food from one end of the enteric canal to the other; its passage through so long, narrow, and coiled a tube can certainly not be accounted for by supposing it to be merely pushed onwards as fresh food is swallowed.

In order to understand the various processes connected with digestion we must make a renewed and more careful examination of the organs concerned, after removing them from the body.

The Digestive Organs.-Arising from the gall-bladder and passing backwards to the duodenum is an extremely delicate tube (Fig. I8, Dc, Dc $c^{1}$ ), the common bile-duct, which opens into the duodenum. By gently squeezing the gall-bladder a drop of greenish fluid may be made to ooze out of the end of the duct $\left(D c^{2}\right)$ into the intestine ; this fluid is the bile.

Very careful dissection shows that the common bile- 
duct is joined by several other tubes which are traceable into the liver and are called hepatic ducts $\left(D h, D h^{1}\right)$. The tubes going to the gall-bladder are called cystic

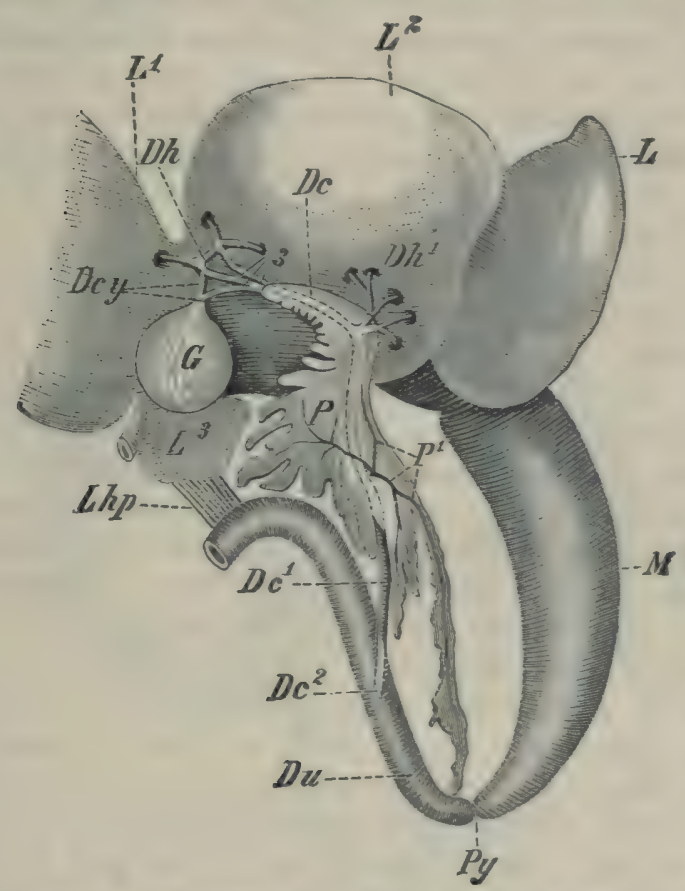

Fic. I8.-Stomach and duodenum of "Edible Frog" (see p. 7) with liver and pancreas. (K 2l.) $D c, D_{i} 1$ common bile-duct; $D c .2$ its opening into the dundenum; D. Cy. cystic ducts; Dh. Dh.' hepatic ducts; Du. duodenum; $G$. gall-bladrier; $L, L^{i}, L^{2}, L^{3}$, lobes of liver, turned forwards ; $L h p$. duodenohepatic omentum, a sheet of peritoneum connecting the liver with the duodenum; $M$. stomach; $P$. pancreas; $P$, pancreatc duct; $P y$. pylcrus. (From Wiedersheim's Comparatite Anatomy.)

ducts (D.cy); by their union with the hepatic ducts the common bile-duct is formed.

The bile is manufactured in the liver, and the gallbladder is merely a reservoir in which it is stored up, 
to be discharged into the intestine when required for digestion.

It requires still more accurate observation to show that the pancreas also discharges a fluid, the pancreatic juice, into the intestine. A very delicate branching tube, the pancreatic duct $\left(P^{1}\right)$, joins the bile-duct, into which it discharges the pancreatic juice, the two fluids entering the intestine together. Both fluids are digestive juices, i.e., liquids which, as we shall see, act in a particular way upon the food.

By cutting open the enteric canal and examining its inner surface under water with a magnifying glass, it is seen that the wall of the canal consists of two layers, easily separable from one another. The outer or muscular layer (Fig. I9, A, musc), covered by the peritoneum (p. 27), is tough and strong, the inner layer or mucous membrane $(m . m)$, is soft and slimy. Between the two is very loose connective-tissue, the submucosa, -really a part of the mucous membrane-which, being easily torn, allows of the ready separation of the muscular and mucous layers.

In the stomach the mucous membrane is raised into longitudinal folds $(r)$, in order to allow of distension; in an empty stomach these are well marked, and give the cavity a star-like cross-section (Fig. 40); in one full of food they are entirely obliterated and the walls of the organ so stretched as to be almost transparent. Anteriorly the ridges thin out and disappear at the cardia or junction between the gullet and stomach; posteriorly they converge, as the stomach narrows, towards the pylorus (Fig. I9, A, py), or junction with the duodenum. Here the muscular coat is greatly thickened in a ring-like form, forming the pyloric valve $(p y . v)$, by which the aperture of communication between the stomach and intestine is greatly narrowed, so that 
only small particles can pass through. In the duodenum (du) the mucous membrane is raised into little tuft-like elevations $\left(r^{\prime}\right)$; in the ileum the ridges $\left(\mathrm{B}, r^{\prime \prime}\right)$ become longitudinal again; in the rectum $(r c t)$ they are absent. Another ring-like muscle, or sphincter, is present round the vent.

When food is taken into the stomach, a fluid, the gastric juice, oozes from the mucous membrane. It is
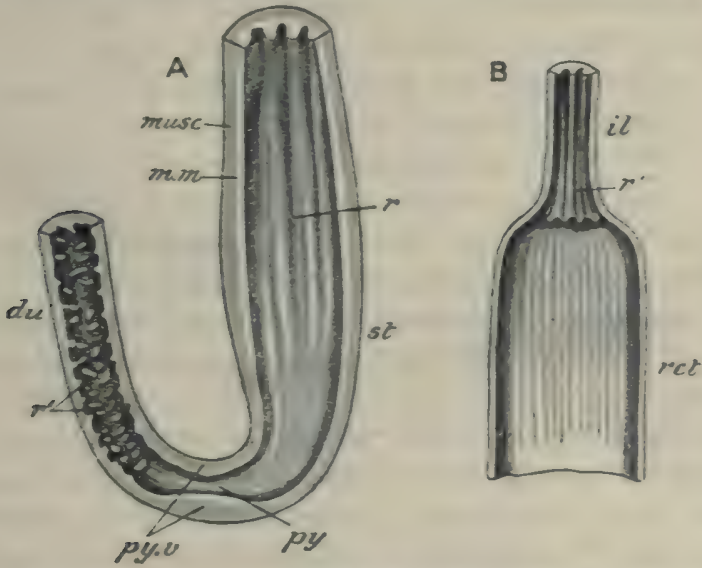

Fic. I9. - Portions of the enteric camal of the Frog in longitudinal section. ( $\because 3$.

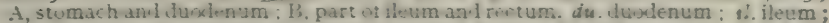
m. $m$. mucous mentorane: inust. muscular layer; $p y$. pylorus; $p: t$. pyloric

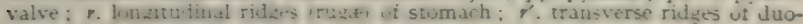
denum; $r$. longitudinal ridges of ilenm: rh. rectum; st. stomach.

this fluid which reduces the slugs, insects, etc., to the pulpy condition referred to above: it is, like the bile and pancreatic fluid, a digestive juice.

General Properties of Food.-We must now devote a little attention to the characters of the food itself and to the precise nature of the changes brought about by the digestive process.

As we have seen, the frog is a carnivorous animal. 
Now the digestible part of the substance of animals consists mainly of two classes of chemical compounds, called proteids and fats. The most familiar example of a proteid is white of egg : other proteids, of varying composition, are found in muscle, in blood, and in other animal tissues. All are composed of the five chemical elements-carbon, oxygen, hydrogen, nitrogen, and sulphur, the five elements being combined in the following proportions:-

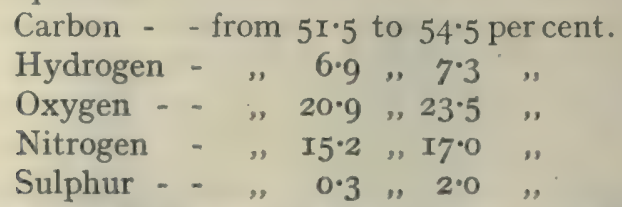

Fats differ from proteids in containing no nitrogen or sulphur : they are formed of carbon, oxygen, and hydrogen, the number of atoms of hydrogen being typically more than twice as great as the number of atoms of oxygen.

It will be noticed that two important articles of diet are absent from the above list, namely sugar and starch - the latter the largest constituent of flour, oatmeal, rice, etc. The vegetable substances, such as corn and grass, used as food by animals, contain these bodies in varying proportions in addition to vegetable proteids, and there is no doubt that the frog must eat a small quantity of such vegetable food, if only in the stomachs of the herbivorous animals upon which it preys. Now starch and sugar belong to a group of compounds called carbohydrates, composed of carbon, oxygen, and hydrogen, but differing from fats in that the number of atoms of hydrogen is always exactly double that of the atoms of oxygen, as in water. Lastly, the food always contains a certain quantity of saline or mineral matters, as well as water. 
Diffusible and Non-diffusible Foods.-These four classes of food materials-proteids, fats, carbohydrates, and minerals-may be arranged in two groups according to a certain physical peculiarity. If a solution of common salt is placed in a vessel with a bottom made of bladder, called a dialyser, which is floated in a larger vessel of pure water, it is found that, after a certain lapse of time, the water in the outer vessel has become salt. The sodium chloride has, in fact, passed by diffusion or osmosis through the bladder. The same thing will happen if a solution of sugar is placed in the inner vessel: salt and sugar are both diffusible substances, capable of passing through an animal membrane.

On the other hand, if the inner vessel contains white of egg, or oil, or starch well boiled in water, no diffusion takes place. Hence proteids, fats, and starch are non-diffusible foods, and are thus sharply distinguished from salt and sugar, which are diffusible.

The mucous membrane of the stomach and intestine are animal membranes having the same physical properties as bladder. We may consequently infer that any salt or sugar contained in the enteric canal will diffuse through the mucous membrane and make its way, as we shall see more particularly hereafter, into the blood, thus serving to nourish the whole body. Proteids, fats, and starch, on the other hand, will be incapable of diffusing, and will, therefore, unless some change happens to them, be absolutely useless as nutriment. For, since the enteric canal communicates with the outer world at both ends, the food, paradoxical as it may sound, is practically outside the body as long as it remains in the canal: it is only when it is absorbed into the blood or lymph that it is actually, in the strict sense, taken into the body. Thus if proteids, fats, and starch are to be of any use to the frog, they must, in some way, be rendered capable 
of being absorbed, in which process the living tissues appear to exercise some degree of selection.

Action of Digestive Juices.-This is exactly what is done by the digestive juices. If white of egg or any other proteid is mixed with gastric juice and kept at a suitable temperature, it is converted into a form of proteid called peptone, which is capable of diffusing through an animal membrane. The change is effected by means of a substance called pepsin, contained in the gastric juice in which there is also a certain proportion of hydrochloric acid. To this the acid reaction of the gastric juice already alluded to (p. 68) is due.

By means of the gastric juice the bodies of the animals swallowed by the frog have their proteids largely converted into peptones, which, being diffusible, pass through the mucous membrane as readily as sugar or salt. Hence the great diminution in the bulk of the food during its sojourn in the stomach: a large proportion of it is absorbed there and then, and only a comparatively small quantity is passed through the pyloric valve into the intestine, where it becomes alkaline, owing to the action of the fluid which enters the duodenum through the bile-duct, and which, as we have seen (p. 70), consists of bile and pancreatic juice.

Pancreatic juice has a similar effect on proteids, the change being effected by a substance called trypsin, which, however, acts in an alkaline solution. It also has the property of converting starch into sugar, and of splitting up fats into fatty acids and glycerine, both of which are diffusible. The substances by which these changes are effected are called by the general name of ferments: pepsin and trypsin are proteolytic or proteinconverting ferments, and the pancreatic juice also contains an amylolytic ferment which converts starch into sugar, and a fat-decomposing ferment. 
The exact mode of absorption of the fats is not thoroughly understood. It is usually supposed that only a small proportion of them are decomposed into fatty acids and glycerine, and that the greater part is merely broken up into particles so small that they can be taken up by the epithelial cells of the intestine. This emulsification of fat is effected by the combined action of the pancreatic juice and bile, the fats being reduced to the condition in which they exist in milk and in the emulsions of cod-liver oil so much used in place of the natural form of that medicine.

Thus during the passage of the food through the intestine the remainder of the prcteids, the whole of the fats, and any starch which may be present, are rendered capable of being absorbed: they pass through the mucous membrane into the blood, and by the time the rectum is reached all the nutriment is extracted from the food, and there remains only a small quantity of indigestible matter which is passed out in the form of fæces.

Peristaltic Movements.-The passage of the food through the enteric canal is effected by the contraction of its muscular layer, which is really double, and which is composed of muscular fibres (see p. III, and Figs. 39 and 40$)$. In the inner layer these fibres have a transverse direction, encircling the tube, and by their contraction narrowing it ; the outer layer consists of longitudinal fibres, which by their contraction shorten it. By the alternate contraction and relaxation of the two layers are produced a series of peristaltic movements, not unlike those by which an earthworm makes its way over the ground: they can be seen in a freshly-killed frog, and still better in a rabbit or rat.

Summary of Chapter.-The body is constantly undergoing waste, and in consequence needs continual reparr. The waste matters chiefly take the form of carbon di- 
oxide, water, and urea. Repair is effected partly by breathing (see Chapter IX), partly by feeding. Food, consisting of proteids, fats, carbohydrates, inorganic substances, as well as water, is taken into the enteric canal, where by the action of the three digestive juicesgastric juice, pancreatic juice, and bile-it is converted partly into a solution (peptones, sugar, fatty acids, glycerine), partly into an emulsion (fats). As it is driven along the canal, from stomach to rectum, by the action of the muscular coat, the dissolved or emulsified substances gradually disappear from the canal, and are absorbed into the system. Finally, the indigestible constituents are expelled as fæces.

Our next task must be to learn something of the process of absorption, and of the means by which the digested food is conveyed to the various parts of the body, so as to supply them with the means of repairing the waste they are constantly undergoing. For this purpose we must now study what is called the vascular system, i.e., the heart, the blood-vessels, and the various cavities containing lymph.

\section{PRACTICAL DIRECTIONS}

The Digestive Organs.-Pin down under water, with the ventral side uppermost, as before, the specimen already dissected, or another in which the body-cavity has been opened in the same way. Note the positions of the postcaval vein (Figs. 3 and $4, p t . c v$ ), the hepatic portal vein (Fig. 3, $h p . p t$ ), the aorta (Fig. $4, d . a o$ ), and the splanchnic or coliacomesenteric artery (Figs. 3 and 4 , coel. mes). Then, taking care not to injure the aorta, remove the greater part of the cligestive organs, including the liver, by cutting through the gullet and rectum and severing the mesentery, cutting through the postcaval also where it enters the liver. (The cloaca will be examined at a later stage.) Pin the organs in the dissecting-dish as nearly as possible in their natural position. Turn the lobes of the liver forwards (i.e., towards 
the head), and after making out the relations of the parts already examined in situ (pp. 22 and 23), note-

The common bile-dust, formed by the union of the hepatic and cystic ducls, and the point at which it enters the duodenum (Fig. I8). Nake a small slit in the duodenum just opposite its entrance and gently squeeze the gall-bladder between your finger and thumb, so as to force a drop of bile into the intestine. (The pancreatic duct and its communication with the bile-duct cannot easily be made out by dissection.) Sketch the whole dissection.

Now remove the liver, unravel the intestine by tearing through the mesentery, and lay open the enteric canal by inserting one blade of the scissors into the gullet, and cutting through the whole tube in a longitudinal direction. Test the contents of the stomach and intestine with litmus paper(for this experiment a freshly-killed frog is of course necessary) -and then pin out your dissection with the inner surface upwards, wash it under the tap, and examine under water with a magnifying glass. Nake out-

I. The cartia, pylorus, and pyloric value.

2. The mucous membrane, and its different appearances in the stomach, small intestine, and large intestine.

3. The muscular layer, covered externally by the peritoneum.

Make a simple dialyser (p. 73) by tying a piece of wet bladder firmly over one end of a wide glass tube about six inches long. Into this put a solution of sugar or salt, and immerse the tube up to the level of the solution in a rather larger vessel of distilled water, and leave it for a short time : taste the water in the outer vessel. Then place some white of egg in the dialyser, and test for albumen by heating some of the water in the outer vessel over a flame: if albumen is present it will become coagulated and form a cloud in the water. 


\section{CHAPTER VI}

THE FROG (continued): THE VASCULAR SYSTEM-THE CIRCULATION OF THE BLOOD

IN our preliminary examination of the frog (Chapter II) we learned one or two facts about the vascular system. We found that there is a heart within a pericardium, two sets of vessels, arteries and veins, containing red blood, and a set of irregular cavities or sinuses, containing lymph. We must now try to get some more accurate and detailed information on these matters.

General Characteristics of Blood and Lymph.-It will be convenient to begin by studying certain easily verified characteristics of the blood. Frog's blood may be used, or, as it is as well to have a considerable quantity, that of some larger, freshly-killed, red-blooded animal, such as a rat or rabbit.

When first drawn from the heart or vessels the blood will be seen to be a fluid, nearly as mobile as water or milk ; it " finds its level," like any other liquid, and can be readily poured from one vessel to another. In a few minutes, however, it undergoes a change; it ceases to be fluid, and coagulates, or "sets" into a jelly, which, if turned out of the vessel, retains the shape of the latter. Before long a further change takes place; the jelly 
begins to shrink, drops of yellowish fluid appear on its surface and gradually run together into larger and larger drops. The jelly contracts still further, and finally draws itself away from the walls of the vessel and floats in the accumulated fluid, still retaining the form of the vessel, but being greatly reduced in size. The process of coagulation of the blood is now complete; the red, jelly-like substance is called the clot, the yellowish fluid the serum.

When first drawn from most veins the blood is deep purple in colour, and the clot retains for a time the same hue. But before long all parts of it which are fully exposed to the air take on a bright scarlet colour. We may therefore distinguish between red, or aërated, and purple, or non-aërated blood.

Lymph also coagulates on standing, producing a colourless clot. It is practically blood minus its peculiar red colouring matter, the properties of which, as well as the real nature of coagulation, will be discussed in the next chapter.

The Heart: external characters.-Some of the divisions of the heart have already been noticed (p. 20). The ventricle (Figs. 3, $4,7,20,2 \mathrm{I}$, and $22, v, v t$ ) is a conical body of a pinkish colour, having its bluntly-pointed apex directed backwards. To its broad base is attached the dark-coloured, thin-walled auricular division, actually consisting of two chambers, the right and left auricles $(r . a u, l . a u)$, but appearing single in the entire heart. Passing obliquely across the auricle is a cylindrical structure, the conus arteriosus (c.art); it starts from the right side of the base of the ventricle, and passes forwards and to the left, finally dividing near the anterior boundary of the auricles into two branches, which extend respectively right and left.

By lifting up the ventricle, or turning it to one side 
(Figs. 3 and 4), there is seen in the dorsal part of the pericardial cavity a thin-walled chamber (Fig. 2I, s.v) of a dark colour, connected with the right side of the auricular division. This is the sinus venosus.

The Arteries. - The two branches of the conus arteriosus just referred to soon branch again. Each divides into three vessels, often spoken of as arterial arches, called respectively the carotid trunk (Fig. 20, car.tr), the systemic trunk (syst.tr), and the pulmo-cutaneous trunk (pul.cu.tr). All these conform to the definition of an artery given on p. 27 , i.e., they are stout, elastic vessels, containing little blood after death, and not collapsing when empty.

The carotid trunk divides immediately into two, a lingual artery $(l g)$, which can be traced to the tongue, and a carotid artery (car), which branches repeatedly, its ultimate ramifications going to various parts of the head. At the origin of the carotid is a little rounded mass with a sponge-like interior, the carotid labyrinth (car. gl).

The systemic or aortic trunk extends outwards, in contact with the gullet, then sweeps upwards, backwards, and inwards-i.e., towards the middle line-and finally joins with its fellow of the opposite side to form a single median vessel, the dorsal aorta (Figs. 4, 5, and 20, d. ao), which passes backwards just beneath the vertebral column and between the kidneys.

As it sweeps round the gullet, the systemic trunk gives off a vertebral artery (Fig. 20 , vert) to the vertebral column and part of the head, a subclavian artery (scl), passing into the fore-limb as the brachial, and an cesophageal artery (os) to the gullet.

From the point of union of the two aortic trunks springs a single splanchnic or coliaco-mesenteric artery (col. mes); it divides into several branches, which are 


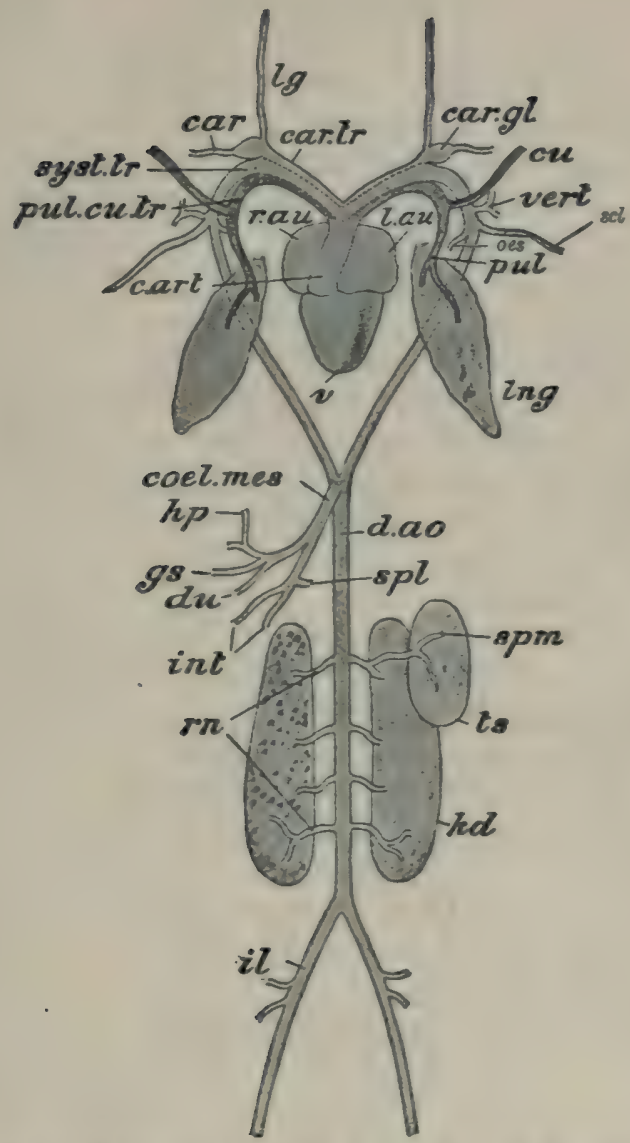

FIG. 20.-The arterial system of the Frog, with the heart, lungs, hidneye, ant left spermary, supposed to be removed from the body and viewed from the ventral aspect. ( $x$ 2.)

car. carotid artery; car. gl. carotid labytinth; $c$. art. conus arteriosus: car. Ir. carotid trunk; coll. mes. splanchnic or coeliaco-mesenteric artery; cu. cutanew artery ; $d$. ao. dorsal aorta ; du. duodenal artery ; gs. gavtric arters; $h p$. hepatic artery; $i l$. iliac artery ; int. intestinal arteries : $k d$. kidney ; $l$. aw. left auric le ; lg. lingual artery; ling. lung: res. cesophayeal artery: pul. pulmonary artery; pul.cu.tr. puimo-cutaneous trunk : $r$. au. right auricle: rn. renal arteries: sit. subclavian artery; $s p$ !. splenic artery; syst. tr. svstemic trunk: spm. spermatir artery: ts. spermary; 0 . ventricle; vert. vertebral artery. (From Parker and Haswell's Zoology.)

Pracr. Zool. 
traceable to the liver $(h p)$, stomach $(g s)$, duodenum $(d u)$, spleen $(s p l)$, and ileum $(i n t)$.

The dorsal aorta gives off on either side four renal arteries $(m)$ to the kidneys, and spermatic arteries (spm) in the male, or ovarian in the female, to the reproductive organs, and at the posterior end of the abdominal cavity divides into the right and left iliac arteries (il), which go to the hind-limbs.

The pulmo-cutaneous trunk divides into two main branches, the pulmonary artery ( $p u l)$ which goes to the lung, and the cutaneous artery (cu). which forms an extensive system of branches over the skin.

With proper care all these arteries can be traced into the various organs to which they are distributed, when they will be found to branch repeatedly, sending ramifications to all parts. The iliac artery, for instance, may be followed along the whole length of the leg, giving off branches to all the muscles, to the skin, and to the digits, with their intervening web.

The Veins.--Since every part of the body has its vein as well as its artery, there is a rough correspondence between the two kinds of blood-vessel. The arrangement of the principal trunks is, however, very different in the two cases.

On either side of the base of the heart is a large vein called the precaval or vena cava anterior (Figs. 3 and 2I, $p r . c v)$ : by turning the ventricle aside, the two precaval veins can be seen to join the anterior end of the sinus venosus (s. v). Each precaval is formed by the confluence of several veins, of which the most important are the external jugular (ext. ju) from the lower jaw and tongue, the internal jugular (int. ju) from the brain, eye, etc., and the subclavian ( $(s c)$, formed by the union of the brachial (br), from the fore-limb, and the musculo-cutaneous ( $m s . c u$ ), already noticed, from the superficial parts of 


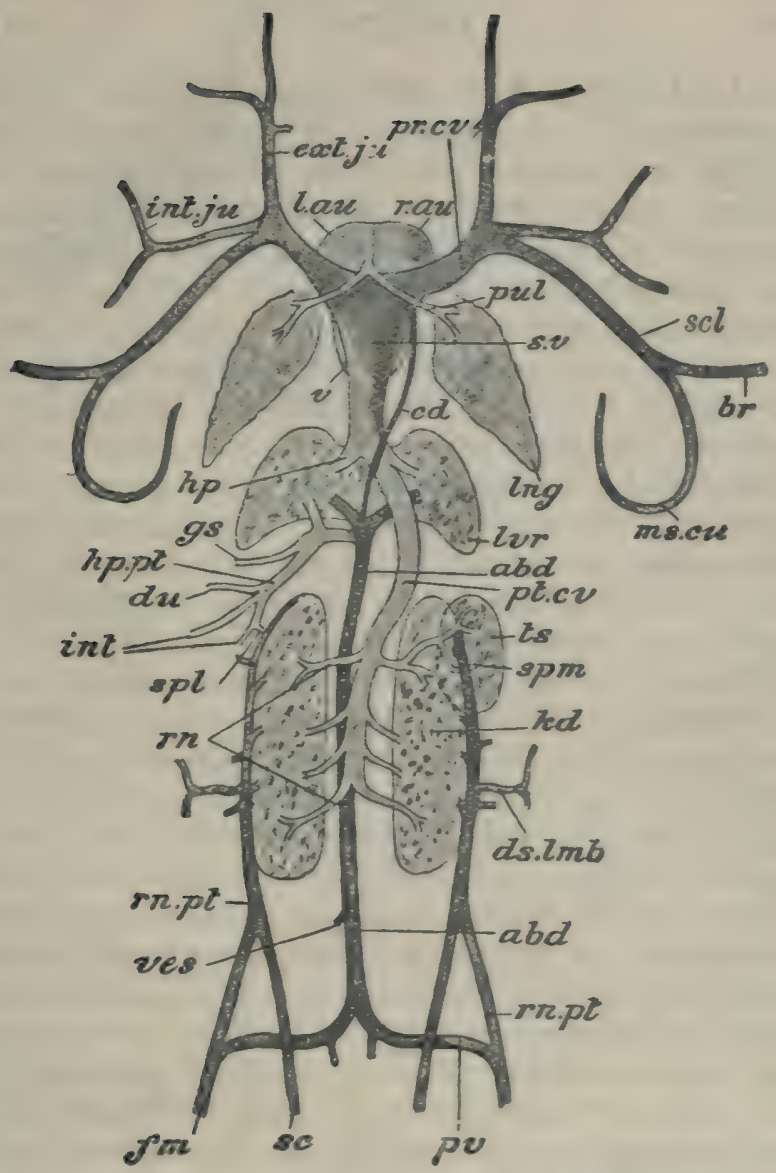

Fic. 21. - The vennus system of the Frng, with the heart, lungs, liver, kiciners, and risht spermary, supposed to be removed from the tody and viewed from the dorsal aspect $\left(x_{2}\right)$.

clud. abchominal vein : br. brachial vein : cd. cardiac vein ; ds. lmb. dorsn-lumbar

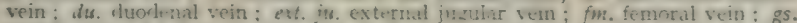
gastric vein : hp hepatie vein; hp. th. hertic portal vein ; int. intestinal veirs ; int. ju. Internal juaular vein; kil. hilnev; 1 . au. left atrirle; Ing. lung: wer. liver; ms. ca. musculo-cutaneous vein : pr. co. precaval veia ; pt. cu. postcaval vein; pul. pulmonary vein; pr. pelvic rein: $\%$. au. risht auricle; $m$. re:hal veins: m. pt. renal portal velr: se. sciatic vein; scl. subclavian veia : spl. splenic vein; spm. spermatic vein: s. $"$. sinus venosus: is. spermary; ves. vesical veins (from bladder). (From Parker and Haswell's Zcology.) 
the head and part of the skin and muscles of the abdomen.

With the posterior end of the sinus venosus is connected a single large vein, the postcaval or vena cava posterior (pt.cv), a wide vessel lying between the kidneys and extending forwards to the liver (Fig. 4). It runs parallel with and beneath, i.e., ventral to, the dorsal aorta (Fig. 5), from which it is at once distinguished by its greater diameter. Posteriorly it is formed by the confluence of four renal veins (Fig. 2I, $r n$ ) from each kidney, and it also receives, in the male, spermatic veins $(s p m)$ from the spermaries, and in the female, ovarian veins from the ovaries. Anteriorly it perforates the liver $(l v r)$, receiving two hepatic veins $(h p)$ from that organ, and finally enters the sinus venosus.

We have now to consider a striking want of correspondence between the arterial and venous systems of the frog. As you will remember, the dorsal aorta, after giving off the renal arteries, passes backwards and divides into the two iliac arteries for the legs. You might naturally expect a somewhat similar arrangement with the veins, especially if you have studied human physiology and learnt how the posterior (or inferior) vena cava of man is formed by the confluence of the veins from the legs, and receives higher up those from the kidneys. In the frog, as we have just seen, the postcaval does not reach to the hinder boundary of the kidneys, and the renal veins are the only vessels entering it posteriorly.

In the frog, as a matter of fact, the connections of the veins of the legs are very peculiar. You remember the abdominal vein seen in our preliminary dissection (Figs. 2, 3, and 4, $a b d . v$ ). This vessel, if traced backwards, is found to fork at the posterior end of the abdomen-or more accurately, the single abdominal 
vein is formed by the confluence of two pelvic veins (Fig. 2I, pv), which can be traced along the base of the leg (compare Fig. 3). Towards the front of the thigh is the principal vein of the leg, the femoral vein (Fig. 2I, $f m$ ), which on emerging from the leg, divides into two branches. One of these is the pelvic vein already seen ; it unites with the corresponding vessel of the opposite side to form the abdominal vein $(a b d)$. The other branch of the femoral is called the renal portal vein $(r n . p t)$; it passes directly forwards, receiving the sciatic vein (sc) from the back of the leg, and then goes along the outer border of the kidney, finally branching out in that organ. It also receives a large vein $(d s . l \mathrm{mb})$ from the muscles of the back.

The abdominal vein receives, near its posterior end, small veins (ves) from the urinary bladder. It passes forwards, as already seen, receiving veins from the abdominal muscles, and quite at its anterior end, a small vein $(c d)$ from the heart. It then divides into two branches which enter the liver and branch out in that gland.

The veins from the stomach $(g s)$, the intestine $(d u, i n t)$, spleen $(s p l)$, and pancreas run in the mesentery alongside the corresponding arteries. Near the liver they all unite to form a large vessel, the hepatic portal vein ( $h p . p t)$, which enters and branches out into the liver, first sending off a branch which joins the abdominal.

Thus the veins from all parts of the body, except the lungs, ultimately discharge into the sinus venosus. The veins from the lungs are quite singular in their course; they unite, in each lung, to form a single pulmonary vein (Figs. 3 and $2 \mathrm{I}, p u l$ ), which passes dorsal to the sinus venosus, and discharges, with its fellow of the opposite side, into the left auricle.

Character of the Blood in the Arteries and Veins.There are certain differences between the arteries and 
veins in respect of the blood they contain. As a rule, the veins contain purple or non-aerrated blood, the arteries more or less thoroughly aërated or scarlet blood. But there are certain exceptions. As we shall see in a later chapter, the blood is aërated in the lungs and skin ; hence the blood returned from those organs by the pulmonary and musculo-cutaneous veins is aërated. On the other hand, the blood in the pulmo-cutaneous artery is non-aërated.

Flow of Blood.-We must now try to understand the function of this complicated blood-system, and the reason why every part of the body has two vessels, an artery and a vein. That there is some kind of movement of the blood has been hinted in the foregoing description, in which arteries have been described as branching out to various parts, veins as formed by the confluence of smaller veins from various parts.

If an artery were cut in a living frog, the blood would be found to flow out in a series of jerks corresponding with the beats of the heart. Moreover, the blood would flow from the side of the cut nearest to the heart, and the flow might be stopped by tying or compressing the artery on that side, i.e., between the heart and the cut. Evidently, then, the blood in the living animal flows from the heart along the arteries to the various parts of the body, and is propelled by the pulsation of the heart, a pulse being observable in the arteries.

If a vein were cut the result would be very different. The blood would flow in a comparatively slow stream and without jerks; it would flow, moreover, from the side of the cut furthest from the heart, so that, in order to stop the bleeding, the vein must be tied or compressed on the far side of the cut. The blood in the veins flows, therefore, towards the heart in an even stream, unaffected by the heart's pulsations. 
Thus the blood is driven by the heart to the various parts of the body through the efferent arteries, and is returned from the various parts of the body to the heart by the afferent veins. Two questions thus naturally arise : how is it that the blood takes this direction and

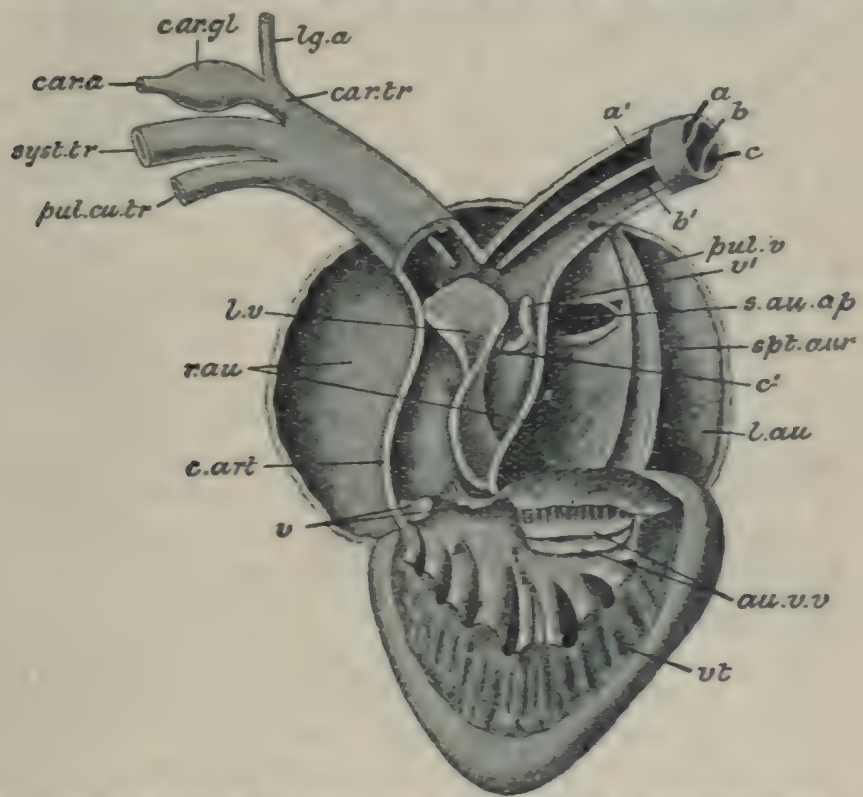

F1G. 22.- The heart of the Frog from the ventral aspect, with the cavities laid open. (x6.)

$a, a^{\prime}$, bristle passed into left carotid trunk: au. $v$. $\tau$. auriculo-ventricular valves:

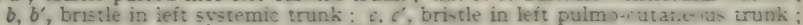
car. $a$. carotid artery; car. ol. car tid habyintb : c. art. conus artercinu : car. tr.

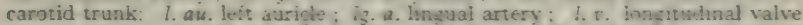

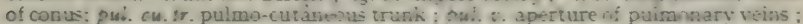

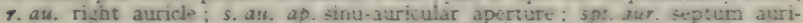
cularum; t. v', valves; t. ventriele. IFrom Parker and Haswell's Zooingy.)

not the other, and how does it make its way from the artery of a given organ into the vein?

Internal Structure of the Heart.- To answer the first question-why the blood leaves the heart by the arteries 
and returns to it by the veins, and not vice vers $\hat{a}$ - we must examine the heart itself in some detail.

The ventricle is a hollow structure with thick spongy walls and a small cavity (Fig. 22,vt), and there are two perfectly distinct auricles, the right $(r . a u)$, considerably larger than the left $(l$. $a u)$, separated from one another by a vertical partition (spt. aur).

You have already seen that the conus arteriosus arises from the right side-the frog's right, not yoursof the base of the ventricle. A little to the left of this point there is an aperture through which a bristle can be passed from the ventricle into either of the auricles. Both auricles, then, communicate with the ventricle, by a single auriculo-ventricular aperture. This is guarded by two little membranous flaps (au.v.v), which spring, one from the dorsal, one from the ventral edge of the aperture, and hang down into the ventricle, to the walls of which they are attached by little tendinous cords, represented in the figure by white streaks. Thus the flaps have the character of folding doors or valves opening only one way; they readily flap backwards, i.e., into the ventricle, but are prevented from flapping forwards, or into the auricles, by the tendinous cords attached to them. The two flaps are the auriculo-ventricular valves. Their mode of action is easily understood. If the auricles, being full of blood, contract and squeeze themselves together, the pressure will force aside the valves and allow the blood a free passage into the ventricle. On the other hand, if the ventricle contracts, the blood, getting behind the valves, will force them together and close the aperture, the tendinous cords preventing their being driven into the auricles by undue pressure.

In the interior of the conus is a longitudinal fold or valve $(l . v)$, which traverses it obliquely, attached to 
its dorsal wall and free ventrally; and there are also three little semilunar or watch-pocket shaped valves $(v)$ guarding the aperture between the ventricle and the conus: they are arranged with their edges turned forwards or towards the cavity of the conus, so that any pressure of fluid from the side of the ventricle must force them back and allow ready ingress into the conus, while pressure in the opposite direction must fill them, bringing their edges together, and so barring the passage.

Anteriorly the longitudinal valve projects in the form of a free flap, and at the same level is a semilunar valve $\left(v^{\prime}\right)$ : the two together separate the conus proper from a small chamber, the bulbus aorte, from which the right and left carotid $\left(a, a^{\prime}\right)$ and systemic $\left(b, b^{\prime}\right)$ trunks arise. The pulmo-cutaneous trunks $(c)$ spring from the conus by an aperture $\left(c^{\prime}\right)$ situated just posteriorly to the valve $v^{\prime}$, and itself guarded by a small valve.

In the dorsal wall of the right auricle is a large transverse aperture $(s, a u . a p)$. This leads into the sinus venosus: it is therefore called the sinu-auricular aperture; its two edges are produced into flaps, the sinu-auricular valves, which allow free passage from the sinus to the right auricle, but prevent any flow in the opposite direction. The aperture of the pulmonary veins was referred to on page 85 .

Valves of the Veins.-In addition to the valves of the heart, many of the veins contain small watch-pocket valves, all arranged with their concavities directed towards the heart, so as to allow of a free passage in that direction. Any attempt to flow in the opposite direction, i.e., from the larger to the smaller veins, will result in filling the valves, bringing their edges into contact with the opposite wall of the vein and thus effectually blocking the passage. 
Circulation of the Blood.-We see then that an investigation of the structure of the heart shows that fluid can traverse it in one direction only, viz., from the sinus to the right auricle, from the auricles to the ventricle, from the ventricle to the conus, and from the conus to the bulbus aortæ, and so to the arteries. The valves in the veins are so arranged as to allow the blood in these vessels to flow only towards the heart. The experiment of cutting the vessels shows that the blood in the arteries does actually flow from the heart, that in the veins towards the heart. We thus demonstrate that there is not merely a movement but a true circulation of the blood, the current starting from the heart, passing by the arteries to all parts of the body, and being returned to the heart by the veins.

Action of the Heart.-The circulation of the blood is effected by the pulsation of the heart. This organ is made of muscle ; each of its cavities is to be considered as a bag, the walls of which are formed of muscular fibres crossing one another in various directions and encircling the cavity. We have seen that when an ordinary spindle-shaped muscle contracts, its two ends are brought nearer together. When a hollow muscular bag contracts the effect will be to squeeze the walls together and so diminish the cavity. Hence when any chamber of the heart contracts it must expel a part or the whole of its contained blood. The contraction of the chambers of the heart takes place in regular order: first the sinus, then the two auricles together, then the ventricle, and lastly the conus. The contraction (systole) in each case is visible as a sort of throb and is followed by a period of rest, during which the chamber regains its former dimensions (diastole).

The course of the blood through the heart will now be clear. When the sinus (Fig. 23, s.v) contracts, the 


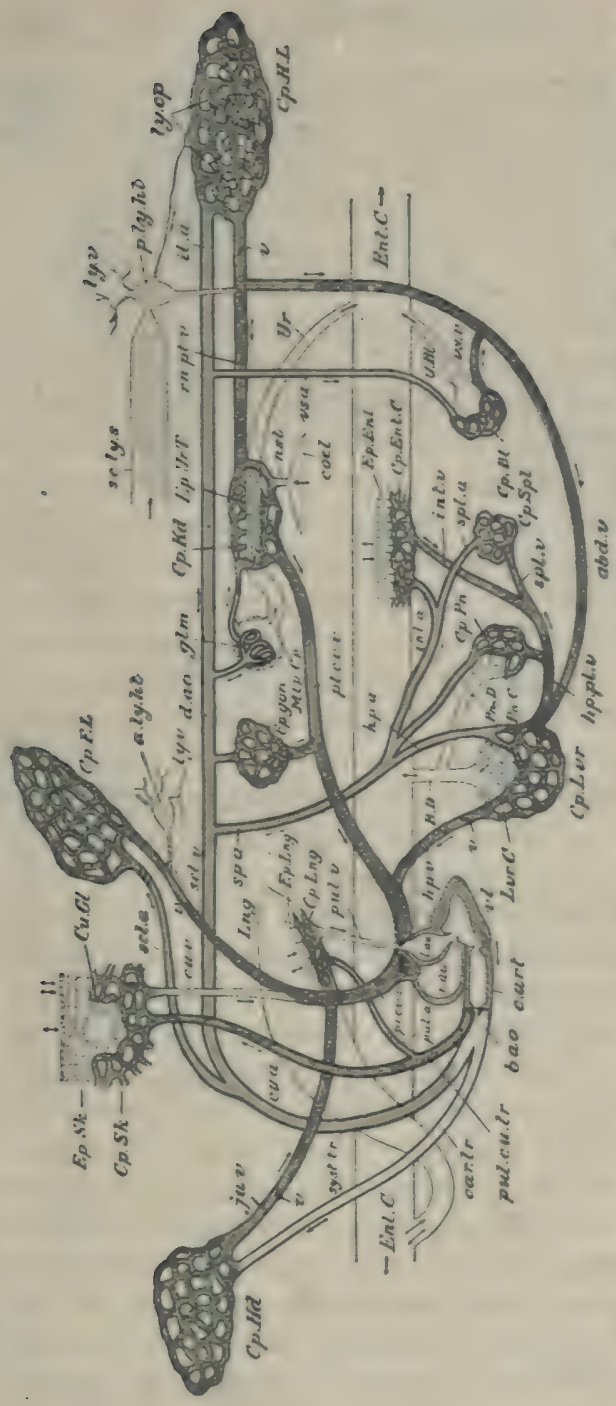

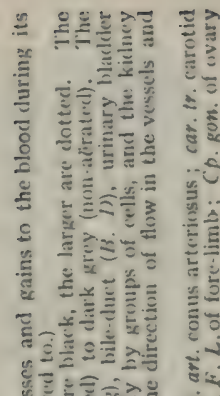

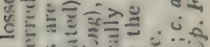

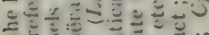
x.․․

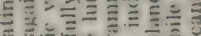
.

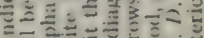

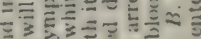
है a c

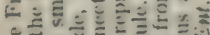

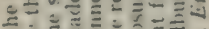

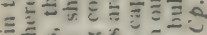

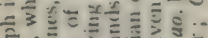

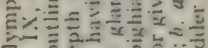
흘. E. है 굴

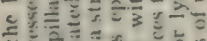

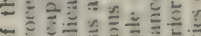
- 0 늘

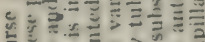

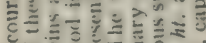
일

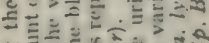

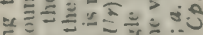

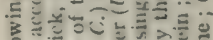
政

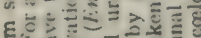

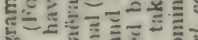
क्ष

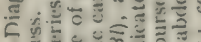
14 1 하의

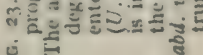
这 
or spermary; $C p . H d$. of head; $C p . H . L$. of hind-limb; $C p . K d$. of kidney; $C p$. Lng. of lung; $C p$. Lvr. of liver; $C p$. $P n$. of pancreas; $C_{p}$. Sk. of skin; $C p$. Spl. of spleen; $c u . a$. cutaneous artery; $c u$. v. cutaneous vein; $C u$. $G l$. cutaneous gland; d. ao. dorsal aorta; Ent. C. enteric canal; Ep. Ent. epithelium or enteric canal; Ep Lng. of lur.g; Ep. Sk. of skin; Ep. Ur.T. of urinary tubule; glm. glomerulus; il. e. iliac artery; int. a. artery to stomach and intestine; int. $v$. vein from stomach and intestine; $j u . v$. jugular vein; $h p . a$. hepatic artery (to liver) ; $h p . p t . v$. hepatic portal vein; $h p . v$. hepatic vein ; $l$. aus. left auricle ; Lng. lung; Lvr. C. liver-cells; $l y . c p$. lymph capillaries; $l y . v$. lymphatic vessels ; $M l p . C p$. Malpighian capsule ; $n s t$. nephrostome ; $p . l y . h t$. posterior lymph-heart; $P n$. $C$. cells of pancreas; $P n . D$. pancreatic duct; pr.cv. v. precaval vein; pl. cv. v. postcaval vein ; pul. a. pulmonary artery; pul. cu. $t r$. pulmo-cutaneous trunk; pul. $v$. pulmonary vein ; r. au. right auricle; $m$. pt. v. renal portal vein; scl. a. subclavian artery; $s . c . l y$. s.sub-cutaneous lymph-sinus; scl. $v$. subclavian vein ; $s p . a$. splanchnic artery; spl. a. splenic artery; spl. v. splenic vein; s. $v$. sinus venosus; syst. $t r$. systemic trunk; $U . B l$. urinary bladder; $U \boldsymbol{r}$. ureter; $v$. valve in vein; $v s . a$. vesical artery (to bladder) ; vs. $v$, vesical vein; $v l$. ventricle.

contained blood, which, coming by the precavals and postcaval, is non-aërated, is acted upon in all directions and might therefore be forced either into the three great veins $(p r . c v . v, p t . c v . v)$ or into the right auricle $(r . a u)$. But the veins are full of blood steadily flowing towards the heart, and any regurgitation is further prevented by their valves: the right auricle, on the other hand, has finished its contraction and is now relaxing ; it is therefore empty. Thus, on the principle of least resistance, the contraction of the sinus fills the right auricle with blood from the great veins, and the sinus itself is refilled from the same source as soon as it begins to relax.

Immediately after the sinus has ceased to contract the two auricles contract together : the right, as we have seen, has just been filled from the sinus, the left (l.au) is full of aërated blood brought to it by the pulmonary vein (pul.v). The presence of the sinu-auricular valves prevents the blood in the right auricle from being forced back into the sinus : that in the left auricle is prevented from being forced back into the pulmonary veins by the steady onward flow in the latter. On the other hand the ventricle is beginning to relax and is empty. Consequently the auriculo-ventricular valves are forced back into the ventricle $(v l)$ and the blood from both auricles flows into and fills that chamber, the right half of which 
becomes filled with non-aërated, the left with aërated blood, the two taking an appreciable time to mingle, owing to the spongy character of the ventricular wall.

The instant it is thus filled, the contraction of the ventricle begins. As it does so the blood, getting behind the auriculo-ventricular valves, forces them together, and thus prevents any backward flow into the auricle. At the same time the semilunar valves at the entrance of the conus (c. art) are pushed aside and the blood flows into that chamber. Since the conus opens from the right side of the ventricle, the blood first entering it will be non-aërated; there will then follow a certain amount of mixed blood; and finally, as the ventricle reaches the limit of its contraction, the aêrated blood from its left side will be forced into the conus (compare Fig. 22).

Last of all the chambers of the heart, the conus begins its contraction. The semilunar valves are immediately filled with blood, and, closing together, stop all backward flow into the ventricle. Two alternative courses are now open to the blood: it can pass either directly from the conus into the pulmo-cutaneous trunk ( $p u l . c u$. $t r$ ), or make its way into the bulbus aortæ $(b, a o)$. As a matter of fact it takes the former course owing to the circumstance that there is little resistance in the limited blood-system of the lungs, while that in the systemic and carotid trunks is very great. Hence the blood just received into the conus from the ventricle, which, as we have seen, is non-aërated, goes immediately to the lungs and skin to be aërated.

Before long-in a fraction of a second-the flow of blood into them increases the pressure in the pulmonary vessels, and at the same time the blood is continually flowing onwards-i.e., away from the heart-in the systemic and carotid trunks. Consequently the pressure 
in these vessels rapidly diminishes, and the blood soon forces aside the valves between the conus and the bulbus and fills the latter. Here again the question of pressure comes in. It is easier for the blood to make its way into the wide systemic trunks (syst. $t r$ ) uniting immediately into the long dorsal aorta $(d . a 0)$ than into the comparatively narrow carotid trunks (car. tr), obstructed by the carotid labyrinths. Hence, the nonaërated blood having been mostly driven into the pulmocutaneous trunk, the mixed blood, from the middle part of the ventricle, goes into the systemic trunk, and thence to the various arteries supplying the limbs ( $s c l$. $a$, $i l$. a) and the viscera (sp. $a$, etc.). Finally, when the pressure is sufficiently raised in the systemic trunks the remaining blood, which, coming from the left side of the heart, is aërated, is pumped into the carotid trunks (car. $t r$ ), and thence to the head.

Thus, owing to the arrangement of the valves, and to the varying pressures in different parts of the vascular system, the non-aërated blood returned from the various parts of the body to the heart is mostly sent to the lungs and skin to be aërated. Mixed blood is sent to the trunk, limbs, and viscera, while for the head with its contained brain-the directing and controlling organ of the whole animal-a special supply of pure, aërated blood is reserved.

We see then that the course of the circulation may be proved, as a simple matter of induction, from the structure of the heart and its valves, the direct observation of its beat, and the manner in which the flow from cut vessels takes place. It was by observation and experiments of this kind that the circulation of the blood in the higher animals was demonstrated by William Harvey in the seventeenth century. But the final and most conclusive proof of the circulation-from 
directly observing the flow-became possible only after the invention of the microscope. This instrument, by furnishing a sufficiently high magnifying power, allows us to see for ourselves the actual movement of the blood in an animal or organ of sufficient transparency ; and at the same time clears up the question, previously insolvable, of how the blood, having reached a given part or organ by the arteries, finds its way into the veins to begin its return journey.

The Circulation in the Frog's Web.-There are three parts in the frog transparent enough to allow of the blood-flow being seen in them-the web of the foot, the tongue, and the mesentery. Of these the weo is the most convenient, and can be examined under the microscope without any injury to the animal.

The Capillaries.-If you have the makings of a naturalist, you will acknowledge the sight to be one of the most wonderful you ever saw. In the thickness of the web is an irregular network of minute blood-vessels, called capillaries (Fig. 24), and through them the blood is seen to flow with great rapidity, its course being made especially evident by the minute particles or corpuscles it contains, the structure of which we shall study later on. You will also notice much larger vessels, the smallest arteries and veins. The arteries $(a)$ are distinguished by the fact that the blood in them flows in the direction from the leg towards the margin of the web, while in the veins $(v)$ it takes the opposite direction. You must remember, however, that under the microscope everything is reversed; right appears left and left right, and a current actually flowing towards the observer appears to go in the opposite direction.

By careful examination you will see that both arteries and veins are in connection, by minute branches, with 
the capillary network, and will be able to trace the blood from an artery, through the capillaries, into a vein. The same thing can be seen in other transparent

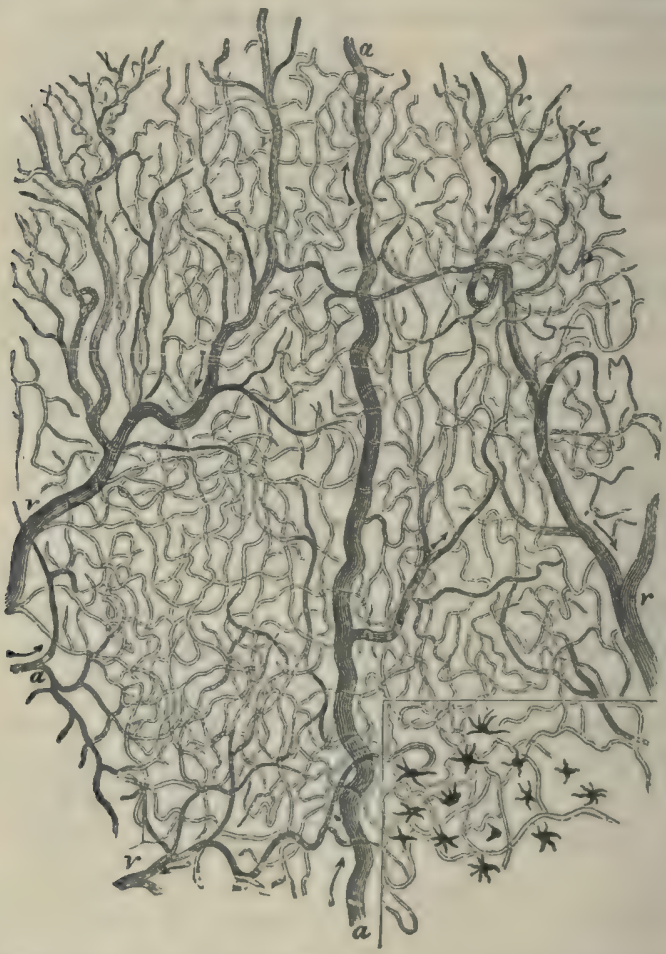

Fig. 24-- Blood-vessels of the web of a Frog's foot seen under a low magnifying power.

a. small arteries; $v$. small veins. The minute tubes joining the arteries to the veins are the capillaries. The arrows show the direction of the circulation. In the small portion marked off, the pigment cells of the skin, which occur throughout the web, are also represented. (From Huxley's Phy'siology'.)

organs; and by injecting the vascular system with a fluid injection-mass, such as gelatine suitably coloured, it can be proved that all parts of the body are permeated with a capillary network into which the blood is passed 
by the arteries, and from which it is received into the veins.

Thus by means of the microscope we are able to take the final step in demonstrating the circulation. The fact that the blood can flow in one direction only is proved by the disposition of the valves of the heart and of the veins, but the passage of the blood from the smallest arteries to the smallest vein by a connecting system of minute tubes or capillaries can be proved only by the employment of considerable magnifying powers. We see that the vascular system of the frog is a closed system of vessels: the blood is everywhere confined within definite tubes through which it flows in a definite direction, never escaping, as in some of the lower animals, into large irregular spaces among the tissues.

The Lymphatie System.-Included in the vascular system are certain cavities and vessels containing lymph, and together constituting the lymphatic system. We have already noticed the subcutaneous lymph sinuses (p. 18, and Fig. 23, s.c.ly.s) and the sub-vertebral lymph-sinus (p. 27, Fig. 5, s.v.ly.s). There are also found in nearly all parts of the body delicate, thin-walled, branching tubes, the lymphatic vessels (Fig. 23, ly. v). Unlike the blood-ressels, the lymphatics are all of one kind, there being no distinction into anything of the nature of arteries and veins. They arise in lymph-capillaries (ly. $c p)$, which are, as it were, interwoven with the bloodcapillaries, but have no connection with them. By the lymph-capillaries the fluid which has exuded from the blood in its passage through the tissues is taken up and passed into the lymphatic vessels or sinuses, and these in their turn finally communicate with certain transparent muscular organs called lymph-hearts. Of these there are two pairs. The anterior lymph-hearts (a. ly. $h t$ ) lie, one on either side, beneath the supra-scapula 
and just behind the transverse process of the third vertebra: the posterior pair $(p . l y . h t)$ are situated one on each side of the posterior end of the urostyle. These organs pulsate regularly, like miniature hearts, and pump the lymph into the veins, the anterior pair communicating with the subclavian, the posterior with the renal portal vein.

The lymphatics of the enteric canal have an important function to perform in that they absorb the fatty portions of the food (p. 75). The fluid they contain has a milky appearance, owing to the presence of minute suspended fatglobules, and for this reason they receive the name of lacteals.

The colome is really a great lymph-sinus (Fig. 23, $\mathrm{coll})$. It communicates with the veins of the kidneys through certain microscopic apertures called nephrostomes ( $n s t)$.

The spleen (p. 23, and Fig. 3, spl) has important relations with the blood-and lymph-vessels, and probably acts as a blood-filter, removing particles in the blood which are no longer wanted.

\section{PRACTICAI DIRECTIONS}

\section{The Vascular System.}

$a$. Let some blood from a frog-or better from the veins of some larger, freshly-killed, warm-blooded animal, such as a rat or a rabbit - flow directly into a white cup or porcelain capsule. Note that it soon coagulates, and shortly afterwards separates into clot and serum. Notice also the difference in colour between the blood freshly drawn from a vein (see p. 85), and that soon assumed by exposed portions of the clot.

b. Pin a freshly-killed frog to the dissecting-board, dorsal side upwards, and cut through the skin of the back along the urostyle. The posterior lymph-hearts (p. 98, Fig. 23) will then be seen. To make out the anterior lymph-hearts, carefully separate the supra-scapulæ from the vertebral column. Some of the chief lymph-sinuses have already been seen: special methods are required to trace the lymph-vessels. 
c. Now turn the frog the other way upwards, pin it down in the dissecting-dish, and open the body-cavity as before (p. 32), taking great care not to cut the abdominal, musculocutaneous, and other veins. Slit open the pericardium and remove as much of it as possible, so as to expose the entire heart. The structure of the heart and the course of many of the blood-vessels can also be made out in the specimen from which you have already removed the alimentary canal. In the following dissections, use a dissecting lens whenever necessary.

I. In the heart (Figs. 3, 4, 7, 20 and 21), notice again the ventricle, and the right and left auricles (appearing single in the entire heart), and make out also the conus arteriosus, dividing into two distally, and the sinus venosus (dorsal).

If the heart is still beating, notice the order of contraction of its different chambers (p. 90).

Injection of the Arteries.-The tracing of the arteries is greatly facilitated by filling them with some coloured substance. The operation requires, therefore, a coloured fluid or injection-mass capable of traversing the arteries, and some contrivance by which it can be injected into them.

The most convenient injection-mass is made as follows :-

I. Grind up in a mortar 4 grammes of "French blue" (to be had at the oilman's) with 4 cubic centimetres of glycerine and the same quantity of methylated spirit.

2. Grind up 50 grammes of common laundry-starch, with 50 cubic centimetres of water and 25 of methylated spirit, and add to the mixture the colour prepared as above. Mix thoroughly and strain through muslin.

This injection-mass will keep for an indefinite period in a stoppered bottle, requiring only to be stirred up when used. If it is considered too troublesome to make, a simpler but less satisfactory mass may be made by simply stirring up some French blue in water in the proportion of a teaspoonful to a tumblerful.

For injecting the mass into the blood-vessels, the most satisfactory instrument is a brass injecting syringe, holding about one ounce, provided with nozzles of various sizes. This is, however, expensive, and an ordinary glass syringe, to be had of any druggist, will answer the purpose very fairly if provided with a proper nozzle or cannula. This latter is made by drawing out one end of a piece of glass tubing about two inches long until it is fine enough to pass into the conus arteriosus; a piece of india-rubber tubing is then used to connect it with the fixed nozzle of the syringe. 
A still simpler injecting apparatus is furnished by a common "medicine dropper" (see p. I2, and Fig. 25). By alternative squeezing and releasing the cap, fluid is drawn into or expelled from the tube.

Having provided these requisites, proceed as follows.

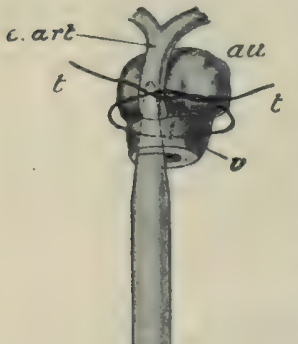

$a$

b

FIc. 25.-Sketch showing the method of injecting the Frog's arteries.

a. glass "medicine dropper" with india-rubbercap $(b)$; its pointed end (dotted) is passed through the cut end of the ventricle $(v)$ into the conus (c. art); au. auricular division of the heart; $t$. thread. Open the abdomen of a freshlykilled frog in the usual way, taking great care not to injure the blood-vessels. Remove the middle portion of the shoulder-girdle, so as to expose the heart, lay open the pericardium, and with the scissors make a snip into the ventricle, allowing the blood to escape freely. Pass a piece of fine thread, about six inches long, round the heart, at about the junction of the auricles and ventricle, and give it a single loose tie, as shown in Fig. 25. When the bleeding has ceased, fill the medicine dropper, or syringe, with injection-mass and pass the narrow end of the former, or the nozzle of the latter, through the cut end of the ventricle into the conus - take care not to push it into one of the auricles instead-and tighten the thread so as to keep it in place. Then squeeze the cap of the medicine dropper, or push in the piston of the syringe, and if the operation is successful, you will see the blue injection pass from the conus into the arterial trunks, and thence into the various arteries of the body. The contrast between the arteries, filled with the blue mass, and the veins, filled with blood, is then very striking, particularly in the mesentery. When the arteries are well filled, withdraw the nozzle from the heart and instantly draw the thread tight and knot it so as to prevent escape of the injection. Then place the whole frog in spirit (methylated spirit 3 parts, water I part) for a few hours, after which time the injection-mass will be found to have set hard 
enough to allow of the arteries being conveniently traced.

Injection of the Veins.-The veins are much more difficult to inject than the arteries, but if you wish to make a double injection on the same specimen, colour the injection-mass with vermilion or carmine in the case of the arteries instead of with French blue, using the latter for the veins. The operation is hest performed by inserting the nozzle into an incision in the abdominal vein (by directing the nozzle forwards, the portal vein will be injected: by directing it backwards the pelvic and renal portal veins), and also into one of the subclavians. But for a really satisfactory preparation, it is best to inject from the heart through the conus, as directed above, with a weak, warm solution of gelatine (in the proportion of one part of gelatine to two parts of water), coloured with precipitated carmine. In this case the injection-mass, containing only microscopic particles, passes from the arteries through the capillaries into the veins, keeping throughout to the course taken by the blood during life, and therefore unimpeder by the valves of the veins. $A$ syringe must be used, since the medicine dropper will not give sufficient pressure, and the animal should be placed in warm water during the process.

II. Now pin down under water and make out the course of the chief veins (p. 82, Fig. 21 ) :-

I. The two precavals, and the external jugular, internal jugular, subclavian, and musculo-cutaneons.

2. The postcaval, to see which turn the viscera on one side (Figs. 3 and 4). Note the renal, spermatic or ovarian, and hepatic veins.

3. The hepatic portal vein and its factors.

4. The abdominal vein and pelric veins.

5. The veins from the hind-legs can be more easily seen at a later stage, after the alimentary canal is removed, and so their examination is best left until certain of the arteries have been traced (or use the specimen you have dissected previously for this purpose). Remove the skin from the thigh, place the frog on its side, and make out the femoral, pelvic (already seen), renal portal, and sciatic veins, as well as a large vein from the muscles of the back.

6. The two pulmonary veins.

Make a sketch of the heart and as many of the veins as you have followed out up to this point, inserting the others after removing the alimentary canal (see p. IOz). 
III. The chief arteries may now be followed out (p. 8o, Fig. 20) :-

Note the carotid, the systemic, and the pulmo-cutaneous trunk, arising from the conus arteriosus, and then trace each of these out as follows:-

I. The carotid trunk gives off a lingual artery and is continued into the head as the carotid artery, having at its origin the carotid labyrinth.

2. The systemic trunk unites with its fellow to form the dorsal aorta, first giving off vertebral, subclavian, and asophageal arteries. From the point of union of the two systemic trunks arises the splanchnic or coliaco-mesenteric artery. After following this out to its distribution, remove the alimentary canal as directed on p. 76 , when the following branches of the dorsal aorta will be more plainly seen :- the renal, spermatic or ovarian, and iliac arteries.

3. The pulmo-cutaneous trunk divides into a pulmonary artery, passing along the outer side of the corresponding lung, and a cutaneous artery.

Sketch the heart and chief arteries, and then make out and sketch the renal portal system (p. IOI), if you have not already done so.

IV.1 Cut out the heart of a frog preserved in formaline, taking great care not to injure it. Fasten it down in a dissecting-dish, with the ventral surface upwards, by sticking very small pins through the arteries and veins-not through the heart itself. Pinch up the ventricle with fine forceps, and with small scissors gradually snip away its ventral wall, noting that it is a hollow structure with thick, spongy walls and a small cavity, which will probably be full of clotted blood. Wash this out and then proceed to open the auricles in a similar way, and to wash out the blood they contain. Observe the right and left auricles, separated by a partition. Slit open the conus arteriosus, and continue the cut forwards to the origin of the main arteries. Examine with a lens and make out (p. 87, Fig. 22) :-

I. The auriculo-ventricular aperture and its valves.

2. The longitudinal valve and the small semilunar valves in the conus arteriosus.

3. The origins of the carotid and systemic trunks from the

1 On account of its small size, the examination of the structure of the frog's heart is somewhat difficult, and the student is advised to dissect first the heart of a larger animal, such as a dogfish (pp. 449 and 477 ). 
bulbus aorta, and the small aperture leading into the pulmocutaneous trunks.

4. The sinu-auricular aperture and its valves.

5. The aperture in the left auricle leading into the pulmonary veins.

Sketch.

Turn over the heart, so that its dorsal surface is upwards, and cut away enough of the dorsal wall of the sinus venosus to show the sinu-auricular aperture from the other side.

$\mathrm{V}$. Get a piece of thin board-e.g., the side of a cigar-box -about six inches long by three wide. Towards one end make a round hole about half an inch in diameter, and opposite this. on either side, make a notch, or rather slit, with a penknife. This is called a " frog-board."

Next get as light coloured a frog as possible. The web may be examined in the living animal without hurting it, or the animal may be first chloroformed as directed on $p .3 I$, but removed from the influence of the anrsthetic as soon as it is insensible, so that there may be no suspicion of the frog feeling any inconvenience from this harmless experiment. Lay the frog on the frog-board with a piece of wet rag wrapped loosely round the body, and take one or two turns around both frog and board with a piece of tape-you must avoid tying it tightly or the circulation will be impeded. Stretch out one leg, and selecting the most transparent web, tie a piece of thick soft silk round each of the two toes by which it is bounded. Adjust the leg so that the web comes just over the hole in the frog-board, and bring the two pieces of silk through the slits, regulating them until the web is evenly stretched out over the hole. Lastly, place the frogboard on the stage of the microscope, ${ }^{1}$ with the hole over the aperture in the stage, and either fix it with the clips or rest the opposite end on some support : adjust the mirror so as to illuminate the web from beneath, and examine it with the low power. Note the network of capillaries and the circulation of the bloor. through the arteries, capillaries and veins (Fig. 24).

1 A brief description of the compound microscope will be given at the end of the next chapter (P. II9). 


\section{CHAPTER VII}

THE FROG (continued): THE MICROSCOPICAL EXAMINATION OF THE SIMPLE TISSUES

BEFORE carrying our inquiries any further into the anatomy and physiology of the frog it will be necessary to devote some consideration to its microscopic structure or histology, since there are many matters in connection with the various organs which can be further elucidated only by the examination of the minute structure of the organs as revealed by the microscope (see p. Irg).

Let us, first of all, examine a drop of the frog's blood under the low power of the microscope. It will at once be seen that the blood is not a simple homogeneous fuid, but that it contains a large number of minute solid bodies floating in it. These are called by the general name of blood-corpuscles: the fluid part of the blood in which they float is called the plasma. At first, owing to currents in the fluid, the corpuscles will be found to move to and fro, but after a time they come to rest. Under the high power you will notice that the corpuscles are of two kinds. The greater number of them are regularly oval in form (Fig. 26, C), and of a yellow colour. If the drop of blood is thick enough in one part for the corpuscles to lie over one another, so that the light passes through two or three layers of them to reach the 
eye, they will appear red: they are hence called red corpuscles. Frequently they are seen turned on edge (D), and their appearance in this position shows them to be flat, oval discs with a swelling in the centre. They are about $\frac{1}{40}$ th of a millimetre (about $\frac{1}{1100}$ th inch) in long diameter.

Among the red corpuscles are found, in much smaller numbers, bodies not more than half the long diameter of the red corpuscles in size, quite colourless, distinctly granular - so as to have the appearance of ground glass -and with a slightly irregular outline (Fig. 26, A). These are the colourless corpuscles or leucocytes. They
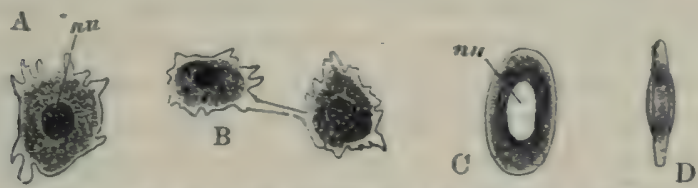

Fig. 26.-Blood-corpuscles of the Frog. ( $\times$ 525.)

$A$, colourless corpuscle; $B$, the same in process of division; C. red corpuscle, surface view ; D, the same, edge view. nu. nucleus. (Fron Parker's Biology.)

are not flat. like the red corpuscles, but have the form of irregular humps.

The plasma, like the leucocytes, is quite colourless, so that the colour of the blood is seen to be due entirely to the large number of red corpuscles it contains.

If the drop of blood has been prepared and examined under the high power with sufficient rapidity, a remarkable phenomenon can be made out with regard to the colourless corpuscles. This can be most easily demonstrated by making a series of outline sketches of the same leucocyte at intervals of a minute or two. You will then notice that the sketches all differ from one another: in one there will perhaps be a little projection going off to the right ; in the next this will have disappeared and a similar projection will have appeared on the left, and so 
on. As a matter of fact, as long as the blood is quite fresh, the leucocytes are in constant movement, sending out and withdrawing little processes of their substance called pseudopods or "false feet," by means of which they can crawl slowly along like independent living things. These very peculiar and characteristic movements are called amœboid movements. Occasionally a leucocyte may be seen to elongate itself and divide into two (Fig. 26, B) : this is a case of what is called simple fission. The red corpuscles neither move nor divide.

If a drop of some dye or staining fluid (p. I2I) be run in under the cover-glass, the corpuscles will be seen to become rather faint in outline, transparent, and lightly tinted; but the most obvious effect is that in the middle of each is seen a rounded or oval granular body $(n u)$ deeply stained by the dye, so as to make a very welldefined coloured area in the interior of the corpuscle. This body is called the nucleus : it is present both in the red and the colourless corpuscles.

By adding to a fresh drop of blood, in the same manner, a drop of weak acetic acid, the nucleus again becomes distinct, while the body of the corpuscle is rendered very transparent and almost invisible : indeed it finally disappears altogether. It is thus proved that the corpuscles, both red and colourless, consist of a substance which is known as protoplasm, but slightly affected by dyes, and soluble in weak acids; and enclosed in this is a nucleus, stained by dyes, and unaffected by weak acids. Both nucleus and protoplasm consist mainly of proteids (p. 72), together with water and a small proportion of mineral matters.

When distilled water is added to a drop of blood on the slide, the corpuscles are seen to swell up and become partly dissolved: the red colouring matter of the red corpuscles is dissolved out, the plasma becoming tinged 
with yellow. Thus the colouring matter is evidently a distinct substance from the protoplasm, and is called hamoglobin. It is characterised, among other things, by a strong attraction for oxygen: in combination with that gas it assumes a bright scarlet colour; when deprived of oxygen, it becomes purple. This affinity for oxygen accounts for the change undergone by the blood when exposed to the air, as described on p. 79 .

Coagulated blood, as seen under the microscope, is characterised by the plasma being traversed by extremely delicate threads, forming a sort of network in which the corpuscles are entangled. These threads are formed of a substance called fibrin, which is separated from the plasma during coagulation, the remaining or fluid portion of the plasma constituting the serum. We may therefore express the coagulation of the blood in a diagrammatic form as follows:-

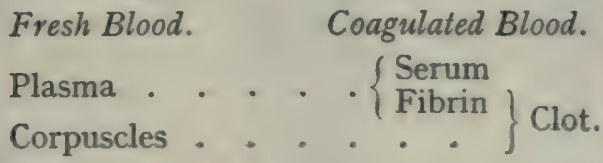

Having observed the microscopic characters of a drop. of blood, let us examine once more the circulation in the web, this time under the high power (Fig. 27). The red corpuscles $(F)$ can be seen streaming through the vessels, those in the capillaries in single file, those in the small arteries and veins, two or more abreast: as they pass through narrow capillaries or round corners, they become bent or squeezed $(G, H)$. The leucocytes $(I)$ travel more slowly and often stick to the sides of the vessels.

Columnar Epithelium.-By carefully teasing out a small piece of the inner surface of the mucous membrane 


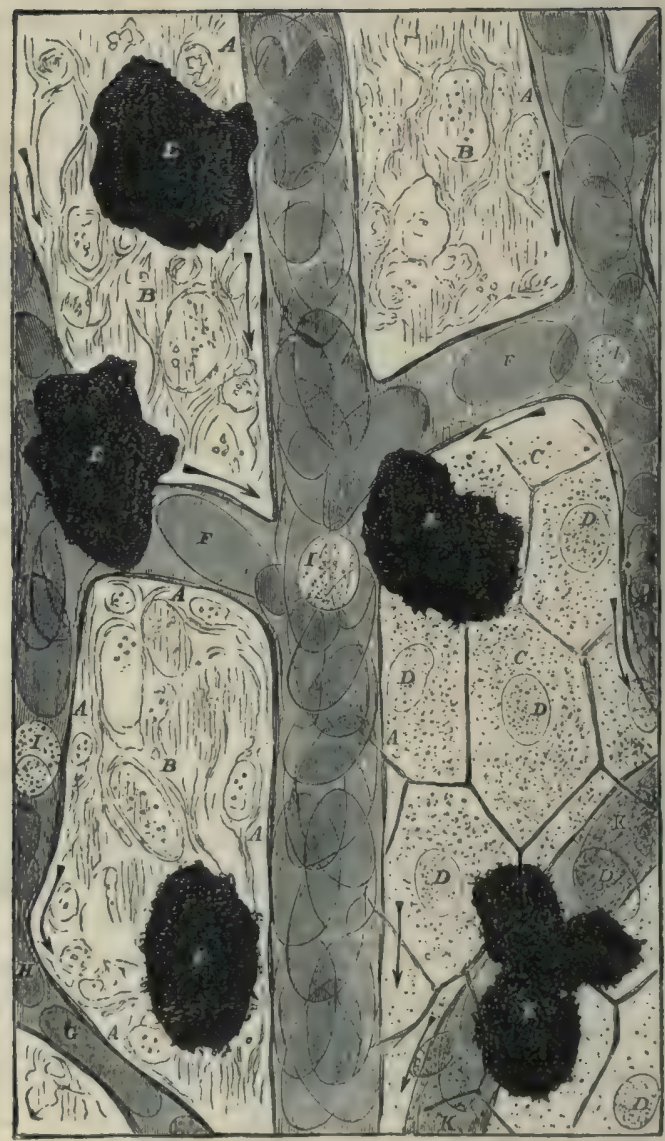

Firc. 27.- The circulation in the Frog's web, under a high power.

$A$, wall of capillaries; $B$, tissue of the web in which the capillaries lie; $C$, epidermcells; $D$, their nuclei; $E$, pigment-cells ; $F$, red corpuscles; $G, H$, red corpuscles being squeezed through a narrow capillary; $K$, capillary seen through the epiderm; I, colourless corpuscles. (From Huxley's Physiology.) 
of the intestine into the smallest possible particles, it will be found that the process has detached numerous, minute, conical bodies, about $\frac{-1}{30} \mathrm{~mm} .\left(\frac{1}{50}\right.$ in. $)$ in length, polygonal in transverse section, and having one end flat and the other more pointed (Fig. 28). These bodies are called epithelial cells: in the natural position they lie closely cemented to one another, like the blocks of a wood-pavement, their flattened ends facing the cavity of the intestine, while

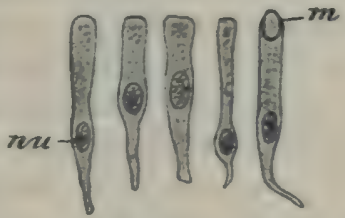

Fig. 28.-Columnar epithelial cells from the Frog's intestine. $(<500$.

$m$. droplet of mucus exuding from cell ; $n u$. nucleus. their narrower ends abut against the connective-tissue layer (p. 70). Thus the epithelial cells together form an epithelium or epithelial layer of the mucous membrane directly bounding the cavity of the enteric canal.

Each cell consists of protoplasm and contains a rounded, granular nucleus $(n u)$, which is made very conspicuous by staining, and in which are one or more small bodies or nucleoli. Cer-

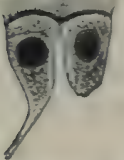

Fic. 29.-Ciliated epithelial cells from the mucous membrane of the frog's mouth. ( $x$ 500.) (From Parker's Biology, after Howes.) tain of these cells have a space towards their free ends containing slime or mucus, and thus have the form of little cups or goblets: they are known as goblet-cells (see right hand cell in Fig. 28).

Ciliated Epithelium.-By the same method the mucous membrane of the mouth is also seen to be lined by an epithelium, but the cells comprising it (Fig. 29) are broader in proportion to their length, and each is produced on its free surface into a number of delicate, transparent threads of protoplasm called cilia, which in the living condition are in constant movement, lashing backwards and forwards like minute 
whip-lashes, or, more accurately, like the blades of grass in a field when acted upon by a strong wind. If you happen to get under the microscope a good-sized bit of mucous membrane with the cells in position, you will see that the cilia produce a strong current by which small particles are swept along, while detached cells swim about, like little independent animals, by the action of their own cilia. These ciliated epithelial cells, like the ordinary columnar cells of the intestine, are made of protoplasm, and each contains a nucleus with one or two nucleoli clearly brought into view by staining.

The action of the cilia can be demonstrated, on a large scale, by placing a freshly-killed frog on its back, turning back or cutting away the lower jaw, and placing a very small cube of cork on the roof of the mouth near to the projection due to the eyes. The cork will be slowly swept back towards the throat.

Squamous or Pavement Epithelium.-By scraping the outer surface of a piece of skin with a sharp knife,

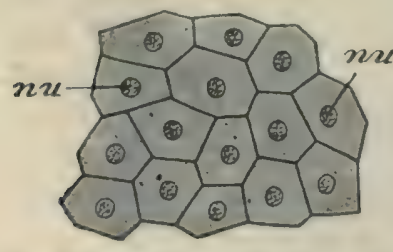

Frg. 30.-Squamous epithelial cells from the Frog's skin. ( $\times$ 300.) nu nuclei.

and examining the scrapings in a drop of water, after staining them, the superficial layer of the skin will be found to be made up of flattened, roughly hexagonal plates (Fig. 30 and Fig. 27, C, D) set closely together, like the tiles of a mosaic pavement. Each plate has a nucleus, and, from its flattened form, is distinguished as a squamous or scale-like epithelial cell.

Meaning of the word "Cell."-We see thus that the body of the frog is partly made up of distinct elements, which, under a considerable diversity of forms, exhibit the same essential characters. Each structure consists 
of a mass of living protoplasm, which appears almost clear or more or less granular, containing in its interior a rounded body, the nucleus, specially distinguished by the affinity of parts of its substance for colouring matters. To a body having this essential structure, whatever its form, the name cell is applied.

Unstriped Muscle.-Examination of a teased preparation of the muscular coat of the intestine, stomach, or urinary bladder will show that it is composed of delicate fibres (Fig. 3I) tapering at both ends, and each with a nucleus in the middle. These are called smooth or unstriped muscular fibres: they are obviously cells which have undergone a great elongation in length.

During the peristaltic movements of the intestine (p. 75) each fibre alternately contracts and relaxes, becoming shorter and thicker during the former process, like the large muscles of the body (p. 6o). The movements in this case, however, are not under the control of the will, and unstriped muscular tissue is therefore often spoken of as involuntary muscle.

\section{Contractility of Protoplasm.} -We have now studied three

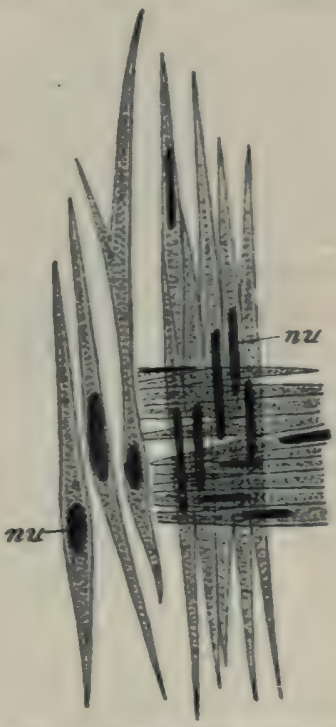

Fig. 31.- Unstriped muscular fibres from the Frog's intestine. $(x$ 500.)

To the right are shown fibres from the longitudinal and circular layers (see Chap. VIII) cross. ing one another; to the left isolated fibres. (After Howes.) different kinds of movement in cells:-muscular movement in the unstriped muscle-fibres, ciliary movement in the ciliated epithelial cells, and amceboid movement in the 
colourless blood-corpuscles. Muscular movement is due to the fibre undergoing a sudden shortening in a particular direction and a consequent approximation of its two ends. Ciliary movement is due to the alternate bending and straightening of the cilia; and the bending of a cilium in a particular direction is caused by the protoplasm of which it is composed shortening or contracting on the side towards which it bends. Amœboid movement is the protrusion and withdrawal of irregular processes of the cell : this results from the protoplasm undergoing a contraction or squeezing in a given cirection, as a consequence of which one part of its substance is drawn in and another pushed out. Hence all three kinds of movement are movements of contraction; and contractility, or the power of contraction, may be considered as a general property of protoplasm.

Striped Musele.-If a small piece of any of the bodymuscles is carefully teased out with the grain, i.e., in the direction of the length of the fibres, so as to break away the connective-tissue binding them together, the fibres, which are much larger than those of smooth muscle, will readily separate from one another, and they will be seen to be long and cylindrical. Under the microscope each fibre shows a delicate transverse striation (Fig. 32), being made up of alternate bright (b) and $\operatorname{dim}(d)$ bands-or more accurately discs, the fibre being cylindrical-set at right angles to its length. Hence the ordinary body-muscles or voluntary muscles are composed of striped muscular fibres. ${ }^{1}$ In addition to the transverse striation a fainter longitudinal striation is more or less distinctly visible.

Each fibre is covered by a delicate membrane (s),

1 The muscles of the heart, although not under the control of the will, are transversely striated; but their structure differs from that of ordinary striped voluntary muscle. 
called sarcolemma, beneath which nuclei $(n)$ occur at intervals. It will be seen that striped muscle, unlike the tissues previously considered, does not appear to be composed of cells, although the occurrence of nuclei seems to indicate their presence. In the embryo, however, the muscle is formed of ordinary nucleated
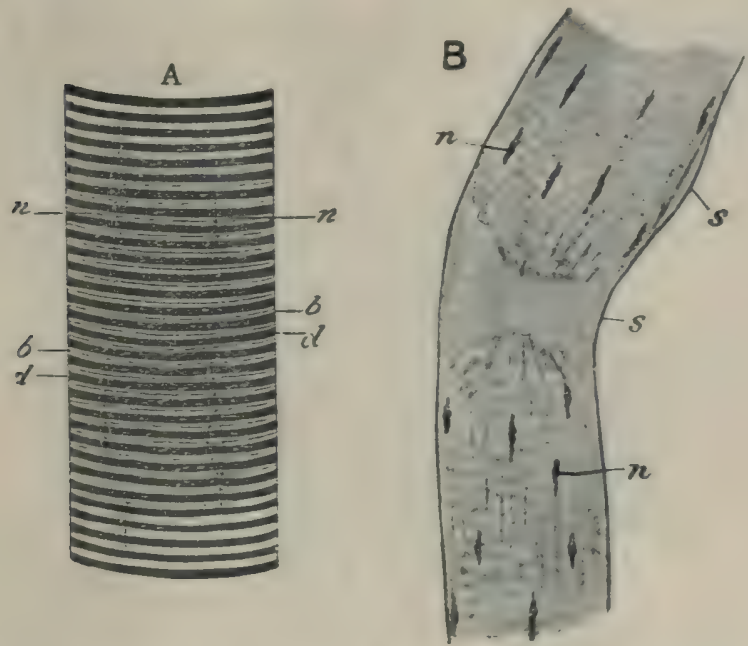

Frc. 32.-A, part of a fresh muscular fibre of a Frog. B, the same after treatment with distilled water followed by methyl-green. ( . about 150.)

$b$. bright bands; $d$. dim bands ; $n$. nuclei ; $s$, s'. sarcolemma, rendered visible as a minute blister $\left(s^{\prime}\right)$ by absorption of water and by the rupture of the muscle-fibre at s. (A, from Huxley's Physiology.)

cells, which, as growth goes on, increase in length while their nuclei multiply by fission, each enormously elongated cell thus containing a considerable number.

Connective-tissue.-We will next examine a piece of the delicate web of connective-tissue which binds the muscles together.

Under the high power, connective-tiss is seen to be 
composed of a sort of irregular network of delicate bundles of wavy fibres called white connective-tissue fibres (Fig. 33, w), which cross one another in all directions. Amongst them are found single fibres sweeping across the field in bold curves and called elastic fibres $(e):$ it is

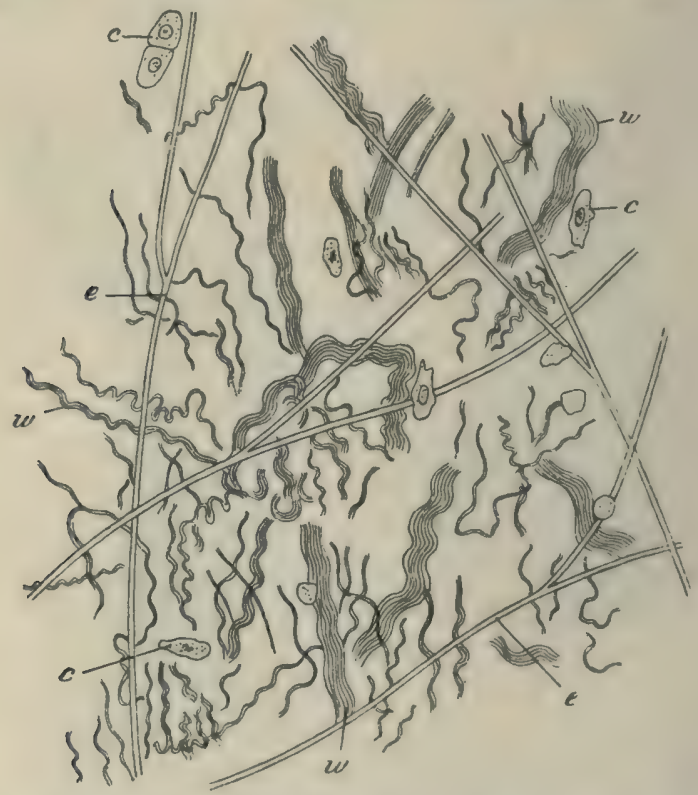

Fyc. 33.-Connective-tissue from between muscles of the Frog's legs. ( $x$ about 200.) $c$. cells; $e$. elastic fibres; $w$. white fibres:- all of which are imbedded in a delicate matrix not specially indicated in the figure.

owing to their elasticity that the tissue cannot be spread out when wet. Scattered among the fibres are numerous nucleated cells $(c)$ of very varied and often irregular form : these are the connective-tissue cells. The fibres, as well as the cells, are imbedded in a soft, homogeneous, ground substance or matrix. 
Thus connective-tissue consists partly of cells, but between these, and forming the main substance of the tissue, is a matrix or intercellular substance, enclosing fibres. In the embryo the tissue consists of closely packed cells, but, as development proceeds, these separate from one another, and the ground-substance is formed between them.

Cartilage.-The ordinary clear or hyaline variety of this tissue is conveniently studied by examining a piece of the thin edge of the omo- or xiphi-sternum, or by

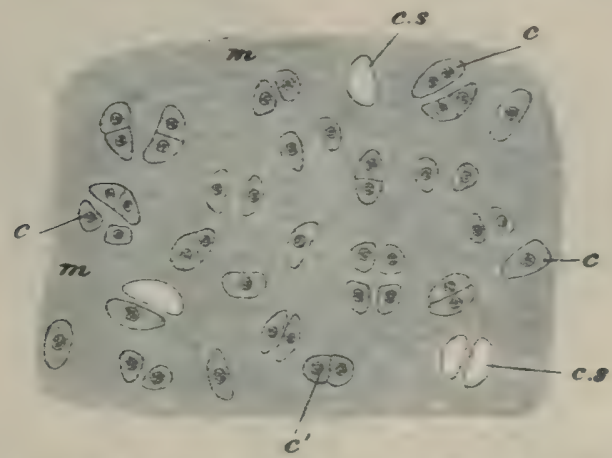

FIG. 34.-Section of cartilage. from the head of the Frog's feraur. ( . 200.) $c$. cells; $c^{\prime}$. cells underzoing fission; c. s. empty cell-sparr; $m$ matrix.

taking a thin section with a razor of the head of the humerus or femur.

Cartilage consists of a tough, . elastic, transparent, homogeneous matrix (Fig. $34, m$ ) containing numerous cavities or cell-spaces (c.s), in each of which is a nucleated cell (c). The cell-spaces, or lacunce, are in many cases arranged in groups of two or four, sometimes clcse together, sometimes with a narrow space of matrix or intercellular substance between them. This is due to the fact that cartilage grows by the cells undergoing 
binary fission, so that two cells are formed in one cellspace: the two then gradually separate from one another an 1 intercellular substance is formed between them. In the embryo, this tissue consists entirely of closely packed cells which gradually separate and form a structureless matrix which is firm and elastic, and which in some parts (pp. 46 and 48) may become calcified.

Bone.-As we have already seen (p. 52) bone is formed of two constituents, a basis of animal matter, in which

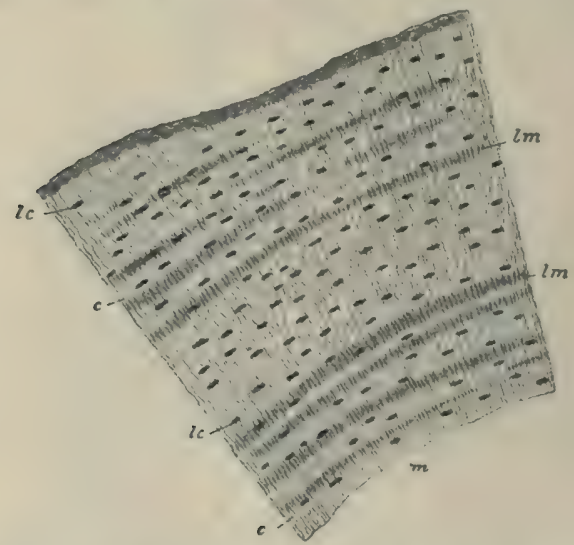

Frg. 35.-Transverse section of dry femur of Frog. ( $x$ about 125.) $c$. canaliculi; $l c$. lacunæ; $l m$. lamella; $m$. marrow-cavity.

mineral matter-calcium phosphate and carbonate-is deposited. In microscopic examination we may therefore investigate either the mineral matter by examining dried bone, or the animal matter by examining decalcified bone.

A thin section of a dried long bone, such as the femur, shows that it is formed of very numerous thin layers or lamella (Fig. 35, lm), surrounding and concentric with the marrow-cavity. The lamellæ contain numerous 
cavities, the lacunce $(l c)$, with delicate, branching tubes, the canaliculi $(c)$, radiating from them in all directions. Both lacunæ and canaliculi commonly appear black, owing to their being filled either with air or with bonedust produced in grinding the section.

In a section of decalcified bone (Fig. 36) the marrow is seen to be surrounded by lamellæ of a delicate fibrous substance, arranged in two layers, an outer $(b)$; having the periosteum $(p)$ closely investing it, and an inner $\left(b^{\prime}\right)$ in contact with the marrow. In the fibrous substance of
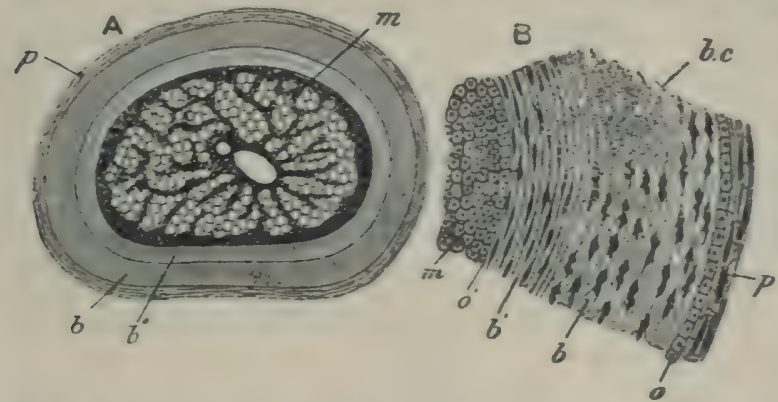

Fic. 36.-A, transverse section of decalcified Frog's femur under a low power.

( $<30$.) B, portion of the same under a high power. (* about I50.)

$b$. outer, and $b^{\prime}$, inner layer of bone ; $b . c$. bone-cells ; $m$. marrow; 0 . layer of osteoblasts in connection with periosteum; $o^{\prime}$. layer of asteoblasts in connection with marrow ; $p$. periosteum. (After Howes.)

the lamellæ are cell-spaces, corresponding with the lacunæ of the dried bone, and each containing a bone-cell (b. c), which sends off delicate branched processes of its protoplasm into the canaliculi. Thus the bone, like connective-tissue and cartilage, consists of cells with an intercellular substance: the latter is in the form of concentric layers and is impregnated with lime-salts.

The long bones of the frog grow in two directions. Between the periosteum and the bone is a layer of cells, the osteoblasts (0), by which new lamellæ of bone are 
formed on the outside of those already existing : thus the outer layer of bone (b) grows from within outwards. Between the marrow and the inner surface of the bone is another layer of osteoblasts $\left(o^{\prime}\right)$ which forms new lam 3 llæ on the inner side of the existing bone, so that the inner layer $\left(b^{\prime}\right)$ grows from without inwards.

Summary.-The various simple tissues studied in the present chapter consist either entirely of cells, or of cells separated by an intercellular substance. Formed entirely of cells are the various kinds of epitheliumcolumnar, ciliated, and squamous, and unstriped muscle. In striped muscle the cells have elongated into fibres and their nuclei have multiplied. Of the supporting tissues, consisting characteristically of cells with intercellular substance, connective-tissue has the matrix soft and homogeneous, with fibres imbedded in it ; in hyaline cartilage it is structureless and tough, though elastic; and in bone laminated and calcified. In the blood, the plasma may be looked upon as a kind of liquid intercellular substance.

Cells, wherever they occur, have the same essential structure, being formed of protoplasm with a nucleus. In nearly all cases they increase by binary fission, first the nucleus and then the protoplasm dividing into two.

The distribution of the various tissues throughout the body is worth noting. Epithelium always bounds a free surface-e.g., that covering the outer surface of the body or lining the inner surface of the enteric canal. Striped muscle forms the "flesh," unstriped muscle, e.g., the outer layer of the enteric canal (p. 70). Bone and cartilage form the framework of the body, while connectivetissue is the packing between the other tissues. 


\section{PRACTICAL DIRECTIONS}

General Strueture of the Compound Microscope.-The compound microscope, with which you must now become acquainted, consists of a strong stand (Fig. 3\%, a) from which rises a vertical pillar $(b)$. To the latter are attached - a horizontal plate or stage $(c)$, perforated in the centre with an aperture $(d)$, the size of which can be varied by means of a diaphragm; an adjustable mirror (e), placed below the stage: and a vertical tube $(\rho)$ attached above the stage by a horizontal arm. Two combinations of lenses are used ; an objective or object-glass $(h)$, consisting of a metal tube with two or more lenses fixed into it, which screws into the lower end of the tube : and an ocular or eyepiece (i), consisting of a metal cylinder with a lens at each end which slides into the upper end of the tube. It is this arrangement of lenses which forms the essential feature of the compound microscope: the object, placed on the stage, is magnified by the objective, and the magnified image, thrown into the interior of the tube, is further enlarged by the ocular.

The object is brought into focus -i.e., placed at such a distance from the objective that a perfectly clear and well-defined image is obtained-in one of two ways. The tube can be raised or lowered either by slicling it up and down in an outer tube or collar $(g)$, or, in the more cxpensive instruments, by a rack and pinion : this movement forms the coarse adjustinent. In addition, all good micro- 
scopes have a fine adjustment, usually consisting of a spring concealed in the pillar, and acting upon the horizontal arm which carries the tube : it is worked by a screw $(k)$, and by means of it the tube can be adjusted to within $1 \frac{1}{1000}$ th of an inch.

When the object is transparent-as in most cases with which we shall have to deal-it is placed over the hole in the stage on a glass slide, and illuminated from below by adjusting the mirror until a beam of light from the window or lamp is reflected vertically upwards: a small hole in the diaphragm should be used with the high power. The object is thus said to be viewed by transmitted light. In the case of opaque substances the mirror is not used, and the object is illuminated by the light falling upon it directly : it is then said to be viewed by reflected light.

A student's microscope should have two objectives, onethe low power-magnifying about 8o, the other-the high porver - about 300 to 400 diameters. One eye-piece is sufficient, and a sliding coarse adjustment is nearly as convenient as a rack and pinion, besides being cheaper and less likely to get out of order. The mistake often made in choosing a microscope is to get one of elaborate construction, the money going largely in brass-work. The proper thing is to get the simplest form of stand consistent with perfect rigidity, fitted with the best possible fine adjustment and lenses: to save on either of the latter is false economy.

Requisites for Microscopic Work.-In addition to the microscope, the following will be required before starting work :-

I. A few slides or slips of glass, 3 inches long by I inch wide, which can be obtained from an optician. They must be thoroughly cleaned before being used.

2. A supply (about $\frac{1}{2} \mathrm{oz}$.) of cover-glasses, small pieces of very thin glass, to be had at the optician's. The most convenient size is 3 inch square. They are best cleaned by being soaked for a few minutes in strong nitric acid, and then thoroughly washed under the tap, after which they should be dried by being placed flat on a clean surface and rubbed with a handkerchief: if held between the finger and thumb they are very liable to be broken.

3. One or two thin glass rods, about 6 inches long and $\frac{1}{8}$ th inch in diameter ; and one or two dipping tubes, or pieces of glass tubing about 6 inches long and 3 th inch in diameter. The ends both of rods and tubes should be smoothed off in the flame of a blow-pipe. 
4. Three or four dissecting needles, made by sticking a fine sewing needle into the end of a wooden penholder, allowing the point to project about half an inch.

5. A few reagent-bottles for holding the various fluids used for applying what are called micro-chemical tests to the tissues. Special bottles can be bought for the purpose, but sufficiently convenient ones can be made from ordinary one-ounce phials, fitted with sound corks. Bore a hole lengthwise through the cork, and insert into the hole a piece of narrow glass rod, pointed at the end, just long enough to reach nearly to the bottom of the bottle when the cork is inserter. This arrangement allows of the ready application of a single drop of fluid to the object under examination.

6. The following micro-chemical reagents :

a. Salt solution. Dissolve 0.75 gramme of sodium chloride in roo c.c. of distilled water, so as to make a per cent. solution.

b. Acetic acid, I per cent. One c.c. of strong acetic acid to 99 c.c. of distilled water.

c. Distilled water.

d. Solutions of one or two aniline dyes. For fresh tissues, dissolve enough methyl-green in distilled water to make a deep bluish-green solution, and add a trace of acetic acid. For preserved (or fresh) tissues, make a saturated solution of magenta or safranin in strong alcohol, and dilute with an equal bulk of water.

e. Glicerine, 50 per cent. Equal parts of pure glycerine and distilled water.

\section{Microseopical Examination of the Simple Tissues.}

For the following work, a freshly-killed frog must be used.

(If you wish to measure each kind of tissue-element, you must learn to use a micromeler, which consists of a circular piece of glass, marked at regular intervals with lines or squares, the distance between which can be calculated by comparing them with a scale engraved on a slide known as a stage-micrometer.)

I. The Blood.- - Have ready a clean dry slide and coverglass. In a freshly-killed frog open a vein or make an incision in the heart, and with a clean glass rod remove a drop of blood to the middle of a slide. Take hold of the edge of the cover-glass with small forceps, and supporting it with a mounted needle, gently lower it on to the drop of blood until the latter is sprear out into an even, transparent, yellowish film. This operation of covering the drop of bloat requires a little practice : if not done quickly (unless a drop of salt solution is added), there is danger of the blood 
coagulating before it is covered, in which case it will not spread out into a transparent layer : if the cover is lowered too suddenly, bubbles of air are commonly included.

Fxamine your preparation, first of all, under the low power, and learn to recognise the appearance of air-bubbles. Note the colourless plasma and the numerous minute bloodcells or corpuscles.

Now replace the low by the high power. Bear in mind that the higher the power, the shorter the focal distance. With the low power you will probably find that the object is in focus when about half an inch from the bottom lens of the objective. The high power, on the other hand, has to be brought to within about $\frac{1}{2}$ th of an inch of the coverglass, which is therefore liable to be broken and the lens to be injured by careless focussing. The safest plan is to screw down the tube, keeping your eye at the level of the stage, until the objective almost touches the cover-glass: then, looking through the microscope, very slowly raise the tube by means of the coarse adjustment, until the object comes into view. Note (Fig. 26) :

a. The numerous flat, oval, red corpuscles, each with a central swelling in which the nucleus is contained. Sketch.

b. The colourless corpuscles or leucocytes, much less numerous, having a granular appearance, and an irregular or rounded outline.

Focus a colourless corpuscle under the high power, and note its amoboid movements: sketch its outline rapidly but accurately, and after a minute or two make another sketch, and then another, until some half-dozen outline drawings of the corpuscle have been obtained: then compare your sketches.

Now place on the slide, against one edge of the cover-glass, a drop of methyl-green, and against the opposite edge a small strip of blotting paper. If the blood is sufficiently fresh-and if it has coagulated you must get another drop - the blotting paper will slowly absorb the blood on one side, and the methyl green will be drawn in and will gradually mingle with the blood. When this has taken place, put a drop of salt solution in the place of the methyl-green, and allow it to be drawn across so as to remove the superfuous stain: then remove the blotting paper, and examine the blood once more under the high power. Notice the nusleus present in each kind of corpuscle, and the surrounding protoplasm. Sketch.

To a fresh drop of blood add, in the same manner, a drop of I per cent. acetic acid. Note that the body of each cor- 
puscle becomes transparent, while the nucleus is rendered distinct.

To another drop of blood add distilled water. The corpuscles become swollen up and partly dissolved, and the colouring matter (hamoglobin) of the red corpuscles is dissolved out into the plasma.

Examine some coagulated blood under the microscope, and note the threads of fibrin in which the red corpuscles are entangled, and the serum.

Examine once more the circulation in the web (p. I03), using the high power, and follow the course of both red and colourless corpuscles through the vessels and capillaries. $\mathrm{By}$ focussing to the surface of the web the flattened epithelial cells of the epiderm or outer skin can be seen, and at a deeper level the black pigment-cells (see p. I28, and compare Figs. 24 and 27).

2. Columnar Epithelium.-Take a small piece of frog's intestine, and place it for 24 hours in a mixture called Ranvier's alcohol, consisting of one part of methylated spirit and two parts of water. With fine scissors snip off a very small piece-not larger than a pin's head-from the inner surface of the mucous membrane, place it on a slide in a drop of water, and with two dissecting needles tease it out by tearing it into the smallest possible particles. The operation is best done under a lens. Then put on a cover-glass, and examine first with the low and then with the high power. (Remember that a cover-glass must always be used with the high power.)

Note the minute, more or less conical cells of columnar epithelium (Fig. 28), each containing a nucleus. Observe the goblet-cells amongst the ordinary columnar cel!s. Stain with magenta, which is more effective than methyl-green in specimens previously treated with alcohol, add a trace of acetic acid, wash with water (salt solution need only be employed in the case of fresh tissues), and add glycerine. Sketch.

3. Ciliated Epithelium.-Snip off a very small bit of mucous membrane from the roof of the mouth of a recentlykilled frog, and tease it out in salt solution (Fig. 29).

Note the form of the cells and their nuclei : they are relatively shorter than the columnar cells just examined, and each bears a number of delicate vibratile cilin at its free end. Observe the movements of the cilia. Sketch.

Treit with methyl-green, magenta, or acetic acid, when the nucleus will become more apparent.

Perform experiment described on $p$. I io to show the action of the cilia as a whole in the entire animal. 
4. Pavement or Squamous Epithelium.-Take a bit of frog's skin which has been kept for a day or two in Ranvier's alcohol, scrape the outer surface with a sharp knife, tease out and examine the edges of the scrapings in a drop of water, afterwards staining with magenta (Fig. 30).

Note the flattened cells fitting together like tiles in a pavement, each one with its nucleus. Sketch.

5. Unstriped Muscle.-Snip off a small piece from an inflated urinary bladder of a frog which has been preserved in formaline, wash with water, and mount. Or, snip off a very small piece-not bigger than a pin's head-from the muscular coat of the intestine or stomach (or from a urinary bladder) which has been in Ranvier's alcohol for at least twenty-four hours; then tease out in a drop of water very thoroughly. Note the elongated unstriped muscular fibres tapering at both ends, each containing a nucleus (Fig. 3I). Stain with magenta. Sketch.

6. Striped Muscle.-Snip off a small piece-about $\frac{1}{8}$ th inch long-from any of the body-muscles of a freshly-killed frog, put it on a slide in a drop of salt solution, and tease it out with the grain, i.e., in the direction of the length of the fibres. The fibres will readily separate from one another: the teasing process must be stopped as soon as they are apart, and care must be taken not to tear or crush the individual fibres, which are large enough to be readily distinguishable with a magnifying-glass.

Observe under the low power of the microscope the long cylindrical fibres (Fig. 32, A), bound together by connectivetissue, and showing a distinct transverse striation and a less distinct longitudinal striation. Examine a single fibre under the high power (Fig. 32, B), and make out the sarcolemma and the numerous nuclei, which will be rendered more distinct by the addition of acetic acid or by staining. Sketch.

7. Connective-tissue.-Carefully separate two of the muscles of the leg in a fresh frog, and note the delicate web of connective-tissue between them : or, note the fine strands of connective-tissue between the skin and the muscles of the body-wall. With fine forceps lift up a small shred of this, snip it off with scissors, and place it on a dry slide. Then, with two needles, spread it out on a thin, even layer, breathing on it occasionally to prevent clrying. Iastly, place a drop of salt solution on a cover-glass and quickly lower it on the preparation. The reason for this procedure is that if connective-tissue is placed in fluid, it contracts into a little lump, which is too opaque for examination and cannot be readily spread out. 
Examine first with the low and then with the high power, and note the bundles of white fibres, and the elastic fibres (Fig. 33). Sketch.

Add acetic acid : the white fibres will swell up, the elastic fibres are more readily distinguished, and the connectivetisswe cells seen : the latter and the delicate ground-substance will be rendered more distinct by staining. (For comparison with this areolar tissue, mount in salt solution a piece of one of the fine tendons of the toes: treat as before, and examine.) Sketch.

8. Cartilage.-Snip off the thin edge of the omo- or xiphisternum or supra-scapula and examine it as before in a drop of salt-solution. Or, cut a thin section of the head of the humerus or femur with a razor.

Note the transparent, homogeneous matrix, containing numerous lacuna, in each of which is a nucleated cell: observe here and there the groups of cells formed by binary fission (Fig. 34). Stain, and sketch.

9. Bone.-For the examination of dried bone, cut a very thin slice of one of the long bones with a fret-saw : fasten it to a slide with Canada balsam (p. I 36). and when the balsam has dried quite hard, rub down the section on a hone until it is thin enough to be quite transparent. Or, a transverse section of a bone from a larger animal may be prepared in the same way or bought trom a dealer in microscopic objects.

a. Examine first a transverse section of dry frog's bone (e.g. femur or humerus), and note the marrow-cavity, the lamella, and the lacunce and canaliculi; the two last will probably appear black (p. II 7 and Fig. 35). A section of human bone, such as is usually supplied ready prepared, or of the bone of some other larger animal, shows a more complicated structure : instead of a single system of lamellæ, the bone consists of a number of such systems, each surrounding a central canal, in which blood-vessels and nerves run, and which corresponds to the marrow-cavity in the simpler frog's bone described above. Sketch.

b. Compare with a section of decalcified frog's bone, ${ }^{1}$ and notice-the fibrous lamella arranged in two layers, the outer of which is closely invested by the periosteum; the lacuna, containing bone-cells; and the outer and inner layers of osteoblasts (Fig. 36). Sketch.

(For the histology of nerious tissue, see Chapter X.)

1 The method of preparing sections of this and other tissues will be described at the end of the next chapter (p. I 36). 


\section{CHAPTER VIII}

THE FROG (continued): THE MICROSCOPIC EXAMINATION OF THE COMPOUND TISSUES-GLANDS-SECRETION AND ABSORPTION

Wrth the exception of the tissues of the nervous system, which will be described in Chapter $\mathrm{X}$, we have now studied the principal simple tissues by the method of dissociation, i.e., by separating their constituent parts. We have now to consider the way in which these tissues are combined in the various organs, and for this purpose must adopt some method of examination by which they are seen in their natural relations.

The method adopted for this purpose is that of sectioncutting. You know how, by cutting sections, in various directions, of a bit of twig, the arrangement and natural relations of its various parts-wood, bark, and pith-can be ascertained. The same thing applies to the organs of the frog and other animals, but, owing to their soft and non-resistant texture, it is impossible to cut them into sections thin enough for microscopic examination without special preparation. The methods employed are by no means easy for the beginner, especially without verbal instruction and the resources of a biological laboratory; but in the event of your wishing to make the preparations described and figured in this chapter for 
yourself, the directions given on pp. $136-139$ are simple enough to be carried out with very limited appliances.

The Skin.-A vertical section of the skin, i.e., one

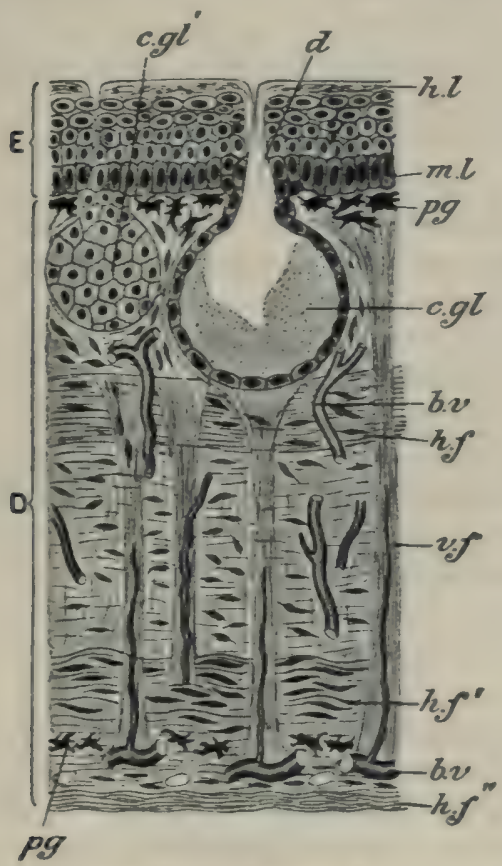

FiG. 38.-Vertical section of the Frog's skin, highly magnified. (. about 200.)

D. derm, formed of $h f, h f^{\prime} h f_{\text {. }}$, horizontal, and $\varepsilon$. $f$. vertical fibres of connective. tissue, and containing $b . v$. blood-vessels, and pg. pigment cells. E. epiderm, with $m . l$. active or Malpighian layer, and $h$. $l$. horny layer of epithelial cells ; c. gl. cutaneous gland in section; c. gl'. in surface view ; $d$. duct. (After Howes.)

taken at right angles to its surface, will be seen to have the following structure.

The skin is clearly divisible into two layers, an outer, 
the epiderm (Fig. 38, E) and an inner, the derm (D). The epiderm is built up of several layers of epithelial cells. These differ greatly in form according to their position, those in the lower or internal layer $(m . l)$ being columnar, while those in the upper or external layer $(h . l)$ are squamous, and have their protoplasm converted into horny matter so as to furnish a comparatively hard and insensitive covering to the body.

The horny layer is cast off periodically in shreds, and to make up for this, the cells of the inner, or deep, layer multiply by binary fission, the increase in their number necessarily resulting in a pushing upwards of the superjacent layers. There is thus a constant travelling of cells from the inner to the outer surface of the epiderm : as they pass towards the outer surface they become more and more flattened, and at last squamous and horny. The whole process takes place in such a way that the multiplication of the columnar cells in the lower layer is just sufficient to make good the loss of the squamous cells in the superficial layer.

The derm (D) is formed of connective-tissue, the fibres of which are mostly horizontal $\left(h . f, h . f^{\prime}, h . f^{\prime \prime}\right)$, or parallel to the surface of the skin, but at intervals are found bands of vertical fibres $(v . f)$. The derm also differs from the epiderm in having an abundant bloodsupply $(b, v)$, capillaries ramifying through it in all directions. It also contains nerves, the ultimate fibres of which have been traced into the deeper layers of the epiderm. Imbedded in the derm, especially in its external portion, are irregular cells $(p g)$, the protoplasm of which contains pigment, often appearing intensely black. It is to these pigment-cells that the coloured patches in the frog's skin are due (Figs. 24 and 27).

In this as well as in the other sections described in 
the present chapter, the structure of the nuclei of the various cells can be more easily made out than in the fresh preparations you have already examined. Each nucleus will be seen to be enclosed by a definite nuclear membrane, and to contain in its interior a number of minute bodies, which take up the stain more deeply than the rest of the nucleus. One or more of these bodies may correspond to the nucleoli already seen (p. I09), but many of them are of a different nature and can often be seen to form a network : the material of which these are composed is known as chromatin, and is surrounded by a semi-fluid substance forming the groundwork of the nucleus.

Cutaneous Glands-Secretion.-In the superficial part of the derm are seen numerous rounded spaces (Fig. $38, c . g l, c . g l^{\prime}$ ), each of which can be proved, by taking sections in various directions, to be a nearly globular cavity, from which a narrow canal $(d)$, like the neck of a flask, passes through the epiderm to open on the external surface. Both the body and the neck of the flask are lined with epithelium, the cells lining the body being nearly cubical, those of the neck squamous.

These structures are called cutaneous glands: they perform the function of manufacturing the slimy fluid which, as we have seen, is constantly exuding on the surface of the skin. The epithelial cells of the gland have the power of forming minute droplets of the fluid out of the materials supplied to them by the blood : the droplets escape from the cells and accumulate in the interior of the gland, whence the fluid is finally discharged by the duct.

The cells lining the duct are continuous on the one hand with those of the gland, and on the other with those of the epiderm. The whole gland with its duct 
is to be looked upon as a depression of the skin, lined by pushed-in epiderm cells.

Epithelial cells having the power of manufacturing and discharging a specific substance are called gland-cells, and the process of manufacture is known as secretion. We have already met with isolated gland-cells in the case of the goblet-cells of the intestine (p. rog), which secrete mucus; but commonly, as in the present in-
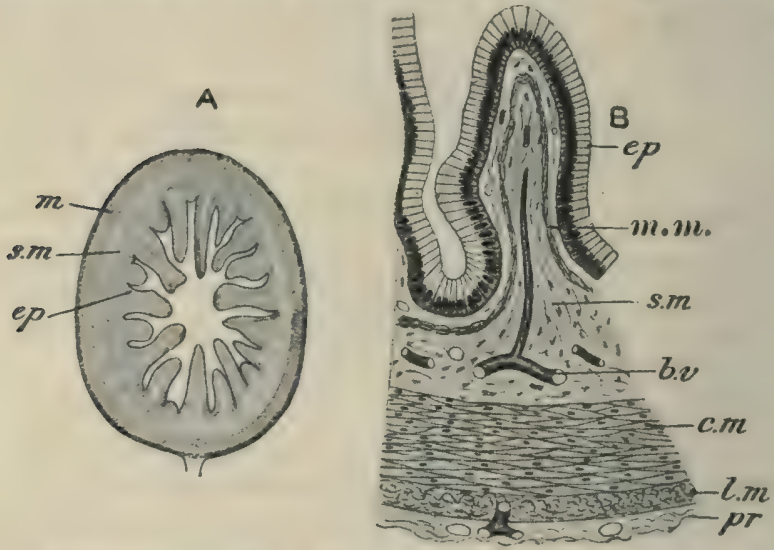

FIG. 39.-A, transverse section of Frog's ileum $(\checkmark 25)$; B, small portion of the same highly magnified. ( $X$ about 125.)

b. $v$. blood-vessel; $c$. $m$. circular layer of muscle-fibres ; ep. epithelium; $1 . m$. longitudinal layer of muscle-fibres; $m$. $m$. muscularis mucosa (sec p. 133 ); pr. peritoneum; m. muscular layer; s. m. submucosa. (After Howes.)

stance, gland-cells are aggregated into a definite organ called a gland.

The Intestine.-A transverse section of the intestine shows also a very definite and characteristic combination of simple tissues. The mucous membrane, like the skin, is composed of two layers, an epithelial layer (Fig. 39, ep), corresponding to the epiderm, and a connective-tissue layer $(s, m)$, corresponding to the derm, its deeper part being called the submucosa (see 
p. 7o). The epithelium consists of a single layer of cells only $(\mathrm{B}, e p)$, all columnar, and with their long axes at right angles to the elevations into which, as we have seen ( $\mathrm{p} .7 \mathrm{I}$ ), the mucous membrane is thrown. Amongst the ordinary epithelial cells numerous mucus-secreting goblet-cells will be recognised. The submucosa, like the derm, contains blood-vessels, lymphatics, and nerves.

The muscular layer $(\mathrm{A}, m)$ is also divisible into two: an outer layer of longitudinal fibres $(\mathrm{B}, l, m)$, running parallel with the long axis of the tube, and an inner, much thicker layer of circular fibres (c. $m$ ) which encircle it, and consequently lie at right angles to the longitudinal fibres. Thus in a transverse section, such as Fig. 39, the fibres of the circular layer are cut longitudinally, those of the longitudinal layer transversely, while the opposite would be the case in a longitudinal section.

The peritoneum ( $p r)$ which, as we have seen (p. 27). forms an outer covering to the intestine, is formed of a thin inner layer of connective-tissue and an outer of squamous epithelium.

The Stomach.--Transverse sections of the stomach (Fig. 40) show it to differ from the intestine not only in the much greater thickness of all its layers, but aiso in the fact that the epithelium, instead of simply forming an even layer over the ridges of mucous membrane, is sunk into the mucous membrane in the form of simple or branched tubes, the gastric glands $(g . g l)$. These differ from the cutaneous glands in being not flask-shaped but test-tube-shaped, each being a long, narrow tube, with an extremely small cavity (B and $C, c)$. They are linęd by a single layer of gland-cells, and open by minute apertures $(m)$ on the surface of the mucous membrane.

The cells of the gastric glands have the power of 
forming, out of the materials supplied to them by the blood, the gastric juice, by which, as we have learnt (p. 74), proteids are digested. Thus, while the raw material supplied to both cutaneous and gastric glands is the same, the manufactured article is entirely different

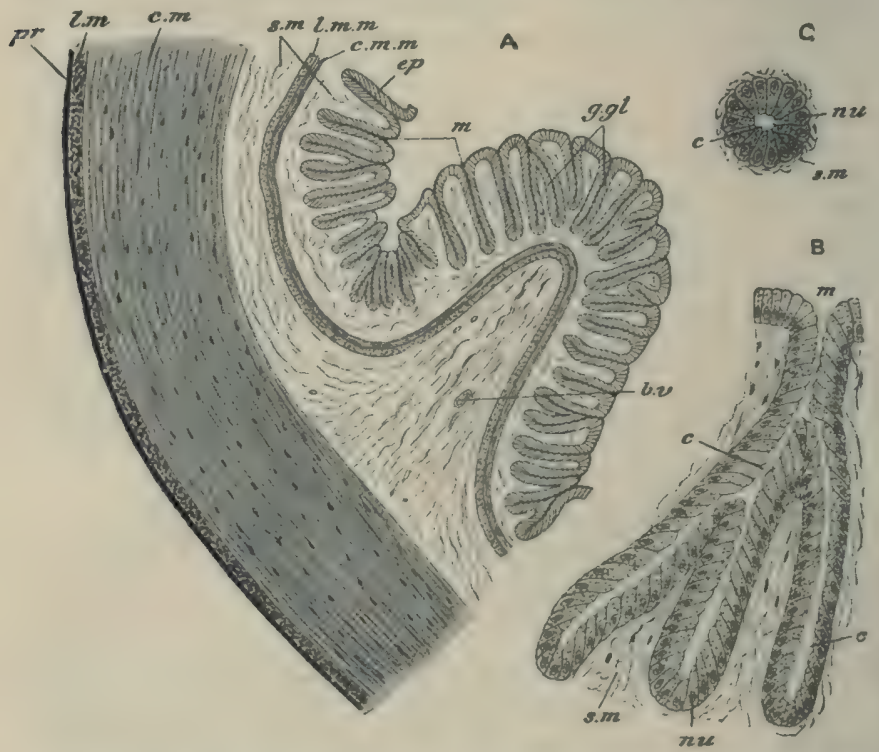

FIG. 40.-A, part of a transverse section of the Frog's stomach ( $x$ about 50); B, one of the gastric glands in longitudinal section ( $X$ about 300$) ; C$, transverse section of a gastric gland.

b. $v$. blood-vessel; $c$. cavity of gastric gland; $c$. $m$. circular muscles; $c . m . m$. circular layer of muscularis mucose; ; ep. epithelium; $g . g l$. gastric glands; $l$. $m$. longitudinal muscles; $l, m$. $m$. longitudinal layer of muscularis mucosæ; m. mouth of gastric gland; nu. nucleus; pr. peritoneum; s. m. mucous membrane: the submucosa is external to the muscularis mucosæ.

in the two cases. Each kind of gland-cell has the faculty of picking and choosing, the material supplied being worked up in the one case into the cutaneous secretion, in the other into gastric juice.

The mucous membrane of the stomach is traversed 
by a narrow band of unstriped muscle, the muscularis mucose, formed, like the main muscular layer, of an outer layer of longitudinal $(l . m . m)$ and an inner of circular (c. m. m) fibres. ${ }^{1}$

The Pancreas.- Sections of this gland (Fig. fI) show it to be made up of microscopic masses or lobules $(l)$, each of which consists of a cluster of gland-cells enclosing a very narrow central space. The cavities of adjacent lobules communicate with one another and
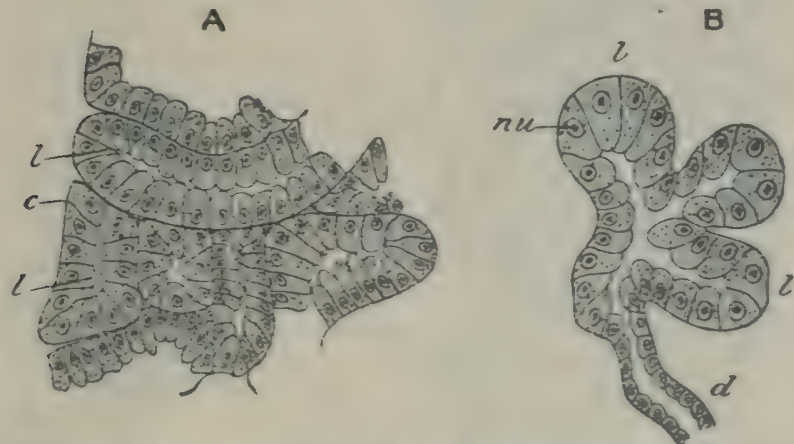

FIG. 41.-A, small portion of a section of the Froy's pancreas ( $<$ about 300$)$; B. diagram showing the connection between the lobules and ducts.

$c$. connective-tissue covering the lobules; $d$. duct; 1 . lobules; $s$ su. nuclei.

open into tubes or ducts (d) lined with epithelium, which join with one another and finally discharge into the bile-duct as it traverses the pancreas (p. 70). The pancreas is distinguished as a racemose or grape-bunch gland: the duct is comparable to the branched stalk and the lobules to the grapes.

The Liver.- Sections of the liver show it to be made up of innumerable large, polyhedral cells (Fig. $42, h p, c$ ), arranged in columns, and bounding extremely fine

1 A very thin muscularis mucosæ is also present in the intes. tine; (compare Fig. 39, B). 
channels or bile-passages $(c . b, b . p)$. These are found to open into one another, and finally to discharge into definite tubes $(B, d)$, lined with epithelium. These, in their turn, unite into larger and larger tubes, which
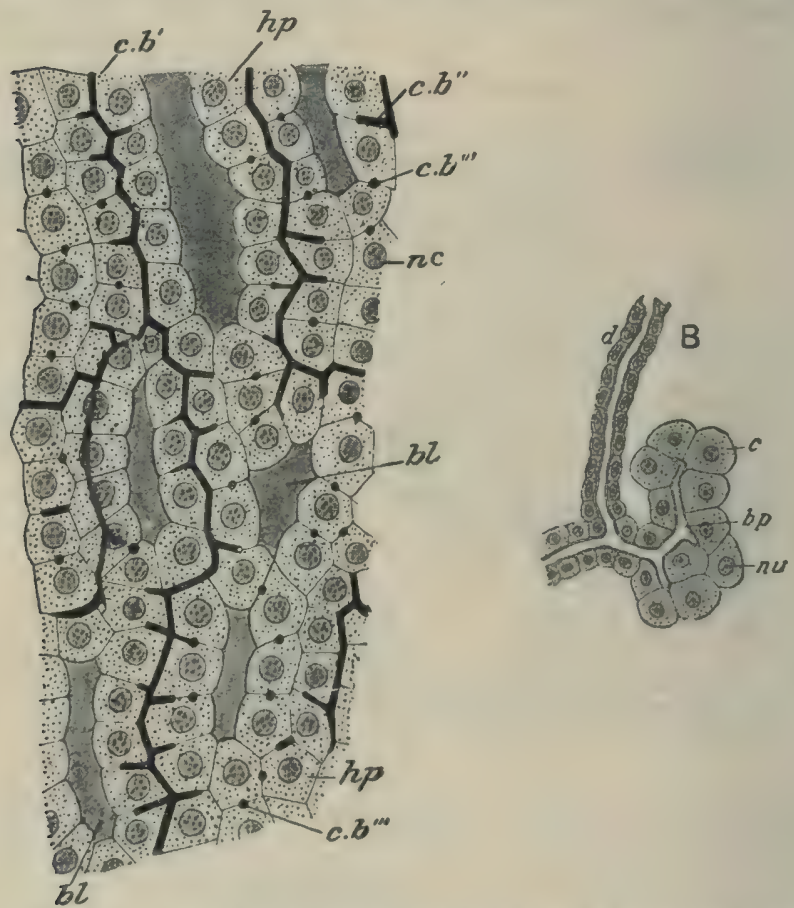

FIG. 42.-A, portion of a section of the Frog's liver $(\times 350) ; B$, small portion of the same, showing the origin of a bile-duct.

bl. blood-capillaries; $b . p ., c . b ., c . b^{\prime}, c . b^{\prime \prime}$, bile-passages; $c . b^{\prime \prime \prime}$, the same in transverse section; $c$., $h p$. liver-cells; $d$. smallest bile-duct ; $n c$, nu. nuclei. (A, after Howes; B, after Hoffmann.)

form the hepatic ducts and ultimately open into the common bile-duct (p. 68).

The liver-cells are glandular and secrete the bile, which, as it is formed, exudes into the bile-passages and 
passes into the hepatic ducts, thence making its way either directly into the intestine or into the gall-bladder (p. 7o). The whole liver, which is the largest gland in the body, is traversed by a complex network of capillaries $(b l)$, arising partly from the hepatic artery, partly from the hepatic portal vein; and from the blood thus supplied, the liver-cells obtain the materials necessary to enable them to discharge their function of secreting the bile.

The liver-cells have, however, other functions, one of which is to manufacture a substance called glycogen or animal-starch. This is stored up in the cells in the form of minute insoluble granules, which, being afterwards transformed into soluble sugar, pass into the blood and so to the tissues.

\section{Connection of the foregoing facts with the Physiology} of Nutrition.- You will now be able to understand more clearly the various processes connected with the nutrition of the frog, hitherto studied without the aid of histology.

When the food enters the enteric canal the various gland-cells are stimulated into activity, and the gastric juice, bile, and pancreatic juice are poured out and mingled with the food, which is digested in the manner already described. The soluble products of digestionpeptones, sugar, salts, fatty acids, and glycerine-diffuse through the epithelium of the enteric canal into the bloodcapillaries of the underlying mucous membrane, and the blood, now loaded with nutriment, is carried by the portal vein to the liver and thence by the hepatic and postcaval veins to the heart (see Fig. 23). At the same time the fats make their way into the lymph-capillaries and are finally pumped, by the lymph-hearts, into the veins. Thus the products of digestion all find their way ultimately into the blood, and are distributed, through the circulatory mechanism, to all parts of the body. 


\section{PRACTICAL DIRECTIONS}

\section{Materials required for the Preparation and Sectioning} of Animal Tissues.--In addition to the requisites mentioned in Chapters I and VII, the following will be required :-

a. Corrosive sublimate: a saturated solution in water. Care should be taken in using this solution, as it is a very virulent poison.

b. Absolute alcohol.

c. Turpentine or xylol.

d. Paraffin: " hard " and "soft." Get an ounce or two of each.

e. A solution of Canada balsam in chloroform, xylol, or turpentine. This should be kept in a small glass bottle with a ground glass cap-not a stopper or cork.

$f$. A solution of alcoholic borax carmine. This (like many other useful staining-solutions, such as Ehrlich's hamatoxylin) may be bought ready prepared : or it may be made as follows :-Grind up in a mortar 2 grammes of carmine and 4 grammes of borax, and dissolve in Ioo c.c. of distilled water : to this solution add an equal volume of 70 per cent. alcohol : allow to stand for a day or two and filter.

g. A water-bath, in which melted paraffin may be kept at a constant temperature. For a makeshift you can use a saucepan with a flat piece of tin over it: fill the saucepan about half full of water, and heat it over a spirit-lamp or a small oil-lamp or gas-burner, regulating the distance of the flame so as to keep the temperature of the water at about $55^{\circ} \mathrm{C} \cdot\left(13 \mathrm{I}^{\circ} \mathrm{F}\right.$.).

$h$. Two or three watch-glasses or other small shallow vessels for containing melted paraffin.

i. A sharp, flat-ground razor.

$j$. A section-lifter, made by beating out flat about half an inch of the end of a piece of stout copper wire, about 6 in. long, and bending the flattened portion at an obtuse angle with the rest.

\section{Preparation of Tissues for Section-Cutting.}

a. Fixing, hardening, and decalcifying.

Sections may be cut from specimens which have been carefully preserved in alcohol :-first in 70 per cent., and after a day or two transferred to 90 per cent. But certain other reagents are more effective for the purpose of fixing the 
tissues-i.e., quickly killing and coagulating the protoplasm of the cells with a minimum of shrinkage,-and of these the one most generally useful is a solution of corrosive sublimate (see $a$, above), in which, from a freshly-killed frog, place small pieces of the various organs and tissues to be examined - e.g., skin, intestine, stomach, liver, pancreas, kidney, ovary, spermary, and spinal cord, as well as the inner half of the eye-ball. The intestine and stomach should be first washed out in salt solution, and then cut into pieces about 1 inch long; the liver should be cut into pieces not more than $\frac{1}{4}$ inch cube. After about half-an-hour to two hours, according to the size of the piece, place in water under a tap, and wash thoroughly for a quarter of an hour or more, until the corrosive sublimate is removed.

After washing, transfer to 50 per cent. alcohol for a few hours, and then to 70 per cent. for twenty-four hours, after which the pieces may be stained at once (see below), or transferred to strong methylated spirit (90-93 per cent.), in which they mav be kept until wanted. This completes the process of hardening : it is done gradually, by alcohols of increasing strength, in order to avoid shrinkage.

In order to decalcify such tissues as bone, from which the lime-salts must be extracted before cutting into sections, place a small piece for a few clays in jo per cent. alcohol to which 2 per cent. of strong nitric acid has been added : then wash thoroughly, transfer to alcohol, and stain.

b. Staining.-Place the organs, cut into convenient sizes for imbedding-i.e., not more than $\frac{1}{4}$ inch long, and, in the case of such organs as the liver, inch in thickness-into borax-carmine or hamatoxylin for one or two days or even more. They will become stained throughout, and the difficulty of staining the sections after cutting will thus be avoided. After staining, place them in weak alcohol $150-70$ per cent.), slightly acidulated with hydrochloric acid : if a watch-glass or some such vessel is used, it is sufficient to dip the end of a glass rod into acid and stir it round in the alcohol. The effect of the acid is to remore much of the colour from the protoplasm of the cells, leaving the nuclei brightly tinted. After half-an-hour or less in the acid alcohol, place the tissues once more in strong methylated spirit.

c. Dehvdrating.-Transfer from the strong methylated spirit to absolute alcohol, which must be kept in a stoppered or tightly corked bottle, as it will otherwise deteriorate by absorption of water from the air. It has the effect of withdrawing the last traces of water from the tissues, an abso- 
lutely necessary step in order that they may be permeated with paraffin.

d. Imbedding.-Transfer the objects from absolute alcohol to turpentine or xylol. Either of these fluids acts as an intermediary between alcohol and paraffin, being freely miscible with both : it gradually replaces the alcohol in the tissues, rendering them transparent. If they are not transparent in the course of an hour or so, the process of dehydration has not been complete, and they must be returned to absolute alcohol. In the meantime, melt some paraffin over the water-bath, using various mixtures of hard and soft according to the season : in a cold room in winter

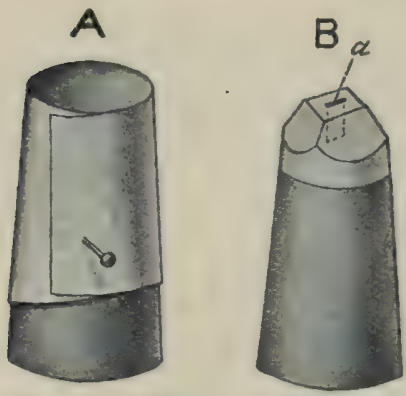

FIG, 43.-A, imbedding box made by wrapping paper round a cork ; $\mathrm{B}$, cork after removal of the paper, showing the paraffin pared down to a convenient size for sectioning. $a$, object to be cut. soft paraffin will be hard enough; in the height of summer hard paraffin alone will be suitable. The temperature of the water-bath must never be allowed to rise more than a degree or two above the meltingpoint of the paraffin. Transfer the objects from turpentine or xylol to melted paraffin and keep them in it for some hours -the time varies according to the size of the pieceuntil they are thoroughly permeated.

If you wish to cut sections by hand, get some ordinary medium - sized bottle-corks : around each wind a piece of paper, allowing it to project about $\frac{1}{2}$ an inch beyond one end of the cork, and fixing it with a pin, as in Fig. 43, A. Into the little cylindrical vessel or imbedding box thus formed pour some melted paraffin, and immediately transfer to it, by means of a warmed sectionlifter or forceps, one of the prepared pieces, adjusting its position by means of a heated needle. When the paraffin is quite cold remove the paper, and you will have fixed to the cork a solid block of paraffin containing the object to be cut.

e. Section-cutting.-Pare away the block of paraffin until the object comes into view: then trim the block, as in Fig. 43, B, until its upper surface, with the object in the 
middle, is not more than $\frac{1}{1}$ inch square. Hold the cork firmly in the left hand, with the wrist resting on the table, and with a razor cut the thinnest possible slices of the paraffin block, including the imbedded object. The razor must be held firmly grasped at the junction of blade and handle, and kept with the surface of the blade parallel with that of the block: use almost the whole length of the edge for each section. With a little practice you will be able to cut sections so thin as to be quite transparent under the high power. ${ }^{1}$

$f$. Clearing and mounting.-Place the section on a slide and warm it gently on the water-bath until the paraffin melts, and then add a large drop of turpentine or xylol in order to dissolve the paraffin. Then draw off the turpentine with blotting-paper and replace it by a fresh drop, repeating the process until all the paraffin is dissolved: put on a cover-glass and examine.

If you wish to be sure that the parts of your sections are not displaced in mounting, or to mount several sections on one slide, the latter should first be smeared over with a very thin laver of a mixture of collodion and oil of cloces, or of glycerine and white of egg, in equal parts: then place the sections on the slide, warm, and immerse the whole slide in a small vessel of turpentine or xylol, leaving it until all the paraffin is dissolved.

In order to make a permanent preparation, remove the paraffin with turpentine or xylol, as above, draw off the turpentine, place a drop of Canada balsam on a coverglass and very gently lower the cover-glass on the object, spreading out the balsam in a thin, even layer. Before long, the balsam will have set quite hard, and the sections may be preserved for an indefinite period: the balsam will set more quickly if you leave your preparations over the water-bath for a short time.

Remember that object, razor, slide, and cover must be kept free from water, the presence of which, from the stage of dehydration onwards, is fatal to success.

\section{Examination of Compound Tissues.}

Examine the following sections, prepared as described above, first with the low, and then with the high power,

1 If you are working in a properly furnished laboratory you will probably learn how to cut sections with a microtsme, or sectioncutting machine, which gives much better results and is absolutely necessary when a complete series of sections of the same object is required. 
noting the parts enumerated in each case, as well as the structure of the nuclei (nuclear membrane, chromatin, and nucleoli).

I. Vertical Section of Skin (Fig. 38).

a. Epiderm, stratified epithelium, divisible into outer (horny) and inner (Malpighian) layers.

b. Derm, connective-tissue fibres, blood-vessels and pigment-cells.

c. Cutaneous glands with their ducts. The apertures of the ducts on the surface you will probably have noticed already in your preparation of the epithelial cells of the skin (p. I24).

Sketch.

\section{Transverse Section of Intestine (Fig. 39).}

a. Mucous membrane: a superficial epithelial layer of columnar cells, with goblet-cells amongst them; and a deeper connective-tissue layer, enclosing vessels and nerves.

b. Muscular layer: an external longitudinal, and an internal circular layer of unstriped muscular fibres.

c. Peritoneal layer. This is very thin, and a careful examination of good preparations is required in order to make out its structure (p. I3I).

Sketch.

3. Transverse Section of Stomach (Fig. 40).-After recognising the layers as above, note :-

a. The gastric glands, and $b$. the muscularis mucose.

Sketch.

4. Sections of Pancreas (Fig. 4I).

a. Lobules, separated by connective-tissue, and each consisting of gland-cells with secretion-granules towards their inner ends; $b$. ducts.

Sketch.

5. Sectiors of Liver (Fig. 42).

a. Polyhedral gland-cells, arranged in columns; $b$. bile. passages and ducts; c. blood-capillaries and vessels.

Sketch. 


\section{CHAPTER IX}

THE FROG (continued): RESPIRATION AND EXCRETION

IN the fifth chapter it was pointed out that a continual waste of substance goes on in the frog's body, the lost material taking the form of three chief waste-products or products of excretion, water, carbon dioxide, and urea. It was further stated that these substances are got rid of by means of the lungs, the kidneys, and the skin.

The Organs of Respiration.-At their anterior ends the two lungs open into a small, thin-walled chamber (Fig. 44, l. $t r . c)$, which, as it corresponds both with the larynx or organ of voice, and the trachea or windpipe in ourselves, is called the laryngo-tracheal chamber: it communicates with the pharynx through the glottis $(g l)$. The walls of the chamber and the edges of the glottis are supported by cartilages (ar).

The structure of the lung is best made out by distending it with air, and then placing it in formaline or spirit until thoroughly hardened: its walls contain so much elastic tissue that if cut when fresh it contracts immensely, and its structure is then difficult to see. The inner surface of the lung is raised up into a conplex network of ridges $(A, r . \operatorname{lng})$, which project into the interior and produce the appearance of an irregular honeycomb. All these ridges are abundantly supplied 
with blood-vessels fed by the pulmonary artery, the blood being carried away by the pulmonary vein.

The main substance of the lung is made of connectivetissue containing elastic fibres and unstriped muscle, and traversed by a network of capillaries. Its cavity is lined by a layer of pavement epithelium, and its outer surface is covered with peritoneum.

Respiratory Movements. - In breathing, the frog depresses the floor of the buccal cavity (Fig. 45, A), and

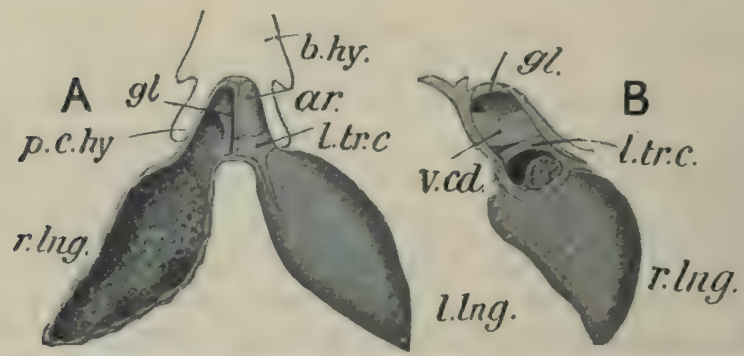

FIG.44.- The respiratory organs of the Frog from the ventral aspect; $B$, the laryngotracheal chamber in longitudinal section, with the right lung. ( $\therefore$ I .)

ar. the arytenoid, or principal cartilage of the larynx; $b . h y$. body of hyoid; $g l$. glottis; $l . \operatorname{lng}$. left lung; l. tr. c. laryngo-tracheal chamber; $p$. c. hy. posterior horn of hyoid; $r$. li:g. right lung, laid open in A to show its internal surface; v. $c d$. vocal cord. (After Howes.)

the mouth being kept shut, air is drawn in through the nostrils. The floor of the mouth is then raised (B) by muscles attached to the hyoid. At the same time the anterior end of the lower jaw presses upon the movable premaxillæ $(p m x)$, the upward processes of which (p. 43 , Figs. 8 and $9, P M X)$ act upon certain cartilages in connection with the external nostrils in such a way as to produce closure of these apertures (Fig. $45, B$ ). The gullet $(g u l)$ is so contracted, except during the act of swallowing, as to be practically closed. Thus when the floor of the mouth is raised, the air contained in it can 
escape in one way only, viz., through the glottis into the lungs.

Thus inspiration, or breathing-in, is produced by the buccal cavity acting as a force-pump ; the lowering of its floor draws in air through the nostrils, the raising of its floor forces the imprisoned air into the lungs.
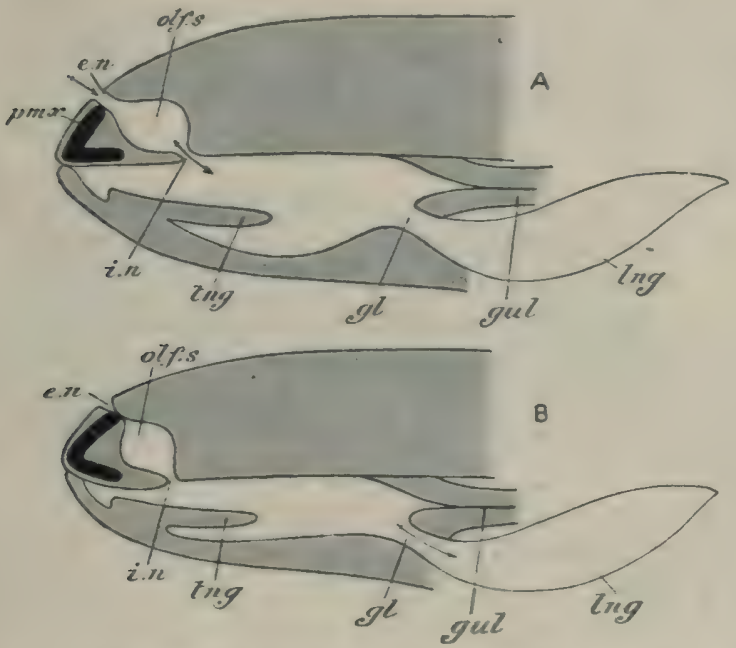

Fic. 45-Diagrams illustrating the respiratory movements of the Frog $(\because z)$. In $\mathbf{A}$ the floor of the mouth is depressed anr air is being drawn in through the nostrils; in $\mathrm{B}$ the floor of the mouth is raised, the nostrils are closed, and air is being forced in to the lungs.

e. n. external nostril; gl. glottis ; gu?. gullet; i. n. internal nostril ; Ing. lung; olf.s. olfactory sac; pmx. premaxilla; $t$ g. tongue.

Expiration, or breathing-out, is due to a contraction of the elastic lungs, accompanied by a slight lowering of the tip of the lower jaw: the latter movement releases the premaxillæ and thus opens the external nostrils.

Respiration.-By the alternate movements of inspira. 
tion and expiration, fresh air passes into the lungs at regular intervals, while part of the air already contained in them is expelled. Now we saw, when studying the blood (p. 79), that dark purple blood drawn from a vein becomes bright scarlet when exposed to air, and we subsequently learnt (p. I07) that this change is due to. the absorption of oxygen by the red corpuscles.

The blood brought to the lungs by the pulmonary artery is, as we have seen (p. 94), non-aërated, being the impure blood returned by the three caval veins to the right auricle. When this blood is pumped into the capillaries of the lungs it is separated from the air contained in those organs only by the extremely thin walls of the capillaries themselves and the equally delicate pavement epithelium lining the lungs (p. I42, and Fig. 23, $C p$. lng, Ep.lng). Under these circumstances an interchange of gases takes place between the air in the lungs and the gases dissolved in the blood: the hæmoglobin of the red corpuscles absorbs oxygen, and the carbon dioxide in the blood, derived from the waste of the tissue, is given off into the cavities of the lungs. The blood in the pulmonary capillaries thus becomes aërated and is returned as red blood to the left auricle: at the same time it loses carbon dioxide, together with a certain amount of water, and these waste substances are expelled from the body with the expired air.

Voice.-It was mentioned above (p. I4I) that the glottis and laryngo-tracheal chamber are supported by cartilages. The largest of these are a pair of semilunar arytenoid cartilages (Fig. 44, ar), which bound the glottis to right and left. The mucous membrane on the inner or adjacent faces of the arytenoids is raised into a pair-right and left-of horizontal folds, the vocal cords $(v$. $c d)$. By means of muscles these folds can be stretched and relaxed, and can be brought into either a 
parallel or a divergent position. When they are parallel the air, passing to and from the lungs, sets their edges in vibration and gives rise to the characteristic croak, the pitch of which can be slightly altered by stretching or relaxing the cords.

Structure of the Kidneys. - The form and situation of the kidneys (Figs. 3, 4,5 , and $7, k d$ ) have already been referred to. Each is a flattened organ of a deep reddishbrown colour, its inner edge nearly straight but for one or two notches, its outer edge curved. Its ventral face

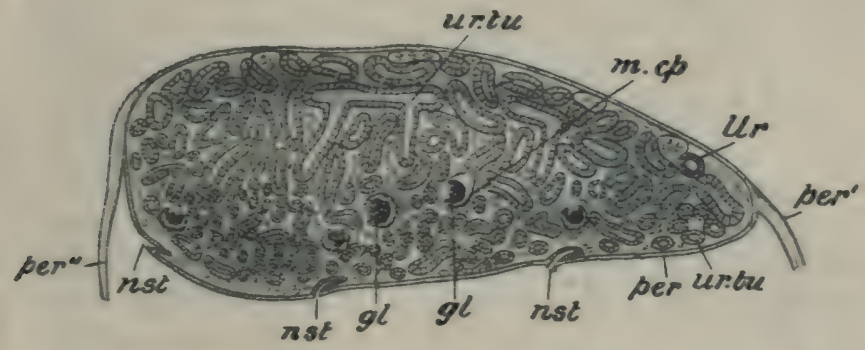

Fig. 46.-Transwerse section of Frog's kiuney. $(x+45$.

gl. glomerulus; $m$. cp. Malpighian capsule; nst. nephrostome (see pp. $9 \$$ and 60I) ; per. peritoneum covering ventral face of kidney : per'. fold of peritoneum supporting its outer border: per". fold supporting its inner border ; $C r$. ureter ; ur. tu. urinary tubules. (After Marshall and Bles.)

is covered by peritoneum (Fig. 46 , per), continued on the one hand into the parietal layer (Fig. $5, p$. per) of that membrane, on the other into the mesentery (mes); its dorsal face is bathed by the lymph of the sub-vertebral sinus (sv. ly.s). From the posterior end of its outer edge a delicate tube, the ureter (Figs. 3,4 , and $7, u r$ ), passes backwards and opens into the cloaca in its dorsal wall. On its ventral face is a singular yellowish-white stripe, the adrenal body, an organ of imperfectly known function (Fig. 7 , between the lines from $k d$ and $t s$ ). 
A thin section shows the whole kidney to be made up of a tangled mass of microscopic tubes (Fig.

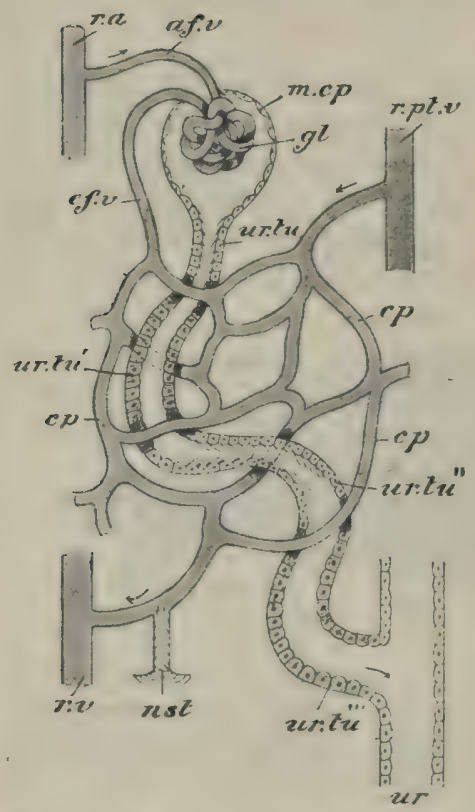

FiG. 47.-Diagram of a single urinary tubule with its blood-vessels, to illustrate the structure of the Frog's kidney.

af. $v$. afferent vessel of glomerulus; $c p$. rapillary network of kiclney; $\epsilon f$. $v$. efferent vessel of glomerulus; gl. glomerulus; m. $c p$. Malpighian capsule, showing epithelium (which in reality also covers the glomerulus); $n s t$. ciliated nephrostome; r. a. renal artery ; $r . p l . v$. ronal portal vein ; $\eta_{v} v$, renal vein; $u r$, ureter; $u r, t u$, ur. $t u^{\prime}$, wr. $t u^{\prime \prime}$, ur. $l u^{\prime \prime}$, different portions of urinary tubule, showing epithelium and cilia. $46, u r . t u)$, so twisted together that any section cuts them in various planes, some transversely, when they appear as circles, others longitudinally or obliquely. Amongst these urinary tubules or nephridia, as they may be called, are seen globular sacs, the Malpighian capsules (m. $c p$ ), each having in its interior a little rounded bunch, known as the glomerulus ( $g l$ ). Very accurate examination of numerous sections, as well as of teased-out specimens, shows that each Malpighian capsule (Fig. $47, m . c p)$ is connected with a urinary tubule (ur. $t u$ ), to which it forms a blind, bulblike end. The tubule itself winds through the substance of the kidney, is joined by other tubules, and finally discharges into the ureter (ur). The tubules are lined with somewhat cubical cells of 
glandular epithelium, which, in some parts ( $u r . t u, u r . t u^{\prime \prime}$ ), are ciliated. The Malpighian capsules are lined with flattened cells of parement epithelium.

The arrangement of the blood-vessels is peculiar. Like other organs, the kidney is permeated by a network of capillaries $(c p)$ which form a close mesh between the urinary tubules, so that the cavity of the tubule is separated from the blood only by the thickness of the gland-cells and of the capillary wall. The capillary network is supplied partly by the renal arteries $(r \cdot a)$, partly by the renal portal vein $(r \cdot p t . v)$, and is drained by the renal veins $(r . v)$. It is in the behaviour of the renal arteries that the chief peculiarity of the kidney-circulation lies. On entering the kidneys they break up into smaller and smaller arteries, but each of the ultimate branches $(a f . v)$, instead of discharging into the general capillary network, passes to a Malpighian capsule, in the interior of which it breaks up into a little bunch of coiled capillaries, the glomerulus $(g l)$. From this the blood is carried off by a minute ressel (ef. $v$ ) by which it is poured into the general capillary network and finally discharged into the renal vein $(r, v)$.

Renal Excretion.-While circulating through the glomerulus, water and certain soluble matters are separated from the blood and make their way into the Malpighian capsule and thence into the urinary tubule. As the blood circulates through the general capillary network, the gland-cells of the tubules excrete, out of the materials brought to them by the blood, the nitrogenous waste matter urea, in the formation of which the liver plays an important part; it is discharged from the cells into the cavity of the tubule, where it is dissolved in the water separated out of the glomerulus. In this way the urine is formed. Accumulating in the tubules, it makes its way into the ureter and thence drop 
by drop into the cloaca, whence it is either expelled at once, or stored for a time in the bladder.

Note that the formation of urine is a process of secretion of a similar nature to the secretion of gastric juice, bile, \&c. The fluid secreted is, however, of no further use to the animal, and would, in fact, act as a virulent poison if retained in the system. It is therefore got rid of as soon as possible. Secretions of this kind, consisting not of useful but of harmful or waste matters, are distinguished as excretions.

Bile is also in part an excretion as it contains pigments due to the disintegration of hrmoglobin, and thus by its means the effete colouring matters of the blood are passed into the intestine and got rid of.

Pulmonary and Cutaneous Excretion.-The lungs, besides being organs of respiration, take their share in excretion, since they get rid of the important waste product, carbon dioxide, together with a considerable quantity of water. Similar functions are discharged by the skin, which is also an organ both of respiration and of excretion. Interchange of gases takes place between the outer air and the blood in the capillaries of the derm: the carbon dioxide of the non-aërated blood brought to the skin by the cutaneous artery (p. 93 and Fig. 23) is exchanged for oxygen, and the blood, in the aërated condition, is returned by the musculo-cutaneous vein to the heart. Moreover, the cells of the cutaneous glands separate water and other constituents from the blood, and the fluid thus formed is poured out on the surface of the body. Here it serves to keep the skin moist, and is finally lost, either by evaporation or by mingling with the water in which the frog is immersed. The cutaneous secretion has also poisonous properties, and so probably serves as a defence against some of the animal's enemies.

Summary of the Processes of Nutrition.-We are 
now in a position to understand the general features of the whole complicated series of processes which have to do with the nutrition of the frog, which are collectively spoken of as metabolism. These processes are illustrated in the diagram (Fis. 23, p. 9I), which should be constantly consulted in connection with the following summary.

All parts of the body are placed in communication with one another by means of the blood-vessels, through which a constant stream of blood is flowing in a definite direction.

In all parts, waste of substance (destructive metabolism) is continually going on, and the waste products - water, carbon dioxide, and some nitrogenous substances which ultimately take the form of urea-are passed either directly into the blood, or first into the lymph and thence into the blood.

At the same time the cells withdraw nutrient materials from the blood, and assimilate them, i.e., form new living substance, whereby the waste is made good, and the tissues adequately nourished (constructive metabolism). Oxygen is also withdrawn from the blood; like the air supplied to a fire, it is essential to the oxidation or low temperature combustion with which the waste of tissues is associated. By the withdrawal of its oxygen the hæmoglobin of the blood alters its colour from scarlet to purple.

Thus the blood as it passes through the body is constantly being impoverished by the withdrawal of nutrient matters and of oxygen, and as constantly being fouled by the discharge into it of waste products. It reaches the capillaries of an organ as bright red, aërated blood, and leaves it as purple, non-aërated blood.

These changes, by which the blood loses nutrient matters and oxygen and gains waste products, takes place all over the body. The converse processes by 
which nutrient matters and oxygen are absorbed and waste products got rid of are carried on in certain definite portions of the circulatory system.

In the walls of the enteric canal (Fig. 23, Ent. C), the products of digestion pass into the blood $(C p . E n t . C)$, or in the case of fats, first into the lacteals and ultimately into the blood. In this way the due proportion of nitrogenous and other food-materials is kept up.

In passing through the capillaries of the lungs $(C p$. $L n g$ ), carbon dioxide is exchanged for oxygen and a certain amount of water is given off. In the capillaries of the skin $(C p . S k)$, a large quantity of water and smaller proportions of other waste matters are got rid of. In the kidneys $(C p . K d)$, a considerable quantity of water, together with the bulk of the urea, are removed from the blood and finally expelled from the body.

Note that all these changes are produced by the special activity of particular groups of epithelial cells which, however alike they may be in general appearance and structure, have a marvellous selective faculty peculiar to themselves. Like all other parts of the body, they are constantly undergoing the usual processes of waste and repair, withdrawing nutrient matters and oxygen from the blood, and passing waste matters into it. But, in addition to the ordinary processes of nutrition, each particular group of cells has the power of withdrawing a specific substance from the blood or of passing substances into it. Thus the epithelial cells of the enteric canal ( $E p$. Ent) pass in digested food, those of the skin $(E p . S k)$ and Malpighian capsules $(M l p . C p)$ withdraw water, those of the urinary tubules (Ep. Ur. T) urea, and so on. Similarly, the various gland-cells, such as those of the liver $($ Lvr. C), pancreas $(P n . C)$, gastric and cutaneous $(C u . G l)$ glands, withdraw specific substances, or secretions, which are 
discharged on the free surface of the epithelium and serve various purposes.

We see that the blood loses-(I), nutrient matters and oxygen all over the body; (2), water in the skin, lungs, and kidneys; (3), carbon dioxide in the lungs and skin; (4), urea, principally in the kidneys; and (5), various substances in the glands. It gains (I), waste products all over the body ; (2), nutrient matters in the enteric canal; (3), liver-sugar (p. I35) in the liver; (4), oxygen in the lungs and skin. It is therefore richest in oxygen and poorest in carbon dioxide as it leaves the lungs and skin, i.e., in the pulmonary and musculocutaneous veins; richest in nutriment as it leaves the enteric canal, i.e., in the portal vein ; poorest in urea as it leaves the kidneys, i.e., in the renal veins ; poorest in water as it leaves the skin and kidneys, i.e., in the cutaneous and renal veins.

In this way a single closed system of pipes not only supplies all parts of the body with everything necessary for their sustenance, but serves also as a drainage system to carry away their various waste products.

Notice that we must distinguish between the nutrition, respiration, and excretion of the frog as a whole, and of its various parts. Every one of the thousands of cells, fibres, \&c., in the entire body is nourished, breathes, and excretes, taking its nourishment and oxygen directly from the blood, and discharging its waste products into it. What are called the organs of nutrition and respiration are special portions of the body set apart for taking in fresh supplies of food or of oxygen for the organism as a whole, such supplies being finally distributed by the blood-system. Similarly, what are called the organs of excretion are special portions of the body by which the waste products, collected by the blood from all parts of the organism, are finally discharged. 
Evolution of Heat.-The oxidation of the tissues, like that of coal or wood in a fire, is accompanied by a rise in temperature. But in the frog, as in other coldblooded animals, the evolution of heat is never sufficient to raise the temperature of the body more than very slightly above that of the surrounding medium. In warm-blooded animals, such as ourselves, the temperature is regulated, according to the season, by a greater or less evaporation of water from the surface of the body. In the frog this is not the case : the temperature of the animal is always nearly the same as that of the air or water in which it lives, and hence the frosts of winter would be fatal to it, but for the habit of hibernation (p. 8).

Death and Decomposition.-The decomposition undergone by a dead frog (p. II) may be looked upon as an excessive process of waste unaccompanied by repair. Owing to the action of certain microscopic plants known as Bacteria, which will be referred to again in Part II. (p. 257), the proteids undergo oxidation, amongst the principal products of which are water, carbon dioxide, ammonia, and certain gases of evil odour, such as sulphuretted hydrogen and ammonium sulphide. Most of the gases escape into the air; while the ammonia is finally converted into nitrous and nitric acids. These, combining with certain substances in the soil, give rise to salts called nitrates and nitrites, which furnish one of the chief sources of the food of plants.

\section{PRACTICAL DIRECTIONS}

The Organs of Respiration and of Voice.-Pin down a frog in the usual way (pp. $3 \mathrm{I}$ and 32 ), remove the heart, and make out the precise relation of the lungs, first distending them with air through the glottis. The specimen already used for the dissection of the vascular system or alimentary canal will serve the purpose. Harden thoroughly in formaline or spirit and note (Fig. ${ }_{4+}$ ) the laryngo-tracheal 
chamber, which communicates on the one hand with the pharynx through the glottis, and on the other with both lungs. Observe also the posterior horns of the hyoid which embrace the glottis, and then separate them from the laryngo-tracheal chamber, so as to remove the latter, together with the lungs, from the body.

Then dissect off what remains of the mucous membrane of the pharynx around the glottis, and notice the small laryngeal muscles in connection with the laryngo-tracheal chamber: remove these, and pin the respiratory organs down under water, dorsal surface of the lungs uppermost, by means of a pin through each lung. Cut away the ventral wall of one lung, so as to expose the cavity and its connection with the laryngo-tracheal chamber. You will very probably find some parasites in the lungs-small worms called Rhabdonema nigroicnosum belonging to the group of Nematode worms. Note:-

I. The two arytenoid cartilages, and a ring-shaped cartilage surrounding the base of the lungs.

2. The nefwork of ridges on the inner surface of the lungs. Examine with a lens. Sketch.

3. The rocal cords. Observe these first in their natural position, and then with the scissors cut through the laryngotracheal chamber along the line of the glottis so as to divide it into right and left halves and thus expose the vocal cords -from their surface. Sketch.

The Kidneys.-a. Examine these organs in situ (Figs. 3, 4, and 7) and note :-

I. Their form and position, and the relations of the peritoneum, which covers them on the ventral side only. (See Fig. 5.)

2. The ureters (their openings into the cloaca may be seen at a later stage).

3. The yellowish adrenals.

Sketch.

b. Examine under the microscope a transverse section of the kidney, prepared as directed on p. 136, and make out (Fig. 46):

1. The urinary tubules, cut through in various planes.

2. The Malpighian (or Bowman's) capsules and their glomeruli.

3. Blood-capillaries and vessels.

4. The nephrostomes (p. 98, Figs. 23,46 , and 47 , and see Part II, p. 60I).

Sketch a portion under the high power. Compare with a section of kidney in which the blood-vessels have been injected with coloured gelatine (p. IOI). 


\section{CHAPTER $\mathrm{X}$}

THE FROG (continued): THE NERVOUS SYSTEM

IN a machine of human construction, such as a steamengine, the proper working of the whole depends, providing the parts of the machine itself are in good order, upon two things-the stoking or regulation of the fires, and the turning of certain cocks and levers by the engineer. In that very complex machine the frog, we have already studied what corresponds to stoking, viz., feeding and breathing. We must now. direct our attention to what may be considered roughly to correspond with the work of the engineer-the means by which the whole complex machinery is kept under control, and its various parts made to work together to a common end.

How does it come about, for instance, that the various digestive glands begin to secrete actively as soon as food is taken into the enteric canal? How is it that a touch on any part of the body, or even the sight of an enemy, is followed instantaneously by a series of vigorous muscular movements so ordered as to facilitate escape from the source of danger?

In the fourth chapter (p. 62) we got so far as to learn that muscular contractions are induced by nervous $i m$ pulses travelling from the brain or spinal cord, along the 
nerves, to the muscles. It may therefore be inferred that the controlling apparatus, by which the functions of the body are regulated, is lodged in either the brain or the spinal cord, or in both.

Divisions of the Nervous System.-The nervous system is divisible into ( $\mathrm{I}$ ) the central nervous system, consisting of the brain and spinal cord (Figs. 6 and 7), and (2) the peripheral nervous system, consisting of the nerves which pass from the central nervous system to the various parts of the body. The nerves are divisible into (I), cerebral nerves (Fig. 53), arising from the brain, (2), spinal nerves (Fig. 5I), arising from the spinal cord, and (3), sympathetic neries (Fig. 5I).

The Spinal Cord.-In form the spinal cord (Figs. 6 and $7, s p . c d)$ is irregularly cylindrical. It is continuous in front with the brain, and tapers off posteriorly into a fine thread-like portion, the filum terminale $(f . t)$, while opposite the fore-limbs, and again just anteriorly to the filum terminale, it presents an enlargement: these are known respectively as the brachial and sciatic swellings. Along its dorsal surface runs a delicate longitudinal line, the dorsal fissure (Fig. $48, d . f$ ), and a distinct groove, the ventral fissure $(v . f)$, extends along its lower surface.

The cord is covered with a delicate pigmented membrane known as the pia mater ( $p . w)$, and the neural canal in which it lies is lined with a stout, tough membrane, the dura mater (Fig. 52, d. m). Between the two is a space filled with a lymphatic arachnoid fuid, which, like the pericardial fluid, preserves the contained organ from shocks.

Examination of a transverse section of the cord under a low power will show that the dorsal fissure is an extremely narrow vertical wall formed by an extersion inwards of the pia mater. The ventral fissure is a distinct cleft. Thus the two fissures divide the cord 
into paired half-cylinders, right and left, joined in the middle by a narrow bridge. This bridge is traversed from end to end by a very narrow longitudinal canal, the central canal (c. c), lined by epithelium, so that the cord is not a solid cylinder, but a tube with an extremely narrow cavity and excessively thick walls.

The section also shows that the cord is not homogeneous, but is composed of two different substances.

Its outer part is pure white and shining in the fresh

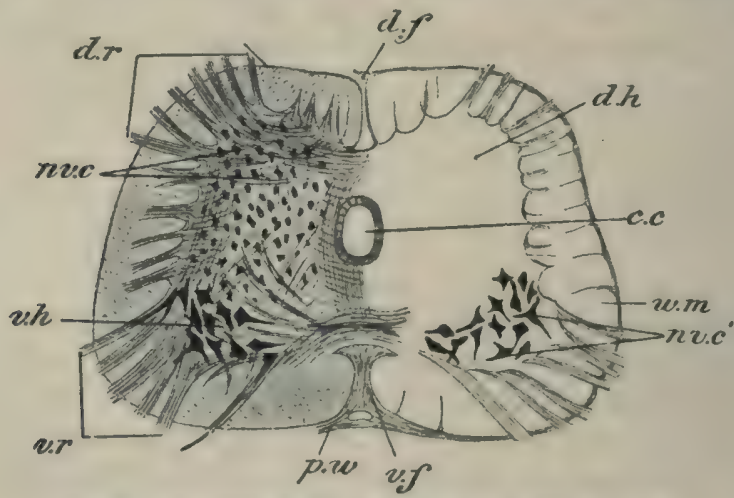

FrG. 48. - Transverse section of spinal cord of Frog. ( $\times 35$.)

$c . c$. central canal ; $d$.f. dorsal fissure; $d . h$. dorsal horn of grey matter; $d$. $r$. fibres of dorsal root of spinal nerve ; $n v . c$. nerve-cells of dorsal horn; $n v . c^{\prime}$ nerve-cells of ventral horn; $p . w^{\prime}$. pia mater; $v$. $f$. ventral fissure; $v$. $h$. ventral horn of grey matter; $v . r$. fibres of ventral root of spinal nerve; $v, m$. white matter. (After Howes.)

cord, and is hence called the white matter $(w . m)$. Its internal substance has a pinkish colour when fresh, and is called the grey matter. The grey matter has a squarish outline in transverse section. It surrounds the central canal, and is continued upwards and downwards, forming what are called the dorsal $(d . h)$ and ventral $(v . h)$ horns of the grey matter.

The Brain.-Anteriorly the spinal cord passes insensibly into the brain (Fig. 49), which is of somewhat 
greater diameter than the cord, and is made up of several very distinct parts or divisions. The hindermost division is called the bulb or medulla oblongata ( $M$ ed. $o b l)$; this appears to be simply a widening of the spinal cord $(S p . c d)$, except that on its dorsal surface is a triangular body $\left(\mathrm{D}, c h . p l x^{2}\right)$, of a reddish colour in the fresh condition, called the posterior choroid plexus; it is simply a thickening of the pia mater containing abundant blood-vessels.

The choroid plexus forms a kind of lid to a triangular cavity ( $\mathrm{A}$ and $\mathrm{D}, v^{4}$ ) excavated in the dorsal region of the medulla oblongata, and called the fourth ventricle. The apex of the cavity, which is directed backwards, opens into the central canal of the spinal cord (Fig. 50, $\left.v^{4}, c . c\right)$, and the fourth ventricle is to be looked upon simply as the anterior part of the central canal which has become widened out and is covered only by a thickened portion of the pia mater.

The fourth ventricle is bounded in front by a narrow ledge of nervous matter (Fig. $49, C b$ ), which would be hardly worthy of being considered as a special division of the brain but for the fact that the corresponding part in many animals - e.g., dogfish, rabbit, man-is a large and important structure. It is called the cerebellum.

In front of the cerebellum comes a pair of rounded elevations, the optic lobes (Figs. 49 and 50, Opt.l). Each contains a cavity, the optic ventricle $(O p t . v)$, communicating with a narrow median passage, the iter (i), which is continuous behind with the fourth ventricle. The bulb is continued forwards beneath the optic lobes as the crura cerebri $(\mathrm{Cr}, \mathrm{C})$.

In front of the optic lobes is an unpaired structure, the diencephalon or 'tween-brain (Di). On its upper surface is a small rounded vascular body, the anterior choroid plexus (Fig. 49, D, ch. plx $x^{1}$ ), formed, 


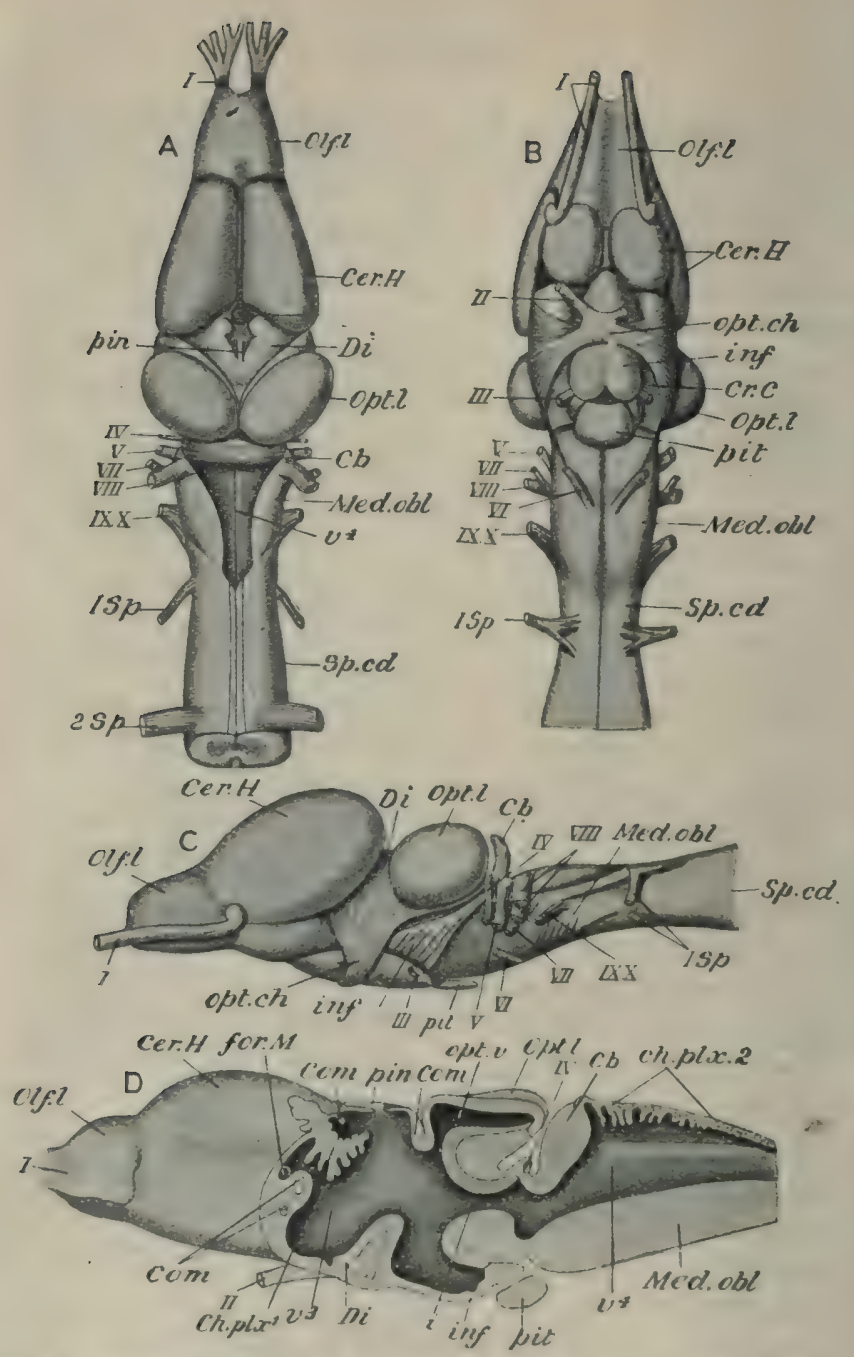

FIG. 49.--Brain of Frog. A, from above: B, from below; C, from the side $\left(x_{4}\right) ; \mathrm{D}$, in longitudinal vertical section. ( $\left.x^{\prime} 5.\right)$

$\mathrm{Cb}$. cerebellum; Cer. $H$. cerebral hemispheres; ch. plx.1 anterior and $c h . p l x .2$ posterior choroid plexus (removed in $A$ ); com. transwerse bands of nerve-fibres or commissures connecting the left and right halves of the brain; $C_{r}, C$. crura 
cerebri : Di. diencephalon; for. M. foramen of Monro; $i$. iter, or aqueduct of Sylvius; inf. infundibulum; Med. obl. medulla oblongata; Olf. $L$. olfactory lobe : opt.ch.optic chiasma : Opt.l.optic lobe ; opt. $\tau$. optic ventricle ; pis.stalk of pineal body: pit. pituitary body ; Sp.cd. spinal cord; $y^{3}$. third ventricle: $\imath+$ fourth ventricle; $1-X$, cerebral nerves; $I S p . z S p$. first and second spinal nerves. (A-C, after Gaupp; D, from Wiedersheim's Comp. Anatomy, after Osborn.)

like the posterior choroid plexus, of a thickening of pia mater, containing numerous blood-ressels. It helps to roof over a narrow slit. like cavity communicating with the iter, the third ventricle $\left(v^{3}\right)$, the sides of which are formed by thickenings of nervous matter, the optic thalami $(D i)$, connected by transverse bands of nervefibres (D, com). On the ventral surface the diencephalon is continued into a funnel-like extension, the infundibulum (Fig. +9, inf), to which is attached a rounded structure, the pituitary body (pit). On the dorsal surface, just behind the choroid plexus, is the delicate stalk (pin) of the pineal body-the vestige of a sensory apparatus between the skull and the skin, which in some lizards, for example, has the structure of a small

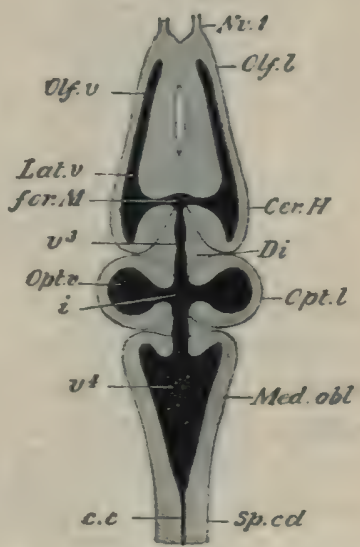

FIG. 50.-Diagrammatic horizontal section of Frog's brain.

c. c. central canal; $C e r . H$. cerebral hemisphere: $D i$. diencephalon; for. $M$. foramen of Monro; i. iter ; Lat. $:$ lateral ventricle; Med. obl. medulla oblongata; $N_{i}, I_{\text {, }}$ olfactory nerve : $O ! f . L$. olfactory lobe; Oif. $v$. olfactory ventricle; Opt. 1. optic lobe; $U_{p}$ t. $v$. optic ventricle: $S p$. cd. spinal cord ; z. 3. third ventricle: $\varepsilon$. 4 , fourth ventricle. (After Ecker \& Wiedersheim.)

eye situated on the top of the

head, and which was probably functional in the ancestors of the frog. We shall meet with other examples of such vestigial organs in the course of our studies. 
In front of the 'tween-brain comes a pair of long, oval bodies, wider behind and narrower in front. These are the cerebral hemispheres (Cer. H). Each contains a cavity, the lateral ventricle (Lat. v), which communicates with the third ventricle by a small aperture, the foramen of Monro (for. $M$ ).

Lastly, each cerebral hemisphere is continued forwards by a rounded olfactory lobe (Olf. l), which is fused with its fellow of the opposite side, the single mass lying in the posterior compartment of the girdle-bone (p. 42). The lateral ventricles are continued forwards into the olfactory lobes, forming the small olfactory ventricles (Fig. 5o, Olf, v).

The brain, like the spinal cord, is formed of grey and white matter, but differently arranged. In the olfactory lobes, cerebral hemispheres, and 'tween-brain the white matter is internal, and the grey forms a thin outer layer or cortex. In the optic lobes and medulla the grey matter is mainly around the ventricles, and the white matter more external.

Like the spinal cord, the whole brain is covered with pia mater, densely pigmented in the region of the optic lobes, and the cranial cavity in which it is contained is lined with dura mater.

The Spinal Nerves.-The spinal nerves arise symmetrically from the spinal cord on the two sides of the body, and pass out from the neural canal through the intervertebral foramina (p. 38).

There are altogether ten pairs of spinal nerves in the adult frog (Fig. 5I, $I-X$ ), each of which on leaving the neural canal divides into a smaller dorsal and a larger ventral branch (Figs. 52 and 53). The first pair leaves the cord through the intervertebral foramina between the first and second vertebra. Each passes at first directly outwards, its large ventral branch, known as the hypo- 
glossal, turning forwards, and going to the muscles of the tongue (Fig. 5I, $I$, Fig. 53, I $S p$ ).

The second pair (Fig. $5 \mathbf{I}, I I)$ is very large; it emerges between the second and third vertebræ, and each is soon joined by the small third nerve $(I I I)$, which emerges between the third and fourth vertebræ, as well as by a small branch or two from the first, thus forming a simple network or plexus-the brachial plexus (br.pl), from which pass off nerves to the fore-limb supplying both skin and muscles.

The fourth, fifth, and sixth nerves take a very similar course. The fourth (IV) emerges between the fourth and fifth vertebræ, the fifth $(V)$ between the fifth and sixth, and the sixth $(V I)$ between the sixth and seventh. They all pass obliquely backwards, and supply the walls of the body, being distributed to both skin and muscles.

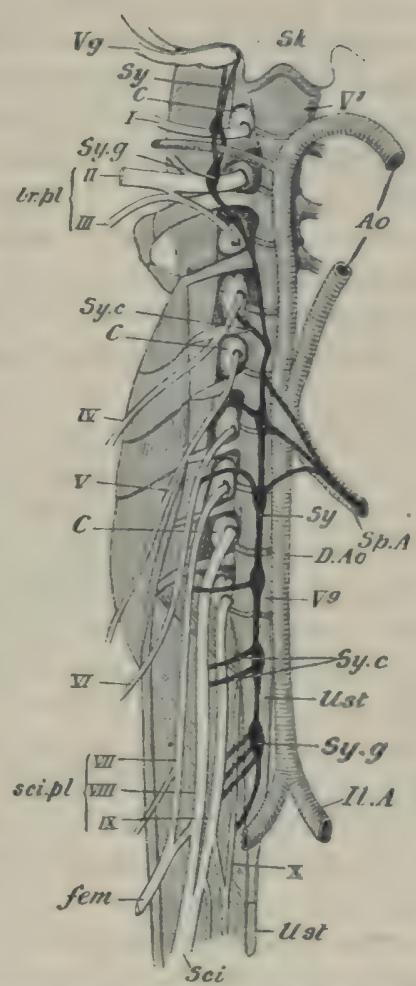

FiG. 5I.-The ventral branches of the spinal nerves and the sympathetic of the Frog, ventral view: shown on the risht side of the animal only. $\left(x_{2}\right)$

$I-X$, spinal nerve: $A 0$. systemic arch ; br. pl. brachial plexus; C. ealcareous bodk's which surround the spinal ganslia; $D A o$. dorsal aorta; fem. femoral nerve; il. A. iliac artery; sci. sciatic nerve; sci. $p i$. sciatic plexus; $S k$. skull; Sp. A. splanchnic artery; Sy. sympathetic cord; $S y$. c. communicating branches between the sympa. thetic and spinal nerves; Sy. g. sympathetic ganglia; Ust. urostyle; $V-V y$, centra of vertebræ; $V_{g}$. vagus nerve, with its ganglion. (After Gaupp, slightly altered.) 
The seventh, eighth and ninth nerves supply the muscles and skin of the hind-limbs. The seventh (VII) leaves the neural canal between the seventh and eighth vertebræ, the eighth $(V I I I)$ between the eighth and ninth, and the ninth $(I X)$ between the ninth vertebra and the urostyle. They all pass almost directly backwards, and are united with one another by oblique cross branches so as to form the sciatic plexus (sci. pl), from which are given off, amongst others, two nerves to the leg, the largest of which, the sciatic nerve (sci), being that already mentioned in the chapter on the muscular system (p. 62).

The tenth $(X)$ is a very small nerve. It emerges through a small aperture in the side of the urostyle (p. 39), and supplies the cloaca, urinary bladder, and adjacent parts. It is connected by cross-branches with the ninth.

It will be noticed that while the large ventral branch of the first spinal nerve-the hypoglossal-supplies muscles only, and is therefore a motor nerve, all the others go to both muscles and skin, and are therefore both motor and sensory, or mixed nerves. They all branch out in a complex manner, and are traceable to the remotest parts of the body.

The Sympathetic Nerves.-On either side of the dorsal aorta is a very delicate nerve, having at intervals little swellings called ganglia, each of which is connected with a spinal nerve by a communicating branch (Figs. $5 \mathrm{I}$ and 53, Sy, Sy. g, Sy. c). In front of the point where the dorsal aorta $(D, A o)$ is formed by the union of the two systemic trunks $(A O)$, these two sympathetic nerves, as they are called, are continued forward, one on either side of the vertebral column, towards the head, when they enter the skull and become connected with certain of the cerebral nerves. 
Each sympathetic nerve has altogether nine or ten ganglia, each connected with one of the spinal nerves, and from the ganglia branches are given off which supply the heart and blood-vessels, the stomach, intestine, liver, kidneys, reproductive organs, and urinary bladder.

Origin of the Spinal Nerves.-The mode of origin of the nerves from the spinal cord is peculiar and characteristic. Traced towards the cord, each nerve is found, on reaching the intervertebral foramen from which it

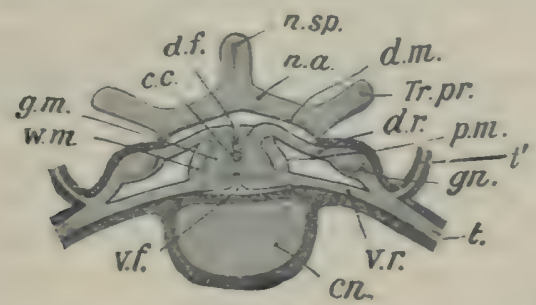

Frg. 32.-Transverse section through the vertebral column and spinal cord of the Frng, to show the mode of origin of the spinal nerves. ( $x$ 6.)

$c$. $c$. central canal; $c n$. centrum with periesteum; $d$. f. dorsal fissure: $d . m$. dura mater; $d . r$. dorsal root; $g$. $m$. grey matter; gn. ganglion of dorsal root; s. a. neural arch: $n . s p$. neural spine; $p . m$. pia mater; $t$. nerve trunk with its sheath (ventral branch) : $t^{\prime}$. dorsal branch; Tr. pr. transverse process; $t$. $f$. ventral fissure; $v$. $r$. ventral root ; $w$. $m$. white matter. (After Howes.)

emerges, to divide into two- a dorsal root which springs from the dorsal, and a ventral root which arises from the ventral region of the cord (Fig. 52, d.r, v.r). The dorsal root is distinguished from the ventral by being dilated into a ganglion ( $g n$ ). In Fig. 5I these ganglia lie hidden within certain calcareous bodies $(C)$ in this region.

Cerebral Nerves.--There are ten pairs of cerebral nerves, some of which are purely sensory, some purely motor, some mixed.

The first or olfactory nevies (Fig. 49,I) arise from the olfactory lobes, and pass through two holes in the trans- 
verse partition of the girdle-bone. Each is distributed to the mucous membrane of the nasal sac or organ of smell of the same side, and is purely sensory.

The second or optic (II) is a large nerve which springs from the ventral surface of the 'tween brain. At their origin the right and left optic nerves have their fibres intermingled, forming a structure something like a St. Andrew's Cross and called the optic chiasma (opt. ch), the other limbs of the cross passing upwards and backwards to the optic lobes. The optic nerve makes its exit from the brain-case through the optic foramen, and is distributed to the retina, a delicate membrane which lines the eye-ball, and is, as we shall see, the actual organ of sight. This nerve also is purely sensory.

The third or oculomotor (III) is a small nerve arising from the crura cerebri beneath the optic lobes. It passes through a small hole in the side of the skull behind the optic foramen, and supplies four out of the six muscles by which the eye-ball is moved (p. I86), and is purely motor.

The fourth or pathetic (IV) is a very small nerve leaving the dorsal surface of the brain between the optic lobes and the cerebellum, and making its exit from the skull above the third nerve. It is also purely motor, supplying one of the muscles of the eye-the superior oblique.

The fifth or trigeminal (Figs. 49 and $53, \dot{V}$ ) is a large nerve arising from the side of the medulla oblongata. Its root dilates to form a ganglion, the Gasserian ganglion, and leaves the skull by the large aperture noticed in the pro-otic bone. It owes its name to the fact that it soon divides into three main branches; one, the ophthalmic (Fig. 53, $V^{1}$ ), going to the skin of the snout; another, the maxillary $\left(V^{2}\right)$, to the upper lip and lower eyelid; and the third, or mandibular $\left(V^{3}\right)$, 
to the muscles and skin of the lower jaw. The trigeminal is a mixed nerve.

The sixth or abducent (Fig. 49, VI) is a very small motor nerve arising from the ventral aspect of the

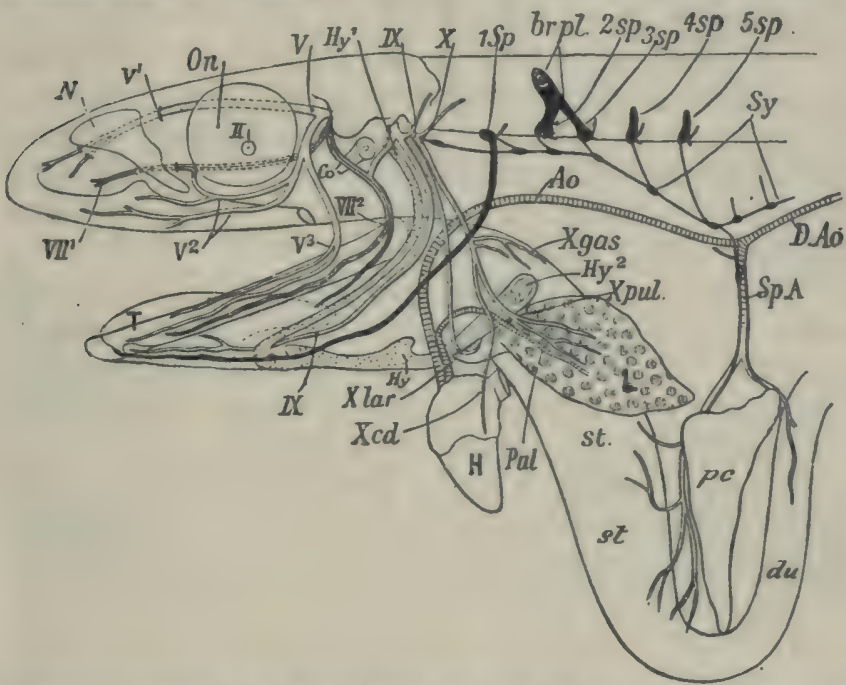

Fig. 53.-Dissection of the head and anterior part of the body of the Frog from the left side, to show the distribution of the fifth, seventh, ninth, and tenth cerebral nerves, as well as of the hypoglossal and part of the svmpathetic. ( $\therefore x_{1}$.)

$A o$. systemic arch; $b r . p l$. brachial plexus ; $c o$. colunella ; $D$. ao. dorsal aorta ; du. duodenum ; $H$. heart ; $H y$. body of hyoid ; $H_{y}$. anterior, and $H_{y}{ }^{2}$. posterior horns of hyoid ; $L$. lung; $N$.olfactory sar: Cn. orbit ; $P u l$. pulmonary artery ; Sp. A. splanchnic artery; st. stomach; Sy. sympathetic; $I I$. cut end of optic nerve; $V^{1}$. ophthalmic, $l \%$ maxillary, and $l \%$ mandibular branch of trigeminal (I) ; $I I^{1}$ palatine, and $V I I^{2}$ hyomandibular branch of faciai; $I X$. glossopharyngeal; $X$. vagus; $X c d$. cardiac, $X$ gas. gastric, Xlar. laryngeal, and $X$ pul. pulmonary branch of vagus; I $S p$. first spinal nerve (hypoglossal); $2 s p .--5 s p$. second to fifth spinal nerves. (After Howes, slightly modified.)

bulb, and supplying one of the muscles of the eye-ball called the posterior rectus.

The seventh or facial nerve (Figs. 49 and 53,VII) arises just behind the fifth and soon joins the Gasserian ganglion. Both it and the sixth leave the skull by the same aperture as the fifth. It divides into two branches, 
one of which, the palatine (Fig. 53, VII ${ }^{1}$ ), supplies the mucous membrane of the roof of the mouth, and the other, or hyomandibular $\left(V I I^{2}\right)$, sends branches to the skin and muscles of the lower jaw and to the muscles of the hyoid. It is a mixed nerve.

The eighth or auditory nerve (Fig. 49, VIII) arises from the medulla just behind the seventh, passes through an aperture in the inner wall of the auditory capsule, and is distributed to the auditory organ or membranous labyrinth (see Figs. 49 and 53). It is the nerve of hearing, and is purely sensory.

The ninth or glossopharyngeal (Figs. 49 and 53,IX) arises behind the auditory nerve. It sends a branch to join the facial, and supplies the mucous membrane of the tongue and pharynx as well as certain small muscles connected with the hyoid. It is also a mixed nerve.

The tenth or vagus (Figs. 49 and $53, X$ ) is a large nerve arising in common with the ninth, and dilating, shortly after leaving the skull, into a vagus ganglion. It supplies the larynx (Xlar), the heart $(X c d)$, the lungs $(X p u l)$, and the stomach (Xgas), and is therefore often known as the pneumogastric. It has - thus an extraordinarily wide distribution, being in fact the only cerebral nerve which supplies parts beyond the head. It is a mixed nerve, and contains many motor fibres, but its branches-some of which have to do with the regulation of the heart's contraction and with respiration-are better described as efferent and afferent than as motor and sensory: the meaning of these terms will be explained later on. The ninth and tenth nerves leave the skull close together through the aperture noticed in the exoccipital bone.

The sympathetic nerve $(S y)$ extends forwards from its junction with the first spinal nerve, joins the vagus, and finally ends anteriorly in the Gasserian ganglion. 
Mieroscopic Structure of Nervous Tissue.-Examination of a piece of nerve under the microscope shows it to be composed, like striped muscle, of cylindrical fibres, bound together by connective-tissue. The latter is much more abundant than in muscle, and in particular forms a thick sheath round the nerve which must be torn off before the nevie-fibres are reached.

Each fibre (Fig. 5t) is a cylindrical cord in which three parts can be distinguished. Running along the axis of the fibre is a delicate protoplasmic strand, the neuraxis or axis-fibre $(n x)$. Around this is a sheath formed of a fatty substance and known as the medullary sheath $(m, s) ;^{1}$ and, finally, investing the whole fibre is a delicate, structureless membrane, the neurolemma (ne). At intervals the medullary sheath is absent, and a node is produced, where the fibre consists simply of the neuraxis covered by the neurolemma.

In the ganglia are found, not only nerve-fibres, but nerve-cells (Fig. 5f): these are cells of a relatively large size, each with a large nucleus and nucleolus. In the spinal ganglia (B) the cell-body is produced into two processes, which come off from the cell by a common stalk. One of these processes (the axon) becomes the neuraxis of a peripheral nerve-fibre; the other (dendron) is also a protoplasmic process which passes into the spinal cord and sends off branches, each branch finally ending in a complicated branch-work or arborisation, which is interlaced, but not actually continuous, with a similar arborisation arising from a nerve-cell in the spinal cord or brain (Fig. 55). The cell with its processes is spoken of as a neurone.

The white matter of the brain and spinal cord consists of nerve-fibres, those in the cord having a longitudinal

1 The medullary sheath may be absent in certain nerve-fibres (e.g., in the sympathetic and olfactory nerves). 
direction; the grey matter contains numerous " multipolar" nerve-cells, each giving off numerous dendrons

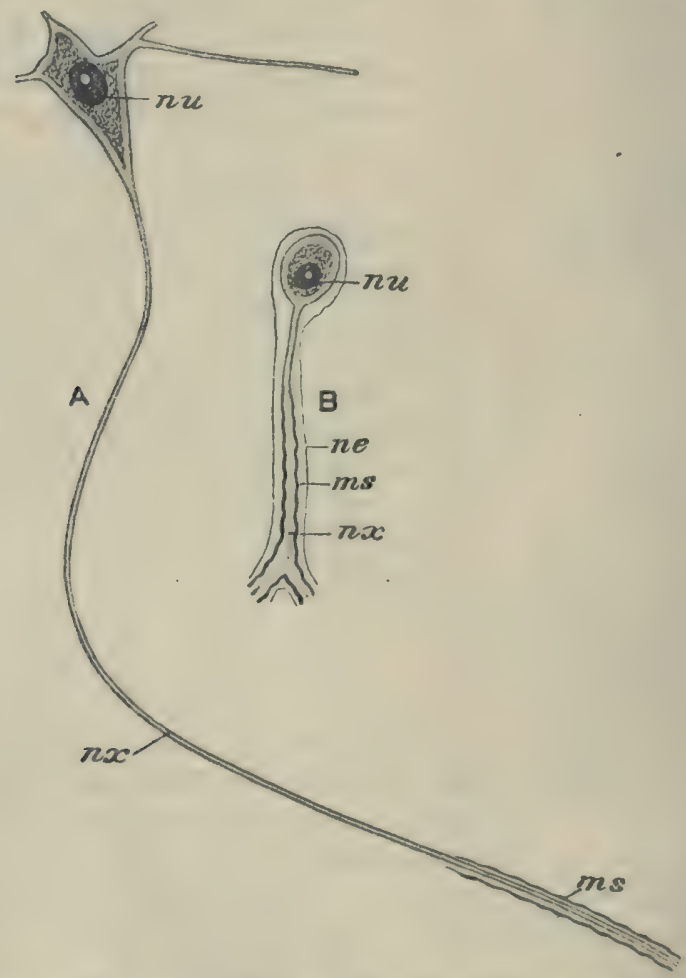

FIG. 54.-A, nerve-cell from the grey matter of the spinal cord of a Frog, and the nerve-fibre arising from it (neurolemma not shown); 13 , cell from the ganglion of a dorsal root. ( $x$ about 200.)

ne. neurolemma; nu. nucleus; $n x$. neuraxis; ms. medullary sheath. (After Howes.)

and one axon, which is continuous with a neuraxis (Figs. 48 and 54, A) ; they are enclosed in a tissue formed partly of the axis-fibres of nerves which enter 
the grey from the white matter, losing their medullary sheath as they do so, and partly of a delicate supporting tissue called neuroglia, in which the other elements are imbedded.

Functions of the Nervous System: Reflex Action.-In the fourth chapter you learned that a muscle may be made to contract by a stimulus applied either to the muscle itself or to its nerve. You are now in a position to pursue the subject of the control of various parts of the body by the nervous system a little further.

A frog is either decapitated or pithed, i.e., the medulla oblongata is severed and the brain destroyed: there can be thus no question either of sensation or of voluntary action on the frog's part. It is then hung up by a hook or string, so that the legs are allowed to hang freely. If one of the toes is pinched with the forceps, the foot will be drawn up as if to avoid the pinch; or, if some very weak acid be applied to a toe, the foot will again be withdrawn, being raised every time it is touched with the acid with the regularity of a machine. Again, if acid be applied to various parts of the body, the foot of the same side will immediately try to rub off the irritating substance; or if that foot be held down, the other will come into play.

Movements of this kind are called reflex actions: the stimulus applied to the skin is transmitted by sensory nerve-fibres to the spinal cord, where it is, as it were, reflected in another form, and passed along motor fibres to one or more muscles, causing them to contract (p. 60).

As already stated, the spinal nerve-trunks are mixed, i.e., contain both sensory and motor fibres, which, however, cannot be distinguished from one another structurally. It has been found by numerous experiments that as the nerve approaches the spinal cord these two sets of fibres separate from one another, the sensory fibres 
passing into the cord by the dorsal root, the motor by the ventral root. As a consequence of this, if the dorsal root be cut and its proximal or central endi.e., the end in connection with the cord-stimulated, muscular contraction will follow just as if the stimulus had been applied to the skin supplied by the nerve in question. If the other cut end-the distal or peripheral end-be stimulated, there is no result. On the

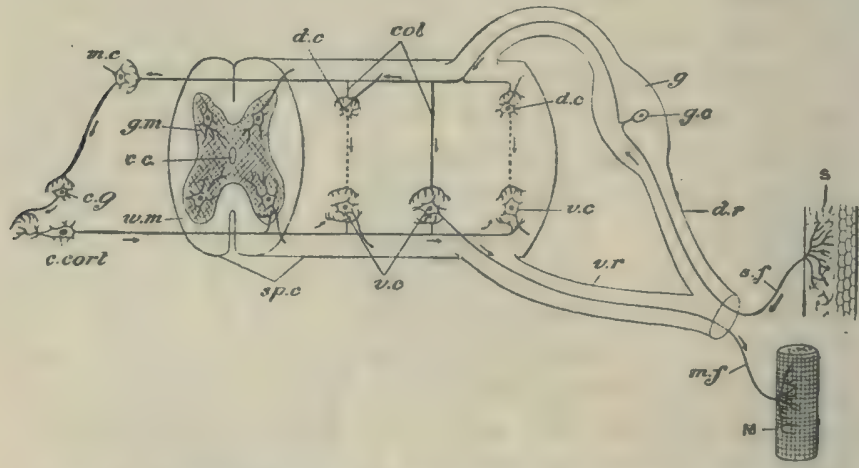

Frg. 55.-Diagram illustrating the paths taken by the nervous impulses.

c. $c$. central canal; col. collaterals ; $c$. cort. cell in rind or cortex of the cerebral hemisphere ; $c . g$. smaller cerebral cell ; d. c. cells in dorsal horn of grey matter; d. $r$. dorsal root ; g. ganglion of dorsal root; g. $c$. cell in ganglion of dorsal ront ; g. $m$. grey matter; M. muscle; $m$. c. cell in medulla oblongata; $m$. $f$. motor fibre; S. skin; s. $f$. sensory fibre; sp.c. spinal cord; $v$. c. cells in ventral horn of grey matter; v. r. ventral root; $w$. $m$. white matter. The argows indicate the direction of the impulses.

other hand, if the ventral root be cut and its distal end stimulated, the muscles supplied by it will contract, while stimulation of the proximal end produces no result.

Very accurate observations have shown that the connection between the motor and sensory fibres is as follows (Fig. 55). A motor fibre $(m . f)$ is traceable from the nerve-trunk through the ventral root $(v, r)$ into the white matter; and then, its medullary sheath 
being lost, passes into the ventral horn of the grey matter, its neuraxis being directly continuous with the axis-fibre process of one of the large motor nerve-cells $(v, c)$ : the remaining processes of these cells simply branch out in the neuroglia. The sensory fibres $(s . f)$ are traceable into the dorsal root $(d . r)$; in passing through the ganglion of the root $(g)$ they are found to be continuous with its simple ("bipolar") nerve-cells (g. c), and then pass into the cord. Instead, however, of entering the grey matter at once, they pass forwards as well as backwards for some distance, along the white matter of the cord, giving off numerous branches, or collaterals (col), which, losing their medullary sheaths, enter the dorsal horn of the grey matter and branch out into a complex series of fine fibres which interlace with the similar arborisations of the nerve-cells in the cord (p. I67).

The path of a nervous impulse known as the reflex arc or chain will now be obvious. The stimulus applied to the skin (Fig. 55, S) is conducted by a sensory fibre to the nerve-trunk and by the dorsal root to a cell in the ganglion and thence to the spinal cord; it then passes along the white matter of the cord, enters the grey matter, and is conducted by the collaterals to the nerve-cells of the ventral horn, either directly, or indirectly after passing through the cells of the dorsal horn : from one of the cells of the ventral horn it is conducted by an axon which becomes the neuraxis of a nerve-fibre, which, leaving the cord by a ventral root, passes along the nerve-trunk and finally goes to a muscle (M) as a motor fibre.

It will be noticed that a single stimulus applied to the skin may result in the contraction of numerous muscles-as, e.g., when the application of a drop of acid to the toe causes the lifting of the leg-and that the 
movements are of such a nature as to withdraw the part stimulated from the irritating substance. Moreover, as shown by the experiment of applying acid to various parts of the body, the movements are varied according to circumstances; if one leg is prevented from rubbing off the irritating substance, the other immediately comes into play. Obviously, then, a simple stimulus reaching the spinal cord may be transmitted to numerous motor cells of the ventral horn, and through these to numerous motor nerves, the particular nerves affected differing according to circumstances (compare Fig. 55). The spinal cord, therefore, is able, in response to a stimulus reaching it by a sensory nerve, to originate motor impulses causing complex muscular movements so adjusted as to serve definite purposes. Without such external stimulus, however, the spinal cord of a brainless frog is quite inactive, and the body of the animal will remain without movement until it dries up or decomposes.

In the uninjured frog, i.e., the frog with its brain intact, the case is very different. The animal no longer acts like an unintelligent machine, each stimulus producing certain inevitable movements and no others; but a single stimulus may produce varied movements, the nature and direction of which cannot be predicted. The frog will probably give a series of leaps, but the number and extent of these vary according to circumstances; its movements are voluntary, not reflex.

This is explained by the fact that certain nerve-fibres of the cord pass forwards to the brain, and that the nerve-cells in the grey matter of the cord are in communication-owing to the interlacing of their branching processes with those of the collaterals-with similar cells in the grey matter of the brain (Fig. 55, m. c, c. g, c. cort). In certain of these brain-cells (c. cort), voluntary 
impulses originate and exercise a controlling effect upon the cells of the spinal cord, so that these latter do not constitute, as in the brainless frog, a machine every movement of which can be accurately predicted.

Moreover, it can be shown by experiment that the process of originating voluntary impulses is not performed by the whole brain, but is confined to the cerebral hemispheres. If the hemispheres and optic lobes are removed so as to leave nothing but the bulb and cerebellum, the frog no longer lies in any position in which it may be placed, exhibiting no movements beyond the beating of the heart, as is the case when the whole brain is removed. It sits up in the ordinary attitude, breathes, swallows food placed in the mouthwhile making no attempt to feed itself, turns over and sits up if placed on its back, and swims if placed in water. If left alone, however, it remains in the sitting posture till it dies. Hence the bulb and cerebellum are evidently concerned with the co-ordination of muscular movements, but have no power of originating impulses. If the optic lobes as well as the medulla oblongata and cerebellum are left, the animal is affected by light, is able to perform complex balancing movements, and will even croak when stroked in a particular way. There is still, however, no voluntary action; without the application of stimuli, the animal sits motionless until it dies.

To sum up in the language of the illustration with which this chapter was begun, comparing the frog with an engine of human construction:- the grey matter of the brain may be compared with the engineer; much of the work of the engine may go on without him, certain levers, valves etc., acting automatically; but it is only by his controlling intelligence that the whole mechanism is adapted to the circumstances of the moment. 
So far we have considered the nervous system only in its relations to the skin or general surface of the body and to the muscles or organs of movement. The other parts of the body are, however, under nervous control.

It has been mentioned that the heart continues to beat in a frog when the brain has been removed: not only so, but it pulsates with perfect regularity when removed from the body. This is due to the fact that the muscles of the heart, like the cilia of ciliated epithelium, have the power of contracting rhythmically quite independently of the nervous system, although the heart contains nerve-cells which were formerly supposed to serve as a special nervous system for this organ, originating all its motor impulses. It is, however, under the control of the central nervous system. We have seen that it is supplied by a branch of the vagus; when this is stimulated, the heart stops in the dilated state and begins to beat again only after a certain interval. A feeble stimulus to the vagus will not actually stop the heart, but will diminish the rate and the strength of its contractions and consequently the amount of blood propelled through the body. The vagus is accompanied by a branch of the sympathetic which has an exactly opposite effect, i.e., stimulation of it accelerates the heart's action. In this way, the general blood-supply of the body is regulated by the central nervous system.

The blood-supply of the various parts and organs is regulated by the vaso-motor nerves. These are traceable through the sympathetic into the spinal cord by the ventral roots : distally they send branches to the muscular coat of the arteries. Under ordinary circumstances a constant succession of gentle stimuli pass along these from a group of nerve-cells in the medulla oblongata and, as the result, the arteries are ordinarily in a state 
of slight contraction. By various circumstances these stimuli may be diminished for any given artery and at the same time stimuli pass down another kind of vaso-motor fibres: the artery will then dilate and the blood-supply of the organ to which it is distributed will be temporarily increased. For instance, the presence of food in the stomach acts, through the central nervous system. upon the cœliac branch of the splanchnic artery, causing a dilatation of its capillaries and promoting an increased secretion of gastric juice. The secretion of other glands is regulated in a similar way. In some cases, however, it has been proved that the nerves of a gland do not act simply by producing dilatation of the capillaries, but have a direct effect upon the gland-cells, causing an increased secretion.

You will thus note that there are nerve-fibres carrying impulses to the central nervous system which have nothing to do with sensation, and fibres carrying impulses from the central nervous system which have nothing to do with motion, but result in increased secretion or in stoppage of motion. It is therefore best to use the term afferent (which includes sensory) for a nerve carrying an impulse to the brain and spinal cord, and efferent (including motor) for one carrying an impulse in the other direction (p. I66).

\section{PRACTICAL DIRECTIONS}

I. The Central Nervous System (Fig. 6).-Lay bare the brain and spinal cord as directed on p. 34, noting the dura mater and pia mater: the latter is densely pigmented over parts of the brain. The specimen on which this operation has already been performed will do, if the dissection has been done carefully.

Observe the origins of the cerebral and spinal nerves, noting the long dorsal and ventral roots of the latter (compare 
Fig. 52) which pass backwards for some distance before making their exit from the neural canal; and also the ganglia on the dorsal roots, lying just outside the canal and each hidden in a whitish calcareous body in this region (Fig. 5I, C). (The ganglia, however, can be more easily made out at a later stage.) Then sever the nerves very carefully from the brain and spinal cord and remove the whole central nervous system from the neural canal : it is best examined after hardening in formaline or spirit. Lay it in a dissecting-dish, under water, and make out its several parts as follows :-

a. The spinal cord.

I. Note its cylindrical form, the brachial and sciatic swellings, the filum terminale, and the dorsal and ventral fissures.

2. Examine a transverse section of the spinal cord, prepared as described on p. I36, under the low power of the microscope, and make out the dorsal and ventral fissures, the central canal, and the relations of the grey and white matter (Figs. 48 and 52 ). Sketch.

b. The brain (Fig. 49).

Beginning from the posterior end of the brain, where it passes into the spinal cord, make out its several divisions as follows :-

I. The bulb or medulla oblongata, with the posterior choroid plexus on its dorsal side : remove the latter, and notice that it roofs over the cavity of the fourth ventricle.

2. The small ledge-like cerebellum.

3. The two rounded optic lobes, and the crura cerebri beneath them.

4. The diencephalon, formed of a right and a left optic thalamus. On its dorsal side is the anterior choroid plexus, roofing in the third ventricle; and on its ventral side the infundibulum, from which the pituitary body easily becomes detached; and, more anteriorly, the optic chiasma.

5. The cerebral hemispheres, continuous in front, with

6. The olfactory lobes, which are fused in the middle line.

Sketch the whole central nervous system from above, and also the brain from below and from the side.

With the small scissors or a sharp scalpel, snip off a small piece of the wall of the hemisphere and optic lobe of one side-say the left, so as to expose the lateral ventricle and the optic ventricle (Fig. 50). Then with a sharp scalpel divide the whole brain into two by a longitudinal vertical 
cut very slightly to the left of the middle line, so as to reduce it to a longitudinal section (Fig. 49, D). Examine the cut surface of the right side under water, and make out as much as possible of the commissures and of the relations of the ventricles:-viz., the fourth ventricle, the iter and optic ventricle, and the third rentricle, which communicates with the lateral ventricle through the foramen of Monro. Sketch.

\section{The Peripheral Nervous System.}

\section{a. The spinal nerves.}

Fasten out a frog with the ventral side uppermost, and remove the heart, enteric canal, reproductive organs, kidneys, and lungs with great care, leaving behind most of the systemic trunk and the dorsal aorta (Fig. 5I). (One of the specimens already dissected will probably serve the purpose if the previous directions have been accurately followed.) Note the spinal neries passing outwards from the vertebral column on either side, and the calcareous bodies close to their points of exit, covering up the ganglia of the dorsal roots (p. 163). If the centra of the vertebræ are removed, the nerve-roots and their origins from the spinal cord can be made out : the removal of the centra is rendered easier if the frog is first decalcified by being placed in 10 per cent. nitric acid for twenty-four hours and then thoroughly washed in running water.

Confine your attention to the large ventral branches of the ten pairs of spinal nerves, as described on Pp. I60-I6z.

b. The sympathetic neres (Figs. 5I and 53).

Examine the systemic trunk and dorsal aorta carefully with a lens. Closely connected with it will be seen on either side a sympathetic nevie-cord, covered by pigmented connective-tissue. Carefully dissect the cord away from the aorta, and note the ganglia and the branches (rami communicantes) connecting them with the spinal nerves. Sketch the spinal nerves and sympathetic.

c. The cerebral neries (Fig. 53).

The dissection of these in a frog is not an easy task for a beginner, and it is best to examine those of a larger animal (e.g., dogfish) before attempting it (see Part II, p. +79). The origin of some of the nerves from the brain and the apertures through which certain of them pass out from the skull, have already been seen.

\section{The Mieroseopie Strueture of Nervous Tissue.}

$a$. Examine your transverse section of the spinal cord 
(Fig. 48) under the high power of the microscope, and observe-

r. The nerve-cells, present in the grey matter only (compare Fig. 54, A). Note their branched form and their nuclei; the larger motor cells are seen in the ventral horns of the grey matter. Sketch.

2. The nerve-fibres, in both grey and white matter, cut across transversely as well as in other directions, and each showing a deeply-stained central neuvaxis. Sketch.

$b$. Tease up a fresh spinal or sympathetic ganglion in salt solution, and stain with methyl-green. Compare the form of the nerve-cells with those in the spinal cord (Fig. 54, B). Sketch.

c. Cut off a very small piece of any fresh nerve (e.g. sciatic), and tease it out carefully, in a longitudinal divection, in salt solution. Note that it is made up of cylindrical, unbranched nerve-fibres, bound together by connectivetissue.

Examine a single fibre under the high power (Fig. 54, A), and make out the neurolemma, the medullary sheath, and the nodes : at the nodes, the neuraxis can also be seen. Sketch.

Tease out another piece of fresh nerve in chloroform, so as partially. to dissolve the medullary sheath, and note the central neuvaxis. Sketch.

Tease out in glycerine a piece of nerve which has been treated with a I per cent. solution of osmic acid in water for an hour or two and then well washed in water. The medullary sheath will appear nearly black, and the neurolemma, as well as the nodes, can be plainly seen. Sketch.

Reflex Action. The experiment described on p. 169 should be seen. 


\section{CHAPTER XI}

THE FROG (continued): THE ORGANS OF SPECIAI SENSE

IN the previous chapter you have learnt how the nervous system controls the various functions of the body, and how voluntary action is absolutely dependent upon the connection of the brain, through the spinal cord, with the nerves. Obviously, in order that the power of voluntary action should be of full use to its possessor, some means of communication with the external world is not only desirable but necessary; the frog, in order to adjust its actions to the circumstances in which it from time to time finds itself, must be able to distinguish friends from enemies, suitable from unsuitable food, darkness from light, heat from cold.

The avenues of communication between the animal and its surroundings are, as in ourselves, the senses of touch, taste, smell, sight, and hearing.

The sense of touch, including that of temperature, is lodged in the whole extent of the skin, which, as you have already learnt, is abundantly supplied with sensory nerves. Many of the nerves terminate in connection with what are known as tactile cells-large flattened cells arranged in groups just below the epiderm and around which the ultimate fibres of the sensory nerves are distributed. Stimuli applied to the skin, either by direct 
touch or by the heat of the sun, are transmitted to the tactile cells and thence through the sensory nerves to the brain. Notice that the stimulus is transmitted to the nerve-ends through the epithelial cells of the skin; if the skin be wounded and a stimulus applied directly to the tactile cells or the nerves, the sensation is one, not of touch, but of pain.

The sense of taste is lodged in the mucous membrane of the mouth, especially on the tongue and in the neighbourhood of the vomerine teeth, but extending also as far back as the gullet. Certain of the epithelial cells have an elongated form and are arranged in groups known as taste-buds, to which the tibres of the ninth and palatine branch of the seventh cerebral nerves, or nerves of taste, are distributed; on the tongue these tastebuds are situated on papille of the mucous membrane. In this case the stimulus is supplied, not by direct touch or by alteration of temperature, but by the contact of sapid or tasty substances. As before, the stimulus is applied to epithelial cells, and by them transmitted to the nerves and so to the brain, when the sensation of taste becomes manifest. Thus, just as common sensation may be abolished, in any part of the body, in three ways -by destruction of the skin, by cutting the sensory nerve, or by destroying the cerebral hemispheres-so the sense of taste is lost if either the mucous membrane of the mouth is injured or if the glossopharyngeal and palatine nerves are cut, or, again, if the cerebral hemispheres are destroyed.

The sense of smell is lodged in the nasal or olfactory sacs, which are enclosed in the olfactory capsules of the skull and separated from one another by a partition, the nasal septum. Each sac has two apertures, the external nostril, opening on the surface of the snout, and the internal nostril, opening into the mouth (p. 17). The 
sacs are lined by a delicate mucous membrane, some of the epithelial cells of which are of the ordinary columnar type, while others are extremely slender and produced into delicate processes at their free ends. With these latter the fibres of the olfactory nerves are connected, and they are distinguished as olfactory cells (Fig. 56). As the epithelial cells of the skin are affected by direct contact or by heat, so the olfactory cells are affected by the minute particles given off from odorous bodies: the contact of these particles acts as a stimulus, which, being transmitted by the olfactory nerves to the brain, gives rise to the sense of smell. This sense can be destroyed-as in the case of feeling and taste-either by destruction of the olfactory mucous membrane or by cutting the olfactory nerves, or by destroying the brain.

The organ of sight or eye of the frog is so similar in structure to that of man that the reader may be referred for details both of structure and of function to the text-books of Physiology, and it will only be necessary to give a brief outline here.

Each eye (Fig. 57) is a nearly globular organ, and when removed from

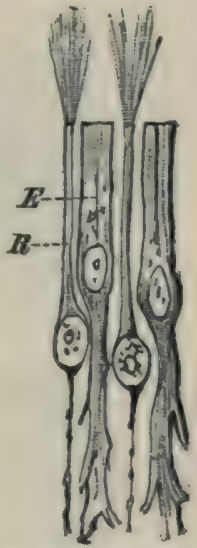

Frg. 56.-Epithelial cells of the olfactory mucous membrane of an Amphibian, highly magnified.

E. interstitial cells ; $\boldsymbol{R}$. olfactory cells. (From Wiedersheim's Comp. A natomy.) the orbit and cleaned by dissecting away the attached muscles, etc., two regions can be distinguished in it-an opaque portion of a dark bluish colour which forms some two-thirds of the entire globe and is hidden within the orbit in the entire animal ; and a clear, transparent portion which is directed outwards and freely exposed between the eyelids in the living frog. The outer coat 
of the concealed portion of the eyeball is the sclerotic (scl) and is formed of cartilage in the frog; the dark colour is due to the presence of a layer of black pigment which forms one of the internal coats; this will be referred to hereafter. Entering the sclerotic on its inner side, i.e., the side next the brain-case, will be seen the cut end of

A

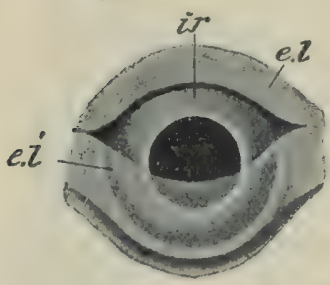

$\mathrm{C}$

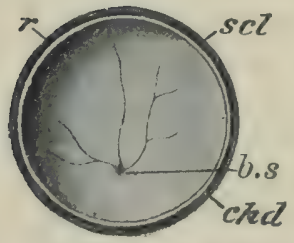

B

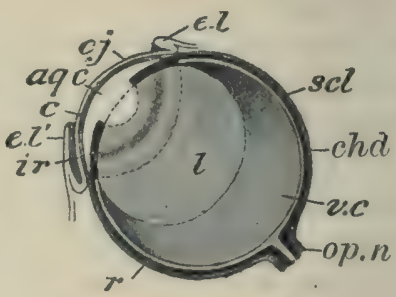

$\mathrm{D}$

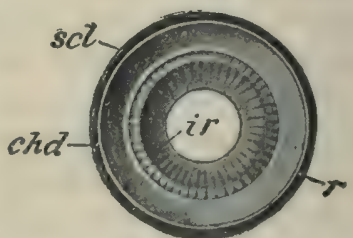

Fig. 57.-The eye of the Frog. A, as seen in the living animal. B, in median longitudinal section, through the plane of the optic nerve. $C$ and $D$, the two halves of the eye, bisected equatorially; the inner half (C) showing the blind spot. ( $x$ 31. $)$

ag. c. aqueous chamber; b. s. blind spot ; c. cornea ; chd. choroid ; cj. conjunctiva ; $e . l$. upper eyelid; $e$. $l^{\prime}$. lower eyelid (nictitating membrane) ; ir. iris ; $l$. crystalline lens; $o p . n$. optic nerve; $r$. retina; scl. sclerotic; $v$. c. vitreous chamber. (After Howes.)

the optic nerve. The transparent, exposed portion of the eyeball is the cornea $(c)$, a superficial thin layer of which, or conjunctiva $(c j)$, is continuous with the lining of the eyelids and thus with the skin covering the head; through it can be seen the coloured part of the eye, or iris (ir), with a black spot-really a hole-in its centre, the pupil. 
The interior of the globe (v.c) is filled with a colourless, transparent jelly, the vitreous humour, surrounding which, everywhere but on its external face, is a thin semi-transparent membrane, reddish when perfectly fresh, but becoming grey soon after death; this is the retina $(\boldsymbol{r})$. Between the retina and the sclerotic is a vascular membrane called the choroid (chd), the inner face of which, i.e., that in contact with the retina, is coloured black. It is this layer of black pigment which gives the dark tint to the semi-transparent sclerotic in the entire eye ; strictly speaking, it belongs to the retina, but actually it adheres to the choroid and appears like the innermost layer of that coat. The retina is readily detachable from the choroid, but at the place where the optic nerve enters (blind spot, $b . s$ ) it becomes continuous with the fibres of the latter, which pass through the sclerotic and choroid. The choroid is made up of connective-tissue and contains numerous vessels as well as pigment-cells.

Lying just internal to the pupil is a nearly globular body, perfectly transparent when fresh, the crystalline lens $(l)$; it is kept in place by a delicate membrane, the capsule of the lens. The iris, which covers the outer face of the lens except where it is perforated by the pupil, is covered on its inner surface with black pigment, and is continuous all round its outer margin with the choroid. Between the iris and the cornea is a space, the aqueous chamber of the eye (aq. c), which contains a watery fluid, the aqueous humour. The main cavity of the eyeball, containing the vitreous humour, is called the vitreous chamber.

The actual relations of these parts in the entire eye are best grasped in a vertical section, such as is represented in Fig. 57, B. The main part of the eyeball forms a chamber, enclosed by the sclerotic, darkened internally 
by the choroid and lined by the retina. Into the outer side of this dark chamber is let a transparent window, the cornea; behind which, and separated from it by a space containing the aqueous humour, is a vertical curtain, the iris, perforated by an aperture, the pupil. Behind the iris and in close contact with it is the lens, and filling the whole of the dark chamber between the lens and iris and the retina is the vitreous humour.

The whole eye thus has the structure of a camera obscura. The cornea, aqueous humour, lens, and vitreous humour are a series of lenses, so arranged that the rays of light from an external object are refracted and brought to a focus on the retina, where they form a greatly diminished and inverted image of the object. The iris is provided with muscles, by means of which the pupil can be enlarged or diminished; it therefore acts as a diaphragm and regulates the amount of light entering the eye. Attached to the capsule of the lens are delicate muscles, by means of which the focus of the entire apparatus can be altered to some extent according to whether the object viewed is nearer to or farther from the eye. This arrangement for accommodation is, however, much less highly developed in the frog than in man and the higher animals, in which the relatively smaller lens is flatter and distinctly biconvex in form. Thus the various parts of this complicated organ are so adjusted as to bring the images of external objects to an accurate focus on the back part of the interior of the eyeball, i.e., on the retina.

A vertical section of the retina (Fig. 58) reveals a very complex structure. On its inner face, i.e., the surface in contact with the vitreous humour, is a layer of nerve-fibres $(n . f)$, formed by the ramifications of the optic nerve, which, passing through the sclerotic and choroid, perforates the retina, and spreads out over its 
inner surface. Next comes'a layer of nerve-cells $(g)$, and then several layers of fibres and nuclei $(g r, n c)$; and finally, forming the outer surface of the retina proper, is a layer of delicate, transparent bodies called, from their form, the rods $(r)$ and cones $(c)$; these are known from their development to be modified epithelial cells. The whole of these structures are supported by a complex framework of connective-tissue. In close contact with the outer or free ends of the rods and cones is a layer of cells the protoplasm of which is filled with a dense black pigment. It is this pigment-layer ( $p . e p)$ which, as we have seen, is often counted as part of the choroid.

In spite of its complex structure, the retina is not much more than $\frac{1}{4}$ th $\mathrm{mm}$. ( $\frac{1}{100}$ th inch) thick, and is perfectly transparent. Hence, when an image is formed on it, the rays of light easily penetrate its whole thickness until they are stopped by the opaque layer of pigment. The rays can thus stimulate the rods and cones, and the stimulus is transmitted through the layers of nuclei and nerve-cells to the fibres of the optic nerve, along which it is con-

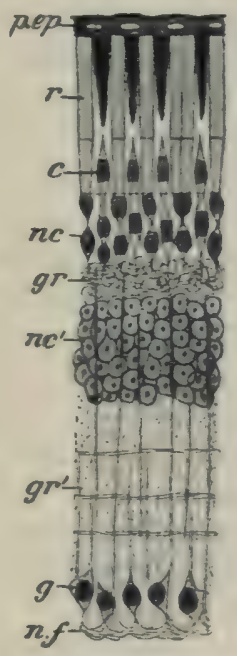

Fig. 58.-Vertical section of Frog's rẹtina. (> 300.)

c. cones; $\boldsymbol{g}$. layer of nerve-celis; $g r, g r^{\prime}$, outer and inner granular layers; $n c$, $n c^{\prime}$, outer and inver nuclear layers; $n$. $f$. nerve-fibre layer: $p$. p. pigmentepithelium ; $r$. rods. (After Howas.) veyed to the brain. Thus the actual organ of sight is not the eye as a whole, but the retina : all the rest is to be looked upon as an accessory apparatus for focussing and for regulating the admission of light. 
As with the other sense-organs, sight may be destroyed by injury to the retina or actual organ of sight, by cutting the optic nerve, or by destroying the brain. But unlike the other sense-organs already considered, that of sight has a complex accessory or focussing apparatus in connection with it, and vision may also be rendered impossible by injury to the cornea or lens.

It is an obvious advantage to an organ of sight such as the frog's that it should be capable of movement in any direction, so as to allow the light from any object to enter the pupil. As a matter of fact, the animal can direct its gaze through a very wide range by means of eight muscles connected with the eyeball in the orbit. One of these, the levator bulbi, raises the whole eye, causing it to project further on the surface of the head. Another, the retractor bulbi, withdraws it, causing it to bulge into the mouth. Four others (compare p. I64 and Fig. 126), the superior, inferior, anterior, and posterior recti, rotate it respectively upwards, downwards, forwards, and backwards. And, finally, two oblique muscles, the superior and inferior, produce a rotation along an axis joining the optic nerve with the middle of the cornea.

The conjunctiva, which covers the outer surface of the eye and lines the eyelids, is kept moist by the secretion of a lacrymal gland, known as the Harderian gland, situated between the eyeball and the orbit in the anteroventral region. The excess of this secretion is carried away into the olfactory chamber by means of a tube, the naso-lacrymal duct.

Each organ of hearing (which also serves for equilibration), like the organ of sight, consists of an essential portion and an accessory apparatus. The essential organ of hearing is a structure called the membranous labyrinth, contained within the auditory capsule of the 
skull (Fig. Io), and consisting of a kind of bag of very peculiar and complicated form (Fig. 59). It is made up, in the first place, of the vestibule-two somewhat ovoid sacs separated by a constriction: the dorsal one is called the utriculus (ut), the ventral the sacculus (s), and from the latter a small process, the cochlea $(c c)$, projects backwards. With the
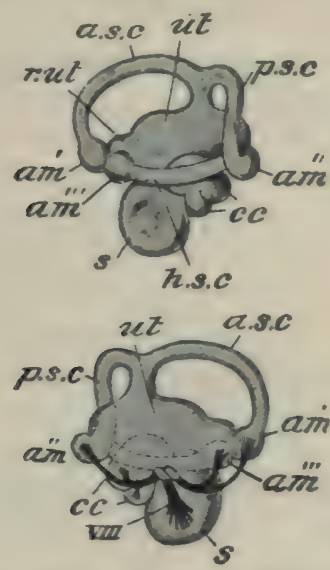

A

B

Fic. 39.-The membranous labyrinth of the Frog's ear. A, outer, and B, inner view. $(x \quad 6$.

$a m^{\prime}, a m^{\prime \prime}, a m^{\prime \prime \prime}$, ampullæ of the semicircular canals; a. s. c. anterior semicircular canal; $c c$. cochlea; h. s. c. horizontal (external) semicircular canal : p. sc. posterior semicircular canal; $r$. ut. recess of the utriculus; s. sacculus; wt utriculus; VIII. branches of auditory nerve. (After Howes.)

utriculus are connected three tubes, called, from their form, the semicircular canals, each of which opens into the utriculus at either end. One of them, the anterior canal (a.s.c), is directed forwards; another, the posterior canal (p.s.c), backwards; both these are vertical in position and are united to one another at their adjacent ends. The third, the external canal (h.s.c), 
is directed outwards and has a horizontal position. Each canal has one end dilated into a bulb-like swelling or ampulla (am); those of the anterior and external canals are at their anterior ends, while that of the posterior canal is at its posterior end.

The whole of this apparatus is filled with a fluid, the endolymph, in which are contained calcareous particles, the otoliths or ear-stones. It is made of connective-

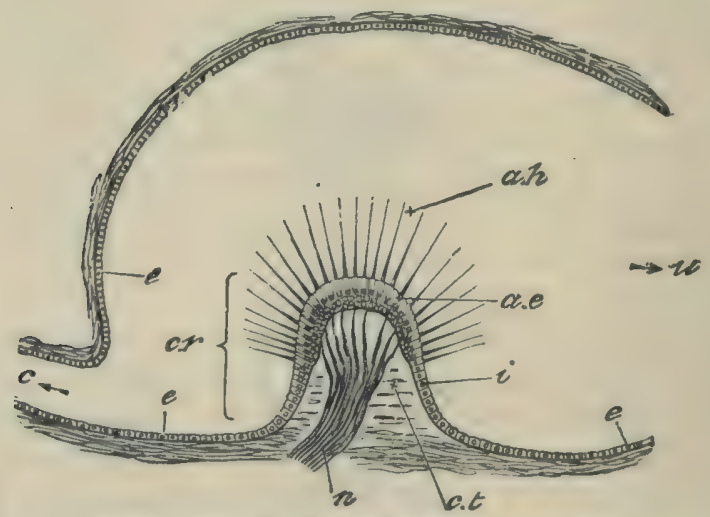

FIG. 6o.--I.ongitudinal section through an ampulla,

a. c. auditory epithelium; $a . h$. auditory hairs ; $c$. part of semicircular canal ; $c, \%$. acoustic spot and ridge; $c . t$. connective-tissue; $e$. epithelium; $n$. nerve; $u$. junction with utriculus. (From Foster and Shore's Physiology.)

tissue and lined with epithelium, the cells of which are cubical for the most part; but in certain places the wall is thickened, forming what are called acoustic spots, of which there is one to each ampulla, situated on a ridge (Fig. 60), while others occur in the utriculus and sacculus. On these acoustic spots the epithelial cells are greatly elongated, and produced at the surface into delicate processes called auditory hairs: to these cells the fibres of the auditory nerve are distributed. 
The membranous labyrinth does not fit tightly into the cavity of the auditory capsule in which it is contained, a space being left between it and the surrounding bone and cartilage (Fig. Io). This space is filled by loose connective-tissue and a fluid called perilymph, by which the membranous labyrinth is surrounded and protected from shocks. As you learnt in studying the skull, the outer wall of the auditory capsule is perforated by a small aperture, the fenestra ovalis (Fig. Io, fen. ov), in which is fixed the stapes $(s t p)$, a small nodule of cartilage connected with a bony rod or columella (col), the cartilaginous hammer-shaped outer end of which, or extra-columella, is fixed to the inner side of the tympanic membrane (tymp. memb). The columella lies in the tympanic cavity (tymp. cav), which is bounded externally by the tympanic membrane, internally by the auditory capsule, and at the sides chiefly by muscles and connective-tissue; while below it communicates with the pharynx by the Eustachian tube (eus. $t$ ).

When sound-waves impinge on the tympanic membrane, the vibrations to which they give rise are transmitted by the columella to the stapes, and so to the perilymph. Thence they are communicated to the endolymph and act as stimuli to the auditory cells of the acoustic spots, and the impulses being carried to the brain by the auditory nerve, give rise to the sensation of sound. Whether or not all the acoustic spots are truly auditory in function is not known: it seems that the semicircular canals are really concerned with the sense of direction and velocity and with the maintenance of equilibrium.

The sense of sound can be destroyed by injury to the membranous labyrinth, by cutting the auditory nerve, by destroying the brain, or-to a great extent at least-by injury to the tympanic membrane or columella. 
Notice that the general plan of all the sensory organs those of the skin, eye, and ear, is the same. They con sist of certain peculiarly modified epithelial cells, speciall sensitive to impulses of particular kinds, and in com munication, by means of an afferent nerve, with nerve cells of the brain. The three things-sensory cell afferent nerve, and brain-form a chain, every link o which is necessary for the performance of the sensor function, so that the particular sense in question may b destroyed, not only by destruction of the sense-organ in the strict sense, but also by section of the afferen nerve or by destruction of the brain.

General Physiology-Summary.-Before going on to the next chapter it will be as well to take a final glanc at the physiological processes of the frog as a whole (com pare Fig. 23). The enteric canal is the manufactory in which the raw material of the food is worked up into form in which it can be used by the various parts of th body. The circulatory organs are the communicating system by which the prepared food is taken to all parts and they also form a drainage system by which wast matters are collected from all parts and finally ejectes by the three main sewers, the skin, lungs, and kidneys The skin and lungs, besides getting rid of waste matters serve for the supply of oxygen-a necessary form o gaseous food. The central nervous system forms a sor of headquarters' staff by which the entire body is con trolled, the means of communication being the nerves and the muscles the executive by which the orders from headquarters are executed. And finally the sense-organ may be looked upon as the various branches of an intelligence department by which the headquarters ar informed of what is going on outside. 


\section{PRACTICAL DIRECTIONS}

\section{The Organs of Special Sense}

I. Olfactory organ. Notice again the external and internal nostrils. Then remove the skin covering the snout, dissect off the nasal bones, and open up the olfactory sacs. Note, using a lens, the pigmented olfactory epithelium lining these, and make out the olfactory neries and nasal septum. Compare with a transverse section through the sacs. Sketch.

II. Eye.

a. Notice again the eyelids, ivis, and pupil. Then remove the skin covering the head so as to expose the nearly globular eyeballs, lying in the orbits. Examine with a lens.

In the antero-ventral region of the orbit make out the Harderian gland, and the eye-muscles passing from the walls of the orbit to the eyeball. The four recti and two oblique muscles can be more easily seen on a larger animal, and directions will be given for their examination in Part II (see Fig. I 26); but if you make a dissection of them in the frog, you should note at the same time the lecator and reiractor bulbi, the latter underlying the eyeball, and the former situated internally to the recti muscles.

$b$. Remove the eyeball from a freshly-killed specimen, noticing as you do so the optic nerne, which is surrounded by the recti and retractor bulbi muscles: dissect away these muscles and note the cartilaginous sclerotic, the cornea, iris, pupil, and the cut end of the optic nerve.

c. Divide the eyeball into an inner and an outer hemisphere by a rapid cut with scalpel or scissors taken vertically, midway between the cornea and the optic nerve, through the vitreous chamber. Place them both in a watch-glass or small dissecting-dish, under water, and examine with a lens (compare Fig. 5i). In the inner hemisphere note the vitreous humour, retina, pigmented choroid, and blind spot or entrance of the optic nerve ; and in the outer hemisphere, the crystalline lens and the margin of the retina, or ora servata. Sketch. Remove the lens, and notice the iriscontinuous with the choroid, the pupil, and the aqueous chamber.

d. Examine sections through the wall of the inner hemisphere of the eyeball, prepared as directed on p. 136, first 
under the low and then under the high power of the microscope. Note :-

I. The cartilaginous sclerotic.

2. The choroid, enclosing pigment-cells and blood-vessels.

3. The retina (Fig. $5^{8}$ ), composed of a number of layers : notice the pigment epithelium, the rod-and cone-layer and the various other layers of the retina, the innermost of which is composed of nerve-fibres continuous with the optic nerve.

Sketch.

The anatomy of the eye can be more easily made out by dissecting that of an ox or sheep, which is essentially similar to that of the frog, and directions for the examination of which will be given in Part II. (p. 556).

III. Auditory organ.

Notice again the tympanic membrane and tympanic ring, and then carefully cut away the former so as to expose the tympanic cavity. Observe the Eustachian tube, the fenestra ovalis, and the relations of the stapes, columella, and extracolumella (Fig. 10).

The essential part of the auditory organ (membranous labyrinth) is very small in the frog, and can be more satisfactorily studied in a good-sized fish (e.g., dogfish or cod). Directions for the preparation of the membranous labyrinth of the former will be given at a later stage (p. 479), but if you have sufficient time and patience to dissect it out in the frog, proceed as follows :-

Place the head of a large frog in nitric acid (about ro per cent.) until the bone is dissolved. Wash well in water so as to remove the acid, and dissect away the muscle, etc., from the auditory capsule until the latter is thoroughly exposed. Then with a sharp scalpel slice away the roof of the capsule until the cavity it contains is seen. Proceed now with great caution, removing the cartilage and decalcified bone, bit by bit, until the cavity is sufficiently enlarged to bring the membranous labyrinth into view (compare Figs. Io and 59). Observe the utriculus, sacculus, otoliths, and the three semicircular canals with their ampulla. Sketch. 


\section{CHAPTER XII}

THE FROG (continued) : REPRODTCTION AND DEVELOPMENT

So far we have considered those parts and organs of the frog which have to do with its welfare as an individual. We have now to consider the organs which are connected with the welfare of the frog as a race, that is, with the propagation of its kind.

The position of the reproductive organs has already been seen (pp. 23 and 25): they must now be examined in more detail. The essential part of these organs in each sex is a pair of bodies known as gonads, called in the male spermaries or testes, and in the female ovaries.

Reproductive Organs of the Male.-The spermaries (Fig. 3, r. spy, Fig. 5, spy, and Fig. 7, ts) are a pair of ovoid bodies, each attached by a fold of the peritoneum to the corresponding kidney, and having connected with it a fat-body $(c p . a d)$. From the inner margin of each spermary spring a number of delicate tubes, the efferent ducis (Fig. 6r, q), which run in the fold of the peritoneum to the kidney. Entering this organ near its inner edge, they open into a longitudinal tube $(L)$ from which transverse tubes pass horizontally across the kidney to open into the ureter $(U r)$. The milt, or spermatic substance (p. q). is thus carried off by the same duct as the urine; the ureter in the male is 
therefore often called the urinogenital duct. On the outer side of the ureter, and communicating with it by numerous short ducts, is a glandular body, the seminal vesicle (Figs. 3 and 7, vs.sm), which serves to store up. the spermatic substance.

The spermary itself contains a narrow, irregular, central cavity, from which the efferent ducts proceed

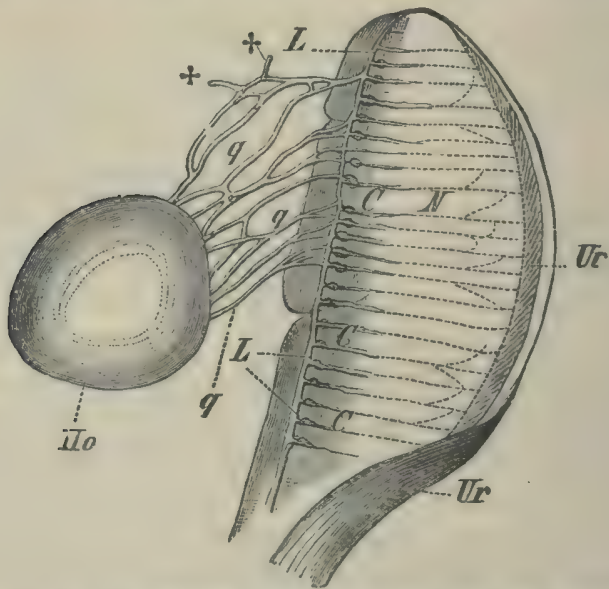

F16, 61-Spermary and kidney of "elible Frog" chowing the relations of the efferent ducts ( $x$ about 3 ; semidiagramnatic).

$C$, transverse tubes in kidney; Ho. spermary; $L$. Jongituclinal tube; $N$. kidney; g. efferent ducts of spermary; Ur. ureter (urinogenital (luct). (From Wiedersheim's Comp. Anatomy.)

and into which open a number of short tubes or crypts (Fig. 62, A). These are lined with epithelium (t.e), the cells of which divide and subdivide, forming groups of smaller cells. Each of the latter undergoes a remarkable change, becoming converted into a rod-like body, produced into a long thread, which performs lashing movements, very much like those of the cilia in ciliated epithelium. These bodies are called sperms or sperma- 
tozoa (Fig. 62, A, sp, and B) ; in spite of their peculiar form, they are cells, the rod-like portion, or heud, being the nucleus, and the delicate vibratile part, or tail, the protoplasm. In the breeding season the cavities of the testes are full of sperms floating in a fluid. Thus the spermatic fluid, like the blood, owes its distinctive character to the cells floating in it.
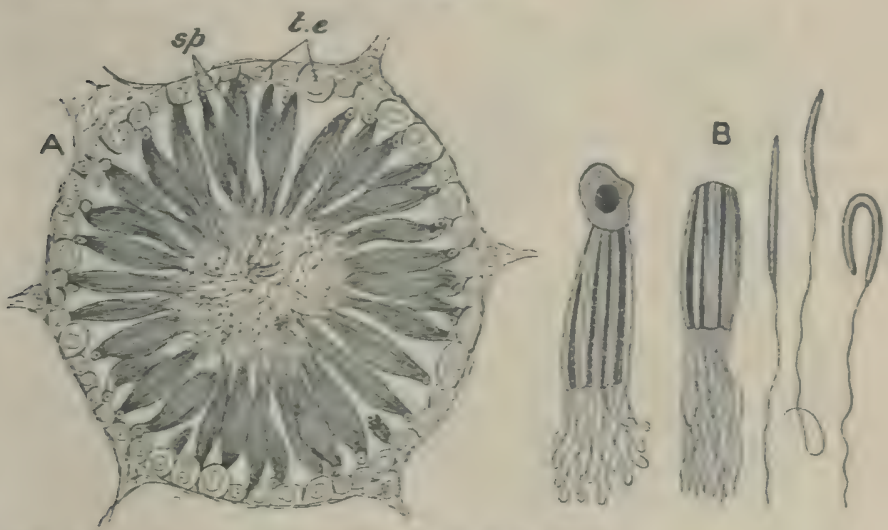

Fic. 62.-A, transverse section of a crypt of the Frog's spermary. ( $\square$ about 350.)

$s p$. bundles of sperms; t.e. germinul epithelium. (A, after Blonutield; B, after Howes.

Reproductive Organs of the Female.--Each ovary (Fig. $4, l$. ovy) is a greatly folded sac with thin walls and a large cavity divided up by partitions. It is attached to the dorsal body-wall by a fold of peritoneum. As we have seen (p. 25), its surface is studded all over with little rounded projections, each of which is an ovisac, and contains an egg. The egg or ovum (Fig. 63) is a large globular cell with a clear nucleus ( $n u$ ) containing numerous nucleoli $\left(n u^{\prime}\right)$, and having its protoplasm ( $p r)$ full of yolk-granules-grains of proteid material which serve as nutriment for the growing embryo. It 
becomes covered with a delicate egg-membrane. By the time the egg is mature a superficial deposit of pigment takes place over one hemisphere.

In the young condition all the epithelial cells forming the walls of the ovary are alike, but as the organ reaches maturity, certain of them (o) enlarge and give

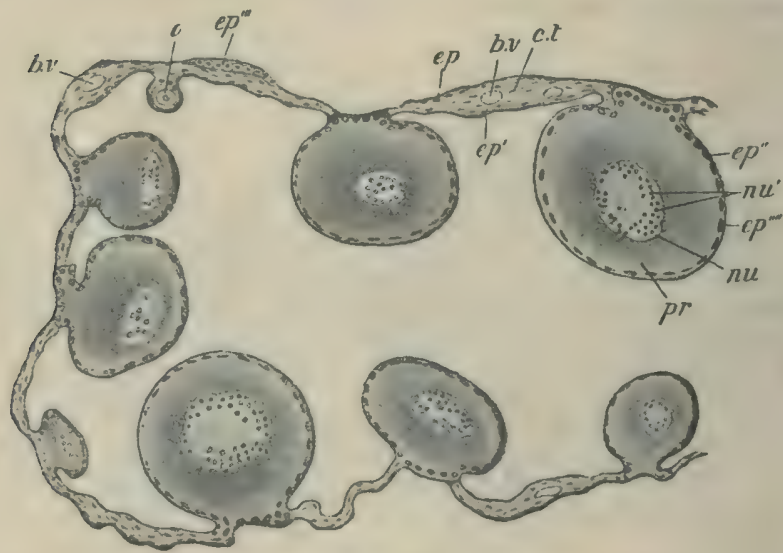

Fig. 63.-Transverse section of Frog's ovary. ( $\times 30$.

l. $v$. blood-vessels: c. $t$. connective-tissue; $e p$. outer layer, and $e p^{\prime}$ inner layer of epithelium; $e p^{\prime \prime}$, outer layer of ovisar, continuous with $e p^{\prime}$; $e p^{\prime \prime \prime}$, germinal epithelium, derived from $e p . ; e p^{\prime \prime \prime}$, follicular epithelium, derived from $c p$. ; $m$. nucl $u$; of nvum : $n u^{\prime}$, ruclesli; n. young ovum; pr. protoplasm of ovim containing yolk-granules. (After Marshall.)

rise to the ova, while others form an investment or follicle (ep $\left.p^{\prime \prime \prime}\right)$ for each ovum.

The oviduct (Fig. $4, l$. ovd, $r$. ovd), as you have seen, is a long and greatly convoluted tube lying above or dorsal to the ovary. Its anterior end narrows considerably, runs parallel with the gullet, passes to the outside of the root of the lung, and then opens into the coelome by a small aperture $(r$. ovd'). The greater part of the oviduct is about as wide as the small intestine, and is thick-walled and lined with gland-cells, which secrete 
the jelly surrounding the eggs when laid (p. 9). Posteriorly it suddenly dilates into a wide, thin-walled chamber $(r$. ovd") which opens into the dorsal wall of the cloaca. Notice that there is thus no connection between the generative organs and the kidneys in the female such as occurs in the male, the ureters serving as renal ducts only.

In the breeding season the ovisacs burst and set free the eggs into the cœlome, whence by some means or other they find their way into the small openings of the oviducts, and so into these tubes, when each becomes surrounded by a little sphere of jelly secreted by the gland-cells. Passing down the oviducts the eggs accumulate in the dilated extremities, which they distend enormously, so that, just before laying, the abdomen of a female frog is nearly filled by these two great eggreservoirs; the ovaries, having lost so many of their eggs, are correspondingly reduced in size, and all the other organs are squeezed out of place.

Fertilisation.-The eggs are now laid, and immediately the spawn is passed from the oviducts of the female into the water, the male sheds over them a quantity of spermatic substance (p. q). The sperms, swimming actively through the water, enter the spheres of jelly and come into contact with the eggs. A single sperm then penetrates the egg-membrane of an ovum, loses its tail, and its head coming into contact with the nucleus of the egg, fuses or conjugates with it, so that a single nucleus is formed by the union of the egg-nucleus with the sperm-nucleus. We may speak of conjugating cells in general as gametes, the sperm in this case being the male gamete, and the ovum the female gamete, the body formed by the fusion of two gametes being known as a zygote.

This process is known as fertilisation or impregnation. 
Without it, as we have seen, the egg is incapable of development ; after it has taken place, the egg-or more strictly, the oosperm, since it is now an ovum plus a sperm-is potentially a young frog, since, if left undisturbed in water, it will in course of time give rise to a tadpole, which in its turn will change into a frog.

It must be remembered in the first place that the fertilised egg is a single cell, comparable with a bloodcorpuscle or an epithelial cell. It is, however, peculiar in two respects; first in having its nucleus derived in part from a sperm, so as to contain matter from both the male and the female parent ; and secondly in having its protoplasm distended with yolk-granules to such an extent that, instead of being a minute body visible only under the microscope, it is easily visible to the naked eye. The yolk is not equally distributed : on one hemisphere it is less abundant than elsewhere, and it is this more protoplasmic hemisphere which is superficially blackened by a layer of pigment, and which always floats upwards in the water when the egg is laid.

Segmentation of the Oosperm.-Almost directly after being laid and fertilised the egg undergoes a remarkable change. A furrow appears all around it, as if made with a blunt instrument, and deepening gradually, at last divides the oosperm into two hemispheres in contact with one another by their flat faces (Fig. 64, A). The examination of sections shows that this process is preceded by the division of the nucleus into two; its final result is the division of the originally one-celled egg into two cells. Now if you refer to Chapter VIII, you will be reminded of the fact that the epithelial cells of the skin multiply by a similar process of simple fission, or division into two; the nucleus in each case dividing first, and afterwards the protoplasm. 


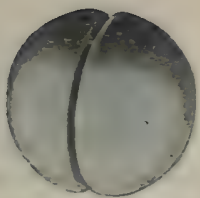

A

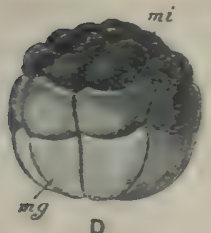

D
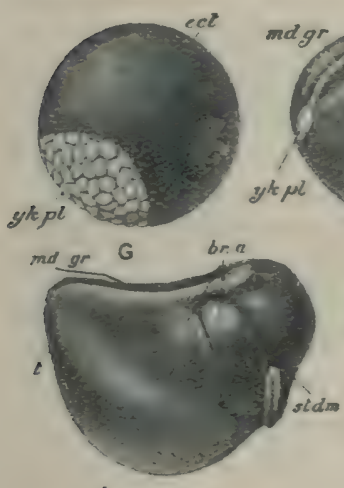

$J$

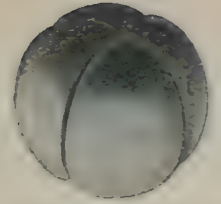

B

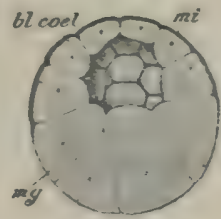

E

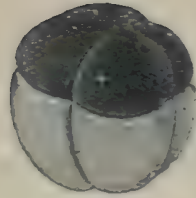

C

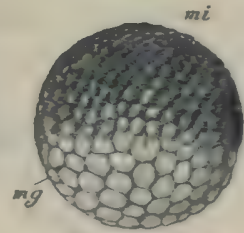

$\mathbf{F}$
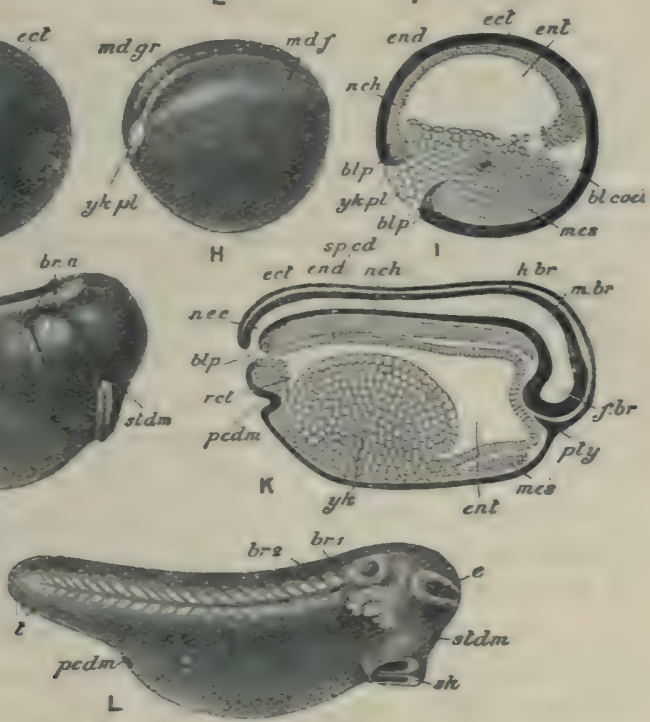

Fig. 64.-Development of the Frog.

A-F, segmentation: G, overgrowth of ectoderm: H. I, establishment of germinal layer: J, K, assumption of tadpole-form and establishment of nervous system, notochord, and enteric canal: L. newly hatched tadpole. (a 10.)

br. col. segmentation cavisy; bip, blp', blastopore: $b^{\prime}{ }^{\prime}, b r^{2}$, gills; $b r$. $a$. branchial arches: $c$. eve: a. ectoderm; end. entoderm; ent. enteron f. br. forcbrain: $h$. br. hind-brain: $m$. br. mici-brain: md. $f$. medullary fold; me. $g r$, medullary groove : mes, messolerm: me. larse liswer cells : ms. small upper cells;

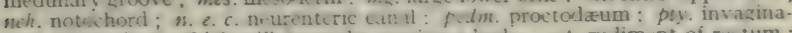

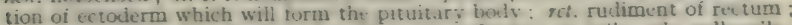
sk. sucker: $s \hat{p}$. $a d$. spinal cord: stim. stomixlaum: $t$. tail:,$k$. yolk-crils: yk. pl. yolk-plug which fills the klastipore. (From Parker and Haswell's Zmogy. A-D, F H, and I from Ziegler's models, E, I, K, and L after Marshall.) 
The furrow which effects this division of the oosperm passes through both black and white poles, so that each of the two cells formed is half black and half white. Soon a second furrow is formed at right angles to the first, being, like it, meridional, i.e., passing through the poles (Fig. 64, B). It divides what we must now call the embryo into four cells, each half black and half white. A third furrow is then formed, passing round the equator, but nearer the black than the white pole (C). It therefore divides the embryo into eight cells, four upper black and four lower white, the latter being obviously the larger.

This process of division or segmentation of the oosperm continues, the black cells dividing more rapidly than the white, so that before long the embryo consists of a mass of cells, the polyplast, somewhat resembling a mulberry, and therefore often called a morula, one hemisphere being composed of small cells containing much protoplasm, and little yolk, and externally pigmented (D-F, $m i)$, and the other of larger cells containing little protoplasm and much yolk, and not pigmented $(m g)$. As segmentation goes on, a cavity appears in the interior of the embryo; it is called the segmentation-cavity $(\mathrm{E}, b l$. coel), and is due to the fact that the work of segmentation produces a waste of substance which there is at present no means of making good, so that for a time, while the size of the embryo remains the same, its bulk diminishes, some of its substance being used up; in other words, it feeds on itself.

Segmentation now proceeds to such an extent that the black cells become too small to be seen except with a lens of tolerably high magnifying power, so that with the amount of magnification used in Fig. 64, G, the black hemisphere shows no division into cells. At the same time the black hemisphere gradually encroaches on 
the white until only a small circular space-the blastopore, filled by a mass of yolk-cells-the yolk-plug, is left uncovered $(\mathrm{G}, \mathrm{H}, y k$. $p l)$.

Development of the Chief Organs.- In the meantime a second cavity appears in the interior of the embryo, and as it increases in size, the segmentationcavity undergoes a proportional diminution and finally disappears. This new cavity, or enteron (Figs. 64, I, and 65

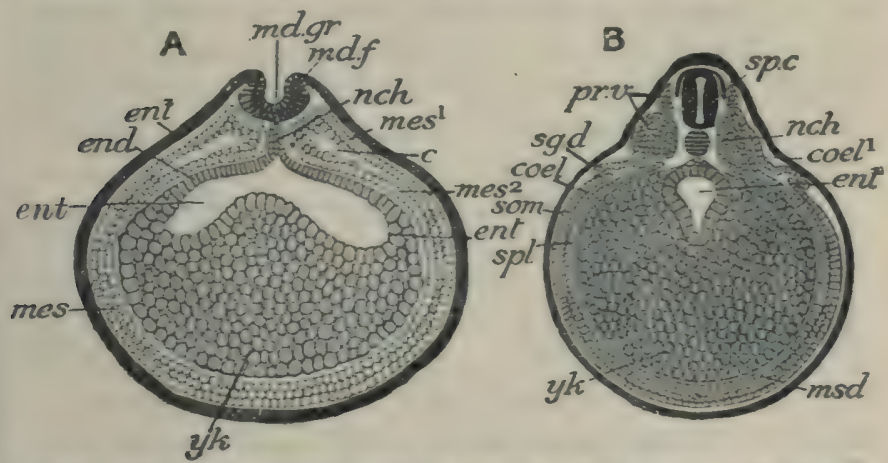

Frg. 65.- Transverse sections through the body of a Frog embryo. A, with open meciullary grnove (compare Fig. 64, J). B, with clnse:l mellullary groove (compare Fig. 64, L.) ( $x$ about 30.)

$c$, crel. coelmme ; crit, prolongation of cuelome intn mesodermal segments (pr. $v$ ) ; coud. endowlerm: ent. enteron; mes. msd. mesorlerm; mes's. som. nuter parietal laver of mesoderm mes ${ }^{2}, s p l$. inner visceral laver of mesoderna ; md. f. mprlullary fold: mul. gr. medullary groove; $n c h$ notochord; pr. $v$. mesodermal segment; $s g . d$. primary kidney duct (pronephric duct) ; $s p . c$. medullary cord; yk. yolkcells. (After Marshall.)

ent), is the rudiment of the enteric canal : it begins to be formed as a narrow slit at the edge of the blastopore through which it at first communicates with the exterioal but it soon becomes closed: at present there is neither mouth nor'anus.

By this time the cells of which the embryo is composed have assumed different forms and become arranged in a very definite manner. First of all there are severa layers of small pigmented cells, derived from the black 
cells of earlier stages, which cover the whole embryo with the exception of the yolk-plug: these constitute the ectoderm (Fig. 64, G, H, I, ect). Forming the roof of the enteric cavity are other layers of cells derived from the yolk-cells of earlier stages and forming the endoderm (I, end): the floor of the enteron is at present formed of unaltered yolk-cells. Between the ectoderm and the yolk-cells on the lower pole are several layers of small cells which gradually spread until they form a complete layer between the ectoderm and the endoderm : these constitute the mesoderm (I, mes). The ectoderm, endoderm, and mesoderm are known as the three embryonic tissues or germinal layers.

While these processes have been procecding, a longitudinal groove appears on the dorsal surface of the embryo: this is the medullary or neural groove, and is bounded by a pair of medullary folds (Figs. 64, H, J, and $65, m d . g r, m d . f)$. The medullary groove extends backwards to the blastopore, which now marks its posterior end. As development goes on, the folds approach one another and unite, so as to convert the groove into a canal; the cells lining this canal give rise to the central nervous system (Fig. 64, K ; compare Part II, Chapter XIII), and its cavity becomes the central canal of the spinal cord and the ventricles of the brain. The anterior end of the hollow medullary cord thus developed enlarges to form three vesicles, one behind the other, and known respectively as the fore-brain, mid-brain, and hind-brain, each containing a ventricle. The fore-brain eventually gives rise to the diencephalon and to the cerebral hemispheres, produced in front into the olfactory lobes; the mid-brain to the optic lobes and crura cerebri; and the hind-brain to the medulla oblongata and cerebellum. It will be seen (Fig. 65) that the medullary groove is lined by ectoderm and that there- 
fore the whole central nervous system is ectodermic in origin; and this is also true of the nerves.

A longitudinal thickening of the endoderm on the dorsal side of the enteron becomes constricted off as a solid rod of cells, lying between the medullary cord and the enteron. This is the notochord (Figs. $64, \mathrm{~K}$, and $65, n \mathrm{ch}$ ) ; it forms the primary axial skeleton around which the vertebral column is subsequently developed. The mesoderm is for some time a solid mass occupying all the space between the ectoderm and endoderm, so that there is no body-cavity. But at a later stage a cavity appears dividing the mesoderm into two layers, one in contact with the ectoderm (parictal laver, Fig. 65, mes ${ }^{1}$, som), the other with the endoderm (visceral layer, mes $^{2}, s p l$ ). The space between them is the calome $(c$, coel). The dorsal part of the mesoderm on either side of the medullary cord and notochord becomes divided transversely, and gives rise to muscle-segments or myomeres (Figs. 64, L, and 65).

The embryo soon begins to elongate in a definite direction (Fig. 64, J) : its dorsal surface, still marked by the medullary groove, which now soon becomes closed, is slightly concave, its ventral surface very convex. The blastopore closes up, the yolk-plug becoming entirely covered by ectoderm ; but for a short time the medullary canal and enteron still communicate by the neurcnteric canal (K, n.e.c). This soon disappears, and at the hinder end of the embryo a conical outgrowth forms the rudiment of the tail $(t)$. The opposite end is rounded, and on its ventral surface appears a little half-moon-shaped groove, the rudiment of the sucker by which the tadpole attaches itself to weeds ( $\mathrm{J}$ and $\mathrm{L}, s k$ ); this afterwards becomes subdivided into two (Fig. 66, C). Just above the sucker a depression-the mouth-pit or stomodaum $(\mathrm{L}, \mathrm{stdm})$-makes its appearance, and is the 
first indication of the mouth. A similar depression below the tail-the proctodceum $(p c d m)$-marks the anus: both at first are mere blind pouches and have no communication with the enteric cavity. The headregion is further marked by two pairs of vertical ridges separated by depressions: the ridges are the branchial or gill arches $(\mathrm{J}, b r . a)$, and the depressions the branchial clefts.

Further elongation takes place (Fig. 64, L), the head becomes distinctly marked off, the tail extends considerably beyond the anus, a thickening appears which marks the position of the eye (e), and a depression that of the ear (just above $b r^{2}$ in the figure) ; and from each branchial arch arises a little tuft, the rudiment of one of the external gills $\left(b r^{1}, b r^{2}\right)$. In this condition the tadpole is hatched : it is still unable to feed, the stomodæum not yet being in communication with the enteric cavity ; it is nourished therefore entirely by the yolk with which a large portion of the body is still filled.

Up to the stage shown in Fig. 64, I, the cells of which the tadpole is composed, although distinguishable into ectoderm, endoderm, and mesoderm, are all more or less similar: there are no muscles, no cartilages, bones, or connective-tissue. But shortly after the stage referred to, the permanent tissues begin to be formed: the outer ectoderm cells take on the form of epiderm, the endoderm cells become the epithelium of the enteric canal, and from offshoots of the latter-all of course lined by endoderm-are formed the lungs, the liver, the pancreas, and the urinary bladder.

The mesodermindergoes much more extensive changes, giving rise to the connective-tissue, cartilage, bone (after metamorphosis), and muscle, as well as to other partsi.e., to by far the greater portion of the permanent tissues. In certain places the embryonic mesoderm cells, hitherto 
in close contact, separate from one another and become branched, while between them appears intercellular substance with fibres crossing one another in various directions : in this way the connective-tissue of the adult body is produced.

The various parts of the skeleton first arise by the conversion of portions of the mesoderm into cartilage. The cells retreat from one another and between them a clear substance, the matrix, makes its appearance. For a considerabie time almost the whole skeleton consists of cartilage, but after metamorphosis much of this tissue is replaced by bone developed from a layer of cells on the inner surface of the perichondrium. The bones thus formed in connection with cartilage are replacing bones: investing bones arise in connective-tissue which has no direct relation to the cartilage (compare p. 43).

In the place where the voluntary muscles are to appear the mesoderm cells elongate, their nuclei multiply by fission, and their protoplasm gradually becomes converted into the striated substance so characteristic of the adult muscular tissue.

These examples will suffice to illustrate the fact, which further study would show to be true, that all the permanent tissues are either-like epithelium-formed entirely of actual cells, or are-like connective-tissue and striped muscle-derived from cells. The entire embryo in its earliest condition-the oosperm stage-is a single cell, which multiplies repeatedly by simple fission, forming a group of cells : and these, by assuming various forms and undergoing various changes, give rise to all the complex tissues-differing from one another both structurally and functionally-of the adult animal. So that every cell, fibre, or what not in the frog is a linear descendant, through repeated fission, of the oosperm; and the oosperm itself is the product of the fusion of two cells, 
one-the ovum-derived from the female, the other-the sperm-from the male parent. Thus in passing from the oosperm to the adult animal there is a gradual structural or morphological differentiation accompanied by a differentiation of function or division of physiological labour.

The expression " division of physiological labour " was invented by the great French physiologist, Henri MilneEdwards, to express the fact that a sort of rough correspondence exists between lowly and highly organised animals and plants on the one hand, and lowly and highly organised human societies on the other. In primitive communities there is little or no division of labour: every man is his own butcher, baker, soldier, doctor, etc., there is no distinction between "classes" and "masses," and each individual is to a great extent independent of all the rest. Whereas in complex civilised communities society is differentiated into politicians, soldiers, professional men, mechanics, labourers, and so on, each class being to a great extent dependent on every other.

\section{Development of other Organs--Structure and Metamor-} phosis of the Tadpole-Metamorphosis.-A sketch of the further development of the tadpole and of its metamorphosis has already been given (pp. 9-II, Fig. I), but it is now necessary to add a few details to those already mentioned.

A third pair of branchial arches appear behind the two already noticed, and on each a third external gill. The first two pairs increase greatly in size and all the gills become branched (Fig. 66, A), The four branchial clefts communicate with the pharynx, and a current of water enters the mouth and passes out by these clefts, thus providing the gills with a constant supply of 
aërated water for purposes of respiration. A thin median foid of the skin, the tail-fin (Fig. I), arises all round the tail, both above and below, and the tail now forms a powerful swimming organ. The stomodæum and, at a still earlier period, the proctodæum (p. 204) open into the enteric cavity (Fig. 66, B-E), so that there is now a complete enteric canal: it grows mucin faster than the body generally, and becomes coiled like a watch-spring $(\mathrm{D}, \mathrm{E})$. The mouth is very small, and is bounded by lips beset with little horny projections or papillæ and provided with a pair of horny jaws (C-E), with which the tadpole now browses upon the water-weeds which form its staple food. It is on account of this diet that the intestine is of such great proportionate length: vegetable diet contains less nourishment, bulk for bulk, than animal, and a longer intestine is required to extract all the nutriment from it.

Soon the external gill's show signs of shrivelling, and on the inner portions of the branchial arches internal gills (Fig. 66, D ${ }^{1}$ ) are developed, like those of a fish. A fold of skin appears on either side in front of the gills and gradually grows backwards, covering the branchial clefts and the external gills, which latter soon disappear entirely; this is the operculum or gill-cover $(\mathrm{B}, \mathrm{C})$; it is similar to that of an ordinary fish, such as a cod or a perch, except that it is not supported by bone. The current of water from the pharynx now, of course, makes its final exit by a wide cleft between the edge of the operculum and the flank. The operculum gradually extends, and its free edge unites with the wall of the body posterior to the gills, so that the originally large aperture through which the water from the pharynx passes is much reduced. After some time a complete union of the operculum with the flank takes place on the right side, the opercular aperture becoming closed: 
on the left side the spout-like aperture remains open for some time longer, and the water from both sides passes out through it (D). All this time the tadpole is to all intents and purposes a fish ; apart from the possession
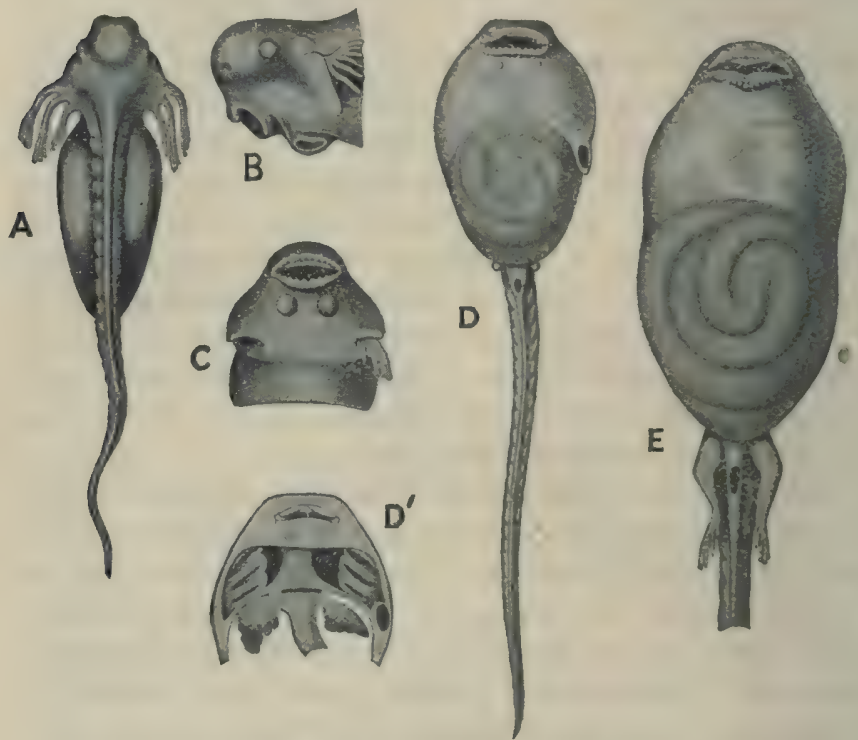

Fic. 66.-Stages in the later development of the Tarlpole. ( $\left.\times 7 \frac{1}{4}\right)$

A, stage with external gills; 13, stage showing the formation of the operculum; C, later stage, in which the external gills are disappearing; 1), stage with internal gills and buckling limbs; 1)1, dissection of stme stage to show the heart, gills, and lungs. $\mathbb{E}$, later stage, in which the limbs are further differentiated. (After Howes.)

of gills and a tail-fin, in the structure of the circulatory organs and various other parts it is more like a fish than a frog.

The lungs $\left(D^{1}\right)$ become functional, and the tadpole is now truly amphibious, rising periodically to the surface to breathe air. At a later stage, however, the gills 
gradually disappear, the single opercular aperture closes, and thenceforth respiration is purely aërial.

In the meantime the limbs are developed. The hindlimbs appear as little rounded buds, one on either side of the root of the tail (D) : they gradually elongate and divide into thigh, shank, and foot (E). The fore-limbs appear beneath the operculum and are therefore hidden at first (E); at a later stage each divides into upper arm, fore-arm, and hand, and emerges from its concealment. As the limbs increase, the tail undergoes a progressive shrinking; its tissues become, as it were, digested, and are carried off by the blood; so that for a time the metamorphosing tadpole is nourished largely by its own tail.

The mouth widens, the horny jaws and papillæ disappear, and teeth are formed. The suckers vanish, and the intestine not only begins to grow less rapidly than the body, but even becomes reduced in length and loses its spiral arrangement, while vegetable is exchanged for animal diet. The dark colour of the tadpole gradually gives place to the bright hues of the frog. The little tailed frog can now leave the water and hop about on land : its tail is soon absorbed, and the metamorphosis is complete (Figs. I, 7,8).

It will be noticed that in the course of development a substitution or replacement of organs occurs. Thus, for instance, the notochord is replaced by a vertebral column, the gills by lungs, and the horny jaws by teeth.

Fate of the Germinal Layers.-In concluding this chapter we may enumerate in rather more detail the chief parts and organs which are derived from each of the three embryonic tissues respectively. From the ectoderm are formed-the epiderm and the cutaneous glands; the whole of the nervous system, central and peripheral, and the essential parts of the sensory organs, as well

Pract, Zools 
as the crystalline lens of the eye; and also the epithelium lining the mouth (stomodæum) and outer part of the cloaca (proctodæum). The endoderm gives rise to the epithelium lining the enteric canal and its various offshoots, including the lungs, urinary bladder, gastric glands, bile and pancreatic ducts, as well as the glandular cells of the liver and pancreas; and to the notochord. From the mesoderm are developed the various parts which are situated between the ectoderm and endoderm with the exception of the notochord, viz., the connective-tissue, cartilage, bone, striped and unstriped muscles, circulatory organs, and peritoneum, as well as the urinary and reproductive organs and the accessory parts of the sensory organs.

\section{PRACTICAL DIRECTIONS}

\section{Organs of Reproduction.}

Open a frog in the usual way: cut through the gullet, rectum, and mesentery, and remove all the digestive organs. In the female, take especial care not to injure the roots of the lungs in severing the gullet. One of the specimens you have already dissected and kept in the preservative fluid should still contain the urinogenital organs intact in the case of the male: in the female, the ovaries and greater part of the oviducts will have been removed, but the relations of the two ends of each oviduct can still be made out.

Examine the reproductive organs, which will now be freely exposed, under water-or in the case of the female, in I per cent. salt solution.

I. Male Organs. (Figs. 3, 5, 7, and 6I.)

I. Notice again the spermaries or testes, each supported by a fold of peritoneum connecting it with the corresponding kidney ; the efferent ducts; the branched fat-body; ; the ureter (uvinogenital duct); and the seminal vesicle. Sketch after examining the cloaca (see next page).

2. Tease up a bit of the spermary of a recently-killed frog in salt-solution, and notice the form and movements of the sperms (Fig. 62, B). Sketch. 
3. Examine under the microscope a transverse section of the spermary, prepared as directed on p. I36, and note (Fig. $62, . A$ ) the numerous seminal tubes or crypts which open into it. Lnder the high power, observe the epithelial cells (germinal epithelium) lining the tubes, and their subdivision into smaller cells, which eventually give rise to the sperms, the tails of which project freely into the cavities of the tubes. Sketch.

\section{Female Organs.}

1. Notice again-(a) the oraries, varying in size and appearance according to the time of year, and each suspended by a fold of peritoneum : they are studded all over with orisacs, each of which contains an egg or orum, pigmented when ripe; and $b$ ) the riducts (Fig. 4 ). Trace the convoluted and glandular middle portion of the oviduct forwards, and make out the anterior thin-walled fortion, running parallel to the gullet and opening into the coelcme by a small aperture at the base of the lung; then trace the middle portion backwards, and notice the thin-walled, dilated, posterior portion, which opens into the cloaca. Sketch after examining the cloaca (see below).

If a female frog is examined in the spring, just before the eggs are laid, the ovaries will be seen to be reduced in size, and the posterior portion of the oviduct filled with eggs, each surrounded with a gelatinous coat secreted by the middle portion.

2. Examine under the microscope a section of the ovary, prepared as directed on p. 136, first with the low, and then with the high power (Fig. 63). Note the epithelial cells (including the germinal epithelium) forming the walls of the ovary, and the ocisacs in difierent stages of development. Each orisac contains an otum, surrounded by follicle-cells. In the ova, note the protoplasm and its contained yolkgranules, the nucleus, and the nucleoli. Sketch.

\section{Cloaca.}

Carefully cut through the pelvic symphysis in the middle line with a scalpel and press apart the two innominate bones, so as to expose the ventral surface of the clcaca. Inflate from the vent, and note that the large intestine is continuous with the cloaca and that the urinary bladder opens into it on the rentral side.

The entire urinogenital apparatus, together with the cloaca, may now be removed from the body, first cutting through the skin round the vent. Pin down under water, 
insert the small scissors into the vent, and slit open the cloaca very slightly to one side of the middle line, so as not to injure the connection between it and the bladder. Notice the openings into the bladder and large intestine respectively, and also:-

In the male, the apertures of the ureters (urinogenital ducts), situated on two small papillæ, lying close to one another on the dorsal side of the cloaca: insert a bristle into the ureter. Sketch.

In the female, the two small apertures of the ureters, and, just in front of these, the two large apertures of the oviducts, all situated on the dorsal wall of the cloaca. Insert bristles into the ureter and oviduct. Sketch.

\section{Impregnation and Development.}

In the early spring (end of February or beginning of March), look about in ponds and ditches for frogs which have taken to the water for the purpose of laying eggs. This process can be watched more conveniently by catching a few frogs, male and female, and putting them in a large vessel of water or aquarium. If you have been unsuccessful in procuring frogs or frogs' spawn, toads will do equally well : their eggs are laid a few weeks later, and are arranged, each surrounded by its gelatinous envelope, not in clumps, like those of the frog, but in a string, like the beads on a necklace.

For purposes of observation, the spawn is best kept in a glass vessel, together with some water-weeds. Put only a small quantity of spawn in one vessel ; if the water begins to get foul, change it at once. Examine the eggs every day with a magnifying-glass-or better, a low power binocular microscope-as development proceeds, and keep some of the tadpoles alive until metamorphosis takes place in June.

If you wish to see some of the stages in the development of the frog during other times of the year, you must obtain some preserved eggs and embryos, and, if possible, you should also examine the series of wax models, made on an enlarged scale, which are to be seen in most zoological museums.

The following are some of the more important things to be noticed. (Sketch a series of stages) :-

Before hatching.

I. The unsegmented oosperm. black above, and white below. 
II. Early stages in segmentation, during which the individual cells can be made out by means of a lens. (Fig. 64, $A-F$.)

III. Later stages in segmentation, during which the individual cells cannot be distinguished without the aid of a microscope. Note the gradual enclosure of the white by the black hemisphere, until only a small rounded yolk-plug is left, filling in an aperture-the blastopore-in the black layer or ectoderm, as it is now called. The yolk-plug is continuous with a mass of yolk-cells, enclosed by the ectoderm, from which the endoderm and mesoderm are developed. (Fig. 64, G-I.)

IV. The gradual flattening and elongation of the embryo, and the position of the blastopore at the posterior end of the dorsal region; the formation of the medullary foids and groo'e along the dorsal side, and the closure of the lilastopore. (Fig. 64, H-J.)

$\mathrm{V}$. The closure of the medullary groove, the formation of the head with its suckers and of the tail with its tail-jin : as well as the appearance of the cyes, ear-sacs, branchial arches, external gills, and the involutions for the mouth and anus, which, however, do not open until after the tadpole is hatched. (Fig. 64, K-L.)

After hatching:

VI. The appearance of the horny jaws and papille, and the further development of the head, external gills, and tail. (Fig. I, I-2, and Fig. 66, A, B.)

VII. The formation of the operculum, the closure of the right opercular aperture, and the disappearance of the external gills. The bud-like rudiments of the hind-limbs, and their further development. The fore-limbs remain for a long time hidden beneath the operculum. (Fig. I, 3-4. and Fig. 66, C-E.)

\section{Metamorphosis.}

VIII. The gradual change in the form and colour of the head and body; the widening of the mouth and loss of the horny jaws and suckers; the appearance of the forelimbs from beneath the operculum, and the shrinking of the tail. (Fig. I, 5-8.)

\section{Dissection of tadpole.}

Pin down a full-sized tadpole under water in a small dissecting-dish, with the ventral surface uppermost, inserting small pins through the tail only. Carefully dissect off the 
ventral body walls, and note the coiled intestine, the heart, the internal gills, \&c. (Fig. 66, D, D1, E.) Sketch.

It is rather a difficult task, and requires much time, to prepare sections of the early stages of the frog-embryo in order to make out the formation of the ectoderm, endoderm, and mesoderm, the relations of the segmentation-cavity and enteron, and the development of the central nervous system and notochord (Figs. $6_{4}, \mathrm{I}, \mathrm{K}$, and $6_{5}$ ); and directions for the practical study of development are given in Part II, Chapter XIII (p. 6I5). But if you wish to make the attempt at this stage, proceed as follows :-

Take a few eggs every day from the time they are laid until the tadpoles are hatched: place these on a tile or piece of glass, and with a pair of needles dissect off the gelatinous covering. Then carefully transfer the segmenting eggs or embryos into corrosive sublimate for a quarter to half an hour, and after washing in running water, put them into weak and then strong alcohol ; stain, imbed, and cut sections as directed on p. 136 . 


\section{CHAPTER XIII}

THE FROG (continued) : MEANING OF THE TERM SPECIESTHE PRINCIPLES OF CLASSIFICATION-EVOLUTIONONTOGENY AND PHYLOGENY-HEREDITY AND VARIATION-STRUGGLE FOR EXISTENCE-NATURAL AND ARTIFICIAL SELECTION-ORIGIN OF SPECIES

THE frog which you have been studying is the only one commonly found in Great Britain. Another kind, very similar to it, is, like the common frog, abundant in Germany and other parts of the European continent, but is rare in this country, only occurring in some places in the eastern counties; and various other frogs, differing in certain minor respects from these are found in different parts of the world. This fact is expressed in the language of systematic zoology by saying that there are various species of frogs, belonging to the same genus, which are distinguished from one another by certain definite characteristics as regards form, structure, and colour.

According to the system of binomial nomenclature introduced by Linnæus, each kind of animal receives two names-one, the generic name, common to all the species of the genus; the other the specific name, peculiar to the species in question. Both generic and specific names are Latin in form, and are commonly Latin or Greek in origin, although frequently modern names of 
persons or places with Latinised terminations are employed. In giving the name of an animal, the generic name is always placed first, the specific name following it, and being written as a rule with a small letter. Thus the common English frog is called Rana temporaria, and the continental form referred to above, often spoken of as the edible frog, Rana esculenta.

You will probably have noticed certain differences in colour and markings in the different individual frogs you have examined, and it is matter of common observation that no two individuals of a species are exactly alike. In the case of human beings and many of the more familiar animals this is very apparent to everyone: in other cases a more careful examination of the individuals is necessary in order to tell them apart; thus, for instance, the individuals in a flock of sheep appear all alike to the casual observer, but the shepherd can easily distinguish them from one another. These differences we designate individual variations, and it is often difficult to decide whether two kinds of animals should be considered as distinct species, or as varieties of a single species, and no universal rule can be given for determining this point. Among the higher animals, mutual fertility is a fair practical test, the varieties of a species (e.g., common pigeon, fowl, dog, horse) usually breeding freely with one another and producing fertile offspring, while distinct species usually do not breed together, or else produce infertile hybrids or mules. Compare for instance the fertile mongrels produced by the union of the various breeds of domestic dog with the infertile mule produced by the union of the horse and ass. But this rule is not without exception, and in the case of wild animals is, more often than not, impossible of application ; failing it, the only criterion of a " good species" is usually the presence of constant differences from 
allied species, whereas if there is a complete series of graduations between two forms, they will be considered to form a single variable species.

In the previous chapters it will have been evident that an animal may be studied from two chief points of view : first, from the point of view of its structure, and secondly from that of the functions performed by its various parts and the way in which these work together for the welfare of the whole. The branch of zoology dealing with the former is known as morphology, and with the latter physiology.

It is evident that a knowledge of morphology is necessary as a preliminary to the study of physiology, and also that, as animals have to be distinguished from one another largely by structural characters, the foundations of a scientific zoology must be laid in morphology, which, as we have seen, deals not only with the external characters and the anatomy and histology of the adult animal, but also with the changes undergone during the development of the egg into the adult form, i.e., with embryology. Given a sound knowledge of the anatomy, histology, and embryology of animals, their elassification may be attempted: that is, we may proceed to arrange them in groups and sub-groups, each capable of accurate definition. In doing so we must be careful to distinguish between homologous parts, or those which correspond structurally (compare p. 39), and analogous parts, which have merely a similar function, and are of no value for purposes of classification. Thus the fore-limbs of a frog are homologous with-i.e., are formed on a similar plan to-the wings of a bird, although differing from them in function; they are only analogous to the limbs of an insect, for though the function is similar in both, the structure is widely different. In the same way the wing of the bird is only analogous to that of an insect. 
The general method of classification employed by zoologists may be illustrated by reference to the different kinds of frogs already referred to in explanation of the terms genus and species.

The common frog (Rana temporaria) is distinguished from the "edible frog" (R. esculenta) by its smaller size and brown colour, by the large dark patch in the tympanic region, and by the rudimentary character of a pair of inflatable vocal sacs at the sides of the head in the male, which are very large and highly distensible in $R$. esculenta. On the other hand, these two frogs agree with one another and with all the other species of the genus Rana, in having teeth on the upper jaw, and in not having the transverse processes of the sacral vertebra dilated. Comparing all these frogs with our English toads, of which there are two species, the common toad and the rarer "natterjack," we find that in them the skin is comparatively dry and covered with glandular warts, the hind-limbs are proportionally shorter, there are no teeth, and the transverse processes of the sacral vertebra are more or less dilated. These differences are so great as not only to necessitate placing the toads in another genus $(B u f o)$, but also to relegate the frogs just mentioned to one family-the Ranidae (including many genera), and the toads to another-the Bufonide. All frogs and toads, however, agree with one another in having no tail in the adult, while the trunk is relatively short and broad, and the hind-limbs are longer than the fore-limbs. They therefore differ fundamentally from such animals as the common English newts and the salamanders, which retain the tail throughout life. and in which the fore- and hind-limbs are of approximately equal size. The differences here are obviously far greater than those between the families mentioned 
above, and are emphasised by placing the frogs and toads in the order Anura, the newts and salamanders in the order Urodela.

The Urodela and Amura, although differing from one another in many important respects, agree, e.g., in possessing gills during part or the whole of their existence, and in nearly always possessing lungs. They usually pass through a metamorphosis, the young being hatched in the form of gilled larvæ; their skin is soft and glandular, and the toes are in nearly all cases without claws. These and numerous other structural characters separate them from reptiles, in which gills are never developed, and the young do not pass through a metamorphosis, while the skin is provided with scales and the toes have claws. The differences here are considerably more important than those between the orders referred to above, and are expressed by placing the latter in the class Amphibia, while reptiles constitute the class Reptilia. In the same way the fishes, which possess fins and gills, form the class $P$ isces, the feathered birds the class Aves, and the hairy animals which suckle their young the class Mammalia.

Mammals, Birds, Reptiles, Amphibians, and Fishes all agree with one another in the possession of red blood and an internal skeleton, an important part of which in the embryo is the notochord (p. 203)-which is nearly always replaced in the adult by a backbone or vertebral column-and in never having more than two pairs of limbs. They thus differ in some of the most fundamental features of their organisation from such animais as Crayfishes, Insects, Scorpions, and Centipedes, which have colourless blood, a jointed external skeleton, and numerous limbs. These differences - far greater than those between classes-are expressed by placing the back boned animals in the phylum or sub-kingdom 
Vertebrata, the many-legged, armoured forms in the phylum Arthropoda. Similarly, soft-bodied animals with shells, such as mussels and snails, form the phylum Mollusca; various worms, such as the earthworm, the phylum Annulata; polypes and jelly-fishes the phylum Coelenterata; the simplest animals, mostly minute, such as Amoeba, the phylum Protozoa. Finally, the various phyla recognised by zoologists together constitute the kingdom Animalia.

Thus the animal kingdom is divided into phyla, the phyla into classes, the classes into orders, the orders into families, the families into genera, and the genera into species; while the species themselves are assemblages of individual animals agreeing with one another in certain definite characteristics. It will be seen that the individual is the only term in the series which has a real existence ; all the others are mere groups, formed, more or less arbitrarily, by man.

Thus the zoological position of the common frog is expressed as follows :-

Kingdom-ANIMALIA.

Phylum-VERTEBRATA.

Class-AMPHiBIA.

Order-ANURA.

Family-Ranida.

Genus-Rana.

Species-temporaria.

Let us now briefly consider some of the reasons which have led zoologists to adopt the system of classification now in general use.

It is obvious that there are various ways in which animals may be classified, and the question arisesWhich of these, if any, is the right one? Is there any 
standard by which we can judge of the accuracy of a given classification, or does the whole thing depend upon the fancy of the classifiers, like the arrangement of books in a library? In other words, are all possible classifications of living things more or less artificial, or is there such a thing as a natural classification?

Suppose we were to try to classify all the members of a given family-parents and grandparents, uncles and aunts, cousins, second cousins, and so on. Obviously there are a hundred ways in which it would be possible to arrange them-into dark and fair, tall and short, curly-haired and straight-haired, and so on. But it is equally obvious that all these methods would be purely artificial, and that the only natural way, i.e., the only way to show the real connection of the various members of the family with one another, would be to classify them according to blood-relationship; in other words, to let our classification take the form of a genealogical tree.

There are two theories which attempt to account for the existence of the innumerable species of living things which inhabit our earth : the theory of special creation and the theory of evolution.

According to the theory of creation, all the individuals of every species existing at the present day-the tens of thousands of dogs, frogs, oak-trees, and what not-are derived by a natural process of descent from a single individual, or from a pair of individuals-in each case precisely resembling, in all essential respects, their existing descendants- which came into existence by a process outside the ordinary course of nature and known as creation. On this hypothesis each species of frog is derived from a common ancestral pair which came into existence, independently of the progenitors of all the 
other species, at some previous period of the earth's history.

Notice that on this theory the various species of frogs are no more actually related to one another than is either of them to a newt, or, for the matter of that, to man. The individuals of any one species are truly related since they all share a common descent, but there is no more relationship between the individuals of any two independently created species than between any two independently manufactured chairs or tables. The words affinity, relationship, \&c., as applied to different species are, on the theory of creation, purely metaphorical, and mean nothing more than that a certain likeness or community of structure exists; just as we might say that an easy chair was more nearly related to a kitchen chair than either of them to a three-legged stool.

We see, therefore, that on the hypothesis of creation, the varying degrees of likeness and unlikeness between the species receive no explanation, and that we get no absolute criterion of classification : we may arrange our organisms, as nearly as our knowledge allows, according to their resemblances and differences, but the relative importance of the characters relied on becomes a purely subjective matter.

According to the rival theory-that of Descent or Organic Evolution, with which the name of Darwin is inseparably connected-every species existing at the present day is derived by a natural process of descent from some other species which lived at a former period of the world's history. If we could trace back from generation to generation the individuals of any existing species, we should, on this hypothesis, find their characters gradually change, until finally a period was reached at which the differences were so considerable as to 
necessitate the placing of the ancestral forms in a different species from their descendants at the present day. And in the same way, if we could trace back the species of any one genus, we should find them gradually approach one another in structure until they finally converged in a single species, differing from those now existing, but standing to ail in a true parental relation.

It will be seen that, on this hypothesis, the relative likeness and unlikeness of the various species of frogs are explained as the result of their descent with greater or less modification or divergence of character from the ancestral form: and that we get an arrangement or classification in the form of a genealogical tree, which, on this hypothesis, is a strictly natural one, since it shows accurately the relationship of the various species to one another and to the parent stock. So that on the theory of evolution, a natural classification of any given group of allied organisms is simply a genealogical tree,

Now it is evident that the only way in which we could be perfectly sure of an absolutely natural classification of the species of any kind of animal - the frog, for example-would be by obtaining specimens as far back as the distant period when the genus first came into existence.

Forming part of the solid crust of the earth are a series of sedimentary or stratified rocks, and the researches of geologists have shown that these present a general order of succession, the lowest, when undisturbed, being in every case older than the more superficial layers. Imbedded in these rocks are found the remains of various extinct animals in the form of what are called fossils; and it might perhaps, on first considering the subject, be supposed that, had a process of evolution taken place, we ought to be able to find in the rocks 
belonging to the various geological formations a complete series of animal remains, representing all the stages in the evolution of the highest from the lowest forms. But owing to various causes which we cannot further consider here, the record of the succession of life on the globe is very imperfect and incomplete in the case of any individual species ; but the evidence furnished by palcontology - as the study of fossils is called - is very important in supplying proofs that there has been a gradual evolution of the higher from the lower forms.

Further evidence in the same direction is furnished by morphology and embryology, as will be more apparent to you when you have studied a number of other kinds of animals. You have, however, seen that the frog begins life as a single cell, and that it is possible to trace a series of modifications which gradually convert the unicellular oosperm into a tadpole-which is to all intents and purposes a fish-and that the tadpole subsequently undergoes metamorphosis into the more highly organised frog. According to the theory of recapitulation, these facts indicate that the frog repeats, during its single life, the series of changes passed through by its ancestors in the course of ages. In other words, ontogeny, or the evolution of the individual, is, in its main features, a recapitulation of phylogeny, or the evolution of the race. At the same time you must bear in mind that it is not always an easy matter to determine which characters are of phylogenetic significance, and which have been secondarily acquired owing to various causes. To take an example: the horny jaws of the tadpole might be taken to indicate that frogs were descended from ancestors with horny jaws and without teeth, though in all probability these 
organs have been secondarily acquired as alaptutions in connection with the habits of the tadpole, and have, therefore, unlike the gills, for instance, no ancestral significance.

It is obvious that the evolution of one species from another presupposes the occurrence of variations in the ancestral form. As we have seen, such individual variation is of universal occurrence. This may be expressed by saying that heredity, according te which the offspring tends tc resemble the parent in essentials, is modified by variability, according to which the offspring tends to differ from the parent in detail. If, from any cause, any well-marked individual variation is perpetuated, there is produced what is known as a variety of the species, and according to the theory of the origin of species by evolution, such a variety may, in course of time, become a new species. Thus a variety is an incipient species, and a species is a (relatively) permanent variety.

One other important factor in evolution must be briefly referred to before concluding this chapter. In order that every species of animal and plant may flourish, certain conditions are necessary. Thus the frog requires, for example, a moist place to live in, and water in which to lay its eggs. For spots presenting the necessary favourable conditions, there is constantly going on a competition between individuals of one species and between the members of different species. The nature of this struggle is well seen when a piece of garden ground is allowed to run to waste. Its surface is soon overgrown by weeds of many kinds, which kill out nearly all the original garden-plants by depriving them of light and food. By and by the more hardy weeds exterminate and replace such weaker forms as may first 
have obtained a footing, till an entirely new set of weeds may take the place of those that first appeared.

A struggle for existence goes on on all sides among animals. To begin with, before there is any struggle for existence in the strict sense, there is-particularly in animals which, like the frog, produce eggs in great numbers annually - a very great indiscriminate destruction of ova and young embryos. Only a few of them reach maturity; a large proportion are destroyed at one stage or other, some failing to reach a spot favourable for their further development, others becoming the food of other animals. But such of the young as are less adapted to escape the various dangers to be encountered, and less fitted to procure the necessary food, are more likely to be destroyed. This is one phase-and the most important, perhaps, of all-of the struggle for existence amongst animals. But there is also a struggle for existence, not only between individual animals of the same kind, but between animals of different kinds. This struggle, in so far as it relates to the competition for food and shelter, is more severe between nearly related species; for in such a case the food and the favourable conditions required are the same, or nearly so, in the two competitors. Again, a struggle for existence of a constant and severe kind also goes on between carnivorous animals and the animals on which they prey-e.g., between the frog and the insects and worms on which it feeds, and between the snake and the frog; a struggle in which the defensive qualities of the prey - such as swiftness, power of eluding observation, or of resisting attack--are opposed to the predatory powers of the attacker.

There can be little doubt that, in the long run, such individuals will survive as are best fitted to cope with 
the conditions to which they are subject. According to Darwin's theory of natural selection, it is assumed that such surviving individuals would transmit their special properties to their progeny, and there would thus be a gradual approximation towards a perfect adaptation of the species to its surrounding conditions by virtue of this " survival of the fittest."

Let us suppose the conditions to change. Gradual alterations. in climate and other conditions are known to take place, owing to subsidence or elevation of the land. But conditions might be changed in many other ways : some animal or plant previously used as food might become exterminated, or a new enemy might find its way into the district inhabited by the species. Then such individuals as presented variations which enabled them better to cope with the new surroundings would have the advantage over the others, and would have a much better chance of surviving and leaving progeny. The useful variations thus produced and transmitted to the progeny would tend to increase, generation after generation, until a form sufficiently distinct to be regarded as a new species had become developed from the original one.

That very different varieties of animals and plants can, and have been produced, in a comparatively short time, by man selecting those forms which tend to vary in a desired direction, is a well-known fact. All the breeds and varieties of our domestic animals have been produced by this process of artificial selection; and " if man can by patience select variations useful to him, why, under changing and complex conditions of life, should not variations useful to Nature's living products often arise, and be preserved or selected ?"

It does not come within the scope of the present work 
to discuss either the causes of variability or those which determine the elevation of a variety to the rank of a species: both questions are far too complex to be adequately treated except at considerable length, and anything of the nature of a brief abstract would only be misleading.*

* At a later stage the student is recommended to consult Doncaster's Heredity in the Light of Recent Research (Cambridge Manuals of Science and Literature, I9I0), which deals with the modern developments of these and other questions, such as Mendelian Heredity. 


\section{P A R T I I}

\section{CHAPTER I}

AMCEBA: UNICELLULAR AND MULTICELLULAR ANIMALS

FROM your study of the frog you will have learnt some of the more important facts with regard to the morphology and physiology of a comparatively highlyorganised animal, and will have overcome a number of preliminary difficulties in acquiring a knowledge of zoological terminology and technique. You will now, therefore, be in a better position to undertake a systematic and comparative examination of a number of other animals - some much less complicated, some more complicated, than the frog-working upwards from the simple to the complex forms. In doing so, you must continually bear in mind the deductions in connection with the theory of evolution referred to in the previous chapter.

Let us begin with a very instructive animalcule belonging to the genus Amœba. Amœbæ are often found in the slime at the bottom of pools of stagnant water, adhering to weeds and other submerged objects.

They are mostly invisible to the naked eye, rarely exceeding $\frac{1}{4}$ of a millimetre $\left(\frac{1}{100}\right.$ th inch) in diameter, so that it is necessary to examine them entirely by the aid of the microscope. Though they can be secn and recognised with the low power, the high power is necessary for the accurate examination of their structure. 


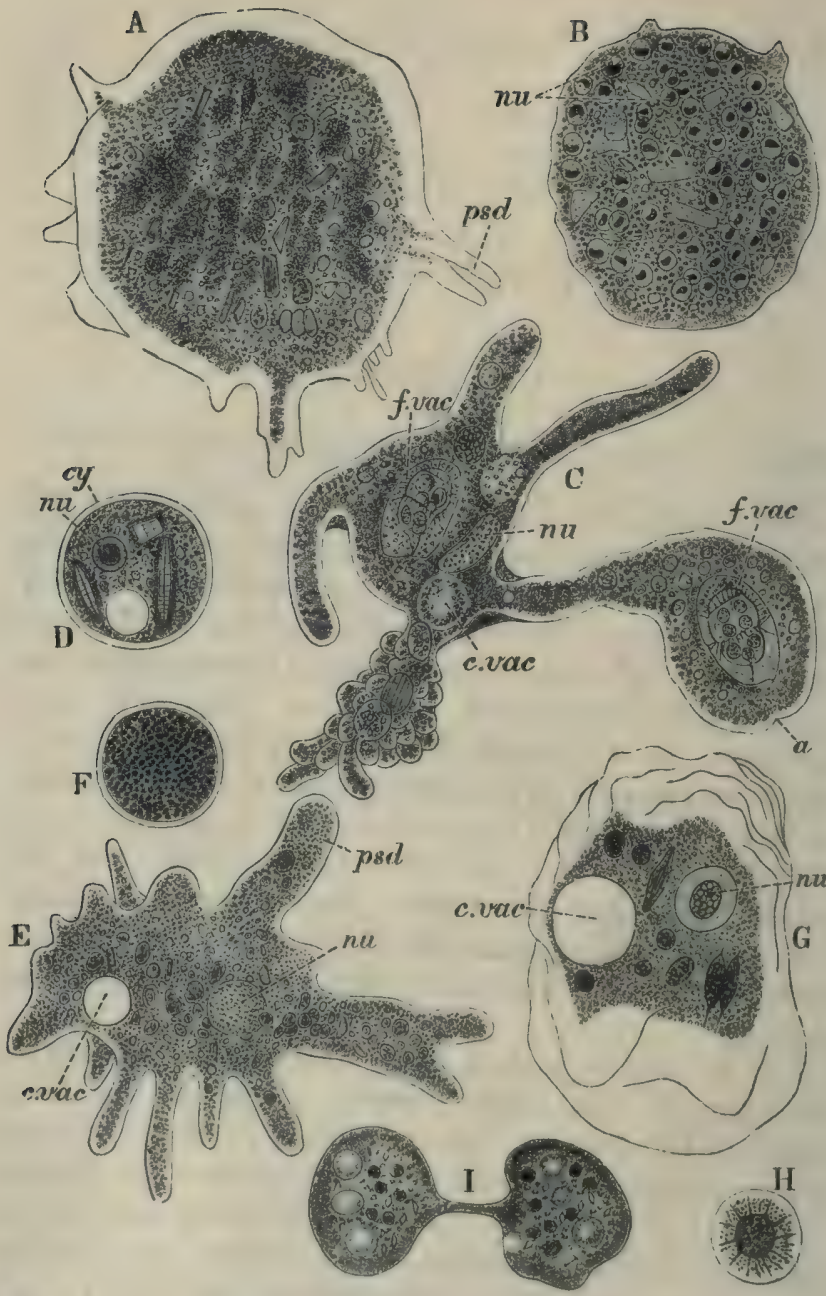

FIG. 67.-A, Amaba quaria, a living specimen, showing granular endoplasm surrounded by clear ectoplasm, and several pseudopods ( $p$ sd $)$, some formed of endoplasm are mostly food-particles. $\mathrm{B}$, the same species, killed and stained with carm

(nu) present in this species (compare Fig. 73$)$. ( $(x 300$.$) the numerous nurlei$ 
C, Amaba proteus, a living specimen, showing large urregular pseudopods, nucleus (ru), contractile vacuole $(c . v a c$ ), and two food-vacuoles ( $f$. vac, , each containing a small infusor (see Chapter III) which has been ingested as food. The letter $a$ to the right of the figure indicates the place where two pseudopods have united to enclose the food-vacuole. The contractile vacuole in this figure is supposed to be seen through a layer of granular protoplasm, whereas in D, E, and $G$ it is seen in optical section, and therefore appears clear. ( $X$ about joo.)

$D$, an encysted Amoba, showing cell-wall or cyst (cy), nucleus (nu), clear contractile vacuole, and three microscopic plants (diatoms) ingested as food.

E, $A$ moeba proteus, a living specimen, showing several large pseudopods ( $p$ sd). single nucleus $(n \mu)$ and contractile vacuole $(c . v a c)$, and numerous food-particles emberdded in the granular endoplasm. $(x \quad 330$.

$F$, nucleus of the same after staining, showing a ground substance containing deeply-stained granules of chromatin, and surrounded by a distinct membrane. ( $\times$ 1010.)

$\mathrm{G}$, A maba verrucosa, living specimen, showing wrinkled surface, nucleus (nu), large contractile vacuole (c. vac), and several ingested organisms. ( $<330$.

$\mathbf{H}$, nucleus of the same, the chromatin aggregated in the centre. ( $x$ roro.)

I, A maba proteus, in the act of multiplying by binary fission. ( $\times 500$.

(From Parker's Biology : A, B, E, F, G, and H after Gruber; $C$ and I after Leidy ; D after Howes.)

Examined under the high power (Fig. 67), the Amøba appears like a little shapeless blob of jelly, nearly or quite colourless, and closely resembling a colourless blood-corpuscle or leucocyte of one of the higher animals (p. 105). The central part of it, or endoplasm, is granular and semi-transparent-something like ground-glass-while surrounding this inner mass is a border of perfectly transparent and colourless substance-the ectoplasm.

One very noticeable thing about the Amœba is that, like the leucocyte, it is never of quite the same form for long together, owing to the protrusion of pseudopods $(p s d)$, by means of which it creeps along slowly. The occurrence of amoboid movements is alone sufficient to show that it is an organism, or living thing, and no mere mass of dead matter. Moreover, it consists of protoplasm, and encloses a nucleus $(\mathrm{C}-\mathrm{H}, n u)$ containing chromatin and rendered more apparent by staining. The Amœba is therefore a cell (compare pp. 106 and Iro).

A very important difference is thus at once seen between the Amœba and the frog: the Amoba is unicellular, i.e., it consists of a single cell, while the 
frog is, as we have seen, a multicellular animal, built up of innumerable cells which are incapable of an independent existence for any length of time.

Besides the nucleus, there is another structure frequently visible in the living Amœba and not present in the leucocyte. This is a clear, rounded space in the protoplasm (c. vac), which periodically disappears with a sudden contraction and then slowly reappears, its movements reminding one of the beating of a minute colourless heart. It is called the contractile vacuole, and consists of a cavity containing a watery fluid.

We must now study the physiology of our animal. cule. First of all, as we have already seen, it is contractile: although it has no muscles, it can move about from place to place. Its movements, like the voluntary movements of the frog (pp. 7,172$)$, may occur without the application of any external stimulus, i.e., they are spontaneous or automatic; or they may be induced by external stimuli-by a sudden shock or by coming in contact with an object suitable for food. Movements of this latter kind, like those resulting from the stimulation of the nerves in a brainless frog, are the result of the irritability of the protoplasm ; the animalcule is therefore both automatic and irritable, although it possesses neither nerves nor sense-organs.

Under certain circumstances an Amœba temporarily loses its power of movement, draws in its pseudopods, and becomes a globular mass around which is formed a thick, shell-like coat, called the cyst or cell-wall (Fig. 67, D, cy). This is formed by the protoplasm by a process of secretion (p. I30); its composition is not known; it is certainly not protoplasmic, and very probably consists of some nitrogenous substance allied in composition to horn and to the chitin (see Chapters VI-VIII) which forms the external shell of crustaceans, insects, \&c. 
The formation of the cyst is probably of great importance in preserving the animalcule from destruction by drought, so that should the pool in which it is living dry up, it may still remain alive, protected by its shelllike case, until the conditions for its active life are once more restored; it escapes by the rupture of the cyst, sometimes having first divided into numerous young Amœbæ.

Very often an Amœba in the course of its wanderings comes in contact with a still smaller organism of some kind or other. When this happens the Amœba may be seen to extend itself round the lesser organism until the latter becomes sunk in its protoplasm in much the same way as a marble might be pressed into a lump of clay (Fig. 67, C, a). The diatom (D) or other organism becomes in this way completely enclosed in a cavity or food vacuole $(f . v a c)$, which also contains a small quantity of water necessarily included with the prey. The latter is taken in by the Amœba as food: so that the Amœba, like the frog, feeds. It is to be noted that the reception of food takes place in a particular way, viz., by ingestion-i.e., it is enclosed entire by the organism.

When the prey is thus ingested, its protoplasm becomes digested, any insoluble portions being passed out or egested, as fæces (pp. 8 and 75), from the surface of the Amoba as it creeps slowly on. Note that all this is done without either ingestive aperture (mouth), digestive cavity (stomach), or egestive aperture (anus): the food is simply taken in by the flowing round it of protoplasm, digested as it lies enclosed in the protoplasm, and those portions for which it has no further use are got rid of by the Amœba flowing away from them.

We have seen that the frog possesses certain digestive glands, the function of which is to secrete digestive fuids which have an important chemical action on the food 
swallowed, rendering it soluble and diffusible before it passes through the epithelial cells of the enteric canal into the blood: the gastric juice, for example, has the power of converting proteids into peptones by means of the ferment pepsin (p. 74); the digestion here takes place outside the cells, i.e., is extracellular. There can be little doubt that the protoplasm of Amœba is able to render that of its prey soluble and diffusible by the agency of some substance analogous to pepsin, and that the dissolved matters diffuse through the body of the Amœba until the latter is, as it were, soaked through and through with them. The process of digestion in Amœba thus takes place within a single cell, i.e., it is intracellular.

It has been proved by experiment that proteids are the only class of food which Amœba can make use of : it is unable to digest either starch or fats (p. 72). Mineral matters must, however, be taken with the food in the form of a weak watery solution, since the water in which the animalcule lives is never absolutely pure.

The Amœba being thus permeated, as it were, with a nutrient solution, the elements of the solution, hitherto arranged in the form of peptones, mineral salts, and water, become rearranged in such a way as to form new particles of living protoplasm, which are deposited among the pre-existing particles. In a word, the food is assimilated, or converted into the actual living substance of the Amœba, which must therefore grow, if nothing happens to counteract this formation of new protoplasm.

We have seen, however, that work results in a proportional amount of waste (p. 66), and just as in the frog or in ourselves, every movement of the Amœba, however slight, is accompanied by a proportional oxidation or low temperature combustion of the protoplasm, i.e., the constituents of the protoplasm combine with oxygen 
forming waste or excretory matters-carbon dioxide, water, and certain nitrogenous substances of simpler constitution than proteids, such as urea. These products of excretion, formed in the case of Amœba without the agency of any special excretory organs (e.g., kidneys), are given off partly from its general surface, but partly, it would seem, by the agency of the contractile vacuole, by means of which the water taken in with the food is also got rid of.

With this breaking down of proteids the vital activities of all organisms are invariably connected. Just as useful mechanical work may be done by the fall of a weight from a given height to the level of the ground, so the work done by the organism is a result of its complex proteids falling, so to speak, to the level of simpler substances. In both instances potential energy or energy of position is converted into kinetic or actual energy.

The statement just made that the protoplasm of Amœba constantly undergoes oxidation presupposes a constant supply of oxygen. The water in which the animalcule lives invariably contains that gas in solution, and diffusion takes place, oxygen passing into the interior of the Amœba while carbon dioxide passes out into the water. This is the process of breathing or respiration (p. I.43), and it occurs in Amœba without the agency of special respiratory organs. Thus the carbon dioxide is got rid of, and at the same time a supply of oxygen is obtained for further combustion. The oxidation of the protoplasm of the Amoba is doubtless accompanied by an evolution of heat, as in higher animals (p. 152), although this has never been proved.

We thus see that a very elaborate series of chemical processes is constantly going on in the interior of Amœba, as in the frog; the whole series of which is 
spoken of collectively as metabolism-constructive and destructive (p. I49). Living protoplasm is thus the most unstable of substances; it is never precisely the same thing for two consecutive seconds; its existence, like that of a waterfall or a fountain, depends upon the constant flow of matter into it and away from it.

It follows from what has been said that if the income of an Amœba, i.e., the total weight of substances taken in (food plus oxygen plus water), is greater than its expenditure or the total weight of substances given out (fæces plus excreta proper plus carbon dioxide), the animalcule will grow : if less it will dwindle away: if the two are equal it will remain of the same weight or in a state of physiological equilibrium:

It is evident that Amœba must also be able to perform the function of reproduction. You have learnt that the cells of the frog multiply by simple or binary fission (p. 106): the nucleus first divides into two, and then the surrounding protoplasm; and precisely the same thing occurs in Amoba, the reproduction of which therefore takes place by the simplest method known, without any special reproductive organs. The animalcule simply divides into two Amœbæ, each exactly like itself, and in doing so ceases to exist as a distinct individual. Instead of the successive production of offspring from an ultimately dying parent, we have the simultaneous production of offspring by the division of the parent, which does not die, but becomes simply merged in its progeny. There can be no better instance of the fact that reproduction is discontinuous growth.

From this it seems that an Amœba, unless suffering a violent death, is practically "immortal," since it divides into two completely organised individuals, each of which begins life with half of the entire body of its parent, there being therefore nothing left of the latter to die: 
it therefore appears certain that " death has no place as a natural recurrent phenomenon" in that organism. In multicellular forms it is only the reproductive cells which are, physically, potentially immortal.

It is said that occasionally two Amœbæ come into contact and undergo fusion, just as the gametes of the frog (sperm and ovum) unite in the processes of fertilisation (p. I97). This process of conjugation-which probably precedes encystment and multiple fission (see p. 233)will be referred to again in the following chapters, and it is important to bear in mind that reproduction can take place quite independently of such a process.

Amœbæ may also be propagated artificially. If a specimen is cut into pieces, each fragment is capable of developing into a complete animalcule provided it contains a portion of nuclear matter, but not otherwise. From this it is obvious that the nucleus exerts an influence of the utmost importance over the vital processes of the organism.

If an Amœba does happen to be killed and to escape being eaten it will, like a dead frog, undergo gradual decomposition, becoming converted into various simple substances of which carbon dioxide, water, and ammonia are the chief (p. 152).

Death results if the temperature to which an Amøba is exposed reaches about $40^{\circ} \mathrm{C}$, and at freezing point its movements cease entirely and it becomes inert.

We thus see that complex organs, composed of various tissues, each consisting of cells of characteristic form, are not necessary in order that the vital functions may be performed: the only essential is nucleated protoplasm. As we pass from the unicellular Amoba to the higher multicellular animals we shall find-just as we found in tracing the development of the frog from the unicellular oosperm $(0,205)$-that a differentiation of 
structure accompanied by a division of physiological labour becomes more and more marked, some cells giving rise to organs of locomotion, others to organs of reproduction, and so on. But every function necessary for the life of an animal-or a plant-is due in the first instance to protoplasm, and a simple cell, like the Amœba, can perform them all.

In the next two chapters we shall study certain other unicellular organisms which show an advance on Amœba in possessing a certain amount of morphological and physiological differentiation. But the structural differentiations, as they are merely parts of one cell, cannot be spoken of as " organs" in the sense in which we have used the word hitherto, as they are not composed of numerous cells. They are, however, organs in the physiological sense, as they perform different functions.

\section{PRACTICAL DIRECTIONS ${ }^{1}$}

\section{Amœba.}

Examine a drop of water from the bottom of a pond, with the low power, first putting on a cover-glass, and look for Amœbæ: if the water does not contain small particles of sand or mud, place a small piece of thin paper under the edge of the cover so as to avoid crushing the organisms. When you have found a specimen, note-

1. The irregular and changing form of the animal, the protoplasm running out into blunt pseudopods.

1 You should, if possible, try to obtain specimens of Amœbæ and the other fresh-water organisms described in this and the two following chapters for yourself, by collecting stagnant pond-water, together with a little of the mud at the bottom and some waterweeds, and latting it stand for a few days in a glass jar or bottle. If you are unable to find the organisms you require, they, as well as most of the other animals described in this book, may be obtained from dealers in Natural History objects (see, e.g., the advertisements in Nature). 
2. The granular character of the protoplasm, the granules usually not extending to the periphery, so that a clear ectoplasm can be distinguished from a granular endoplasm. The granules render the flowing movements of the protoplasm visible.

3. The food-vacuoles in the protoplasm, containing fluid, and often also food-particles.

4. The contractile racuole, containing fluid, and its rhythmical contractions.

5. The protrusion and retraction of the pseudopods. Sketch a specimen several times at short intervals, noting the direction in which the granules flow. Then put on the high power. Go over \$\$ I-5 again, and make a detailed sketch.

6. I.ook out for specimens undergoing multiplication by binary fission, and also for ency'sted individuals.

7. Run a little dry carmine or indigo under the coverglass, and note that the particles can be taken in at all parts of the surface.

8. Treat with methyl-green, or magenta and acetic acid (see p. I21). This will kill the animal, and render the nucleus distirct.

9. Permanent preparations, showing the nucleus, may be made on the slide as follows:

Place a drop of water containing Amøbæ on a slide, and soak up with blotting-paper as much of the water as is possible without carrying the Amœbæ along with it. Fix (see p. 136) with a drop of absolute alcohol, stain (a stainingfuid called picrocarmine is better than borax-carmine for this purpose), wash carefully with weak and then with absolute alcohol, and add a drop of turpentine or xylolor better, oil of cloves. Soak off the excess of oil of cloves with blotting-paper, and mount in Canada balsam. 


\section{CHAPTER II}

SPHÆRELLA AND EUGLENA: MONADS AND BACTERIADIFFERENCES BETWEEN ANIMALS AND PLANTSSAPROPHYTES

THE rain-water which collects in puddles, open gutters, $\& c$., is frequently found to have a green or red colour. The colour is due to the presence of various organismsplants or animals - one of the commonest of which is Spharella (or, as it is sometimes called, Hamatococcus or Protococcus) pluvialis.

Like Amœba, Sphærella is so small as to require a high power for its examination. Magnified three or four hundred diameters it has the appearance (Fig. 68, A) of an ovoidal body, somewhat pointed at one end, and of a bright green colour, more or less flecked with bright red.

Like Amœba, moreover, it is in constant movement, but the character of the movement is very different in the two cases. An active Sphærella is seen to swim about the field of the microscope in all directions and with considerable apparent rapidity. We say apparent rapidity because the rate of progression is magnified to the same extent as the organism itself, and what appears a racing speed under the microscope is actually a very 
slow crawl when divided by 300 . It has been found that such organisms as Sphrrella travel at the rate of one foot in from a quarter of an hour to an hour; or, to

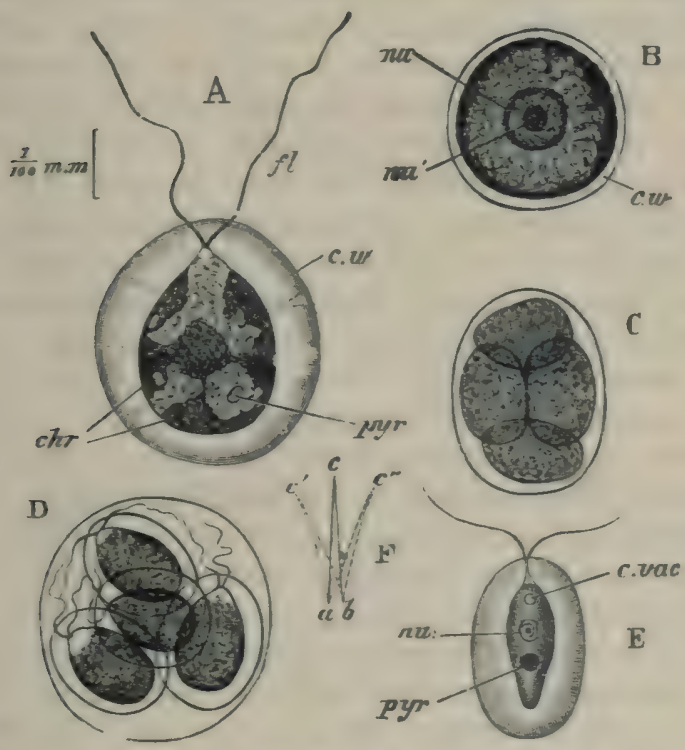

F16. 68.-A, Spherella plurialis, motile phase ( $x$ about 350). Living specimen. showing protoplasm with chromatophore (chr) and pyrenoids ipyr). cell-wall (c. $a)$ connected to cell-body by protoplasmic filaments, and flagella $(f)$. The scale to the left applies to Figs $\mathrm{A}-\mathrm{D}$.

$\mathrm{B}$, resting stage of the same, showing nucleus ( $n u)$ with "nucleolus" ( $n u^{\prime}$ ) and thick cell-wall (c. $w$ ) in contact with the protoplasm.

C, the same, showing division of the cell-body in the resting stage into four daughter-cells.

D, the same, showing the development of flagella and detached cell-wall by the daughter-cells before their liberation from the enclosing mother cell-wall.

E, Spherelue lacustris, showing nucleus (nw), single large pyrenoid $(p y r)$. and contractile vacuole (c. vac).

$F$, diagram illustrating the movement of a flagellum; $a b$. its base : $c, c^{\prime}, c^{*}$, different positions assumed by its apex. (From Parker's Biology; E, after Butschli.)

express the fact in another and fairer way, that they travel a distance equal to two and a half times their own diameter in one second. In swimming the pointed

Pract. Zool. 
end is always directed forwards, and the forward movement is accompanied by a rotation of the organism upon its longer axis.

Careful watching shows that the outline of a swimming Sphærella does not change, so that there is evidently no protrusion of pseudopods, and at first the cause of the movement appears rather mysterious. Sooner or later, however, the little creature is sure to come to rest, and there can then be seen projecting from the pointed end two excessively delicate colourless threads (Figs. 68, A, $f$ ), each about half as long again as the organism itself : these resemble the cilia on the epithelial cells lining the frog's mouth (p. rog) except that they are few in number, and do not vibrate rhythmically ; they are therefore usually distinguished as flagella. In a Sphærella which has come to rest these can often be seen gently waving from side to side: when this slow movement is exchanged for a rapid one the whole organism is propelled through the water, the flagella acting like a pair of extremely fine and flexible fins or paddles. Thus the movement of Sphærella is not amœboid, i.e., produced by the protrusion and withdrawal of pseudopods, but is ciliary, i.e., due to the rapid vibration of cilia or flagella.

By staining and other tests it is shown that Sphærella, like Amœba, consists of protoplasm, and that the flagella are simply filamentous processes of the protoplasm.

The green colour of the organism is due to the presence of a special pigment called chlorophyll, the substance to which the colour of leaves is due. That this is something quite distinct from the protoplasm may be seen by treatment with alcohol, which simply kills and coagulates the protoplasm, but completely dissolves out the chlorophyll, producing a clear green solution. The solution, although green by transmitted light, is red 
under a strong reflected light, and is hence fuorescent; when examined through the spectroscope $1 i$ has the effect of absorbing the whole of the olue and violet end of the spectrum as well as a part of the red. The red colour which occurs in so many individuals, sometimes entirely replacing the green, is due to a colouring matter closely allied in its properties to chlorophyll, and called hamatochrome.

At first sight the chlorophyll appears to be evenly distributed over the whole body, but accurate examination under a high power shows it to be lodged in a structure called a chromatophore (Fig. 68, A, chr), which forms a layer immediately beneath the surface, and in this case is relatively large and urn-shaped. It consists of a protoplasmic substance impregnated with chlorophyll.

After solution of the chlorophyll with alcohol a nucleus ( $\mathrm{B}, m u$ ) can be made out; like the nucleus of Amœba, it is rendered more distinct by staining. Other bodies which might easily be mistaken for nuclei are also visible in the living organism. These are small ovoidal structures (A, pyr), with clearly defined outlines, occurring in varying numbers in the chromatophore. When treated with iodine they assume a deep, apparently black, but really dark blue colour. The assumption of a blue colour with iodine is the characteristic test of the carbohydrate starch (p. 72), as can be seen by letting a few drops of a weak solution of iodine fall upon some ordinary washing starch. The bodies in question have been found to consist of a proteid substance covered with a layer of starch, and are called pyrenoids.

In $S$. pluvialis a distinct contractile vacuole is wanting, but in another species, $S$. lacustris, this structure can be recognised as a minute space near the anterior or pointed end (Fig. 68, E, c. vac). 
There is still another characteristic structure to which no reference has yet been made. This appears at the first view something like a delicate haze round the red or green body, but by careful focussing is seen to be really an extremely thin globular shell (A, c. w), composed of some colourless transparent material, and separated, by a space containing water, from the body, to which it is connected by very delicate radiating strands of protoplasm. It is perforated by two extremely minute apertures for the passage of the flagella. Obviously we may consider this shell as a cyst or cell-wall, differing from that of an encysted Amøba (Fig. 67, D) in not being in close contact with the protoplasm.

A more important difference, however, lies in its chemical composition. The cyst or cell-wall of Amœba; as stated in the preceding chapter (p. 232), is very probably nitrogenous; that of Sphærella, on the other hand, is formed of a carbohydrate called cellulose, allied in composition to starch, sugar, and gum, and, like starch, having the formula $\mathrm{C}_{6} \mathrm{H}_{10} \mathrm{O}_{5}$. Many vegetable substances, such as cotton, consist of cellulose, and wood is a modification of the same compound. Cellulose is stained yellow by iodine, but iodine and sulphuric acid together turn it blue, and a similar colour is produced by a solution of iodine and potassium iodide in zinc chloride known as Schulze's solution. These tests are quite easily applied to Sphærella: the protoplasm stains a deep yellowish-brown, around which is seen a sort of blue cloud, due to the stained and partly-dissolved cell-wall.

After leading an active existence for a longer or shorter time, the Sphærella comes to rest, losing its flagella, and its protoplasm being closely surrounded by the cellulose cell-wall (Fig. 68, B). So that, as in Amœba, there is an 
alternation of an active or motile with a stationary or resting condition.

In the matter of nutrition, the differences between Sphærella and Amøba are very marked, and indeed fundamental. As we have seen, Sphærella has no pseudopods, and therefore cannot take in solid food after the manner of Amœba; moreover, even in its active condition it is usually surrounded by a cell-wall, which of course quite precludes the possibility of ingestion. As a matter of observation, also, however long it is watched it is never seen to feed in the ordinary sense of the word. Nevertheless it must take food in some way or other, or the decomposition of its protoplasm would soon bring it to an end.

Sphærella lives in rain-water. This is never pure water, but always contains certain mineral salts in solution, especially nitrates, ammonia salts, and often sodium chloride or common table-salt. These salts can and do diffuse into the water which is a constituent part of the protoplasm of the organism, so that we may consider its protoplasm to be constantly permeated by a very weak saline solution, the most important elements contained in which are oxygen, hydrogen, nitrogen, potassium, sodium, calcium, sulphur, and phosphorus. It must be remarked, however, that the diffusion of these salts does not take place in the same uniform manner as it would through parchment or other dead membrane. The living protoplasm has the power of determining the extent to which each constituent of the solution shall be absorbed.

If water containing a large quantity of Sphærella is exposed to sunlight, minute bubbles appear in it, and these bubbles, if collected and properly tested, are found to consist largely of oxygen. Accurate chemical analysis 
has shown that this oxygen is produced by the decomposition of the carbon dioxide contained in solution in rain-water, and indeed in all water exposed to the air; the gas, which is always present in small quantities in the atmosphere, being very soluble in water.

As the carbon dioxide is decomposed in this way, its oxygen being given off, it is evident that its carbon must be retained. As a matter of fact it is retained by the organism but not in the form of carbon ; in all probability a double decomposition takes place between the carbon rioxide absorbed and the water contained in its proto. plasm, the result being the liberation of oxygen in the form of gas and the simultaneous production of some extremely simple form of carbohydrate, i.e., some compound of carbon, hydrogen, and oxygen with a comparatively small number of atoms to the molecule.

The next step seems to be that the carbohydrate thus formed unites with the ammonia salts or the nitrates absorbed from the surrounding water, the result being the formation of some comparatively simple nitrogenous compound. Then further combinations take place, substances of greater and greater complexity are produced, sulphur from the absorbed sulphates enters into combination, and proteids are formed. From these, finally, fresh living protoplasm arises.

From the foregoing account, which only aims at giving the very briefest outline of a subject as yet imperfectly understood, it will be seen that, as in Amœba, the final result of the nutritive process is the manufacture of protoplasm, and that this result is attained by the formation of various substances of increasing complexity. But it must be noted that the steps in this process of constructive metabolism are widely different in the two cases. In Amoba we start with living protoplasmthat of the prey-which is killed and broken up into 
diffusible proteids, these being afterwards re-combined to form new molecules of the living protoplasm. of Amœeba. So that the food of Amœba is. to begin with, as complex as itself, and is first broken down by digestion into simpler compounds, these being afterwards re-combined into more complex ones. In Sphærella, on the other hand, we start with extremely simple compounds, such as carbon dioxide, water, nitrates, sulphates, \&c. Nothing which can be properly called digestion, i.e., a breaking up and dissolving of the food, takes place, but its various constituents are combined into substances of gradually increasing complexity, protoplasm, as before, being the final result.

To express the matter in another way: Amcba can only make protoplasm out of proteids already formed by some other organism: Sphærella can form it out of simple liquid and gaseous inorganic materials.

Speaking generally, it may be said that these two methods of nutrition are respectively characteristic of the two great groups of living things. Animals require solid food containing ready-made. proteids, and cannot build up their protoplasm out of simpler compounds. Green plants, i.e., all the ordinary trees, shrubs, weeds, \&c., take only liquid and gaseous food, and build up their protoplasm out of carbon dioxide, water, and mineral salts. The first of these methods of nutrition is conveniently distinguished as holozoic, or wholly-animal, the second as holophytic, or wholly-vegetal.

It is important to note that only those plants or parts of plants in which chlorophyll is present are capable of holophytic nutrition. Whatever may be the precise way in which the process is effected, it is certain that the decomposition of carbon dioxide which characterises this form of nutrition is a function of chlorophyll, or, to speak more accurately, of chromatophores, since there 
is reason for thinking that it is the protoplasm of these bodies and not the actual green pigment which is the active agent in the process.

Moreover, it must not be forgotten that the decomposition of carbon dioxide is carried on only during daylight, so that organisms in which holophytic nutrition obtains are dependent upon the sun for their very existence. While Amœba derives its energy from the breaking down of the proteids in its food (see p. 235), the food of Sphærella is too simple to serve as a source of energy, and it is only by the help of sunlight that the work of constructive metabolism can be carried on (photosynthesis). This may be expressed by saying that Sphærella, in common with other organisms containing chlorophyll, is supplied with kinetic energy (in the form of light or radiant energy) directly by the sun.

As in Amœba, destructive metabolism is constantly going on side by side with constructive. The protoplasm becomes oxidised, water, carbon dioxide, and nitrogenous waste matters being formed and finally got rid of. Obviously, then, absorption of oxygen must take place, or, in other words, respiration must be one of the functions of the protoplasm of Sphærella as of that of Amœeba. In many green, i.e., chlorophyllcontaining, plants, this has been proved to be the case : respiration, i.e., the taking in of oxygen and giving out of carbon dioxide, is constantly going on, but during daylight is obscured by the converse process-the taking in of carbon dioxide for nutritive purposes and the giving out of the oxygen liberated by its decomposition. In darkness, when this latter process is in abeyance, the occurrence of respiration is more readily ascertained.

Owing to the constant decomposition, during sunlight, of carbon dioxide, a larger volume of oxygen than of carbon dioxide is evolved; and if an analysis were made 
of all the ingesta of the organism (carbon dioxide puns mineral salts plus respiratory oxygen) they would be found to contain less oxygen than the egesta (oxygen from decomposition of carbon dioxide plis water, excreted carbon dioxide, and nitrogenous waste); so that the nutritive process in Sphærella is, as a whole, a process of deoxidation. In Amœba, on the other hand, the ingesta (food plus respiratory oxygen) contain more oxygen than the egesta (fæces plus carbon dioxide, water, and nitrogenous excreta), the nutritive process being therefore on the whole one of oxidation. This difference is, speaking broadly, characteristic of plants and animals generally; animals, as a rule, take in more free oxygen than they give out, while green plants always give out more than they take in.

Destructive metabolism is, however, manifested not only in the formation of waste-products, but in that of substances simpler than protoplasm which remain an integral part of the organism, viz., cellulose and starch. The cell-wall is probably formed by the conversion of a thin superficial layer of protoplasm into cellulose, the cyst attaining its final thickness by frequent repetition of the process. The starch of the pyrenoids is apparently formed by a similar process of decomposition or destructive metabolism of protoplasm.

We see then that destructive metabolism may result in the formation of $(a)$ waste products and $(b)$ plastic products, the former being got rid of as of no further use, while the latter remain an integral part of the organism.

Let us now turn once more to the movements of Sphærella, and consider in some detail the manner of their performance.

Each flagellum (Fig. 68, $\mathbf{A}, A$ ) is a thread of protoplasm of uniform diameter except at its distal or free 
end, where it tapers to a point. The lashing movements are brought about by the flagellum bending successively in different directions (F.). Thus the ciliary movement of Sphærella, like the amœboid movement of Amœba, is a phenomenon of contractility. Imagine an Amøeba to draw in all its pseudopods but two, and to protrude these two until they became mere threads: imagine further these threads to contract rapidly and more or less regularly instead of slowly and irregularly; the result would be the substitution of pseudopods by flagella, i.e., of temporary slow-moving protoplasmic processes by permanent rapidly-moving ones.

To put the matter in another way: in Amoeba the function of contractility is performed by the whole organism ; in Sphærella it is discharged by a small part only, viz., the flagella, the rest of the protoplasm being incapable of movement.

Sphærella multiplies, after becoming quiescent, in the encysted condition (Fig. 68, C, D); as in the active Amœba (p. 236), its protoplasm undergoes simple or binary fission, but with the peculiarity that the process is immediately repeated, so that four daughter-cells are produced within the single mother-cell-wall. By the rupture of the latter the daughter-cells are set free as the ordinary motile form, acquiring their flagella and detached cell-wall before making their escape (D).

Under certain circumstances the resting form divides into numerous smaller daughter-cells, and these when liberated are found to have no cell-wall. Sphærella therefure occurs, in the motile condition, under two distinct forms, i.e., is dimorphic: the larger or ordinary form with detached cell-wall is called a megazooid, the smaller form without a cell-wall a microzooid. The microzooids, which are all similar to one another, are (in S. bütschlii) liberated as gametes which conjugate in 
pairs to form zygotes (pp. 197, 237), each of which becomes surrounded with a cell-wall, and after a time again undergoes multiplication

We will now examine another small organism which is often found in puddles and pools, frequently in such vast numbers as to give the water a green colour. This organism is known as Euglena viridis.

Euglena is also microscopic, its length varying from $\frac{1}{24} \mathrm{~mm}$. to $\frac{1}{6} \mathrm{~mm}$. The body is spindle-shaped, wide in the middle and narrow at both ends (Fig. 69, A, C-F) : one extremity is blunter than the other, and from it proceeds a single long flagellum $(f)$ by the action of which the organism swims with great rapidity, the flagellum being, as in Sphærella, directed forwards. Besides its rapid swimming movements, Euglena frequently performs slow movements of contraction and expansion, something like those of a short. worm, the body becoming broadened out first at the anterior end. then in the middle, then at the posterior end, twisting to the right and left, and so on $(\mathrm{C}-\mathrm{F})$. These movements are so characteristic of the genus that the name euglenoid is applied to them.

The organism consists of protoplasm covered with a very delicate membrane or cuticle which is often finely striated, and is to be looked upon as a superficial hardening of the protoplasm. The colour is due to the presence of chlorophyll, which tinges all the central part, the two ends being colourless. It is difficult to make out that the chlorophyll is lodged in a number of distinct chromatophores (ch).

In Sphærella we saw that chlorophyll was associated with starch (p. 243). In Euglena there are, near the middle of the body, a number of grains of paramylum $(A, p)$, a carbohydrate of the same composition as 
starch $\left(\mathrm{C}_{6} \mathrm{H}_{10} \mathrm{O}_{5}\right)$, but differing from it in remaining uncoloured by iodine.

Water containing Euglenæ gives off bubbles of

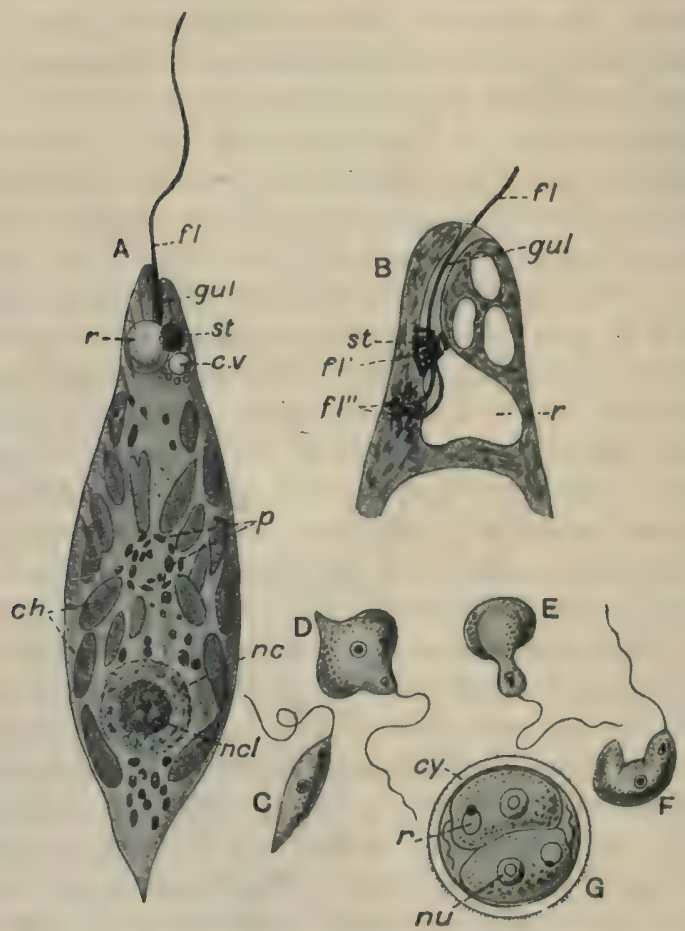

Fic. 69,-Euglema viridis.

A, view of entire organism, showing details of structure ( $x$ about rooo).

$\mathrm{B}$, anterior end, to show origin of flagellum, etc. ( $x$ about 3000 ).

$\mathrm{C}-\mathrm{F}$, four views of the living organism, showing the changes of form produced by the characteristic euglenoid movements.

$\mathrm{G}$, resting form after binary fission, showing cyst or cell-wall, nuclei, and reservoirs of the daughter-cells.

ch. chromatophores; $c v$. contractile vacuole ; $c y$. cyst or cell-wall ; fl. flagellum ; $f^{\prime}$ thickening on flagellum; $A^{\prime \prime}$. bifurcated base of flagellum; gul. gullet ; m., nu. nucleus; ncl. " uucleolus"; p. paramylum bodies; r. reservoir ; st. eye-spot or stigma.

A, from Doflein; $B$, from Lothein, after Wager; $C-G$, after Saville Kent. 
oxygen in sunlight: as in Sphrerella the carbon dioxide in solution in the water is decomposed in the presence of chlorophyll, its oxygen evolved, and its carbon combined with the elements of water and used in nutrition. For a long time Euglena was thought to be nourished entirely in this way, but there is reason for thinking that this is not the case.

When the anterior end of a Euglena is very highly magnified, it is found to have the form shown in Fig. 69, A, B. It is produced into a blunt snout-like extremity, at the base of which is a conical depression ( $g u l)$ :just the sort of depression one could make in a clay model of Euglena by thrusting one's finger or the end of a pencil into the clay. The bottom of this tube opens into a space, the reservoir $(r)$, from one side of which the flagellum arises by a bifurcated base, and by its continual movement gives rise to a sort of whirlpool in the neighbourhood. By the current thus produced, minute solid food-particles are said to be swept down the tube and forced into the soft internal protoplasm, where they doubtless become digested in the same way as the substances ingested by an Amœeba. That solid particles are so ingested by many unicellular organisms (see e.g., pp. 266 and 272) has been proved by diffusing finely powdered carmine in the water, when the coloured particles were seen to be swallowed in the way described.

The depression in question serves therefore as a gullet, and its external aperture or margin $(m)$ as a mouth. Euglena, like Amœba, may take in solid food, but instead of ingesting it at any part of the body, it can do so only at one particular point where there is a special ingestive aperture or mouth. This is clearly a case of specialisation or differentiation of structure : in virtue of the possession of a mouth and gullet, Euglena is more highly organised than Amœba. 
It thus appears that in Euglena nutrition is both holozoic and holophytic (p. 247) : very probably it is mainly holophytic during daylight and holozoic in darkness (compare also p. 256).

Near the centre or somewhat towards the posterior end is a nucleus $(\mathrm{A}, n c)$ with a well-marked " nucleolus" ; at the anterior end is the clear space $(r)$ already referred to, looking very like a contractile vacuole, but which is in reality a non-contractile cavity or reservoir into which the true contractile vacuole $(c . v)$ opens, and which itself communicates with the gullet.

In close relation with the reservoir is found a little bright red speck composed of pigment granules and called the eye-spot or stigma (st), its colour being due to hæmatochrome (p. 243). Recent experiments seem to show that it is specially sensitive to light, and is therefore a true eye in the sense of a light-perceiving organ, although having no actual visual function.

As in Sphærella a resting condition alternates with the motile phase: the organism loses its flagellum and surrounds itself with a cyst of cellulose (G, cy, see p. 244), from which, after a period of rest, it emerges to resume active life.

Reproduction takes place by simple binary fission of the resting form, the plane of division being always longitudinal $(G)$. Sometimes each product of division or daughter-cell divides again : finally the two, or four, or sometimes even eight daughter-cells emerge from the cyst as active Euglenæ. A process of multiple fission (p. 237) has also been described, numerous simple, minute, active bodies or spores being produced, which gradually assume the ordinary form and size.

We have seen that typical animal-cells, such as those 
of the frog (Part I, Chap. VII) are not provided with a cellulose cell-wall and do not contain chlorophyll. It is characteristic, on the other hand, of most plant-cellswhich also consist of nucleated protoplasm-that they are surrounded with a cellulose cell-wall, and that, in the case of green plants, they contain chlorophyll. Speaking generally, the nutrition of animals is holozoic, and that of green plants holophytic; and in correspondence with this difference in the character of the food, most animals have an ingestive aperture or mouth for taking in the solid food, and some kind of digestive cavity, either permanent (stomach) or temporary (food-vacuole) ; they also have, as a rule, some kind of excretory apparatus. Moreover, animals are usually capable of automatic movement, while in most plants the organism, as a whole, exhibits no automatism, but only the slow movements of growth.

Let us now apply these definitions to the simple forms described above and see how far they will help us in placing those organisms in one or other of the two " kingdoms" (p. 220) into which living things are divided.

Amœba has a cell-wall, probably nitrogenous, in the resting condition; it ingests solid proteids, its nutrition being therefore holozoic: it has a contractile vacuole: and it performs amoboid movements. It may therefore be safely considered as an animal.

Sphærella has a cellulose wall that contains chlorophyll and its nutrition is purely holophytic: a contractile vacuole is present in S. lacustris, though not distinct in S. pluvialis: and its movements are ciliary.

Euglena has a cellulose wall in the encysted state : in virtue of its chlorophyll it is nourished by the absorption of carbon dioxide and mineral salts, but it may also ingest solid food through a special mouth and gullet : it has a 
contractile vacuole, and performs both euglenoid and ciliary movements.

In both these organisms we evidently have conflicting characters: the cellulose wall and holophytic nutrition would place them both among plants, while from the contractile vacuole and active movements of both genera, and from the holozoic nutrition of Euglena, we should group them with animals. That the difficulty is by no means easily overcome may be seen from the fact that both genera are claimed at the present day by zoologists and by botanists.

Another mode of nutrition, which under certain circumstances may also be adopted by Ëuglena. occurs

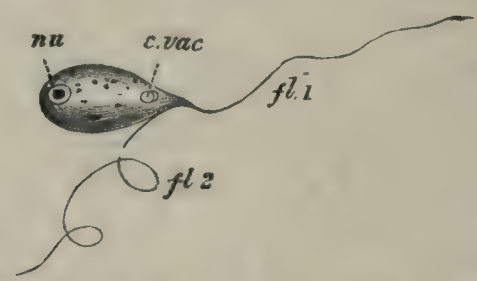

FIg. 70.-A Monad (Heteromita rostrata), showing nucleus (nu), contractile vacuole (c. vac), and two flagella $\left(f 1, f^{2}\right)$. ' ( $x$ about 1500$)$. (From Parker's Biology. after Dallinger.)

in certain organisms which must now be referred to very briefly.

When animal or vegetable matter is placed in water and allowed to stand at the ordinary temperature, the well-known process of decomposition or putrefaction (pp. II, I52, and 237) sooner or later sets in, the water becoming turbid and acquiring a bad smell A drop of it examined under the microscope is then found to teem with very minute unicellular organisms, some of which are known as Monads, much smaller than Euglena, the form represented in Fig. 70 (Heteromita) being only 
about $\frac{1}{1 \frac{1}{2}} \mathrm{~mm} \cdot\left(\frac{1}{3000}\right.$ inch) in length. Like Sphærella, the Monad swims about by means of two flagella, but it contains no chlorophyll. The putrefying infusion in which it lives contains proteids in solution, in part split up by the process of decomposition into simpler compounds, some of which are diffusible. As the Monad contains no chlorophyll, its nutrition is evidently not holophytic; and, apart from the fact that it possesses neither mouth nor pseudopods, observation seems to show pretty conclusively that it is not holozoic.

There remains only one way in which nutrition can take place, namely, by absorption of the proteids and other nutrient substances in the solution: the Monad may be said to live immersed in an immense cauldron of broth which it is for ever imbibing, not by its mouth, for it has none, but by the whole surface of its body. This is the saprophytic mode of nutrition, and resembles that which occurs in certain plants which contain no chlorophyll-the fungi. It will be seen that the main difference between saprophytic and holozoic nutrition is that in the former digestion, i.e., the process of rendering food-stuffs soluble and diffusible takes place outside the body, so that constructive metabolism can begin at once.

In the Monad, the characters are again conflicting (compare p. 255): the probable absence of cellulose, the presence of a contractile vacuole, and the flagella all have an "animal " look, but the mode of nutrition is that of a fungus.

Decomposition, as already stated (p. I52), is due in the first instance to the action of certain other minute organisms, known as Bacteria, which appear in the earlier stages of the putrefaction of an organic infusion. The nutrition of these organisms, like that of Monads, is usually saprophytic and the movements are generally ciliary; but as they have a definite cell-wall and no 
contractile vacuole, they are almost universally classed amongst plants, while Monads are as constantly included in the animal kingdom.

We see, then, that while it is quite easy to divide the higher organisms into the two distinct groups--plants and animals, any such separation is by no means easy in the case of the lowest forms of life. It was in recognition of this fact that Haeckel proposed, many years ago, to institute a third "kingdom" called Protista, to include all unicellular organisms. Although open to many objections in practice, there is a great deal to be said for the proposal. From the strictly scientific point of view it is quite as justifiable to make three subdivisions of living things as two: the line between animals and plants is quite as arbitrary as that between protists and plants or between protists and animals, and no more so: the chief objection to the change is that it doubles the difficulties by making two artificial boundaries instead of one.

The important point for the student to recognise is that these boundaries are artificial, and that there are no scientific frontiers in Nature. As in the liquefaction of gases there is a "critical point " at which the substance under experiment is neither gaseous nor liquid: as in a mountainous country it is impossible to say where mountain ends and valley begins : as in the development of an animal it is futile to argue about the exact period when, for instance, the egg becomes a tadpole or the tadpole a frog: so in the case under discussion. The distinction between the higher plants and animals is perfectly sharp and obvious, but when the two groups are traced downwards they are found gradually to merge, as it were, into an assemblage of organisms which partake of the characters of both kingdoms, and cannot without a certain violence be either included in 
or excluded from either. When any given " protist" has to be classified the case must be decided on its individual merits: the organism must be compared in detail with all those which resemble it closely in structure, physiology, and life-history; and then a balance must be struck and the doubtful form placed in the kingdom with which it has, on the whole, most points in common.

It will no doubt occur to the reader that, on the theory of evolution (p. 222), we may account for the fact of the animal and vegetable kingdoms being related to one another like two trees united at the root, by the hypothesis that the earliest organisms were protists, and that from them animals and plants were evolved along divergent lines of descent.

\section{PRACTICAL DIRECTIONS}

Sphærella.-Examine a drop of water containing Sphærellæ (see note on p. 238), first with the low power, and then, after putting on a cover-glass, with the high power. Note-

I. Therr rounded form and green (or red) colour; the cellulose cell-wall; the protoplasm, enclosing (a) a chromatophore containing chloropkyll (the red colour is due to another colouring matter, hamatochrome), and (b) a central nucleus, seen better later; and in the active forms, the two flagella. Sketch.

2. Dissolve out the chlorophyll by adding alcohol; the nucleus will then be visible, and may be made more distinct by staining with magenta, or a solution of potassium iodide in water saturated with iodine. After treatment with the iodine solution, a bluish coloration will often be seen around the small starch-containing pyrenoids.

3. Treat some specimens either with strong iorline solution and then with sulphuric acid ( 75 per cent.), or with Schulze's solution, which is prepared as follows:-make about 25 c.c. of a syrupy solution of zinc chloride, and let it stand for a short time; then pour off the clear fluid, saturate it with potassuim iodide and add iodine until the solution has about the colour of sherry. Note the blue coloration of the cell-wall.

4. In the living specimens note also the mode of division 
into four megazooids or into numerous microzooids, and observe their movements when set free. Sketch.

Euglena.-Examine a drop of water containing Euglena with the low power, then cover, and put on the high power. Note-

I. The spindle-shaped form of the body, and its changes of form in contraction and expansion.

2. The long flagellum.

3. The superficial cuticle, and the mouth and conical depression (gullet) at the anterior end.

4. The central part of the body, which contains chlorophyll, except at the two ends. Near the middle is a nucleus enclosing a " nucleolus," and near the anterior end a noncontractile space into which the gullet and the contractile vacuole open, close to which a red eye-spot is situated. Grains of paramylum may be recognised near the centre of the body. Sketch before and after staining as above.

5. Look for specimens in the encysted condition, and observe if any of them are undergoing division. Sketch.

Monads and Bacteria.--Examine a drop of water containing some putrefying animal or vegetable substance. An infusion of hay is convenient for this purpose :-pour boiling water on a handful of hay and strain the fluid through blotting-paper; shortly afterwards decomposition will set in.

The first organisms to appear in such a putrefying infusion are Bacteria, which are so minute as to appear as mere specks under the high power-some of them being only $\frac{1}{3} \frac{\mathrm{mm}}{\mathrm{n}}$. ( $\frac{1}{2} \frac{1}{500} \mathrm{inch}$ ) in length, or even less. Careful examination will show globular, rod-like, and spiral forms, all capable of movement at one phase of their existence. Monads will appear somewhat later, and may be recognised by their ovoidal form and their flagella (Fig. 70), which, however, like those of Bacteria, can only be seen under a very high power. 


\section{CHAPTER III}

PARAMGECIUM : VORTICELLA AND ITS AILIES-COLONIAL ORGANISMS

WE have now to consider certain organisms in which differentiation has gone much further than in the unicellular forms already considered: which have, in fact, acquired many of the characteristics of the higher animals and plants while remaining unicellular (compare p. 238). The study of several of these more or less highly differentiated though unicellular forms will occupy the present chapter.

It was mentioned above that, in the earlier stages of the putrefaction of an organic infusion, Bacteria only were found, and that, later, Monads made their appearance (p. 257). Still later, organisms much larger than Monads are seen, generally of an ovoidal form, moving about very quickly and seen by the use of a high power to be covered with innumerable fine cilia. These are called ciliate Infusoria, in contradistinction to Monaảs, which are often known as flagellate Infusoria: many kinds are common in putrefying infusions, some occur 
in the intestines of the higher amnials, while others are among the commonest inhabitants of both fresh and salt water.

A very common ciliate infusor is the beautiful "slipperanimalcule," Paramœcium, which from its comparatively large size and from the ease with which all essential points of its organisation can be made out is a very convenient and interesting object of study.

Compared with the majority of the organisms which have come under our notice it may fairly be considered as gigantic, being no less than $\frac{1}{5}-\frac{1}{4} \mathrm{~mm} .(200-260 \mu)$ in length : in fact it is just visible to the naked eye as a minute whitish speck.

Its form (Fig. 7I, A) can be fairly well imitated by making out of clay or stiff dough an elongated cylinder rounded at one end and bluntly pointed at the other; then giving the broader end a slight twist; and finally making on the side rendered somewhat concave by the twist a wide, shallow groove beginning at the broad end and gradually narrowing to about the middle, where it ends in a tolerably deep depression.

The groove is called the buccal groove (A and B, buc. gr): at the narrow end, as in Euglena (Fig.69), is a small aperture, the mouth ( $m t h)$, which, however, leads directly into the soft internal protoplasm. The surface of the creature on which the groove is placed is distinguished as the ventral surface, the opposite surface being upper or dorsal; the broad end is anterior, the narrow end posterior, the former being directed forwards as the animalcule swims. These descriptive terms being decided upon, it will be seen that the buccal groove begins on the left side, and gradually curves over to the middle of the ventral surface. 

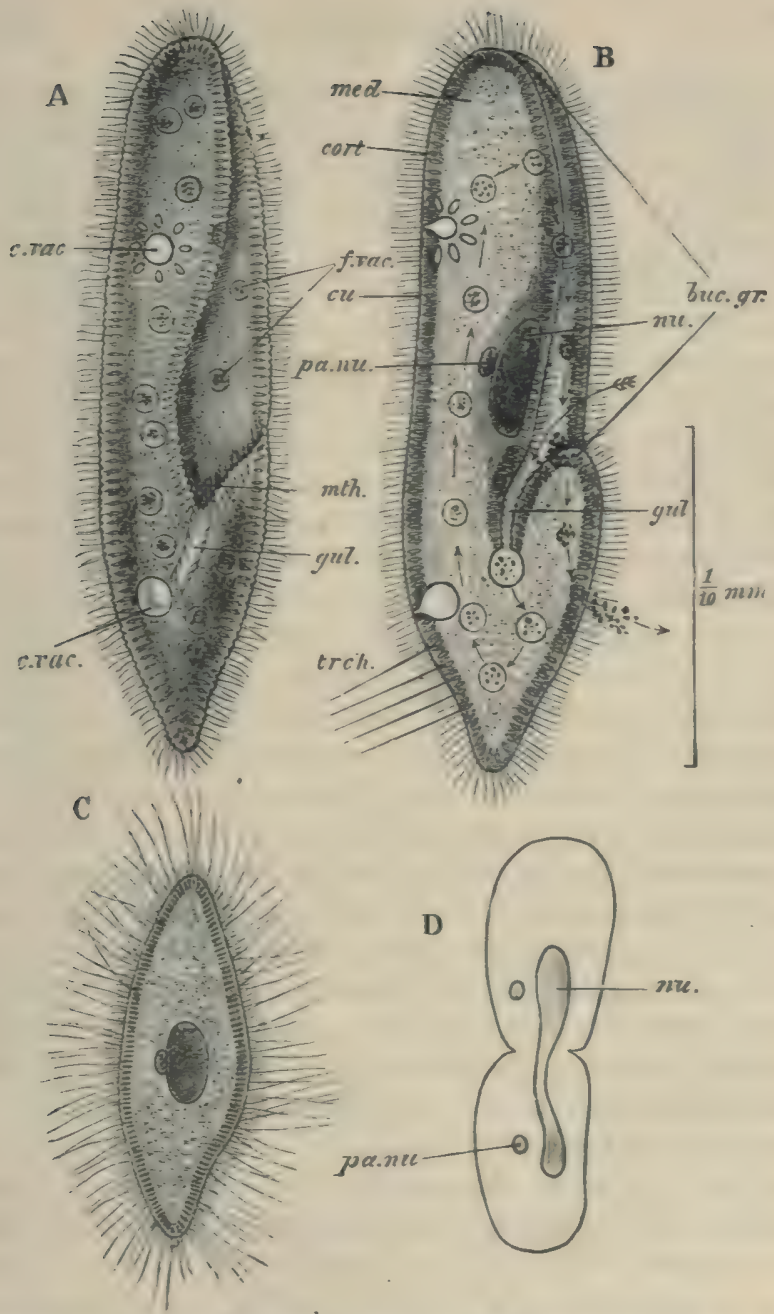

Fic. 7I,-Peramacium coudatum.

A, the livint animal from the ventral aspect. shewing the cuvering of clia, the bucual groove (to the rjoht) ending posteriorly in the nouth (mith) and zullet (gul) ; several food vacuoles (f. vac) and the two contractile vacuoles (c. iuc). ( $\times$ 300.) 
B, the same in optical section showing cuticle (cu), cortex (corl), and medulla (med) ; buccal groove (buc. gr), mouth, and gullet ( $g u l$ ) ; numerous food vacuoles ( $f$. vac) circulating in the direction indicated by the arrows, and containing particles of indigo, which are finally ejected at an anal spot; meganucleus $(n u)$, micronucleus (pa. $n u)$, and trichocysts, some of which (trch) are shown with their threads ejected. The scale to the right of this figure applies to $A$ and $B$.

C, a specimen killed with osmic acid, showing the ejection of trichocyst-threads, which project considerably beyond the cilia.

D, diagram of binary fission; the micronucleus ( $p a, n u$ ) has already divided, the meganucleus $(\mathrm{mu})$ is in the act of dividing. (From Parker's Biology: D, after Lankester.)

As the creature swims its form is seen to be permanent, exhibiting no contractions of either an amœboid or a euglenoid nature. It is, however, distinctly flexible, often being bent in one or other direction when passing between obstacles, such as entangled masses of weed. This permanence of contour is due to the presence, as in Euglena, of a tolerably firm though delicate cuticle $(\mathrm{B}, c u)$ which invests the whole surface.

The protoplasm thus enclosed by the cuticle is distinctly divisible into two portions-an external somewhat dense layer, the cortical layer or cortex (cort), and an internal more fluid material, the medullary substance or medulla (med). It will be remembered that a somewhat similar distinction of the protoplasm into two layers is exhibited by Amœba (p. 23I), the ectoplasm being distinguished from the endoplasm simply by the absence of granules. In Paramocium the distinction is a far more fundamental one: the cortex is radially striated and is comparatively firm and dense, while the medulla is granular and semi-fluid, as may be seen from the fact that food-particles (f. vac, and see p. 266) move freely in it, whereas they never pass into the cortex. The medulla has a reticular structure similar to that of the protoplasm of the ordinary animal-cell, consisting of a delicate granular network the meshes of which are filled with a transparent material. In the cortex the meshes of the network are closer, and so form a comparatively dense substance. The cortex also exhibits a superficial oblique striation. 
The mouth ( $m$ th) leads into a short funnel-like tube, the gullet $(g u l)$, which is lined by cuticle and passes through the cortex to end in the soft medulla, thus making a free communication between the latter and the external water.

The cilia with which the body is covered are of approximately equal size, quite short in relation to the entire animal, and arranged in longitudinal rows over the whole outer surface. They consist of prolongations of the cortex, and each passes through a minute perforation in the cuticle. They are in constant rhythmical movement, like the cilia on the epithelial cells of the frog's mouth (p. I09), and are thereby distinguished from the flagella of Sphærella, Euglena, \&c., which exhibit more or less intermittent lashing movements.

Near the middle of the body, on the inner boundary of the cortex, is a large oval nucleus $(B, C, n u)$, and against one side of it, in $P$. caudatum, is a small oval structure (pa. nu) which is also deeply stained by, e.g., magenta or carmine. This is the micronucleus: it is to be considered as a second, smaller nucleus, the larger body being distinguished as the meganucleus. In the closely allied $P$. aurelia there are two micronuclei.

There are two contractile vacuoles (c. vac) in relation with the cortex, one situated at about a third of the entire length from the anterior end of the body, the other at about the same distance from the posterior end.

The action of the contractile vacuoles is very beautifully seen in a Paramœcium at rest : it is particularly striking in a specimen subjected to slight pressure under a cover-glass, but is perfectly visible in one which has merely temporarily suspended its active, swimming movements. It is then seen that during the diastole, or phase of expansion of each vacuole a number-about 
six to ten-of delicate, radiating, spindle-shaped spaces filled with fluid appear round it, like the rays of a star (upper vacuole in A and B): the vacuole itself contracts or performs its systole, completely disappearing from view, and immediately afterwards the radiating canals flow together and refill it, becoming themselves emptied and therefore invisible for an instant (lower vacuole in $\mathrm{A}$ and $\mathrm{B}$ ) but rapidly appearing once more. There seems to be no doubt that the water taken in with the food is collected into these canals, emptied into the vacuole, and finally discharged to the exterior.

The process of feeding can be very conveniently studied in Paramœcium by placing in the water some finely-divided carmine or indigo. When the creature comes into the neighbourhood of the coloured particles, the latter are swept about in various directions by the action of the cilia: some of them, however, are certain to be swept into the neighbourhood of the buccal groove and gullet, the cilia of which'all work downwards, i.e., towards the inner end of the gullet. The grains of carmine are thus carried into the gullet, where for an instant they lie surrounded by the water of which it is full : then, instantaneously, probably by the contraction of the tube itself, the animalcule performs a sort of gulp and the grains with an enveloping globule of water or food-vacuole are forced into the medullary protoplasm. This process is repeated again and again, so that in any well-nourished Paramœcium there are to be seen numerous globular spaces filled with water and containing particles of food-or in the present instance of carmine or indigo. At every gulp the newly-formed food-vacuole pushes, as it were, its predecessor before it : contraction of the medullary protoplasm also takes place in a definite direction, and thus a circulation of food-vacuoles is produced, as indicated in Fig. 71, B, by arrows. 
After circulating in this way for some time the water of the food-vacuoles is gradually absorbed, being ultimately excreted by the contractile vacuoles, so that the contained particles come to lie in the medulla itself (refer to figure). The circulation still continues, until finally the particles are brought to a spot situated about half-way between the mouth and the posterior end of the body: here if carefully watched they are seen to approach the surface and then to be suddenly ejected. The spot in question is therefore to be looked upon as a potential anus, or aperture for the egestion of fæces or undigested food-matters. It is a potential and not an actual anus, because it is not a true aperture, but only a soft place in the cortex through which, by the contractions of the medulla, solid particles are easily forced.

Of course when Paramoecium ingests, as it usually does, not carmine but minute living organisms, the latter are digested as they circulate through the medullary protoplasm, and only the non-nutritious parts cast out at the anal spot. It has been found by experiment that this infusor can digest not only proteids but also starch and perhaps fats. The nutrition of Paramœeium is therefore characteristically holozoic.

It was mentioned above that the cortex is radially striated in optical section. Careful examination with a very high power shows that this appearance is due to the presence in it of minute spindle-shaped bodies (A and B) closely arranged in a single layer and perpendicular to the surface. These are called trichocy'sts.

When a Paramocium is killed, either by the addition of some poisonous reagent or by simple pressure of the cover-glass, it frequently assumes a remarkable appearance. Long delicate threads suddenly appear, projecting from its surface in all directions (C) and looking very much as if the cilia had suddenly protruded to many 
times their original length. But these filaments have really nothing to do with the cilia; they are contained under ordinary circumstances in the trichocysts, probably coiled up; and by the contraction of the cortex consequent upon any sudden irritation they are projected in the way indicated. In Fig. $7 \mathrm{I}, \mathrm{B}$, a few trichocysts (trch) are shown in the exploded condition, i.e., with the threads protruded. Most likely these bodies are weapons of offence like the very similar structures (nematocysts) found in polypes (see Chapter V., Fig. 77).

Paramœecium multiplies by simple fission, the division of the body being always preceded by the elongation and subsequent division of the mega- and micro-nucleus, accompanied by the regeneration of the parts wanting in each product (Fig. 7I, D).

Conjugation (pp. 237, 25I) also occurs, usually after multiplication by fission has gone on for some time. Two Paramocia come into contact by their ventral faces, and in each of these conjugating individuals or gametes the meganucleus takes no part in the process and degenerates, while the micronucleus undergoes a somewhat complicated series of changes; the essential part of the process being the fusion of two products of the division of the micronuclei, one from each gamete, each of which then contains a single nuclear body, the conjugationnucleus, formed by the union of nuclear matter derived from two distinct individuals, and therefore comparable to the nucleus of the oosperm in the higher animals (p. 197). The other products of division of the micronucleus disappear (compare Chapter XII., p. 572), and a new mega- and micro-nucleus arise from the conjugationnucleus. In this case, however, the two entire gametes do not unite to form a zygote, but separate after the process is complete and begin once more to lead an 
independent existence, when ordinary transverse fission again takes place.

It will be noticed that, in the present instance (see also p. 25I), conjugation is not a process of multiplication: it has been ascertained that during the time two infusors are conjugating each might have produced a very large number of offspring by continuing to undergo fission at the usual rate. The importance of the process lies in the exchange of nuclear material between the two conjugating individuals.

The next organism we have to consider is a ciliate infusor, even commoner than that just described. It is hardly possible to examine the water of a pond with any care without finding in it, sometimes attached to weeds, sometimes to the legs of water-fleas, sometimes to the sticks and stones of the bottom, numbers of exquisitely beautiful little creatures, each like an inverted bell with a very long handle, or a wine-glass with a very long stem. These are the well-known "bell-animalcules," the commonest among them belonging to various species of the genus Vorticella.

The first thing that strikes one about Vorticella (Fig. 72, A) is the fact that it is permanently fixed, like a plant, the proximal or near end of the stalk being always firmly fixed to some aquatic object, while to the distal or far end the body proper of the animalcule is attached.

But in spite of its peculiar form it presents certain very obvious points of resemblance to Paramœcium. The protoplasm is divided into cortex $(\mathrm{C}$, cort $)$ and medulla (med), and is invested with a delicate cuticle (cu). There is a single contractile vacuole (c. vac) the movements of which are very readily made out owing to the ease with which the attached organism is kept under 


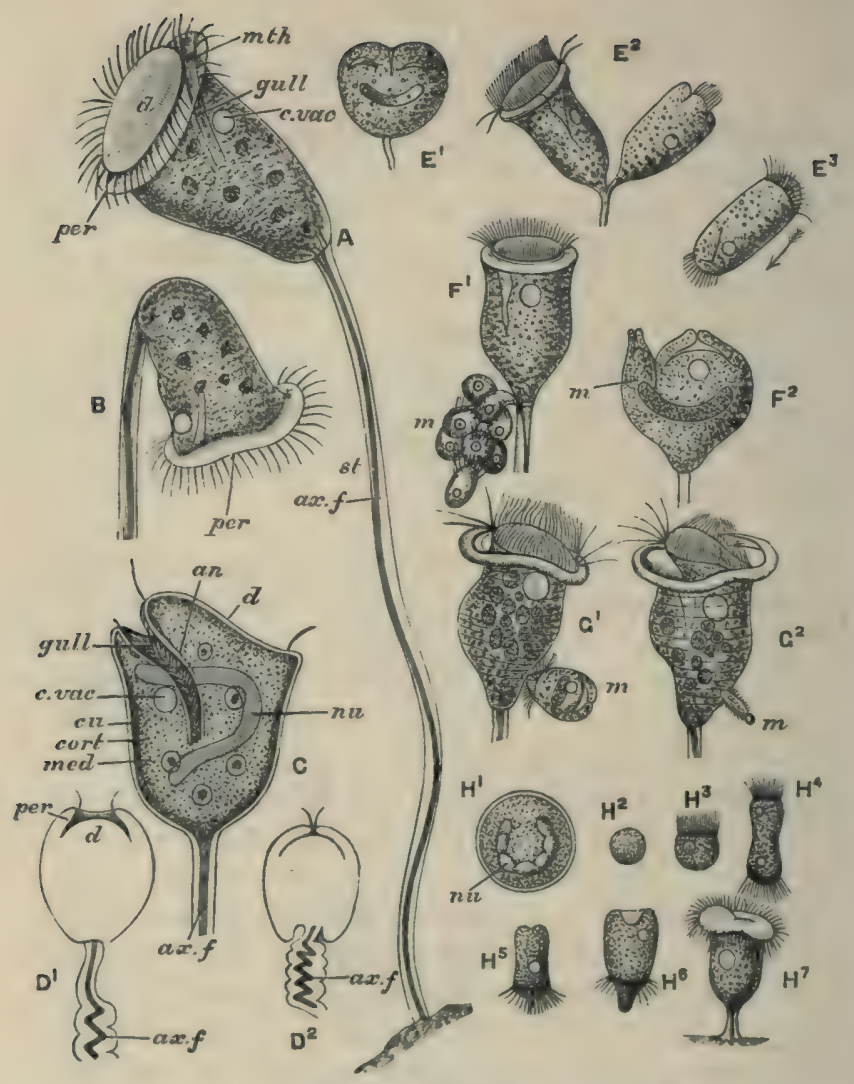

Fig. 72.-Voricella.

A, living specimen fully expanded, showing stalk (st) with axial fibre ( $a x . f)$, peristome (per), disc $(d)$, mouth ( $m t h)$, gullet (gull), and contractile vacuole (c. vac). ( $\times$ 250.)

$B$, the same, bent on its stalk and with the disc turned away from the nbserver.

C, optical section of the same, showing cuticle (cu), cortex (corl), medulla (med). meganucleus (nu), gullet (gull), several food vacuoles and anal spot (an), as well as the other structures shown in $\mathrm{A}$.

D1, a half-retracted, and $\mathrm{D}^{2}$ a fully-retracted specimen, showing the coiling of the stalk and overlapping of the disc by the peristome.

$\mathrm{E}^{1}$ commencement of binary fission; $\mathrm{E} 2$, completion of the process; $\mathrm{E}^{3}$, the product of division swimming freely in the direction indicated by the arrow.

F1, a specimen dividing into a megazooid and several microzooids $(m) ; F 2$, division into one mega- and one micro-zooid.

GI, G², two stages in conjugation, showing the gradual absorption of the microgamete $(m)$ into the megagamete.

$\mathrm{H}^{1}$, multiple fission of encysted form, the nucleus dividing into numerous masses ; $\mathrm{H}^{2}$, spore formed by multiple fission: $\mathrm{H}^{3}-\mathrm{H}^{7}$, development of the spore $-\mathrm{H}^{\mathrm{s}}$ is undergoing binary fission. (From Parker's Biology : F-H after Savile-Kent.) 
observation. There is a meganucleus (nu) remarkable for its elongated band-like form, and having in its neighbourhood a minute micronucleus. Cilia are also present, but the way in which they are disposed is very peculiar and characteristic. To understand it we must study the form of the body a little more closely.

The conical body is attached by its apex or proximal end to the stalk: its base or distal end is expanded so as to form a thickened rim, the peristome (per), within which is a plate-like body elevated on one side called the disc $(d)$, and looking like the partly raised lid of a chalice. Between the raised side of the disc and the peristome is a depression, the mouth $(m t h)$, leading into a conical gullet (gull).

There is reason for thinking that the whole proximal region of Vorticella answers to the ventral surface of Paramocium, and its distal surface with the peristome and disc to the dorsal surface of the free-swimming genus: the mouth is to the left in both.

A single row of cilia is disposed round the inner border of the peristome and continued on the one hand down the gullet, and on the other round the elevated portion of the disc; the whole row of cilia thus takes a spiral direction. The rest of the body is completely bare of cilia.

The movements of the cilia produce a very curious optical illusion: as one watches a fully-expanded specimen it is hardly possible to believe that the peristome and disc are not actually revolving - a state of things which would imply that they were discontinuous from the rest of the body. As a matter of fact the appearance is due to the successive contraction of all the cilia in the same direction, and is analogous to that produced by a strong wind on a field of corn or long grass. The bending down of successive blades of grass produces a series 
of waves travelling across the field in the direction of the wind. If instead of a field we had a large circle of grass, and if this were acted upon by a cyclone, the wave would travel round the circle, which would then appear to revolve.

Naturally the movement of the circlet of cilia produces a small whirlpool in the neighbourhood of the Vorticella, as can be seen by introducing finelypowdered carmine into the water. It is through the agency of this whirlpool that food particles are swept into the mouth, surrounded, as in Paramœecium, by a globule of water : the food-vacuoles (C) thus constituted circulate in the medullary protoplasm and the nonnutritive parts are finally egested at an anal spot (an) situated near the base of the gullet.

The stalk (A, st) consists of a very delicate, transparent, outer substance, which is continuous with the cuticle of the body and contains a delicate axial fibre $(a x . f)$ running along it from end to end in a somewhat spiral direction. This fibre is a prolongation of the cortex of the body $(\mathrm{C})$ : under a very high power it appears granular or delicately striated, the striæ being continued into the cortex of the proximal part of the body.

A striking characteristic of Vorticella is its extreme irritability, i.e., the readiness with which it responds to any external stimulus. The slightest jar of the microscope, the contact of some other organism, or even a current of water produced by some free-swimming form like Paramœecium, is felt directly by the bell-animalcule, and is followed by an instantaneous change in the relative position of its parts. The stalk becomes coiled into a close spiral so as to have a mere fraction of its original length, and the body from being bell-shaped becomes globular, the disc being withdrawn and the peristome closed over it $\left(D^{1}, D^{2}\right)$. 
The coiling of the stalk leads us to the consideration of the particular form of contractility called muscular, which is met with in multicellular animals, e.g., the frog (p. 6o). It was mentioned above that while the stalk in its fully expanded condition is straight, the axial fibre is not straight, but forms a very open spiral, i.e., it does not lie in the centre of the stalk, but at any transverse section is nearer the surface at one spot than elsewhere, and this point as we ascend the stalk is directed successively to all points of the compass.

Now suppose that the axial fibre undergoes a sudden contraction, that is to say, a decrease in length accompanied by an increase in diameter, since, as we have already seen, there is no decrease in volume in protoplasmic contraction. There will naturally follow a corresponding shortening of the elastic cuticular substance which forms the outer layer of the stalk. If the axial fibre were entirely towards one side of the stalk, the result of the contraction would be a flexure of the stalk towards that side, but, as its direction is spiral, the stalk is bent successively in every direction, that is, is thrown into a close, spiral coil.

The axial fibre is therefore a portion of the protoplasm which possesses the property of contractility in a special degree ; in which, moreover, contraction takes place in a definite direction-the direction of the length of the fibre-so that its inevitable result is to shorten the fibre and consequently to bring its two ends nearer together. This is the essential characteristic of a muscular contraction, and the axial fibre in the stalk of Vorticella is therefore to be looked upon as the first instance of a clearly differentiated muscle which has come under our notice amongst unicellular animals.

There are some interesting features in the repro- 
duction of Vorticella.. It multiplies by binary fission, dividing through the long axis of the body $\left(\mathrm{E}^{1}, \mathrm{E}^{2}\right)$. Hence it is generally said that fission is longitudinal. not transverse, as in Paramœcium. But on the theory (p. 27I) that the peristome and disc are dorsal and the attached end ventral, fission is really transverse in this case also.

It will be seen from the figures that the process takes place by a cleft appearing at the distal end $\left(\mathrm{E}^{1}\right)$ and gradually deepening until there are produced two complete and full-size individuals upon a single stalk $\left(E^{2}\right)$. This state of things does not last long: one of the two daughter-cells takes on a nearly cylindrical form, keeps its disc and peristome retracted, and acquires a new circlet of cilia near its proximal end $\left(\mathrm{E}^{3}\right)$ : it then detaches itself from the stalk, which it leaves in the sole possession of its sister-cell, and swims about freely for a time. Sooner or later it settles down, becomes attached by its proximal end, loses its basal circlet of cilia, and develops a stalk, which ultimately attains the normal length.

The object of this arrangement is obvious. If, when a Vorticella divided, the plane of fission extended down the stalk until two ordinary fixed forms were produced side by side, the constant repetition of the process would so increase the numbers of the species in a given spot that the food-supply would inevitably run short. This is prevented by one of the two sister-cells produced by fission leading a free existence long enough to enable it to emigrate and settle in a new locality, where the competition with its fellows will be less keen. The production of these free-swimming zooids is therefore a means of dispersal: contrivances having this object in view are a very general characteristic of fixed organisms.

Conjugation occasionally takes place, and presents 
certain peculiarities. A Vorticella divides either into two unequal halves $\left(\mathrm{F}^{2}\right)$ or into two equal halves, one of which divides again into from two to eight daughtercells $\left(F^{1}\right)$. There are thus produced from one to eight microzooids which resemble the barrel-shaped form $\left(E^{3}\right)$ in all but size, and like it become detached and swim freely by means of a basal circlet of cilia. After swimming about for a time, one of these microzooids comes in contact with an ordinary form or megazooid, when it attaches itself to it near the proximal end $\left(G^{1}\right)$, and undergoes gradual absorption $\left(\mathrm{G}^{2}\right)$, the mega- and micro-zooids becoming completely and permanently fused to form a zygote (p. 25I).

Notice that in this case the conjugating bodies or gametes are not of equal size and similar character; but one which is conveniently distinguished as the microgamete (= microzooid) is relatively small and active, while the other or megagamete (= megazooid, or ordinary individual) is relatively large and passive. As we have seen in the case of the frog (pp. I95 and I96), this differentiation of the gametes is precisely what we get in the higher animals, and, in fact, in almost all organisms with two sexes: the microgamete being the male, the megagamete the female conjugating body (p. Ig7).

The result of conjugation is somewhat different in the two cases just studied: in Paramœecium no zygote is formed, conjugation being a mere temporary union (p. 268) : in Vorticella the zygote is an actively moving and feeding body, indistinguishable from an ordinary individual of the species.

Vorticella sometimes encysts itself (Fig. $7^{2}, \mathrm{H}^{1}$ ), and the nucleus of the encysted cell has been observed to break up into a number of separate masses, each doubtless surrounded by a layer of protoplasm. After a time 
the cyst bursts, and a number of small bodies or spores $\left(\mathrm{H}^{2}\right)$ emerge from it, each containing one of the products of division of the nucleus. These acquire a circlet of cilia $\left(\mathrm{H}^{3}\right)$, by means of which they swim freely, and they are sometimes found to multiply by simple fission $\left(\mathrm{H}^{\mathbf{q}}\right)$. Finally, they settle down $\left(\mathrm{H}^{5}\right)$ by the end at which the cilia are situated, the attached end begins to elongate into a stalk $\left(\mathrm{H}^{6}\right)$, this increases in length, the basal circlet of cilia is lost, and a ciliated peristome and disc are formed at the free end $\left(\mathrm{H}^{7}\right)$. In this way the ordinary form is assumed by a process of progressive differentiation or development (p. 9); and, moreover, the freeswimming young $\left(\mathrm{H}^{3}\right)$, to which the spores formed by division of the encysted protoplasm give rise, differ strikingly in form and habits from the adult. This is expressed by saying that development is in this case accompanied by a metamorphosis, this word, literally meaning simply a change, being always used in zoology to express a striking and fundamental difference in form and habit between the young and the adult; as, for instance, between the tadpole and the frog, or between the caterpillar and the butterfly. It is obvious that in the present instance metamorphosis is another means of ensuring dispersal.

In Vorticella, as we have seen, fission results not in the production of equal and similar daughter-cells, but of one stalked and one free-swimming form. It is, however, quite possible to conceive of a Vorticella-like organism in which the parent-cell divides into two equal and similar products, each retaining its connection with the stalk. If this process were repeated again and again, and if, further, the plane of fission were extended downwards so as to include the distal end of the stalk, the result would be a branched, tree-like stem with a Vorticella-like body at the end of every branch. 
As a matter of fact, this process takes place not in Vorticella itself, but in some nearly allied infusors, such as Carchesium and Epistylis. Each of these forms consists of a main stem attached by its proximal end and giving off at its distal end several branches, on each of which numerous bell-animalcules with short stalks are borne, like foxgloves or Canterbury bells on their stem.

We see, then, that Carchesium and Epistylis differ from all our previous types in being compound organisms. The entire "tree" is called a colony or stock, and each separate bell-animalcule borne thereon is an individual or zooid, morphologically equivalent to a single Vorticella or Paramoecium. The colony is therefore an individual of a higher grade than the zooid; and such a multicellular animal as a frog, the cells of which differ markedly in structure and function, is an individual of a higher grade still.

As in Vorticella, the stem of Carchesium consists of a cuticular sheath with an axial muscle-fibre which, at the distal end of the main stem, branches like the stem itself, a prolongation of it being traceable to each zooid; so that the muscular system is common to the whole colony, and any shock causes a general contraction of all the zooids. The stalk of Epistylis, on the other hand, is non-contractile.

\section{PRACTICAL DIRECTIONS}

Paramœeium.-Spread a little cotton-wool on a slide over a drop of water containing Paramœcia (see note on p. $23^{8}$ ), in order to entangle them in its meshes, and put on a cover-glass. Examine first with the low power and then with the high power. Note-

I. The elongated form of the animal ; its anterior (more rounded) and posterior (more pointed) end; its flattened dorsal and ventral surfaces; and its mouth, near the middle of the ventral surface. 
2. The active movements, due to the cilia covering the body.

3. The marked distinction between cortex and medulla.

4. The characters of the elastic cortex:-(a) the superficial cuticle, and deeper striated layer; (b) the cilia arising from the deeper layer, and projecting through the cuticle; (c) the trichocysts-small oval sacs, imbedded in the deeper layer; $(d)$ the two spherical contractile vacuoles, in close relation with the deeper layer on the dorsal side: note that canals radiate from them when they contract; (c) the cilia lining the buccal groove. (The potential anus behind the mouth can only be seen at the moment of defæcation.) Compare the mode of feeding with that of Amociba.

5. The characters of the medulla:-(a) the food-vacuoles and their circulation; $(b)$ the meganucleus and micronucleus, which can be better seen when stained. Sketch.

6. Add methyl-green or magenta. Then note again the structure of cortex and medulla, as well as-(a) the oval meganucleus, near the middle of the body; (b) the micronucleus, a smaller body, close to the meganucleus: (c) the extruded trichocysts. Sketch.

7. Look out for specimens undergoing transverse fission, and also for others in process of conjugation. Sketch.

Permanent preparations may be made as directed in the case of Amœba (p. 239).

Vorticella.-Mount some specimens in a drop of water, and examine with the low power. The Vorticella will be seen to have the form of a wine-glass or bell with a long stem. The stem frequently contracts spirally, the edge of the bell being at the same time turned in, so as to give the animal a rounded form. Put on the high power and note-

x. The form of the bell, its thickened rim or peristome, and the disc, which forms a cover to the bell.

2. The mouth and gullet, opening on one side between the peristome and disc, which is here slightly raised. (The anal spot is in the oral depression, but can only be seen at the moment of defæcation.)

3. The single row of cilia round the peristome and extending down the gullet on the one hand, and on to the raised portion of the disc on the other. Run in a little fincly powdered indigo or carmine under the cover-glass, and note the currents produced by the cilia : the granules of pigment will be carrierl down the gullet.

4. The contractions of the bell and stalk. 
5. The structure of the cortex, which is similar to that of Paramocium, except that the cilia have a restricted distribution, and there are no trichocysts. Moreover, in the stalk (into which the merlulla does not extend) the deeper layer of the cortex gives rise to a central contractile axial fibre, by means of which the stalk can contract spirally. The contractile ractoole is single.

6. The medulla, which circulates and contains foodvacuoles, as in Paramocium. In it are situated an elongated and curved meganucleus and a minute micronucleus, which is not easy to distinguish. Sketch.

7. Make preparations as directed under Paramœcium. Sketch.

8. Look out for specimens undergoing fission, noting the different stages, and the second, proximal ring of cilia on one of the daughter individuals, which eventually becomes detached. Note these free-swimming forms, and also search for conjugating individuals - a small free Vorticella uniting with a large stationary one. Sketch.

Carchesium or Epistylis.-You will very likely find specimens of one of these, or of an allied genus, amongst the Vorticellæ. Note that several individual zooids are borne upon a branched stalk, together forming a colony. 


\section{CHAPTER IV}

OPALINA : MONOCYSTIS-PARASITISM-BIOGENESIS AND ABIOGENESIS-CLASSIFICATION OF THE UNICELLULAR ORGANISM EXAMINED.

Amongst the Protozoa (see p. 220) are found certain forms which are parasites. Parasites are organisms which live in association with other organisms, the ready digested food of which they utilise, or even nourish themselves from the tissues of the forms they infest. It will be interesting to compare Paramocium with a ciliate infusor which lives in the intestine of the common frog, and is known as Opalina ranarum.

Opalina has a flattened body with an oval outline (Fig. 73, A, B), and full-sized specimens may be as much as I $\mathrm{mm}$. in length. The protoplasm is divided into cortex and medulla, and is covered with a cuticle ; the cilia are equal-sized and uniformly arranged in longitudinal rows over the whole surface.

On a first examination no nucleus is apparent, but after staining, a large number of globular nuclei can be seen $(B, n u)$ : these nuclei multiply within the body of the infusor.

The presence of numerous nuclei in Opalina is a fact worthy of special notice. The unicellular organisms we 
have studied are mostly uninucleate as well as unicellular (compare, however, Fig. $67, \mathrm{~B}$, and pp. 265 and 269) : the higher animals consist of numerous cells each with a nucleus: Opalina, on the other hand, is multi-

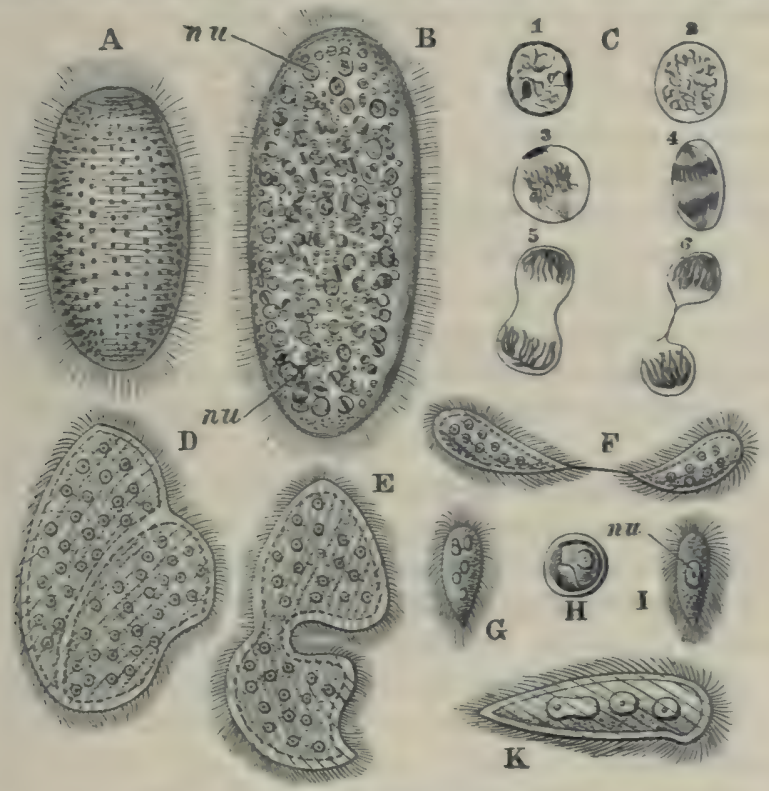

Fis. 73,-Opalina rananum.

A, living specinen, surface view, showing longitudinal rows of cilia. ( $\times 35$.

$\mathrm{B}$, the same, stained, showing numerous nuclei $(n u)$ in various stages of division. $(\times 50$.

C, $1-6$, stages in the division of a nucleus.

$\mathrm{D}$, longituơinal fission.

$\mathbf{E}$, transverse fission.

$F$, the same in a specimen reduced in size by repeated division.

$\mathrm{G}$, final product of successive divisions.

$\mathrm{H}$, encysted form.

1, uninucleate form produced from cyst.

$\mathbf{K}$, the same after multiplication of the nucleus has begun. (From Parker's Brology: A- C. after Ptitzner; D- K, from Savile-Kent, after Zeller.)

nucleate but its protoplasm is undivided, so that it presents a condition of things intermediate between the unicellular and the multicellular types of structure (see 
There is no contractile vacuole, and no trace of either mouth or gullet, so that the ingestion of solid food is impossible. The creature lives, as already stated, in the intestine of the frog: it is therefore, like the worms you have probably noticed in the frog's urinary bladder and lungs (pp. 33 and I53), an internal parasite, or endoparasite, having the frog as its host. The intestine contains the partially-digested food of the frog, and it is by the absorption of this that the Opalina is nourished. Having no mouth it feeds solely by imbibition: whether it performs any kind of digestive process itself is not certainly known, but the analogy of other mouthless parasites leads us to expect that it simply absorbs food ready digested by its host, upon which it is dependent for a constant supply of soluble and diffusible nutriment.

Thus Opalina, in virtue of its parasitic mode of life, is saved the performance of certain work-the work of digestion, that work being done for it by its host. This is the essence of internal parasitism : an organism exchanges a free life, burdened with the necessity of finding food for itself, for existence in the interior of another organism, on which, in one way or another, it levies blackmail.

Note the close analogy between the nutrition of an internal parasite like Opalina and the saprophytic nutrition of a monad (p. 257). In both, the organism absorbs proteids rendered soluble and diffusible, in the one case by the digestive juices of the host, in the other by the action of putrefactive Bacteria.

The reproduction of Opalina presents certain points of interest largely connected with its peculiar mode of life. It is obvious that if the Opalinæ simply went on multiplying, by fission or otherwise, in the frog's in- 
testine, the population would soon outgrow the means of subsistence: moreover, when the frog died there would be an end of the parasites. What is wanted in this, as in other internal parasites, is some mode of multiplication which shall serve as a means of dispersal (compare p. 274), or, in other words, enable the progeny of the parasite to find their way into the bodies of other hosts, and so start new colonies instead of remaining to impoverish the mother-country.

Opalina multiplies by a somewhat peculiar process of binary fission: an animalcule divides in an oblique direction:(Fig. 73, D), and then each half, instead of growing to the size of the parent-cell, divides again transversely $(\mathrm{E})$. The process is repeated again and again (F), the plane of division being alternately oblique and transverse, until finally small bodies are produced $(G)$, about $\frac{1}{20}-\frac{1}{30} \mathrm{~mm}$. in length, and containing from two to six nuclei.

If the parent-cell had divided simultaneously into a number of these little bodies the process would have been one of multiple fission (p. 254) : as it is, it forms an interesting link between simple and multiple fission.

Opalina multiplies in this way especially rapidly in the spring-i.e., during the frog's breeding season. The majority of the small products of division $(G)$ become encysted $(\mathrm{H})$, and in this passive condition are passed out with the frog's excrement, probably falling on to a water-weed or other aquatic object. Nothing further takes place unless the cyst is swallowed by a tadpole, as must frequently happen when these creatures, produced in immense numbers from the frog's eggs, browse upon the water-weeds which form their chief food.

Taken into the tadpole's intestine, the cyst is burst or dissolved, and its contents divide up into lanceolate gametes each containing a single nucleus and covered 
with cilia (I): these conjugate with one another, and the zygote becomes encysted. On emergence from the cyst, as the young Opalina absorbs the digested food in the intestine of its host, it grows, and at the same time its nucleus divides repeatedly $(K)$ in the way already described, until by the time the animalcule has attained the maximum size it has also acquired the large number of nuclei characteristic of the genus.

Here, again, we have an interesting case of differentiation or development (p. 276 ): the organism begins life as a very small uninucleate mass of protoplasm, and as it increases in size, increases also in complexity by the repeated binary fission of its nucleus.

In another group of the Protozoa known as the Sporozoa parasitism occurs without exception, and the relation between parasites and host is much more intimate than in the case of Opalina: instead of living within the enteric canal and merely absorbing the contained products of digestion, these penetrate into the tissues of the host, even passing into the interior of its cells, from the contents of which they absorb fluid nutriment. As is the rule in parasites, they are able to multiply very rapidly.

Sporozoan parasites occur in most classes of animals : many seem to be comparatively innocuous, so that even when infested with large numbers the host apparently suffers no harm. In other cases they, like some other parasitic Protozoa (e.g., the flagellate Trypanosoma in "sleeping sickness") and certain Bacteria, may cause dangerous diseases and epidemics (compare p. 287).

As an example which is easily obtainable we will examine Monocystis (Fig. 74), one of those Sporozoa commonly known as Gregarines, which occurs abundantly in earthworms. When the body-wall of an 
earthworm is cut open, certain white, lobed organs known as sperm-sacs are very apparent a short distance from the anterior end of the animal (compare Chapter VI., p. 347 ). In these, the sperms of the worm undergodevelopment, and amongst and within the spermmother-cells Monocystis lives and passes through the
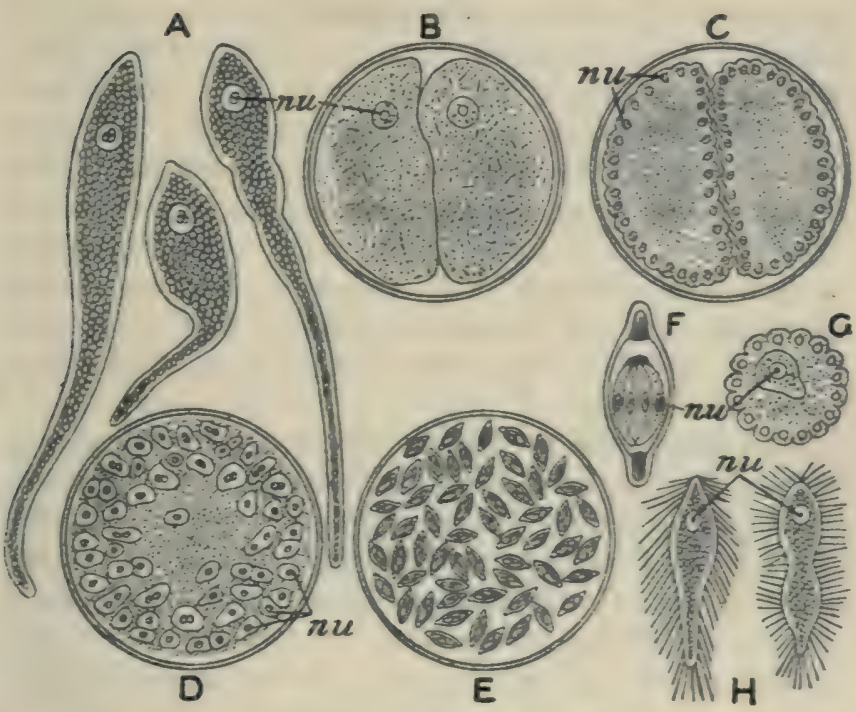

FIG. 74-Monocystis agilis. ( $x$ about 250.)

A, trophozoites in different stages of contraction. B, encysted gametocytes. $\mathrm{C}$, division of gametocytes into gametes. D (semi-diagrammatic!, conjugation of gametes to form zygotes. E, cyst enclosing ripe spores formed from the zygotes. F, single spore, drawn to a larger scale, showing the (\$) sporozoites in its interior. G, group of developing sperm-cells of the earthworm, enclosing a sporozoite in the centre. $H$, young trophozoites still surrounded with the tails of the degenerated sperms. nu, nuclei.

various stages of its life-history. The two commonest species are known as $M$. agilis and M. magna.

The encysted stage is the most easily recognised. and a number of spherical cysts, varying between about $\frac{1}{6}$ and 
$\frac{1}{15} \mathrm{~mm}$. in diameter, can usually be seen if a small piece of a sperm-sac is examined under the microscope. When ripe each cyst contains a large number of small spindleshaped or boat-shaped spores (E). Before encystment, Monocystis has a somewhat elongated form, and is capable of slow movements amongst the cells of the sperm-sacs, contractions of the organism as a whole, or constrictions in various parts, causing its shape to vary considerably at different times (A). There is a clear cortical layer covered by a cuticle (p. 264), and a very granular medullary portion, enclosing the spherical nucleus $(n u)$ in which one or more nucleolus-like bodies can be distinguished. No pseudopods or cilia are formed, and, as in Opalina, there is no mouth, gullet, or contractile vacuole, fluid nutriment being absorbed from the cells of the host. During this stage the organism feeds and grows, and is spoken of as a trophozoite.

After a time two trophozoites become applied together, without fusing, and a common resistant cyst is formed around them: each is now known as a gametocyte (B). The nucleus of each gametocyte then undergoes successive divisions $(C)$, the ultimate products of which, each surrounded by protoplasm, constitute small gametes, part of the protoplasm of the gametocytes remaining undivided and serving as nutrient material for the developing spores. These gametes then conjugate in pairs (D), probably those derived from one gametocyte uniting with those derived from the other. The result is the formation of numerous zygotes, each of which becomes spindle-shaped and surrounded by a tough cell-wall, so that the cyst $(\mathrm{E})$ now contains a number of the characteristic spores already referred to, each of which is about $\frac{1}{40}-\frac{1}{80} \mathrm{~mm}$. in length.

The contents of each spore then undergo division (F) 
forming eight elongated and nucleated bodies or sporozoites, which become set free by the bursting of the enclosing cyst-membrane: apparently, however, they must enter a fresh host before this takes place. It is probable that earthworms become infected by swallowing spores which have been set free into the earth, either directly, owing to injury to the host, or by means of the excrement of worm-eating birds. The spore-membrane is probably acted upon by the digestive juices of the worm, the liberated sickle shaped and motile sporozoites passing through the wall of the intestine and so reaching the sperm sacs, when they enter some of its cells $(G)$, absorbing so much of the contents of the latter that there is not sufficient left to form complete sperms. They then become set free among the cells of the sperm-sacs, surrounded by the tails of the degenerated sperms which look like an investment of stiff cilia $(\mathrm{H})$; they continue to grow until the fully developed trophozoite-stage is again reached and the life-cycle thus completed.

It will be seen that in Monocystis there is no special method for the parasite to be regularly transferred from one host to another. In some Sporozoa, as in many parasites belonging to other and higher groups, infection is provided for, and the life-history is still further complicated owing to the fact that two hosts, belonging to different groups, are necessary for the completion of the cycle. A good example is the malaria-organism (Laverania), which lives within the red blood-corpuscles of man. This sporozoan multiplies rapidly by multiple fission, thus producing very numerous merozoites, which attack other blood-corpuscles of the same host. Should a drop of blood, containing merozoites, be sucked up by a mosquito (Anopheles), the parasites undergo a different mode of development. From two different kinds of gametocytes, ovum-like megagametes and sperm-like microgametes are respectively formed (p. 275 ). After conjugation, the active zygotes penetrate the epithelium of the mosquito's stomach, become encysted, and give rise to an enormous number of sporozoites, which reach the salivary ducts of the mosquito. If the insect then bites another human being, the sporozoites are injected 
into the wound and again attack the red corpuscles, and thus spread the infection. Thus the mosquito is not a mere germ-carrier, like the common house-fly.

The study of the foregoing living things and especially of Bacteria, the smallest and probably the simplest of all known organisms (p. 257), naturally leads us to the consideration of one of the most important problems of Biology - the problem of the origin of life.

In all the higher organisms we know that each individual arises in some way or other from a preexisting individual: no one doubts that every bird now living arose by a process of development from an egg formed in the body of a parent-bird, and that every tree now growing took its origin either from a seed or from a bud produced by a parent-plant. But there have always - until quite recently, at any rate-been upholders of the view that the lower forms of life, Bacteria, Monads, and the like, may under certain circumstances originate independently of pre-existing organisms : that, for instance, in a flask of some organic infusion boiled so as to kill any living things present in it, fresh forms of life may arise de novo-may in fact be created then and there.

We have therefore two theories of the origin of the lower organisms, the theory of biogenesis, according to which each living thing, however simple, arises by a natural process of budding, fission, spore-formation, or what not, from a parent organism : and the theory of abiogenesis, or as it is sometimes called spontaneous or equivocal generation, according to which fully-formed living organisms sometimes arise from not-living matter.

In former times the occurrence of abiogenesis was universally believed in. The expression that a piece of meat has "bred maggots"; the opinion that parasites such as the gall-insects of plants or the tape-worms in 
the intestines of animals originate where they are found ; the belief still held in some rural districts in the occurrence of showers of frogs, or in the transformation of horse-hairs kept in water into eels ; all indicate a survival of this belief.

As accurate enquiries into these matters were made, the number of cases in which equivocal generation was supposed to occur was rapidly diminished. It was not surprising, however, considering the rapidity with which Bacteria and Monads were found to make their appearance in organic substances and infusions, that many men of science imagined them to be produced abiogenetically. The rapid multiplication of these forms means, of course, that a certain amount of fresh living protoplasm has been formed out of the constituents of the hay-. infusion, through the agency in the first instance of a single living Bacterium. The question naturally arises, why may not the formation of protoplasm take place independently of this insignificant speck of living matter?

It must not be thought that this question is in any way a vain or absurd one. That living protoplasm has at some period of the world's history originated from not-living matter seems a necessary corollary of the doctrine of evolution, and is obviously the very essence of the doctrine of special creation (p. 22I) ; and there is no a priori reason why it should be impossible to imitate the unknown conditions under which the process took place. But at present we are quite unable to solve this fundamental problem.

Experiments conducted with proper precautions, however, all tell the same tale : they prove conclusively that in putrescible infusions that have been properly sterilised -i.e., thoroughly boiled so as to kill any organisms they may contain-and adequately protected from the 
entrance of atmospheric germs, no micro-organisms ever make their appearance. So that the last argument for abiogenesis has been proved to be fallacious, and the doctrine of biogenesis shown, as conclusively as observation and experiment can show it, to be of universal application as far as existing conditions known to us are concerned. It is also necessary to add that the presence of microbes in considerable quantities in our atmosphere has been proved experimentally.

There is another question intimately connected with that of biogenesis, although strictly speaking quite independent of it. It is a matter of common observation that, both in animals and plants, like produces like: that a cutting from a willow will never give rise to an oak, nor a snake emerge from a hen's egg. In other words, ordinary observation teaches the general truth of the doctrine of homogenesis.

But there has always been a residuum of belief in the opposite doctrine of heterogenesis, according to which the offspring of a given animal or plant may be something utterly different from itself, a plant giving rise to an animal or vice versa, a lowly to a highly organised plant or animal and so on. Perhaps the most extreme case in which heterogenesis was once seriously believed to occur is that of the " barnacle-geese." Buds of a particular tree growing near the sea were said to produce barnacles (p. 386), and these falling into the water to develop into geese. This sounds absurd enough, but within the last forty years two or three men of science have described, as the result of repeated observations, the occurrence of quite similar cases among microscopic organisms. For instance, the blood-corpuscles of the silkworm have been said to give rise to fungi, Euglenæ to thread-worms, and so on.

It is proverbially difficult to prove a negative, and it 
might not be easy to demonstrate, what all competent naturalists must be firmly convinced of, that every one of these supposed cases of heterogenesis is founded either upon errors of observation or upon faulty inductions from correct observations.

It is obvious that the only way in which a case of heterogenesis could pe proved would be by actually watching the transformation, and this no heterogenist has ever done; at the most, certain supposed intermediate stages between the extreme forms have been observed-say, between a Euglena and a thread-worm -and the rest of the process inferred. On the other hand, innumerable observations have been made on these and other organisms, the result being that each species investigated has been found to go through a definite series of changes in the course of its development, the ultimate result being invariably an organism resembling in all essential respects that which formed the starting point of the observations: Euglenæ always giving rise to Euglenæ and nothing else, Bacteria to Bacteria and nothing else, and so on.

There are many cases which imperfect knowledge might class under heterogenesis, such as the origin of frogs from tadpoles or of jelly-fishes from polypes (see Chapter V), but in these and many other cases the apparently anomalous transformations have been found to be part of the normal and invariable cycle of changes undergone by the organism in the course of its development: the frog always gives rise ultimately to a frog, the jelly-fish to a jelly-fish. If a frog at one time produced a tadpole, at another a trout, at another a worm: if jelly-fishes (p. 3I 4 ) gave rise sometimes to polypes, sometimes to infusorians, sometimes to cuttlefishes (p. $4 \mathrm{II}$ ), and all without any regular sequencethat would be heterogenesis. 
It is perhaps hardly necessary to caution the reader against the error that there is any connection between the theory of heterogenesis and that of organic evolution. It might be said - if, as naturalists tell us, dogs are descended from wolves and jackals, and birds from reptiles, why should not, for instance, thread-worms spring from Euglenæ or Infusoria from Bacteria? To this it is sufficient to answer that the evolution of one form from another takes place by a series of slow, orderly, progressive changes going on through a long series of generations (p. 222); whereas heterogenesis presupposes the casual occurrence of sudden transformations in any direction-i.e., leading to either a less or a more highly organised form-and in the course of a single generation. ${ }^{1}$

Each of the organisms which we have studied in this and the two previous chapters consists of a single cellor in the case of Carchesium and Epistylis of a colony of cells to a large extent independent of one another. They are therefore placed in the lowest primary divisior of the animal kingdom-the phylum Protozoa (p. 220). This phylum is subdivided into a number of classes, examples of certain of which we have examined. Those in which, like the Amœba, the amoboid form is predominant constitute the class Rhizopoda: those in which, like the Monads and Euglenæ (Flagellata), the flagellate form is predominant are often included with the ciliated forms (Ciliata)-such as Paramœecium, Vorticella, and Opalina-in a single class, the Infusoria; and those in which, like Monocystis, the encysted form is predominant, are known as the Sporozoa.

1 Apart from such fluctuating or continuous variations, others (mutations), which may be described as discontinuous, do sometimes appear with apparent suddenness, but not to the extent which would be required by the theory of heterogenesis. 
The animals above the Protozoa are placed, as we have seen, in a number of different phyla, but as they are all multicellular they are often spoken of collectively as the Metazoa, one of the simplest of which we must next examine.

\section{PRACTICAL DIRECTIONS}

Opalina.-Make an incision in the rectum or small intestine of a freshly-killed frog, and mount a little of its contents in water on a slide. Having found an Opalina, note its oval and flattened form, the uniform covering of cilia, the cuticle, cortex, and medulla, and the absence of a. mouth and contractile vacuole. Stain with methyl-green or magenta, and make out the numerous nuclei. Look out for the produnts of division, free and encysted.

Monocystis.- Kill an earthworm by placing it for a few minutes in methylated spirit. Then, with the scissors, slit through the body-wall along the mid-dorsal line through about the anterior third of the animal, when some white, lobed bodies, the sperm-sacs (see p. 347) will be apparent. Snip off a very small portion of one of these, place it on a slide in salt solution and examine with the low power. Amongst the cells of the sperm-sac you will probably be able to distinguish :

(a) Numerous cysts of Monocystis, many of which will be seen to contain a number of spindle-shaped spores (Fig. 74, E), and in some others, two gametocytes (B); (b) trophozoites (A) in various stages of growth and contraction.

Put on a high power, and after observing the groups of cells of the sperm-sacs and sperms in various stages of development, examine in detail :

I. The trophoroites (A), noting the cuticle, cortex, medulla, and nucleus. (Observe their movements.)

2. The encysted gametocytes, before and after division into gametes $(\mathbf{B}, \mathbf{C})$.

3. Further stages in the formation of gametes and zygotes, and the fully-developed spores (D, E).

4. The structure of an individual spore, which contains eight sporozoites (F).

5. Sporozoites which have entered a clump of sperm-cells (G); and later stages after they have become free, surrounded by the tails of the sperms $(H)$.

Stain a preparation with methyl-green or magenta, and go over I-5 again. 


\section{CHAPTER V}

HYDRA : OBELIA-SYMBIOSIS-ALTERNATION OF GENERATIONS-CHARACTERS OF THE PHYLUM CCELNTERATA

A CAREFUL search in ponds will often result in the capture of some small organisms known as "freshwater polypes," belonging to the genus Hydra.

Although far from uncommon in pond-water, Hydra is not always easy to find, being rarely abundant and by no means conspicuous. In looking for it the best plan is to fill either a clear glass bottle or beaker, or a saucer, with weeds and water from a pond, and to let it remain undisturbed for a short time. If the gathering is successful there will be seen adhering to the sides of the glass, the bottom of the saucer, or the weeds, little white, tawny, or green bodies, about as thick as fine sewing-cotton, and 2-10 $\mathrm{mm}$. in length. They adhere pretty firmly by one end, and examination with a pocket lens shows that from the free extremity a number of very delicate filaments, barely visible to the naked eye, are given off.

Under the low power of a compound microscope a Hydra (Fig. 75) is seen to have a cylindrical body attached by a flattened base to a weed or other aquatic object, and bearing at its opposite or distal end a conical structure, the hypostome $(\operatorname{lo} y p)$, at the 


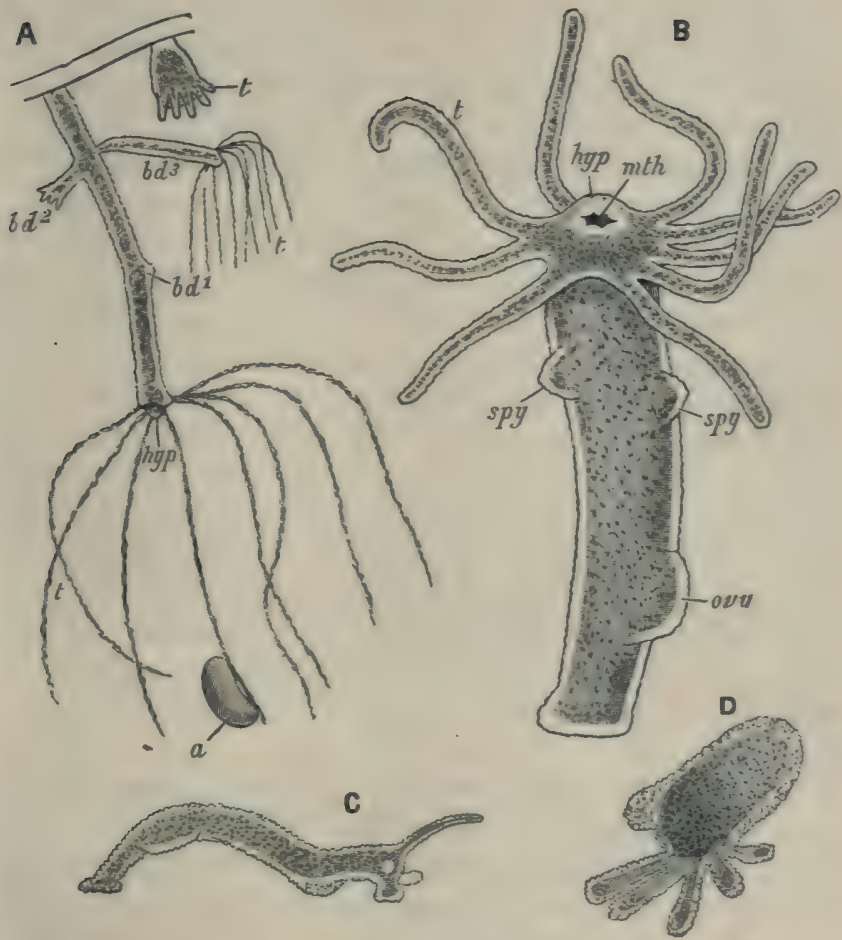

Fic. 75.-Hydra.

A, two living specimens of $H$. viridis attached to a bit of weed. The larger specimen is fully expanded, and shows the elongated body ending distally in the hypostome (hyp), surrounded by tentacles $(t)$, and three buds $\left(b d^{l} b d^{2}\right.$, bdth in different stages of development; a small water-flea $(a)$ has been captured by one tentacle. The smaller specimen (to the right and above) is in a state of complete retraction, the tentacles $(t)$ appearing like papillæ. (. 6.)

B, $H$. fusca, showing the mouth (mth) at the end of the hypostome (hyp), the circlet of tentacles $(t)$, two spermaries (spy), and an ovary (oty). ( $\therefore 12$.

$C$, a Hydra creeping on a flat surface by looping movements.

D, a specimen crawling on its tentacles. (From Parker's Biology : C and D after W. Marshall.)

apex of which is a circular aperture, the mouth ( $m$ th). At the junction of the hypostome with the body proper are given off from six to eight long delicate tentacles 
( $t$ ) arranged in a circlet or whorl. A longitudinal section shows that the body is hollow, containing a spacious cavity, the enteron (Fig. 76, A, ent. cav), which communicates with the surrounding water by the mouth. The tentacles are also hollow, their cavities communicating with the enteron.

Thus it will be seen that the Hydra is not bilaterally symmetrical, like the frog-i.e., equally divisible into two lateral halves by a median vertical plane passing through the axis of the body-but is radially symmetrical, i.e., the body is divisible into similar parts radiating from a common central axis.

There are three kinds of Hydra commonly found : one, $H$. vulgaris, is colourless or nearly so ; another, $H$. fusca, is of a pinkish-yellow or brown colour; the third, $H$. viridis, is bright green. In the two latter it is quite evident, even under a low power, that the colour is in the inner parts of the body-wall, the outside of which is formed by a transparent, colourless layer (Fig. 75).

An examination of the living animal shows, in the first place, that its form is continually changing. At one time (Fig. 75, A, left-hand figure) it extends itself until its length is fully fifteen times its diameter and the tentacles appear like long delicate filaments: at another time (right-hand figure) it contracts itself into an almost globular mass, the tentacles then appearing like little blunt knobs.

Besides these movements of contraction and expansion, Hydra is able to move slowly from place to place. This it usually does after the manner of a looping caterpillar $(\mathrm{C})$ : the body is bent round until the distal end touches the surface: then the base is detached and moved nearer the distal end, which is again moved forward, and so on. It has also been observed to crawl 
like a cuttle-fish (D) by means of its tentacles, the body being kept nearly vertical.

It is also possible to watch a Hydra feed. It is a very voracious creature, and to see it catch and devour its prey is a curious and interesting sight. In the water in which it lives are always to be found numbers of "water-fleas," minute animals of about a millimetre or less in length, belonging to the class Crustacea (see p. 386).

Water-fleas swim very rapidly, and occasionally one may be seen to come in contact with a Hydra's tentacle. Instantly its hitherto active movements stop dead, and it remains adhering in an apparently mysterious manner to the tentacle. If the Hydra is not hungry it usually liberates its prey after a time, and the water-flea may then be seen to drop through the water like a stone for a short distance, but finally to expand its limbs and swim off. If, however, the Hydra has not eaten recently, it gradually contracts the tentacles until the prey is brought near the mouth, the other tentacles being also used to aid in the process. The water-flea is thus forced against the apex of the hypostome, the mouth expands widely and seizes it, and it is finally passed down into the digestive cavity. Hydræ can often be seen with their bodies bulged out in one or more places by recently swallowed water-fleas.

The precise structure of Hydra is best made out by cutting it into a series of extremely thin sections and examining them under a high power of the microscope. The appearance presented by a vertical section through the long axis of the body is shown in Fig. $76, \mathrm{~A}$.

The whole animal is seen to be built up of cells, each consisting of protoplasm with a large nucleus (B-D. $m u$ ), and with or without vacuoles. As in the case of most animal cells, there is no cell-wall. 
The essential feature in the arrangement of the cells is that they are disposed in two layers round the central digestive cavity or enteron (A, ent. cav) and the cavities of the tentacles (ent. cav'). So that the wall of the body is formed throughout of an outer layer of cells, the ectoderm (ect), and of an inner layer, the endoderm (end), which bounds the enteric cavity (compare p. 202). Between the two layers is a delicate transparent membrane, the mesogloea, or supporting lamella ( $m s g l$ ). A transverse section (B) shows that the cells in both layers are arranged radially.

Thus Hydra is a two-layer or diploblastic animal, and may be compared to a chimney built of two layers of radially arranged bricks with a space between the layers filled with mortar or concrete.

Accurate examination of thin sections, and of specimens teased out or torn into minute fragments with needles, shows that the structure is really much more complicated than the foregoing brief description would indicate.

The ectoderm-cells are of two kinds. The first and most obvious (B, ect, and $\mathrm{C}$ ) are large cells of a conical form, the bases of the cones being external, their apices internal. Spaces are necessarily left between their inner or narrow ends, and these are filled up with the second kind of cells (int. c), small rounded bodies which lie closely packed between their larger companions and are distinguished as interstitial cells.

The inner ends of the large ectoderm-cells are continued into narrow, pointed prolongations (C, $m . p r)$ placed at right angles to the cells themselves and parallel to the long axis of the body. There is thus a layer of these longitudinally-arranged muscle-processes lying immediately external to the mesogloea (B, $m . p r)$. They appear to possess, like the axial fibre of Vorticella 


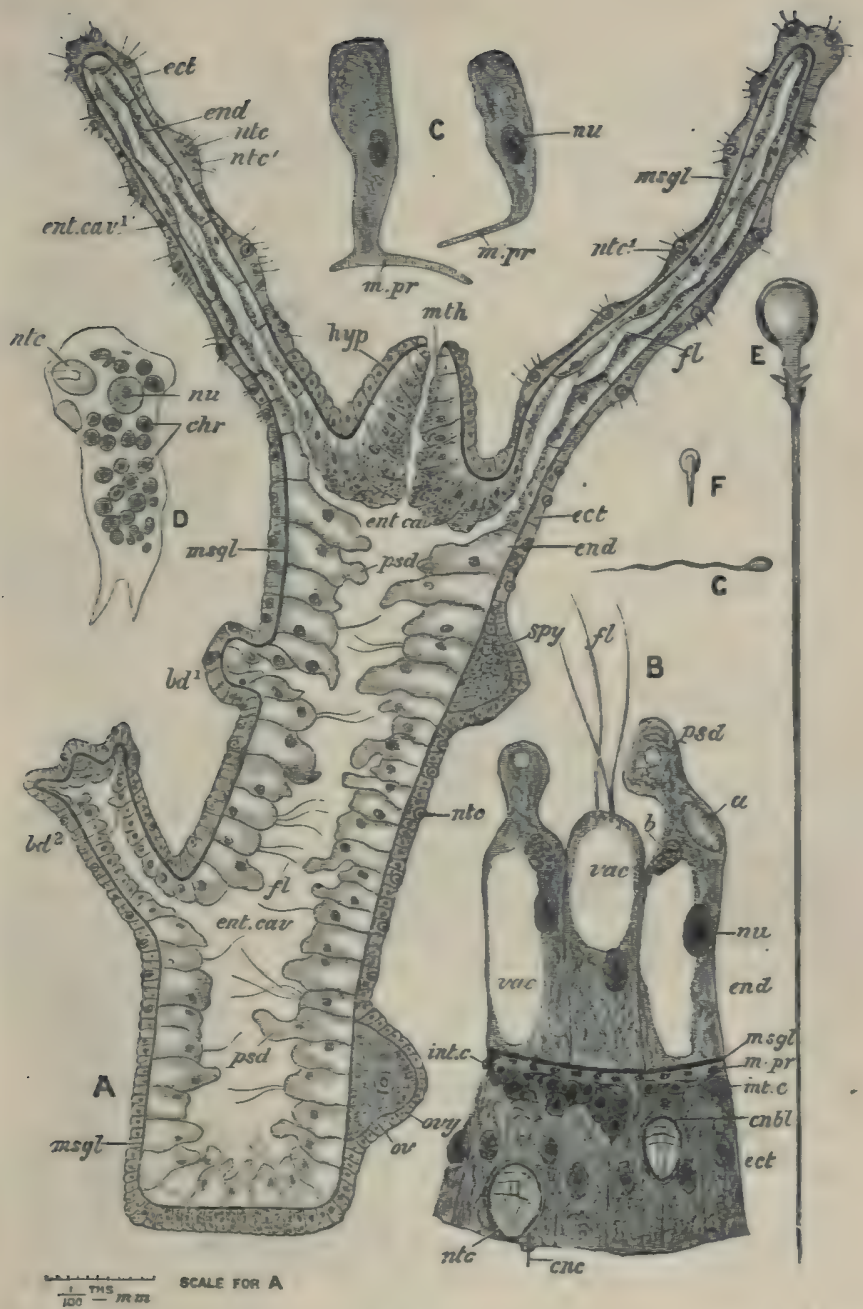

Fig. $76,-H y d r a$.

A, vertical section of the entire animal, showing the body-wall composed of ectoderm (ect) and entoderm (end). prolosing an enteric cavity (cnt. cat), which, as well as the two layers, is continued (ens. cav) into the tentacles, and opens 
externally by the mouth $(m t h)$ at the apex of the hypostome (hyp). Between the ectoderm and endoderm is the mesngloa ( $\mathrm{ns} g \mathrm{l}$ ), represented by a black line. In the ectoderm are seen large (ntc) and small (ntc) nematocysts; some of the endoderm-cells are putting out pseudopods ( $p s d)$, others flagella $(f t)$. Two buds $\left(b d .1, b d .2^{2}\right)$ in different stages of development are shown on the left side, and on the right a spermary $(s p y)$ and an ovary (ovy) containing a single ovum (ov). $(x$ 20.)

$B$, portion of a transverse section more highly magnified, showing the large ectoderm-cells (ect) and interstitial cells (int. c); two cnidoblasts (cnbl) enclosing nematocysts (ntc) and one of them produced into a cnidocil (cruc); the layer of muscle-processes ( $m . p r$ ) cut across just external to the mesogloea (msgl); endoderm-cells (end) with large vacuoles and nuclei (nu), pseudopods ( $p s d$ ), and flagella $(f t)$. The endoderm-cell to the right has ingested a diatom $(a)$, and all enclose minute black granules. ( $x$ roo.)

$\mathrm{C}$, two of the large ectoderm-cells, showing nucleus (nu) and muscle-process $(m . p r)$.

$\mathrm{D}$, an endoderm-cell of $H$. viridis, showing nucleus $(n u)$, numerous Zoochlorellæ $(c h r)$, and an ingested nematocyst (ntc).

$\mathrm{E}$, one of the larger nematocysts with extruded thread, barbed at the base.

$\mathrm{F}$, one of the smaller nematocysts.

G, a single sperm. (From Parker's Biology: D after Lankester; F and G after Howes.)

(p. 273), a high degree of contractility, the almost instantaneous shortening of the body being due, in great measure at least, to their rapid and simultaneous contraction. It is probably correct to say that, while the ectoderm-cells are both contractile and irritable, a special degree of contractility is assigned to the muscleprocesses, the cells themselves being eminently irritable, the slightest stimulus applied to them usually being followed by immediate contraction of the whole body.

Imbedded in and between some of the large ectodermcells are found clear, oval sacs $(n t c)$, with very well defined walls, called "thread-cells" or nematocysts. Both in the living specimen and in sections they ordinarily present the appearance shown in Figs. $76, \mathrm{~B}$, and 77 , $\mathrm{A}$, but are frequently met with in the condition shown in Figs. $76, \mathrm{E}$, and $77, \mathrm{~B}$, that is, with a short, conical tube protruding from the mouth of the sac, armed near its distal end with three recurved barbs besides several similar processes of smaller size, and giving rise distally to a long, delicate, flexible filament.

Accurate examination of the nematocysts shows that the structure of these curious bodies is as follows. Each consists of a tough sac (Fig. 77, A), one end of 


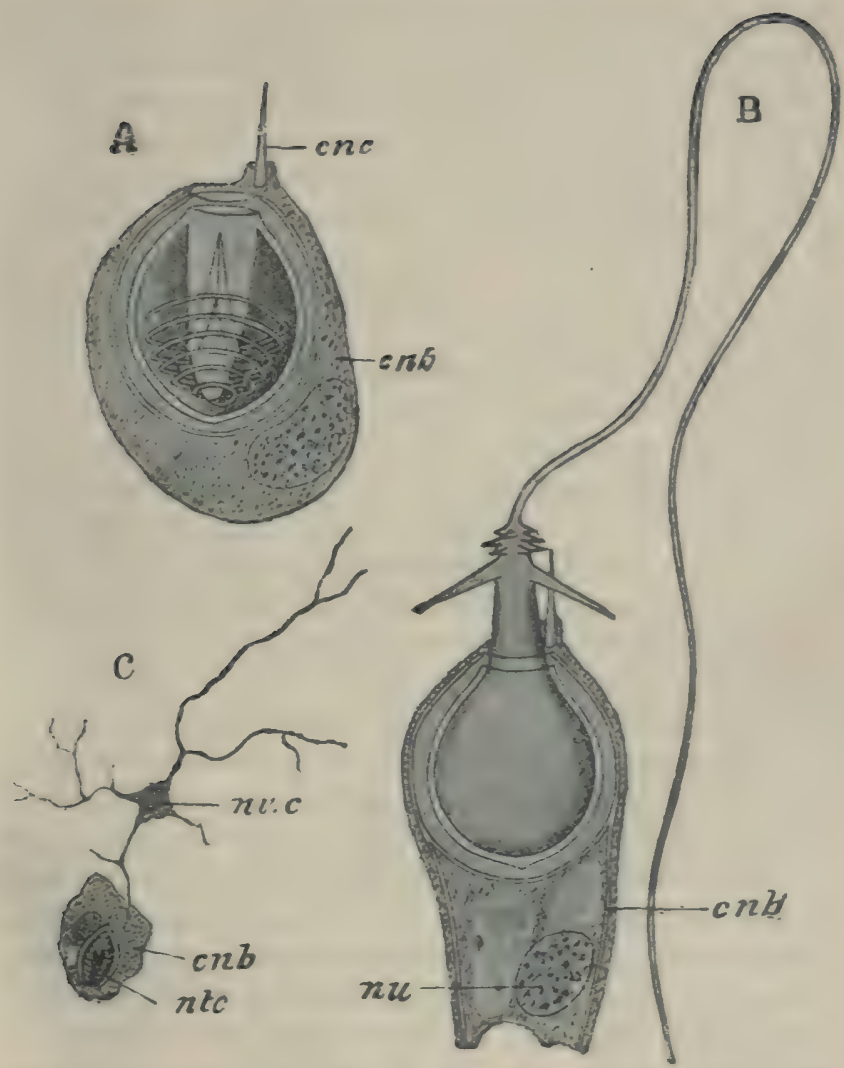

Frg. 77.-Hydra.

A, a nematoryst containerl in its cnidoblast (cnb), showing its coiled filament and the enifincil (cm). ( $(* 00)$

$B$, the same aiter extrusion of the tbread, showing the larger and smaller barbs at the base of the thredi : $n$ s. the nucleus of th.. enichblast.

$\mathrm{C}$, a cridoblast, with its contained nematocist, connected with one of the processes of a nerve-cell (wv. c). (From Parker's Biology: after Schneicler.)

which is turned in as a hollow pouch: the free end of the latter is continued into a hollow, coiled filament, and from its inner surface project the barbs. The 
whole space between the wall of the sac and the contained pouch and thread is tensely filled with fluid. When pressure is brought to bear on the outside of the sac the whole apparatus goes off like a harpoon-gun (B), the compression of the fluid forcing out first the barbed pouch and then the filament, until finally hoth are turned inside out.

It is by means of the nematocysts-the resemblance of which to the trichocysts of Paramcecium (p. 267) should be noted - that the Hydra is enabled to paralyse its prey. Probably some specific poison is formed and ejected into the wound with the thread: in the larger members of the group to which Hydra belongs, such as jelly-fishes, the nematocysts produce an effect on the human skin quite like the sting of a nettle.

The nematocysts are formed in special interstitial cells called cnidoblasts (Figs. 76, B, and 77, $c n b$ ), and are thus in the first instance at a distance from the surface. But the cnidoblasts migrate outwards, and so come to lie quite superficially either in or between the large ectoderm-cells. On its free surface the cnidoblast is produced into a delicate pointed process, the cnidocil or "trigger-hair" (cnc). In all probability the slightest touch of the cnidocil causes contraction of the cnidoblast, and the nematocyst, thus' compressed, instantly explodes.

Nematocysts are found in the distal part of the body, but are absent from the foot or proximal end, where also there are no interstitial cells. They are especially abundant in the tentacles, on the knob-like elevations of which-due to little heaps of interstitial cells-they are found in great numbers. Amongst these occur small nematocysts with short threads and devoid of barbs (Fig. 76, A, ntc', and F).

In connection with the cnidoblasts small irregular cells with large nuclei occur (Fig. $77, \mathrm{C}, n v . c$ ) ; they are 
supposed to be nerve-cells, and to constitute a rudimentary nervous system (compare p. I67).

The ectoderm-cells of the foot differ from those of the rest of the body in being very granular (Fig. $76, \mathrm{~A}$ ). The granules are probably the material of the adhesive secretion by which the Hydra fixes itself, and these cells are therefore glandular (p. 130).

The endoderm consists for the most part of large cells which exceed in size those of the ectoderm, and are remarkable for containing one or more vacuoles, sometimes so large as to reduce the protoplasm to a thin superficial layer containing the nucleus (Fig. 76, A and $\mathrm{B}$, end). Then, again, their form is extremely variable, their free or inner ends undergoing continual changes of form. This can be easily made out by cutting transverse sections of a living Hydra, when the endodermcells are seen to send out long blunt pseudopods ( $p s d)$ into the digestive cavity, and now and then to withdraw the pseudopods and send out from one to three long, delicate flagella $(f)$. Thus the enduderm-cells of Hydra illustrate in a very instructive manner the essential similarity of flagella and pseudopods already referred to (p. 250). In the hypostome the endoderm is thrown into longitudinal folds, so as to allow of the dilatation of the mouth in swallowing.

Amongst the ordinary endoderm-cells are found lorg narrow cells of an extremely granular character. They are specially abundant in the distal part of the body, beneath the origins of the tentacles and in the hypostome, but are absent in the tentacles and in the foot. There is no doubt that they are gland-cells, their secretion being a fluid used to aid in the digestion of the food.

In Hydra viridis the endoderm-cells (Fig. 76, D) 
contain chromatophore-like bodies (chr) coloured green by chlorophyll (p. 242), the function of which we have already considered (p. 247). It has been proved, however, that these are not actual parts of the endodermcells, but are distinct Sphærella-like organisms known as Zoochlorella, which are passed on from one generation of the Hydra to another by entering its developing eggs. Such an intimate living-together of two organisms is known as symbiosis. It differs essentially from parasitism (see p. 280), in which one organism preys upon another, the host deriving no benefit but only harm from the presence of the parasite. In symbiosis, on the contrary, the two organisms are in a condition of mutually beneficial partnership. The carbon dioxide and nitrogenous waste given off by the cells of the Hydra serve as a constant food-supply to the Zoochlorella: at the same time the latter by decomposing the carbon dioxide provides the Hydra with a constant supply of oxygen, and also with two important foodstuffs-starch and proteids, which, after solution, diffuse from the protoplasm of the Zoochlorella into that of the endodermcells. The latter may therefore be said to keep the Zoochlorellæ constantly manured, while the Zoochlorellæ in return supply them with oxygen and ready-digested food. In the endoderm of $H$. fusca bodies of an orange or brown colour are present which are devoid of chlorophyll.

Muscle-processes also exist in connection with the endo. derm-cells, and they are said to take a transverse or circular direction, $i$ e., at right angles to the similar processes of the ectoderm cells.

When a water-flea or other minute organism is swallowed by a Hydra, it undergoes a gradual process of disintegration. The process is begun by a solution of the soft parts due to the action of a digestive 
fluid secreted by the gland-cells of the endoderm; it is apparently completed by the endoderm-cells seizing minute particles with their pseudopods and engulfing them quite after the manner of Amœbæ. It is often found that the protrusion of pseudopods during digestion results in the almost complete obliteration of the enteric cavity.

It would seem, therefore, that in Hydra the process of digestion or solution of the food is to some extent intracellular, i.e., takes place in the interior of the cells themselves, as e.g., in Amœba or Paramœcium: it is, however, largely extra cellular or enteric, i.c., is performed in a special digestive cavity lined by cells (pp. 67 and $13 \mathrm{I}$ ).

The ectoderm-cells do not take in food directly, but are nourished entirely by diffusion from the endoderm. Thus the two layers have different functions: the ectoderm is protective and sensory-it forms the external covering of the animal, and receives impressions from without ; the endoderm, removed from direct communication with the outer world, performs a nutrient function, its cells alone having the power of digesting food.

The essential difference between digestion and assimilation (p. 234) is here plainly seen : all the cells of Hydra assimilate, all are constantly undergoing waste, and all must therefore form new protoplasm to make good the loss. But it is the endoderm-cells alone which can make use of raw or undigested food: the ectoderm has to depend upon various products of digestion received by diffusion or osmosis from the endoderm.

It will be evident from the preceding description that Hydra is comparable to a colony of Arnœbæ in which particular functions are made over to particular individuals-just as in a civilised community the 
functions of baking and butchering are assigned to certain members of the community, and not performed by all. Hydra is therefore an example of individuation: morphologically it is equivalent to an indefinite number of unicellular organisms: but, these acting in concert, some taking one duty and some another, form, physiologically speaking, not a colony of largely independent units (compare p. 277), but a single multicellular individual.

Hydra has two distinct methods of reproduction, asexual and sexual.

Asexual multiplication takes place by a process of budding. A little knob appears on the body (Fig. 75, $\left.A ; b d^{1}\right)$, and is found by sections to arise from a group of ectoderm-cells; soon, however, it takes on the character of a hollow outpushing of the wall containing a prolongation of the enteron and made up of ectoderm, mesoglœea, and endoderm (Fig. $76, \mathbf{A}, b d^{1}$ ). In the course of a few hours this prominence enlarges greatly, and near its distal end six or eight hollow buds appear arranged in a whorl (Figs. 75, A, and 76, A bd ${ }^{2}$ ). These enlarge and take on the characters of tentacles, and a mouth is formed at the distal end of the bud, which thus acquires the character of a small Hydra (Fig. 75, A, $b d^{3}$ ). Finally the bud becomes constricted at its base, separates from the parent, and begins an independent existence. Sometimes, however, several buds are produced at one time, and each of these buds again before becoming detached : in this way temporary colonies are formed. But the buds always separate sooner or later, although they frequently begin to feed while still attached.

It is a curious circumstance that Hydra can also be multiplied by artificial division: the experiment has 
been tried of cutting the living animal into pieces, each of which was found to undergo regeneration into a perfect individual (compare p. 268).

The sexual organs or gonads (p. 193) are of two kinds, spermaries and ovaries. Both are found in the same individual, Hydra being hermaphrodite or monacious.

The spermaries (Figs. 75, B, and 76, A, spy) are white conical elevations situated near the distal end of the body: as a rule not more than one or two are present at the same time, but there may be as many as twenty. They are perfectly colourless, even in the green and brown species, being obviously formed of ectoderm alone.

In the immature condition the spermary consists of a little heap of interstitial cells covered by an investment of somewhat flattened cells formed by a modification of the ordinary large cells of the ectoderm. When mature each of the small internal cells becomes converted into a sperm (p. I94), consisting of a small ovoid head formed from the nucleus of the cell, and of a long vibratile tail formed from its protoplasm (Fig. 76, G). By the rupture of the investing cells or wall of the spermary the sperms are liberated and swim freely in the water.

The ovaries (Figs. 75, B, and $76, \mathrm{~A}$, ovy) are found nearer the proximal end of the body, and vary in number from one to eight. When ripe an ovary is larger than a spermary, and of a hemispherical form. It begins, like the spermary, as an aggregation of interstitial cells, so that in their earlier stages the sex of the gonads is indeterminate. But while in the spermary each cell is converted into a sperm, in the ovary one cell (Fig. 76, A, ov) soon begins to grow faster than the rest, and becomes amœboid in form, sending out pseudopods amongst its companions and ingesting the 
fragments into which they become broken up, thus continually increasing in size at their expense. Ultimately the ovary comes to consist of this single amoboid ovum and of a layer of superficial cells forming a capsule for it. As the ovum grows, yolk granules (p. I95) are formed in it, and in Hydra viridis it also acquires Zoochlorellæ (p. 304).

When the ovary is ripe the ovum draws in its pseudopods and takes on a spherical form : the investing layer then bursts so as to lay bare the ovum and allow of the free access to it of the sperms. One of the latter conjugates with the ovum, producing an oosperm (p. I98) or unicellular embryo.

The oosperm undergoes segmentation, dividing into a number of cells which constitute a morula or polyplast (p. 200), the outermost cells of which become changed into a hard shell or capsule, which eventually bursts and sets free the embryo. The embryo develops into a Hydra, its cells becoming differentiated into ectoderm and endoderm, the enteron and mouth being formed, and the tentacles budding out around the latter.

It was stated on p. 306 that in a budding Hydra the buds do not always become detached at once, but may themselves bud while still in connection with the parent. temporary colonies being thus produced.

Suppose the state of things to continue indefinitely: the result would be a tree-like colony or compound organism consisting of a stem with numerous branchlets each ending in a Hydra-like zooid. Such a colony would bear much the same relation to Hydra as Carchesium or Epistylis bears to Vorticella.

As a matter of fact this is precisely what happens in a great number of animals allied to Hydra and known by the name of Zoophytes or Hydroid polypes 
Everyone is familiar with the common Hydroids known as Sertularians of the sea-coast, often mistaken for sea-weeds: they are delicate, much-branched, semitransparent structures of a horny consistency, the branches beset with little cups, from each of which, during life, a Hydra-like body is protruded.

A very convenient genus of Hydroids for our purpose is Obelia, which occurs in the form of a delicate, whitish or light-brown, almost fur-like growth on seaweed, the wooden piles of piers, etc. It consists of branched filaments about the thickness of fine sewingcotton : of these, some are closely adherent to the timber, and serve for attachment, while others are given off at right angles, and present at intervals short lateral branches, each terminating in a bud-like enlargement.

The structure is better seen under a low power of the microscope. The organism (Fig. 78 ) is a colony, consisting of a common stem or axis, on which are borne numerous zooids (compare p. 277). The axis consists of a horizontal portion, resembling a root or creeping stem, and of vertical axes, which give off short lateral branches in an alternate manner, bearing the zooids at their ends. At the proximal ends of the vertical axes the branching often becomes more complex: the offshoots of the main stem, instead of ending at once in a zooid, send off branches of the third order on which the zooids are borne. In many cases, also, branches are found to end in simple club-like dilatations $(B d, I, 2)$ : these are immature zooids.

The large majority of the zooids are little Hydra-like bodies, the polypes or hydranths, each with a hypostome or manubrium and a circlet of about two dozen tentacles. Less numerous, and found chiefly towards the proximal region of the colony, are long cylindrical 


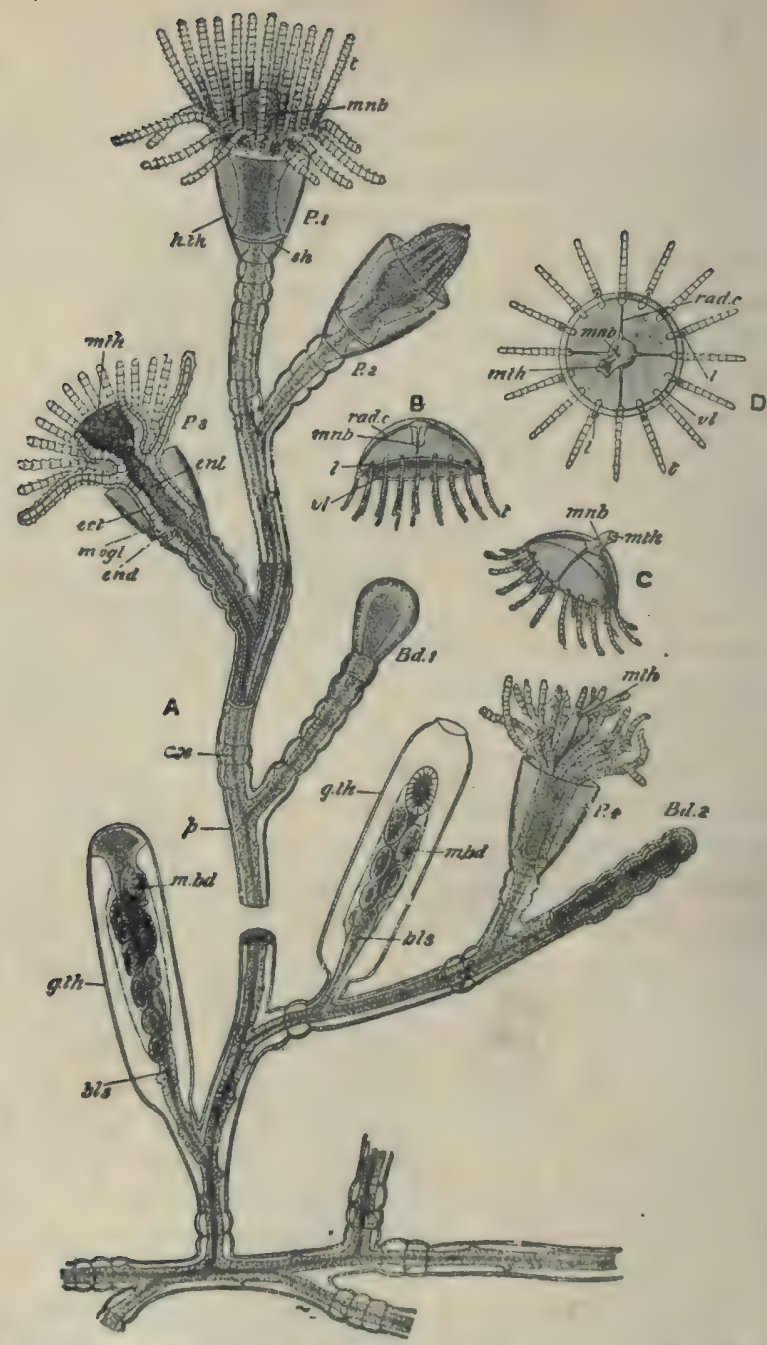

Fig. 78. - Obelia. A, portion of a colony with certain parts shown in longitudinal section ( $\times$ IO) ; $\mathrm{B}$, medusa $(<5) ; C$, the same with reversed umbrella; $I$ ), the same, oral aspect. $B d . I, 2$, buds ; bis. blastost yle ; $c \alpha$. cuenosare ; ect. ectoderm ; end. endoderm; ent. enteric cavity; $g . t h$. gonotheca; $h$. th. hydrotheca; $l$. lithecyst ; $m$. bd. medusa-bud; $m n b$. hypostome or manubrium; msgl. mesoglooa; mth. mouth; $p$. perisarc; $P .1,2,3$, polypes; rad. $c$. radial canal; $t$. tentacle; vl. velum (From Parker and Haswell's Zoology, reduced.) 
bodies or blastostyles (bls), each bearing numerous small lateral offshoots, varying greatly in form according to their stage of development, and known as medusa-buds $(m . b d)$; these will be considered presently.

Examination under a high power, either of an entire branch or of sections, shows that the polypes have essentially the structure of a Hydra, consisting of a double layer of cells-ectoderm (with nematocysts) and endoderm-separated by a supporting lamella or mesoglœa and enclosing a digestive cavity (ent) which opens externally by a mouth placed at the summit of the hypostome. The mouth is capable of great dilatation and contraction, and accordingly the hypostome appears now conical, now trumpet-shaped.

The tentacles, however, differ from those of Hydra in two important respects. In the first place they are solid : the endoderm, instead of forming a lining to a prolongation of the enteron, consists of a single axial row of large cells with thick cell-walls and vacuolated protoplasm. Then in the position of the muscle-processes of Hydra there is a layer of spindle-shaped fibres many times longer than broad, and provided each with a nucleus. Such muscle-fibres are obviously cells greatly extended in length (p. III), so that the ectoderm-cell of Hydra with its continuous muscle-process is here represented by an ectoderm-cell with an adjacent musclecell. We thus get a partial intermediate layer of cells between the ectoderm and endoderm, in addition to the gelatinous mesogloea ; and so, while a hydroid polype is, like Hydra, diploblastic (p. 298), it shows a tendency towards the assumption of a three-layered or triploblastic condition (compare p. 202).

The part of the stem and branches continuous with the bases of the polypes, which is known as the conosarc, is formed of the same layers and contains a cavity con- 
tinuous with those of the hydranths : thus the structure of a hydroid polype is, so far, simply that of a Hydra in which the process of budding has gone on to an indefinite extent and without separation of the buds.

There is, however, an additional layer added for protective and strengthening purposes. It is evident that such a colony would, if formed only of soft ectodermal and endodermal cells, be so weak as to be hardly able to bear its own weight even in water. To remedy this a layer of transparent, yellowish substance of horn-like consistency, called the perisarc, is developed outside the ectoderm of the cœnosarc, extending on to the branches and continuous with a glassy, cup-like investment, or hydrotheca, around the base of each polype, and with a transparent case, or gonotheca, enclosing each blastostyle. Each hydrotheca $(h . t h)$ has the form of a vase or wine-glass, and is perfectly transparent and colourless. A short distance from its narrow or proximal end, it is produced inwards into a sort of circular shelf $(s h)$, perforated in the centre: upon this the base of the polype rests, and through the aperture it is continuous with the common stem. When irritated-by a touch or by the addition of alcohol or other poison-the polype undergoes a very marked contraction: it suddenly withdraws itself more or less completely into the ineca, and the tentacles become greatly shortened and curved over the manubrium $(P .2)$. At the base of each zooid or branch the perisarc presents several annular constrictions, giving it a ringed appearance: for the most part it is separated by an interval from the cœnosarc, but processes of the latter extend outwards to it at irregular intervals, and at first $(B d .2)$ the two layers are in close apposition.

It is this layer which, when the organism dies and decays, is left as a semi-transparent, branched structure 
resembling the living colony in form except that polypes, blastostyles, and medusa-buds are wanting. The perisarc is therefore a supporting organ or skeleton, not, like our own bones, formed in the interior of the body (endoskeleton), but, like the shell of a crayfish or lobster, lying altogether outside the soft parts (exoskeleton).

As to the mode of formation of the perisarc:-we saw that many organisms, such as Sphærella and Amœba, are able to form a cyst or cell-wall, by secreting or separating from the surface of the protoplasm a succession of layers either of cellulose or of a transparent horn-like substance (pp. 232 and 244). But Amœba and Sphærella are unicellular, and are therefore free to form this protective layer at all parts of their surface. The ectoderm-cells of Obelia, on the other hand, are in close contact with their neighbours on all sides and with the mesoglœea at their inner ends, so that it is not surprising to find the secretion of skeletal substance taking place only at their outer ends. As the process takes place simultaneously in adjacent cells, the result is a continuous layer common to the whole ectoderm instead of a capsule to each individual cell. It is to an exoskeletal structure formed in this way, i.e., by the secretion of successive layers from the free faces of adjacent cells, that the name cuticle is in strictness applied in multicellular organisms.

In the blastostyles both mouth and tentacles are absent, the zooid ending distally in a flattened disc: the hydrotheca of a polype is represented by the gonotheca (g. th), which is a cylindrical capsule enclosing the whole structure, but ultimately becoming ruptured at its distal end to allow of the escape of the medusa-buds. These latter are, in the young condition, mere hollow offshoots of the blastostyle: when fully developed they have the appearance of saucers 
attached by the middle of the convex surface to the blastostyle, produced at the edge into sixteen very short tentacles, and having a blunt process, the manubrium, projecting from the centre of the concave surface. They are ultimately set free through the aperture in the gonotheca as little medusa or jelly-fish (B-D).

The structure of a medusa must now be described in some detail. The saucer-shaped "bell" or umbrella (Figs. 78, B-D, 79, and 80) is formed of a gelatinous

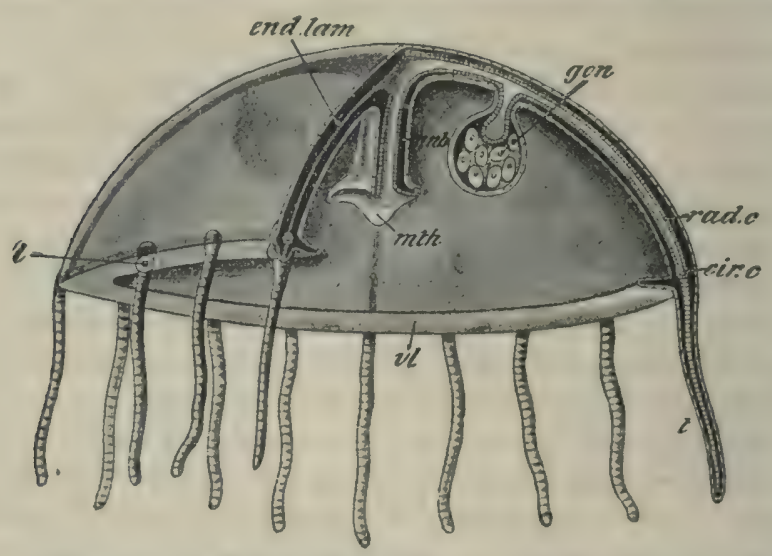

Frc. 79.-Dissection of a Medusa $(\times 25)$ with rather more than one-quarter of the umbrella and manubrium cut away (diagrammatic). The ectoderm is dotted, the endoderm striated, and the mesogloea black. circ. c. circular canal; end. lam. endoderm-lamella; gon. gonad; $l$. lithocyst; mmb. manubrium; mih. mouth; rad. c. radial canal; $v l$. velum.

substance (Fig. 80, D, $m s g l$ ) coverad both on its inner surface, or sub-umbrella, and on its outer surface, or ex-umbrella, by a thin layer of delicate cells $(e c t)$. The clapper-like manubrium $(m n b)$ is formed of two layers of cells, precisely resembling the ectoderm and endoderm of Hydra, and separated by a thin mesoglœa; it is hollow, its cavity (ent. cav) opening below, i.e., at its distal or free end, by a four-sided aperture, the mouth 
( $m$ th), used by the medusa for the ingestion of food. Very commonly as the medusa swims the umbrella becomes turned inside out, the sub-umbrella then forming the convex surface and the manubrium springing from its apex (Fig. $78, \mathrm{C}$ ). At its upper (attached or proximal) end the cavity of the manubrium is continued into four narrow, radial canals (Figs. $78, \mathrm{~B}, \mathrm{D}$, and 79 , rad. c, and Fig. So, D, and $\mathrm{D}^{\prime}$, rad) which extend through the gelatinous substance of the umbrella at equal distances from one another, like four meridians, and finally open into a circular canal (cir.c) which runs round the edge of the umbrella. By means of this system of canals the food, taken in at the mouth and digested in the manubrium, is distributed to the entire medusa. The canals are lined by a layer of cells (Fig. 8o, D and $\mathrm{D}^{\prime}$, end) continuous with the inner layer or endoderm of the manubrium; and extending from one canal to another, in the gelatinous substance of the umbrella, is a delicate sheet of cells, the endoderm-lamella (D', end. lam).

The edge of the umbrella is produced into a very narrow fold or shelf, the veluin (Fig. 79, vl, Fig. \&o, v), and gives off the tentacles $(t)$, which are sixteen in number in the newly-born medusa, very numerous in the adult. At the bases of eight of the tentacles-two in each quadrant -are minute globular sacs $(l)$, each containing a calcareous particle or lithite. These are the marginal senseorgans or lithocysts: they were formerly considered to be organs of hearing, and are hence frequently called " otocysts" : in all probability their function is to guide the medusa by enabling it to judge of the direction in which it is swimming. The marginal organs in this case may therefore be looked upon as organs of the sense of direction or of equilibration, and may be spoken of as statocysts (compare p. I89). The velum consists of a 


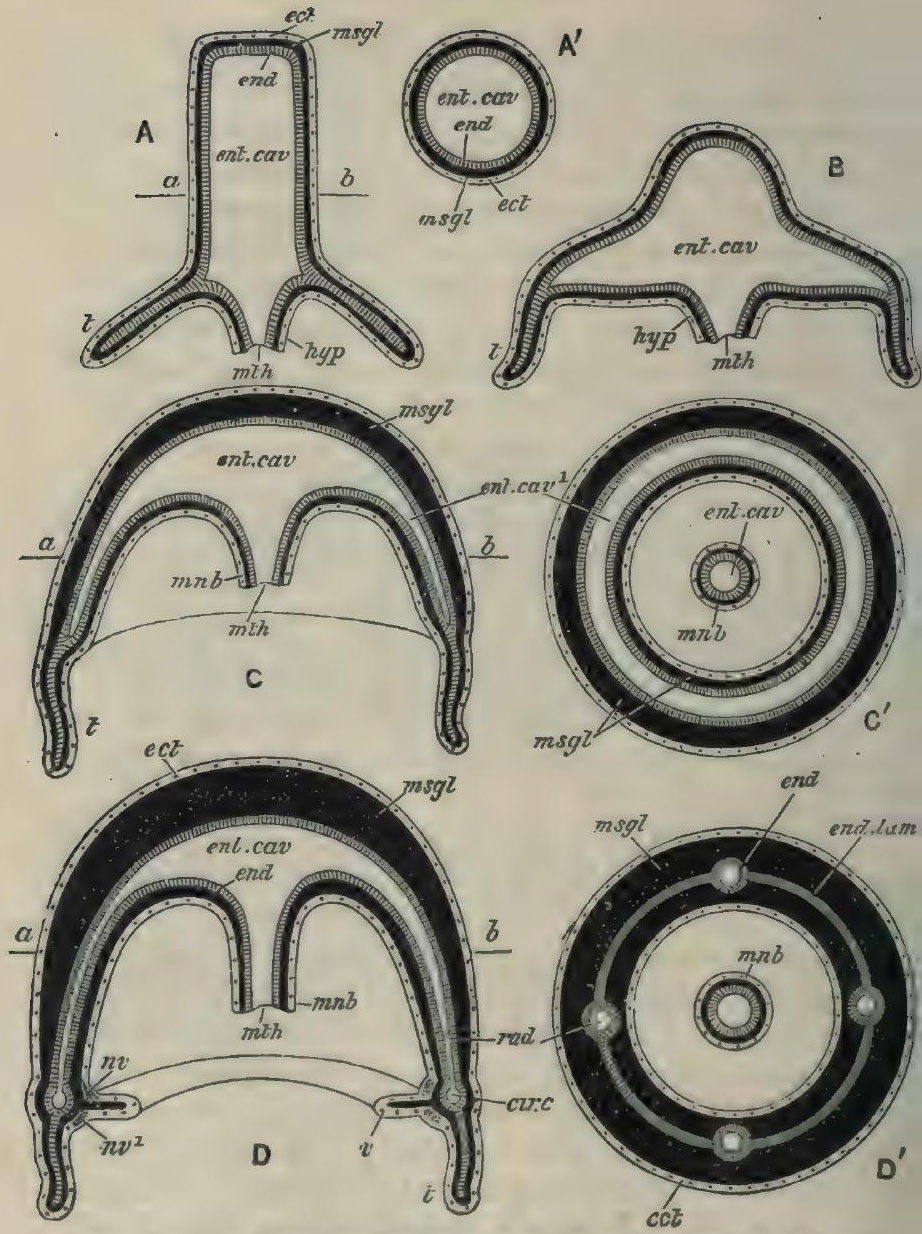

Frg. 80.--Diagrams illustrating the derivation of the medusa from the hydranth. In the whole series of figures the ectorlerm (ect) is dotted, the endoderm (end) striated, and the mesogloea (msgl) black.

A, longitudinai section of a simple polype showing the tubular body, with enteric cavity (ent. cav), hypostome (hyp), wouth (mth), and tentacles ( $t$ ).

$A^{\prime}$, transverse section of the same through the plane $a b$.

$\mathrm{B}$, the tentacular region is extended into a hollow disc.

$\mathrm{C}$, the tentacular region bas been further extended and bent into a bell-like form, 
the enteric cavity being continued into the umbrella (ent. cav'); the hypostome now forms a manubrium (mnb).

$C^{\prime}$, transverse section of the same through the plane $a b$, showing the continuous cavity (ent. cat) in the umbrella.

$D$, fulli formed medusa: the cavity in the umbrella is reduced to the radial (raci) and circular (car. c.) canals, the velum (v) is formed, and a double nervering ( $n v$. m) is produced from the ectoderm.

$D^{\prime}$. transverse section of the same through the plane $a b$, showing the four radial canals (rad) united by the endoderm-lamella (end. lawn), produced by partiai obliteration of the continuous cavity (ent. rav') in C. (From Parker's Builogy).

middle layer of mesoglœa with ectoderm on either side : there is no extension of endoderm into it. The tentacles, like those of the polype, are formed of a core of endoderm covered by ectoderm, which encloses numerous stingingcapsules.

At first sight there appears to be very little resemblance between a medusa and a polype, but it is really quite easy to derive the one form from the other.

Suppose a simple polype or Hydra-like body with four tentacles (Fig. 80, A, $\mathrm{A}^{\prime}$ ) to have the region from which the tentacles spring pulled out so as to form a hollow, transversely extended disc (B). Next, suppose this disc to become bent into the form of a cup with its concavity towards the hypostome, and to undergo a great thickening of its mesoglœa. A form would be produced like $\mathrm{C}$, i.e., a medusa-like body with umbrella and manubrium, but with a continuous cavity $\left(C^{\prime}\right.$, ent. cav') in the thickness of the umbrella instead of four radial canals. Finally, suppose the inner and outer walls of this cavity to grow towards one another and meet, thus obliterating the cavity, except along four narrow radial areas (D, rad) and a circular area near the edge of the umbrella (cir.c). This would result in the substitution for the continuous cavity of four radial canals opening on the one hand into a circular canal and on the other into the cavity of the manubrium (ent.cav), and connected with one another by a membrane-the endoderm-lamella ( $\mathrm{D}^{\prime}$, end. lam)indicating the former extension of the cavity.

It follows from this that the inner and outer layers of 
the manubrium are respectively endoderm and ectoderm: that the gelatinous tissue of the umbrella is an immensely thickened mesoglœa: that the layer of cells covering both inner and outer surfaces of the umbrella is ectodermal : and that the layer of cells lining the system of canals, together with the endoderm-lamella, is endodermal.

Thus the medusa, the polype, and the blastostyle are similarly constructed or homologous structures (p. 2I7), and the hydroid colony is trimorphic (compare p. 250), bearing zooids of three kinds. In some allied forms this individuation may go still further, the zooids being of very various forms and performing diverse functions: such a colony is said to be polymorphic.

Sooner or later the medusæ separate from the hydroid colony and begin a free existence. Under these circumstances the rhythmical contraction-i.e., contraction taking place at regular intervals- of the muscles of the umbrella causes an alternate contraction and expansion of the whole organ, so that water is alternately pumped out of and drawn into it. The obvious result of this is that the medusa is propelled through the water by a series of jerks. The movement is due to the contraction of the muscle-processes and muscle-fibres of the sub-umbrella and velum, some of which differ from the similar structures in the polype in exhibiting a delicate transverse striation.

There is still another important matter in the structure of the medusa which has not been referred to. At the junction of the velum with the edge of the umbrella there lies, immediately beneath the ectoderm, a layer of peculiar, branched cells, containing large nuclei and produced into long fibre-like processes. These nervecells (pp. I67 and 303) are so disposed as to form a double 
ring round the margin of the bell, one ring (Fig. 80, D, $n v)$ being immediately above, the other $\left(n v^{\prime}\right)$ immediately below the insertion of the velum. An irregular network of similar cells and fibres occurs on the inner or concave face of the bell, between the ectoderm and the layer of muscle-fibres. The whole constitutes the nervous sy'stem of the medusa; the double nerve-ring is the central, the network the peripheral nervous system (p. I55).

Some of the processes of the nerve-cells are connected with ordinary ectoderm-cells, which thus as it were connect the nervous system with the external world : others, in some instances at least, are probably directly connected with muscle-fibres.

We thus see that while the manubrium of a medusa has the same simple structure as a polype, the umbrella has undergone a very remarkable differentiation of its tissues. Its ordinary ectoderm-cells, instead of being large and eminently contractile, form little more than a thin cellular skin or epithelium (p. I09) over the gelatinous mesoglœa: they have largely given up the function of contractility to the muscle-processes or fibres, and serve merely as a protective and sensitive layer.

Similarly the function of automatism, possessed by the whole body of Hydra, is made over to the group of specially modified ectodermal cells which constitute the central nervous system. If a Hydra is cut into any number of pieces each of them is able to perform the ordinary movements of expansion and contraction, but if the nerve-ring of a medusa is removed by cutting away the edge of the umbrella, the rhythmical swimming morements stop dead: the umbrella is in fact permanently paralysed.

It is not, however, rendered incapable of movement, for a sharp pinch, i.e., an external stimulus, causes a single contraction, showing that the muscles still retain 
their irritability. But no movement takes place without such external stimulus, each stimulus giving rise infallibly to one single contraction: the power possessed by the entire animal of independently originating movement, i.e., of supplying its own stimuli, is lost with the central nervous system (compare p. I72).

Another instance of morphological and physiological differentiation is furnished by the marginal sense-organs situated at the base of the tentacles (p. 3I5).

The polype and medusa are respectively nutritive and reproductive in function, the reproductive zooids becoming detached and swimming off to found a new colony elsewhere: the polypes are purely nutritive zooids; the medusæ, although capable of feeding, are specially distinguished as reproductive zooids. Hanging at equal distances from the sub-umbrella, in immediate relation with the radial canal, are fourovoid gonads (Fig. 79, gon), each consisting of an outer layer of ectoderm continuous with that of the sub-umbrella, an inner layer of endoderm continuous with that of the radial canal and enclosing a prolongation of the latter, and of an intermediate mass of cells which have become differentiated into ova or sperms. As each medusa bears organs of one sex only (spermaries or ovaries, as the case may be), the individual medusæ are dicecious, and not, like Hydra, monœcious. It will be noticed that the gonad has the same general structure as an immature zooid-an out-pushing of the body-wall consisting of ectoderm and endoderm, and containing a prolongation of the enteric cavity.

The medusæ, when mature, become detached and swim away from the hydroid colony. The sperms of the males are shed into the water and carried to the ovaries of the females, where they fertilise the ova, converting them, as usual, into oosperms. 
The oosperm undergoes segmentation, forming a polyplast or morula (p. 200): ectoderm and endoderm become differentiated, and the ectoderm-cells acquire cilia, by means of which the embryo now swims freely in the water. An enteron appears in the endoderm, and in this stage the embryo, which has an elongated form, is known as a planula. It then loses its cilia and settles down on a rock, shell, sea-weed, or other submarine object, assuming a vertical position, with its broader end fixed to the support.

The attached or proximal end widens into a disc of attachment, a dilatation is formed a short distance from the free or distal end, and a thin cuticle is secreted from the whole surface of the ectoderm. From the dilated portion short buds arise in a circle: these are the rudiments of the tentacles: the narrow portion distal to their origin becomes the hypostome. Soon the cuticle covering the distal end is ruptured so as to set free the growing tentacles: an aperture, the mouth, is formed at the end of the hypostome, and the young hydroid has very much the appearance of a Hydra with a broad disc of attachment, and with a cuticle covering the greater part of the body. Extensive budding next takes place, the result being the formation of the ordinary hydroid colony.

Thus from the oosperm or impregnated egg-cell of the medusa the hydroid colony arises, while the medusa is produced by budding from the hydroid colony. We have what is called an alternation of generations, the asexual generation or agamobium (hydroid colony) giving rise by budding to the sexual generation or gamobium (medusa), which in its turn produces the agamobium by a sexual process, i.e., by the conjugation of ovum and sperm (compare p. 287). This form of alternation of generations is known as metagenesis. 
Hydra and Obelia both belong to the simplest classthe Hydrozoa - of the phylum Colenterata : this phylum includes all the polypes or zoophytes, the jelly-fishes, and the anemones and corals. In all there is an ectoderm and an endoderm, separated by a mesogloa, which may consist, as in Hydra, of a structureless membrane containing no cells, or may be gelatinous as in the medusa, and may even contain cells, thus assuming more the character of an intermediate celllayer or mesoderm. There is no body-cavity or colome (p. 20) surrounding the digestive cavity or enteron, and tentacles are present round the mouth. Organs of offence occur in the form of thread-cells or nematocysts.

In all the higher phyla a definite mesoderm is developed in the embryo in addition to the ectoderm and endoderm (triploblastic condition), and in nearly all cases there is a definite cavity or colome present in the mesoderm: hence all these animals are often included together as the Coolomata.

\section{PRACTICAL DIRECTIONS}

\section{Hydra.}

Examine some living Hydræ in a vessel of water, with the naked eye or with a pocket lens, and note the differences in form according to the degree of contraction. The animal is usually attached to foreign bodies (weeds, $\& c$.) at one end, and at the other end a number of tentacles (usually six to eight) are given off. In the expanded state the body and tentacles are greatly elongated and threadlike, while when contracted the body is more globular, and the tentacles appear like small knobs. Note the brown colour in $H$. fusca, and the green colour in $H$. viridis. Observe the methor of seizing food. Place a specimen on a slide in a drop of water, together with a small piece of water-weed or paper to prevent crushing, and then put on a cover-glass. Wait till the animal is fully expanded, and then examine with the low power. Note (Fig. 75) :-

I. The body, enclosing the digestive cavity or enteron, which opens by the mouth at the distal end of the animal, at the 
summit of a conical hypostome. At the proximal end is the foot, or disc of attachment.

2. The tentacles, arranged in a single circlet or whorl around the base of the hypostome. They are hollow, and their cavities communicate proximally with the general digestive cavity of the body. On their surface are a number of small knobs.

\section{The contractions of the body and tentacles.}

4. The structure of the body-wall, which is made up of (a) an outer layer of colourless cells (ectoderm), and $(b)$ an inner layer (brown in $H$. jusca, and green in $H$. viridis) of cells (endoderm) lining the digestive cavity. Between these two layers is a thin gelatinous non-cellular supporting lamella or mesoglcea, not easily seen with the low power. (The tentacles have a similar structure, the details of which cannot be made out with the low power.) Sketch.

Put on the high power and examine a tentacle, focussing on to the surface as well as deeper, so as to get an optical section (see p. $55^{8}$ and Fig. 76, A).

5. The relations of the ectoderm, endoderm, and supporting lamella, and the nuclei of the ectoderm and endoderm cells.

6. The structure of the ectoderm:-(a) large conical cells with their broader ends outwards, arranged in a single row, and differing in form according to the state of contraction. The spaces between the inner narrower ends of these are filled up with $(b)$ smaller rounded interstitial cells (absent on the foot); (c) thread-cells or nematocysts (Fig. $7 j$ )-oval capsules containing a spirally-wound thread, developed within certain of the interstitial cells called cnidoblasts, and when fully formed, found imbedded in or between the large ectoderm-cells; they are much more numerous on the tentacles than on the body, causing the knobs referred to above. Each cnidoblast gives rise to a small process-the trigger-hair or cnidocil, which projects from the surface. Notice the discharged thread-cells, and observe that each consists of a flask-like base (to which part of the protoplasm and the nucleus of the burst cnidoblast usually remains attached) and a long filament, with three large and several smaller spines or barbs at its proximal end. (Smaller thread-cells, with thicker threads and no spines, are aiso present; some of these have long. spirally-coiled threads, others shorter, straight threads. These can be seen later on.)

7. The endoderm, consisting of a single layer of large amœboid cells, which in $H$. viridis contain green $Z$ oo- 
chlorella (p. 304). Note the currents in the tentacles, which are produced by long vibratile flagella present on many of the endoderm-cells.

8. The thin transparent supporting lamella. Sketch.

9. Treat a specimen with methyl-green. A slight pressure on the cover-glass will crush the animal, and render the interstitial cells and thread-cells especially distinct. Note also other isolated cells of the ectoderm and endoderm. Sketch.

Io. Examine a specimen with buds in different stages of development, and note as much as possible of the mode of asexual reproduction by gemmation. Sketch.

II. If none of your specimens bears sexual organs, try to procure a mounted preparation which shows them, and examine first with the low, and then the high power. Note-(a) the spermaries-several conical swellings near the bases of the tentacles; they are covered with large ectodermcells, and contain numerous interstitial cells, each of which eventually gives rise to a sperm with a " head " and vibratile "tail." These are discharged at the apex of the cone, which when ripe may be ruptured by a slight pressure on the cover-glass. (b) The ovaries (sometimes only one), generally situated nearer the proximal end of the body. They are larger than the spermaries and more spherical, but at first have a similar structure. When ripe a single ooum is found in each. Sketch.

Place some Hydra in a watch-glass with a very small amount of water, and when they have expanded, pour quickly over them a warm saturated solution of corrosive sublimate in alcohol or water. Wash several times with weak alcohol, stain for a few minutes with borax-carmine or hæmatoxylin, and wash with weak and then with stronger alcohol. Place in absolute alcohol for a few minutes, and afterwards in turpentine, xylol, or oil of cloves ; mount in balsam. Work through $\$ \S 5-8$ again, noting especially the characters of the various cells and their nuclei, as well as -

I2. The contractile processes coming off from the inner ends of the large ectoderm-cells (Fig. 76, C). These extend longitudinally, and lie against the outer surface of the supporting lamella. Sketch.

Examine transverse sections through the body or tentacles (Fig. 76, B) prepared as directed on P. I36, after killing and fixing the specimens as above. Work through 
$\S \S 6-8$ again, noting the various cells and their nuclei, \&c. Observe especially-

13. (a) The contractile processes of the ectoderm-cells, which will be cut across transversely, so as to appear as dots just outside the supporting Iamella; (b) the amoeboid and vacuolated character of the endoderm-cells. (Special methods of preparation are necessary in order to show the flagella.) Sketch.

\section{Obelia. ${ }^{1}$}

If possible, examine first alive, and then kill and stain as directed in the case of Hydra.

I. Examine under the low power and note :-

(a) The polypes, with their tentacles and hypostome, expanded and contracted; and the immature polypes. (b) The blastostyles and medusa-buds. (c) The conosarc and the perisarc, hydrotheca, and gonothece (Fig. 78). Sketch.

2. Then stain, put on the high power and make out the minute structure of the polypes, noting the-

(a) Mouth, (b) enteron, (c) ectoderm, supporting lamella, and endoderm (solid in the tentacles). On the blastostyles examine the medusa-buds. (If you wish to make permanent preparations, mounted in balsam, use the method given for Hydra.)

Sketch an optical section of a polype and blastostyle.

Sections of an entire branch, prepared in the usual way (see p. 136), should also be made. Select for examination those which pass as nearly as possible through the vertical and transverse axes of a polype, and compare with your sections of Hydra.

3. Place a medusa (Figs. $78 \mathrm{~B}-\mathrm{D}$ and 79 ) on a slide with the sub-umbrella surface uppermost, stain, and mount carefully in glycerine. Note-

(a) The umbrella, (b) manubrium and mouth, (c) tentacles, (c) radial and circular canals, (d) velum, (e) gonads, and (f) lithocysts (often difficult to recognise in preserved specimens). Sketch.

1 Specimens living or preserved, both of the colonial and medusa-stage of Obelia or some other Hydroid (as well as other marine animals described in this book) can be obtained from any Marine Biolngical Laboratory; or the fresh-water Cordylophora will answer the purpose as far as the colony is concerned, but it has no medusa-stage. 


\section{CHAPTER VI}

THE EARTHWORM : NEREIS-CHARACTERS OF THE PHYLUM ANNULATA

THE general form and appearance of an earthworm are familiar to everyone. In this country there are a number of different species of earthworms belonging to several genera, the commonest of which are Lumbricus and Allolobophora; but the differences between these are of minor importance to the beginner, and any one of the common forms will serve our purpose.

Earthworms burrow into the soil and live on decaying leaves and other organic matter, which they swallow together with a considerable quantity of earth. This earth, mingled with the undigested portions of the food, is passed from the body on to the surface of the ground in the form of the well-known little heaps or " castings" which you must have noticed in gardens and fields, especially after rain, when the worms come more frequently to the surface. In this way a quantity of finely divided earth, mixed with the fæces of the worms, is constantly being spread out on the surface of the soil, and Darwin calculated that on an average a layer of earth about one-fifth inch in thickness, or about ten tons an acre, is thus brought to the surface in the course of a year. Earthworms are therefore good friends to the 
gardener and agriculturist, as they are continually ploughing and manuring the soil, and in doing so they gradually cover up stones and other objects lying on the surface.

The body of the earthworm is long and narrow, approximately cylindrical in shape, and bilaterally symmetrical (p. 296): in the common forms it reaches a length of about six inches. Anteriorly it is bluntly pointed, while more posteriorly it is somewhat flattened, its greatest diameter being reached at about a third of the entire length from the anterior end. In the ordinary creeping movements of the animal, which are effected by the alternate contraction and extension of its body, the anterior end is directed forwards. The colour is pinkish in most species, and is paler on the lower or ventral than on the upper or dorsal side.

The surface of the body is distinctly marked by transverse annular grooves into body-segments or metameres (Fig. 82), the number of which is about $\mathrm{I}_{5} \mathrm{O}$, more or less : the segments are rather longer towards the anterior end than they are further back. At the extreme anterior end is a small finger-shaped head-lobe or prostomium, which overhangs the mouth, situated on the anteroventral surface of the next segment, which is therefore called the peristomium, and is counted as the first metamere. The anus is a slit-like aperture on the hinder surface of the last or anal segment. The earthworm is thus a metamerically segmented animal, and the segments are serially homologous with one another (p. 39).

In adult worms a prominent glandular swelling is noticeable on the dorsal and lateral surfaces of the body extending through from five to ten segments beginning at about the thirtieth ; this is known as the clitellum, and, as we shall see, it is important in the process of impreg- 
nation and in forming a case or cocoon for the eggs. On the ventral part of this region are some small glandular swellings, which are more conspicuous in young worms before the clitellum is developed.

The whole of the body is invested with a delicate, iridescent membrane or cuticle (p. 3I3), formed as a secretion of the epiderm or outer epithelial layer of the body (p. I28). Every segment, except the first and the last, is provided with eight small cuticular spines or sete (Fig. 8I, set)-slightly curved bodies with tapering ends, composed of a horn-like substance called chitin (compare p. 232)-each of which is developed in a small sac formed as an involution of the epiderm and is provided with muscles by means of which it can be protruded and retracted. These setæ are arranged in couples, forming two double rows along each latero-ventral region of the body, and their points can be distinctly felt on drawing the worm through the fingers : they serve to prevent the animal from slipping backwards as it moves along on the surface of the ground or in its burrows.

We have seen that the earthworm takes in its food, together with quantities of earth, by the mouth, and after retaining it for a longer or shorter time in the body expels it by the anus. It is obvious, therefore, that there must be some kind of digestive cavity into which the food passes by the mouth, and from which effete matters are expelled through the anus. Sections (Fig. 8I) show that this cavity is not a mere space excavated in the interior of the body, but a definite tube, the enteric or alimentary canal (p. 23), which passes in a straight line from mouth to anus, and is separated in its whole extent from the walls of the body by a wide space, the body-cavity or coelome (coel), as in the frog (p. 20). So that the general structure of the earthworm might be imitated by taking a wide tube, stopping the ends of it 
with corks, boring a hole in each cork, and then inserting through the holes a narrow tube of the same length as the wide one. The outer tube would represent the body-wall, the inner the enteric canal, and the cylindrical space between the two the cœlome. The inner tube would communicate with the exterior by each of its ends, representing respectively mouth and anus; the space between the two tubes, on the other hand, would have no such communication with the outside. A transverse section of the body has, therefore, the general character of two concentric circles.

It will be remembered that a transverse section of Hydra has the character of two concentric circles, formed respectively of ectoderm and endoderm (Fig. 76, p. 298), the two layers being, however, only separated by the thin mesogloea. At first sight, then, it seems as if we might compare the earthworm to a Hydra in which the ectoderm and endoderm, instead of being in contact, were separated by a wide interval; we should then compare the body-wall of the earthworm with the ectoderm of Hydra, and its enteric canal with the endoderm. But this comparison would only express part of the truth.

A thin transverse section (Fig. 8r) shows the bodywall of the earthworm to consist of several distinct layers. Outside is a thin transparent cuticle (cut) showing no structure beyond a series of intersecting oblique lines. Next comes a layer of epithelium, the epiderm or deric epithelium (epid). Within this is a very thin connective-tissue layer representing the derm (p. I28), and a double layer of muscle-fibres by means of which the movements of the body are produced-an outer, in which the fibres extend transversely round the body (circ. mus), and a much thicker inner layer consisting of. longitudinal fibres, in section arranged like the barbs of 
a feather on a central axis (long. mus). Finally, within the muscular layer and lining the colome is a thin peritoneal membrane (parietal layer, compare p. 26), on

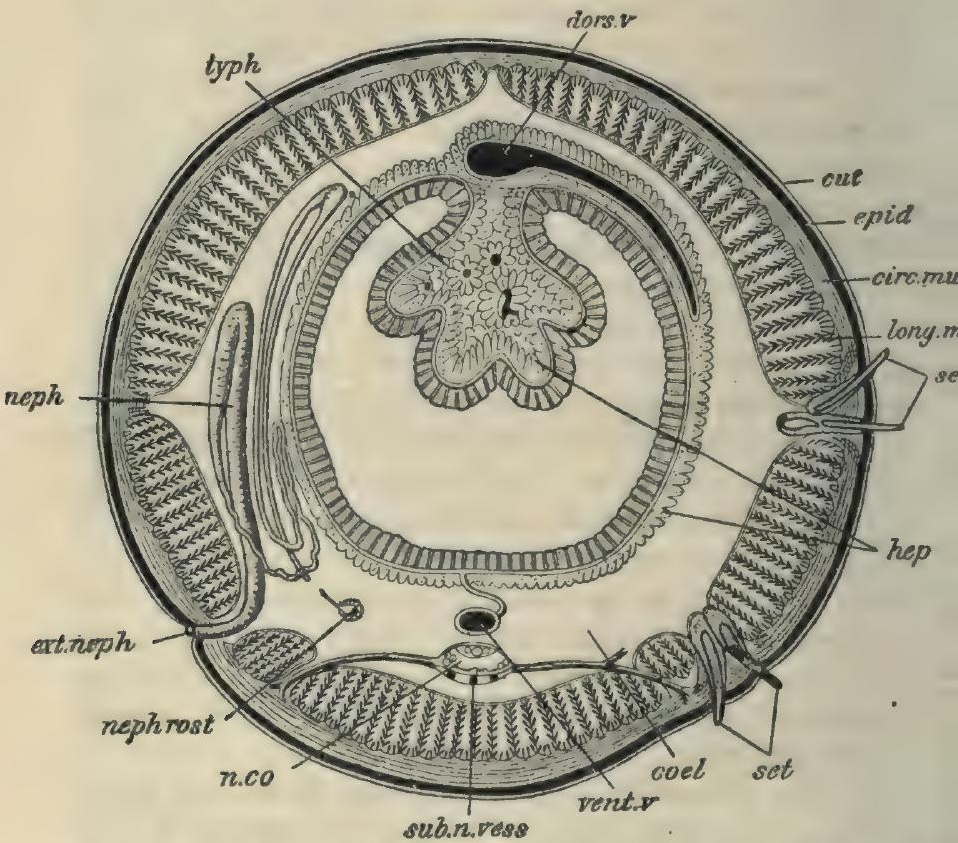

Frg. 8r.-Lumbricus, semidiagrammatic transverse section of the middle region of the body. ( $X$ about 18 .)

circ. mus. layer of circular muscular fibres; cael. coelome ; cut. cuticle ; dors. $v$. dorsal vessel ; epid. epiderm; ext. neph. nephridiopore; hep. layer of yellow cells; long. mus. longitudinal muscles; neph. nephridium (shown entire) : nephrost. nephrostome; $n$. co. nerve cord ; set. seta ; sub. n. vess. sub-neural vessel; typh. typhlosole; vent. v. ventral (sub-intestinal) vessel. (From Parker and Haswell's Zoology, after Marshall and Hurst.)

the inner surface of which is a very thin layer of cells, the colomic epithelium.

A transverse section of the intestine shows an inner layer of ciliated, columnar enteric epithelium (compare p. I09), a very thin middle layer composed of muscle- 
fibres and connective-tissue, and an outer layer of large yellow cells, the function of which is said to be excretory, and which correspond to a special development of the colomic epithelium covering the visceral layer of the peritoneal membrane which invests the intestine.

We are now in a better position to compare the transverse section of the Hydra and the earthworm. The epiderm of the earthworm being the outermost cell-layer is to be compared with the ectoderm of Hydra, and its cuticle with the layer of the same name which, though absent in Hydra, is present in the stem of hydroid polypes, such as Obelia (viz., the perisarc). The enteric epithelium of the earthworm, bounding as it does the digestive cavity, is clearly comparable with the endoderm of Hydra. So that we have the double layer of muscle-fibres and the two layers of peritoneum not represented in Hydra, in which their position is occupied merely by the mesogløa. The muscle-fibres are not of the striped kind, like those in the corresponding position in the frog ( $p$. II2).

But it will be remembered that in medusæ there is sometimes found a layer of separate muscle-fibres between the ectoderm and the mesogloea, and it was pointed out (P. 3II) that such fibres represented a rudimentary intermediate cell-layer or mesoderm. We may therefore consider the muscular layer and the peritoneum of the earthworm as mesoderm, and we may say that in this animal, as in the frog (p. 203. and Fig. 65), the mesoderm is divisible into an outer or parietal layer, and an inner or visceral layer.

The parietal layer is in contact with the ectoderm or deric epithelium, and with it forms the body-wall; the visceral layer is in contact with the endoderm or enteric epithelium, and with it forms the enteric canal. The 
cœlome separates the parietal and visceral layers from one another, and is lined throughout by colomic epithelium.

The relation between the diploblastic polype and the triploblastic worm may therefore be expressed in a tabular form as follows:

Hydroid.

Cuticle

Ectoderm.

\section{Earthworm. ${ }^{1}$}

Cuticle.

Deric epithelium or epiderm. Connective-tissue and muscle-fibres.

Peritoneum with its cœlomic epithelium (parietal layer). (rudimentary)

Endoderm . . . Enteric epithelium. Peritoneum with its colomic Visceral epithelium (visceral layer). layer Connective-tissue and muscle-fibres.

Strictly speaking this comparison does not hold good of the anterior and posterior ends of the worm : at both mouth and anus the deric passes insensibly into the enteric epithelium, and the study of development shows that the cells lining both the anterior and posterior ends of the canal are ectodermal (compare pp. 204 and 207). For this reason the terms deric and enteric epithelium are not mere synonyms of ectoderm and endoderm respectively.

It is important that you should, before reading further, understand clearly the general composition of a triplo-

1 It will be seen that the relations of these layers in the earthworm and frog are similar, except that in the latter the cuticle is wanting (compare Figs. 5, 38, and 39). 
blastic animal as typified by the earthworm, which may be summarised as follows. It consists of two tubes formed of epithelial cells, one within and parallel to the other, the two being continuous at either end of the body where the inner tube (enteric epithelium) is in free communication with the exterior; the outer tube (deric epithelium) is lined by a layer of connectrve-tissue and muscle-fibres, within which is a thin peritoneum lined by cœlomic epithelium, the three together forming the bodywall; the inner iube (enteric epithelium) is covered externally by a layer of muscle-fibres and connectivetissue and a thin peritoneum covered by cœlomic epithelium, which form with it the enteric canal ; lastly, the body-wall and enteric canal are separated by a considerable space, the colome.

The enteric canal is not, as might be supposed from the foregoing description, connected with the body-wall only at the mouth and anus, but is supported in a peculiar way. There is no dorsal mesentery as in the frog (p. 27), but a series of transverse vertical partitions or septa (Figs. 82 and 83 ) extends right across the body-cavity, each being perforated by the canal. The septa are regularly arranged and correspond in position with the external grooves by which the body is divided into metameres. Thus the transverse or metameric segmentation affects the cœlome as well as the body-wall, the former being divided up into a series of chambers, which, however, communicate with one another ventrally, where the septa are incomplete (Fig. 83, n. a). Each septum is composed of a sheet of connective-tissue and muscle-fibres, and is covered on both sides by cœlomic epithelium. The colome communicates with the exterior by a series of dorsal pores situated in the grooves between all the segments except about the first ten. 


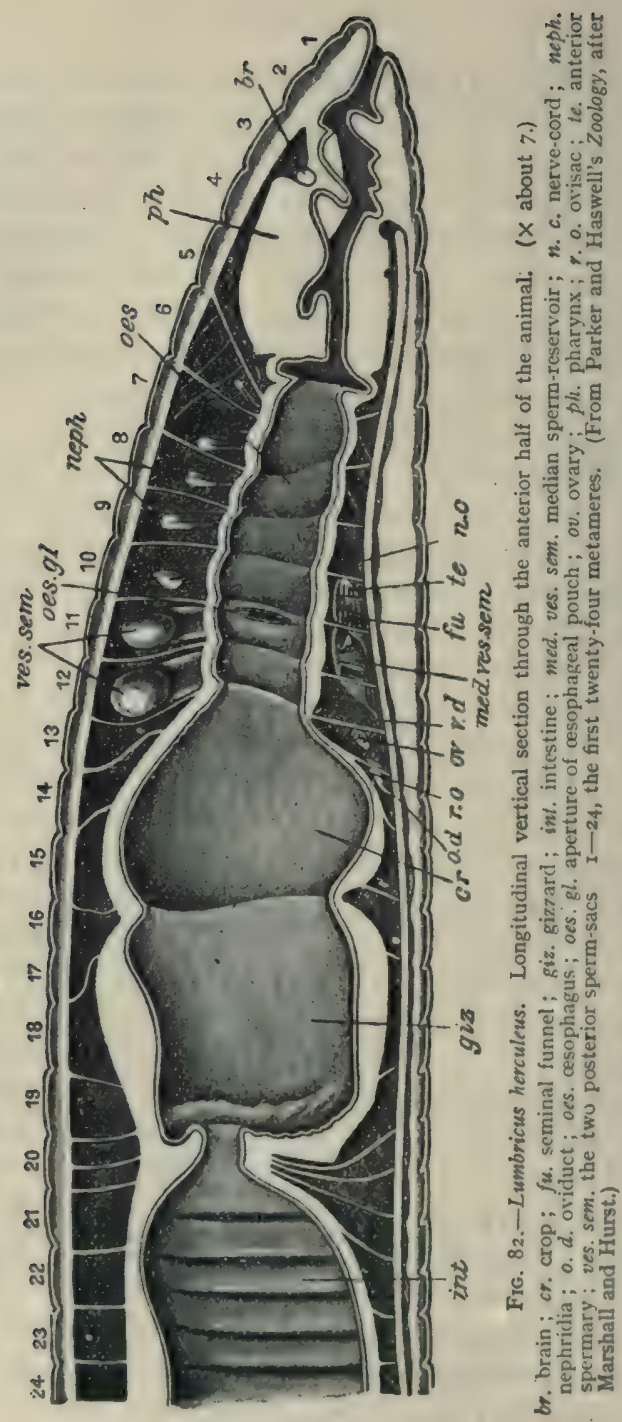


The digestive canal is not a simple tube of even calibre throughout, but is divisible into several portions. The mouth is bounded by a soft lip and leads into a small buccal cavity, which communicates with a thickwalled pharynx (Figs. 82 and 83 , ph), extending through about five segments and connected with the body-wall by a number of radially arranged muscle-fibres, the septa being absent in this region. When the worm feeds, the buccal cavity is everted, and the muscles serve to draw it and the pharynx back again, as well as to dilate the pharynx. The latter is followed by a narrow gullet or asophagus (œs, œ), extending through about eight segments, and provided at about the middle of its course with a pair of lateral pouches (Fig. 83, $\propto$. $g$ ), with each of which, in Lumbricus, two yellowish cesophageal or calciferous glands communicate posteriorly: these contain a calcareous substance which may neutralise the organic acids present in the food swallowed. The pouches open into the gullet (Fig. 82, oes. gl), which pass posteriorly into a dilated, thin-walled receptacle, the crop (cr), and this, again, communicates posteriorly with a large gizzard (giz) with thick and muscular walls, which in about the 2oth segment communicates with the intestine (int). The intestine has a similar character throughout, and extends from the gizzard to the anus; it is ciliated and its dorsal wall is folded inwards so as to produce a longitudinal ridge or typhlosole (Fig. 8I, typh), which serves to increase the absorptive surface and in the interior of which the yellow cells are very numerous.

Certain of the cells lining the enteric canal, and especially those along the typhlosole, are very granular, and, like the endoderm cells of the hypostome of Hydra (p. 303), are to be considered as unicellular glands. They secrete a digestive juice which-mixing with the 
various substances containing organic acids taken in by the mouth and said to be neutralised by the calcareous secretion of the œsophageal glands-dissolves the proteids and other digestible parts so as to allow of their absorption. It is very probable that the process is purely extra-cellular or enteric, the food being dissolved and rendered diffusible entirely in the cavity of the canal (p. 305). By the movements of the canal-caused partly by the general movements of the body and partly by the contraction of the muscles of the canal and septa aided by the action of the cilia-the contents are gradually forced backwards, and the earth and other indigestible matters expelled at the anus.

The cœlome is filled with a colourless transparent coelomic fluid in which are suspended amœboid corpuscles or leucocytes like those of the frog's blood and lymph (p. I05). The function of this colomic fluid is probably to distribute the digested food in the enteric canal to all parts of the body. In Hydra, where the lining wall of the digestive cavity is in direct contact with the simple wall of the body, the products of digestion can pass at once by diffusion from endoderm to ectoderm; but in the present case a means of communication is wanted between the enteric epithelium and the comparatively complex and distant body-wall. The peptones and other products of digestion diffuse through the enteric epithelium into the colomic fluid. and by the continual movement of the latter-due to the contractions of the body-wall--are distributed to all parts. Thus the external epithelium and the muscles, as well as the nervous system, reproductive and other organs not yet described, are wholly dependent upon the enteric epithelium for their supply of nutriment.

The earthworm, like the frog, possesses a series of 
blood-vessels, containing red blood, the whole of which form a single closed vascular system, there being no communication between them and any of the other cavities of the body. The main trunks have a longitudinal direction, the chief ones being a large dorsal vessel, running along the dorsal surface of the enteric canal, and a ventral or sub-intestinal vessel, below the canal (Figs. 8r, dors. v, vent. $v$, and 83 ), In addition to these there are three smaller longitudinal trunks in relation to the nerve-cord, which, as we shall see, extends along the ventral side of the cœlome: these are a median subneural and two lateral neural vessels (Figs. 8I and 83). All these longitudinal trunks give off branches to the various parts of the body, and certain of them are connected with one another by a series of pairs of lateral commissural vessels : in the region of the gullet there are five (Lumbricus) or six (Allolobophora) pairs of large vessels connecting the dorsal and subintestinal trunks; and the dorsal and subneural trunks are also connected in each segment all along the body by a pair of smaller commissural vessels, running in the inner surface of the body-wall (Fig. 83).

Notice that there is here no heart and no distinction into arteries and veins, as in the frog (p. 27). The vessels gradually divide up into smaller and smaller branches in the various parts of the body, and then again unite to form larger and larger vessels which eventually open into one or other of the main trunks.

The circulation of the blood is effected by the rhythmical, peristaltic contraction (p. 75) of certain of the larger vessels: thus the dorsal trunk contracts from behind forwards, and the large commissural vessels-often spoken of as " hearts "-which connect it anteriorly with the sub-intestinal trunk, from above downwards, 
so that the blood passes forwards in the dorsal, backwards in the ventral vessel. The series of vessels of
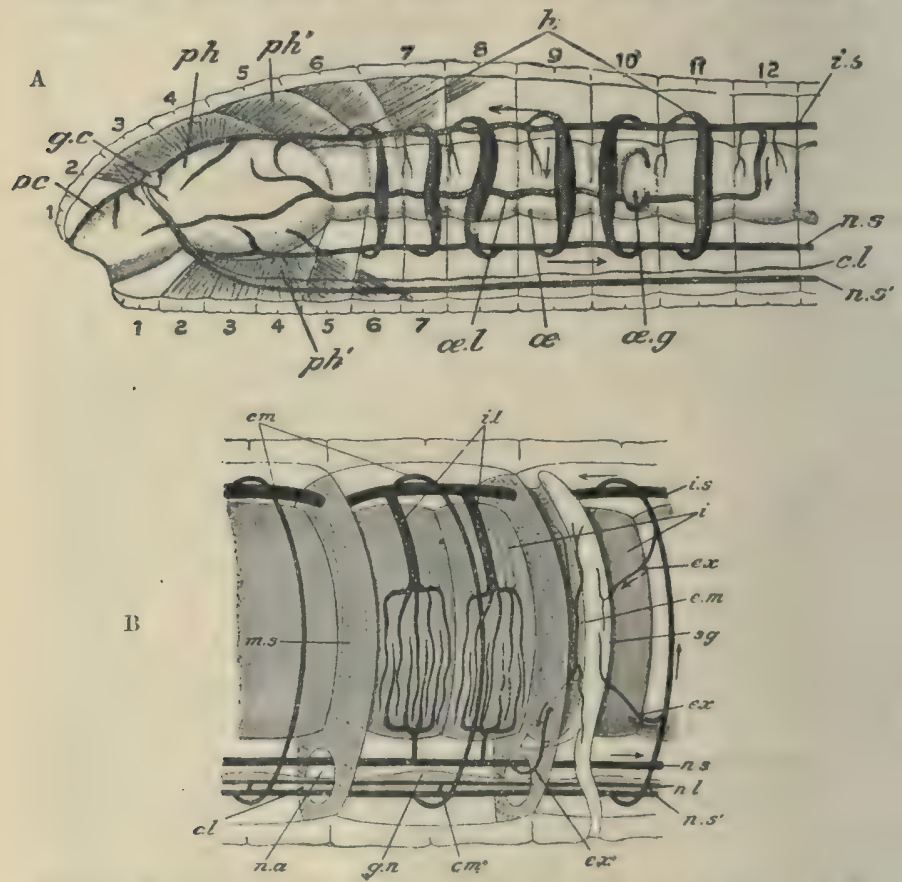

FIG. 83.-Blood-vessels of Allolobophora. Diseertions from the left side of --A, the twelve anterior segments ( $\leqslant 5$ ), and $B$, three segments in the intestinal region ( $\times$ 10.) The arrows inclicate the course of the circulation. (From Howes's Allas.)

$b c$, buccal sac; $c$. $l$, nerve-cord; $c m$, commissural vessels connceting the dorsal and subneural vessels ; $\mathrm{cm}^{\prime}$, neural commissural vessels ; $e$. $m$, mesentery, and $e x$, vessel of nephridium; g. $c$, cerebral ganglion; $g . n$, ganglionic swelling of nerve-cord ; $h$, commissural vessels (" hearts ") connerting the dorsal and subintestinal vessels anteriorly; $i$, intestine; $i, l$, lateral intestinal vessels ; $i . s$, dorsal (supra-intestinal) vessel; $m . s$, septum; $n . a$, ventral perforation of septum; $u$. $l$, lateral neural vessel ; $n$. $s$, sub-intestinal vessel; $n$. $s^{\prime}$, subneural vessel; $\alpha$, gullet; $\alpha$. , , œsophageal pouch; $\alpha . l$, lateral oesophageal vessel; ph, pharynx; sg. nephridium.

the enteric canal are connected with the dorsal and subintestinal trunks, and those of the excretory organs, to be described presently, with the sub-intestinal trunk 
and the commissural vessels in the body-wall which connect the dorsal and subneural trunks. By means of branches of these parietal vessels the body-wall is plentifully supplied with blood.

The red colour of the blood is due to hamoglobin (p. 107), which is not, as in the frog, contained in red blood-corpuscles, but is dissolved in the plasma, in which, however, minute colourless corpuscles can be recognised. The function of hæmoglobin in the process of respiration has already been described (p. I44) ; but in the earthworm, as in many other lower animals, there are no specialised respiratory organs (lungs or gills), the necessary exchange of gases being performed by the entire surface of the body, the minute branches of the blood-vessels in the body-wall being only separated from the air by the single layer of epidermic cells-and even penetrating amongst the latter in the region of the clitellum: this is an exceptional occurrence, for, as we have seen, capillaries do not, as a general rule, extend amongst epithelial cells (compare, e.g., Figs. 38-40).

In discussing in a previous chapter the differences between plants and animals (p. 255), we found that in the unicellular organisms previously studied the presence of an excretory organ in the form of a contractile vacuole was a characteristic feature of such undoubted animals as Amœba and many other Protozoa. But the reader will have noticed that Hydra and its allies have no specialised excretory organ, waste-products being apparently discharged from any part of the surface. In the earthworm we meet once more with an animal in which excretory organs are present, although, in correspondence with the complexity of the animal itself, they are very different from the simple contractiie vacuoles of Paramœcium or Vorticella, and are more nearly comparable with those of the frog (p. I46). 
The excretory organs of the earthworm consist of

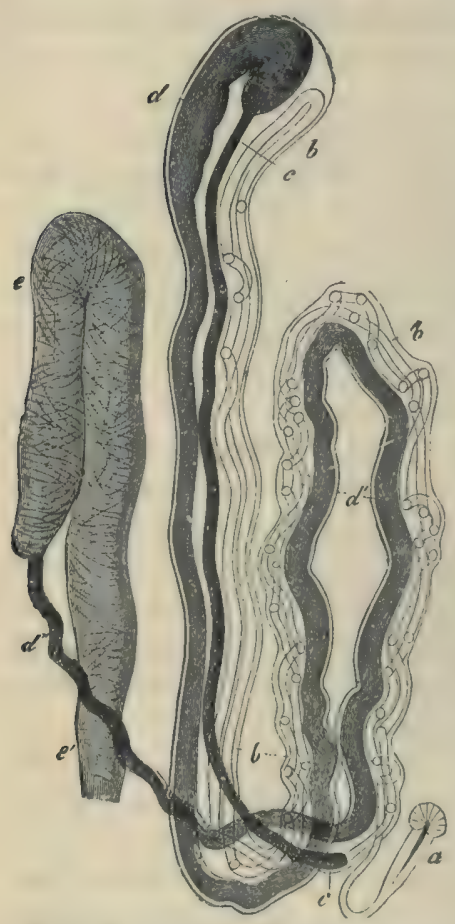

Frg, 84.-A nephridium of Lumbricus, showing the three main loops into which the different parts of the tubule are arranged, as well as the different portions of the tubule. ( $x$ about 30 .)

$a$. nephrostome $; b, b, b$, slender portion of the tubule into which the nephrostome opens; $c, c$, second ciliated portion; $\boldsymbol{d}$, glandular portion; $e$, muscular portion; $\varepsilon$ end of $e$ at which the nephridiopore opens. (From Gegenbaur.) little tubes called nephridia, of which each metamere-except the first three and the last possesses a pair, one on either side (Figs. 8I-84). You will remember that in the frog all the urinary tubules are connected together to form a pair of kidneys, each with a single duct communicating with the cloaca. In the earthworm each nephridium is a long and extremely delicate tube, arranged in three main loops (Fig. 84), opening at one end into the colome by a nephrostome and at the other communicating with the exterior directly (Fig. 8I). ${ }^{1}$ The tubes are attached to the posterior faces of the septa. Each nephrostome $(a)$ is ciliated, and projects through the corresponding septum so as to communicate with the segment of the body-cavity next in front of that in which the main part

1 In the frog the nephrostomes lose their connection with the nephridia, and open in the adult into the renal veins (Fig. 47 , p. 146$)$. 
of the tubule is situated. The nephrostome opens into a long and slender transparent part of the tube, lined with ciliated cells in part of its course and extending along the first and second loops $(b)$; this part is succeeded by a wider, ciliated portion in the second loop $(c)$, which communicates with a still wider portion (d) lined by granular, non-ciliated, glandular cells, also lying in the second loop; the glandular portion opens into a much wider muscular part of the tube $(e)$, which constitutes the third loop and communicates with the exterior by a small pore-the nephridiopore-near the outer seta of the inner couple. The muscular part is lined by an epithelium, while the rest of the nephridium is formed of a single row of hollowed cells, set end to end, like a series of drain-pipes, so that their cavity is intracellular, not intercellular.

Thus the nephridia, which are abundantly supplied with blood-vessels, are lined in part by gland-cells and in part by cilia which work towards the exterior. Water and nitrogenous waste from all parts of the body pass by diffusion inte the blood and are conveyed to the nephridia, the gland-cells of which withdraw the waste products and pass them into the cavities of the tubes, whence they are finally discharged from the body. The granular yellow colomic cells on the wall of the intestine also appear to contain excretory products, ${ }^{1}$ which become set free in the body-cavity and are thence got rid of by means of the nephridia. It will be noticed that a certain amount of loss of the cœlomic fluid must take place through the dorsal pores as well as through the nephridia.

In discussing the hydroid polypes we found that one of the most important points of difference between the

1 It is probable that in primitive forms the whole cœlomic epithelium was excretory in function. 
locomotive medusa and the fixed polype was the presence in the former of a well-developed nervous system (p. 319) consisting of an arrangement of peculiarly modified cells, to which automatic action was seen to be due. It is natural to expect in such an active and otherwise highlyorganised animal as the earthworm a nervous system

of a considerably

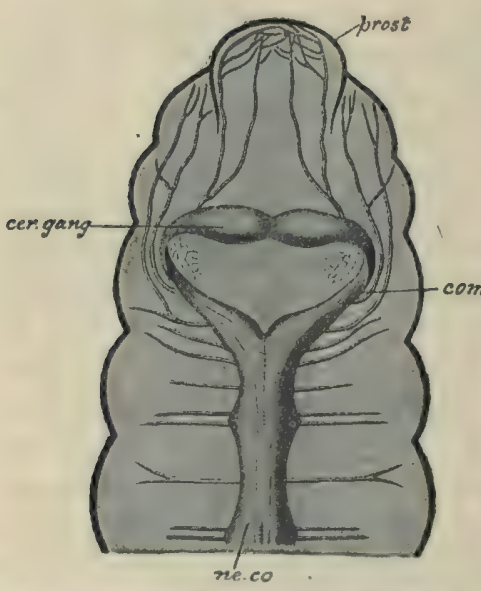

Fig. 85.-Anterior portion of nervous system of Lumbricus. ( $x$ about 8 .)

cer. gang. cerebral ganglia or brain ; com. œesophageal connectives; ne. co. ventral nervecord; prost. prostomium. (From Parker and Haswel!'s Zoology, after Leuckart.)

higher degree of complexity than that of a medusa.

The central nervous system (Figs. 82,83 , and 85) consists of two parts, the brain and the ventral nerve-cord. The brain consists of a pair of white pear-shaped swellings or ganglia situated on the dorsal side of the buccal sac, where it is continued into the pharynx. The ventral nerve-cord is a longitudinal band consisting of two cords surrounded by a common sheath extending along the whole middle ventral line of the body, internally to the longitudinal muscular layer, from the third to the anal segment, and slightly swollen in each segment. The brain is connected with the anterior end of the ventral nerve-cord by a pair of nervous bands, the pharyngeal connectives which pass respectively right and left of the buccal sac and thus form a nerve-collar: the cord is also primarily paired. 
It is to be noted that one division of the central nervous system-the brain-lies altogether above and in front of the enteric canal, the other division-the ventral nerve-cord-altogether beneath it ; and that, in virtue of the union of the two divisions by the pharyngeal connectives, the enteric canal perforates the nervous system. Both brain and cord are composed of delicate nerve-fibres and nerve-cells, the latter being situated in the ventral and lateral regions of the cord along its whole length, so that there is here hardly any distinction into ganglia and connectives, although the swellings are often spoken of as ganglia. Along the dorsal side of the cord are three transparent tube-like structures, known as giant-fibres, the function of which is not known (Fig. 8r). The whole cord is enclosed in a sheath consisting of connective-tissue and muscular fibres.

The peripheral nervous system consists of a number of nerves, both sensory and motor (p. I62), which arise from the central nervous system and supply the various parts of the body. From the brain a number of nerves are given off to the prostomium, and from each ganglionic enlargement two pairs of nerves can be traced into the body-wall, while between these enlargements one pair is given off which supply mainly the septa.

Comparing the nervous system of the earthworm with that of a medusa, it is important to notice the concentration of the central nervous system in the higher type, and the special concentration at the anterior end of the body to form a brain. When, again, we compare the central nervous system of the earthworm with that of the frog (pp. 28 and I55) several important points of difference are noticeable. In the former it lies freely in the colome, and, with the exception of the brain, is situated on the ventral side of the body ; while in the frog it is enclosed in a neural canal and is dorsal 
in position. The brain of the frog is a complicated structure, and the whole nervous system is hollow, there being ventricles in the brain and a central canal in the spinal cord; while in the earthworm the brain consists merely of a pair of cerebral ganglia, and it and the ventral cord are solid.

The whole nervous system is capable of originating automatic action. It is a well-known fact that if the body of an earthworm is cut into several pieces each performs independent movements ; in other words, the whole body is not, as in the higher animals, paralysed by removal of the brain (p. I72). There can, however, belittle doubt that complete co-ordination, i.e., the regulation of the various movements to a common end, is lost when the brain is removed.

The earthworm is devoid of organs of sight or hearing. It exhibits sensitiveness to bright light, which may be due to direct action on the central parts of the nervous system. The sense of hearing appears to be absent; but a faculty analogous to taste or smell enabling the animal to distinguish between different kinds of food is well developed. Groups of narrow sensory cells in the epiderm, which are most abundant on the prostomium and peristomium, have probably to do with this faculty.

There are two matters of general importance in connection with the structure of the earthworm to which special attention must be drawn.

Notice in the first place how in this type, far more than in Hydra, we have, as in the frog, certain definite parts of the body set apart as organs (p. 30) for the performance of particular functions: it is clear that differentiation of structure and division of physiological labour play a far more obvious and important part than 
in any of the lower organisms described in the five previous chapters.

Notice in the second place the vastly greater complexity of microscopic structure, the body being divisible into tissues (p. II 8), each clearly distinguishable from the rest. We have epithelial tissue with its cuticle, muscular tissue, and nervous tissue, as well as blood and cœlomic fluid. One result of this is that, to a far greater extent than in Hydra, we can study the morphology of the earthworm, as we have done that of the frog, under two distinct heads: anatomy and histology (p. I04).

Asexual reproduction does not take place normally in the earthworm, but it frequently happens by accident that a worm is cut into two or more parts. When this occurs, each end is able to reproduce the missing portion : this process is known as regeneration (compare pp. 268 and 307$)$.

The earthworm, like Hydra, is monœcious or hermaphrodite (p. 307), and besides the essential organs of sexual reproduction-ovaries and spermaries-which are, as in the frog, developed from certain parts of the cœlomic epithelium (p. 604), it possesses various accessory organs. The whole reproductive apparatus is situated in segments $9-15$.

The cvaries (Figs. 82, ov, and 86,o) are a pair of minute bodies about I mm. in length, attached by a short stalk, one on either side, to the posterior face of the septum separating segments $I 2$ and I3, not far from the nerve-cord. The proximal end of each ovary, nearest the stalk, is composed of a mass of undifferentiated cells of germinal epithelium (compare Figs. 62 and 63): nearer its middle, certain of these are seen to increase in size so as to be recognisable as young ova: while the 
distal end contains the ripe ova, arranged in a single row,

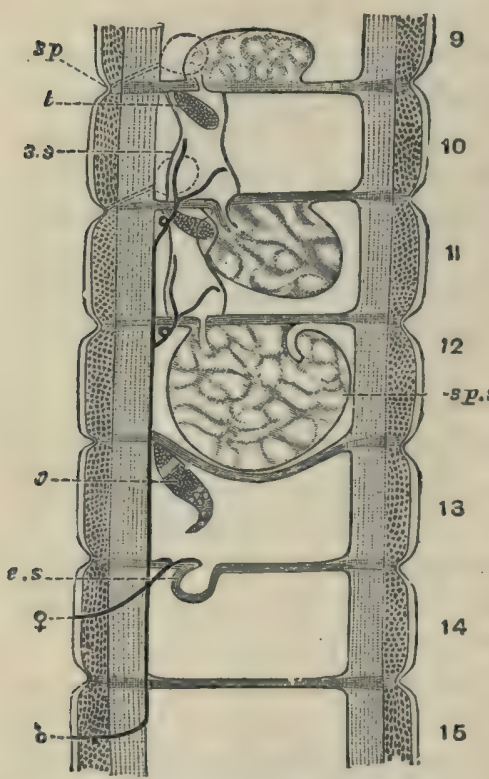

Fig. 86.-Diagrammatic longitudinal section of part of a Lumbricus, showing segments 9-I5 and the contained generative organs of one side $: \times 7$. In the body-wall the cuticle is indicated by a clear space, the circular muscles by irtegular dots, the longitudinal muscles by dotted longitudinal lines, and the peritoneal membrane by a thin line.

e. s. egg-sac; 0 . ovary ; sp. aperture of anterior spermotheca-both spermotheca are indicated by dotted lines; $s p . s$. posterior sperm. sac, the anterior and middle sacs, are not lettered; s8. sperm-reservoir; $t$. anterior spermary-the posterior is not lettered; \$. aperture of oviduct; $\delta$, aperture of spermiduct. The oviduct, spermiduct, and seminal funnels are indicated by thick lines. (From the Cambridge Natural History, after Hesse.) septum and extending back into the cavity of segment fourteen. vitelline membrane and containing a large nucleus and nucleolus and a number of granules of food-yolk (p. 195). The eggs are discharged into the cœlome and are received into the female gonoducts or oviducts (Fig. 82, o.d, and Fig. 86) - two short tubes, each with a wide ciliated mouth, placed opposite the corresponding ovary. The oviduct perforates the next following sep$\operatorname{tum}(i . e .$, that between segments thirteen and fourteen) to open by a minute aperture on the fourteenth segment, near the ventral couple of setæ. Connected with the mouth of each oviduct is a small egg-sac (Fig. $82, r .0$, Fig. $86, e . s)$, developed as an outgrowth from the same

each enclosed in a 
Certain globular sacs called spermothece (Fig 86) also belong to the female part of the reproductive apparatus. Of these there are usually two pairs (sometimes more than two in Allolobophora) situated in the ninth and tenth segments, and opening to the exterior between the ninth and tenth, and tenth and eleventh segments respectively ; their function will be mentioned presently.

The earthworm possesses two pairs of very minute spermaries attached to the posterior face of the septum between the ninth and tenth, and tenth and eleventh segments respectively (Figs. $82, t e$, and $86, t$ ). They have a flattened form and their free or distal ends are produced into finger-shaped processes. Behind each spermary, and in the same segment, is a ciliated seminal funnel opening into the cœlome and produced backwards through the septum next behind into an efferent duct, the two ducts of either side communicating with a main spermiduct or vas deferens. Each of these extends backwards in the ventral body-wall to open by a tumid lip on the fifteenth segment (Fig. 86), near the inner couple of setæ.

The most prominent portions of the reproductive apparatus are certain large whitish bodies-the spermsacs or seminal vesicles (Figs. 82, ves. sem, and 86, sp. s), which are very apparent in the adult worm as soon as the cœlome is cut open in this region. Of these there are three (Lumbricus) or four (Allolobophora) pairs, situated in segments 9-I2. They arise as outgrowths of the septa, and communicate with the cœlome; but in Lumbricus, the two anterior pairs and the posterior pair respectively become joined across the middle line so as to form tivo median sperm-reservoirs (med. ves. sem, and s. $s$ ), each of which encloses a colomic cavity in which one pair of spermaries and seminal funnels becomes enclosed. 
The cells of which the spermaries are composed do not develop into sperms in the testes themselves, but pass into the sperm-sacs, where they undergo division into rounded masses of cells (gametocytes, compare p. 286) looking very much like a segmenting oosperm in the polyplast stage. Each of these products of division of the testicular cells becomes elongated, and gradually takes on the form of a sperm with a rod-like head and a vibratile tail (compare pp. I94 and 307). When set free, the sperms pass into the spermiducts through the ciliated funnels.

It is well known that many flowers (the reproductive organs of higher plants) contain the generative cells of both sexes, enclosed within the ovules and anthers respectively; yet in very many cases self-fertilisation does not occur owing to contrivances of various kinds for its prevention. It has been proved in numerous instances that cross-fertilisation-i.e., the impregnation of the ovum in one individual by the male cell of another -is of great importance in keeping up the strength and vigour of the plant from generation to generation. The same is true amongst animals ; and though in some monœcious forms, such as the Hydra, there is no special arrangement for the prevention of self-impregnation if the male and female gametes of the same individual ripen at the same time, in others, such as the earthworm, the ova are always fertilised by the sperms from another individual.

This is effected in the earthworm in the following way. Two individuals, their anterior ends pointing in opposite directions, become applied together by their ventral surfaces and attached to one another in this position by a viscid secretion from the clitellum. The sperms are then passed from the male apertures of one into the spermothecæ of the other individual, and the 
two worms afterwards separate. The clitellum then secretes a tough chitinous tube or cocoon, which forms a broad ring round the body in this region, and which is gradually slipped forwards. As it passes over the apertures of the oviducts and spermothecæ, ova and sperms (the latter derived from the other individual) are passed into it, as well as albumen secreted by certain glands present in this region. When the worm has entirely withdrawn itself from the cocoon, the latter closes up at the ends in virtue of its elasticity, and the eggs, after fertilisation, undergo segmentation.

The cells of the polyplast soon become differentiated into an outer ectoderm, and an inner endoderm enclosing the enteron, which communicates with the exterior by the blastopore (compare p. 20I). A mesoderm (pp. 202 and 322 ) is then developed, and each layer gradually gives rise to the corresponding parts in the adult animal, much as in the frog, except that the nephridia are apparently derived in the first instance from the ectoderm (compare p. 209); the mesoderm undergoes segmentation, the cœlome appearing in it as a cavity (p. 203)-or rather as a series of cavities, one in each segment. The young worm is then hatched. and it is to be noticed that it passes through no metamorphosis (p. II).

There are a number of different kinds of animals commonly known as "worms," but many of these (e.g., the parasitic worms in the lungs and bladder of the frog, liver-flukes, tapeworms, etc.) are very different from the earthworm in structure, and are placed in several different phyla. The earthworm is a member of the phylum Annulata, which also includes a number of other worms living in the sea and in fresh water, as well as the leeches, etc. In all these the body is elongated, bilaterally symmetrical, and divided into metameres; the 
integument is soft ; the mouth is anterior, and the anus posterior; there is usually an extensive cœlome; and the nervous system and nephridia are essentially similar to those of the earthworm. The class Chretopoda ("bristle-footed" worms), in which the earthworms and their fresh-water and marine allies are included, receives its name from the fact that all its members are provided with cuticular setæ, which in the order to which the marine forms belong (Polychceta) are usually long and of varied forms, and are much more numerous than in the earthworms and fresh-water worms, which constitute the order Oligochata. This order includes several families, both Lumbricus and Allolobophora belonging to the family Lumbricida.

The oligochætous earthworm is, as we have seen adapted to a subterranean mode of life, and feeds on decaying organic matter. For comparison, it will be instructive to consider the external form and mode of life of a predaceous marine polychætous worm, called Nereis, various species of which are of common occurrence between tide-marks on the sea-shore, under stones, and among sea-weed in all parts of the world, sometimes swimming actively through the water. The worm varies considerably in colour even in the same species.

In shape (Fig. 87) the body, which may be about 7 or 8 centimetres in length, is long and narrow, approximately cylindrical, somewhat narrower towards the posterior end. A very distinct head, bearing eyes and tentacles, is recognisable at the anterior end; the rest is divided by a series of ring-like narrow grooves into a corresponding series of metameres, which are about eighty in number altogether; and each of these bears laterally a pair of movable muscular processes called parapods, provided with bundles of setæ. 
The head is constituted by the prostomium and the peristomium (p. 327). The former bears on its dorsal surface four large, rounded eyes, in front a pair of short cylindrical tentacles, and further back a pair of somewhat longer, stout appendages or palps. The peristomium, which has some resemblance to the segments of the body, though wanting the parapods, bears laterally four pairs of long, slender, cylindrical tentacles; on its ventral aspect is a transversely elongated aperture, the aperture of the mouth. The segments of the body differ little in external characters from one another throughout the length of the worm and there is no clitellum; each bears laterally a pair of parapods, which in the living animal are usually in active movement, aiding in creeping or acting as a series of oars for propelling it through the water. When one of the parapods (Fig. 88) is examined more attentively it is found to be biramous, consisting of two distinct divisions-a dorsal, which is termed the notopod (noto), and a ventral, termed the neuropod (neuro). Each of these is further subdivided into several lobes, and bears a bundle of setæ, lodged in a sac formed by invagination of the epiderm and capable of being

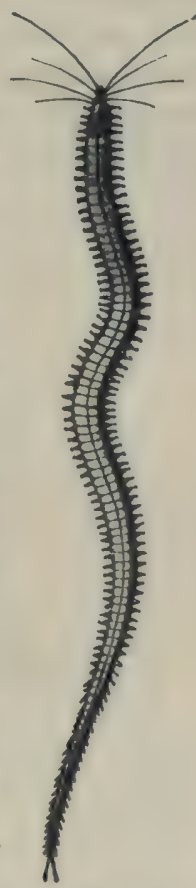

Fig.87.-Nereis dumerilii. Natural size. (From Parker and Haswell's Zoology, after Claparède.) protruded or retracted and turned in various directions by muscular fibres in the interior of the parapod. In each bundle there is, in addition to the ordirary setæ, a stouter, straight, dark-coloured seta (ac), 
the pointed apex of which projects only a short distance on the surface; this is termed the aciculum. The ordinary setæ are exceedingly fine but stiffish, chitinous rods, of which two principal kinds are recognisable; both have a terminal blade articulating with the main shaft of the seta by a distinct joint. On the dorsal side of the parapod is a short, cylindrical, tentacle-like appendage, the dorsal cirrus (Fig. 88, dors. cirr), and a similar, somewhat shorter, appendage, the ventral cirrus (vent. cirr), is situated on its ventral side. The last segment of the body, the anal segment, bears posteriorly a small rounded aperture, the anus; this segment is

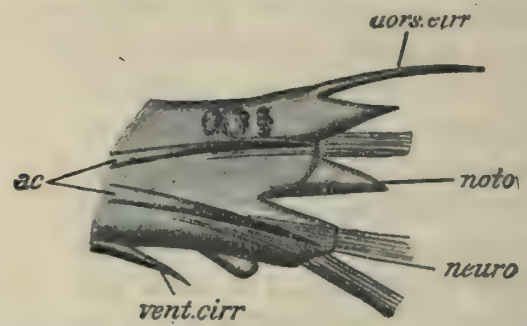

F1G. 88.-Nereis dumerilii. A single parapod ( $x$ about 25). ac, aciculum; dors, cirr, dorsal cirrus; neuro, neuropod; noto, notopod ; vent. cirr, ventral cirrus. (From Parker and Haswell's Zoology, after Claparède.)

devoid of parapods, but bears a pair of appendages, the anal cirri, similar in character to the cirri of the ordinary segments, but considerably longer.

On the ventral surface near the bases of the parapods, there is in each segment a pair of very fine nephridiopores (p. 34I), through which the sperms possibly pass to the exterior, there being no special genital duct. Dorsal pores are also wanting.

The mouth leads into a wide buccal cavity, which is continued back into a pharynx. In the pharynx are a number of very small, dark brown, chitinous denticles, which are very regularly arranged. The posterior part 
of the pharynx has very thick walls composed of bundles of muscular fibres which are concerned in the movements of a pair of laterally placed chitinous jaws. The anterior part of the alimentary canal is capable of being everted, as a proboscis, until the jaws are thrust forth and project freely, so that they can be brought to bear on some small living animal or fragment of animal matter, which is thus seized and swallowed.

In correspondence with its different mode of life,

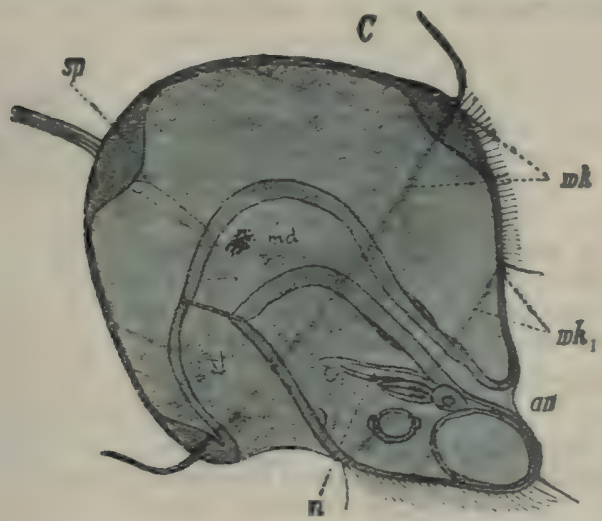

Fic. 89. - Trochosphere of the worm Eupomatus, from the side, highly magnitied. an. anus ; md. mid-gut : n. larval heasl-nephridium : sp. ne-ural plate brain); st. stomodatum ; "र́. pre-oral ciliated rus ; rk.1 pest-oral ciliated ring. (From Lang's Comparatue Anatomy, after Hatschek.)

Nereis is much better provided with sense-organs than is the earthworm. The tentacles and palps, as well as the cirri, are probably organs of touch; and as we have already seen, four eyes are present on the prostomium. Each eye consists of a doubly-pigmented cup, the retina, formed as an invagination of the ectoderm, with a small, rounded aperture, or pupil, and enclosing a mass of gelatinous matter, the lens. The cuticle of the general 
surface passes over the eye, and a continuation of the epiderm, with its cells somewhat flattened, constitutes a cornea (compare p. 182 ).

Almost without exception, the Polychæta further differ from the Oligochæta in being diocious, and in passing through a metamorphosis. The segmented oosperm gives rise to a minute, more or less oval larva known as a trochosphere (Fig. 89), which swims by means of cilia arranged in circles round the body, and gradually undergoes metamorphosis into the adult.

\section{PRACTICAL DIRECTIONS}

\section{EARTHWORM}

Select a large earthworm, and after noting its movements and mode of progression, kill by immersion in spirit for a few minutes and then place in-a dish and let the tap run on it for a short time.

\section{$A$. External Characters.}

I. Note:- $-a$. The form and-colour of the body and its division into metameres: $b$, the anterior end, terminating in the prostomium and followed by the peristomium; $c$. the clitellum; and $d$. the last or anal segment.

2. If the worm be drawn through the fingers-backwards, the setce will be felt: examine with a lens and observe their position and the number in each segment.

3. Make out the following apertures: $a$. the mouth; b. the anus; $c$. the dorsal pores (p. 333) ; $d$. the two apertures of the spermiducts, with thickened lips, on the fifteenth segment.

(It requires careful examination to see the other apertures, viz.--those of the oviducts, spermotheca, and nephridia.)

Sketch from below or from the side.

\section{$B$. Dissection.}

I. Take a freshly-killed worm in the left hand, and carefully insert the point of the fine scissors into the integument about one-third of the way along the body, close to the middle dorsal line. Place a drop of the colomic fuid 
which exudes on a slide, add a drop of salt solution and cover. Examine with the low and high powers, and note the structure and movements of the amaboid corpuscles. Sketch at intervals. (The small granules you will notice in the coelomic fluid are derived from the broken down ye low cells of the intestine.)

II. Continue the cut forwards to the prostomium, keeping very slightly to one side of the median dorsal line, and taking care that the point of the scissors does not penetrate deeper than the integument: note the iridescent cuticle. Place the animal in a dish with just enough water to cover it; and carefully insert a pin between the integument and the yellow intestine on either side, near the posterior end of the incision, so as to expose the calome: note the septa connecting the body-wall with the intestine. Then insert more pins, obliquely, so as to expose the coelome and enteric canal up to the anterior end, taking especial care not to tear the ventral parts of the septa and to stretch the animal longitudinally as much as possible. Then note :-

I. The sperm-sacs - three or four pairs of large white bodies in segments IX-XII, and varying greatly in size and form according to the size of the animal. If your specimen is an adult Lumbricus, you will notice that the two anterior pairs and the posterior pair are respectively united across the middle line, beneath the enteric canal, to form the two sperm-reservoirs.

2. The enteric cana? and its subdivisions:- $a$. buccal sac ; b. pharynx; c. gullet (largely hidden by the sperm-sacs); d. $c r o p$; e. gizzard; and $f$. intestine, covered with a layer of yellow cells.

3. The dorsal blood-ressel, containing red blood and giving off branches to the enteric canal; the large rhythmically contractile commissural ressels connecting the dorsal with the ventral (sub-intestinal) vessel: the latter will be seen subsequently.

4. A pair of small, whitish, coiled bodies, the nephridia, attached to the posterior face of each septum exposed (except the first three), on either side of the enteric canal. Carefully remove one of these in the region of the intestine(take hold of the septum with the fine forceps, and cut around the nephridium with the small scissors)-mount in salt-solution or water, and examine first with the low power, and then with the high power. Note that the nephridium consists of a long, coiled tube, plentifully supplied with blood-vessels, and that long vibratile cilia can be seen in 
parts of it: look for the ciliated nephrostome. (For details see $\S$ VI.)

Add a little methylated spirit to the water in your dissecting dish and sketch your dissection.

5. The ovaries. Examine segment XIII closely, being very careful not to injure its contents, and the ovaries may then be seen projecting backwards into this segment, one on either side just in front of the crop. They can easily be recognised by their shape, and by the fact that they hang freely into the cœlome, as can be seen by touching them with a seeker. Carefully seize the septum between segments XII and XIII with the small forceps, and cut around the attachment of an ovary so as to remove it. Stain with methyl-green or magenta, and mount in glycerine (or else fix, stain, and mount in balsam, as directed on p. 136). Note the mass of undifferentiated cells at the proximal, attached end of the ovary, and the gradual development of the ova towards the distal, narrower end. Examine the ovum, and observe the nucleus, nucleolus, and granules of food-yolk. Sketch the ovary.

6. The globular spermothece (usually two pairs, see p. 347) in segments IX -X.

III. Tease out a small portion of a sperm-sac, stain with magenta, and mount in glycerine. The following stages in the development of the sperms can then be made out:$a$. the sperm-mother-rells or gametocytes (developed in the spermary) in different stages of division; the products of division, each with a nucleus, and arranged in a single peripheral row, the central mass of protoplasm remaining undivided; $b$. the gradual elongation of these small cells ; and $c$. the conversion of each into a sperm, the nucleus forming the rod-like " head," and the protoplasm giving rise to the delicate " tail "; $d$. free sperms (also to be found in the spermothecæ; some of them should be examined fresh and their movements noted). Sketch a series of stages.

It is not easy to make a satisfactory dissection to show in situ the ovaries, oviducts, and ovisacs, as well as the spermaries, seminal funnels, and spermiducts; but an attempt should be made at a later stage with a worm preserved in spirit (see $\S$ VI.) : if possible, transverse sections of the generative region, prepared as directed in $\S C$., should also be examined. Note again the ovaries, and also the oviducts and ovisacs, which latter can be recognised by their red colour. Each seminal funnel appears something like a 
minute cauliflower: look for the spermiducts (partly imbedded in the body-wall) in connection with the funnels, and for the two pairs of spermaries, each projecting backwards from the septum in front of the corresponding seminal funnel. In Lumbricus the spermaries and funnels are enclosed within the median sperm-reserioirs (p. 355), which must be cut open and washed out in order to see them (compare Figs. 82 and 86). Remove and examine an ovary, a spermary, and a seminal funnel.

IV. Remove the sperm-sacs carefully, and make out further details as regards the enteric canal (see § II. 2). Note the osophageal pouches and glands. Cut open part of the intestine along one side, and observe the thick dorsal fold or typhlosole projecting into it. Sketch.

V. I. Note the small cerebral ganglia or brain on the dorsal side of the buccal sac, and then cut through the anterior part of the pharynx just behind the brain. Carefully remove the enteric canal, noting as you do so the ventral sub-intestinal blood-ressel. The nevious system will now be exposed. Observe again the paired cerebral ganglia, from which arise a pair of connectives, forming a small nerve-ring or collar around the buccal sac, and continuous ventrally with the ventral nerve-cord, consisting of fused lateral halves and extending along the whole length of the ventral bodywall, passing through a perforation in each septum, and expanding slightly in each segment, so as to form ganglionic swellings. Three pairs of nerves are given off in each segment. Sketch.

2. Remove the nerve-ring and a small portion of the ventral cord, and examine with the low power. Sketch.

3. A lateral neural vessel can be seen close to the ventral cord on either side. Remove a portion of the cord, and note the sub-neural vessel.

VI. Further details as regards the nephridia and generative organs (see above) are best made out in a worm which has been preserved in spirit. Very carefully cut away the enteric canal from the gullet backwards so as not to injure the septa more than necessary; the nephrostomes can then be seen with a lens, looking like small, whitish dots. Remove an entire nephridium carefully as before (\$ II. 4), stain, and mount in glycerine or balsam. Note the three loops, and$a$. the ciliated nephrostome; $b$. the first, slender part of the tube with its cilia; $c$. the second, wider, ciliated part; $d$. the third, still wider, glandular part; and $e$. the fourth, 
much wider, muscular part which opens on to the exterior by the nephridiopore (Fig. 84). Sketch.

VII. Remove a small piece of the integument containing setce, and separate the latter out with needles. Mount in water and examine. Sketch.

C. Transverse Sections.-For the preparation of these, keep a worm for a few clays in coffee-grounds or small pieces of blotting-paper moistened with water, in order that the gritty contents of its intestine may be replaced by a soft substance which will not blunt the razor. Kill the worm, cut off a small piece about 3 inch in length from the region of the intestine, and fix, stain, and cut into transverse sections as directed on p. 136. Examine a section with the low power, and note:-

I. a. The thin cuticle; $b$. the epiderm, enclosing gobletcells (unicellular glands); $c$. the very thin derm; $d$. the sete, with their sacs and muscles, if your section passes through one or more of them.

2. The muscles of the bodv-wall. a. The external circular layer; and $b$. the thicker longitudinal layer, appearing feather-like in transverse section and broken up into bands in the regions of the dorsal pores and setæe. Note that the muscles are unstriped.

3. The coelome and peritonea! membrane.

4. The intestine, with its thick dorsal typhlosole. It is lined by a single layer of columnar cells (enteric epithelium), outside which is a thin muscular and connective-tissue layer. Externally to this, again, are the elongated and granular yellow cells, which are especially abundant in the typhlosole.

5. The dorsal, ventral, and intestinal blood-vessels.

6. The ventral nevve-cord, just internal to the longitudinal muscular layer. It is enclosed in a muscular and connectivetissue sheath, embedded in which the sub-neural and lateral neural vessels can be seen. Along the dorsal side are three clear-looking "giant fibres." Observe the nerve-cells along the cord ventrally and laterally, the nevies coming off from the cord, and the symmetrical halves of which the cord is composed.

7. The nephridia:-these will be seen cut through in various planes.

The thin septa will be cut through in different directions, and their relations are therefore not easily seen in sections ; note the circular and radial muscular fibres in them. Sketch 
the lateral half of your section, and then put on the high power and work through $\$ \$ I-7$ again. Notice that the cavities in the sections of the nephridia are intra-cellular, except in the muscular part (p. 34I). Sketch as many details as possible.

Sift some earth and look for cocoons.

\section{NEREIS}

Note:-I. The head, consisting of $(a)$ prostomium with its tentacles, palps, and eycs, and $(b)$ peristomium with its tentacles; also the proboscis, mouth, and lateral chitinous jaws.

2. The metameres of the body, each with a pair of (a) torsal and ientral cirri, and (b) lobed parapods, each subclivided into notopod and neuropod, and bearing a bundle of chitinous setce.

3. The anal segment with anal cirri, but no parapods.

Carefully cut out an entire parapod and mount it on a slicle (Fig. 88). Note the two bundles of seta, each made up of an aciculum and of numerous jointed setæ of two different forms. The structure of the seta will be more easily made out if separated from one another with needles, or if the entire parapod is treated with a solution made by dissolving 5 grammes of potassium hydrate in Ioo c.c. of water.

Prepare sections, in the usual way, of the prostomium, passing through the eyes, and note the pigmented optic cup and retina, the pupil, and lens.

Procure some trochosphere larve (see note on p. 325). Mount and compare with Fig. 89. 


\section{CHAPTER VII}

THE CRAYFISH-CHARACTERS OF THE PHYLUM ARTHROPODA

WE have now to study an animal formed on a very similar plan of structure to the earthworm as regards segmentation and arrangement of many of the organs, but which reaches in every respect a far higher grade of organisation.

The common British fresh-water crayfish is usually known as Astacus fuviatilis, and is found in many of the streams and rivers of England and Ireland, hiding under stones or in holes, into which it darts very suddenly on the approach of danger. Its ordinary creeping movements are slow, and are effected by means of a number of jointed limbs, for which reason it is included, together with insects, spiders, scorpions, \&c., in the phylum Arthropoda (p. 220).

In colour, the crayfish is greenish-grey, and in form it is very similar to the marine Lobster, to which the following description will apply almost equally well.

In addition to the presence of paired limbs or appendages, one of the most striking points of difference between an earthworm and a crayfish is the smaller and constant number of segments or metameres in the latter as well as the fact that certain of these are more or less 
firmly united with one another-i.e., have undergone concrescence. The result of this fusion of the segments is that two distinct regions can be distinguished in the body -an anterior cephalothorax and a posterior abdomen (Fig. 93, cth, ab).

The cephalothorax is unjointed and is covered by a cuirass-like structure, the carapace; and the abdomen is divided into distinct segments, movable upon one another in a vertical plane. The cephalothorax is divided into two regions, an anterior-the head, and a posterior-the thorax, by a transverse depression, the cervical groove. The carapace is developed from the dorsal and lateral regions of both head and thorax ; it is free at the sides of the thorax, where it forms a flap or gillcover (Fig. 94, $k d$ ) on each side, separated from the actual body-wall by a narrow space in which the gills are contained.

The limbs spring from the ventral surface. Both trunk and appendages are covered with a sort of shell formed of chiiin (p. 328), strongly impregnated with carbonate of lime in most parts, so as to be hard and but slightly elastic.

The abdomen is made up of seven segments : the first six of these are to be considered as metameres in the sense in which the word is used in the case of the earthworm. Each has a ring-like form, presenting a broad dorsal region or tergum (Fig. 90, T), a narrow ventral region or sternum $(\mathrm{S})$, and downwardly directed lateral processes, the pleura (PL). The seventh division of the abdomen is the telson (Fig. 93,t): it is flattened horizontally and divided by a transverse groove into anterior and posterior portions. All seven segments are calcified and are united to one another by uncalcified articular membranes: the first segment is similarly joined to the thorax. Thus the exoskeleton of the crayfish is a continuous cucicular structure, discontinuously calci- 
fied so as to have the character of a hard, jointed armour. Tufts of minute feather-like cuticular structures, or sete, are present on various parts of the exoskeleton both of the body and appendages.

It has been stated that the abdominal segments are movable upon one another in a vertical plane, i.e., the

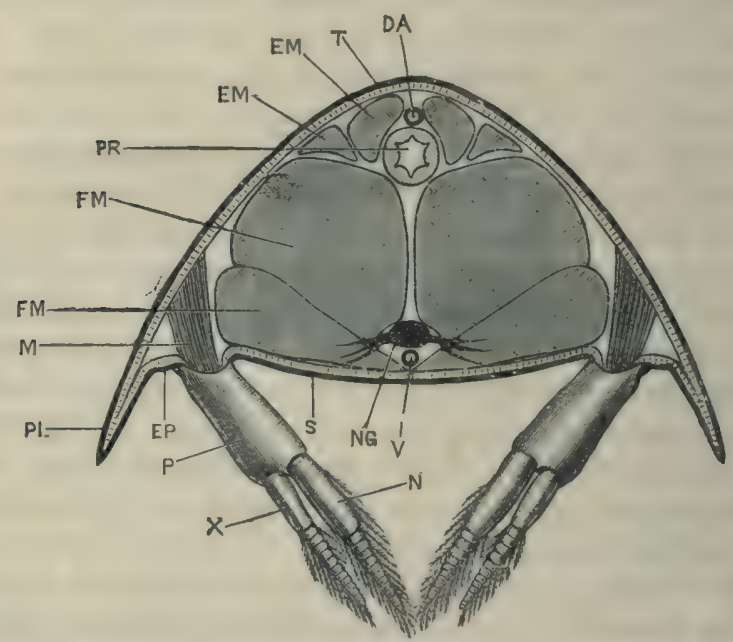

FIc. 90.- Transverse section of abdomen of (rayfish. $(\times 4$.

DA. clorsal abdominal artery; K.M. dorsal muscles of the abiomen; EP. space between the pleuron and the appendage; H.M. ventral muscles of the abdomen; M. muscles of the apnendage; N. endopodite; NG.nerve-ganglion; P. protopodite; I'L. pleuron; I'R. hind-gut; S. stermum; T. tergum; Y. ventral abdominal artery; $\mathbf{X}$. exopodite. (From Marshall and Hurst's Zoology.)

whole abdomen can be extended or straightened, and flexed or bent under the cephalothorax; the segments are incapable of movement from side to side. This is due to the fact that, while adjacent segments are connected dorsally and ventrally by flexible articular membranes, they present at each side a joint, placed at 
the junction of the tergum and pleuron, and formed by a little peg-like process of one segment fitting into a depression or socket in the other. A line drawn between the right and left joints constitutes the axis of articulation, and the only possible movement is in a plane at right angles to this axis.

Owing to the presence of the carapace the thoracic region is immovable, and shows no distinction into segments either on its dorsal (tergal) or lateral (pleural) aspect. But on the ventral surface the sterna of the thoracic segments are clearly marked off by transverse grooves, and the hindmost of them is slightly movable. Altogether eight thoracic segments can be counted.

The ventral and lateral regions of the thoracic exoskeleton are produced into the interior of the body in the form of a segmental series of calcified plates, so arranged as to form a row of lateral chambers in which lie the muscles of the limbs and a median tunnel-like -passage or sternal canal, containing the thoracic portion of the nervous system (Fig. 94). The entire endophragmal system, as this series of plates is called, constitutes a kind of internal skeleton.

The head exhibits no segmentation: its sternal region is formed largely by a shield-shaped plate, the epistoma, nearly vertical in position. The ventral surface of the head is, in fact, bent upwards, so as to face forwards instead of downwards. The cephalic region of the carapace is produced in front into a large median spine, the rostrum: immediately below it is a plate from which spring two movably articulated cylindrical bodies, the eye-stalks, bearing the eyes at their ends.

The appendages have very various forms, and are all, like the abdomen, jointed or segmented, being divisible into freely articulated limb-segments or podomeres. You will at once notice the long feelers attached to the 
head, the five pairs of legs springing from the thorax, and the little fin-like bodies arising from the sterna of the abdomen. It will be convenient to begin with the last-named region.

The third, fourth and fifth segments of the abdomen bear each a pair of small appendages, the swimming feet or pleopods (Fig. 90, P, N, X). A pleopod (Fig. 9I, Io) consists of an axis or protopodite having a very short proximal ( $p r . \mathrm{I})$ and a long distal $(p r .2)$ podomere, and bearing at its free end two jointed plates, fringed with setæ, the endopodite (en) and exopodite (ex). These appendages act as fins, moving backwards and forwards with a regular swing, and probably aiding in the animal's forward movements.

In the female a similar appendage is borne on the second abdominal segment, while that of the first is more or less vestigial (p. I59). In the male the first and second pleopods (Fig. 9I, 9) are modified to form incomplete tubes which serve to transfer the spermatophores (p. 382) to the body of the female. The sixth pair of pleopods (II) are alike in the two sexes: they are very large, both endopodite and exopodite having the form of broad, flat plates: in the natural position of the parts they lie one on either side of the telson, forming with it a large five-lobed tail-fin: they are therefore conveniently called uropods or tail-feet. The telson itself bears no appendages.

The thoracic appendages are very different. The four posterior segments bear long, slender, jointed legs (8), with which the animal walks : in front of these is a pair of very large legs terminating in huge claws or chela, and hence called chelipeds (Fig. 93, bf. 4). The three anterior thoracic segments bear much smaller appendages, more or less leg-like in form, but serving as jaws: they are distinguished as maxillipeds or foot-jaws. 

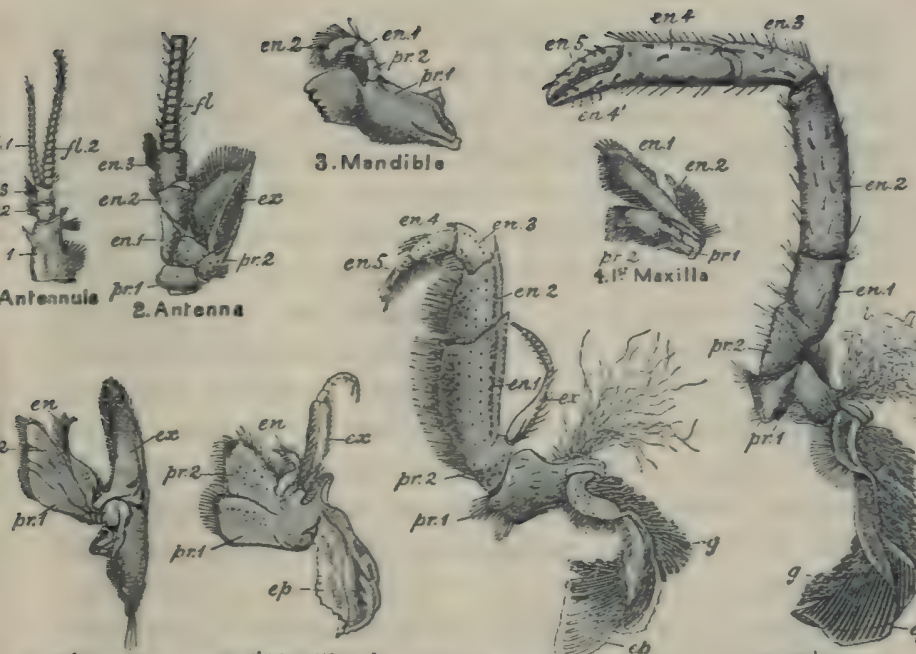

5. ${ }^{2}$ Maxilla

6. 1" Maxilliped

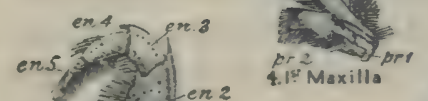

7. 3. Maxilliped
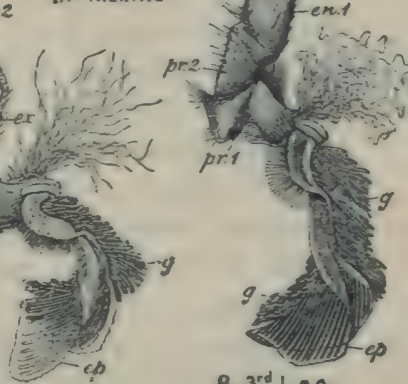

8. 3 ? $^{\text {d }}$ Lo8

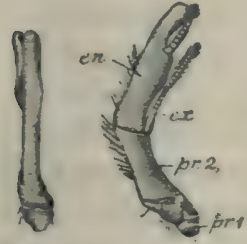

Q. Copulatery Organs

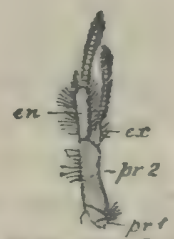

10.Swimming Foof

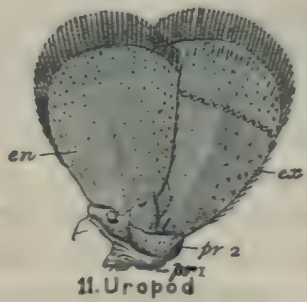

11. Uropod

Frg. 9r-Typical appendages of the Fresh-water Crayfish, placed in the same position, with the protopodite ( $p r)$ and epipodite $(e p)$ downwards, the endopodite $(e x)$ to the left, and the exoporite $(e x)$ to the right. ( $x$ 1 $\%$.)

The protopodite is typically formed of two podomeres (pr. $1, f \%, 2)$, the endopodite of five $(e n .1-e n .5)$ : a gill $(g)$ mav be attached to the epipodite.

The three proximal segments of the antennule are marked $I-3$, its flagella $f$. I and $f .2$; the distal end of the endopodite of the antenna is a flagellum $(f)$. (The tufts of threads in 7 and 8 are very long setæ which extend between the gills.) (From Parker and Haswell's Zoology, after Huxley.)

The structure of these appendages is best understood by a consideration of the third maxilliped (Fig. 9I, 7). The main portion of the limb is formed of seven podo- 
meres arranged in a single series, strongly calcified, and-with the exception of the second and third, which are fused-movably articulated with one another. The second podomere, counting from the proximal end, bears a many-jointed feeler-like organ $(e x)$, and from the first springs a thin, folded plate $(e p)$ having a plume-like gill $(g)$ attached to it. The first two segments of the axis form the protopodite $(p r . I, 2)$, its remaining five segments the endopodite (en. I-5), the base of which is toothed ; and the feeler, which is directed outwards, or away from the median plane, the exopodite $(e x)$. The folded plate is called the epipodite: in the natural position of the parts it is directed upwards, and lies in the gillcavity between the proper wall of the thorax and the gill-cover.

The five legs (8) differ from the third maxilliped in their greater size, and in having no exopodite: in the fifth or last the epipodite also is absent. The first three of them have undergone a curious modification, by which their ends are converted into pincers or chele: the fourth segment of the endopodite (sixth of the entirc limb, en. 4) is produced distally so as to form a claw-like projection $\left(e n .4^{\prime}\right)$, against which the terminal segment (en. 5) bites. The first leg is much stouter than any of the others, and its chela is of immense size and forms an important weapon of offence and defence. The second maxilliped resembles the third, but its endopodite is smaller: the first (6) has its endopodite greatly reduced, the two segments of its protopodite large and leaf-like, and no gill is connected with the epipodite.

The head bears a pair of mandibles and two pairs of maxille in relation with the mouth, and in front of that aperture a pair of antenmules and of antenne. The hindmost appendage of the head is the second maxilla (5), a leaf-like appendage, its protopodite being cut up into 
lobes, while the exopodite is modified into a boomerangshaped plate, which, as we shall see, is an important accessory organ of respiration. The first maxilla (4) is a very small organ having neither exopodite nor epipodite. The mandible (3) is a large, strongly calcified body, toothed along its inner edge, and bearing on its anterior border a little three-jointed, feeler-like structure, the palp, the two distal segments of which represent the endopodite, its proximal segment, together with the mandible proper, the protopodite.

The antenna (2) is of great length, being nearly as long as the whole body. It consists of an axis of five podomeres, the fifth or last of which bears a long, flexible, many-jointed structure, or feeler $(f)$, while from the second segment springs a scale-like body or squame (ex). It is fairly obvious that the two proximal segments represent the protopodite, the remaining three, with the feeler, the endopodite, and the squame the exopodite. On the ventral side of the basal segment of the protopodite is a conical elevation on which the duct of the excretory organ opens (p. 375).

The antennule (I) has an axis of three podomeres ending in two many-jointed feelers ( $f$. I, $f$. 2 ), which are sometimes considered as corresponding to the endopodite and exopodite. But in all the other limbs, as we have seen, the exopodite springs from the second segment of the axis, and the probabilities are that there is no exact correspondence between the parts of the antennule and those of the remaining appendages.

The eye-stalks, already noticed, arise just above the antennules, and are formed each of a small proximal and a large distal segment. They are sometimes counted as appendages serially homologous with the antennæ and legs, but are more properly to be looked upon as articulated processes of the prostomium. It is probable 
that the antennules are also prostomial and not metameric structures: assuming this to be the case, it will be seen that the body of the crayfish consists of a prostomium, eighteen metameres, and a telson, which is probably composed of an anal segment plus a post-anal extension. The prostomium bears eye-stalks and antennules: the first four metameres are fused with the prostomium to form the head, and bear the antennæ, mandibles, first maxillæ, and second maxillæ: the next eight metameres (5th-I2th) constitute the thorax, and bear the three pairs of maxillipeds and the five pairs of legs: the remaining six metameres (I 3 th-I8th), together with the anal segment, constitute the abodmen, and bear five pairs of pleopods and one of uropods.

The articulation of the various podomeres of the appendages is on the same plan as that of the abdominal segments (p. 362). The podomeres are, it must be remembered, rigid tubes: they are connected with one another by flexible articular membranes (Fig. 92, art. $m$ ), but at two points the adjacent ends of the tubes come into contact with one another and are articulated by peg-and-socket joints $(h)$, the two joints being at opposite ends of a diameter which forms the axis of articulation. The two podomeres can therefore be moved upon one another in a plane at right angles to the axis of articulation and in no other direction, the joints being pure hinge-joints (p. 57). As a rule the range of movement is from the perpendicular to a tolerably extensive flexion on one side-the articulations are single-jointed, like our own elbows and knees. The whole limb is, however, capable of universal movement, owing to the fact that the axes of articulation vary in direction in successive joints : the first joint of a limb bending, for instance, up and down, the next obliquely, the next backwards and forwards, and so on. In some cases-e.g., the pleopods--peg-and-socket joints are absent, the articula- 
tion being formed merely by an annular articular membrane, movement being therefore possible in any plane.

Sections show the body-wall to consist of an integument composed of a layer of deric epithelium or epiderm (p. 329) secreting a thick cuticle, and a layer of connective-tissue forming the derm, beneath which is a very thick layer of large and complicated muscles which fill up a great part of the interior of the body. Neither on the epiderm nor elsewhere are there any cilia, the absence of these structures being generally characteristic of Arthropods.

The cuticle is of great thickness, and except at the joints between the various segments of the body and limbs, is impregnated with lime-salts so as to form a hard, jointed armour. It thus constitutes a cuticular exoskeleton, forming a continuous investment over the whole body but discontinuously calcified. It is shed entire and renewed periodically-once a year during adult life---the process being known as ecdysis, growth taking place during the period between ecdysis and renewal while the animal is soft.

The muscular system shows a great advance in complexity over that of the earthworm, and consists entirely of transversely striated fibres (compare p. II2). In the abdomen the muscles are of great size, and are divisible into a smaller dorsal and a larger ventral set. The dorsal muscles (Figs. 90 and 93, em) are paired longitudinal bands, divided into segments called myomeres (p. 203), and inserted by connective-tissue into the anterior border of each segment: in front they are traceable into the thorax, where they arise from the side-walls of that region. When these muscles contract they draw the anterior edge of each tergum under the posterior edge of its predecessor, and thus extend or straighten the abdomen (compare p. 63).

The ventral muscles (Figs. 90 and $93, f m$ ) are 
extraordinarily complex and cannot be described in detail here. They partly aid the dorsal muscles in extending the abdomen, but are cbiefly important in producing an approximation of the sterna, and thus in flexing the abdomen. The ventral muscles are, like the dorsal, traceable into the thorax, where they arise from the endophragmal system (p. 363). The flexor muscles are immensely powerful, and produce, when acting together, a sudden and violent bending of the abdomen upon the cephalothorax, causing the crayfish to dart backwards with great rapidity. There is also a paired rotator of the abdomen.

It will thus be seen that the body-muscles of the crayfish cannot be said to form a layer of the body-wall, as in the earthworm (Fig. 8I), but constitute an immense fleshy mass, filling up the greater part of the.bodycavity (see p. 373), and leaving a very small space around the enteric canal.

In the limbs the essential arrangement of the muscles in relation with the joints in Arthropods is more easily seen (Fig. 92): each podomere is acted upon by two muscles situated in the next proximal podomere. These muscles are inserted, by chitinous and often calcified tendons, into the proximal edge of the segment to be moved, the smaller $(e x t)$ on the extensor, the larger $(f)$ on the flexor side, in each case half-way between the two hinges, so that a line joining the two muscular insertions is at right angles to the axis of articulation.

The digestive organs are constructed on the same general plan as those of the earthworm, but present many striking differences. The mouth lies in the middle ventral line of the head and is bounded in front by a shield-shaped process, the labrum, at the sides by the mandibles, and behind by a pair of delicate lobes, the paragnatha. It leads by a short wide gullet (Fig. 93, oc) into a capacious gizzard, often spoken of as the stomach, 
which occupies a great part of the interior of the head, and is divided into a large anterior division (cs) and a smaller posterior division ( $p s_{\text {) }}$; the latter passes into the intestine, which consists of a narrow and very short mid-gut or mesenteron (md), from which a somewhat wider hind-gut $h d$ ) extends to the anus (an), situated on the ventral surface of the telson. The gullet and gizzard together constitute the fore-gut.

The outer layer of the enteric canal consists of connective-tissue containing striped muscular fibres; within this is a single layer of columnar epitheiial cells, none of them glandular. In the fore- and hind-gut the epithelium secretes a layer of chitin, which thus constitutes the innermost layer of their cavities. It is proved by development that the midgut, which has no chitinous lining, is the only part of

Fic. 92.-A leg of the Fresh-water Crayfish with part of the exoskeleton removed to show the muscles. ( $(\times 5$.

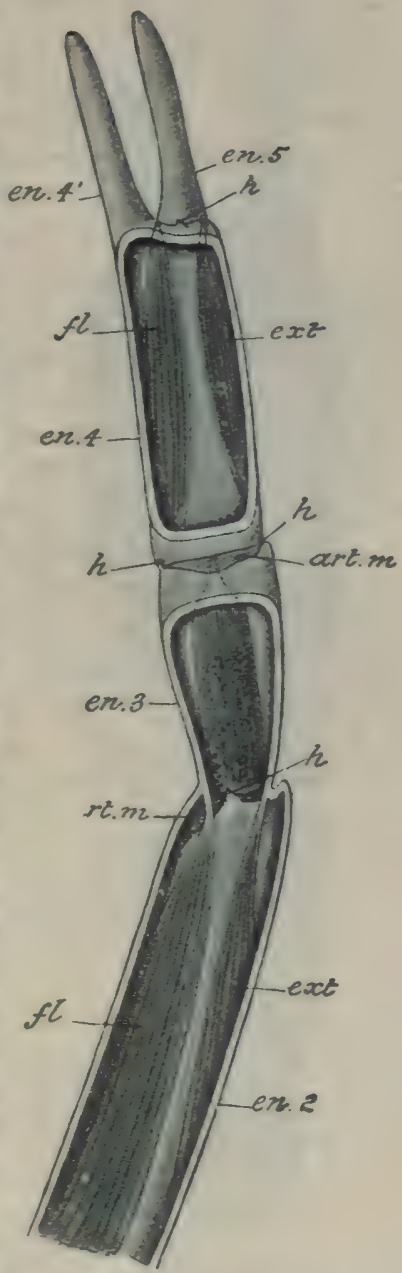
exrensor muscle; $f$. flexor muscle. (From Parker and Haswell's Zoology.) 


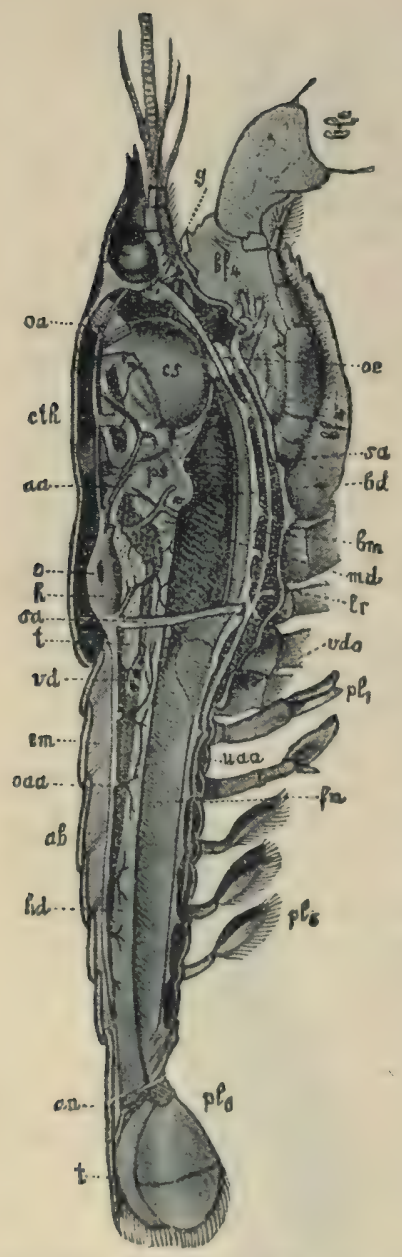

the enteric canal developed from the enteron of the embryo: the gullet and gizzard (fore-gut) arise from the stomodæum, the hind-gut from the proctodæum ( $p$. 204). Thus only a very small portion of the enteric epithelium is endodermal.

In the anterior division of the gizzard the chitinous lining is thickened and calcified in certain parts, so as to form a complex articulated framework, usually known as the " gastric mill," on which are borne one median and two lateral teeth, strongly calcified and projecting into the cavity of the gizzard. Two pairs of strong muscles arise from the carapace, and are inserted on

FIG. 93.-Dissection of Fresh-water Crayfish, made by removing the exoskeleton with the appendages, and the muscles, digestive gland and excretory organ, of the right side. (Nat. size.)

$a a$. antennary artery; $a b$. abdomen; $a n$. anus; $b . d$. aperture of right digestive duct exposed by removal of gland; bf. 4, cheliped; bm. ventral nervecord; cs. anterior division of gizzard; $c t h$. cephalothorax; $\mathrm{cm}$. dorsal muscles; $f m$. ventral muscles; $g$. brain ; $h$. heart; hd. hind-gut; $l$. left digestive gland; md. mid-gut; 0 . right lateral ostium of heart; oa. ophthalmic artery : oaa. dorsal abdominal artery ; oc. gullet ; $p l .1-5$, pleopods ; $p l .6$, uropod ; ps. posterior division of gizzard; s. a. sternal artery; $t$. (near heart), spermary ; $t$. (below an) telson; waa. ventral abdominal axtery; $v$. $d$. spermiduct; vdo. male genital-aperture. (From Lang, after Huxley.) 
the gizzard (Fig. 93): when they contract they move the mill in such a way that the three teeth meet in the middle line and complete the comminution of the food begun by the jaws. The separation of the teeth is effected partly by the elasticity of the mill, partly by delicate muscles in the walls of the gizzard. The posterior division of the gizzard forms a strainer: its walls are thickened and produced into numerous setæ, which extend quite across the narrow lumen and prevent the passage of any but finely divided particles into the intestine. Thus the gizzard has no digestive function, but is merely a masticating and straining apparatusit is therefore not a stomach either in the embryological or in the physiological sense. On either side of its anterior division is found, at certain seasons of the year, a plano-convex mass of calcareous matter, the gastrolith or " crab's-eye," which possibly merely serves to store up reserve calcareous material for use after the next ecdysis.

The digestion of the food, and to some extent the absorption of the digested products, are performed by a pair of large glands (Fig. 93, lr), lying one on either side of the gizzard and anterior end of the intestine. They are formed of finger-like sacs or caca, which discharge into wide ducts opening into the mid-gut, and are lined with glandular epithelium derived from the endoderm of the embryo. The glands are often spoken of as the liver, but as they have a complex function, and the fluid they secrete is able to digest proteids, it is better to call them simply digestive glands. The crayfish is largely carnivorous, its food consisting of decaying animal as well as vegetable matter.

The digestive organs and other viscera are surrounded by an irregular cavity, which is in free communication with the blood-vessels and itself contains blood. This cavity is not lined by epithelium, and is to be looked 
upon as a large blood-sinus, and not as a true cœlome: it may therefore be described as a hremocole.

There are well-developed respiratory organs in the form of gills (Figs. $9 \mathrm{r}, g$, and $94, k)$, contained in a narrow branchial chamber, bounded internally by the proper wall of the thorax, externally by the gillcover or pleural region of the carapace. Each gill consists of a stem giving off numerous branchial filaments, so that the whole organ is plume-like. The filaments are hollow and communicate with two parallel canals in the stem-an external, the afferentbranchial vein, and an internal, the efferent branchial vein (Fig. 96). The gill is to be considered as an out-

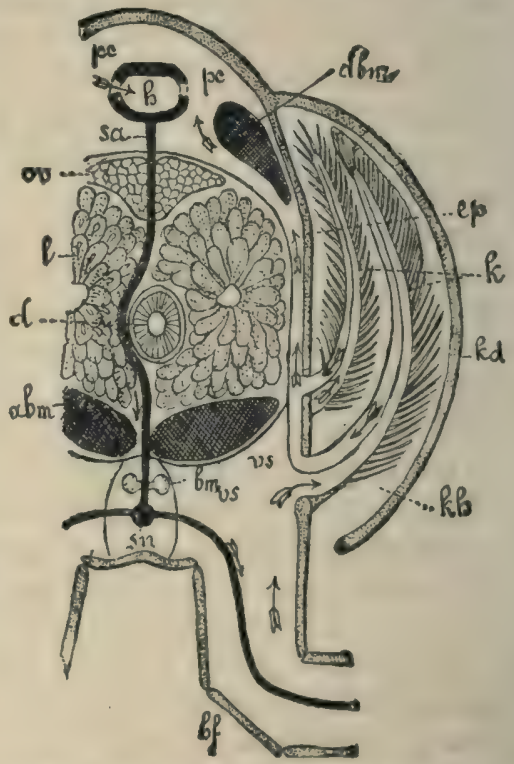

Fra. 94.-Transverse section of thorax of Crayfish, diagramatic. ( $x$ about 4 .) $a b m$. ventral abdominal muscles ; $b f$. leg ; bm. ventral nerve-cord ; $d$. intestine ; abclominal muscles; $c p$. wall of thorax; $h$. heart; $k$. gills; $k d$. gill-cover : $l$. digestive gland; $o v$. ovary; $p c$. pericardial sinus; $s a$, sn. sternal artery; vs. ventral blood sinus, The a:rows show the direction of the bloodcurrent. (From Lang's Comparative Anatomy.)

pushing of the body-wall specially modified for respiration, and it contains the same layers - a thin layer of chitin externally, then a single layer of epithelial cells, and beneath this, connective-tissue, hollowed out for the blood-channels. 


\section{vI RESPIRATORY AND EXCRETORY ORGANS 375}

According to their point of origin the gills are divisible into three sets-first, podobranchs, or foot-gills, springing from the epipodites of the thoracic appendages, from which they are only partially separable; secondly, arthrobranchs, or joint-gills, springing from the articular membranes conrecting the thoracic appendages with the trunk ; and thirdly, pleurobranchs, or wall-gills, springing from the lateral walls of the thorax, above the attachment of the appendages. The total number of gills is eighteen, besides two filaments corresponding to vestigial or vanishing gills, which are represented by functional organs in some allied forms.

The water in the branchial chamber is constantly renewed in the living crayfish by the action of the plate attached to the second maxilla (p. 367), the movement to and fro of which bales out the water in front, and consequently causes fresh water to flow in behind. Thus a fresh supply of water, containing air in solution, is continually being passed over the gills. The fact that the podobranchs are attached to the bases of the limbs must also result in bringing their surfaces more easily in contact with the water when the animal uses its legs in walking.

The excretory organs differ both in position and in form from those of the earthworm. At the base of each antenna is an organ of a greenish colour, the antennary or green gland (Fig. 95), by which the function of renal excretion is performed. The gland is cushion-shaped, and contains canals and irregular spaces lined by glandular epithelium: it discharges its secretion into a thin-walled sac or urinary bladder $(b l)$, which opens by a duct on the proximal segment of the antenna. The green glands are to be looked upon as corresponding to peculiarly modified nephridia (p. $3+0$ ).

The circulatory organs are in a high state of develop- 
ment. The heart (Fig. 93, $h$ ) is situated in the dorsal region of the thorax, and is a roughly polygonal, muscular organ pierced by three pairs of apertures or ostia (o), guarded by valves which open inwards. It is enclosed in a spacious pericardial sinus (Fig. 94, pc) which contains blood. From the heart spring a number of delicate arteries (compare p. 337), which serve to convey the blood to various parts of the body. At the origin of each artery from the heart are valves which allow of

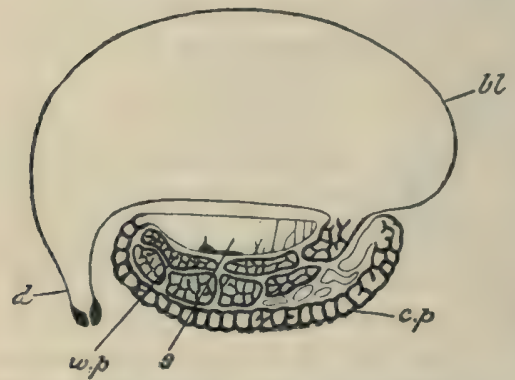

FiG. 95.-Diagram of excretory organ of Crayfish. ( $x$ 5.) bl. bladder ; c. p. outer or cortical green portion; $d$. duct; $s$. yellowish sac-like portion; $w$. $p$. white tubular portion. (From Parker and Haswell's Zoology, after Marchal.) the flow of blood in one direction only, viz., from the heart to the artery. From the anterior end of the heart arise five vessels - the median ophthalmic artery (Fig. 93, od), which passes forwards to the eyes; paired antennary arteries (aa), going to the antennules, antennæ, green glands, \&c., and sending off branches to the gizzard; and paired hepatic arteries, going to the digestive glands. The posterior end of the heart gives off two unpaired arteries practically united at their origin -the dorsal abdominal artery (oaa), which passes backwards above the intestine, sending branches to it and to the dorsal muscles; and the large sternal artery (Figs. 93 and 94, sa), which extends directly downwards, indifferently to right or left of the intestine, passing between the connectives uniting the third and fourth thoracic nerve-ganglia (p. 379), and then turns forwards and runs in the sternal canal, immediately below the 
nerve-cord, as. a ventral thoracic artery sending off branches to the legs, jaws, \&c. At the point where the sternal artery turns forward it also gives off the median ventral abdominal artery (Fig. 93, uaa), which passes backwards beneath the nerve-cord, and supplies the ventral muscles, pleopods, \&c.

All these arteries branch extensively in the various organs they supply, becoming divided into smaller and smaller offshoots, which finally end in microscopic capillaries (p. 95). These latter end by open mouths, which communicate with the blood-sinuses-spacious cavities lying among the muscles arıd viscera, and all communicating, sooner or later, with the sternal sinus, a great median canal running longitudinally along the thorax and abdomen, and containing the ventral nervecord and the ventral thoracic and abdominal arteries. In the thorax the sternal sinus (Fig. 94, vs, and Fig. 96, st. s) sends an offshoot to each gill in the form of a welldefined vessel, which passes up the outer side of the gill, and is called the afferent branchial vein (af. br. v). Spaces in the gill-filaments place the afferent in communication with the efferent branchial vein (ef. br. v.), which occupies the inner side of the gill-stem. The eighteen efferent branchial veins open into six branchiocardiac veins (br. c. v.) which pass dorsally in close contact with the lateral wall of the thorax and open into the pericardial sinus, some of them uniting before doing so.

The whole of this system of cavities is full of blood, and the heart is rhythmically contractile. When it contracts the blood contained in it is prevented from entering the pericardial sinus by the closure of the valves of the ostia, and therefore takes the only other course open to it, viz., into the arteries. When the heart relaxes, the blood in the arteries is prevented from regurgitating by the valves at their origins, and the 
pressure of blood in the pericardial simus forces open the valves of the ostia and so fills the heart. Thus in virtue of the successive contractions of the heart and of the disposition of the valves, the blood is kept constantly moving in one direction-from the heart by the arteries to the various organs of the body, where it receives carbon dioxide and other waste matters; thence by

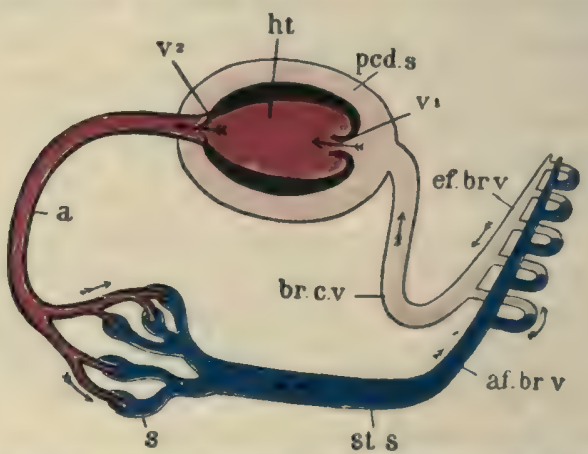

FIG. 96.-Diagram illustrating the course of the circulation of the blood in the Crayfish. Heart and arteries, red; veins and sinuses containing non-aërated blood, blue; veins and sinuses containing aërated blood, pink. The arrows show the direction of the flow.

The blood from the pericardial sinus ( $p c d$. $s$.) enters the heart ( $h t$ ) by a valvular aperture $(v 1)$ and is propelled into arteries $(a)$, the orifices of which are guarded by valves $\left(v^{2}\right)$; the ultimate branches of the arteries discharge the blood into sinuses $(s)$, and the sinuses in various parts of the body debouch into the sternal sinus (st. s.); thence the blood is taken by the afferent branchial veins (af. br. $v$ ) into the gills, where it is purified and is returned by efferent branchial veins $(e f . b r . v)$ into the branchiocardiac veins $(b r . c . v)$ which open in to the pericardial sinus. (From Parker and Haswell's Zoology.)

sinuses into the great sternal sinus; from the sternal sinus by afferent branchial veins to the gills, where it exchanges carbon dioxide for oxygen; from the gills by efferent branchial veins to the branchiocardiac veins, thence into the pericardial sinus, and so to the heart once more.

It will be seen that the circulatory system of the crayfish, like that of the frog, consists of three sections(I) the heart, or organ of propulsion; (2) a system of 
outgoing channels, the arteries, which carry the blood from the heart to the body generally ; and (3) a system of returning channels-some of them, the sinuses, mere irregular cavities, others, the veins, with definite walls: these return it from the various organs back to the heart. The respiratory organs, it should be observed, are interposed in the returning current, so that blood is taken both to and from the gills by veins.

Comparing the blood-vessels of the crayfish with those of the earthworm (Figs. $8 \mathrm{r}$ and 83 ), it would seem that the ophthalmic artery, heart, and dorsal abdominal artery together answer to the dorsal vessel, part of which has become enlarged and muscular and discharges the whole function of propelling the blood. The ventral thoracic artery, together with the ventral abdominal, represent the main ventral vessel, while the vertical portion of the sternal artery is a commissure, developed sometimes on the right sometimes on the left side, its fellow being suppressed.

The blood when first drawn is colourless, but after exposure to the air takes on a bluish-grey tint. This is owing to the presence of a colouring matter called hamocyanin, which becomes blue when combined with oxygen; it is a respiratory pigment, and serves, like hæmoglobin (pp. I07 and 339), as a carrier of oxygen from the external medium to the tissues. The hrmocyanin is contained in the plasma of the blood: the corpuscles are all leucocytes (pp. I05 and 336).

The nervous system consists, like that of the earthworm, of a brain (Fig. 93, g) and a ventral nerve-cord $(b m)$, united by asophageal connectives. But the ganglia of the ventral nerve-cord are more distinct, and to them the nerve-cells are confined, the paired longitudinal connectives between them consisting of nervefibres only. The brain is complex, and supplies not 
only the eyes and antennules, but the antennæ as well : a study of development shows that the ganglia belonging to the antennary segment have fused with it. Hence we have to distinguish between a primary brain or archi-cerebrum - the ganglion of the prostomium, and a secondary brain or syn-cerebrum, formed by the union of one or more pairs of ganglia of the ventral cord with - the archi-cerebrum. A further case of concrescence of ganglia is seen in the ventral nerve-cord, where the ganglia of the last three cephalic and first three thoracic segments have united to form a large compound $s u b$ cesophageal ganglion. All the remaining segments have their own ganglia, with the exception of the telson, which is supplied from the ganglion of the preceding segment. There is a visceral system of nerves supplying the foregut and hind-gut, the nerves of the former originating in part from the brain and in part from the œsophageal connectives, and those of the latter from the last abdominal ganglion.

The eyes (Fig. 97) have a very complex structure. The chitinous cuticle covering the distal end of the eyestalk is transparent, is divided by delicate lines into areas or facets which are mostly square, and constitutes the cornea. Beneath each facet of the cornea is an apparatus called an ommatideum, consisting of an outer segment or vitreous body having a refractive function, and an inner segment or retinula, enclosing a striated body, the rhabdome, and forming the actual visual portion of the apparatus. The ommatidea are optically separated from one another by black pigment, so that each is a distinct organ of sight, with a very limited visual area, and the entire eye is called a compound eye. The optic nerve $(o p . n)$ dilates to form an optic ganglion (op. $g n)$ in close connection with the inner ends of the ommatidea : the latter are thus turned towards the light, and are not, 
like the rods and cones of the vertebrate eye (p. I85), covered by a layer of nerve-fibres, \&c., through which the light must first penetrate.

Each antennule bears two sensory organs, to which the functions of smell and hearing have been respectively assigned. The "olfactory" organ is constituted by a number of delicate spatulate setæ, borne on the external

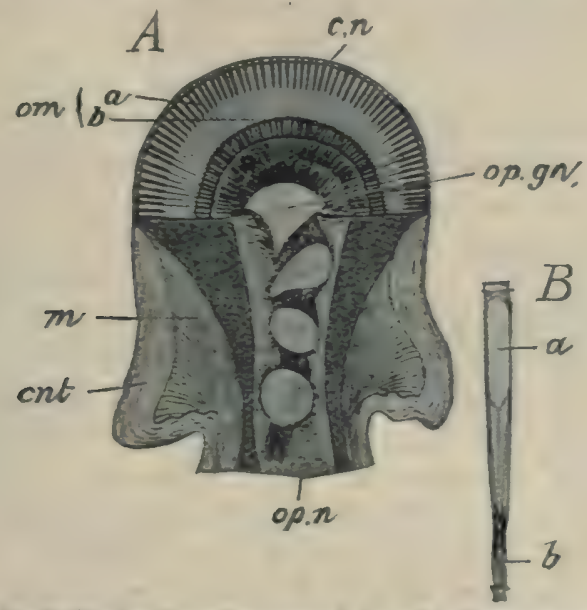

Fic. 97.-A longitudinal section of an evestalk ( 12$) ; \quad B$, a single ommatideum ( 530 ). $a$, vitreous body : $b$, retinula : cn. cornea, mntinucus with cnt, cuticle of eyestalk : $m$, muscles; om, ommatidea ; op. $g n$, optic ganglion; $o p$, $n$, optic nerve. (After Howes.)

feeler, and supplied by branches of the antennulary nerve. The "auditory" organ, which is better described as an organ of equilibration, or statocyst (p. 315), is a sac formed by invagination of the dorsal surface of the proximal segment, and is in free communication with the surrounding water by a small aperture. The chitinous lining of the sac is produced into delicate, feathered, sensory setæ, supplied by branches of the antennulary nerve, and in the water which fills the sac 
are minute sand-grains, which take the place of the lithites or otoliths (pp. I88 and 3I5) found in most organs of this kind; but which, instead of being formed by the animal itself, are taken in after each ecdysis when the lining of the sac is shed. Many of the setæ on the body generally have a definite nerve-supply, and are probably tactile organs.

The crayfish is dicecious (p. 320), and presents a very obvious sexual dimorphism or structural difference between male and female, apart from the actual organs of reproduction. The abdomen of the female is much broader than that of the male: the first and second pleopods of the male are modified into tubular or rather spout-like organs (p. 364); and the reproductive aperture is situated in the male on the proximal podomere of the fifth leg, in the female on that of the third.

The spermary (Fig. 93, $t$ ) lies in the thorax, just beneath the floor of the pericardial sinus, and consists of paired anterior lobes and an unpaired posterior lobe. From each side goes off a convoluted spermiduct or vas deferens $(v d)$, which opens on the proximal segment of the last leg $(v d o)$. The sperms differ greatly from those already described in other animals: they are curious, rounded, non-motile bodies produced into a number of stiff processes: by a secretion of the spermiduct they become aggregated into vermicelli-like spermatophores.

The ovary is also a three lobed body, and is similarly situated to the spermary: from either side proceeds a thin-walled oviduct, which passes downwards, without convolutions, to open on the proximal segment of the third or antepenultimate leg. The eggs are of considerable size and contain a large amount of yolk.

Both ovary and spermary are hollow organs, discharging their products internally. Their cavities, lined by germinal epithelium, represent part of the true 
colome (compare p. 374), and their ducts are organs of the same general nature as nephridia, opening on the one hand into a coelomic cavity, and on the other to the exterior. The ova, when laid, are fastened to the setæ on the pleopods of the female by the sticky secretion of glands occurring both on those appendages and on the segments themselves: they are fertilised immediately after being laid, the male depositing spermatophores on the ventral surface of the female's body just before oviposition.

The process of segmentation of the oosperm presents certain striking peculiarities. The nucleus dirides
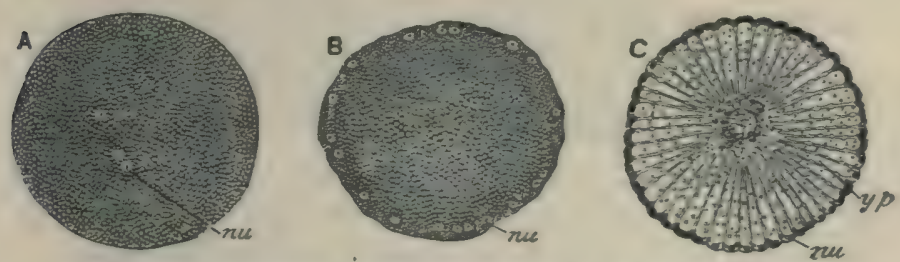

Frg. $9^{9}$. - Three stages in the early development of the Crayfish. ( $x$ ro.)

In $A$ the products of division of the nucleus ( $m$ ) are seen in the centre of the yolk in $\mathrm{B}$ and $\mathrm{C}$ the nurlei have become arranged in a peripheral layer, each sur rounded by protoplasm. so as to form the blastoderm; $y p$. yolk-pyramids. (From Parker and Haswell's Zoology, after Morin.)

repeatedly (Fig. 98, A, $n u$ ), but no corresponding division of the protoplasm takes place, with the result that the polyplast-stage (p. 200), instead of being a heap of cells, is simply a multi-nucleate body (compare p. 28r). Soon the nuclei thus formed retreat from the centre of the embryo, and arrange themselves in a single layer close to the surface $(\mathrm{B}, \mathrm{C})$ : around each of these protoplasm accumulates, the central part of the embryo consisting entirely of yolk-material. We thus get a superficial segmentation, characterised by a central mass of yolk, and a superficial layer of cells collectively known as the 
blastoderm. From this the ectoderm and endoderm are derived, the latter enclosing a relatively small enteron formed by an inpushing or invagination of the blastoderm into the yolk, and communicating with the exterior through a blastopore (p. 20I).

Very soon the embryo becomes triploblastic, or threelayered, by the budding off of cells from the endoderm in the neighbourhood of the blastopore: these accumulate between the ectoderm and endoderm, and constitute the mesoderm.

Before long the blastopore closes, and a stomodæum and proctodæum (p. 20.4) are formed as invaginations of the ectoderm, which eventually communicate with the enteron, forming a complete enteric canal (p. 37r). On each side of the mouth or aperture of the stomodæal depression (Fig. 99) three elevations appear, the rudiments of the antennules $\left(a^{1}\right)$, antennæ $\left(a^{2}\right)$, and mandibles $(m)$ : in front of them is another pair of elevations on which the eyes $(A)$ subsequently appear. An unpaired elevation $(T A)$ behind the mouth, having the anus or aperture of the proctodæal depression at its summit $(A)$, is the rudiment of the thorax and abdomen. The embryo is now in the nauplius stage.

In many allied forms the embryo is hatched at this stage in the form of a free-swimming nauplius larva (Fig. IOO), characterised by the presence of three pairs of appendages used for swimming (the first simple, the second and third forked), and becoming the antennules, antennx, and mandibles of the adult: the eye is median and sessile. In the crayfish there is no free larva, and the young are hatched at a much later stage.

The embryo is gradually transformed into the crayfish by the appearance of fresh appendages, in regular order, behind the first three; by the elongation of the rudiment of thorax and abdomen; and by the gradu- 


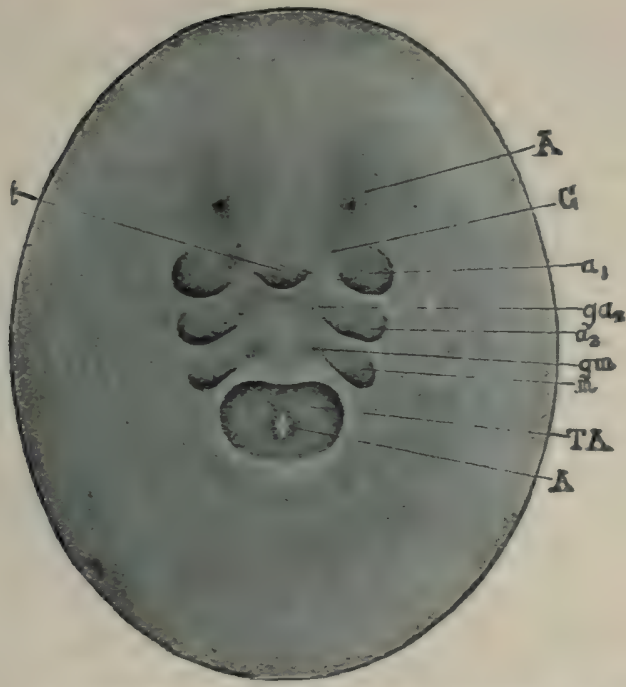

FIG. 99.-Early embryo of the Craytish in the nauplius stage. ( $x$ about 20.) $A$ in the upper part of the figure is the eve ; $l$. the labrum overhanging the mouth, on either side of which are the rudiments of the antennules $\left(a^{1}\right.$.), antenna $\left(a^{2}.\right)$, and mandibles $(m$.$) ; behind them is the rudiment of the thorax and abdomen$ $(T A)$ with the anus $(A)$. The rudiments of the first three pairs of ganglia $\left(G, g q^{2}, g m\right)$ are seen through the transparent ectoderm. (From Lang's Comp. Anatomy, after Reichenbach.)

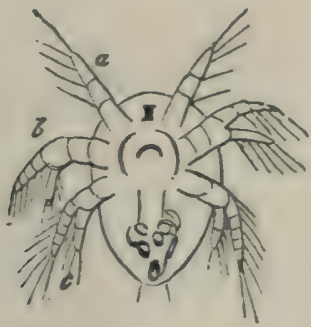

FiG. roo. - Nauplius larva of Water-flea (Cyclops). ( $\nless$ about 20.)

$a, b, c$, the three pairs of swimming appendages, representing the antennules, antennz, and mandibles of the Crayfish; the median eye is seen between the bases of the first pair. (From Gegenbaur's Comb. Anatomy.) 
differentiation of the appendages. When hatched the young animal agrees in all essential respects with the adult, but its proportions are very different, the cephalothorax being nearly globular and the abdomen small. For some time after hatching the young crayfishes cling in great numbers to the pleopods of the mother by means of the peculiarly hooked chelæ of the first pair of legs.

All the members of the phylum Arthropoda (p. 360) are characterised by each typical segment of the body bearing a pair of appendages divisible into podomeres ; in addition to this, there is an almost universal absence of cilia in the phylum, the sperms are usually non-motile, the muscles are nearly always of the striped kind, and the body-cavity, which does not correspond to a true cœelome, is largely represented by blood-spaces in free communication with the circulatory system.

The phylum is divided into several classes, which are all air-breathing except the Crustacea, the class to which the crayfish belongs and which aiso includes the Lobsters, Crabs, Shrimps, Wood-lice, Barnacles, Waterfleas, \&c. : in these, when special respiratory organs are present, they have the form of gills.

\section{PRACTICAL DIRECTIONS}

\section{CRAYFISH}

In a living specimen note the cephalothorax, abdomen, jointed appendages, and exoskeleton, as well as the mode of walking and swimming. Holding the animal out of water between the thumb and finger, observe that bubbles are continually being formed on either side of the lower part of the head (respiratory movements, see $\S \mathrm{B}, \mathrm{I})$. Kill with chloroform (p. 3I), and, at the end of the day's work, preserve your dissection in 70 per cent. spirit or $2-3$ per cent. formaline. 


\section{A. External Characters.}

I. Note again the cephalothorax and abdomen, and that the abdomen consists of seven movable segments or metameres. Examine the ventral side of the cephalothorax, and note that it also is composed of a number of segments all fused except the rentral part of the last.

2. Examine the third or fourth abdominal segment closely, and note that it is connected with the segments in front and behind by a sort of peg and-socket joint on either side, and that the chitinous exoskeleton at the joints is soft and pliable, forming an articular membrane, while elsewhere it is calcified. Distinguish between the dorsal convex tergum, the ventral stermum, and the pleuron projecting downwards on either side from the tergum.

3. Fxamine the appendages of the same segment: they are attached to the sterna, near the pleura, by articular membranes. Each consists of a basal or proximal portion - the protopodite, to which two distal, many-jointed parts are attached-an inner endopodite, and an outer exopodite. The cuticle covering the segments of the limb, or podomeres, is more or less calcified, and the distal segments are covered with feathery sete.

4. The second to the sixth abdominal segments are essentially similar to one another except as regards the appendages of the second in the male and of the sixth in both sexes (p. 364 ). The first abdominal segment is smaller than the others, and its pleura are reduced. The sixth abdominal appendages are very large, anch together with the anal segment or telson, form the tail-fin.

5. The cephalothorax is reckoned as consisting of a prostomium and I 2 metameres, which are completely fused dorsally and laterally, forming a large calcified shield-the carapace. Thus the entire number of segments is 20 (prostomium +18 metameres + telson). The sternal region of the head is bent upwards.

6. Note:- $a$, the transverse cerical groove on the dorsal surface of the carapace, extending forwards laterally and forming the boundary between the head (prostomium +4 metameres) and the thorax ( 8 metameres); $b$, the two longitudinal branchio-pericardial grootes on the tergal region of the thoracic portion of the carapace, about $\frac{1}{2}$ inch apart: the part of the exoskeleton between these covers the heart, and the part below each groove forms a large plate, the gill-cover, at the side of the thorax; $c$, the rostrum, movable eje-stalks, and epistoma (p. 363). 
7. Note the following apertures: Median-a, the mouth, on the ventral surface of the head, between the jaws; $b$, the anus, on the ventral side of the telson: Paired$c$, the auditory aperture, on the dorsal side of the basal joint of the smaller feeler or antennule (it will be seen later on); $d$, the renal aperture, on a conical ventral elevation of the basal joint of the larger feeler (antenna); $e$, the genital aperture, in the male on the basal joint of the last thoracic leg, and in the female on the last thoracic leg but two.

\section{$B$. Respiratory Organs.}

I. Carefully cut away the left gill-cover with scissors, and fix the animal under water on its right side, so as to expose the left gill-chamber containing the feathery-looking gills. The inner wall of the chamber is formed by the proper wall of the thorax, and the chamber is open behind and below. In front of the gills is a groove, in which a flattened plate (see p. 367) works backwards and forwards during life, driving the water out in front, and causing the bubbles already noticed.

2. The gills are $\mathbf{r} 8$ in number, and each has the form of a bottle-brush. The six podobranchs are external to the arthrobranchs and pleurobranchs (p. 375), and each is attached to a large folded and corrugated epipodite (p. 366). The gills are related to different metameres, as will be seen from the following table, in which ep stands for epipodite, and $v$ for the vestige of a gill. Note that the first pair of thoracic limbs bears a simple large epipadite only.

\begin{tabular}{|c|c|c|c|c|c|c|c|c|c|}
\hline $\begin{array}{c}\text { Thoracic } \\
\text { SEgments. }\end{array}$ & I. & II. & III. & IV. & r. & VI. & VII. & VIII. & Total. \\
\hline Podobranchs.. & $0+e p$ & $1+e p$ & $1+c p$ & $1+e p$ & $1+e p$ & $x+e p$ & $I+e p$ & o & $6+7 e p$ \\
\hline Arthrobranchs & o & $x$ & 2 & 2 & 2 & 2 & 2 & - & II \\
\hline Pleurobranchs & o & o & 0 & $\circ$ & - & $\vartheta$ & $\boldsymbol{v}$ & $\mathbf{r}$ & $x+2 v$ \\
\hline Total....... & $0+c \beta$ & $2+c p$ & $3+e p$ & $3+e p$ & $3+c p$ & $3+v+c p$ & $3+v+e p$ & 1 & $18+2 z+7 i t$ \\
\hline
\end{tabular}

3. Turn down the podobranchs and make out the relations of the arthrobranchs from the above table. Then turn these down also, or cut them off, and note the single complete pleurobranch and the two vestigial ones. Cut off an arthrobranch and examine its structure, noting the afferent and efferent blood-vessels in its stem. Sketch. 
4. Note the branchiocardiac veins on the inner side of the thoracic wall. Blow air or inject French blue (see p. 99) into the cut bases of the gills removed, and note that the branchiocardiac trunks extend upwards to the pericardial sinus (see below) from the gills.

\section{General Dissection.}

Holding the animal in your left hand, insert a scalpel carefully beneath the hinder edge of the carapace on the dorsal side, so as to separate the exoskeleton from the soft integument, and then with the large scissors cut along the outer side of each branchio-pericardial groove, and remove the median portion of the carapace. Note the pigmented integument and then remove it, when some of the nearly colourless blood will ooze out.

I. I. Examine a drop of blood under the microscope, adding salt-solution. Note the nucleated amoboid corpuscles. Sketch.

2. The pericardial sinus will now be exposed, containing the heart with three pairs of valvular ostia (only the dorsal ostia can be seen at present), through which the blood enters the heart from the pericardial sinus.

Inject some French blue into the heart through one of the ostia, so as to fill the arteries (tying is unnecessary). Then remove the dorsal part of the exosheleton and integument bit by-bit, all along the thorax and abdomen, as weil as the pair of longitudinal extensor muscles lving just beneath the dorsal integument of the abdomen. Pin down under water, dorsal surface uppermost, and note :-

3. The absence of a continuous muscular layer in the bodywall and of a true coelome, and the presence of irregular spaces (blood sinuses) between the viscera and muscles.

4. The delicate arteries arising from the anterior and posterior ends of the heart : $-a$, the anterior median ophthalmic artery, running forwards to the eye-stalks; $b$, the paired antennary artery, on either side of $a$, and passing forwards and downwards to supply the gizzard, renal organ, feelers, \&c. : $c$, the hepatic artery (also paired), rather further back and more ventral, extending into and supplying the digestive gland; $d$, the median dorsal abdominal artery, arising from the posterior end of the heart, and running along the dorsal side of the intestine, giving off branches in each metamere ; $e$, the sternal artery, arising just beneath the anterior end of $d$, and passing directly ventralwards to one side of the intestine (this will be seen better later on : 
compare Figs. 93 and 94): it perforates the ventral nervecord, and divides into (a) a ventral thoracic artery supplying the segments and appendages of the thorax, and $(b)$ a ventral abdominal artery, supplying the segments and appendages of the abdomen (this artery can be seen in injected specimens through the transparent cuticle).

5. Note the position of the following parts before dissecting further:- $a$, the gizzard, a large sac in the head, with two pairs of muscles passing to the integument (now cut through); (b), the adductor muscles of the mandible, just external to a; $c$, the paired, brownish or greenish digestive gland on either side of, and extending further back than the gizzard; above it are $d$, the gonads, on either side of and behind the pericardial sinus. In the male, the spermary is small and whitish, and each spermiduct is a coiled, densely white tube; in the female, the ovary is a larger, brownish organ, containing prominent ova. (In both sexes, the paired character of the gonads is partly lost by fusion : a pair of anterior lobes and a single posterior lobe can be seen in each.) Sketch. By slightly raising the surrounding parts the gonaducts can be seen to pass ventralwards to their external apertures (p. 388), the oviducts being thin-walled and straight. White masses, the spermatophores (p. 382 ), will very likely be found stuck on to the sternal region of the body.

6. Tease up a small portion of the spermary or of a spermatophore; stain, and mount in glycerine. Examine under the microscope and note the rounded and flattened sperms, each with a number of stiff, curved processes coming off from the periphery. The sperms are non-motile. Sketch.

Remove the heart and reproductive organs carefully, noting the sternal artery (see above) as you do so, and taking especial care not to injure the surrounding parts. Examine the heart under water, and note the six ostia.

II. The enteric canal. Note-I. The oval mouth, bounded by the labrum in front, leading into a short and wide gullet (this will be seen later on), which dilates to form the large gizzard (Fig. 93), filling up a considerable portion of the head and extending into the thorax: a transverse constriction divides it into an anterior and a posterior portion; both gullet and gizzard are lined by chitin. The chitinous cuticle of the gizzard is calcified in places to form the sclevites or so-called "ossicles" of the "gastric mill." Note the two median sclerites bounding the transverse constriction in front and behind respectively: to them the anterior and posterior pairs of muscles (mentioned in $\$ 5$ ) are attached. 
2. Following on the gizzard is the short, thin-walled mid-gut. on the dorsal side of which is a small cacum. It has no chitinous lining, and the large duct of the digestive gland opens into it on either side. Each digestive gland is made up of three main lobes, and consists of a number of small blind tubes.

3. The hind-gut, which runs straight to the anus. Its inner surface is raised into longitudinal ridges which take a slightly spiral course, and it is lined by a thin chitinous cuticle.

4. Carefully press the gizzard backwards, and note $a$, the brain, just behind the bases of the small feelers: $b$, the gullet; and $c$, a pair of white nerve-cords (connectives) coming off from the brain and embracing the gullet. Taking care not to injure these parts of the nervous system, cut through the gullet, just above the connectives, and then gently remove the whole enteric canal, together with the digestive gland, from the body, cutting through the intestine just in front of the anus. Examine the whole digestive system under water.

5. Note again the mid-gut and the digestive glands and ducts; then remove the digestive gland of one side, and sketch the enteric canal from the same side. Slit up the hind-gut so as to see the ridges and cuticle.

6 . Clean the walls of the gizzard and note the "gastroliths " and the other sclerites of the " gastric mill ":-

Articulated to each end respectively of the two median sclerites already referred to, in each lateral wall of the gizzard, is a lateral sclerite, the two articulating with one another at their other ends, so that these six sclerites together form a sort of hexagonal frame. Twn other median sclerites, arising respectively from the median ones mentioned above, extend downwards into the constriction between the two portions of the gizzard, and these join below at an angle, where they bear a median tooth. Each of the posterior lateral sclerites bears a lateral tooth.

Cut open the anterior end of the gizzard, and note the strongly calcified, brownish, median tooth, and the two large lateral teeth. Seize hold of the two median ossicles with two pairs of forceps, one in each hand, and pull gently backwards and forwards (in the direction in which the muscles pull). It will then be seen that the median and lateral teeth come together in the middle line so as to act as a "gastric mill." Note the slit-like lumen of the part of the gizzard behind this and the arrangement of the seto which act as strainers. Make sketches as you proceed. 
III. I. The chief muscles of the body: $-a$, the paired and segmented dorsal extensor, arising from the side walls of the thorax, and extending into the abdomen above the intestine, giving off slips.to each segment of the abdomen (this muscle has already been removed); and $b$, the large and complex ventral muscles, the lateral halves of which are not separate from one another, the fibres being interwoven, somewhat like those of a rope; slips are given off to the abdominal sterna. These act mainly as a flexor of the abdomen (compare p. 370).

2. Muscles pass from the body to the proximal joints of the appendages: those between successive podomeres will be examined at a later stage $(\S D)$.

3. Note again the paired adductor of the mandible (p. 390), and trace its calcified tendon downwards to its insertion on to the mandible.

4. Tease out a small piece of muscle so as to separate its fibres from one another. Stain, and mount in glycerine. Note the transverse striations, sarcolemma, and nuclei (compare Fig. 32). Sketch.

Remove the muscles of the body described above, noting the sternal artery (p. 389), and taking especial care to leave the abdominal nerve-cord in situ when removing the large ventral muscles. Note that in the thorax, the nerve-cord passes into a sternal canal, formed by a series of ingrowths of the exoskeleton-the endophragmal system-from which the muscles passing to the thoracic limbs arise. Insert the scissors into the sternal canal, and cut away and remove its roof, bit by bit. The whole of the central nervous system will then be exposed.

IV. Observe that a more marked distinction into ganglia and connectives is seen than in the case of the earthworm, and that the fusion of the two lateral halves of the cord or chain has only affected the ganglia, the connectives being double all the way along.

1. Note: $a$, the brain, or compound supra-cesophageal ganglia; $b$, the esophageal connectives; and $c$, the postoral ventral nerve-cord, consisting of a large compound subasophageal ganglion and of I I segmental ganglia, united by paired connectives. Beneath the cord, the ventral thoracic and abdominal arteries (p. 390) will be seen, the main sternal artery passing between the connectives joining the fourth and fifth postoral ganglia.

2. The brain gives off nerves to the eyes and the two pairs of feelers: the sub-cesophageal ganglion supplies the 
mandibles and five following pairs of appendages and their segments. Each of the other ganglia supplies one segment (with its appendages) only, except in the case of the last or ith ablominal ganglion, which gives off nerves to the telson. (The small anterior visceral nerie, arising from the brain and ossophageal connectives, and supplying the foregut, will have been removed; the posterior isceral nerie, supplying the hind-gut, arises from the last abdominal ganglion.) Sketch.

3. Tease up a ganglion in salt-solution, stain, and examine for nerve-cells.

V. The antennary or green-glands are situated just behind the bases of the large feelers. Blow through the renal aperture of one side (p. 388) and note the duct and urinary bladder on the dorsal side of the gland. Then cut through the duct, remove the whole gland, and examine under water. Sketch.

$D$. The Appendages.-Remove the appendages on one side, beginning with the last, one by one, cutting through the articular membrane with a scalpel, and then taking hold of the basal joint with the forceps and pulling the appendage away. Work through the description on pp. $364-367$ and sketch typical appendages from each region. Note the delicate paragnatha behind the mouth and the labrum in front of it (p. $3 \%$ o).

Procure some pond-water containing specimens of the small " water-flea" known as Cyclops, which will be recognised by its pear-shaped'body, and by the two oval egg-sacs of the female. Examine drops of this water until you find some nauplius-laria of Cyclops, noting the three pairs of appendages and the median eye (p. $38+$ and Fig. IOo).

The arrangement of the joints and muscies of the limbs can be well seen by examining the large first leg or cheliped. Note that each joint works in a different plane, and then cut away the exoskeleton from one side of the two or three distal podomeres, so as to expose the muscles (compare Fig. 92). Then remove these, and note their chitinous tendons. Observe that the adductor muscle and its tendon, which closes the pincers, is much larger than the abductor muscle.

\section{$E$. Sensory Organs.}

I. Tactile organs. Snip off some setæ from the body or appendages, and examine under the microscope. Sketch.

2. "Olfactory" organs. Examine the outer feeler of the 
antennule under the low power, and note the tufts of spatula-like " olfactory" setee on the ventral surface. Sketch.

3. "A uditory" organ (statocyst). Carefully cut away the convex ventral side of the basal segment of the antennule with scissors, so as to expose the statocyst. Cut this out and place it on a slide, carefully removing the muscles surrounding it, as well as the setæ around its aperture. Note the contained grains of sand, and then wash them away. Stain with magenta and mount in glycerine, flattening the sac out with a cover-glass. Note that the sac is an involution of the integument lined by cuticle, and that it contains simple jointed sensory setce of various sizes, arranged in rows, and that branches of the antennulary nerve run up the stem of each seta. Sketch.

4. The eyes. Remove one of the eye-stalks, and note the apparently black, uncalcified, oval portion of the cuticle (cornea) at its distal end. Strip this off, and note that it is transparent. Then wash off any pigment which may bave come away with it and mount in water. Observe the corneal facets. Then cut the eyestalk into two longitudinal halves with a knife, and examine with a lens under water. Note the optic nevve entering the stalk, and enlarging to form the optic ganglion, from which a number of bodies (ommatidea) radiate outwards to the corresponding facets of the cornea. The ommatidea are separated from one another by pigment. Sketch.

Examine longitudinal sections of the eye-stalks, decalcified and prepared as directed on p. I36, and note in detail the above parts. Each ommatideum lies beneath the corresponding corneal facet, and is made up of an outer vitreous body or crystalline cone, and an inner retinula formed of sensory cells and enclosing a transversely-striated, spindle-shaped, refractive body or rhabdome, and closely connected with the optic ganglion. Note also the pigment between the ommatidea. Sketch.

\section{F. Structure of the Exoskeleton.}

In order to follow this out in greater detail, proceed as follows :-

I. Cut through the thorax and abdomen of a crayfish transversely, and note the relations of the hard and soft parts. (Compare Figs. 90 and 94.)

2. Dip a crayfish into hot water, so that the soft parts come away easily. Open up the cephalothorax from the dorsal side, separate some of the abdominal segments, and 
clean thoroughly. Examine the joints and make out the relations of the endophragmal system (p. 392), looking something like a lattice-girder.

3. Examine your preparation of the eye once more under the microscope and notice the part where the section passes through the outer wall of the eye-stalk, so as to make out the microscopic structure of the integument and exoskeleton, which, apart from the calcification, is similar in all parts. Notice the epiderm, and the thick, laminated chitinous cuticle, the superficial layer of which is uncalcified throughout.

\section{$G$. Dissection from the Side.}

Cut through the carapace on the dorsal side as before, but be careful to keep your cut very slightly to one sidesay the left - of the middle line, and when the pericardium is exposed inject the arteries through the heart as before (p. 389). Then remove the gill-cover of the same side and examine the gills once more.

Remove and examine again all the appendages of the same side, and continue cutting longitudinally through the exoskeleton, both dorsally and ventrally, close to the middle line, taking care you do not injure any median organs. Remove the entire exoskeleton of this side, as well as the dorsal and ventral muscles, cutting through the latter in the median line very carefully.

After your dissection has been pinned down under water, the antennary gland, digestive gland, and gonad of the same side should also be removed, and the gizzard cut open. Then tidy up the dissection, which will now be reduced to a longitudinal section like that represented in Fig. 93.

Once more carefully follow out the structure and relations of all the organs exposed, and sketch your dissection. 


\section{CHAPTER VIII}

THE FRESH-WATER MUSSEL-CHARACTERS OF THE PHYLUM MOLLUSCA - ENUMERATION OF THE CHIEF PHYLA OF THE ANIMAL KINGDOM

IN the mussel we meet with an entirely new type of structure: the animal, like the worm and crayfish, is bilaterally symmetrical, but there is no trace of metameric segmentation; the power of locomotion is greatiy restricted, and food is obtained passively by ciliary action, as in Infusoria, not by the active movements of definite seizing organs-tentacles, limbs, or protrusible mouth-as in most of the Mctazoa (p. 292).

Fresh-water mussels are found in rivers and lakes in most parts of the world. Anodonta cygnea, the swanmussel, is the commonest species in England; but another kind, Unio pictorum, occurs in many places, and the pearl-mussel, Unio margaritifera, is found in mountain streams, while other species of the same genus are universally distributed.

The mussel is enclosed in a brown, calcified shell, formed of two separate halves or valves hinged together along one edge. It lies on the bottom, partly buried in the mud or sand, with the valves slightly gaping, and in the narrow cleft thus formed a delicate, semi-transparent substance is seen, the edge of the mantle or pallium. The mantle really consists of separate halves or lobes corresponding with the valves of the shell 
(compare Fig. I03), but in the position of rest the two lobes are so closely approximated as to appear simply like a membrane uniting the valves. At one end, however, the mantle projects between the valves in the form of two short tubes, one (Fig. IOI, ex. sph) smooth-walled, the other (in. $s p h$ ) beset with delicate processes or tentacles. By diffusing particles of carmine or indigo in the water it can be seen that a current is always passing in at the fringed tube-hence called the inhalant siphon, and out at the smooth or exhalant siphon. Frequently a semi-transparent, tongue-like body $(f t)$ is protruded between the valves at the opposite side from the hinge and at the end furthest from the siphons: this is the foot, by means of which the animal is able slowly to plough its way through the sand or mud. When the mussel is irritated the foot and siphons are withdrawn and the valves tightly closed. In a dead animal, on the other hand, the shell always gapes, and it can then be seen that each valve is lined by the corresponding lobe of the mantle, and that the exhalant siphon is formed by the union of the lobes above and below it and is thus an actual short tube; but that the boundary of the inhalant siphon facing the gape of the shell is simply formed by the approximation of the mantle-lobes, so that this tube is a temporary one.

The hinge of the shell is dorsal, the gape ventral, the end bearing the siphons posterior, the end from which the foot is protruded anterior: hence the valves and mantle-lobes are respectively right and left.

In a dead and gaping mussel the general disposition of the parts of the animal is readily seen. The main part of the body lies between the dorsal regions of the valves : it is produced in the middle ventral line into the keel-like foot: and on either side between the foot and the corresponding mantle-lobe are two delicate, striated 
plates, constituting a gill or ctenidium, as it is often called. Thus the whole animal has been compared to a book, the back being represented by the hinge, the covers by the valves, the fly-leaves by the mantle-lobes, the two first and the two last pages by the gills, and the remainder of the leaves by the foot (Fig. I 03).

When the body of the mussel is removed from the shell the two valves are seen to be united, along a straight hinge-line, by a tough, elastic substance, the hinge-ligament (Fig. I03, lg) passing transversely from valve to valve. It is by the elasticity of this ligament that the shell is opened; it is closed, as we shall see, by muscular action: hence the mere relaxation of the muscles results in opening the shell. In Anodonta the only junction between the two valves is afforded by the ligament, but in Unio each is produced into strong projections and ridges, the hinge-tecth, separated by grooves or sockets, and so arranged that the teeth of one valve fit into the sockets of the other.

The valves are marked externally by a series of concentric lines parallel with the free edge or gape, and starting from a swollen knob or elevation, the umbo, situated towards the anterior edge of the hinge-line. These lines are lines of growth. The shell is thickest at the umbo, which represents the part first formed, and new layers are deposited under and concentrically to this original portion as secretions from the mantle, the shell being, like the armour of the crayfish, a cuticular exoskeleton (p. 369). As the animal grows each layer projects beyond its predecessor, and in this way successive outcrops are produced, giving rise to the markings in question. In the region of the umbo the shell is usually more or less eroded by the action of the carbonic acid in the water.

The inner surface of the shell also presents characteristic markings. Parallel with the gape, and at a short 
distance from it, is a delicate streak caused by the insertion on to the shell of muscular fibres from the edge of the mantle: the streak is hence called the pallial line. Beneath the anterior end of the hinge the pallial line ends in an oval mark, the anterior adductor impression, on to which is inserted one of the muscles which close the shell. A similar but larger posterior adductor impression lies beneath the posterior end of the hinge (compare Fig. Ior, $a$. $a d, p$. ad). Two smaller markings close behind the anterior adductor impression, dorsal and ventral respectively, mark the insertion of the anterior retractor and of the protractor muscle : one just anterior to the posterior adductor impression, that of the posterior retractor. From all these impressions faint converging lines can be traced to the umbo: they mark the gradual shifting of the muscles during the growth of the animal.

The shell consists of three layers, the outer layer, as in the crayfish, being uncalcified. Outside is a brown horn-like layer, the periostracum, composed of conchiolin, a substance allied in composition to chitin. Beneath this is a prismatic layer formed of minute prisms of calcium carbonate, separated by thin layers of conchiolin; and, lastly, forming the internal part of the shell is the nacre, or " mother-of-pearl," formed of alternate layers of carbonate of lime and conchiolin arranged parallel to the surface. The periostracum and the prismatic layer are secreted from the edge of the mantle only, the pearly layer from the whole of its outer surface. The hingeligament is continuous with the periostracum, and is to be looked upon simply as a median uncalcified portion of the shell, which is therefore, in strictness, a single continuous structure.

By the removal of the shell the body of the animal is seen to be elongated from before backwards, narrow from side to side, produced on either side into a mantlelobe, and continued ventrally into a keel-like visceral 
mass, which passes below and in front into the foot (Fig. IOI, $f t$ ). Thus each valve of the shell is in contact with the dorso-lateral region of the body of its own side together with the corresponding mantle-lobe, and it is from the epithelium covering these parts that the shell is formed as a cuticular secretion. The whole space between the two mantle-lobes, containing the gills, visceral mass, and foot, is called the mantle-cavity.

A single layer of epithelial cells, the deric epithelium or epiderm, covers the whole external surface-i.e., the body proper, both surfaces of the mantle, the gills, and foot; that of the gills and the inner surface of the mantle is ciliated. Beneath the epiderm come connective and muscular tissue, which occupy nearly the whole of the interior of the body not taken up by the viscera, the cœlome being, as we shall sce, much reduced. The muscles are all unstriped, and are arranged in distinct bands or sheets, many of them being very large and conspicuous. The largest are the anterior and posterior adductors (Figs. IOI and I03, $a . a d, p . a d$ ), great cylindrical muscles which pass transversely across the body and are inserted at either end into the valves of the shell, which are approximated by their contraction. Two muscles of much smaller size pass from the foot to the shell, which they serve to draw back: they are the anterior and posterior retractors. A third extrinsic muscle arises from the shell close to the anterior adductor, and has its fibres spread fan-wise over the visceral mass, acting as a protractor. The substance of the foot itself consists of a complex mass of intrinsic muscles, which aid in withdrawing the foot: its protrusion is largely due to vascular turgescence. Lastly, all along the border of the mantle is a row of delicate pallial muscles, which, by their insertion into the shell, give rise to the line already seen. 
The colome is reduced to a single oroidal chamber, the pericardium (Figs. IOI and I03. pc), lying in the dorsal region of the body and containing the heart and part of the intestine: it is lined by coelomic epithelium, and does not correspond with the pericardial sinus of the crayfish, which is a blood-space (p. 376). In the remainder of the body the space between the epiderm and the viscera is filled by the muscles and connective-tissue.

The mouth (Fig. InI, mth) lies in the middle line just below the anterior adductor. On either side of it are two triangular flaps, the internal and external labial palps; the external palps unite with one another in front of the mouth, forming an upper lip ; the internal are similarly united behind the mouth, forming a lower lip : both are ciliated externally. The mouth leads by a short gullet (gul) into a large stomach (st), which receives the ducts of a pair of irregular, dark brown, digestize glands $(d . g l)$. The intestine (int), which is lined by a ciliated epithelium, comes off from the posterior end of the stomach, descends into the visceral mass, where it is coiled upon itself, then ascends parallel to its first portion, turns sharply backwards, and proceeds, as the rectum $(\boldsymbol{r c t})$, through the pericardium-where it traverses the ventricle of the heart, and above the posterior adductor, finally discharging by the anus (a) into the exhalant siphon or cloaca. The wall of the rectum is produced into a longitudinal ridge, or yphlosole (Figs. IOI and IO3, ty), and two similar ridges begin in the stomach and are continued into the first portion of the intestine. The stumach contains at certain seasuns of the year a gelatinous rod, the crystalline style.

The gills consist, as we have seen, of two plate-like bodies, which represent a single ctenidium (see Fig. I0 3 P), on either side between the risceral mass and the mantle: we have thus right outer and inner gill-laminee and left 
outer and inner gill-lamince (Fig. I03). Seen from the surface (Fig. IOI), each lamina presents a delicate double striation, being marked by faint lines running parallel

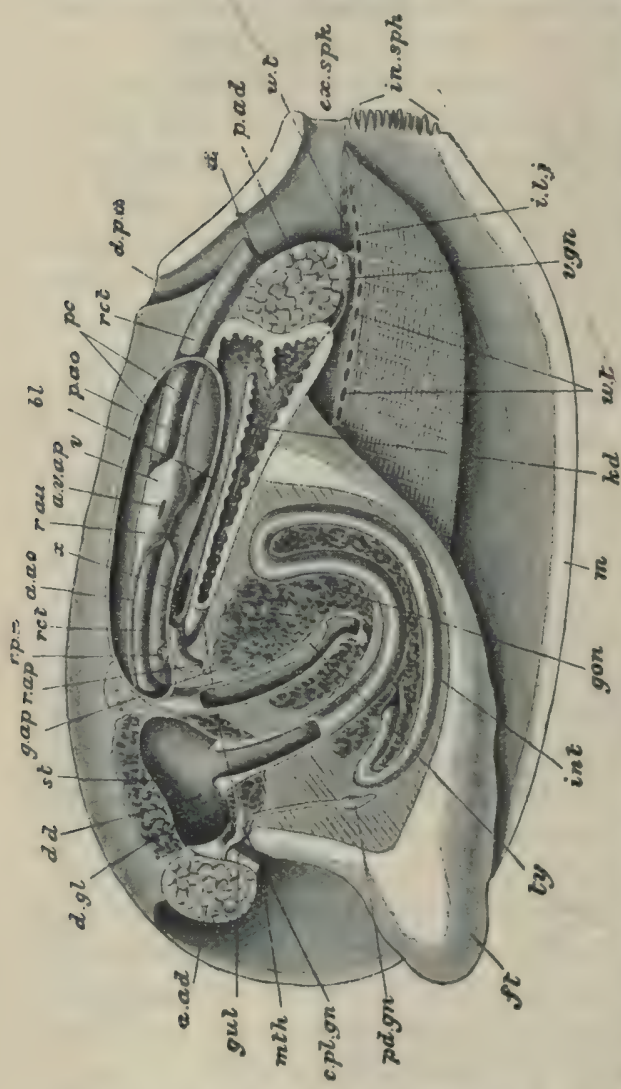

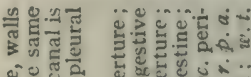

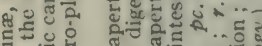

घ. 는 三.

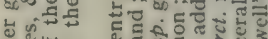
훙ㅎㅇ

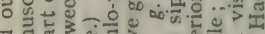

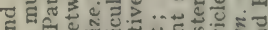
\% व के कु

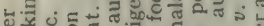
ถ้ง

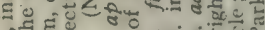

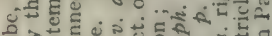
을 त o 을 हี ส n 7 강 장

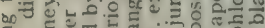

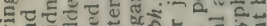

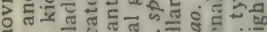

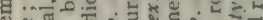
पू >iñ D. 0 \% ชำ व क्षे डू० जि 군

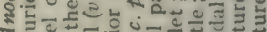
से क्षे ले ले

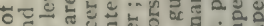
\% 응 है ल चु 远

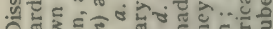
. 1.

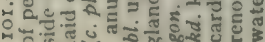
乌.

with, and by more pronounced lines running at right angles to, the long axis of the organ. Moreover, each lamina is double, being formed of two similar plates, the inner and outer lamilla, united with one another along 
the anterior, ventral and posterior edges of the lamina, but free dorsally. The lamina has thus the form of a long and extremely shallow bag, open above (Figs. I02 and ro3): its cavity is subdivided by vertical plates of tissue, the inter-lamellar junctions $(i . l . j)$, which extend between the two lamellae and divide the intervening

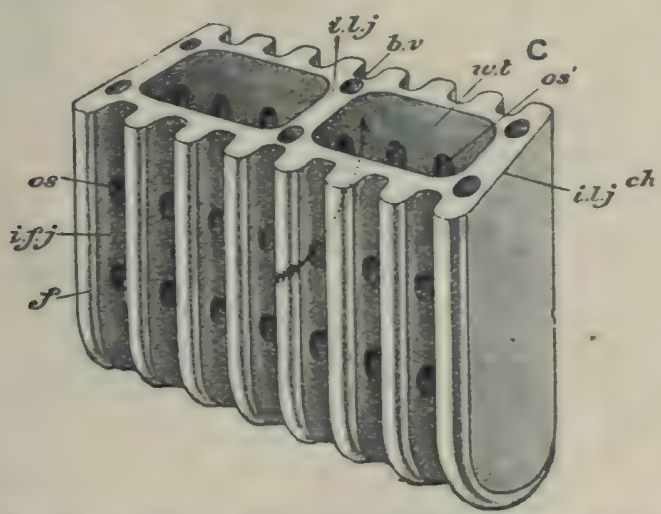

FiG. I02.-Diagram of the structure of a piece of gill of Anodonta.

The gill-lamina is made up of $V$-shaper gill-filament $=(f)$ arranged in longitudinal series and bound tngether by horizontal inter-flamentar junctions $(i . f . j)$ which cross them at right angles, forming a kind of basket-work with apertures, the ostia (os) leading from the outside and opening $\left(0 s^{\prime}\right)$ into the cavity of the gill. The latter is divided by vertical partitions, the inter-lamellar junctions (i...$j$, into compartments or water-tubes $(w, t)$, which open also into the suprabranchial chamber; b. v. blood-vessels. (From Parker and Haswell's Zoology.)

space into distinct compartments or water-tubes (Figs. IOI and $102, w . t)$, closed ventrally, but freely open along the dorsal edge of the gill. The vertical striation of the laminæ is due to the fact that each lamella is made up of a number of close-set gill-filaments (Fig. I02, $f$ ): the longitudinal striation to the circumstance that these filaments are connected by horizontal bars, the interfilamentar junctions $(i . f . j)$. At the thin free or ventral edge of the lamina the filaments of the two lamellæ are continuous with one another, so that each lamina has 
actually a single set of $\mathrm{V}$-shaped filaments, the outer limbs of which go to form the outer lamella, their inner limbs the inner lamella. Between the filaments, and bounded above and below by the inter-filamentar junctions, are minute apertures or ostia (os), which lead from the mantle-cavity through a more or less irregular series of cavities into the interior of the water-tubes. The filaments themselves are supported by chitinous rods, and covered with ciliated epithelium, the large cilia of which produce a current running from the exterior through the ostia into the water-tubes, and finally escaping by the wide dorsal apertures of the latter. The whole organ is traversed by blood-vessels $(b . v)$.

The mode of attachment of the gills presents certain features of importance (compare Fig. I03, A, B, C). The outer lamella of the outer lamina is attached along its whole length to the mantle: the inner lamella of the outer and the outer lamella of the inner lamina are attached together to the sides of the visceral mass a little below the origin of the mantle: the inner lamelia of the inner lamina is also attached to the visceral mass in front, but is free further back. The gills are longer than the visceral mass, and project behind it, below the posterior adductor (Figs. IOI and I03, C), as far as the posterior edge of the mantle: in this region the inner lamellæ of the right and left inner laminæ are united with one another, and the dorsal edges of all four laminæ constitute a horizontal partition between the pallial cavity below and the exhalant chamber or cloaca above. Owing to this arrangement it will be seen that the watertubes all open dorsally into a supra-branchial chamber (Fig. I03, s. br. c), continuous posteriorly with the cloaca and thus opening on the exterior by the exhalant siphon.

The physiological importance of the gills will now be obvious. By the action of their cilia a current is produced which sets in through the inhalant sivhom into 


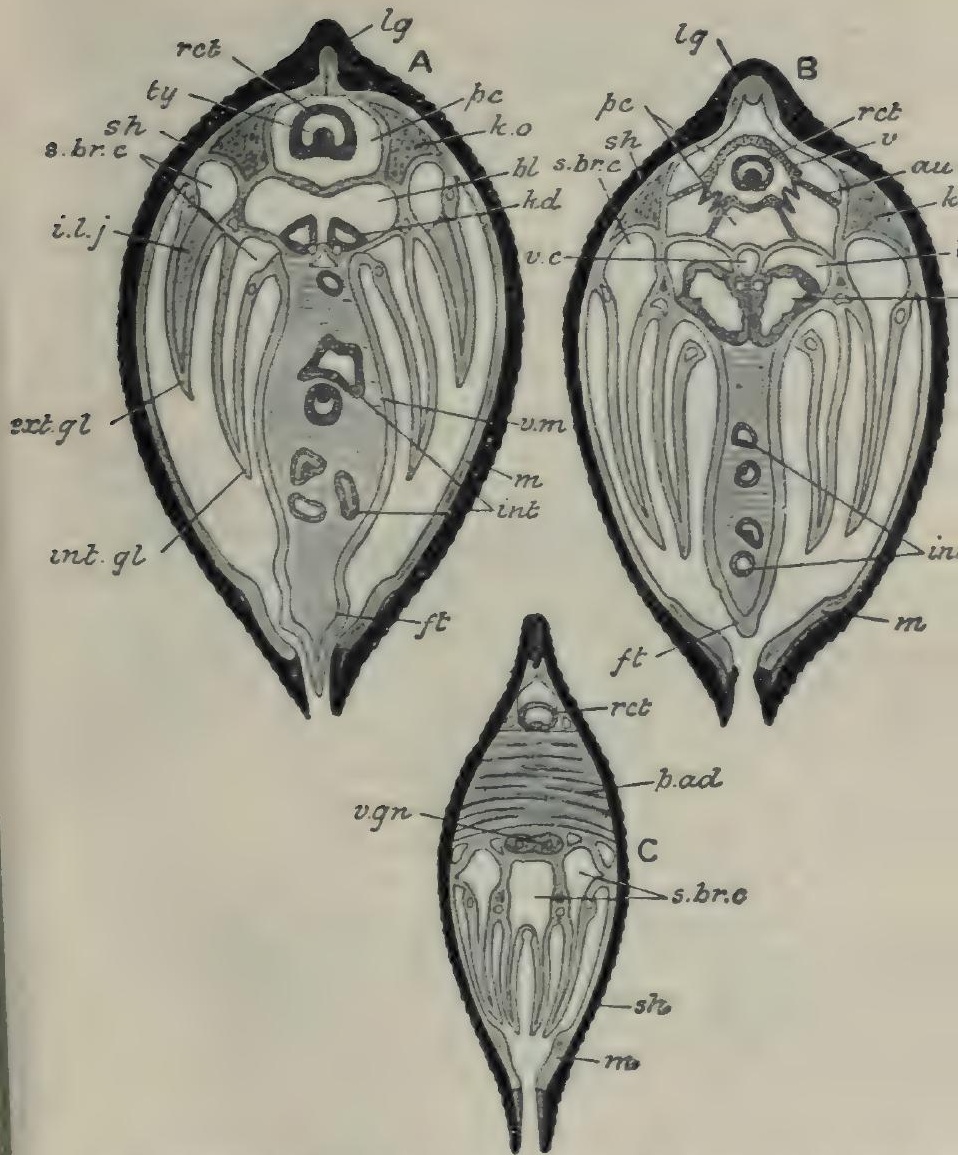

Fig. 103.-Anodonts. Three transverse sections $\left(X \mathrm{I} \frac{1}{1}\right)$. A, through the anterior part of the visceral mass. D, through the posterior part of the visceral mass. C, through the posterior adductor muscle.

au. auricle; bl. urinary bladder; ext. gl. external gill-lamina ; ft. foot ; i. $l . j$. inter-lamellar junction ; ist. intestine; int. gl. internal gill-lamina; $k d$. kidney; $k$. o. pericardial gland; $l g$. ligament; $m$. mantle; $p$. ad posterior adductor; pc. pericardium ; ret. rectum : s. br. c. supra-branchial chamber ; sh. shell ; $t y$. typhlusole ; $v$. ventricle ; v.c. vena cava ; v. gr. visceral ganglion; $v$. m. visceral mass. (From Parker and Haswell's Zoology, after Howes, slightly altered.) 
the pallial cavity, through the ostia into the water-tubes, thence into the supra-branchial chamber, and out at the exhalant siphon. The in-going current carries with it not only oxygen for the aëration of the blood, but also diatoms, infusoria, and other microscopic organisms, which are swept into the mouth by the cilia covering the labial palps. The out-going current carries with it the various products of excretion and the fæces passed into the cloaca. The action of the gills in producing the food current is more important than their respiratory function, which they share with the mantle.

The excretory organs are a single pair of curiously modified nephridia, situated one on each side of the body just below the pericardium. Each nephridium consists of two parts, a brown, spongy, glandular portion or kidney (Figs. IOI and I03, $k d$ ), and a thin-walled, non-glandular part or bladder $(b l)$. The two parts lie parallel to one another, the bladder being placed dorsally and immediately below the floor of the pericardium : they communicate with one another posteriorly, while in front the kidney opens into the pericardium $(r, p, a)$, and the bladder on the exterior by a minute aperture $(r . a p)$ situated between the inner gill-lamina and the visceral mass. Thus the whole organ, often called, after its discoverer, the organ of Bojanus, is simply a tube bent upon itself, opening at one end into the colome (see p. 40I), and at the other on the external surface of the body: it has therefore the normal relations of a nephridium (p. 340). The two bladders communicate anteriorly (Fig. ror, $x$ ), and their epithelium is ciliated, producing an outward current.

It seems probable that an excretory function is also discharged by a large glandular mass of reddish-brown colour, called the pericardial gland or Keber's organ (Fig. I03, $k$. o). It lies in the anterior region of the body just in front of the pericardium, into which it discharges. 
The circulatory system is well developed. The heart lies in the pericardium, and consists of a single ventricle (Figs. Ior and I03, B, v) and of right and left auricles (au). The ventricle is a muscular chamber which has the peculiarity of surrounding the rectum $(r c t)$ : the auricles

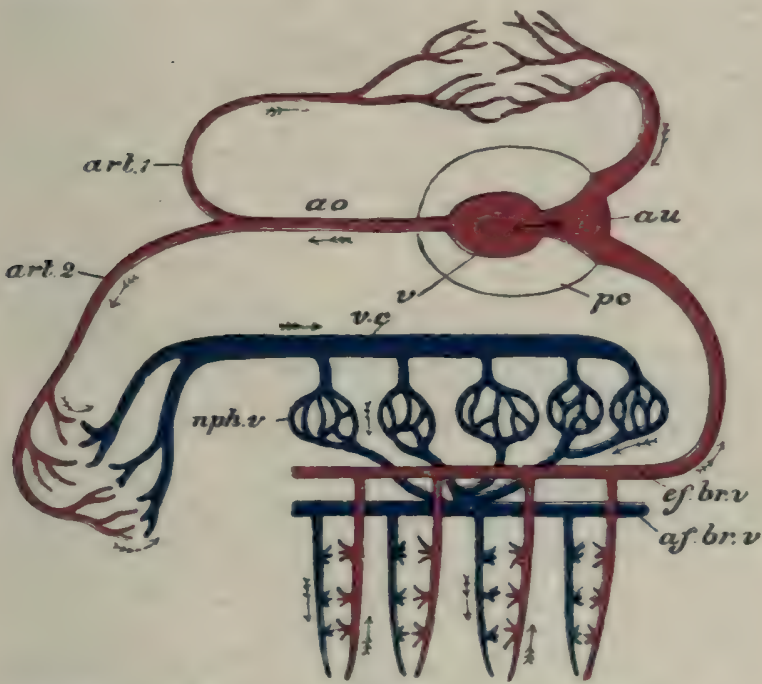

Frg. I04.-Diagram of the circulatory system of A rodonta.

The blnod received from the auricles $(a \omega)$ is pumped by the ventricle $(v)$ into the aorta $(a 0)$ and thence passes to the mantle $\left(a r b^{1}\right)$ and to the body generally (art 2). The blood which has circulated through the mantle is returned directly to the auricle : that from the body generally is collected into the vena cava $(v . c)$, passes by nephridial veins ( $r p h . v$.) to the kidners, thence by afferent branchial veins $(a f . b r . v)$ to the gills, and is returned by efferent branchial veins (ef. br. v) to the auricles; pc. pericardium. (From Parker and Haswell's Zoology.)

are thin-walled chambers communicating with the ventricle by valvular apertures opening towards the latter. From each end of the ventricle an artery is given off, the anterior aorta (Fig. Ior, $a . a 0$ ) extending above, the posterior aorta ( $p$. ao) below the rectum. From the aortæ the blood passes into arteries (Fig. Io4, 
$\left.a r t^{1}, a r t^{2}\right)$ which ramify all over the body, finally forming an extensive network of vessels, many of which are devoid of proper walls and have therefore the nature of sinuses. The returning blood passes into a large longitudinal vein, the vena cava (Figs. I03 and I04, v. c), placed between the nephridia, whence it is taken to the kidneys themselves (Fig. I04, nph. v), thence by afferent branchial veins $(a f . b r . v)$ to the gills, and finally returned by efferent branchial veins (e. br. v) to the auricles. The mantle has a very extensive blood-supply, and probably acts as the chief respiratory organ (p. 406): its blood $\left(\operatorname{art}{ }^{1}\right)$ is returned directly to the auricles without passing through either the kidneys or the gills. The blood is colourless and contains leucocytes. There is no communication between the blood-system and the pericardium.

The nervous system is formed on a type quite different from anything we have yet met with. On either side of the gullet is a small cerebro-pleural ganglion (Fig. IOI, c. $p l . g n)$, united with its fellow of the opposite side by a nerve-cord, the cerebral commissure, passing above the gullet. Each cerebro-pleural ganglion also gives off a cord, the cerebro-pedal connective, which passes downwards and backwards to a pedal ganglion ( $p d$. gn) situated at the junction of the visceral mass with the foot; the two pedal ganglia are so closely united as to form a single bilobed mass. From each cerebro-pleural ganglion there further proceeds a long cerebro-visceral connective, which passes directly backwards through the kidney and ends in a visceral ganglion (Figs. Ior and $103 \mathrm{C}, v . g n$ ) situated on the ventral side of the posterior adductor muscle. The visceral, like the pedal ganglia, are fused with one another. The cerebro pleural ganglia supply the labial palps and the anterior part of the mantle; the pedal, the foot and its muscles; the visceral, the 
enteric canal, heart, gills and posterior portion of the mantle. The nerve-cells are confined to the ganglia.

It will be seen that the cerebral commissures and cerebro-pedal connectives, together with the cerebropleural and pedal ganglia, form a nerve-ring which surrounds the gullet; the cerebro-pleural ganglia may be looked upon as a supraœsophageal nerve-mass corresponding with the brain of the earthworm and crayfish, and the pedal ganglia as an infraøesophageal mass representing the ventral nerve-cord.

Sensory organs are poorly developed, as might be expected in an animal of such sedentary habits. In connection with each visceral ganglion is a patch of sensory epithelium forming the so-called " olfactury organ," or, better, osphradium, the function of which is apparently to test the purity of the water entering by the respiratory current. Close to the pedal ganglia a minute paired statocyst, containing a calcareous lithite (pp. 315 and 38I), is found, the nerve of which is said to spring from the cerebro-pedal connective, being probably derived from the cerebral ganglion. Sensory cells, probably tactile, also occur round the edge of the mantle, and especially on the tentacles of the inhalant siphon.

The sexes are separate. The gonads (Fig. IoI, gon) are large, paired, racemose (p. r33) bodies, occupying a considerable portion of the visceral mass amongst the coils of the intestine: the spermary is white, the ovary reddish. Each gonad has a short duct which opens (g. $a p$ ) on the surface of the visceral mass, just in front of the renal aperture.

In the breeding season the eggs, extruded from the genital aperture, pass into the supra-branchial chamber, and so to the cloaca. There, in all probability, they are impregnated by sperms introduced with the respiratory current. The oosperms are then passed into the cavities 
of the outer gill-laminæ, which they distend enormously. Thus the outer gill-laminæ act as brood-pouches, and in them the embryo develops into the peculiar larval form presently to be described.

As in the frog and earthworm, the celis formed by the segmentation of the oosperm are of two sizes, small cells composed entirely of protoplasm, and large cells loaded with yolk-granules. The large become enclosed by the small cells, but the enteron thus formed is very small and quite unimportant during early larval life, the
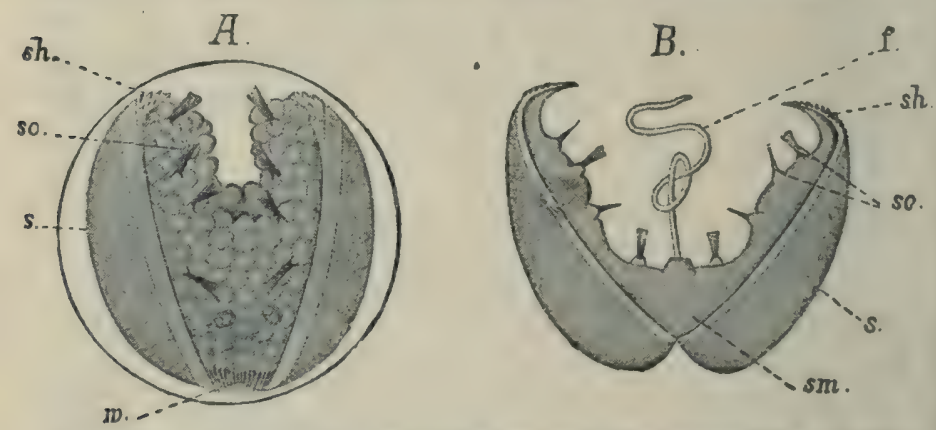

FIG. I05.-A, advanced embryo of Anodonta enclosed in the egg-membrane. B, free

$f$, byssus; $s$. shell ; sh. hooks ; sm. adductor muscle ; so. sensory hairs; $w$. ciliated area. (From Korschelt and Heider's Embryology.)

young mussels being nourished, after the manner of parasites (p. 280), by a secretion from the gills of the parent.

The dorsal surface of the embryo is soon marked out by the appearance of a deep depression, the shell-gland, which secretes, in the first place, a single, median shell. This is, however, soon replaced by a bivalved larval she!l (Fig. I05, s) of triangular form, the ventral angles being produced into hooks $(s h)$. The body at the same time becomes cleft from below upwards (A), forming the right and left mantle-lobes. On the ventral surface, 
between the lobes of the mantle, is formed a glandular pouch, which secretes a long thread, the byssus $(f)$. The larva is now called a glochidium, the subsequent history of which furnishes another example of a means of ensuring dispersal in a sedentary animal (compare pp. 274,283 , and 320 ).

The glochidia, entangled together by means of their byssal threads, escape from the gills of the parent by the exhalant siphon, and eventually attach themselves by their hooked valves to the body of a passing fish, such as a stickleback, becoming enclosed by an overgrowth of the skin of the fish. Here they live for a time as external parasites, gradually undergoing metamorphosis, and finally dropping from the host and assuming the sedentary habits of the adult.

This mode of development is exceptional amongst bivalves, in most of which (e.g., oyster) the egg gives rise to a larva resembling the trochosphere of many annelids (Fig. 89), the prostomial region then growing out into a thickened rim or velum which bears the circlet of cilia in front of the mouth, the larva at this stage being distinguished as a veliger.

The mussel belongs to the phylum Mollusea, which includes, in addition to the bivalved "shell-fish" (such as mussels, oysters, and cockles), the snails, slugs, whelks, periwinkles, \&c. (most of which possess a univalved shell), as well as the cuttle-fishes and their allies. These are all sharply distinguished from the Arthropods by the absence of segmentation, and by having, as a rule, an exoskeleton in the form of a shell. The bivalves are included in the class Pelecypoda or Lamellibranchiata, the essential structure of which you will have learnt from your study of the mussel.

You have now examined examples of the following chief divisions or phyla of the animal kingdom (compare p. 219):
1. Protozoa.
3. Annulata.
2. Cœlenterata.
4. Arthropoda.
5. Mollusca.
6. Vertebrata. 
In addition to these, you will probably have seen, when dissecting the frog, certain parasites. One of these, Polystomum (p. 33), belongs to a lowly group of worms of a flattened form, constituting the phylum Platyhelminthes, which includes the parasitic liver-flukes and tape-worms as well as certain free-living forms; another, Rhabdonema (p. I 53), belongs to the phylum Nemathelminthes, in which the mostly parasitic thread-worms are placed. Apart from certain other smaller groups, which include such animals as "wheel-animalcules," "sea-mats," "lampshells," \&c., there only remains one other of the larger phyla of which you will not have studied an example, viz., the phylum Echinodermata, which is constituted by the star-fishes, sea-urchins, sea-cucumbers, \&c. : these are all inhabitants of the sea, and are peculiar in exhibiting in the adult a more or less pronounced radial arrangement of their parts (p. 296), and in possessing a curious calcareous exoskeleton developed within their integument, consisting of small particles or of definitely-shaped plates. All the phyla with the exclusion of the Vertebrata are spoken of collectively as the Invertebrata.

\section{PRACTICAL DIRECTIONS}

FRESH-WATER MUSSEL

Examine a living specimen in a vessel of water with some sand at the bottom, and note the greenish-brown bivalve shell, the foot, and the manner in which the animal buries itself, anterior end downwards, with the pallial openings projecting posteriorly. Observe the currents of water passing in at the fringed inhalant apertuiv, and out at the exhalant aperture: these can be more easily observed if a little finely-powdered indigo or carmine is placed in the water.

In order to kill the animal in as fully-extended a condition as possible, place it in a saucepan or beaker of water, and heat over a gas-burner or spirit-lamp until the water is warmed up to a temperature of about $40^{3} \mathrm{C}$., when the foot will be protruded. Keep at this temperature until the mussel is dead. The animal is best preserved from day to day in 70 per cent. spirit, or $2-3$ per cent. formaline.

\section{A. External Characters.}

Compare the shell with the carapace of the crayfish, and observe that it covers the whole body and is densely calcified 
except along the dorsal hinge-line, where it forms an elastic ligament connecting the two lateral ialves of the shell. Note the lines of growth, and the umbo near the hinge-line. The anterior end is rounded, the posterior end more pointed. In dead specimens the valves gape, owing to the elasticity of the ligament and to the relaxation of the adductor muscles (see below), and they can then be seen to be lined by a fold of the integument, the mantle or pallium. By wedging the valves open still further, the anterior and posterior adductor muscles are seen connecting the two valves; also the foot, iisceral mass, and gills, between the two mantle folds.

\section{$B$. Dissection.}

I. Carefully insert a scalpel between the left valve and mantle-edge, and separate them all round the margin of the valve. Then cut through the anterior and posterior adductor muscles close to the shell, and remove the left valve, cutting through the ligament.

I. Compare the positions of the cut muscles on the body with the muscular impressions on the shell: $-a$, the large anterior adductor near the dorsal and anterior end; and close behind it $-b$, the protractor, and $c$, the antevior retractor, the latter dorsal to the former; $d$, the large posterior adductor near the dorsal and posterior end, on the upper and anterior margin of which is $e$, the posterior retractor. Note also the thickened ventral edge of the mantle, the corresponding pallial line on the valve, as well as the lines from the muscular impressions converging towards the umbo, the smooth longitudinal hinge (with hinge-teeth in Unio), the ligament, and the iridescent inner layer of the shell. Sketch the inner surface of the valve.

2. Break the shell across, and examine the edge with a lens, noting the three layers of which it is composed: $a$. the thin outer uncalcified periostracum; $b$. the thicker middle prismatic layer; and $c$. the inner pearly or nacreous layer.

Pin down the animal firmly under water, leaving it in the right valve: insert the pins through the ligament and muscular part of the foot, and obliquely against the edges of the shell. Note:-

3. The left mantle-lobe uniting with the right above the middle of the anterior adductor muscle anteriorly, and behind the posterior adductor posteriorly. Just behind the lat ter muscle the margins of the mantle are much thickened ; and in life, the two approximated mantle-edges here separate 
to form the exhalant and inhalant apertures, the latter of which is provided with short tentacles.

4. Turn back the mantle-lobe and note the mantle-cavity and its contents (Fig. IOI) :- $a$. the foot and visceral mass; $b$. the left pair of gill-lamina at the sides of the visceral mass ; and anteriorly $c$. the left couple of small, triangular labial palps. (Note that there is no distinct head.) These parts are situated between the two mantle-folds in the large ventral mantle-cavity. Note also the position of the pericardium on the dorsal side of the gills, the pericardial gland (p. 406), and the left excretory organ (nephridium), between the pericardium and gill, and extending back to the posterior adductor muscle.

5. Pass a seeker into the exhalant aperture and note that it enters a short exhalant siphon or cloaca, separated from the mantle-cavity by the bases of the gills. Slit this chamber open, and note that it extends above the posterior adductor muscle, as well as above the gills. The hinder end of the rectum will be seen just above the posterior adductor muscle, opening by the anus into the cloaca. Insert a seeker into the mouth, between the anterior adductor muscle and the anterior edge of the foot.

II. I. Remove the left mantle-lobe, cutting very carefully along the bases of the palps and gills. Then slit open the pericardium longitudinally, a short distance dorsally to the gills, and note the rectum, ventricle, and delicate left auricle. Cut away sufficient of the wall of the pericardium to expose these parts, taking care not to injure the auricle. Note-

2. The rectum, which runs straight through the pericardium, and in its middle is surrounded by the elongated muscular ventricle, which is bilobed posteriorly. The thinwalled, triangular left auvicle can be seen opening into the ventricle by a valvular aperture: the base of the auricle is attached along the dorsal border of the gills. (Note the right auricle on the other side.) Inject French-blue (p. 99) into the left auriculo-ventricular aperture, and note $a$. the anterior aorta above the rectum, and $b$. the posterior aorta below the rectum. In the middle line of the floor of the pericardium the vena cava can be seen.

3. Examine the gills. Note the left outer and inner lamina, and that in the female the former is often distended with eggs or lavee. (Fig. I05): save some of these for subsequent examination ( $(I V)$. Each lamina consists of an outer and an inner lamella, forming a kind of trellis-work, 
with small meshes or ostia, separated by horizontal and vertical bars. Cut away a piece of the outer lamella, noting that the two lamella of each gill are united at intervals by interlamellar junctions.

4. Make out the mode of attachment of the gills (see p. 404 , and Fig. 103). Sketch your dissection with the gill-lamina in their natural position.

5. Pass a seeker forwards from the exhalant siphon along the supra-branchial passages-two longitudinal canals at the bases of the gills communicating with the interlamellar spaces or water-tubes. The inner canal unites with its fellow behind the visceral mass (see Fig. I03, C), and also communicates with the branchial chamber through the slit between the visceral mass and the middle part of the lamella of the inner gill-lamina. Cut open the canals carefully, and examine their relations.

6. Turn up the gill-lamina, and insert the scissors in the slit just mentioned; cut forwards through the inner lamella of the inner lamina for a short distance. The renal aperture or nephridiopore can then be seen opening into the supra-branchial passage, and just below and in front of it is the genital aperture. Then note the paired reno-pericardial aperture (nephrostome) in the anterior end of the pericardium, just beiow the point at which the rectum enters it : this can be more easily seen if the rectum is cut through and raised up.

7. Blow through the nephridiopore, and note the thinwalled, sac-like bladder into which it opens: this lies just below the pericardium, and communicates with its fellow anteriorly. Just beneath and internal to the bladder is the glandular portion of the kidney, which is dark in colour and extends further back than the bladder, beyond which it forms a large mass just in front of the posterior adductor muscle : it communicates with the hinder end of the bladder. Sketch.

III. I. Remove both gill-lamina carefully, cutting along their bases; stain and mount a small piece of one lamella. Note that the lamella is made up of close-set vertical bars or gill-filaments, connected by numerous transverse interfilamentar junctions; the bars are covered with ciliated epithelial cells, and each is strengthened by pairs of small chitinous rods. The whole gill is traversed by bloodvessels. Sketch.

2. A small portion of the gill should be preserved, stained, embedded, and cut into sections (see p. 136) passing trans- 
versely through the gill-filaments. Compare with Fig. IO2, and sketch.

3. Mount in salt-solution a small piece of the edge of a fresh gill, and also of the mantle, and observe the movements of the cilia.

IV. If your specimen is a female, and contains ova or larvæ in the outer gill-lamina, examine some under the microscope. The ova are provided with a canal or micropyle perforating the egg-membrane, for the entrance of the sperms. Note the form of the larvæ or glochidia (Fig. 105). Sketch.

$\mathrm{V}$. The nervous system consists of three pairs of small orange-coloured ganglia, with connectives between them.

I. Cut away the left labial palps very carefully and look for the left cerebro-pleural ganglion, situated just below the attachment of the mantle-lobe close to the mouth and directly beneath the epithelium :-it may already have been exposed by removing the mantle-lobe. The two cerebro-pleural ganglia are connected by a commissure above the gullet.

2. On the under surface of the posterior adductor muscle are the two closely-apposed visceral ganglia, which will be at once seen when the membranous covering of this muscle is dissected off.

3. The pedal ganglia are more difficult to find, as they are deeply situated, at the junction of the foot and visceral mass. Slit up the foot by a median longitudinal incision, and carefully dissect the two halves apart until the two closely apposed ganglia are seen; then remove the left half of the foot.

4. Carefully dissect out the cerebro-pedal connective, which runs straight from the cerebro-pleural to the pedal ganglion. Then follow out the cerebro-visceral connective from behind forwards:- each extends forwards from the corresponding visceral ganglion along the inner surface of the renal organ, and then passes slightly downwards across the visceral mass to the cerebro-pleural ganglia. Sketch.

VI. The sensory organs are not easy to make out. In order to see the osphradium and tactile-cells, it is necessary to cut microscopic sections. The statocyst may be found by examining under the microscope a portion of the tissue just behind the pedal ganglion; but it is much more easily observed in the small fresh-water bivalve Cyclas, often to be found in ponds : it is only necessary to examine the entire 
animal in water under a low power after removing the shell in order to see the two globular statocysts, each lined by epithelial cells and containing a lithite, which is in constant, trembling motion.

VII. The alimentary organs are embedded in solid tissue in the visceral mass, the hinder part of the intestine or rectum alone being surrounded by a cœlomic cavity (pericardium). The anus has already been seen.

I. Remove the animal entirely from the shell, and pin down through the adductor muscles and foot under water. Dissect away the epiderm to the left side of and above the stomach (if this has not already been done), and note the brown digestive gland in the anterior dorsal part of the visceral mass. Then insert a seeker into the stomach through the mouth, to act as a guide, and slit up the short gullet and stomach. Note the ducts of the digestive gland opening into the stomach.

2. In the same manner slit up the whole intestine, either from the stomach backwards or from the rectum forwards, using the seeker as a guide the whole way, and first examining Fig. ror to see the direction which the coils take. Note the ventral typhlosole in the rectum, beginning at the last coil. Sketch.

VIII. I. The gonads appear very similar in both sexes, and fill up a large part of the visceral mass between the coils of the intestine : their ducts open at the genital apertures $(\$ \mathrm{II}, 6)$.

2. Tease up a bit of the ovary or spermary in salt-solution, stain with magenta, and examine.

\section{Transverse Sections (Fig. IO3).}

With a sharp scalpel or razor cut a specimen hardened in spirit or formaline into transverse sections about $\frac{1}{t}$ inch thick, passing obliquely through-

I. The anterior part of the pericardial cavity, visceral mass, and foot. Note the relations of the mantle-folds, the gonads, the enteric canal (including the rectum with its typhlosole), the gills and supra-branchial canals, the anterior aorta and iena caia, and the kidne's and bladders.

2. The ventricle, auricles, and hinder part of the visceral mass. Note the relations of the mantle-folds, enteric canal, gills, supra-branchial canals, zena caía, kidneys, and bladders.

3. The posterior adductor muscle, hinder part of gills, and rectum. Note the relations of the various parts. Sketch the three sections. 


\section{CHAPTER IX}

CHARACTFRS OF THE PHYLUM VERTEBRATA-THE LANCELET

From your study of the frog you have already learnt a good deal about a vertebrate animal, and we will now examine a few more examples of the phylum Vertebrata, which, as we have seen (p. 2r9), includes several classes, the Pisces, Amphibia, Reptilia, Aves, and Mammalia. Leaving aside the lampreys and their allies, which present certain peculiarities and are therefore placed in a class by themselves, these all agree with one another and resemble the frog in the following essential characters.

They all possess :- a vertebral column-or at any rate a notochord (p. 203), which is nearly always replaced by a vertebral column in the adult--and a skull with upper and lower jazes; a dorsal, hollow, nervous system, consisting of brain and spinal cord; paired olfactory organs, eyes, and auditory organs, which take on a close connection with the skull; a pharynx, which at an early stage, at any rate, is perforated by a small number (never more than seven) of paired gill-clefts (p. 204); a mouth which is ventral and anterior, and an anus which is ventral and posterior; kidneys which are composed of numerous urinary tubules or nephridia; a chambered heart and 
red blood-corpuscles; a liver, and a hepatic portal system; usually ti'o pairs of limbs, and never more than two pairs ; and a series of body-muscles which are divided into segments or myomeres (p. 203), at any rate in early stages, and are composed of striped fibres.

Now in various parts of the world, occurring only rarely off the English coasts, certain small fish-like animals called lancelets are found, the commonest species of which is known as Amphioxus lanceolatus (Fig. I06). This animal, which possesses a median fin like that of the tadpole ( $p$. 207), is not more than a couple of inches in length, and lives in the sea near the shore, burrowing in the sand ; it is especially interesting owing to the fact that it presents certain characteristics indicating a near relationship to the primitive ancestors of Vertebrates. It possesses a notochord, a dorsal hollow nervous system, a pharynx perforated by gill-slits, a hollow outgrowth of the intestine representing a simple liver, with a hepatic portal system, and a
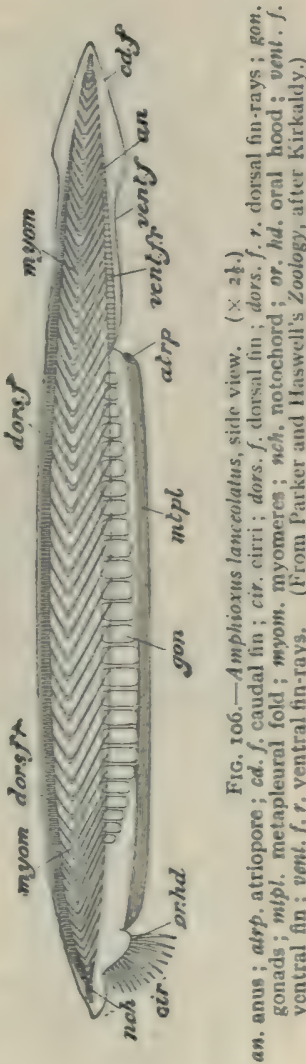
series of nephridia. But it differs from all the higher Vertebrates in the following important points.

The epiderm consists of a single layer of cells. There is no distinct head and no skull ; the persistent notochord 


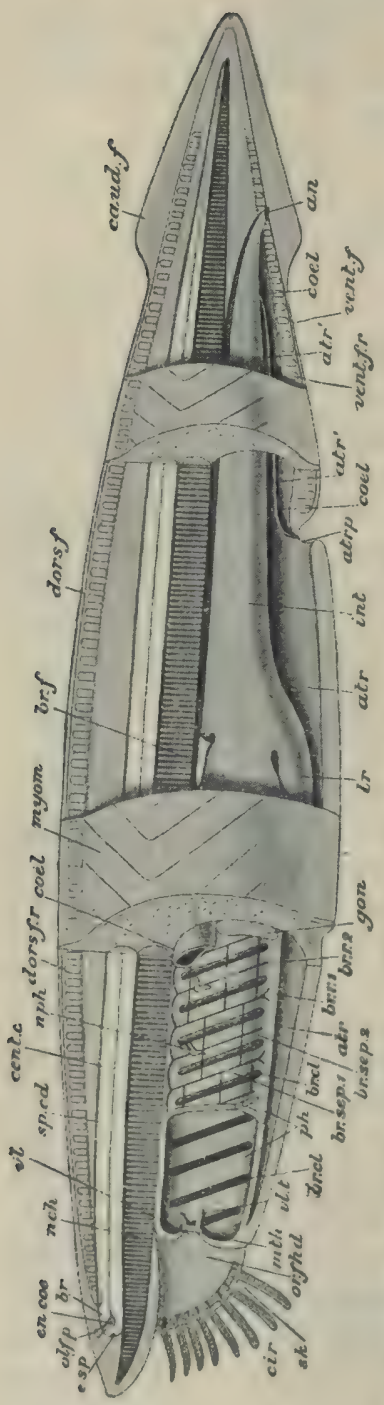

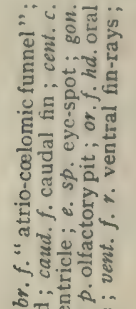

के 루.

엉

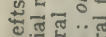
을

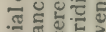
궁 है कि म 눙ำำ ㄷํㅇ 성 इ… कू च

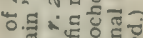
र造证 토을 을 牙.

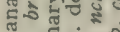
- है + . त ह \&

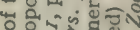
.동

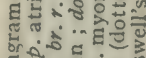

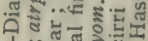
1

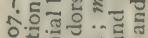
됴 들 ن들 स응 के के है छ ㄴ. $23 \div$

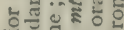

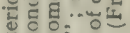

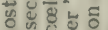

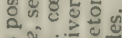

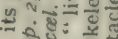
$25 . .20$ कै

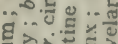

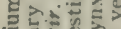

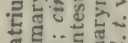
ข้․ํ를 들ㄷ… ปิธูป

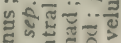

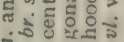

(Fig. I0\%, nch) extends to the anterior end of the animal, and there are no paired limbs. A brain (br) can hardly be said to be present, and there are no paired olfactory, optic, or auditory organs. The pharynx is relatively very large, and is perforated by very numerous oblique gill-slits (br. cl), which do not open on the exterior directly, but aresurrounded by a chamber, the atrium (Figs. I07, atr, and I09, $\mathrm{A}$,

b) ; this opens externally by a pore (Figs. 106 and $10 \%$, atrp), and though differing in its mode of development from the branchial chamber of the tadpole (p. 207), has somewhat 
similar relations. There is no heart (Fig. IIO), and the colourless blood apparently contains no corpuscles of any kind. The nephridia remain distinct, not being united into a single kidney on either side: they are situated anteriorly, in the neighbourhood of the pharynx (Figs. I07, $n p h$, and I09, A, n); the gonads (Figs. I06 and 107, gon, and Fig. I09, A, g) are metamerically arranged, and have no ducts.

In certain of its characters Amphioxus resembles the members of a group of animals - the Tunicata, commonly known as "sea-squirts," in which the body is enclosed in a "test" or mantle, consisting largely of cellulose (p. 244). These, like Vertebrates, possess a notochord and a dorsal, hollow nervous system in young stages, and in the adult retain numerous pharyngeal gill-slits; they are almost certainly degenerate descendants of primitive animals from which the Vertebrata also arose.

These numerous and marked differences between the lancelet and the higher Vertebrates make it necessary to place Amphioxus in a separate division of the Vertebrata; called-from one important negative characterthe Acrania, while all the other Vertebrates, which possess skulls, are included in the division Craniata.

The external appearance of Amphioxus is represented in Fig. I06. In addition to the points already referred to, it will be seen that the mouth is surrounded by a fold, the oral hood (or. hd), from which a number of tentacles or cirri (cir) are given off; and that there is a lateral or metapleural fold $(m t p l)$ along either side of the body extending backwards as far as the atrial pore, in addition to the median fin-fold (dors.f, cd.f, vent.f) extending round the tail as a caudal fin. In the young animal the gill-slits open directly to the exterior, but a median canal is subsequently formed along the ventral side of the body, and as this extends inwards and its edges unite to form the atrium (Fig. I08, $s l, p$ ), leaving 
only the atriopore open, it gradually surrounds the pharynx at the sides, pushing the coelome before it, so that the latter becomes greatly reduced in this region (Fig. I09, A, co).

The skeleton is very simple; besides the notochord there are rod-like bars of a substance somewhat
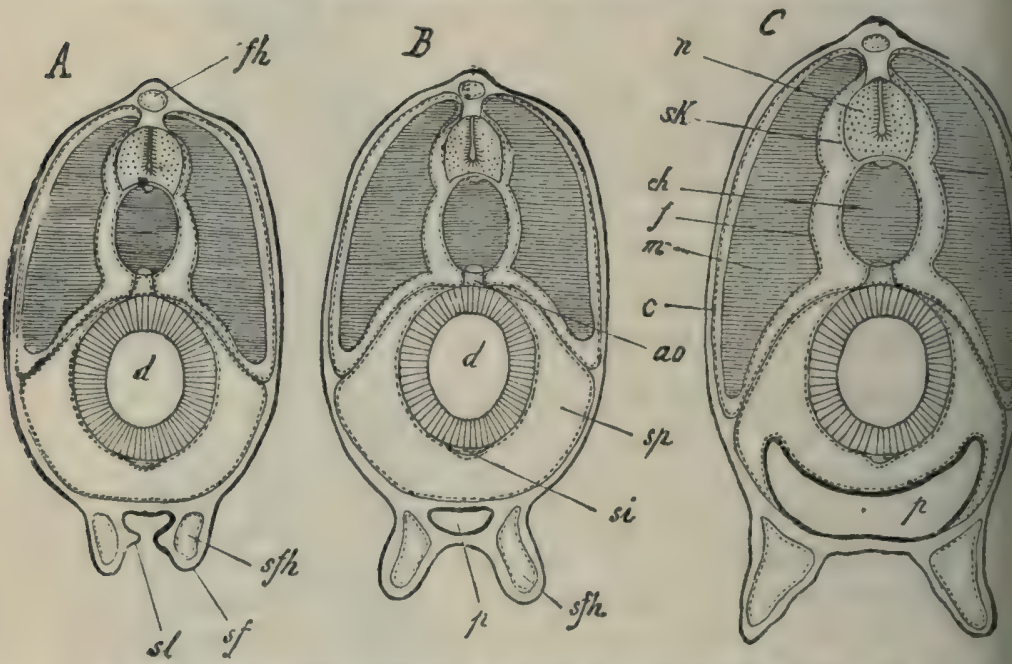

Fic. I03.-Amphioxus lanceolatus. Diagrammatic transverse sections of larva in three different stages to show the development of the atrium.

ao. aorta ; $c$. derm; $c h$. notochord; $d$. intestine; $f$. fascia ; $f h$. cavity for dorsal fin-ray; $m$. myomere; $n$. spinal cord; $p$. atrium: $s f$. metapleural folds ; $s f h$. metapleural cavity; si. sub-intestinal vein; $s k$. wall of neural canal and sheath of notochord; sl. sub-atrial ridge; $s p$. coelome. (From Korschelt and Heider's Embryology, after Lankester and Willey.)

resembling cartilage supporting the oral hood and cirri (Fig. IO7, $s k$ ), chitinoid rods (br. $r$ ) supporting the gillbars between the clefts, and short rods of connectivetissue, the fin-rays, in the dorsal and ventral fins (Figs. I06 and I07, dors. $f$. $r$, vent. $f . r$ ).

In the course of development, each primary gill-slit becomes divided into two by the growth, from above 
On the ventral wall of the pharynx is a longitudinal groove, the endostyle (Fig. I09, A, e), lined by ciliated and glandular cells; and a somewhat similar groove, the epipharyngeal groove, extends along the dorsal aspect of the pharynx: these serve as channels along which the particles of food pass into the intestine. The minute mouth (Fig. IO7, mth) leads from the cavity of the oral hood into the pharynx, and is surrounded by a membrane, the velum $(v l)$, produced at its edge into a number of tentacles $(v l . t)$.

The intestine is straight (Fig. I07, int) and the hepatic cacum, or rudimentary liver, arises from its anterior end on the ventral side, and extends forwards to the right of the pharynx (Fig. I07, lr, and Fig. Iog, A, $l$ ).

Although no heart is present, the blood-vessels, some of which are contractile, present certain undoubted homologies with the more complex vessels of the Craniata, and so a distinction is made between arteries and veins. The arrangement of the chief of these may be seen from the diagram (Fig. IIO), which should be compared with Fig. 83 and later on with Fig. II9. Blood occurs in certain spaces as well as in the vessels.

The numerous nephridial tubes (Fig. I07, $n p h$ ) are situated above the pharynx on either side in relation with the reduced coelome in this region. The internal end of each is provided with bunches of peculiar knoblike cells projecting from closed nephrostomes: the other end opens into the atrium (Fig. I09, A, n).

The spinal cord has a dorsal fissure but no ventral fissure (p. I55). Its central canal widens out in front (Fig. $107, b r$ ) to form a small cerebral ventricle (en.coe) in the front wall of which is an unpaired pigment-spot, representing a simple kind of eye (e. $s p)$, and at its anterior end is a ciliated depression usually known as the olfactory pit (olf. p). There is no auditory organ. 
The dorsal and ventral nerves (Fig. Ing, A, $s n$ ) remain separate, and do not unite to form a nerve-trunk (compare p. I63).

The sexes are distinct, and the metamerically arranged gonads (Figs. 106, 107, and 109, A, gon, g) are situated in pouches, the cavities of which represent part of the coelome and which project into the atrium on either side of the pharynx. When ripe, the minute eggs and

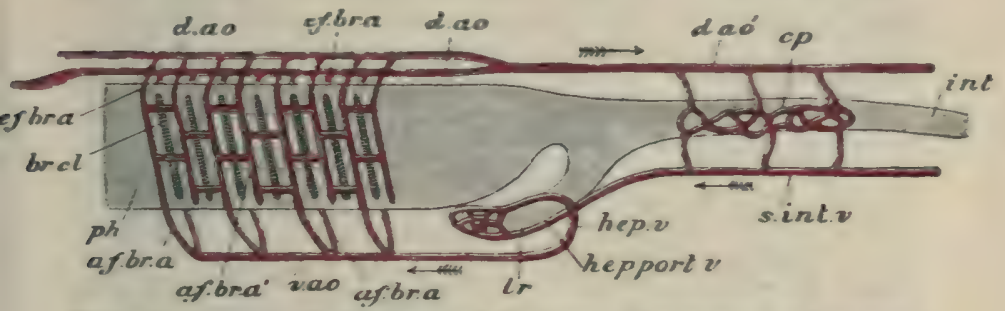

Frg. Iro.-Diarram of the chief versels of A mphiorus. The arrows show the course taken by the blood.

af. $b$ r. a. afferent branchial arteries: $b r$. $c l$. gill-slits : $c p$. intestinal capillaries; d. ao. paired dorsal aortx: d. ao'. median dorsal aorta; ef. br. a. efferent branchial arteries: hep. port. $\mathrm{t}$. hepatic portal vein : hep. $r$. hepatic vein : int. intestine: Ir. "liver "; ph. pharynx ; s. int. $v$. sub-intestinal vein ; $v$. co ventral aorta. which contracts from behind forwards. (Representatives of the precaval and cardinal reins of fisbes are also said to be present.) (From Parker and Haswell's Zoology.)

sperms burst through into the atrium and find their way out through the atriopore.

The oosperm undergoes segmentation, and the embryo is hatched at a relatively early stage as a simple larva, which gradually develops into the adult form. The embryology of Amphioxus, which is very instructive, will be referred to in greater detail in Chapter XIII. 


\section{PRACTICAL DIRECTIONS}

\section{AMPHIOXUS}

\section{A. External characters.}

Examine an entire specimen and note :- 1 , the form of the body; 2, the continuous median fins-dorsal, caudal, and ventral ; 3 , the paired lateral fin-fold or metapleure, extending along the body anteriorly to the ventral fin ; 4 , the oval hood, anterior and ventral, with its cirri; 5 , the anus, a short distance from the posterior end, just on the left side of the caudal fin ; 6 , the median ventral atriopore, at the junction of the lateral and ventral fins; 7 , the myomeres.

\section{$B$. Anatomy.}

The dissection of Amphioxus is too difficult a task for the beginner, and so it is better to proceed as follows:-

1. Place a young specimen for a short time in absolute alcohol, and then transfer to oil of cloves : if the specimen is a small one, it may with advantage be slightly stained first. Then transfer to a hollow slide, or, if you use an ordinary slide, support the cover-glass on two small pieces of wood of the thickness of the specimen, and mount in thick balsam: or the preparation may be examined in oil of cloves. (Many details are better seen in very young specimens, not more than half an inch long, which may be purchased ready mounted.) Examine and note in addition to the above points:-

I. The notochord, extending from the anterior to the posterior end of the body, rather nearer the dorsal than the ventral side.

2. The neural canal enclosing the spinal cord, lying just above the notochord, and having pigment in its walls.

3. The oral skeleton, consisting of a segmented basal bar along the sides of the oral hood, with a rod passing down each cirrus.

4. The longitudinal series of connective-tissue compartments, which are filled with gelatinous substance, forming the fin-rays.

5. The elastic horny rods supporting the gill-bars, and the arrangement of the gill-slits in pairs :- the primary gillbars separating the successive pairs, the rods supporting them being forked ventrally; and the secondary gill-bars 
between the members of a pair, the rods of these being unsplit ventrally. Note also the horizontal bars connecting the primary and secondary gill-bars and making the walls of the pharynx appear like a meshwork.

6. The cavity of the oral hood, bounded by lateral folds, the muscular ielum between it and the pharynx, and the minute mouth.

7. The intestine, running straight from the hinder end of the pharynx to the anus, and giving off near its anterior end the hepatic cacum or "liver" extending forward on the right side of the pharynx.

8. The myomeres and the intermuscular septa between them, arranged like a series of V's, the apices of which point forwards. The muscular fibres run longitudinally from septum to septum, and are of the striped kind.

9. The gonads (ocaries or spermaries), arranged in a single row on either side of and also rather further back than the pharynx extending as far as the atriopore. Note in the female the large $o v a$, which, when ripe, cause a great distension of the body; and in the male the minute sperm-cells, the structure of which cannot be made out in entire specimens. Sketch.

II. Cut a specimen into short pieces, about an eighth of an inch in length, and select portions from- $a$, between the hinder end of the atrium and the anus (Fig. I09, B), or through the latter; $b$, just in front of or thrcugh the atriopore; $c$, through the anterior part of the pharynx; and $d$, through the posterior part of the pharynx (Fig. I09, A). Stain and embed these, and prepare a few sections from each (see p. 136). Examine in the order given above, first with the low, and then with the high power. Sketch a section from each region.

a. I. The oval form of the section, the median dorsal and ventral fins, and the integument (columnar epiderm and thin derm).

2. The myomeres cut across in various planes, and appearing as squarish masses separated by the septa.

3. The central notochord, oval in transverse section, with transverse wavy lines indicating the boundaries of the vacuolated notochordal cells. It is surrounded by a connective-tissue sheath, continuous with the connectivetissue investment of the neural canal above and with the intermuscular septa at the sides: the latter pass into the derm peripherally. 
4. The spinal cord, lying in the neural canal. It is ovoid in transverse section, and has a dorsal fissure extending downwards nearly to the central canal, which is nearer the ventral than the dorsal surface. See if your section happens to pass through a dorsal or ventral nevve. The dorsal nerves arise by large single roots from about the middle of the sides of the cord, while the ventral nerves have numerous fine roots, and arise from the ventro-lateral angles of the cord. The dorsal and ventral nerves are not in the same transverse plane (compare Fig. 52), but have an alternating arrangement.

5. The gelatinous connective-tissue forming the fin-rays.

6. The intestine, with its single layer of long epithelial cells. If the section passes through the anus, the latter will be seen opening on the left side of the ventral fin.

7. The colome, surrounding the intestine, and also enclosing the subintestinal veins, which are continuous anteriorly with the hepatic portal vein (Fig. IIO).

8. The dorsal aorta, just below the notochord.

$b$. Go over $2,3,4,5,6$, and 8 again-all much as in $a$. Note-

I. The dorsal and lateral (metapleural) fin-folds, ventral body-wall, and small metapleural canals.

2. The ccelome, a narrow space round the intestine, in which three or four subintestinal (portal) veins can be seen.

3. The atrial cavity just outside the colome, and separated from it by the atrial membrane. If the section passes through the atriopore, it will be seen in the mid-ventral line, putting the atrial cavity in communication with the exterior.

c. Note-1. The triangular form of the body in transverse section; the dorsal fin, the ventral surface, with its folded integument and thin transverse ventral muscles; the metapleural folds and their contained large canals. Then go over a. 2, 3, 4, and 5 again, which are all much as in $a$ and $b$.

2. The pharynx, lying just below the notochord, and extending nearly to the ventral body-walls. Note(a) the deep, dorsal epibranchial groove; (b) the ventral endostyle, projecting into the pharyngeal cavity and enclosing a colomic canal; (c) the gill-bars, a great number of which will be cut through obliquely, larger primary alternating with smaller secondary ones. Note the skeletal rods near their outer surfaces, and, in the primary bars, the small 
calomic spaces on the outer sides of the rods; (d) the dorsal suspensory folds of the pharynx, which ericlose the two dorsal coelomic canals.

3. The atrial caity, surrounding the pharynx, except at the mid-dorsal line, where the pharynx is attached to the sheath of the notochord, and communicating with the cavity of the pharynx through the gill-slits. It is enclosed by the two atrial folds, which are united in the mid-ventral line: the atrial epithelium is often much folded ventrally.

4. The single ventral aorta in the endostylar cœlomic canal, and the paired dorsal aoria; on either side of the epibranchial groove.

d. Note again $a .2,3,4,5 ; c . \mathbf{I}, 3,4$, and also :-

I. The pharynx, much as in c. 2, but laterally compressed. The endostyle is now converted into a groove.

2. The hepatic cacum, on the right side of the pharynx, and covered by the atrial epithelium. Note the coplomic space around it, in which are the portal reins and the hepatic veins (dorsal side).

3. The gonads (ovaries or spermaries)-large masses on either side of the pharynx, projecting into the atrial cavity and covered by the atrial folds. The spaces around them represent portions of the colome. Note the small spermcells in the male, and in the female, the large ova.

(The nephridia can only be made out satisfactorily in good sections of very well preserved specimens.) 


\section{CHAPTER $\mathrm{X}$}

CHARACTERS OF THE CLASS PISCES-THE DOGFISH

The class Pisces (see p. 4r8) includes a number of aquatic Vertebrates which present a considerable amount of difference in form and structure, and which are distinguished from the Amphibia, as a whole, by certain constant characteristics, of which the following are the chief.

The organs of respiration and locomotion are adapted for life in the water. The former consist, as in later stages of the tadpole (p. 207), of a series of vascular internal gills, attached to the septa separating the gillclefts, and they persist throughout life : only in a very few instances (viz., in the small sub-class of the Dipnoi or "Mud-fishes ") are lungs and internal nostrils also present. The pectoral and pelvic limbs have the form of paddle-like fins, which, like the median fins (p. 42I), are supported by skeletal fin-rays :- the median fin is usually subdivided into separate dorsal, ventral, and caudal portions. In addition to the endoskeleton, there is usually an exoskeleton, developed in the derm and consisting of scales; and peculiar integumentary sense-organs, occurring also in aquatic and larval Amphibians, and supplied by special nerves not represented in terrestrial Vertebrates, are always present. The cerebellum is relatively large; a tympanic cavity 
and membrane are not present, and except in the Dipnoi, there is only one auricle in the heart and no postcaval vein. A urinary bladder developed as an outgrowth of the enteric canal (p. 2ro) is wanting. There is never such a marked metamorphosis as in the case of the frog.

The two most important sub-classes of the Pisces are the Elasmobranchii and the Teleostomi. The Elasmobranchs are all marine forms, and include the dogfishes, sharks, rays, and skates: their endoskeleton is composed of cartilage, like that of the tadpole. The Teleostomi, in which the skeleton is mainly or to a large extent bony, include by far the greater number of fishes-both marine and fresh-water forms-such as the Salmon, Cod, Herring, Perch, as well as the Sturgeon and its allies.

The dogfishes are small sharks, of which there are a number of genera and species. They are all powerful swimmers, and feed voraciously on other fishes, crustaceans, \&c.

The commonest British forms are the Rough Hound (Scyllium canicula), the Lesser Spotted Dogfish (S. calulus), the Piked Dogfish (Acanthias vulgaris), and the Smooth Hound (Mustelus vulgaris). The following description, though referring mainly to Scyllium, will apply, in essential respects, to any of these.

External Characters and General Strueture.-The dogfish has a spindle-shaped body, ending in front in a bluntly-pointed snout and behind tapering off into an upturned tail. On the ventral surface of the head is the large, transversely elongated mouth (Fig. II 7), supported by a pair of jaws which work in a vertical, and not, like those of the crayfish, in a transverse plane: they are, in fact, like those of the frog, portions of the 
skull, having nothing to do with limbs. They are covered with numerous rows of teeth which vary in form in the different species. In front of the mouth, on the ventral surface of the snout, are the paired nostrils, each leading into a cup-like nasal or olfactory sac, and (in Scyllium) connected with the mouth by a groove. The eyes are situated one on either side of the head, above the mouth : they are protected by thick folds of the skin forming upper and lower eyelids, which can be partially closed over the eye. Behind the mouth are five pairs of slit-like apertures arranged in a longitudinal series: these are the gill-clefts or external branchial apertures (p. 4r8). Just behind each eye is a small aperture, the spiracle: like the gill-clefts, it communicates with the pharynx, and it is found by development to be actually the functionless first gill-cleft.

On the ventral surface, about half-way between the two ends of the fish, is the vent or anus, leading into the cloaca (p. 23), and on either side of it a small pouch into which usually opens a minute hole, the abdominal pore (Fig. I27), communicating with the cœlome, which is therefore not a completely closed cavity, as in the frog. The region from the end of the snout to the last gillcleft is considered as the head of the fish ; that from the last gill-cleft to the vent as the trunk; and the rest as the tail.

A number of symmetrically-arranged, minute apertures on the skin of the head, particularly numerous on the snout, lead into a series of tubes known as sensory canals, which are situated beneath the skin in this region; and a single tube, known as the lateral-line canal, the position of which is indicated by a very faint longitudinal line, extends along either side of the body and tail. The whole apparatus constitutes an important, but imperfectly understood, integumentary sense-organ: as 
already mentioned, it is represented in the tadpole but disappears at metamorphosis.

Springing from the body are a number of flattened folds, called the fins, divisible into median and paired. The median folds are not continuous, as in the tadpole and lancelet, but are subdivided into several distinct parts, viz., into two dorsal fins along the middle line of the back, a caudal fin lying along the ventral edge of the upturned or heterocercal tail, and a ventral fin behind the anus. The paired folds are the pectoral fins, situated one on either side of the trunk just behind the last gillcleft, and the pelvic fins, one on each side of the vent: these correspond to the pectoral and pelvic limbs of the frog. In the male there is connected with the inner border of each pelvic fin a grooved, rod-like structure known as the clasper, which serves as a copulatory organ.

It is very possible that the paired fins, like the median fins, are specialised portions of a primarily continuous fin-fold which extended along either side of the body, like the lateral or metapleural fold of Amphioxus (p. 42I).

The fish swims by vigorous strokes of the tail: the pectoral fins are used chiefly for steering, and the dorsal and ventral fins serve, like the keel of a boat, to maintain equilibrium.

The skin or external part of the body-wall consists, as usual, of two layers, an outer layer of deric epithelium or epiderm (Fig. III, Der. Epthm), formed, like that of the frog, of several layers of cells; and an inner layer of connective-tissue, the derm. In the derm are innumerable close-set, calcified bodies, each consisting of a little irregular bony plate produced into a short spine - composed, like the teeth present in the frog and most Vertebrates, of a calcified tissue harder than bone known 
as dentine, capped with a still harder tissue called enamel -which projects through the epiderm and gives a rough, sandpaper-like character to the skin. These placoid scales or dermal teeth together constitute the exoskeleton of the dogfish : it is a discontinuous, mainly dermal, exoskeleton (compare p. 445), and not a continuous cuticular one like that of the crayfish (p. 369).

Beneath the derm is the muscular layer, which, as in Amphioxus and in the tail of the tadpole, is metamerically segmented. - The muscles are divided into myomeres, following one another from before backwards, and having a zigzag disposition. The fibres composing them are longitudinal, and are inserted at either end into fibrous partitions or myocommas which separate the myomeres from one another. ${ }^{1}$ The muscular layer is of great thickness, especially its dorsal portion. The fibres of all the body-muscles are, as in the frog and Vertebrates generally, of the striped kind.

There is a large colome (Figs. III and $\mathrm{I} \mathrm{I} 7$ ), which, as in other Vertebrates, is confined to the trunk. The cavity is divisible into two parts: a large abdominal cavity, containing most of the viscera, and a small anterior and ventral compartment, the pericardial cavity (Fig. II $7, p c d . c a v$ ), containing the heart and communicating with the abdominal cavity by a canal which opens on the ventral surface of the gullet. Both are lined by colomic epithelium (Fig. II , Cal. Epthm) underlain by a layer of connective-tissue, a strong lining membrane being thus produced, called, as in the frog, peritoneum in the abdominal, pericardium in the pericardial cavity.

Another very characteristic Vertebrate feature is that

1 In the adult frog a segmentation can still be seen in the rectus muscle of the abdomen (Figs. 2 and I6). 
the dorsal body-wall is tunnelled, from end to end, by a median longitudinal neural cavity, in which the central nervous system is contained. The greater part of the cavity is narrow and cylindrical, and contains the spinal cord (Figs. III and II 7 , $s p . c d)$ : its anterior or cerebral portion is dilated, and contains the brain.

Skeleton.-Embedded in the body-wall and extending into the fins are the various parts of the endoskeleton. This characteristic supporting framework is mainly composed, as in the tadpole and in embryos of Vertebrates generally, of cartilage, which may be more or less impregnated with lime salts (p. 46), so as to have, in part, the appearance of bone but differing in structure from true bone: this calcification is well seen in the vertebral centra (p. 439). - The entire skeleton consists of separate pieces of cartilage, calcified or not, and connected with one

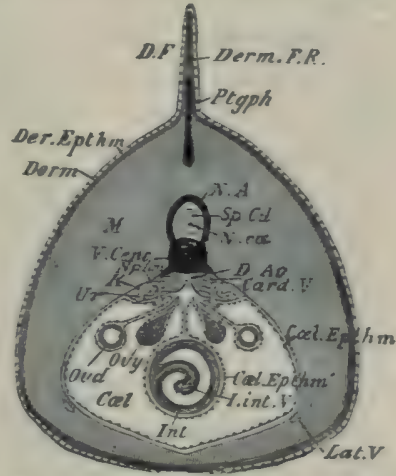

I IG. III.-Diagrammatic transverse section through the trunk of a female dog fish (compare Fig. 5). The ectoderm is dotted, the endoderm radially striated, the mesoderm evenly shaded, and the coelomic epithelium represented by a beaded line. $(x+$ i. $)$

Card. V. cardinal vein ; Col. coelome; Cal. Epthw. parietal, and Col. Epthm". visceral layer of coelomic (peritoneal) epithelium; D. Ao. dorsal aorta; Des. Epthm. epiderm; Derm. derm; Derm. F. R. dermal fin-ray; $D$. F . dorsal fin ; $\boldsymbol{I}$. int. $\boldsymbol{V}$. intra-intes tinal vein; $\boldsymbol{I n f}$. intestine; $\boldsymbol{K}$. kidney; Lat. $\boldsymbol{V}$. lateral vein; $M$. myomeres; $N$. $A$ : neural arch; $N$. ce. central canal of spinal cord; $N p h$. nephridium ; Ood. oviduct; Ooy. ovary; $S p$. Cd. spinal cord; $U r$. ureter; V. Cent. vertebral centrum. (From Parker's Elementery Biology.)

another by ligaments (p. 57): as in the frog, it is divisible into skull, vertebral column, and skeleton of the paired fins, with their arches or girdles; in connection with the skull are certain cartilaginous visceral arches, forming the upper and lower jaws and supporting the 
gills; and there are also skeletal parts in the median fins.

The cranium or brain-case (Fig. I12, $\mathrm{Cr}$ ) is an irregular cartilaginous box containing a spacious cavity for the brain, very similar to the chondrocranium of the frog (p. 43). It is produced into two pairs of outstanding projections : a posterior pair, called the auditory capsules (aud. $c p$ ), for the lodgment of the organs of hearing, ridge-like projections on which indicate the position of the semicircular canals (p. 187); and, in front of the brain-cavity, an anterior pair, the olfactory capsules (olf. $c p$ ), for the organs of smell, open below, and separated from one another by a septum. Between the olfactory and auditory capsules, on either side, the cranium is hollowed out into an orbit (or), bounded by a supraorbital and a sub-orbital ridge, for the reception of the eye. In front the brain-case is produced into three cartilaginous rods, forming the rostrum $(r)$ and supporting the snout. On its posterior face is the foramen magnum (p. 40), on either side of which is an oval condyle for articulation with the first vertebra. On the roof of the skull, between and behind the olfactory capsules, there is a fontanelle (p. 43), closed over by connective-tissue only ; and between the two auditory capsules is a depression into which open the two endolymphatic ducts of the ears (p. 465). The nerve-apertures will be referred to at a later stage.

In the human and other higher vertebrate skulls the upper jaw, as we have seen to be the case in the frog (Fig. 9), is firmly united to the cranium, and the lower alone is free. But in the dogfish both jaws $(u p . j, l . j)$ are connected with the cranium by ligaments $\left(l g, l g^{\prime}\right)$ only, and each consists of strong, paired (right and left) moieties, united with one another by fibrous tissue. The upper jaw, which corresponds to the palatoquadrate 


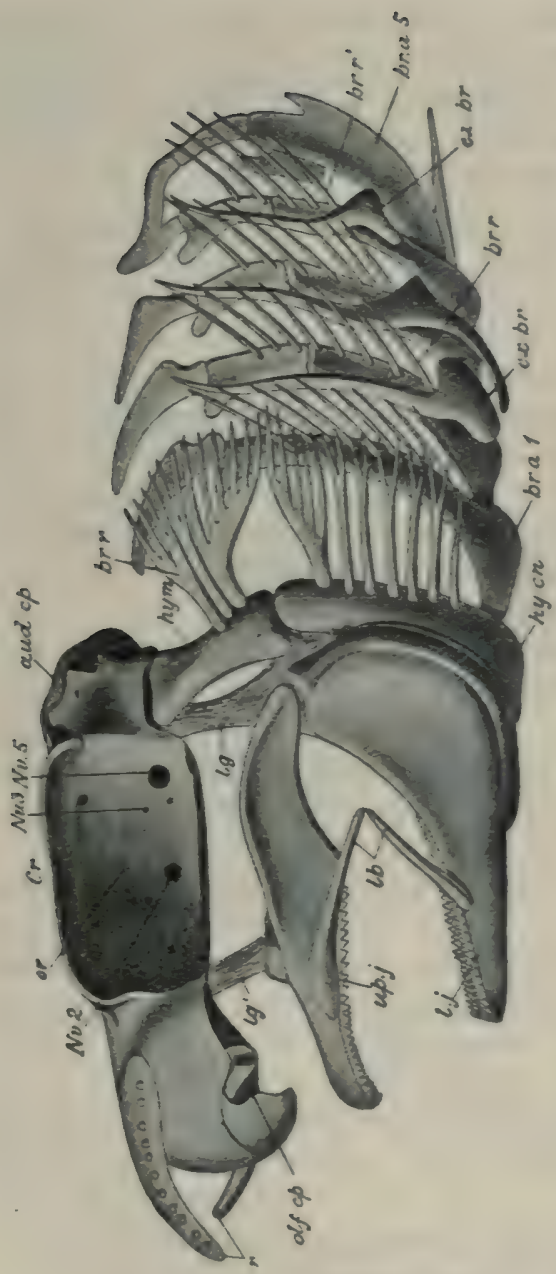

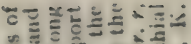

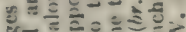
뜨르를 늘 닫을

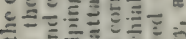
늘 ํㅡㄹ E.

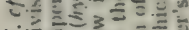
늘 c 늘 늘

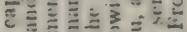
굴

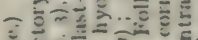
글 일

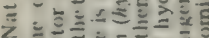

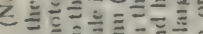

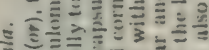
들 츨

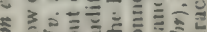

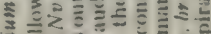

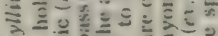

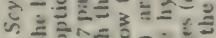
Ј

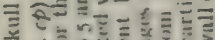

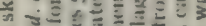
을 3 를

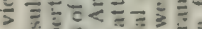

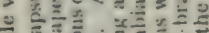

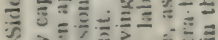
\& $>2 \div \geq=\div$ ? $\bar{c} \frac{y}{3} \bar{x} \bar{x}$

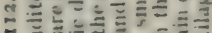
운 뜰

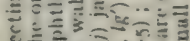
¿ํㅡㄹ

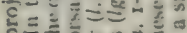

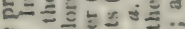

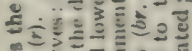

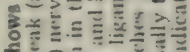

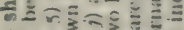

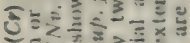

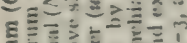
Ji

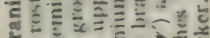
항

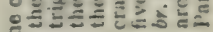
F 
cartilage of the frog (p. 44), presents at its posterior end a rounded surface against which fits a corresponding concavity on the lower jaw, so that a free articulation is produced, the lower jaw, or mandibular cartilage (p. 44), working up and down in the vertical plane.

The upper and lower jaws correspond to the first of a series of seven pairs of visceral arches, and are therefore often spoken of as the mandibular arch. The remaining six pairs have on either side the form of cartilaginous half-hoops, lying in the walls of the pharynx, and united with one another below so as to form a basket-like apparatus supporting the gills. The second of these arches is distinguished as the hyoid, and is situated immediately behind the jaws. It consists of two parts: a strong, rod-like hyomandibular (Fig. II2, hy. $m$ ), which articulates above with the auditory capsule and is connected below by fibrous tissue with the jaws, thus helping to suspend them to the cranium and serving as a suspensorium-formed in the frog by the quadrate (pp. 40 and 44); and a hyoid cornu or horn, which curves forwards inside the lower jaw, and is connected with its fellow of the opposite side by a median basi-hyal plate which supports the tongue (compare Fig. 9).

The remaining five arches (br.a. I-br.a.5) are called the branchial arches. Each is formed of several separate pieces, united by fibrous tissue so as to render possible the distension of the throat during swallowing: the fifth is connected below with its fellow by a large median basi-branchial plate, which supports the roof of the pericardial cavity. Both they and the hyoid give attachment to delicate cartilaginous branchial rays (br. $r$, $b r . r^{\prime}$, Fig. II8, $r$ ) which support the gill-filaments.

In the frog-tadpole the gills are similarly supported by cartilaginous arches, which become greatly reduced and 
modified at metamorphosis: the hyoid cartilage of the adult (p. 44) represents the hyoid and first branchial arch.

In addition to the parts of the dogfish's skull described above there are certain small cartilages of minor importance in relation with the nostrils, spiracle, mouth, and outer sides of the branchial arches (e.g., $l b$ and $e x . b r$ in Fig. I12).

The vertebral column has the general character of a jointed tube surrounding the spinal portion of the neural
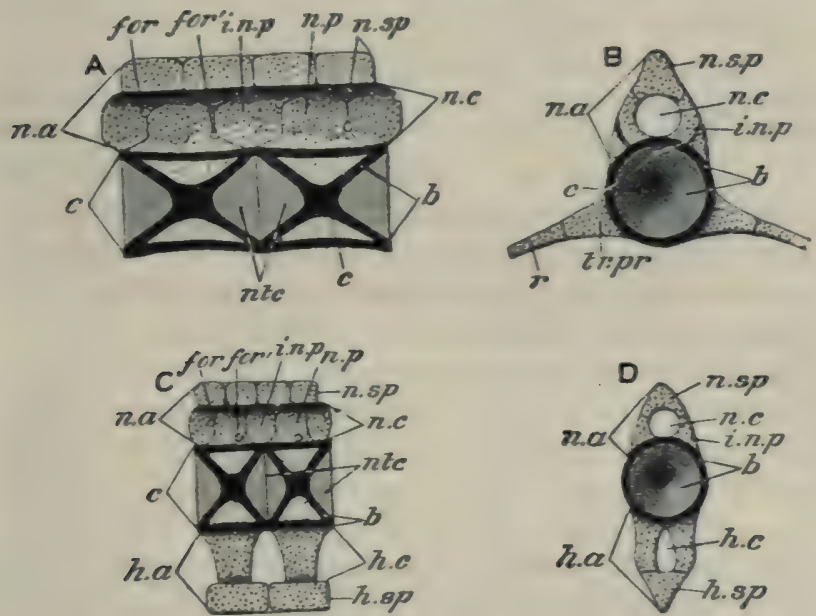

Fic. It3.-Vertebra of Scylliwin canicula. ( $\times$ 2.)

$A$ and $B$ from the trunk, $C$ and $D$ from the middle of the tail ; $A$ and $C$, two vertebra in longitudinal section; $B$ and $D$, single vertebræ viewed from one end.

b. calcified portion of centrum : $c$. centrum; for. foramen for dorsal, and for'. for ventral root of spinal nerve; $h . a$. hæmal arch; $h . c$. hæmal canal; $h . s p$. hæmal spine; $i . n . p$. interneural plate; $n$. $a$. neural arch; $n$. $c$. neural canal; $n$. $p$. neural process and plate; $n$. $s p$. neural spine; $n c$. intervertebral substance (remains of notochord); $i r$. pr. transverse process $r$. proximal portion of rib.

canal. Lying beneath this cavity, i.e., between it and the cœlome, is a longitudinal row of biconcave or amphicolous discs, the vertebral centra (Fig. II3, c; Fig. II7, cn): they are formed of cartilage, but have their anterior and posterior faces denselv calcified; 
calcified bars also extend longitudinally through cach centrum from face to face, so as to show a radial arrangement in transverse section. The biconcave intervals between the centra are filled with a soft intervertebral substance (Fig. II3, ntc), which is also present between the first vertebra and the skull and which represents part of the embryonic notochord (see pp. 203 and $4 \mathrm{r} 8$ ). The centra are united by ligament, so that the whole vertebral chain is very flexible.

In the frog it will be remembered that the completely ossified centra are procoelous, and are articulated with one another (pp. 36 and 57 ).

Connected with the dorsal aspect of the series of centra is a cartilaginous tunnel consisting of the neural arches (Figs. II 3 and II $7, n$, a), enclosing the spinal cord : it is divided into segments, corresponding with, but usually twice as numerous as the centra, owing to the presence of intercalary pieces. Arising from each centrum on either side is a neural process which is fused with a neural plate (Fig. II3, $n$. $p$ ) and perforated posteriorly for the exit of the ventral root of a spinal nerve $\left(f \circ r^{\prime}\right)$; and fitting between two consecutive neural plates is an intercalary piece, the interneural plate $(i . n . p)$, perforated by an aperture for the dorsal root of a spinal nerve (for). The arch is completed above by the neural spines ( $n . s p$ ), which fit in between the neural and interneural plates respectively, and are thus, like the lateral elements of the arch, twice as numerous as the centra. The first vertebra has facets for articulation with the condyles of the skull.

In the anterior part of the vertebral column the centra give off paired, outstanding transverse processes (Fig. II3, B, tr. pr), to the end of each of which is articulated a short, cartilaginous rod, the rib ( $r$ ). Further back ribs are wanting, the transverse processes 
are directed downwards, instead of outwards, and in the whole caudal region they unite below, forming hremal arches and spines (Fig. II 3, D, h. $a, h . s p$, and Fig. II7, $h . a$ ), which together constitute a kind of inverted tunnel in which lie the artery and vein of the tail. In the region of the caudal fin the hæmal spines are elongated and act as supports for the fin. A centrum together with the corresponding neural arch and transverse processes, or hæmal arch, represent a vertebra or single segment of the vertebral column.

In the frog we have seen that there are no independent ribs, and that the caudal vertebræ are represented by a single bone, the urostyle (p. 39).

It should he noticed that in the vertebral column we have another instance of the metameric segmentation of the vertebrate body. The vertebræ do not, however, correspond with the myomeres, but alternate with them. The myocommas (p. 43t) are attached to the middle of the vertebræ, so that each myomere acts upon two vertebræ and thus produces the lateral flexion of the body.

In the embryo dogfish, as in the tadpole, before the development of the vertebral column, an unsegmented, cellular rod with an elastic sheath, the notochord, resembling that of Amphioxus (p. 4I9), lies beneath the neural cavity in the position occupied in the adult by the line of centra, by the development of which it is largely replaced. Segmentally arranged cartilages appear above and below the notochord, which on the one hand give rise to the arches, and on the other invade the notochord and constrict it at regular intervals, so as to replace it completely in those regions which will form the middle parts of the vertebral bodies, leaving the vacuolated notochordal cells in the biconvex spaces between the centra (Fig. II $3, n t c$ ). Thus much of the notochord persists as the soft intervertebral substance.

The skeleton of the median fins consists of a series of parallel cartilaginous rods, the fin-rays or pterygio- 
phores, the proximal ends of which are more or less fused together to form basal cartilages or basalia. The free edges of the fins are supported by a double series of delicate horn-like fibres, the dermal fin-rays.

The paired fins are also supported proximally by cartilaginous pterygiophores, fused at the bases to form basal cartilages which articulate with the corresponding arch or girdle, and distally by horny, dermal fin-rays (Fig. II5, d. f. r). The pelvic arch (Fig. II4, BP) is a transverse bar of cartilage situated just in front of the vent, and representing the pubic and ischiatic portions

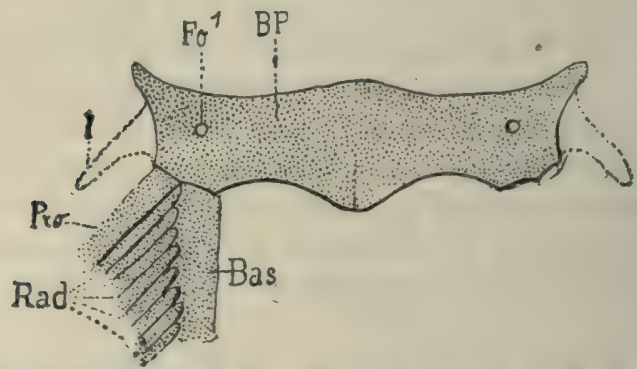

Fig. I I4.-Diagram of the Elasmobranch pelvic arch $(B P)$ and fin.

$B$ as. basal cartilage; $F 0^{\prime}$. nerve-foramen; $I$. iliac process; Pro. anterior ray articulating directly with the arch; $\mathrm{Rad}$. the remaining radial cartilages. (From Wiedershein's Comparative Anatomy.)

of the girdle in the frog (p. 5o); an iliac region, extending dorsally, and coming into connection with the vertebral column, being hardly represented (I). On its posterior edge are articular facets for the pelvic fins, each of which has a single very large basal cartilage (Bas), but one or two of the anterior rays (Pro) may articulate separately with the arch. In the male, the skeleton of the clasper (p. 433) is connected with the distal end of the basal cartilage.

The shoulder-girdle (Fig. II5) is a strong, inverted 
arch of cartilage situated just behind the last branchial arch. On its outer surface it presents three articular facets on either side for the corresponding pectoral fin ; the presence of these allows of the division of each side of the arch into a narrow, pointed, dorsal portion corresponding to the scapular region of the frog (pct.g),

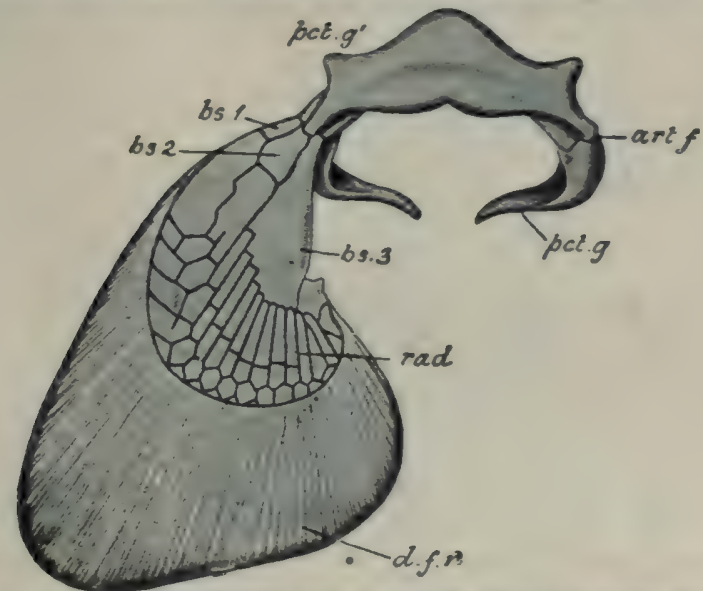

Fic. Ir5.- Tentral view of pectoral arch of Scyllium caricula with right pectoral fin.

The pectoral arch is divisible into dorsal or scapular (pct. $g$ ), and ventral or coracoid ( $p c t . g^{\prime}$ ) portions, continuous at the articular facets (art. $f$ ) for the fin. The pectoral fin is formed of three basal cartilages (bs. $1-3)$ and numerous radials $(\mathrm{rad})$ : its free edge is supported by dermal rays $(d . f . r$.$) (After Marshall and$ Hurst, slightly modified. ( $x$ s.)

and a broader ventral portion, answering to the coracoid (pct. $\left.g^{\prime}\right)$, united in the middle line with its fellow of the opposite side (compare p. 46): there is no sternum. The pectoral fin is formed of pterygiophores $(\mathrm{rad})$, fused proximally to form basals which are three in number (bs. $\mathbf{r}-3$ ), the third, like the main basal of the pelvic fin, being the largest and supporting the greater number of the cartilaginous rays, which give rise distally to a series of polygonal plates.

It will be noticed that while the skeleton of the crayfish is a series of articulated tubes with the muscles inside 
them, that of the dogfish and of the frog is a series of articulated rods with the muscles outside. The joints, formed by two rods applied at their ends and bound together by ligament, are not all confined to movement in one plane, like the hinge-joints of the crayfish, but may be capable of more or less rotary movement.

Digestive Organs. - The mouth, as we have seen, is a transverse aperture bounded by the upper and lower

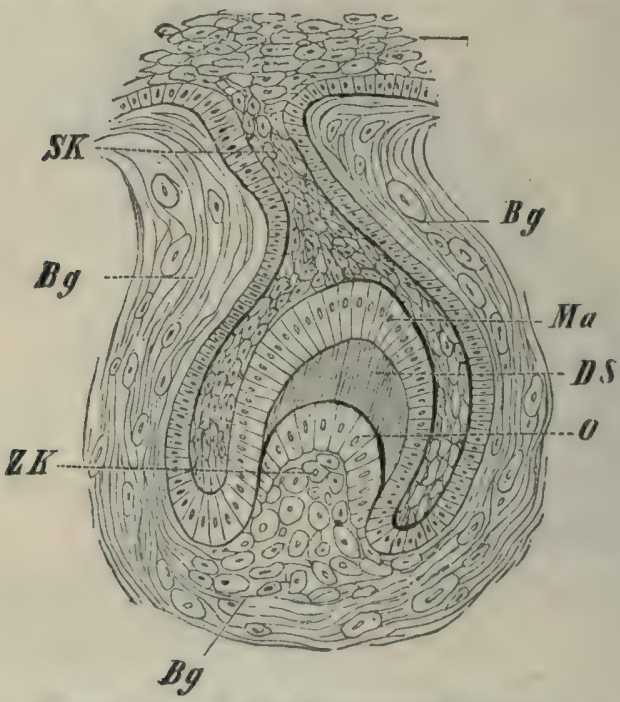

FiG. II6.-Diagram of the development of a tooth.

$B g, B g$. mesoderm; $D S$. dentine; $E M$. epithelium of mouth; $M a$. epithelium of enamel organ; $O$. odontoblasts; $S K$. dental lamina; $Z K$. dental papilla. (From Wiedersheim's Comparative Anatomy.)

jaws. In the mucous membrane covering the jaws are embedded large numbers of teeth-conical, calcified bodies, with enamelled tips, arranged in transverse rows. They are to be looked upon as special developments of the placoid scales or dermal teeth (p. 433) enlarged for the purpose of seizing prey, and are continually renewed on the inner sides of the jaws as they are worn away on the outer sides. 
The teeth, in Vertebrates generally, are developed in the following manner. The ectodermal epithelium of the mouth (stomodaum, p. 204) - or in the case of the dermal teeth of the dogfish that covering the body generallygrows inwards to form a ridge or dental lamina (Fig. II6, $S K$ ) which projects into the underlying mesodermal connective-tissue and becomes enlarged distally to form a bellshaped enamel-organ, into the base of which a mesodermal dental papilla $(Z K)$ extends: the superficial part of this papilla forms a layer of cells known as odontoblasts $(O)$. The dentine $(D S)$ is formed, in successive layers, from the odontoblasts, and gradually accumulates between them and the epithelium lining the interior of the enamel organ (Ma), which gives rise, also in successive layers, to the enamel, the hardest, external part of the tooth. Around the base of the papilla more or less bone-like substance-the cement -is formed (compare p. 510). The apex of the tooth eventually breaks through the overlying epithelium and comes to the surface. It will thus be seen that while the teeth are mainly mesodermal structures, a part of themthe enamel--is ectodermal in origin.

The mouth leads into an oral cavity-on the floor of which is a rudimentary tongue (Fig. II $7, t n g$ ) capable of very little movement-which passes insensibly into the throat or pharynx $(p h)$, distinguished by having its walls perforated by five pairs of slits, the internal branchial apertures $(i, b r . a)$, as well as by the inner openings of the spiracles $(s p)$. The pharynx is continued by a short gullet (gul) into a capacious U-shaped stomach, consisting of a wide cardiac division $(c d$. st) and a narrow pyloric division $(p y l . s t)$. The pyloric division communicates by a narrow valvular aperture with the intestine (int), a wide, nearly straight tube having its lining membrane produced into a spiral fold, the spiral valve $(s p . v l)$, which practically converts the intestine into a very long, closely-coiled tube, and greatly increases the absorbent surface. Finally the intestine opens into a large chamber, the cloaca $(\mathrm{cl})$, which communicates with the exterior by the vent.

From the gullet backwards the enteric canal is con- 


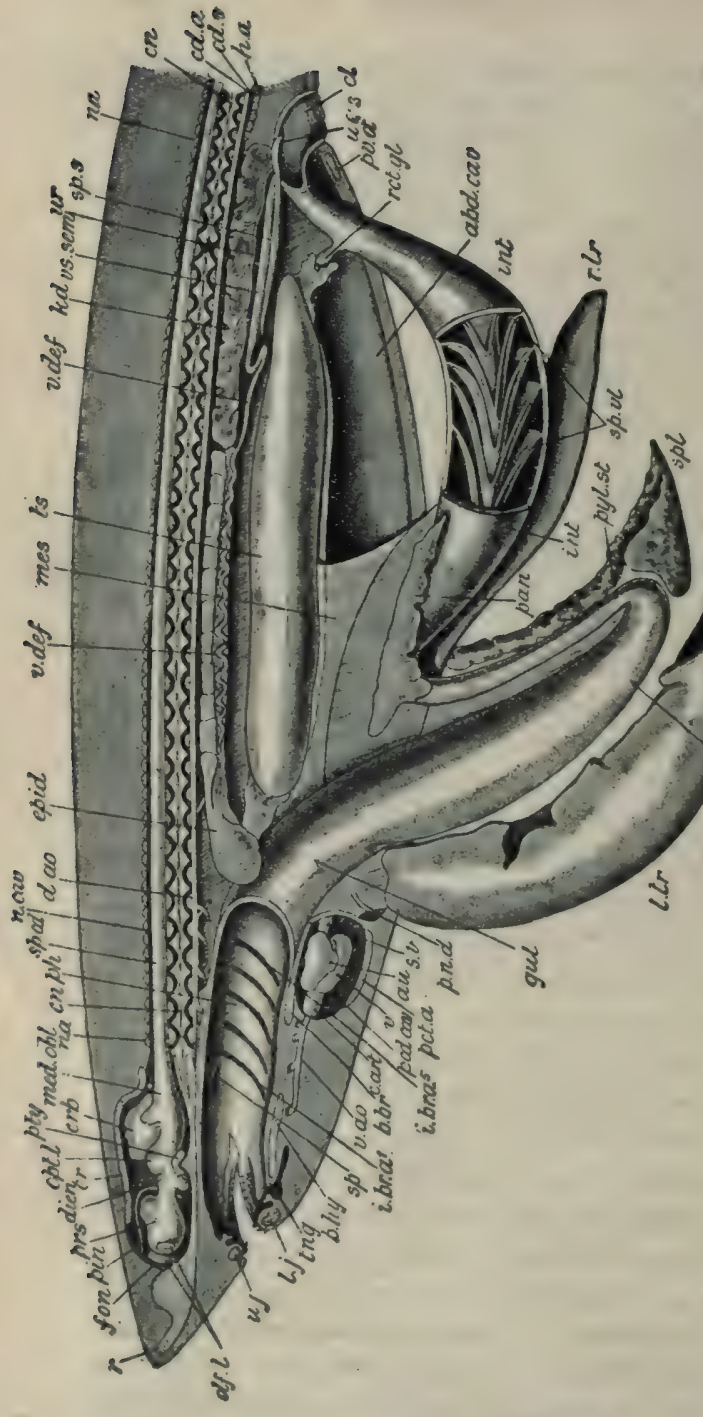

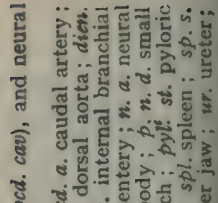

छे ชु

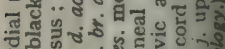

รึ $8.0 . \cdots$

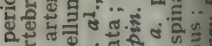

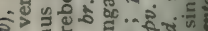

-

$x$ ชैं

的

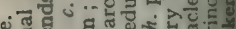

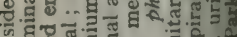

म

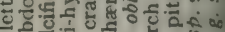

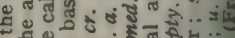
स

ह

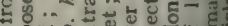

앙ㅎㅇ

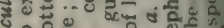

8 웡

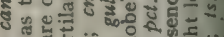

S

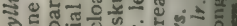

है정

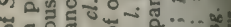

of

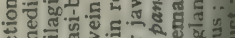

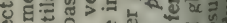

.

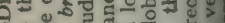

1 율.

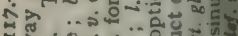

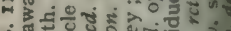

0 of

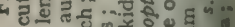

ต

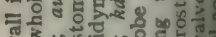

त...

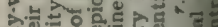

कृ

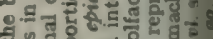

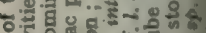

政

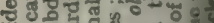

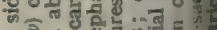

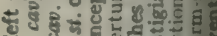

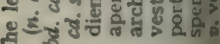


tained in the crelome, to the dorsal wall of which it is suspended by a median incomplete mesentery (Figs. II I and $\mathrm{II} 7$, mes). The greater part of the canal is developed from the enteron of the embryo, and is consequently lined by endoderm; the oral cavity is formed from the stomodæum, and the cloaca from the proctodæum (p. 20.4). Outside the enteric epithelium are connectivetissue and muscular layers, the latter formed of unstriped fibres: it is generally characteristic of vertebrates that the voluntary muscles are striped, the involuntary unstriped (compare pp. III and II 2 ).

The immense liver, divided into two lobes (l. lr, r. lr), is situated below the stomach along the whole length of the abdomen, to the front wall of which it is attached by a fold of peritoneum. It discharges its secretion, the bile, into the anterior end of the intestine by a tube, the bile-duct, which gives off a blind offshoot terminating in a gall-bladder (p. 69).

The pancreas (pan) lies against the anterior end of the intestine, with which it communicates independently by the pancreatic duct. Opening into the hinder part of the intestine or rectum is a small finger-like rectal gland $(r c t . g l)$, the function of which is not known. In addition to these there are, as in all Vertebrates, minute tubular gastric glands sunk in the mucous membrane of the stomach (p. I3I).

The spleen $(s p l)$ is an irregular, dark-red, gland-like body, of considerable size, attached by peritoneum to the stomach (compare pp. 23 and 98).

Other so-called " ductless glands"-which are also represented in the frog, giving rise to internal secretions the functions of which are imperfectly understood-are the inyroid in the throat (Fig. I 22) and the thrmus in connection with the dorsal ends of the branchial arches ; there are also suprarenal and interrenal bodies in the neighbourhood of the kidneys, corresponding to the adrenals of the frog (p. I +5 ). 
Respiratory Organs.-The respiratory organs consist of five pairs of pouches, each opening by one of the internal branchial apertures (Fig. II $7, i, b r . a)$ into the pharynx, and by one of the external branchial apertures on the exterior. The walls of the pouches, or interbranchial septa, which form the bars separating the clefts, are supported by the cartilaginous visceral arches

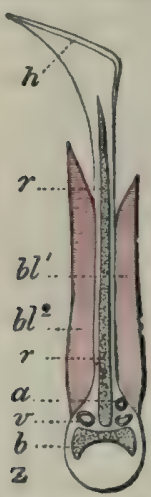

Fig. II8.-Transverse section through a gill of an Elasmobranch.

a. afferent branchial artery ; b. branchial arch; bll. anterior, and ble. pose terior hemibranch; $h$. septum ; $r$. branchial ray ; $v$. efferent branchial arteries. (From R. Hertwig's Zoology.) and branchial rays (Figs. II2, br. $r$, and $\mathrm{II} 8, r)$ and are lined with mucous membrane raised into horizontal ridges, the branchial filaments, which are abundantly supplied with blood-vessels and are the actual organs of respiration. As the fish swims, water enters the mouth and passes by the internal clefts into the branchial pouches, and thence outwards by the external clefts, a constant supply of oxygen being thus ensured. The gill-pouches are developed as offshoots of the pharynx, and the respiratory epithelium is therefore endodermal, not ectodermal, as in the crayfish, mussel and young tadpole (compare pp. 204 and 207).

As already mentioned, the walls of the pharynx are supported by the cartilaginous visceral arches, which surround it like a series of incomplete hoops, each halfarch being embedded in the inner or pharyngeal side of an interbranchial septum. Thus the visceral arches alternate with the gill-pouches, each being related to the posterior set of filaments of one pouch and the anterior set of the next. An entire gill or holobvanch therefore consists of two half-gills or hemibranchs-the sets of branchial filaments belonging to the adjacent sides of two consecutive gill-pouches (Fig. 118). On the other hand, a gill-pouch 
encloses the posterior hemibranch of one gill and the anterior hemibranch of its inmediate successor.

The first pouch is situated between the hyoid and the first branchial arch, and the hyoid thus bears a hemibranch only. The first four branchial arches bear each a holobranch, and the fifth is without gill-filaments. There is a vestigial hemibranch, or pseudobranch, on the anterior (mandibular) wall of the spiracle.

Now it is known that parts which have become useless tend to disappear more or less completely (e.g., pineal body of the frog-p. I 59, and certain of the gills in the crayfishp. 375). In some cases, however, such vanishing parts take on new relations with other organs and so once more become useful in other ways, undergoing a change of function. Thus in higher Vertebrates the spiracle is utilised in connection with the auditory organ, and instead of disappearing entirely, as do the other gill-clefts, it gives rise to the tympanic cavity and Eustachian tube (compare p. 45).

Cireulatory Organs. - The heart is situated in the pericardial cavity or anterior compartment of the colome (p. 434) and is a large muscular organ composed of four chambers. Posteriorly and dorsally is a small, thinwalled sinus venosus (Figs. II 7 and II $9, s . v$ ), opening in front into a single, capacious, thin-walled auricle (au) ; two auricles are present only in those Vertebrates which possess lungs. The auricle communicates with a very thick-walled ventricle $(v)$, from which is given off in front a tubular chamber, also with thick muscular walls, the comus arteriosus (c. art). There are valves between the sinus and the auricle, and between the auricle and ventricle, and the conus contains two transverse rows, each of three valves: all the valves are arranged so as to allow of free passage of blood from sinus to auricle, auricle to ventricle, and ventricle to conus, but to prevent any flow in the opposite direction (compare pp. 79 and 87 ).

The conus gives off in front a single blood-vessel $(v, a 0)$ having thick elastic walls composed, like other arteries, of connective and elastic tissue and unstriped muscle. This vessel, the ventral aorta, passes forwards beneath the 


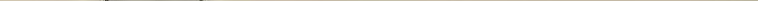


gills, and from it arise on either side paired lateral branches, the afferent branchial arteries (Fig. II9, a. br. a, and compare Fig. Iro). Each afferent artery passes to the corresponding gill, and there branches out into smaller and smaller arteries, which finally open into a network of delicate capillaries (p. 95), with which the connective-tissue of the branchial filaments is permeated. The blood in these respiratory capillaries is therefore brought into close relation with the surrounding water, and as the blood flows through them it exchanges its carbon dioxide for oxygen, obtained from the air dissolved in the surrounding water.

From the respiratory capillaries the blood is collected into minute arteries which join into larger and larger trunks, and finally unite into efferent branchial arteries (e. br.a) by which the purified blood is carried from the gills. The efferent arteries of the right and left sides communicate with a median longitudinal artery, the dorsal aorta $(d . a o)$, which passes backwards, immediately beneath the vertebral column, to the end of the tail.

From the efferent branchial arteries and the dorsal aorta are given off numerous arteries supplying the various parts with blood. The most important of these are paired carotid arteries (c. a) to the head, and subclavians (scl.a) to the pectoral fins ; unpaired splanchnic arteries ( $c l . a, m s . a)$ to the enteric canal, liver, pancreas, and spleen; numerous paired renals $(r . a)$ to the kidneys, spermatic $(s p . a)$ or ovarian arteries to the gonads, and a pair of iliacs (il. a) to the pelvic fins. The posterior part of the dorsal aorta, supplying the tail, is contained in the hæmal canal of the caudal vertebræ, and is often spoken of as the caudal artery $(c d . a)$.

The arrangement of the arteries in the tadpole is very similar to that described above, and the diagram (Fig. II9) would serve almost equally well for a tadpole as for a fish. 
In the former there are four pairs of afferent and efferent branchial arteries (corresponding to the second to the fifth in Fig. II9) in relation with the corresponding branchial arches and their gills. The afferent and efferent vessels at first communicate with one another through the respiratory capillaries, but later on each afferent becomes directly connected with the correspond-

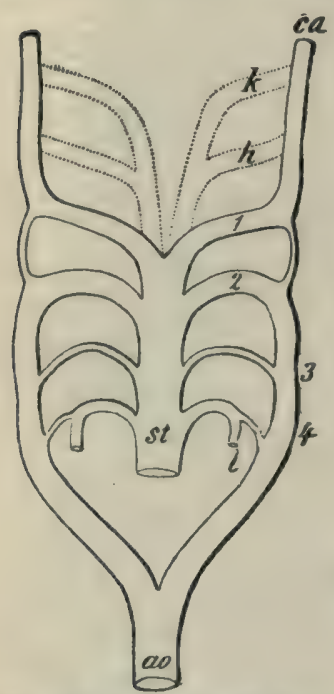

FIC. 120.-Diagram of the arterial arches of an Amphibian.

I-4, the four arterial arches which pass up the corresponding branchial arches; ao. dorsal aorta ; c, a. carotid artery : $k$. embryonic arterial arch of the mandibular arch ; and $h$. of the hyoid; $l$. pulmonary artery: st. ventral aorta. (From Wiedersheim's Comp. Analomy, after Boas.) ing efferent artery; and at metamorphosis, when the gills gradually disappear, all the blood thus passes directly from the ventral to the dorsal aorta, through the four arterial arches (Fig. I20). The first arch (I) gives rise in the adult frog to the carotid trunk $(c a)$, and loses its connection with the second at the dorsal end; the second (2) forms the systemic trunk; the third (3) disappears; and the fourth (4), losing its connection with the dorsal aorta, forms the pulmo-cutaneous trunk (l) (compare p. 8o).

Having now traced the main course and arrangement of the chief arteries, there are a few minor points of detail to be noticed in the dogfish (Fig. I2I).

The five afferent branchial arteries (af. br $I-5)$ of either side do not arise regularly and symmetrically from the ventral aorta, as represented in the diagrammatic Fig. Ir9. The anterior end of the ventral aorta divides into right and left branches, each of which again subdivides to form the first two afferent branchial arteries, which supply respectively the hemibranch of the hyoid arch and the holobranch of the first branchial arch. The third afferent branchial artery arises from about the middle of the ventral aorta, and supplies the holobranch of the second branchial arch; a short distance behind it the fourth and fifth come off 
close together, and supply the gills on the third and fourth branchial arches respectively: it will be remembered that the fifth branchial arch bears no gill-filaments. After aëration the blood from each hemibranch passes into an efferent branchial artery (ef. br), which joins with its fellow of the same cleft (except in the case of $e f . b r^{2}$ ), and thus forms a loop surrounding the cleft, the two halves of adjacent

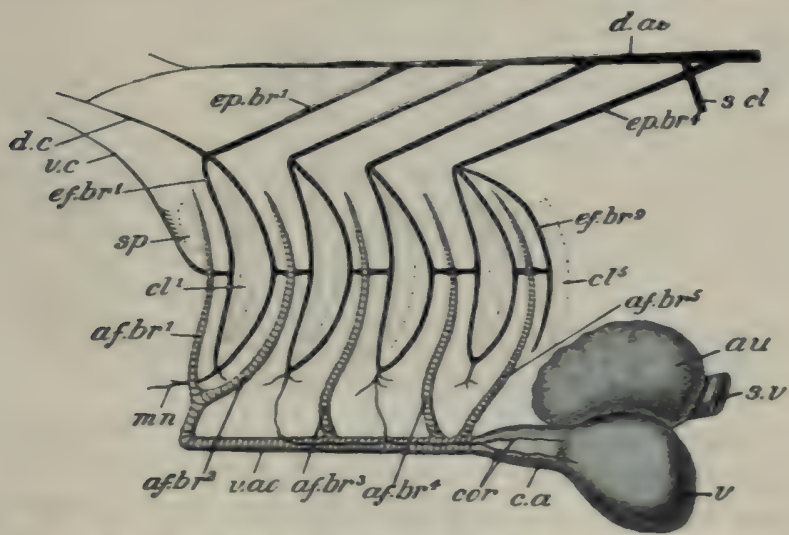

Fig. 121.-Diagram of the heart and Sranchial arteries (of one side only) of Scylium; lateral view. ( . $1 \frac{1}{4}$.)

af. br. ${ }^{1-5}$, afferent branchial arteries : au. auricle; c. a. conus arteriosus : cl. $1-5$. branchial clefts : cor. coronary artery; $d$. ao. dorsal aorta : $d$. c. dorsal carotid artery ; of. br. ${ }^{l}-9$, efferent branchial arteries; $c p$. br. L. epibranchial arteries; ms. mandibular artery: sp. spiracle: s. $c$. subclavian artery : s. $t$. sinus venosus ; $v$. ventricle ; $v$. 20 . ventral aorta ; $v$. c. ventral carotid artery.

loops being connected in the middle by a commissural vessel. From the upper ends of each of the four loops arises an epibranchial artery' (ep. br) which is connected with the dorsal aorta $(d . a o)$, the blood from the last hemibranch passing into the fourth loop.

From the dorsal end of the first efferent branchial, a dorsal carotid artery $(d . c)$ is given off; this passes forwards and inwards, gives off a branch to the upper jaw and snout, and then runs inwards in a groove on the skull-floor, which it penetrates in the middle line so as to reach the cranial cavity. A vessel arises from the middle of the first efferent branchial and supplies the pseudobranch, and is then continued into a zentral carotid artery (i.c), which passes through the orbit into the cranium, giving off branches to the brain and anastomosing with the dorsal carotid. From 
the ventral end of the first efferent branchial a small mandibular artery $(m n)$ passes to the lower jaw. The dorsal aorta is continued forwards, anteriorly to the first epibranchial artery, as a slender vessel which soon bifurcates and anastomoses with the dorsal carotid. From the ventral ends of the efferent branchial loops small arteries are given off which supply the lower parts of the head, the branchial region, and the heart (cor). The subclavian arteries (s. cl) arise from the aorta just. before it is joined by the last epibranchials.

In the short-bodied frog we have seen (p. 8o) that there is only a single splanchnic or cœliaco-mesenteric artery, which soon divides into a coeliac and a mesenteric. In the dogfish there are four splanchnic arteries, arising separately from the dorsal aorta, viz., a coeliac, supplying the proximal limb of the stomach and the liver; an anterior mesentevic, arising a short distance further back and supplying the intestine, \&c. ; a lienogastric, coming off from the aorta close behind the anterior mesenteric and going to the spleen and part of the stomach and pancreas; and a small posterior mesenteric supplying the rectal giand.

As in all Vertebrates, the arteries branch and branch again in the various parts to which they are distributed, their ultimate ramifications opening into a capillary network with which all the tissues except the cartilages and epithelia are permeated. From these systemic capillaries the blood is collected into larger and larger afferent thin-walled trunks or veins, parts of many of which are greatly dilated to form sinuses.

Most of the blood from the head is brought back by a pair of jugular sinuses (Figs. II 9. j. v, and I22, jug) : each of these enters a cardinal simus (card), which will be referred to again directly. The blood from the tail is returned by a caudal vein ( $c d . v$, caud) lying immediately beneath the caudal artery in the hæmal canal; this vessel enters the colome and then divides into right and left branches, the renal portal veins $(r . p . v, r . p)$, which pass to the kidneys and join with the capillaries of these organs, impure blood brought from the tail mingling with the pure blood of the renal arteries (Fig. II9, $r . a$ ). From 
the kidneys the blood is returned into a pair of partially united cardinal veins (crd. v) which pass forwards, become swollen to form large sinuses anteriorly (Fig. I22, card), receive veins from the reproductive organs, muscles, \&c., and finally each communicates with a precaval sinus $(p r . c v)$, which passes vertically downwards and enters the sinus venosus.

From the stomach, intestine, spleen, and pancreas the blood is collected by numerous veins, which all join to form a large hepatic portal vein (Fig. II9, h.p.v). This behaves in the same way as the renal portal: instead of joining a larger vein on its way to the heart, it passes to the liver and breaks up to connect with the capillaries of that organ; its blood, deprived of oxygen but loaded with nutrient matters from the enteric canal, mingling with the oxygenated blood brought to the liver by a branch of the coliac artery. After circulating through the capillaries of the liver the blood is taken by a pair of hepatic veins $(h, v)$, which enlarge to form a large sinus $(h . s)$, and this opens into the sinus venosus (compare p. 85).

The course and arrangement of the veins, like that of the arteries, is very similar to that existing in the tadpole, in which several important changes occur at metamorphosis. With the disappearance of the tail and caudal vein, the renal portal veins receive their blood from the hind-limbs only. The hinder parts of the two cardinal veins, situated between the kidneys, fuse into one, and their anterior parts disappear, a new vessel being developed which conducts the blood from the fused cardinals to the sinus venosus: the whole of the great vein thus formed is the postcaval (p. 82, Fin. 2I), which is present in the Dipnoi (p. 43o) and in all Vertebrates above the fishes.

The iliac veins of the dogfish (Fig. II9, il) pour the blood from the pelvic fins into the lateral veins (Figs. II I, II9, and I22, lat)-paired trunks running forwards in the side walls of the body to the sinus venosus, and communicating at their anterior ends with the subclavian 
veins $(s c l . v, s c)$ from the pectoral fins. In addition to the dorsally situated jugular sinuses, there are paired inferior jugulars (Fig. II2, inf. jug), bringing back the blood from the ventral parts of the head and each opening into the corresponding precaval together with the subclavian.

As we have seen, several of the veins, e.g., the precavals, jugulars, cardinals, and the genital veins, are dilated into spacious cavities called sinuses (Fig. I22). These are, however, of a totally different nature from the sinuses of the crayfish, which are mere spaces among the tissues devoid of proper walls (pp. 374 and 377 ). In the dogfish, as in the frog and Vertebrates generally, the blood is confined throughout its course to definite vessels : the heart, arteries, capillaries, and veins invariably forming a closed system of communicating tubes.

The general course of the circulation will be seen to agree with that already described in the frog as well as in the crayfish and mussel, i.e., the blood is driven by the contractions of the heart through the arteries to the various tissues of the body, whence it is returned to the heart by the veins or sinuses (Fig. I 23). But whereas in both crayfish and mussel the respiratory organs are interposed in the returning current -both their afferent and efferent vessels being veins, in the dogfish they are interposed in the outgoing current-their afferent and efferent vessels being arteries. An artery, it must be remembered, is a vessel taking blood from the heart to the tissues of the body and having thick walls; a vein is a thin-walled vessel bringing back the blood from the tissues to the heart.

Moreover, the circulation in the dogfish is, as in the frog, complicated by the presence of the two portal systems, renal and hepatic. In both of these we have a vein, renal portal or hepatic portal, which, instead of joining with larger and larger veins and so returning its blood directly to the heart, breaks up, after the manner of an artery, in the kiclney or liver, the blood finding its way into the ordinary renous channels after having traversed the capillaries of the gland in question.

Thus an ordinary artery arises from the heart or from an artery of higher order and ends in capillaries; an ordinary vein arises from a capillary network, and ends in a vein of 
higher order or in the heart. But the hepatic and renal portal veins end in capillaries after the manner of arteries, and the efferent branchial arteries begin in capillaries after the manner of veins.

With regard to the general morphology of the bloodsystem, the dorsal aorta with the caudal artery may be considered as a dorsal vessel (compare Earthworm, p. 337 , Crayfish, p. 379, and Amphioxus, Fig. IIo); the caudal vein, hepatic portal vein, and ventral aorta as together representing a ventral vessel; the afferent and efferent branchial arteries as commissural vessels; and the lateral veins as Iateral vessels. It will be seen that the heart of Vertebrates is a muscular dilatation of the ventral vessel, as is also shown by a study of its development (p. 597).

The blood, like that of the frog (pp. I04-Io6), consists of a colourless plasma containing red corpuscles (the

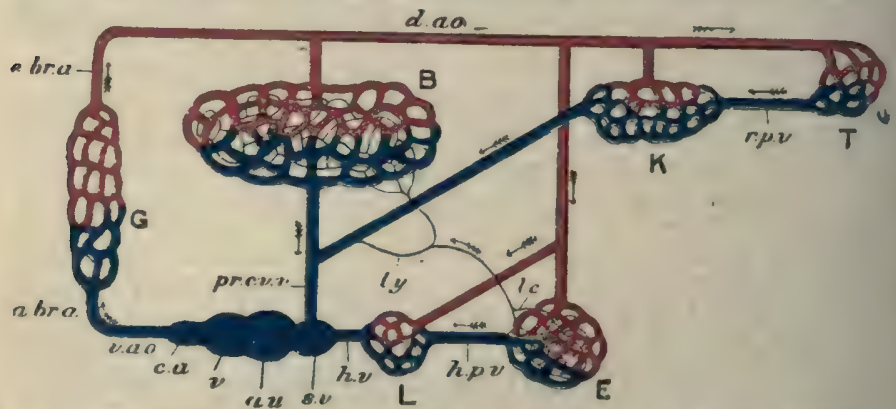

FIG. I23,-Diagram illustrating the course of the circulation in the Dogfish. Vessels containing oxygenated blood red, non oxygenated blood blue.

$B$, capillaries of the body generally; E. of the enteric canal; $G$, of the gills ; $\mathbf{K}$. of the kidneys; L. of the liver; T. of the tan. a. br. a. afferent branchial arteries; au. auricle; $c$. a. conus arteriosus; $d$. ao. dorsal aorta ; $e . b r . a$. efferent branchial arteries; $h . p . v$. hepatic portal vein; $h$. $v$. hepatic vein ; $l c$. lacteals $(p .98) ; l y$. other lymphatics; $p r . c v . v$. precaval vein ; r. p. v. renal portal veins; s. $v$. sinus venosus; $v$. ventricle; $v$. ao. ventral aorta. The arrows show the direction of the current. (From Parker and Haswell's Zoology.)

colour of which is due to hæmoglobin) and leucocytes. It must be remembered that the ventral aorta and the afferent branchial arteries (Fig. I23), like the pulmonary artery of the frog (p. I 44 ), contain venous blood. As in the frog, there is, in addition to the bloodvessels, a system of lymphatic vessels 
Nervous System.- The nervous system is constructed on a similar plan to that of the frog (compare Part I, Chapter X) and of Vertebrates in general. The central nervous system is dorsal in position and consists of a brain, contained within the cranial cavity, and continuous posteriorly with a spinal cord, enclosed in the neural canal of the vertebral column: it consists of grey and white matter, and its cavity or neurocele, lined with epithelium, gives rise to the ventricles of the brain and to the central canal of the spinal cord.

In correspondence with the form of the body, the spinal cord is relatively much longer than in the frog; it is not swollen opposite the paired appendages.

In the brain (Figs. II7 and I24) the bulb or medulla oblongata (. $\mathrm{Med}$. obi) broadens out anteriorly to form lateral swellings, and its contained fourth ventricle $\left(v^{4}\right)$ is roofed over by the "pia mater." The cerebellum $(C b)$, which is very small in the frog, is here relatively enormous, and its surface is marked by slight grooves: it overlaps the bulb behind and the optic lobes in front, and contains a ventricle communicating with the fourth ventricle. The oval optic lobes $(O p t . l)$ are hollow, their cavities communicating with the median ventricle or iter, and ventrally to them are the crura cerebri. The diencephalon $(D i)$ is relatively narrower than in the frog. From its thin roof, which covers over the third ventricle, is a delicate tube-like structure ( $p i n)$, which extends upwards and forwards and ends in a small knob attached to the roof of the skull : this is the pineal body. From the ventral surface of the diencephalon arises the infundibulum, with an oval swelling on each side, and to it is attached the pituitary body (Fig. II 7 , pty), with a vascular sac on either side of it. In front of the infundibulum is the optic chiasma (compare Figs. 49 and 50$)$. 
Apart from the large size of the cerebellum, the most marked difference between the brain of the dogfish and that of the frog is seen in its anterior portion. In the

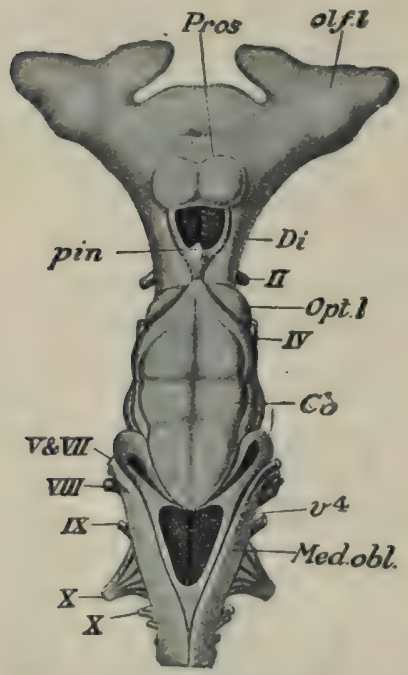

FIG. 124.-Dorsal view of the brain of Sycllium canicula. ( $X \mathrm{I}\}$.)

The posterior division of the brain is the medulla oblongata ( $\mathrm{Med}$. obl.), enclosing the fourth ventricle $(v i)$. The large cerebellum $(C b)$ nearly covers the optic lobes $(O p t$. $l)$. The diencephalon $(D i)$ shows, in the middle, the third ventricle, and the place of attachment of the pineal stalk (pin). The prosencephalon (Pros) gives off the olfactory lobes (olf. $l$ ). The origins of the following nerves are shown :- optic $(I I)$, trochlear $\left(I^{*}\right)$, trigeminal (V), facial (III), auditory (VIII), glossopharyngeal $(I X)$, and vagus $(X)$. (From Wiedersheim's Comp. Anatomy.) frog, the diencephalon is continuous anteriorly with the paired cerebral hemispheres; in the dogfish there is in this region a relatively smaller, unpaired portion of the brain, marked in front by a slight groove, and known as the prosencephalon (Pros), which represents the cerebral hemispheres of the higher Vertebrates but which does not becomesubdivided externally into paired lobes. Anteriorly it gives off, right and left, a large, oval olfactory lobe (olf. l), each connected with the prosencephalon by a short, stout stalk and applied distally to the corresponding olfactory capsule. The prosencephaion contains paired lateral ventricles, which communicate posteriorly with the third ventricle and anteriorly are continued into the olfactory lobes. 
Each spinal nerve arises, as in the frog (p. I63), by two roots, which, however, are not in the same transverse plane, the dorsal root being slightly anterior to the corresponding ventral root, so that successive dorsal and ventral roots of either side alternate with one another. As already mentioned (p. 440), the two roots of each nerve pass out from the neural canal independently, uniting on the outside of the canal to form the spinal nerve-trunk. Plexuses in connection with the nerves supplying the paired appendages are much simpler than in the frog (pp. I6r and 162). A sympathetic system (p. I62) is represented.

The origin and distribution of the cerebral nerves is in the main similar to that already described in the case of the frog (p. I63), the chief differences, characteristic respectively of pulmonate and of branchiate Vertebrates in general, being as follows. ${ }^{1}$

In fishes, there are certain nerves, usually counted as belonging to the facial and vagus, which supply the sensory canals of the integument (Fig. I25, VII $o p, b, e . m$, and $\mathrm{X} l$ ) : these organs (pp. 432 and +64 ) are not present in terrestrial forms, and their nerves are consequently also wanting. The vagus, mureover, gives off a series of branchial branches ( $b r$. $^{1-4}$ ) to the gills instead of a pulmonary branch, and the glossopharyngeal (IX) is also a branchial nerve.

The olfactory nevies (Fig. I25, I) arise from the olfactory lobe, which is situated in a large aperture in the skull communicating between the cranial and olfactory cavities. The optic nerie (II) is continued outwards from the optic chiasma, and passes through a foramen in about the middle of the orbit, towards the ventral side (compare Fig. I12). The oculomotor (III), arising from the crura cerebri, makes its exit from the skull a short distance behind and slightly

1 The nature of a small paired nerve in dogfishes and certain other fishes which arises from the prosencephalon and passes to the nasal mucous membrane is not understood. 


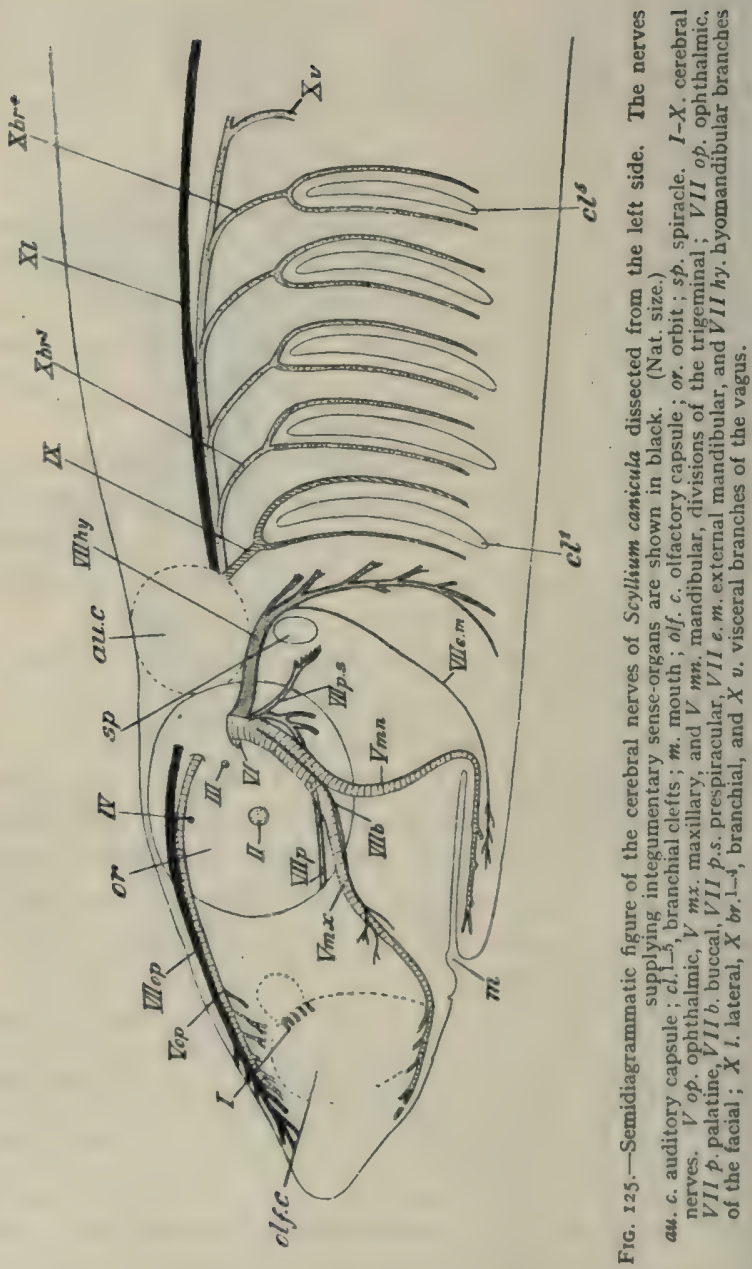


above the optic nerve. The pathetic (IV), coming off from the dorsal side of the front end of the medulla oblongata and supplying the superior oblique muscle, pierces the cranial wall almost directly above the optic foramen(compare also Fig. I26). All the other nerves arise from the ventrolateral regions of the medulla oblongata, the abducent, supplying the external rectus muscle, coming off nearer the middle line than, and anterior to, the others. The abducent (VI) and the greater part of the Irigeminal (V) and facial (TII) nerves pass out through a single foramen in the skull in the posterior and ventral part of the orbit, just anterior to the auditory capsule. A short distance above this foramen are two others, the ventral slightly anterior to the dorsal: these transmit the ophthalmic branches (see below) of the trigeminal and facial (Vop, VII $o p$ ) respectively, and from them grooves pass along the dorsal side of the orbit to an aperture just behind the olfactory capsule, the nerves emerging again on the dorsal side of the skull. The auditory neri'e passes through a large foramen on the inner side of the auditory capsule to supply the membranous labrrinth. The glossopharyngeal (IX) emerges behind the auditory capsule at the posterior end of a horizontal groove in this region, and the vagus (X) passes out through a foramen between the glossopharyngeal and the foramen magnum.

The nerves supplying the integumentary sense organs are as follows: (I) The ophthalmic branch of the facial (VII op) runs, as we have seen, dorsally to the similarly named branch of the trigeminal, close under the skin, and supplies the sensory tubes and ampulla (see p. $4^{\sigma_{4}}$ ) of the upper part of the snout; those of the lower part of the snout are innervated by (2) a buccal branch (VII b), which extends along the floor of the orbit just above the maxillo-mandibular division of the trigeminal; and those in the region of the hyomandibular by a small (3) external mandibular branch (VII $e, m$ ), arising from the large hyomandibular nerve (see below). The lateral-line canal, extending along the body and tail, is supplied by (4) the lateral branch of the iag!os (X $t$ ) which runs backwards to the inner side of the rest of the nerve and dorsally to the spinal nerves, along the inner side of the body-wall, giving off branches which extend outwards between the great lateral muscles to the lateral canal.

The other branches of the facial are :-a small palatine (VII p), which extends along the floor of the orbit, just behind the trigeminal, and supplies the roof of the mouth; and a large hyomandibular (VII $h y$ ) which passes behind the spiracle-first giving off small prespiracular branches 
(VII $p . s$ ) to its anterior wall-and extends along the anterior border of the auditory capsule and the posterior wall of the orbit, and just beneath the skin, to the anterior side of the hyoid arch : it thus forks over the spiracular or mandibulo-hyoid cleft.

The glossopharyngeal (IX) forks above the first gill-cleft, thus giving rise to two branches, one passing down the posterior side of the hyoid, and the other down the anterior side of the first branchial arch. The main part of the vagus extends backwards to the outer side of the lateral nerve and gives off four branchial nevves ( $\mathrm{X} \mathrm{br}{ }^{1 \_4}$ ) forking over the second to the fifth gill-clefts respectively, and is then continued into the visceral nerves ( $\mathrm{X} v$ ), which supply the stomach and heart.

Sensory organs. - The dogfish possesses, as we have seen, a series of peculiar integumentary sense-organs supplied by the nerves just described, the exact function of which is not known with certainty. They are situated within a number of epithelial canals, developed from the epiderm, the openings of which on the head have already been noticed (p. 432).

These tubes are of two kinds, known respectively as sensory and ampullary canals: the former, which are at present in all Vertebrates with gills (p. 430), are all continuous with one another and are situated along certain definite tracts on the head and jaws, a canal extending along the body and tail as the lateral-line canal. The ampullary canals, which are peculiar to Elasmobranch fishes, and which contain a gelatinous material, are not continuous with one another, but run side by side, and form large masses in the snout and at the sides of the head; at their blind ends they are swollen to form ampulle, to which the nerves are distributed. The sensory cells are arranged in little conical masses in the lining epithelium of the sensory canals and of the ampullæ, a section of one of which nearly resembles that of an ampulla of a semicircular canal of the ear (Fig. 60).

The olfactory organs are a pair of cup-like sacs in the snout, enclosed by the olfactory capsules and opening externally on the ventral side by the nostrils. Notice that there are no internal nostrils, as in the frog: these 
are only present in Vertebrates which possess lungs. The sacs are lined by the olfactory epithelium, which is supplied by the olfactory nerves and is raised up into ridges so as to increase the surtace.

The structure of the eye, as well as of the accessory apparatus in connection with it, is in all essential respects the same as in the frog (p. I8I), except for the differences in the eyelids (pp. 5 and 432), the absence of a lacrymal apparatus (p. I86), and for the fact that the four recti muscles (Fig. I26) do not ensheath the optic nerve, which emerges into the orbit a short distance in front of their point of origin.

The membranous labyrinth of the ear (compare Fig. 59, p. I87) is also very similar to that of the frog, but being larger, and the auditory capsules being composed entirely of cartilage, it can be dissected

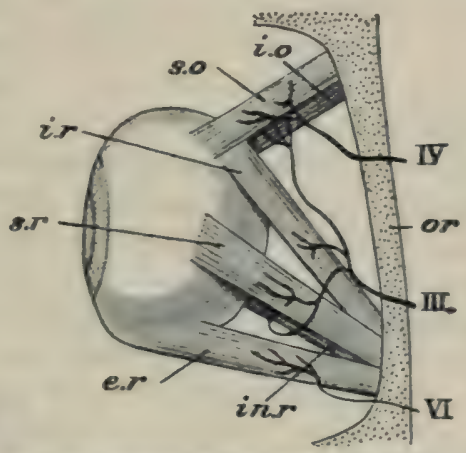

FIG. I26.-Semidiagrammatic figure of the eyemuscles and their nerves of an Elasmobranch. $I I I$. oculomotor, $I V$. pathetic, and $V I$. abducent nerve; $e$. $r$. posterior rectus muscle; $i . o$. inferior oblique; in. $\boldsymbol{r}$. inferior rectus; $\boldsymbol{i}_{\text {. }} \boldsymbol{r}$. anterior rectus; or. wall of orbit : s. o. superior oblique ; s. r. superior rectus. (From Parker and Haswell's Zoology.)

out with comparative ease by slicing away the capsule with a knife.

A tube given off from the sacculus, called the endolymphatic duct, which in the frog communicates with the lymphatic system, opens to the exterior on the top of the head in the dogfish, and thus the endolymph is in free communication with the surrounding sea-water.

As we have seen, the membranous labyrinth is the essential part of the ear, and it, together with its enclosing 
capsule, is often spoken of as the internal ear. In the frog there is also an accessory apparatus-the tympanic cavity and membrane, together with the columella-which is called the middle ear (compare Fig. Io and pp. I 89 and 449).

Urinogenital organs.--The kidneys of the dogfish are narrow, lobulated organs, lying close to the vertebral column on either side and covered ventrally by the thick peritoneum. They extend primarily along almost the whole length of the coelome, but in the course of development (see p. 6or) certain important modifications take place in them and their ducts.

It will be remembered that in the male frog the efferent ducts from each spermary enter the comparatively short kidney of the same side, and that the duct of the latter serves as a urinogenital duct (p. I93). In the dogfish, about the anterior half of the primary kidney loses its excretory function (Fig. I27, $k^{\prime}, k^{\prime \prime}$ ); in the male it becomes connected with the anterior end of the spermary by means of a small number of fine efferent ducts (ef. $d$ ) and gives rise to a glandular body, the so-called epididymis (A, $\left.k^{\prime}, k^{\prime \prime}\right)$, through which the sperms pass in order to reach the original kidney-duct, which serves as a spermiduct only $(s p d)$. This duct remains in close connection with the epididymis ventrally and becomes greatly convoluted. In the female the whole of the anterior part of the primary kidney and its duct are merely represented by vestiges $\left(\mathrm{B}, k^{\prime}\right)$. The posterior half of the embryonic kidney in each sex is the only part which serves as a renal organ; this is somewhat swollen posteriorly, and in connection with it special ducts or ureters are developed to carry off the urine $(u r)$. In the female these open separately into the persistent posterior ends of the primary kidneyducts, which unite together at their bases to form a median urinary sinus $(\mathrm{B}, u, s)$, opening by a single 
aperture into the cloaca; while in the male, four of them on either side unite to form a wide main ureter before communicating with a similar median sinus, which, as it receives the products both of the spermaries and kidneys, is called the urinogenital simus (A, ug. s).

The spermaries are a pair of soft, elongated organs, suspended to the dorsal body-wall by delicate peritoneal folds and invested by lymphoid tissue (see pp. 507 and 5I5) which unites them together posteriorly. From the anterior end of each the delicate efterent ducts referred to above pass to the epididymis to become connected with the convoluted spermiduct. The latter dilates posteriorly, where it underlies the functional kidney, forming an elongated, spindle-shaped seminal vesicle (s.v.), which opens $\left(s . v^{\prime}\right)$ into the base of a thinwalled, blind reservoir of about the same length, the sperm-sac (sp. s) ; just to the inner side of its aperture are the openings of the ureters $\left(u r^{\prime}\right)$. The sperm-sac is continuous posteriorly with the urinogenital sinus, the opening of which into the cloaca is situated on a papilla.

The female Scyllium has a single ovary $(B$, ov) suspended by a fold of peritoneum, its fellow having become aborted. In the adult it is studded all over with rounded ova in different stages of development, varying in diameter from I2-I4 mm. downwards: in other Vertebrates which produce large eggs, a similar reduction of one ovary may take place (e.g., Birds). The paired oviducts (ovd) are not coiled as in the frog ; they extend along the whole length of the dorsal wall of the colome, below the kidneys, and anteriorly unite with one another below the gullet and just in front of the liver, where they communicate with the cœlome by a common aperture $\left(o v d^{\prime}\right)$; posteriorly they open together by a single aperture $\left(o v d^{\prime \prime}\right)$ into the cloaca, behind the rectum $(\boldsymbol{r})$. About the anterior third of each oviduct is 


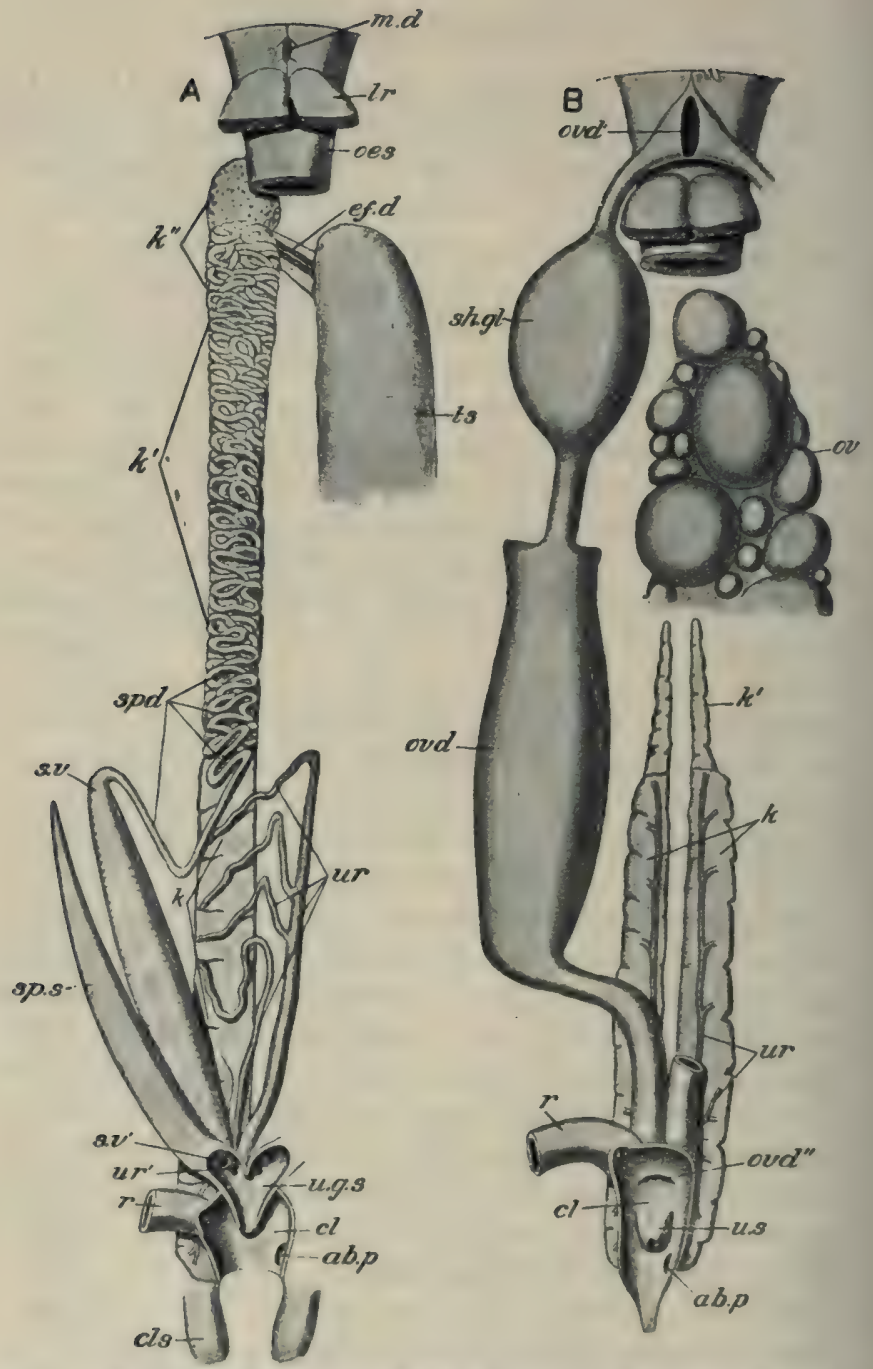

FIG. 127.- The urinogenital organs of Scyllium canicula from the ventral side. ( \& $)$

$\mathbf{A}$, male, and $\mathbf{B}$, female. Oniy the anterior end of the gonal is represented in each figure, and except that in $\mathrm{B}$ both kidneys are shown, the organs of the right 
side only are drawn. In A the seminal vesicle and sperm-sac are dissected away from the kidneys and displaced outwards, and the ureters inwards.

ab. p. depression into which the abdominal pore opens : cl. cloaca : cls. clasper ; ef. $d$. efferent ducts of spermary : $k$. kidney; $k^{\prime}$ (in B) vestigial portion of the kidney ; $k^{\prime}, k^{\prime \prime}$ (in A) epididymis : $i r$. anterior portion of liver; $m$. $d$. vestigial oviduct in the male; oes. gullet; or ovary: ovd. oviduct containing egg ; ovd its coelomic aperture ; ovd". the common aperture of the oviducts into the cloaca; r. rectum ; sh. gl. shell-gland : spd. spermiduct : $s p$. $s$. sperm sac : $s$. $v$. seminal vesicle: $s . \tau^{\prime}$. its aperture into the urinogenital sinus; $t s$. spermary ; u. g. s. urinogenital sinus; $w r$. ureters; $u r^{\prime}$. their apertures into the urinogenital sinus; w. s, urinary sinus.

narrow and thin-walled; the posterior two-thirds is wide and distensible, and at the junction of the two parts is a yellowish glandular region, the shell-gland $(s h . g l)$. A vestige of the anterior ends of the oviducts can be recognised in the male $(A, m . d)$.

Development.-Impregnation is internal, and is effected through the agency of the claspers of the male (p. 433). The eggs, when ripe, break loose from the surface of the ovary into the coelome, and thence pass, through the common oviducal aperture, into one or other of the oviducts, where fertilisation occurs. On reaching the dilated portion of the oviduct, the oosperm of Scyllium becomes surrounded first by a gelatinous substance, and then by a horny egg-shell or " Mermaid's purse" 1 secreted by the shell-gland, and having the form of a pillow-case produced at each of its four angles into a long, tendril-like process. The eggs are laid among sea-weed, to which they become attached by their tendrils. In Acanthias and Mustelus (p. 43I) a mere vestige of the egg-shell is formed, and the eggs undergo the whole of their development in the oviducts, the young being eventually born alive with the form and proportions of the adult.

The great size of the egg is due to the immense quantity of yolk it contains : its protoplasm is almost entirely aggregated at one pole, where it forms a small

1 An egg is contained in the oviduct shown in Fig. 127, B. 
disc. When segmentation of the oosperm takes place it affects the protoplasmic part alone, the inactive yolk taking no part in the process (compare crayfish, p. 383). The polyplast stage consequently consists of a little mass of cells, the blastoderm (Fig. I28), at one pole of an undivided sphere of yolk. The cells of the blastoderm become differentiated into the three embryonic layers - ectoderm, mesoderm, and endoderm. At the same time the blastoderm extends in a peripheral direction so as gradually to cover the yolk, and its middle part becomes raised up into a ridge-like thickening, which is moulded, step by step, into the form of the embryo fish. The head, trunk, and tail acquire distinctness, and become more and more completely separated off

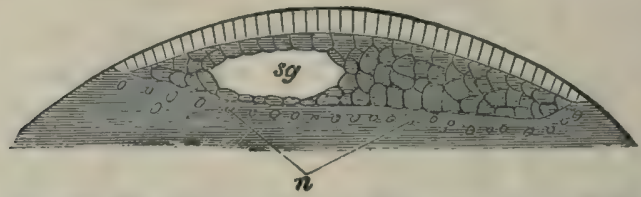

Fic. 128.-Magnified section of the upper part of the oosperm of a Dogfish which has undergone segmentation to form the blastoderm. The blastoderm is formed of a single layer of ectoderm cells (white), and of several rows of cells (shaded) which subsequently give rise to endoderm and mesoderm.

sg. segmentation cavity ; below the blastoderm is the unsegmented yolk containing scattered nuclei $(n)$. (From Balfour's Embryology.)

from the bulk of the egg, the latter taking the form of a yolk-sac (Fig. I29, A, yk. s) attached by a narrow stalk to the ventral surface of the embryo and supplied with blood-vessels (see p. 599).

In this condition the various parts of the adult fish can be recognised, but the proportions are different and the head presents several peculiarities. The gill-filaments $(b r . f)$ are so long as to project through the external branchial apertures and spiracles in the form of long threads abundantly supplied with blood-vessels, and 
apparently serving for the absorption of nutrimentthe albumen in the egg-shell in the case of Scyllium, secretions of the oviduct in the viviparous forms referred to on p. 469. Besides this mode of nutrition, the yolksac communicates with the intestine by a narrow duct (st), through which, as well as by means of the blood-vessels, absorption of its contents is constantly going on. By the time the young fish is ready to be hatched, the greater part of the yolk-sac has been drawn into the cœlome, a mere remnant of it still dangling from the ventral surface of the body.

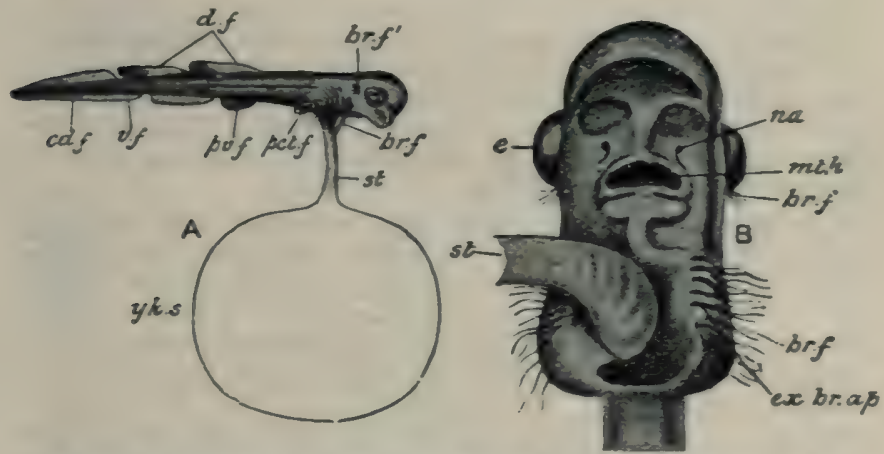

Fic. I29.-A, embryo of Scyllium with yolk-sac $(\times \quad \mathrm{r}) ; \mathrm{B}$, underside of head, enlarged. $b r . f$. branchial filaments protruding through gill-clefts; $b r . f$. branchial filaments protruding through spiracle ; $c d$. $f$. caudal fin; $d$. $f$. dorsal fins; e. eye : $e x$. br. ap. external branchia! apertures; mth. mouth; na. nostril; pct. $f$. pectoral fin: po. $f$. pelvic fin : st. stalk of yolk-sac; $v$. $f$. ventral fin ; yk. s. yolk-sac. (From Parker's Biology; after Balfour slightly aitered.) 


\section{PRACTICAL DIRECTIONS}

Dogfishes are best preserved in 5 per cent. formaline, which, unlike spirit, does not coagulate the blood, so that the vessels can be injected in preserved specimens. They can be obtained, fresh or ready preserved, from any Marine Biological Station.

$A$. External Characters: see pp. 43I-434. Sketch from the side.

Examine a small piece of the skin under the low power with reflected light and note the form and arrangement of the dermal teeth. Isolate some of these by boiling a small piece of skin in caustic potash (p. 359), and make out the bone-like basal plate, and the spine composed of dentine tipped with enamel. Sketch.

13. Skeleton. It is advisable to have one skeleton prepared entire, and one in which the parts have been disarticulated. Obtain a common butcher's or cook's pointed knife (a strong pocket-knife will do) for cutting through the rough skin and for the coarser work of preparation. Prepare as directed on p. 53, dipping into hot water occasionally, or macerating in 2 per cent. nitric acid for a day or two. When the greater part of the muscles has been removed, disarticulate the skull from the vertebral column, leaving the branchial apparatus attached to it, and also remove the paired fins and their arches. Disarticulate the hyomandibular cartilage from the cranium so as to separate the visceral arches, including the jaws (compare Fig. II2): these should then be thoroughly cleaned without further immersion in hot water, as the cartilages of which they are composed come apart very easily. The other parts may be dipped into hot water for a few seconds from time to time, but care should be taken that the more delicate elements do not thereby become separated. It is useful to prepare a second cranium as well as a few additional trunk- and caudal vertebræ, which should be bisected vertically into right and left halves. When prepared, the skeleton should be kept in weak spirit, and 
not allowed to dry, or the cartilages will of course shrink, unless the following methorl is resorted to:-

Thoroughly clean a skeleton, or typical parts of it (e.g., skull, limb-skeleton, and a few trunk-and caudal vertebric), and then transfer from weak into strong methylated spirit for a day or so, and afterwards into absolute alcohol for a few hours. Place in a vessel filled with turpentine for another day, and then transfer into melted paraftin in the water-bath until the parts are thoroughly permeated, after which they should be suspencled in the water-bath in orcler to drain off the superfluous paraffin, and then allowed to cool. Any superfluous paraffin still remaining may then be removed with a hot wire.

With the specimens before you, work through pp. 435-443, noting first of all the relations of the parts in the entire skeleton, (viz., cranial and visceral portions of the skull, trunk-and caudal vertebræ, and the skeleton of the median and paired fins). When examining the skull, note the nerve foramina (pp. 46r and 463 ).

Sketch-(a) the skull (including visceral arches) from the side, and the cranium in longitudinal section; (b) trunkand caudal vertebre from the side and in longitudinal section, and from the anterior or posterior face ; $(c)$ the pectoral arch, from the side, with the pectoral fin attached; and (d) the pelvic arch and fin.

\section{General dissection: Enteric Canal, \&e.}

I.-Fix the animal down on the dissecting board with the ventral surface uppermost, by means of fine nails inserted through the paired fins, and make a median longitudinal cut with a common knife through the skin and underlying muscular layer-which is closely connected with the skin, from the pectoral to the pelvic arch. At each end of this incision cut through the body-walls transversely, and reflect and pin down the two flaps. Cut through the pelvic arch slightly to one side of the median line, so as not to injure the cloaca. The abdominal caity', lined by the peritoneum, will then be exposed. (In the course of your dissection you will probably find many parasitic thread-worms belonging to the phylum Nemathelminthes-see p. 4I2.) Malre out (compare Fig. II7) :-

I. The liver, with the gall-bladder partly embedded in it close to the junction of its two lobes; the gullet, U-shaped stomach, and the branches of the ragus nere on its walls; the wide intestine, narrowing into a short rectum posteriorly; 
the cloaca; the pancreas, spleen, and rectal gland; and the incomplete mesentery. Pass a seeker lackwards, on one side of the cloaca, through an abdominal pore.

2. In the male, the spermaries, fused posteriorly ; and in the female, the single orary, and the oriducts and shellglands. The peritoneum covering the kidneys is so thick that at present they can only be recognised as slightly convex ridges.

II.-Remove the skin from the dorsal surface of the head between and slightly in front of and behind the eyes, and then slice away part of the roof of the skull with a knife until the brain is exposed, being careful not to injure some nerves which you will see close beneath the skin on either sicle of the brain-case in front. Then cut off the tail transversely, a short distance behind the pelvic fins, and on the cut surface note-

I. The integument, in which runs the sensory canal of the lateral line; the lateral cutaneous vein (Fig. 122, l.c.).

2. The centrum and the neural and hemal arches of the vertebra, and the soft intervertebral substance (remains of the notochord); the spinal cord; and the caudal artery and vein.

3. The myomeres and myocommas (see p. 480); and, if your section passes through a dorsal fin, the cartilaginous pterygiophores and the horny fin-rays (compare lig. III). Sketch.

III.- The ciorsal aorta and its branches may now be injected (see p. o9) through the cut end of the caudal artery, into which a cannula should be inserted for some distance (tying is unnecessary). Now return to the examination of the abdominal viscera, and make out:-

I. The bile-duct, opening into the intestine just behind the pylorus. The pancreatic duct runs in the wall of the intestine, and careful dissection is required to make out its course (see §IV, I).

2. The hepatic porlal vein and its factors, entering the liver near the median plane. If the blood has escaped from it, try to blow it up with a blowpipe.

3. The position of the dorsal aoria, which will be seen better at a later stage, but the chief branches of which should now be tracel to their distribution, as follows: $a$, the coliac artery, extending downwards and backwards along the stomach from above the posterior end of the gullet; $b$, the anterior mesenteric artery, arising about 
$I \frac{1}{2}$ inches behind the coliac; $c$, the lienogastric artery, arising close behind, and then crossing, the anterior mesenteric; and $d$, the small posterior mesenteric artery, passing downwards to the rectal gland.

4. The large hepatic sinus, immediately in front of the liver, below the gullet: slit it open, and note the veins entering it from the liver. On either side of the gullet in this region along the dorsal surface of the cœlome, a capacious cardinal sinus will be seen : make an aperture in this, and pass a seeker backwards, noting that the sinus narrows into the cardinal vein, which passes along the inner side of the corresponding kidney and parallel to the aorta (Figs. II9 and 122). The genital (spermatic or ovarian) sinus communicates with the cardinal.

5. The lateral abdominal veins (Fig. 122, lat.), running on either side of the body just beneath the peritoneum. Cut through the body-wall on one side, a short distance behind the pectoral fin; insert a cannula, directed forwards, into the cut end of the lateral vein (see Fig. III), and inject. The vein will then be seen running forwards as far as the pectoral arch, when it turns towards the dorsal side.

6. In the female, the united anterior ends of the oviducts, and their coelomic aperture, ventral to the gullet and just in front of the liver.

IV.-Taking care not to injure the anterior ends of the oviducts and to leave part of the hepatic sinus in situ, remove the liver, together with the stomach and intestine, without injuring the bile-duct, cutting through the stomach at its junction with the gullet and the intestine just in front of the rectal gland. Wash out the portion of the enteric canal thus removed under the tap, fill it with water, and place the whole under water in a dissecting-dish. Cut away portions of the wall of both stomach and intestine, and make out-

I. The course of the bile-duct and pancreatic duct, and their apertures into the intestine.

2. The pyloric valie, and the spiral valie of the intestine, which makes about seven or eight close turns, appearing like a series of cones one within the other.

3. The characters of the mucous membrane of the stomach and intestine.

Sketch your dissection.

\section{Urinogenital organs.}

I.- After noting again the gonads, and in the male the delicate efferent ducts of the spermaries (Fig. I 27, A), remove 
in the male all but the anterior ends of the latter, and in the female the entire ovary; then carefully dissect away the thick peritoneum covering the kindeys, noting as you do so the dorsal aorta and its various branches, and once again the cardinal veins $(C, \S I I I, 4)$, which may be inflated with air. The renal portal veins are not easily traced without injection : this may be done from the cut end of the caudal vein.

Note-

I. The brownish kidneys, and in the male the whitish forward continuation of each into the anterior sexual part or epididymis.

2. In the male: $a$, the convoluted spermiduct, indistinguishable from the epididymis anteriorly and enlarging posteriorly to form the elongated seminal vesicle; and $b$, the grooved claspers, which are supported by cartilages and have each a gland at the base of the groove.

II.-Cut through the skin round the vent, and clissect the entire cloaca and the kidneys (together with the epididymes in the male) away from the body, noting as you do so some small paired and median bodies in close relation to the dorsal aspect of the kidneys; these are respectively the suprarenal and interrenal bodies (p. 447). Pin your dissection down, ventral side uppermost, under water. Clear away with great care the connective-tissue which binds the ureters and generative ducts to the kidneys posteriorly, slit open the cloaca and make out-

I. In the male (Fig. I27, A) the aperture of the rectum, and the urinogenital papilla. Insert the small scissors into the aperture at the apex of the latter, and slit open the urinogenital sinus, continuing the cut into the two sperm-sacs; make out the apertures of the seminal vesicles and ureters. Pass a seeker or probe into these apertures (the main ureter may be injected), and then dissect out, on one side- $a$, the elongated and pointed, thin-walled sperm-sac; and $b$, the delicate ureters, four of which unite to form a wiclish common tube, situated towards the inner border of the kidney, before opening into the urinogenital sinus. Sketch.

2. In the female (Fig. $127, B$ ), the thin-walled anterior united ends, of the oviducts, their thick-walled posterior portion, the shell-glands, the apertures of the rectum and oviducts into the cloaca, and the urinary papilla. Insert the point of the scissors into the aperture on the apex of the latter, and slit open the urinary sinus, in which several openings of the ureters will be seen on either side. Cut 
open the oviducts, and note, if present, the eggs enclosed in horny egg-cases. Sketch.

\section{E. Circulatory ${ }^{1}$ and Respiratory organs, \&c.}

I.-After noting the spinal neries, exposed by the removal of the kidneys, the body may be cut through just behind the pectoral arch, and the posterior portion thrown away. Pin down the head and anterior portion of the body, ventral side uppermost, make a median longitudinal incision through the skin from the lower jaw to the pectoral arch, and dissect it away on either side as far as the gill-clefts. Then, without injuring the lateral vein $(\mathrm{p} .475)$, remove the middle portion of the pectoral arch and expose the pericardial carity and heart. Insert a seeker, pointing backwards, along the dorsal side of the heart, through the pericardio-peritoneal canal which communicates between the pericardial and abdominal cavities (p. 434): it opens on the ventral side of the gullet by two apertures. Nake out-

$\mathrm{r}$. The form and relations of the chambers of the heart (sinus ienosus, auricle, ventricle, and conus arteriosus).

2. The ientral aorta, to expose which the muscles in front of the pericardium must be carefully removed; but before doing so, it is better to inject the ventral aorta, cutting a small hole in the ventricle, and inserting and tying the cannula into the conus arteriosus : use a blue injection if you have already used red for the dorsal aorta.

3. The five afferent branchial arteries (compare Fig. I2I) : trace these outwards, and note their distribution. Sketch your dissection.

II.-Cut through the ventral aorta at its junction with the conus arteriosus, and through both ends of the sinus venosus, carefully separating the latter from the walls of the pericardium and noting the entrance of the hepatic sinus (p. 475). Remove the entire heart, pin it down under water, ventral side uppermost. Cut open the ventricle and conus arteriosus and note-

I. Their cavities and walls; the auriculo-ventricular aperlure and values: the iales in the conus arteriosus, of which there are two sets, consisting of three in each set. Sketch.

2. The sinu-auricular a perture is best made out by turning the heart over with the dorsal sicle uppermost, before cutting open the auricle from the ventral side. Sketch.

3. Insert a seeker into one of the cut distal ends (still left in situ) of the sinus venosus, and slit it up so as to expose

1 See also C. $\S$ III. 
the precaval sinus of the same side: by means of a seeker find the apertures of the following veins or sinuses (compare Fig. I22)-a, the cardinal and jugular; $b$, the inferior jugular; and $c$, the lateral vein.

III.-Insert the scissors into the external gill-clefts of one side, one by one, and extend the clefts by cutting dorsally and ventrally, so as to expose the gill-pouches, communicating with the pharynx by the internal gill-clefts. Make out-

1. The branchial filaments, and observe that there are four complete gills on the first four branchial arches, and a half-gill or hemibranch on the posterior face of the hyoid arch. Note also the pseudobranch on the anterior side of the spiracle.

2. The structure of the gills. Remove two entire gills; dissect one, and cut the other across transversely (compare Fig. II8), noting the relations of the septum, cartilaginous branchial arch and rays, branchial filaments, and single afferent and paired efferent branchial artery. Sketch.

IV.-Cut through the floor of the pharynx and mouth close to the middle line, just on one side of the ventral aorta, and extend the cut through the lower jaw. On one side, turn the floor outwards, and fix it back in this position so as to expose the roof of the mouth and pharynx and the internal gill-clefts; dissect away the mucous membrane lining the roof, and trace out on one side (Fig. I2I)-

I. The epibranchial and efferent branchial arteries, and the dorsal aorta.

2. The carotid, subclavian, coronary arteries, \&c.

\section{$F$. Nervous System and Sense-organs.}

I.-Remove with the knife the rest of the skull-roof and a few of the anterior neural arches so as to expose the entire brain and the anterior part of the spinal cord. In doing so, be careful not to injure the contents of the orbit, the nerves referred to on p. 474 , $\S$ II., or the auditory capsule of one side. After noting the "pia mater," make out-

I. The subdivisions of the brain (olfactory lobes, prosencephalon, diencephalon, optic lobes, cerebellum, and medulla oblongata). Sketch.

2. The origins of the cevebral neves from the brain and the points at which they penetrate the walls of the skull (pp. 46I-464).

3. The spinal cord, and the alternating dorsal and ventral roots of the spinal nerves. Then cut through the spinal 
cord just behind the medulla oblongata, and through the origins of the cerebral nerves. Remove the brain and place it in formaline or spirit.

II.-Carefuliy dissect away the skin covering the head and pharyngeal region on the unclissected side, expose the orbit, and remove the delicate comnective-tissue surrounding its contents. Pin down firmly, and dissect out the following from the side (compare Fig. 125 and Pp. $46 \mathrm{I}-464$ ).

I. The ophthalnic division of the facialnerve, and immediately below it that of the trigeminal; trace them backwards to their foramina in the skull-wall and forwards, through a canal between the olfactory capsule and the cranium, to their distribution.

2. The large mass of sensory (ampullary) canals on the dorsal side of the snout.

3. The four recti and the two ablique eye-muscles (Fig. I z(i), and the nerves (III, IV, VI) supplying them.

4. The eve, and the optic nerie-anterior to the recti muscles. The eye may now be removed by cutting throngh the muscles and optic nerve, and dissected as directed on p. I9I.

5. The large, flat, maxillo-mandibular division of the trigeminal, running forwarls and outwards along the floor of the orbit, and there dividing into maxillary and mandibular branches.

6. The facial neve, entering the orbit close behind the maxillo-mandibular nerve, and giving off :-behind the spiracle-a large hyomundibular branch, passing along the anterior border of the auditory capsule and posterior wall of the orbit, and down the anterior side of the hyoid arch just beneath the skin: and in front of the spiracle-a palatine and prespiracular branches. Of the branches to the sensory canals, the ophthalmic has already been seen; the buccal and external mandibular require very careful dissection in order to make them out satisfactorily.

7. The glossopharingeal and vagus neres. To expose these, slice away sufficient of the auditory capsule noting as you do so the semicircular cands and the indrlymphatic duct, p. $\left.46_{5}\right)$ to expose the foramina by which they emerge from the skull, behind the auditory capsule, and separate the mass of muscles lying alongsicle the vertebral criumn from the branchial apparatus, by dissecting away the connective-tissue. Trace the glossopharrngeal to its bifurcation over the first gill-cleft, and in the vagus follow out$a$, the four branchial branches, forking over the remaining 
gill-clefts; $b$, the visceral branch; and $c$, the lateral line branch, above and to the inner side of the branchial branches.

8. Separate some of the ampullary sensory tubes from one another, and note the ampullce and the nerves supplying them.

9. Carefully slice away the cartilage of the auditory capsule of the side you have not already dissected so as to expose the membranous labyrinth. Examine under water, and make out the vestibule (utriculus and sacculus) with its contained otolithic mass, the three semicircular canals with their ampulla, and the branches of the auditory nerve (compare Fig. 59).

III. Now examine the preserved brain from above, from below, and from the side, making out, in adclition to the parts already noticed $(F, \S I, I)-$

I. The optic chiasma, infundibulum (with an oval lobe and a vascular sac on either side), pituitary body, crura cerebri, and, as far as possible, the origins of the nerves. Sketch from above and below.

2. On one side of the brain, cut into the olfactory lobe, prosencephalon, optic lobe, and cerebellum from above, so as to expose the olfactory ventricle, lateral ventricle, oftic ventricle, and cerebellar ventricle. Then bisect the entire brain into right and left halves with a sharp scalpel, and examine the uninjured half in longitudinal section, noting, in addition to the parts mentioned above, the third ventricle, foramen of Monro, iter, and fourth ventricle. Sketch.

G. Transverse Sections.-Cut thick transverse sections of an entire dogfish with a knife through- $a$, the anterior, and $b$, the posterior part of the head (pharyngeal region) ; $c$, about the middle of the body; and $d$, the tail. Make out the relations of the various parts and organs, and sketch the lateral half of each section.

A more satisfactory method than this is to obtain a very young dogfish, not more than $\frac{1}{2}$ inch in diameter, and after cutting it transversely into pieces about $\frac{1}{8}$ inch in thickness in the regions named above, stain, embed, and mount a few sections from each piece (see p. 136). These can first be examined with a lens or with the low power of the microscope, and then, by putting on the high power, important points in the histology can be made out. In adclition to the minute structure of the tissues and organs described in P'art I. of this book, the structure of the notochord (p. 44 I), integumentary sense-organs (j) $) .432$ and $f^{(0.4)}$, dermal teeth (pp. +33 and 444 ), \&c., should be studied. 
$H$. Side Dissection.-It is very instructive to supplement and recapitulate your work on the anatomy of the dogfish by dissecting another specimen from the side (compare Fig. II7), as in the case of the crayfish. Dissect off the skin from the left side for an inch or so and observe the myomeres and myocommas. Cut open the abdominal cavity as before, very slightly to the left side of the middle line. Continue to cut forwards through the pectoral arch and backwards through the pelvic arch; the arteries may be injected at this stage. Then dissect off the left half of each arch with the corresponding fin, and cut away the left bodywall. Cut through the skin in the mid-dorsal line: and then, working from the left side, remove the left half of the skull and visceral arches, as well as the left side of the vertebral column, so as to expose the brain and spinal cord. Remove the left kidney (and left spermary if your specimen is a male; left oviduct if it is a female). Pin down under water, and tidy your dissection so as to reduce it to a neat longitudinal section, in which all the unpaired soft organs are left intact. Without tearing the mesentery, pin out the liver, stomach, and intestine beyond the ventral limits of the body-wall, so that the other abdominal organs are not hidden. Follow out the relations of the various organs as before, and sketch your dissection.

It is important at this stage to refresh your memory of the anatomy of the frog, and to compare it with the dogtish, by making a similar dissection of it from the side (compare Fig. 7). Sketch. 


\section{CHAPTER XI}

CHARACTERS OF THE CLASS MAMMALIA-THE RABBIT

BEFORE examining a rabbit, as an example of the highest class of Vertebrates-the Mammalia, it will be well to recapitulate some of the characters of the frog, the organisation of which is higher than that of a fish.

The frog, taken as an example of the class Amphibia, differs from a fish in the following points amongst others. Its paired limbs have not the form of paddle-like fins, but the fore-limb consists of upper arm, fore-arm, wrist, and hand, and the hind-limb of thigh, shank, ankle, and foot, each with characteristic skeletal parts; it has no median fin in the adult, and that of the tadpole is not supported by fin-rays; there is no hard, dermal exoskeleton; respiration in the adult is pulmonary, and internal nostrils are present; there are two auricles in the heart, and the cardinal veins are replaced by a postcaval; there is a urinary bladder formed as an outgrowth of the cloaca. Moreover, the endoskeleton, unlike that of the dogfish, is in the adult composed mainly of bone.

In all these characters the frog resembles the rabbit. But the Mammal differs from the Amphibian in many important respects, some of the chief of which are:the presence of an epidermic exoskeleton consisting of hairs; the high temperature of the blood, which remains 
almost uniformly within a few degrees of $100^{\circ} \mathrm{Fahr}$., and does not vary to any appreciable extent with the temperature of the air ; the absence of nuclei in the red corpuscles of the blood; the presence of mammary glands beneath the skin in the female which secrete milk for nourishing the young; the subdivision of the body-cavity into two portions-thorax and abdomen-by a transverse partition, the diaphragm; the presence of two ventricles as well as of two auricles in the heart, and of a single systemic aortic arch (that of the left side); the higher differentiation of the brain, and also of the skeleton; and the mode of articulation of the lower jaw. Moreover, in the large majority of Mammals, the teeth are differentiated into front-teeth for biting or seizing the food and cheek-teeth or grinders, and their succession is limited to two functional sets; an external ear or pinna is present; there is no cloaca, the anus and urinogenital apertures opening separately on the exterior, while the ureters open directly into the bladder ; the ova are minute; the young undergo their early development in the oviduct, where they are nourished by diffusion from the blood-system of the parent by means of an organ known as the placenta, and after birth they are suckled by the mother.

Bearing in mind these essential characters of the higher Mammalia as compared with the Vertebrates previously studied, we can now proceed to examine the structure of the rabbit in greater detail.

External Characters.-The rabbit (Lepus cuniculus) is a very abundant and widely distributed animal which in the wild state makes burrows in the earth, and the practically hairless young are born in these or in special nests. There are a number of varieties, the habits and general appearance of which have been modified by domestication (compare p. 227). 
In addition to head, trunk, and short tail, the rabbit possesses a distinct neck, and the whole animal, including the limbs and even the soles of the feet, is covered with a soft fur consisting of hairs (Fig. I30). In the wild rabbit, the fur is of a brownish colour, lighter below, and white under the tail : in the many domesticated varieties the colour is very varied.

The hairs correspond to modified epidermic cells which become converted into a horny material ; they are developed in tube-like involutions of the epiderm called hair-sacs, into

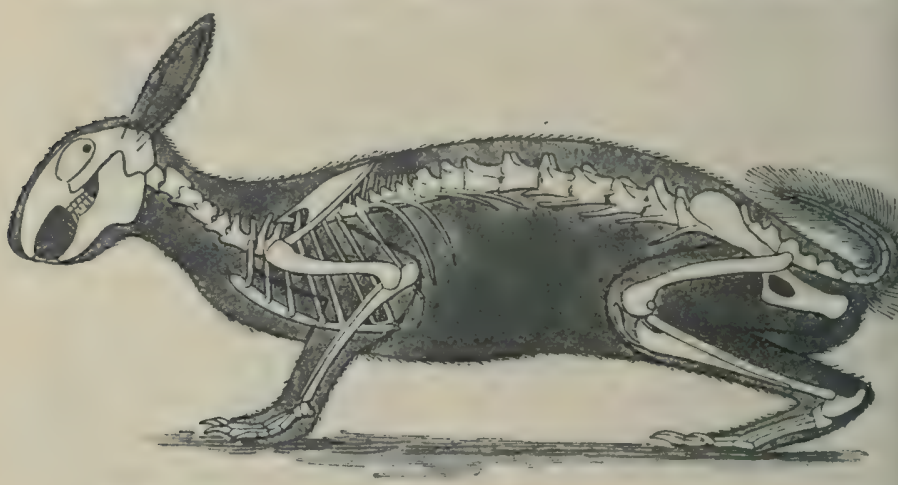

Frg. 130.-Lateral view of skeleton of Rabbit with outline of body. ( $x$ t.) (From Parker and Haswell's Zoology.)

the swollen base of each of which a mesodermal hair-papilla projects, the substance of the hair, with its cortex and medulla, being formed from the epidermic cells covering the papilla (Fig. I3I) Into the hair-sacs open the ducts of sebaceous glands (HBI)), the secretion of which serves to lubricate the hairs, which can be erected by means of muscles (Ap).

There are five digits in the hand or manus, and four in the foot or pes, each terminated by a pointed and curved horny claw, developed, like the hairs, from the 


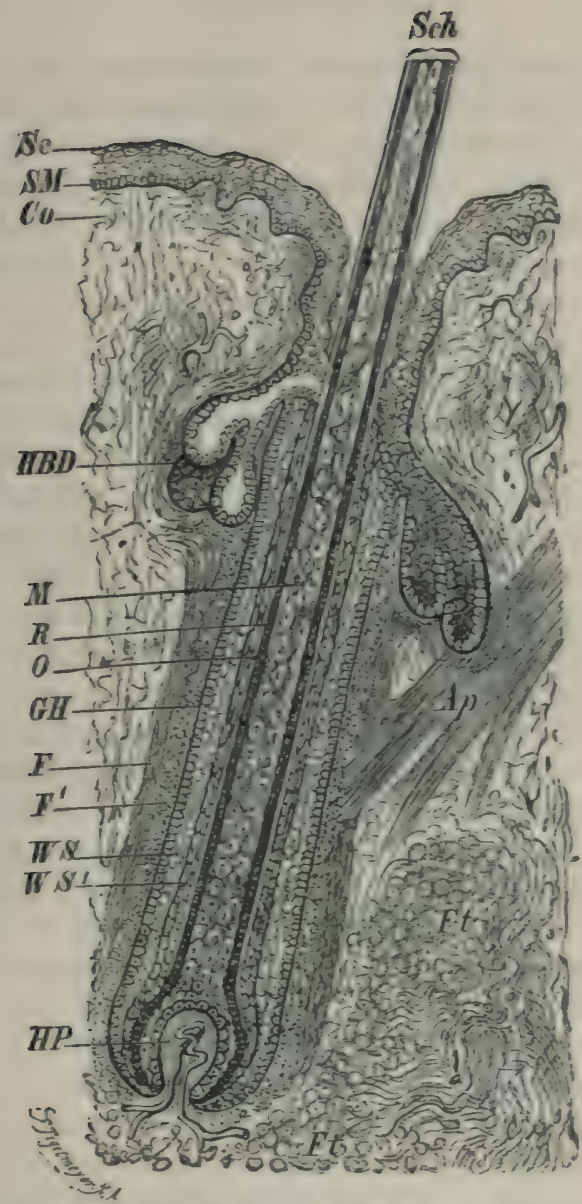

Fig. 13r.-Longitudinal sestion through a hair-sac. (Diagrammatic.) (From Wiedersheim's Comp. Anatomy.)

$A p$, muscles for erecting the hair ; $C o$. derm; $F$. outer longitudinal layer, and $F 1$. inner transverse layer of connective-tissue fibres of hair-sac; $F t$. adipose tissue: $G H$. hyaline layer, which lies between the inner and outer hair-sheaths, i.eon between the root-sheath and the bair-sac: $H B D$. sebaceous glands; $H P$. hairpapilla, containing vessels; $M$. medulla; $O$. cuticle of shaft; $R$. cortex; Sc. horny layei of epi-lerm; Sith. hair-shaft; S.M. Malpighian layer of epiderm; WS, WSi. external and internal root-sheath. 
epiderm. Along the ventral surface of the body in the female are four or five pairs of papillæ-the teats, on which open the ducts of the milk-glands, which correspond to modified integumentary glands. The various parts of the skeleton (Fig. I30) can be felt through the skin, and it will be noticed that the anterior part of the trunk, or thorax, is surrounded by ribs, many of which meet below with a breast-bone or sternum, and which are absent in the posterior part of the trunk, or abdomen.

Beneath the anterior end of the snout is the transverse mouth, which has a narrow gape and is bounded by upper and lower lips: the upper lip is divided by a longitudinal cleft which is continuous with the oblique, slit-like external nostrils. Just inside the lips are the upper and lower front teeth or incisors, which are chiselshaped, and behind them the hairy integument is continued on either side into the cavity of the mouth. The eyes are protected by movable hairy upper and lower eyelids, as well as by a hairless third eyelid or nictitating membrane (compare p. 5), supported by cartilage and situated in the anterior corner of the eye, over which it can be partly drawn: it corresponds to the little red lump in the inner corner of the human eye. On the upper lip and above and below the eye are certain very long and stiff hairs - the "whiskers" or vibrisse, and behind the eyes are a pair of long and movable external ears or pinna: these are supported by cartilage and are somewhat spout-shaped, leading to the external auditory openings.

Below the root of the tail is the anus, and in front of and below this the urinogenital aperture, the space between them being known as the perineum. On either side of these apertures is a hairless depression of the skin on which open the ducts of the perineal glands, the secretion of which has a strong and characteristic odour. 
In the female the slit-like urinogenital aperture is called the vulva; in the male the aperture is smaller and is situated on the conical apex of a cylindrical organ, the penis, which can be retracted within a fold of skin, the foreskin or prepuce. On either side of the penis is an oval pouch of the skin, the scrotal sac, not very apparent in young animals, in each of which a spermary or testis is contained.

Skeleton.-The skeleton of the adult rabbit consists almost entirely of bone, but it must be remembered that, in addition to certain cartilages described below, all articular surfaces are covered or lined by a thin layer of cartilage, and that the various parts of the skeleton are connected together by ligaments.

In the skull, both replacing and investing bones (p. 43) are much more numerous than in the frog, and the structure of the entire skull is far more complicated and highly differentiated. A posterior, relatively large cranial region, in the side walls of which the auditory capsules are embedded, can be distinguished from an anterior, somewhat conical facial region, constituting the skeleton of the snout (Fig. I,32). Just behind the junction of these two regions on either side is a large orbit, separated from its fellow by a thin interorbital septum, perforated by a foramen for the optic nerve (opt. fo).

At the sides of the foramen magnum are the two rounded occipital condyles; the auditory apertures (aud. me) are situated at the sides of the posterior part of the cranium, and the external nostrils open at the anterior end of the snout. Most of the bones remain more or less distinct throughout life, and are in contact along lines or sutures, many of which are wavy or zig-zagged: others, again, become completely fused in the adult, so that their limits are no longer distinguishable. 


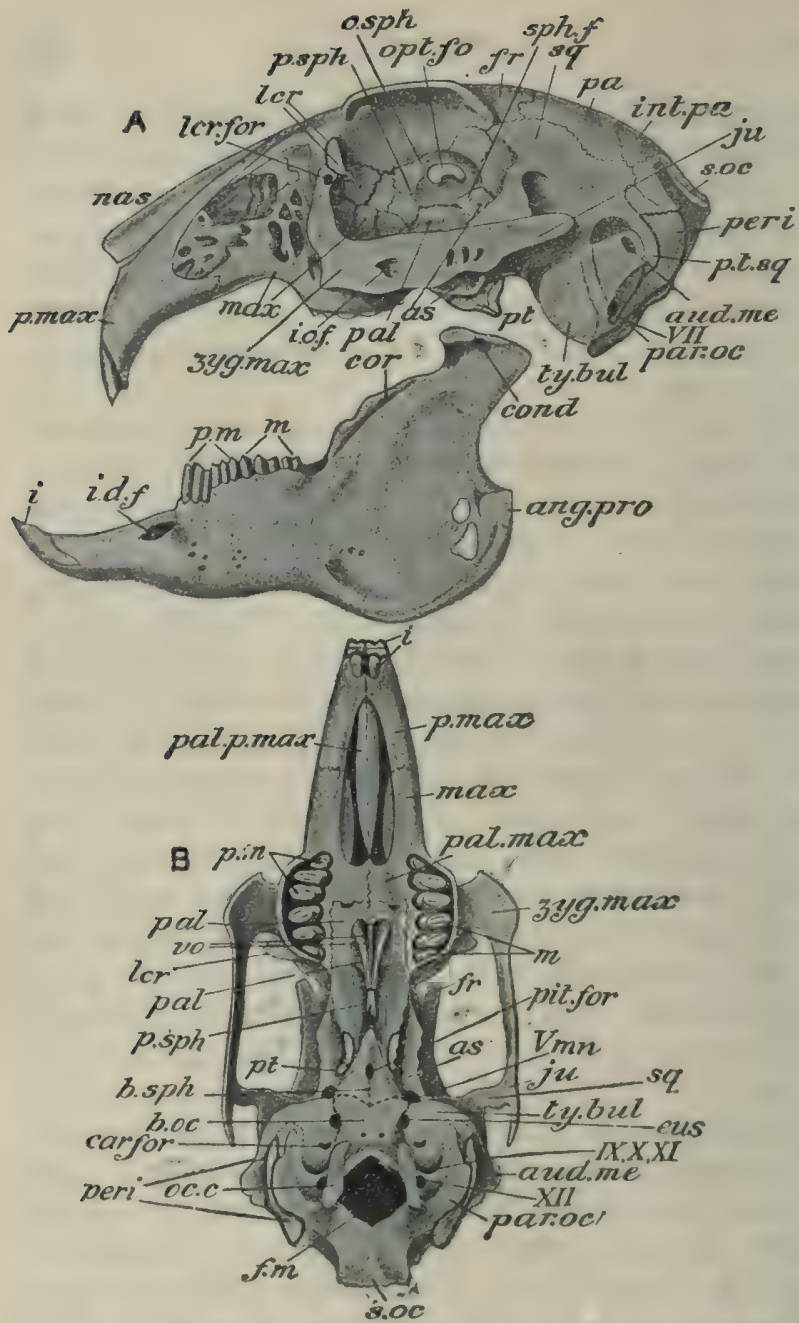

Fig. I32.-Skull of Rabbit. (Nat. size.) A, latera view with lower jaw;

$B$, ventral view.

ang. proc. angular process of mandible; as. alisphenoid (external pterygoid process below); aud. me. external auditory aperture in tympanic bone; $b$. oc. basioccipital; $b$. sph. basisphenoid; cond. condyle of lower jaw ; car. for. foranen for internal carotid artery; eus. Eustachian canal; $f$. m. forameu magnum; cor. coronoid process; $f r$. frontal; $i$. incisors ; $i$. $d$. $f$. inferior dental foramen for vassage of the manclibular division of the trigeminal nerve; int. pa. inter- 
parietal ; i. o. f. infraorbital foramen for passage of the maxillary Nivision of the trigeminal nerve; $j u$. jugal; kr. lacrvmal: $k r$. for. lacrvmal foramen: $m$. melars; max. maxilla : nus. nasal: oc. $c$. occipital condyle; opt. fo. optic foramen; $o$. sph. orbitosphenoid : pa. parietal : pal. palatire; pm. premalars; pa: max. palatine process of maxilla : par. oc. paruccipital preves of exocupital ; pa!. p. mar. palatine precess of premaxilla ; p. max. prennwila : peri. Furitic ;

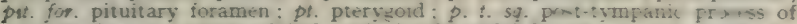
squamonal : s. oc. supraoccipital ; spls. $f$. spheroidal fissure : so. squam $=a 1$ : $t \%$. bul. tympanic bulla : to. vimer: 5 . mar $z$ yzumatic process of maxilia; $i$. mr. foramen for mandibular division of triseminal nerve : 11 . for factal nerve: $I X . X . X l$. for glossopharyngeal, vagus, and spinal accessory; $X I I$. for bypoglossal. (From Parker and Haswell's Zoology.)

The upper jaw forms part of the facial region, which encloses the olfactory chambers; and the lower jaw, consisting of a single bone on either side, articulates directly with the sides of the cranium without the intervention of a hyomandibular as in the dogfish (p. 438 ) or of a quadrate cartilage as in the frog $(\mathrm{p} .+4)$. The rest of the visceral portion of the skull, representing the hyoid and first branchial arch, forms the so-called hyoid bone which is embedded in the base of the tongue (Fig. I35. $h y)$.

The bones ${ }^{1}$ which form the walls of the brain-case are arranged in three rings or segments, between the middle and posterior of which are intercalated the auditory capsules (Figs. I33 and I32 A, peri).

The posterior, or occipital segment, consists of three bones, which in the adult become completely united with one another. The lower of these is the basioccipital ( $b$. oc), a flattened bone bounding the foramen magnum below, and forming the hinder part of the base of the skull and the lower part of each occipital condyle (oc. c). The two exoccipitals (e.oc) bound the foramen magnum at the sides, and form the upper part of the occipital condyles: each is produced downwards into a paroccipital process (par. oc) which fits closely against the posterior surface of a swollen bone $(t y . b u l)$ to be described presently, which is continuous with a tube surrounding

1 In the following description, the investing bones are distinguished by an asterisk from the replacing bones (see p. 43). 
the auditory aperture (aud. me). The occipital segment is completed above by the supraoccipital (s.oc), bounding the foramen magnum above; it has a pitted surface and is marked externally by a shield-shaped prominence.

The middle, or parietal segment, consists of five bones -a basisphenoid (b. sph) below, an alisphenoid (a. sph, as) on either side, and two parietals* $(p a)$ above. The broad posterior end of the basisphenoid is connected

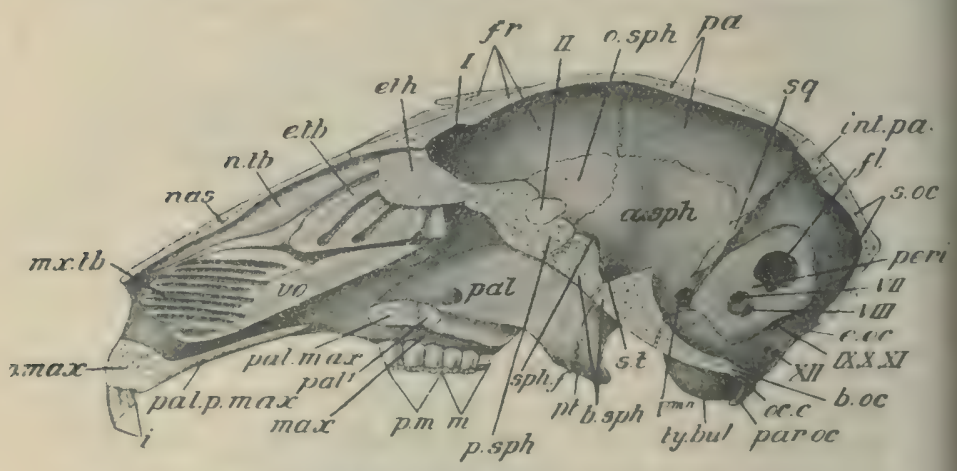

F1G. I33. - Skull of Rabhit in longitudinal vertical section. (Nat. size.) The cartilaginous nasal septum is removed.

a. $s p h$. alisphenoid; $e$. oc. exorripital; $\epsilon$. tb. ethmo-turbinal; $e t h$. ethmoid ; $f$. fossa for floceulus of hrain; $i$. incisors; $m x$. $t b$. maxillary turbinal; $n$. ib. nasoturbinal : pul'. palatine portion of the bony palate; p. sph. prespleteneil ; s. $t$. sella turcica, or depression in which the pituitary bouly lies; I, region at which the olfactory nerves leave the skull; II, optic foraren; $V$ mn. foramen for manclibular division of triseminal; VII, for facial nerve; VIII, for auclitory nerve: $\mathbf{I X}, \mathbf{X}$, XI, for glnswpharyngeal, vagus, and spinal accessory; XII for hypoglossal. Other letters as in Fig. 132 .

with the basioccipital by a thin plate of cartilage, and tapers in front to a blunt point : it is perforated at about its middle by an oval pituitury foramen, and on its upper surface is hollowed out to form a depression in which the pituitary body lies (s.t). The alisphenoids are wing-like bones, directed upwards and outwards, and firmly united with the basisphenoid: each is produced ventrally into a pterygoid process (as), consisting of two 
laminæ which converge and unite with one another anteriorly. The parietals are a pair of thin, slightly arched bones forming a considerable part of the roof of the brain-case and united with one another by suture along the middle line; the outer edge of each gives off a thin, ventral process which is covered by the squamosal ( $s q$ ), a bone which will be referred to presently (p. 495) and which, on the external face of the skull, separates the parietal from the alisphenoid. Interposed between the parietals and the supraoccipital is a small median interparietal* (int.pa).

The frontal segment also consists of five bones-a presphenoid (p.sph), two orbitosphenoids (o.sph), and two frontals* $(f r)$. The small presphenoid is laterally compressed and is connected with the basisphenoid by cartilage, so that in the dry skull there is a considerable interval between the two bones; it forms the inferior and anterior boundary of the optic foramen (opt. fo, II), which puts the two orbits in communication with one another and both in communication with the cranial cavity. The orbitosphenoids are two wing-like laminæ directed outwards and slightly backwards, and completely fused with the presphenoid; they surround the rest of the optic foramen. The frontals form the roof and side-walls of the anterior part of the brain-case, and are united by suture with one another in the middle line and with the parietals behind ; below they meet with one another anteriorly on the floor of the brain-case and unite with the presphenoid by suture; the outer part of each forms a prominent crescentic ridge, the supra-orbital process.

The brain-case is closed in anteriorly by a bone riddled with numerous small holes for the passage of the olfactory nerves (I): this is the cribriform plate of the ethmoid (eth). 
It will be remembered that in the frog the occipital region is ossified by exoccipitals only, the parietals and frontals of either side are fused, there are no ali- or orbito-sphenoids, the cartilaginous walls of the anterior part of the cranium are ossified as a sphenethmoid, and that the floor of the skull is supported by an investing bone, the parasphenoid (pp. $40-44$ ).

The auditory capsules are comparatively loosely wedged in laterally between the parietal and occipital segments; in the embryo each is ossified from three centres, instead of one (the pro-otic) as in the frog, but these early unite to form the periotic bone (peri), as the ossified auditory capsule is called. The internal or petrous portion of this bone (Fig. I33) encloses the membranous labyrinth of the ear and is very dense and hard; posteriorly it is produced outwards as the porous mastoid portion, which is visible on the outer side of the skull (Fig. I32, A). Closely applied to the outer surface of each periotic is a bone called the tympanic*, consisting of a tubular portion above-the edge of which surrounds the auditory opening ( $a u d . m e$ ) to which the cartilage of the pinna is attached, and of a swollen portion, or tympanic bulla (ty. bul), below: this encloses the tympanic cavity, and in it, at the base of the tubular portion, is an incomplete bony ring to which the tympanic membrane is attached (Fig. I 43 ). The tympanic is incomplete on its inner side, where its cavity is closed by the outer wall of the periotic, and between the two, at the antero-inferior angle of the former, is the aperture by which the Eustachian tube leaves the tympanic cavity (compare p. 45). When the tympanic is removed, two small holes are seen on the outer wall of the periotic: the anterior of them is the fenestra ovalis and is plugged by the stapes-which, together with two small bones, the malleus and incus (Fig. I43), forms the chain of auditory ossicles to be described later in connection 
with the organ of hearing; the posterior aperture is called the fenestra rotunda. On the internal or cranial surface of the periotic is a large depression (A) which lodges the flocculus of the cerebellum (Fig. I4I).

The olfactory capsules are roofed in by two long and narrow nasal bones* (nas), which meet together in the middle line and unite by suture with the frontals posteriorly. Their side walls are formed by the bones which bear the teeth of the upper jaw-the premaxill $e^{*}$ (p. max) and maxille* $(\max )$, and in the median line below is a single long and slender bone, deeply grooved on its upper surface, and formed by the fusion of the two vomers* $(v o)$. The two nasal chambers are separated from one another in the middle line by a median vertical plate of cartilage, the nasal septum (Fig. I35, n. s), embraced below by the vomer. This cartilage, together with the cribriform plate and a median vertical plate of bone (eth) extending forwards from the latter into the septum, constitutes the mesethmoid. Within the nasal chambers certain scroll-like folds of the mucous membrane (Fig. I35) are present in order to increase the surface, and in these cartilages are developed. The cartilages become ossified, and the resulting turbinal bones unite with certain of the bones enclosing the olfactory organs, and are named accordingly. The ethmoid turbinals (Fig. I33, e. $t b$ ), or true olfactory scrolls, are two complicated, folded bones united to the cribriform plate of the ethmoid, and are covered in the fresh condition by the olfactory epithelium ; the maxilloturbinals $(m x . t b)$ are similar but more complex bones in the antero-ventral part of the nasal cavities; and the naso-turbinals $(n . t b)$ are thin, folded bones, much less complex, and fused with the inferior surface of the nasals. 
In the front wall of each orbit, fitting comparatively loosely between the frontal and maxilla, is a small bone, the lacrymal* (Fig. I32, A, lcr), with a notch near its outer border through which the naso-lacrymal duct passes (p. I86).

As in the frog, the chief bones of the upper jaw on either side are the premaxilla* $(p$. max) and the maxilla* (max), and nearer the middle line are the palatine* (pal) and "pterygoid"* $(p t)$ : in the embryo the position of the two last-mentioned bones is taken by cartilage representing the upper jaw of the dogfish (compare Figs. II 2 and 9). The premaxillæ, in which the sockets for the front or incisor teeth are situated, form the anterior boundary of the snout, and articulate with one another in the median line and with the maxilla behind: each gives off a nasal process passing backwards between the nasal and maxilla to the frontal, and a palatine process (pal. p. max) extending backwards along the palate in contact with its fellow of the opposite side. The maxillæ are large and irregular bones, parts of the sides of which are fenestrated, and in which the cheek-teeth are situated. From the inner and inferior edge of each, opposite the first two cheek-teeth, a horizontal palatine process ( $p$ al. $\max$ ) is given off, which, articulating with its fellow of the opposite side, forms the anterior part of the bony support of the hard palate-this is of much less extent in the rabbit than in most mammals: from its outer side arises a zygomatic process (zyg. max), which forms the anterior part of the strong zygomatic arch extending below and externally to the orbit.

The palatines are thin, nearly vertical, bony laminæ, internal to the maxillæ to which they are attached in front, while above they join the presphenoid and the pterygoid process of the alisphenoid. They bound the passage of the internal nostrils, and from the inner and 
anterior region of each is given off, opposite the third cheek-tooth, a horizontal, inwardly directed process $\left(p_{a l}\right)$, which, articulating in the middle line with its fellow of the opposite side and in front with the palatine process of the maxilla, forms the posterior part of the bony support of the hard palate. The bones usually known as pterygoids are small irregular plates attached to the posterior edge of the corresponding palatine and the pterygoid process of the alisphenoid; each ends ventrally in a backwardlycurved process.

The squamosals* $(s q)$ are a pair of plates which overlap and complete the side-walls of the brain-case (p. 49I) in front of the periotics: they articulate with the frontals, parietals, orbitosphenoids, and alisphenoids. From the outer face of each is given off a strong zygomatic process, which bears on its under surface the articular facet for the lower jaw, and further back a slender process ( $p . t . s q)$ arises which is applied to the outer surface of the periotic. .

The zygomatic processes of the squamosal and maxilla respectively are united by a flat bar of bone, the jugal* (ju), which in the adult is fused with the latter. All these three bones therefore take part in forming the zygomatic arch.

Most of the apertures for the transmission of the cerebral nerves have so far not been mentioned: the branches of the olfactory nerve, as we have seen, pass out through the numerous apertures in the cribriform plate (Fig. I33, eth, I), and the optic foramen (opt. fo, II) is situated between the orbitosphenoid and presphenoid. Behind and below the optic foramen is a vertical aperture-the sphenoidal fissure $(s p h . f)$-between the basisphenoid and alisphenoid, which transmits the third, fourth, and sixth nerves, as well as the ophthalmic and maxillary divisions of the fifth. Between the periotic and alisphenoid is a large space $(\mathrm{V} m n)$, through the anterior part of which the mandibular division of the 
trigeminal leaves the skull. ${ }^{1}$ Between the mastoid portion of the periotic and the posterior border of the tympanic, at the junction of the tubular and bulbous portions of the latter bone, is a small aperture-the stylomastoid foramen, which transmits the seventh nerve: this and the eighth (VII, VIII) enter the periotic just below the depression for the flocculus of the cerebellum $(f)$. A space (IX, X, XI) between the occipital condyle and tympanic bulla gives exit to the ninth and tenth, as well as to the eleventh-which is not represented as a distinct nerve in the dogfish and frog ; and the hypoglossal (p. I6o), which in Mammals is counted as the twelfth cerebral nerve, passes out through two small apertures (XII) in the exoccipital, just anterior to the condyle. Various other apertures will be noticed in the skull and jaws: through some of these branches of certain of the above-mentioned nerves pass, while others transmit bloodvessels.

The lower jaw or mandible (Fig. I32, A) consists of two halves or rami, each corresponding essentially to the dentary of the frog, which unite with one another in front, at the symphysis, by a rough surface, while behind they diverge like the limbs of the letter $\mathrm{V}$. Each ramus is a vertical plate of bone, broad behind and tapering towards the front, where it bears the incisor teeth : further back, on its upper margin, are the sockets for the cheek-teeth, and behind them is an ascending portion which bears the condyle (cond) for articulation with the facet on the squamosal: in front of the condyle is a curved coronoid process (cor). The postero-inferior border, which is rounded and inflected, is known as the angular process (ang. pro).

The hyoid is a small bone situated at the root of the tongue, anterior to the larynx (Fig. I35, hy). It consists of a stout body or basi-hyal, a pair of small anterior horns, representing the ventral ends of the hyoid arch

1 In many Mammals (e.g., dog, cat) the maxillary division of the trigeminal passes out through a separate foramen, behind the sphenoidal fissure; and the anterior part of the space referred to above is separated off as a distinct foramen for the mandibular division. 
of lower Vertebrates, and a pair of longer, backwardlyprojecting posterior horns or thyro-hyals, attached to the larynx and representing the lower ends of the first branchial arch (compare p. 44).

The vertebral column includes about forty-five bony vertebræ, each consisting of a centrum, a neural arch, and various processes (compare pp. 36-38), but becoming simplified towards the end of the tail. The centra have flat anterior and posterior surfaces, and are not connected by synovial articulations, as in the frog, but interposed between them are elastic iniervertebral discs of fibrocartilage. In addition to the ossification which gives rise to the main part of the centrum, a separate flat disc of bone (Fig. I34,ep) is formed on the anterior and posterior surface of each. These epiphyses are characteristic of the vertebræ of all or nearly all Mammals: they unite comparatively late with the centrum proper, and so in disarticulated skeletons of young animals they often come away from the main mass of the centrum and remain attached to the intervertebral discs.

In correspondence with the differentiation of the parts of the body, the vertebral column is divisible into five regions (Fig. I30): the cervical in the neck, including seven vertebræ, the first two of which-called respectively the atlas and axis-are peculiarly modified in order to allow the skull free movement; the thoracic in the thorax, twelve or thirteen in number, and bearing ribs; six or seven lumbar in the abdominal region; three or four sacral in the sacral region; and about fifteen or sixteen caudal in the tail.

Examining one of the anterior thoracic vertebra first (Fig. I 34 ), we see that the centrum $(c)$ is continuous ahove with the neural arch $(n, a)$, the lower part of which, on either side, presents an anterior and a posterior notch (i. $v \cdot n)$, so that when the vertebræ are in their natural position, an 
intervertebral foramen is formed for the passage of a spinal nerve. The roof of the arch is continued into a long neural spine (n. sp) projecting upwards and backwards, and just above the intervertebral notches are a pair of anterior and posterior articular processes or zygapophyses (pr.z, pt. z), which articulate synovially with the vertebra next in front and behind respectively. The articular surface of each pre-zygapophysis looks upwards and outwards, that of the

post - zygapo physis downwards and inwards.

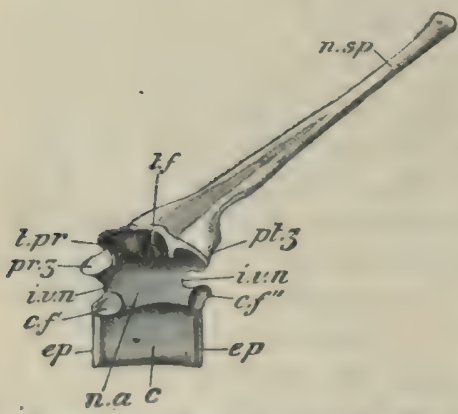

Fig. 134--Fifth thoracic vertebra of the Rahbit, from the left side. ( $1 \frac{1}{2}$.)

c. centrum; $c$. $f^{\prime}$ capitular half-facet for fifth and $c . f^{\prime \prime}$ for sixth rib; ep. epiphysis: i. $v$. n. intervertebral notch; $n$. $a$. neural arch ; n. sp. neural spine ; pr. z. pre-zygapophysis ; pt. $z$. post-zygapophysis ; $t . f$. tubercular facet for fifth rib ; $t$. pr. transverse process. Arising laterally from either side of the arch is an outstanding transverse process $(t . p r)$, on the under surface of which is an articular tubercular facet (t. $f)$, with which the upper fork of the rib (p. 50o) articulates. The lower fork or head of the rib articulates with a facet (c. $f$ ) formed partly by the anterior edge of the corresponding centrum just at the base of the neural arch, and partly by the posterior edge of the centrum next in front, so that each centrum bears half a capitular facet, as it is called,

on either side, both anteriorly and posteriorly $\left(c . f^{\prime}, c . f^{\prime \prime}\right)$. There are no free ribs in relation with the vertebric of other regions, in which, however, they are represented in the embryo, but early fuse with the corresponding transverse processes.

The first cervical vertebra, or atlas, is ring-shaped, and its lower portion is narrow and unlike the other centra. The neural spine is small, and the transverse processes are broad horizontal plates, each perforated at its base by a verlebrarterial canal through which the vertebral artery runs. On the anterior face of the lateral parts of the atlas are two concave articular facets for articulation with the occipital condyles of the skull, and on its posterior face are two small facets for articulation with the second vertebra. The second cervical vertebra, or axis, has its centrum produced 
anteriorly into a oncical edivenchid pruess, which fits into the lomer gurt of the rug ot the atias and is hesi in uts flacte by a liza- ent exteris z cranswersely wcross the larter: it is ossits: from a distinct esentme which resliy brelonss to the centrum of the atlas. The cemal sptue of the axis is eiongated and oompretssed. and its tra-sverse grouessets small and periorated each by a vertebrartertal ciamal : cyeapoFhyses art presen: ouly on the pustenur tace of the arch. In all the fither cerr.coal vertebre, the tracsverse privessses $a r=$ alsin perforated by vertubcarteral ca-als, $\mathrm{s}=\mathrm{d}$, exbept in the sevorth or last. are vivited into dorsal and vatral

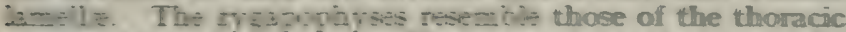
vertebrs issuribil abive. The seventh cervwcal wertubra

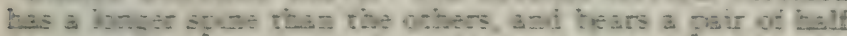

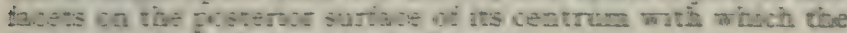

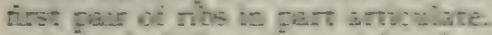

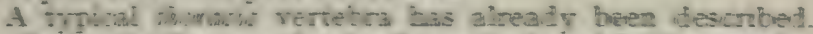
In the teati, the ceural squne ss wertual, $\mathbf{a}=\mathrm{d}$ in the reman.

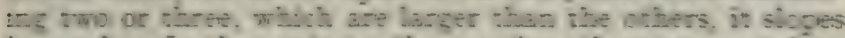
¿orwands. In the pustertur three wr thor thene are wo twher-

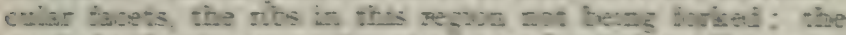

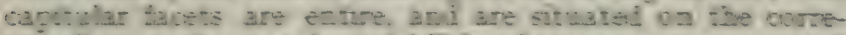
sgioning certrum ouls. Adintional prowesses ane pressedt above the per-tycapoghyses inum phe manth enoracic verteber onwaris.

The homilum vestebr:e are relatively large, imcreasiag in sine trom boture foe ikwaris, ant theht varows prowesses are

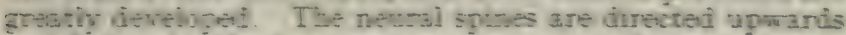
aud ijruanis, the trazsuerse grobesses ane large and project octwants, downmanis and fucwaris. As in the poveterour thoracio vertebre, tatre are stowt processes above the

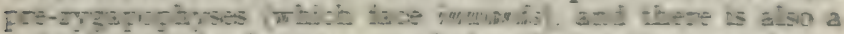

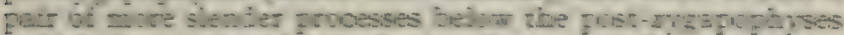

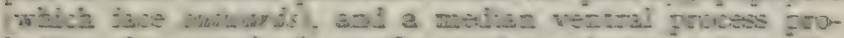

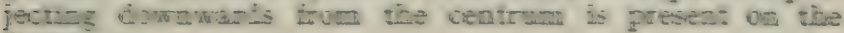
Etrst two.

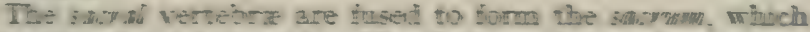
supports the pelvac anch. The frit-and to a less exteat the stovod alsj-las large, expundei, transverse provesises whic arvaculate woth tho dia : these are the sacrai vertebre

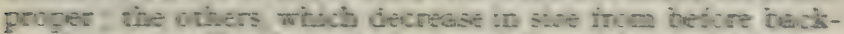

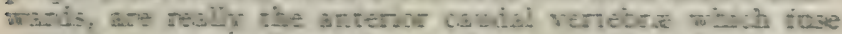

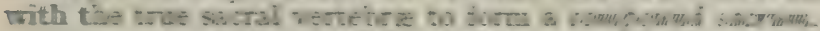

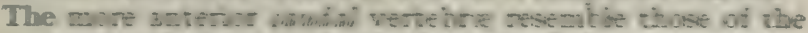

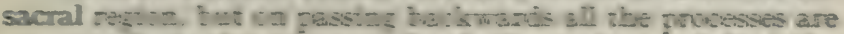

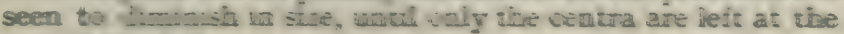
end of the tail. 
There are twelve or occasionally thirteen pairs of ribs, which have the form of curved rods, situated in the walls of the thorax, and articulating with the thoracic vertebræ above and-in the case of the first seven-with the breast-bone or sternum below: the remaining ribs do not reach the sternum (Fig. I30).

Each rib consists of a bony, dorsal vertebral portion, and of a ventral, sternal portion consisting of cartilage which is calcified or only incompletely ossified. The dorsal end-the head or capitulum of the rib-articulates with the capitular facet on the centra, and the first nine have also a iubercle, a short distance from the capitulum, which articulates with the tubercular facet; just externally to the tubercle is a short, vertical process (compare pp. 498 and 499).

The sternum, which is developed in the embryo by the fusion of the ventral ends of the ribs (and therefore has a different morphological significance from the sternum of Amphibians, see p. 48), consists of six segments or sternebre, the first of which, or manubrium, is larger than the rest, and has a ventral keel. With the last is connected a rounded, horizontal, cartilaginous plate, the xiphisternum. The ribs articulate between the successive sternebræ except in the case of the first pair, the articulations of which are on the manubrium.

The chief bone of the pectoral arch is the flat, triangular scapula, the coracoid portion (compare p. 47) becoming early fused with it and forming a small, inwardly curved, coracoid process, situated anteriorly to the glenoid cavity at the lower end or apex of the scapula : the apex lies over against the first rib, and the bone inclines upwards and backwards to its dorsal base, which in the fresh condition consists of a strip of cartilage, the supra-scapula. On its outer surface is a prominent ridge or spine, the free ventral edge of which is called the acromion, from which a process, the metacromion, projects backwards. The collar-bone or 
clavicle is never strongly developed in those Mammals in which the fore-limb only moves in one plane-forwards and backwards : in the rabbit it is a small, curved, rodlike bone, attached by fibrous tissue at one end to the sternum and at the other to the coracoid process of the scapula, there being small cartilages at either end of it.

The relative positions of the bones of the fore-limb are at first sight somewhat difficult to understand owing to their having become altered in the course of development. In your own fore-arm the bones can be rotated on one another, so that the thumb can be made to point outwards or inwards: while in the rabbit the first digit has permanently the same position, pointing inwards. To understand this, extend your arm outwards with the thumb pointing away from the ground. The back of the hand and arm, continuous with the dorsal surface of the body, or back, is its dorsal surface ; the palm of the hand, and the surface of the arm continuous with the chest, is its ventral surface; the border of the arm and hand continuous with the thumb is the preaxial border; and that continuous with the little finger the postaxial border. This position is called the position of supination; if the fore-arm and hand be now rotated, so that the thumb points inwards, the position is that of pronation. While in this position, bend the elbow at right angles and bring it inwards close to the body ; the preaxial border of the hand will now be on the inner side, and an examination of the bones of the fore-arm shows that they cross one another. It is in this position that the bones of the rabbit's fore-limb are permanently fixed (Fig. . 30 , and compare Fig. 8).

The proximal extremity of the humerus bears a rounded head for articulation with the glenoid cavity, in front of which is a groove for the tendon of the biceps muscle (p. 6r) ; certain tuberosities for the attachment of muscles will also be observed. Its distal extremity presents a large, pulley-like surface or trochlea for the articulation of the bones of the fore-arm, and a deep depression or fossa, perforated by a foramen, on its posterior side, for the reception of the end of the ulna. The radius is the shorter, inner (preaxial) bone of the 
fore-arm, and is slightly curved. Its head presents a large double surface for articulation with the trochlea of the humerus, and its distal extremity a pair of slight concavities for the bones of the carpus : the shaft is flattened where it abuts against the corresponding flattened surface of the unna. Near the proximal end of the lastmentioned bone is a cavity for the articulation of the humerus, and proximally to this, at the elbow, the ulna is produced to form a large olecranon process, which is received into the fossa on the humerus when the limb is extended : its small distal end articulates with the carpus.

The carpus, as in the frog (p. 50), consists of a proximal and a distal row of small, nodular bones, which articulate with one another where they are in contact. The bones of the proximal row, beginning at the inner (preaxial) side, are the radiale and intermedium, articulating with the radius, and the unare, articulating with the ulna. In the distal row are five bones, the middle one of which is distinctly proximal to the other four, so as really to lie in the middle of the carpus: this is the centrale, the others constituting a row of distal carpals. Of these the first three articulate with the corresponding digits, the fourth, on the outer (postaxial) side, supporting the fourth and fifth digits and really consisting of two carpals fused with one another.

A small bone, the pisiform, articulating with the ulna and ulnare on the ventral side, is usually looked upon as a sesamoid bone, i.e., an ossification in the tendon of a muscle ; but it probably represents the vestige of a sixth digit.

The hand or manus consists of five digits, each made up of a metacarpal and of phalanges, articulating with one another. The innermost (preaxial) digit-the thumb or pollex-is the shortest, and the third the longest : the former has two phalanges, the others three each, the distal or ungual phalanx of all the digits 
having a conical form, its dorsal surface being grooved for the firmer attachment of the horny claw.

The ends of the long bones in both limbs are separately ossified as epiphyses (compare p. 497), which eventually unite with the shaft of the bone in question. Small sesamoid bones are situated on the under or palmar side of the joints of the digits.

The pelvic arch consists of two lateral halves or innominate bones, the long axis of which is almost parallel with that of the vertebral column (Fig. I30), and which are firmly united anteriorly and internally with the transverse processes of the sacral vertebræ by a rough surface, while ventrally they are connected together by cartilage at the pelvic symphysis. On the outer surface of each innominate bone, at about the middle of its length, is a deeply concave cup, the acetabulum, for articulation with the head of the femur: in it, in young rabbits, a triradiate suture can be seen, marking the boundaries of the three bones of which the innominate is composed (p. 5I). Of these, the antero-dorsal is the ilium, which is connected with the sacrum. The postero-ventral portion of the innominate is perforated by a large aperture - the obturator foramen, through which a nerve of that name passes (p. 532), the bone above and behind it being the ischium, and that below and in front of it the pubis. Behind the obturator foramen the ischium has a thickened posterior edge or tuberosity, and then curves round and becomes continuous with the pubis, both bones taking part in the symphysis.

In young rabbits it will be noticed that the part of the pubis which enters the acetabulum consists of a small, distinct epiphysis.

The hind-limb has undergone rotation forwards (Fig. I30), so as to be brought, like the fore-limb, into a plane parallel with the median vertical plane of the body ; but the rotation being forwards, and the bones of the shank not being crossed, 
the preaxial border is internal in the whole limb, and the original dorsal surface looks, on the whole, forwards.

Close to the proximal end of the femur, on its inner (preaxial) border, is a rounded projecting head for articulation with the acetabulum : the actual end of the bone is formed by a strong process, the great trochanter, while just distal to the head is a lesser trochanter, and opposite this, on the outer (postaxial) side, a third trochanter. The distal end of the bone bears two large condyles, separated from one another by a notch, for articulation with the tibia: this notch is continuous with a groove extending for a short distance along the anterior (dorsal) surface of the femur in which a large sesamoid bone (p. 502), the knee-cap or patella, slides: the patella lies in the tendon of the extensor muscles of the leg, and is connected by ligament with the tibia. Two other sesamoid bones, the fabella, occur on the opposite side of the knee-joint.

The tibia, or inner (preaxial) bone of the shank, is much larger than the fibula, the distal half of which in the adult becomes completely fused with it. The proximal end of the tibia bears two slightly concave articular surfaces for the condyles of the femur, and distally it articulates with the tarsus: a prominent ridge-the cnemial crest-extends along the proximal end of its anterior (dorsal) surface. The slender fibula is attached proximally to the tibia.

The tarsus consists of six bones arranged in three rows. In the proximal row (compare p. 5I) are two tarsals, of which the inner (preaxial) or astragalus-probably corresponding to two bones fused together, the tibiale and intermedium - has a large pulley-like surface for articulation with the tibia ; while the outer (postaxial) calcaneum or fibulare articulates with the fused end of the fibula, and is produced into a strong heel or calcaneal process. In the 
middle row is a single bone, the centrale (navicular) of the tarsus, and the distal row is made up of three bones, the true first, together with the corresponding digit (hallux), being absent as a distinct bone. The second (apparent first) distal tarsal articulates proximally with the centrale, and distally with the innermost (preaxial) metatarsal: the third (apparent second) with the centrale and the corresponding metatarsal: the fourth (apparent third), which corresponds to the two fused outer (postaxial) tarsals, with the centrale, calcaneum, and the remaining two digits.

The foot or pes consists of four metatarsals with their phalanges, of which there are three to each digit. The metatarsal of the hallux, together with the corresponding distal tarsal, is probably represented by a distinct ossification which in the adult becomes fused with the second (apparent first) metatarsal, and forms a process on that bone which articulates with the centrale. The phalanges are similar to those of the manus, and sesamoid bones are also present on the under surface of the foot.

\section{Museles and Body-wall.-It will be remembered} that in the lancelet and dogfish the muscles of the trunk are divided up into myomeres (pp. $4 \mathrm{Ig}$ and 434 ), while in the adult frog the only indication of such a segmentation of the muscles is seen in the recti of the abdomen. In the rabbit nearly all trace of a segmentation of the muscles has also disappeared, and the muscular system, although similar in its general arrangement to that of the frog (compare Fig. I6), is more highly differentiated and complicated. We shall have occasion to notice certain of the muscles in the course of our examination of other organs.

Immediately beneath the skin, which consists of epiderm and derm (Fig. I3I), the whole ventral region of the trunk and neck is covered by a thin cutaneous muscle, 
by means of which the rabbit is able to twitch its skin. Internally to this muscle in the female are the mammary glands (p. 483), which, when secreting, appear as whitish branched masses, the ducts of which can be traced to the teats, on the apices of which they open by numerous small apertures.

A whitish band of connective-tissue passes along the midventral line of the abdomen from the xiphisternum to the pubis : this separates two longitudinal bands of muscle, the recti abdominis, from one another; and laterally to them, the abdominal wall consists of three thin layers of muscle with their fibres running in different directions - the external oblique, the internal oblique, and the transiersalis, the latter being lined on its inner surface by the peritoneum. A fibrous cord, known as Poupart's ligament, beneath which the blood-vessels and nerves pass outwards to the leg, extends upwards and forwards from each pubis to the corresponding ilium. In the thorax the muscles of the body-wall are broken up into separate portions by the ribs, and thus form a series of intercostal muscles, which, like the oblique muscles of the abdomen, are arranged in two layers, external and internal, and are important in respiration.

Extending from the thorax to the fore-limb of either side are the large pectoral muscles; and a number of other muscles can be seen in the neck, in the ventral middle line of which, covered by the cutaneous muscle, the windpipe or trachea is visible (Fig. 135). The trachea is strengthened by a series of cartilaginous rings and ends in front in the larynx, situated between the two rami of the mandible; and just in front of the larynx is the hyoid bone (p. 496), embedded in a mass of muscle.

The Colome and its Contents.-On cutting open the body-cavity, it will be seen to be divided into two main chambers - the thoracic and abdominal cavities - by means of the diaphragm (Fig. I35, d). The relatively small thorax-which is lined by a serous membrane corresponding to the peritoneum of the abdomen and known as the pleura-contains the lungs, as well as the heart 
enclosed in a pericardium, on the ventral surface of which latter is an organ known as the thymus (see p. 447): the gullet and main blood-vessels also pass through the thorax. The abdomen encloses the greater part of the enteric canal, together with the liver and pancreas, the spleen, and the urinogenital organs. The diaphragm is convex on its anterior side, towards the thorax: it consists of a central, thin, tendinous portion into which radial muscles are inserted. These arise from the vertebral column and posterior ribs, and are especially strong on the dorsal side, where they form two bands known as the pillars of the diaphragm. When the muscles contract, the diaphragm is made flatter, and thus the thoracic cavity is enlarged.

Digestive Organs. - The mouth-cavity (Fig. I35) is large, and the small gape is bounded by upper and lower lips, behind which are the incisor teeth $(i)$. On either side of the cavity are the borders of the upper and lower jaws from which the cheek-teeth project: these are separated from the incisors by a considerable interval or diastema. Close behind the upper incisors are a pair of very small openings leading into the nasopalatine canals $(n, p . c)$, which communicate with the nasal cavities but must not be confounded with the internal nostrils. The roof of the oral cavity is formed by the palate, the anterior part of which, or hard palate $(h . p)$, is transversely ridged and partly supported by bone (h. $\left.p^{1}, p .49 t\right)$; while the posterior part, or soft palate (s. $p$ ), is smooth, its hinder, free edge forming a pendulous flap, the velum palati, on either side of which is an organ known as the tonsil, consisting of connective and lymphoid tissue and resembling the "lymphatic glands" (compare p. 5I5) which occur along the lymphtrunks; it has the form of a small pit with a broad 


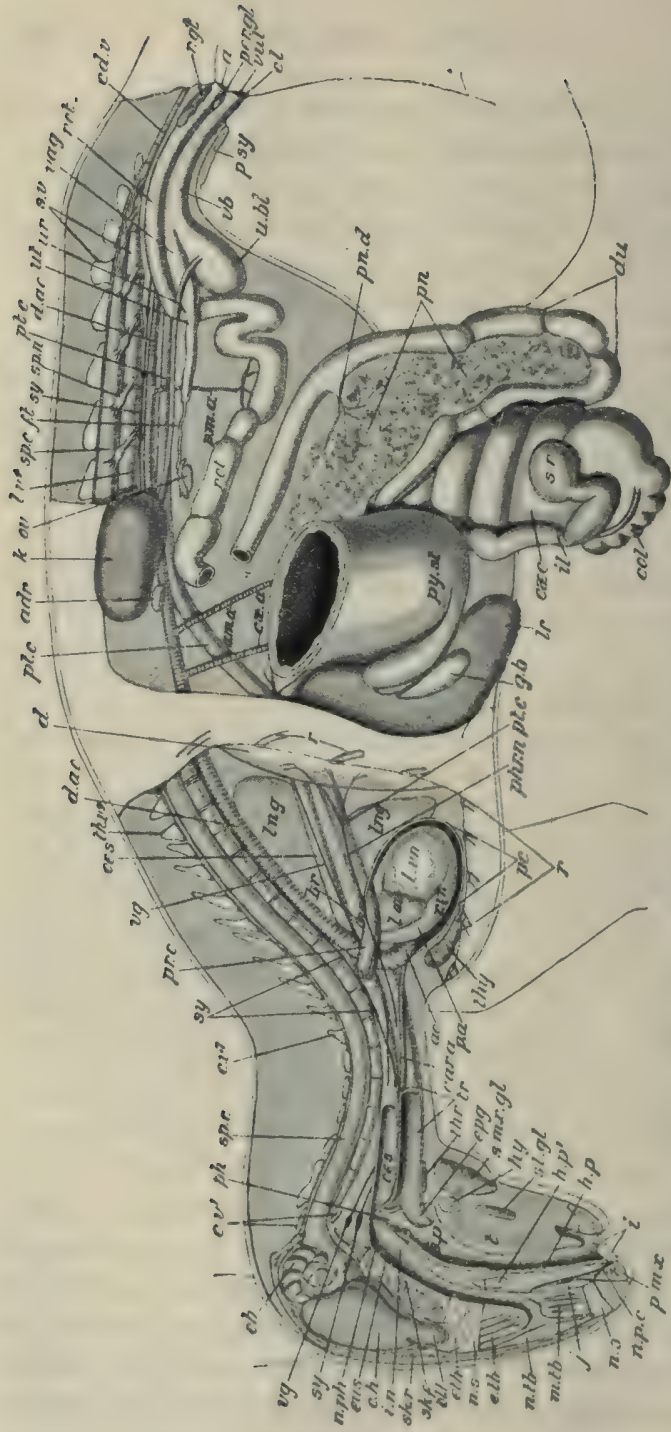

뜡류- 월

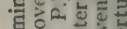

近的乐

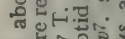

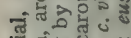

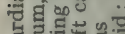

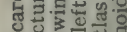

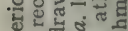

का च

डल लै है ज

ठु हैं... खै

㐘

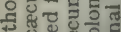

- 4 द्य

-

$\times 88 \leq 0$

\& $\cdots$

๑

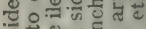
ज政政

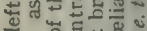
용 ई0

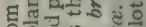

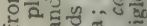


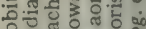
ณ ๑ ㅎํ웡 을 है ज्ञात

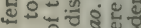
๓กิข

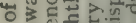
त क्षेट है

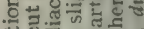

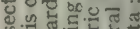

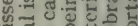

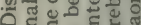
了 ำ

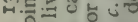
0 की 约 ฮั ธั

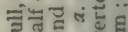
근

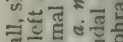

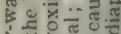

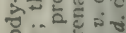

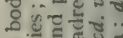
을 है 톨롱

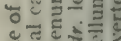
ช้ के टे‥ ल 플

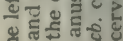
हैं 
of Eustachian tube; $f . t$. uterine tube; $g . b$. gall-bladder; $h . p$. hard palate; $h$. $p^{l}$. bones of hard palate; $h y$. hyoid; i. incionrs ; il. ileum; $i$. $n$. passage of internal nostrils; $j$. Jacobson's organ; $k$. left kidney; $l$. au. left auricle; Ing. left lung, seen through pleura; $l r$. liver; $l$. $v^{4}$. fourth lumbar vertebra; $l$. $v$. left ventricle; $m . t b$. maxillary turbinal; $n . p . c$. naso-palatine canal; $n$. ph. naso-pharynx ; $m$. tb. nasn-turbinal ; n. s. nasal septum (middle part cut away); ces. gullet; ol. $l$. olfactory lobe; ov. left ovary; p. a. pulmonary artery; $p$. pericardium; per. gl. perineal gland; ph. pharynx; phr. $s$. phrenic nerve; (origin not shown); $p . m . a$. posterior mesenteric artery; $p . m x$. premaxilla; $p$. pancreas; $p n$. d. pancreatic duct; $p r . c$. left precaval; $p$. sy. pelvic symphysis; pt. c. postcaval ; py. st. pyloric region of stomach; $r$. ribs ; rct. rectum; $r$. $g l$. rectal gland : $r . t ' n$. right ventricle; $s k$. $f$. floor, and $s k$. $r$. roof of skull; sl. gl. sublingual gland; s. $m x$. gl. submaxillary gland; s. p. soft palate, ending in the velum palati, on the lower side of which a tonsil is seen : $s p . c$. spinal cord: $s p$. n. spinal (lumbar) nerves; $s . r$. sacculus rotundus of ileum; $s$. $v$. first sacral vertebra; sy. sympathetic (the anterior end shown on the right side, the rest on the left); $t$. tongue; thr. thyroid; tho $v^{3}$. ninth thoracic vertebra; thy. thymus; tr. trachea; $u . b l$. urinary bladder; ur. ureter; ut. uterus; tag. vagina; $v b$. vestibule; t'g. vagus (the anterior end shown on the right side, the rest on the left) ; vul. vulva.

papilla on its outer margin. The tongue $(t)$ lies on the floor of the mouth to which it is attached below, its anterior, rounded end being free: the surface of its posterior part is elevated, and elsewhere-but more particularly on the tip-its covering of mucous membrane is produced into minute, finger-shaped pupilla, on which some of the microscopic organs of taste are situated (compare p. I80). Taste-organs are also present on a pair of circumvallate papilla on the dorsal side of the tongue towards its posterior end, and on a pair of transversely ridged areas-the foliate papille, situated laterally, slightly anterior to the former. The main substance of the tongue is composed of muscles, some extrinsic, or arising from other parts, and others intrinsic, or entirely confined to the organ in question.

The teeth (Figs. I32 and I33), as we have seen, are not all alike, as in the dogfish and frog; there are incisors and cheek-teeth or grinders, the latter being divisible into two series-the premolars and the molars. In most Mammals there is also a pair of canine teeth situated between the incisors and premolars, and these are especially long and pointed in such carnivorous animals as the dog and cat. In the dogfish and frog, again, 
the teeth are continually renewed as they become worn out, but in Mammals there are only two functional sets, which are known respectively as the deciduous or " milk" teeth, and the successional or permanent teeth: certain of the former may even be absorbed before birth, as is

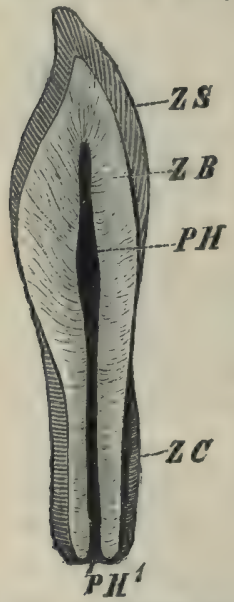

FrG. 136.-Longitudinal section of a mammalian tooth, semidiagrammatic.

$P H$. pulp-cavity; $P H^{\prime}$. opening of same; $Z B$. dentine; $Z C$. cement; ZS. enamel. (From Wiedersheim's Comp. Anutomy.) the case with the incisors of the rabbit. The incisors and premolars (and in Mammals in which they are present the canines also) have deciduous predecessors, the molars developing behind the premolars and having no predecessors.

All the teeth are embedded in deep sockets or alveoli of the jawbones, and each contains a pulpcavity (Fig. 136, PH) extending into it from the base and containing blood-vessels and nerves. In the case of the rabbit, the aperture of the pulp-cavity $\left(P H^{1}\right)$ remains wide open in each tooth, and the substance of the tooth is continually added to at its base as it wears away at the other end: in many Mammals, however (e.g., dog, cat, man), the aperture becomes narrowed and growth ceases after a time, the base of the tooth forming one or more roots or fangs. The main substance of each tooth is formed of dentine $(Z B)$, into which the pulp-cavity extends for a considerable distance and round which the enamel (ZS) forms an external layer, which may become more or less folded inwards (as in the cheekteeth and front upper incisors of the rabbit), the cement ( $Z C$ ) extending into the folds (compare pp. 445 and $5 \mathrm{II}$ ). 
The number of the various teeth in the jaws is conveniently expressed by a dental formula, in which the kind of tooth (incisor, canine, premolar, or molar) is indicated by the initial letter, $i, c, p m$, or $m$; and the whole formula has the arrangement of four vulgar fractions, in each of which the numerator indicates the number of teeth in the upper, the denominator that of those in the lower jaw, only those of one side being indicated, since the teeth of the right and left sides are always the same. Thus the dental formula of the rabbit is $i \frac{2}{1}, c \frac{0}{0}, p m_{\frac{s}{2}}^{s}, m_{s}^{s}=28$.

The anterior incisors in the upper jaw of the rabbit are long and greatly curved. They are surrounded by enamel, which is much thicker on the anterior surface, where it presents a median groove ; the posterior upper incisors are much smaller and are situated behind the anterior ones. In the lower jaw the single pair of long and curved incisors have no median groove, and they bite between the anterior and posterior upper incisors: owing to the thick layer of enamel anteriorly, they, like the large upper incisors, wear away less quickly in this region, and thus remain sharp, like a chisel, at their biting edges. The premolars and molars, on the other hand, are modified for grinding the food, to do which satisfactorily it is necessary that they should have broad crowns with a surface which wears unevenly. This is effected in most of the cheek-teeth by the enamel becoming involuted along the outer side in a longitudinal direction, so as to form a groove extending into the dentine almost to the other side, the groove becoming filled up with cement. As the enamel is harder than the dentine and cement, it thus gives rise to ridges as the crown wears. The first upper premolar and the last upper and lower molars are simpler than the others, and the first lower premolar has two grooves.

Connected with the mouth-cavity are several pairs of salivary glands, not present in the other Vertebrates we have examined, the secretion of which-saliva-contains a ferment called ptyalin, which is capable of converting starch into sugar (compare p. $7 f$ ). The food taken into the mouth is ground up or masticated and mixed with 
the saliva before passing down the gullet, and thus digestion begins in the mouth.

The infra-orbital salivary gland is a large, lobulated pinkish mass situated in the antero-ventral region of the orbit, below and in front of the eyeball : its duct passes downwards to open into the mouth nearly opposite and externally to the second premolar. The parotid gland is a soft, irregular, flattened organ, lying close beneath the skin, just below and in front of the base of the external ear; its duct passes forward and opens close to the duct of the infra-orbital gland. The sub-maxillary gland (Fig. $\mathrm{I} 35, s . m x . g l$ ) is a reddish, ovoid, compact body situated inside the angle of the lower jaw and near the middle line, somewhat anterior to the larynx : its duct runs forward to open into the mouth a short distance behind the lower incisors. The sublingual gland $(s l . g l)$ is an elongated structure situated on the inner side of the mandible, and having several ducts opening independently into the mouth.

The oral cavity is continued backwards as the pharynx $(p h)$ : this begins at the velum palati, above which it extends forwards as the naso-pharynx (n.ph); the latter is continuous with the passage of the internal nostrils, and into it open the Eustachian tubes (eus, compare pp. I7 and 45). On the floor of the pharynx, behind the base of the tongue, is the glottis, which leads into the larynx and is guarded in front by an elastic, leaf-like, cartilaginous flap, the epiglottis $(e p g)$ : this projects upwards towards the velum palati and is capable of being pressed backwards over the glottis during the passage of food from the mouth to the gullet.

The gullet (Figs. I35 and 137 , oes) is a narrow but dilatable tube which passes backwards along the neck and through the thorax, entering the abdomen through an aperture in the diaphragm and then opening into the stomach ( $p y . s t, c d . s t)$, a wide, curved sac, elongated transversely and greatly dilated at the cardiac end, which lies towards the left side of the body: the pyloric end, from which the duodenum arises, towards the animal's 


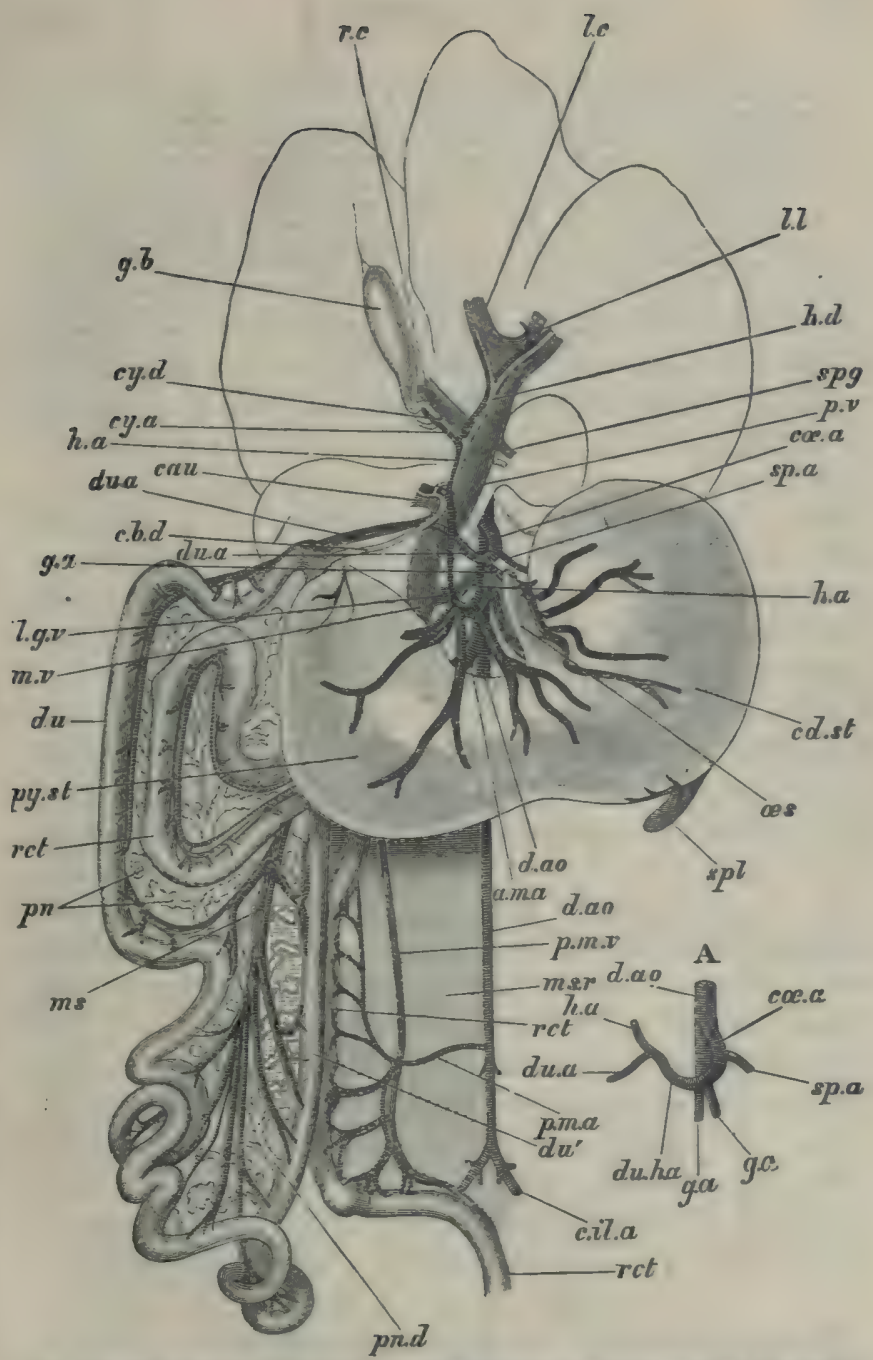

Fig. 137. - The stomach, duodenum, posterior portion of rectum, and liver (in outline) of the Rabbit, with their arteries, veins, and ducts. ( ${ }^{3}$.) $A$, the coeliac artery of another specimen. The gullet is cut through and the stomach some- 
what displaced backwards to show the ramifications of the coliac artery $(c \propto, a)$; the duodenum is spread out to the right of the subject to show the pancreas $(p n)$; the branches of the bile-duct $(c, b, d)$, portal vein $(p, v)$ and hepatic artery $(h, a)$ are supposed to be traced some dlistance into the various lobes of the liver.

a. $m . a$. anterior mesenteric artery; cau. caudate lobe of liver with its artery, vein and bile-duct; $c, b . d$. common bile-duct; $c d$. st. cardiac portion of stomach ; c. il. a. common iliac artery; $c a, a$. coeliac artery; $c y$. a. cystic artery; $c y, d$. cystic duct; $d$. ao. dorsal aorta ; $d u$. proximal, and $d u^{\prime}$. distal limbs of duodenum; $d u$. a. duodenal artery; du. $h . a$. (in $A$ ), duodeno-hepatic artery; $g . a$. gastric artery and vein; $g . b$. gall-bladder; $h . a$. hepatic artery; $h . d$. left hepatic duct; $l . c$. left central lobe of liver, with its artery, vein, and bile-duct; $l . g . v$. lieno-gastric vein; $l$. $l$. left lateral lobe of liver, with its artery, vein, and bileduct; $m s$. branch of mesenteric artery and vein to duodenum ; ms. $r$. mesentery of the rectum; $m . v$. chief mesenteric vein; aes. gullet; $p . m$. $a$. posterior mesenteric artery; $p . m$. v. posterior mesenteric vein; pn. pancreas; $p n . d$. pancreatic duct; $p$. $v$. portal vein; $p y$. st. pyloric portion of stomach; rct. rectum; $r$. c. right central lobe of liver, with artery, vein, and bile-duct; $s p g$. Spigelian lobe of liver with its artery, vein, and bile-duct; $s p l$. spleen; $s p . a$. splenic artery. (From Parker's Zootomy.)

right, is less dilated and has much thicker muscular walls. The mucous membrane of the stomach, in which the microscopic gastric glands (p. I3I) are contained, is raised into ridges or ruge, and there is a circular pyloric valve at the entrance to the intestine.

The duodenum $(d u)$ extends backwards along almost the whole length of the abdomen and then turns forwards again, forming a slightly coiled U-shaped loop, and becoming continuous with the very long and coiled second portion of the small intestine or ileum (Fig. I35, $i l)$, which finally dilates to form a rounded sac $(s . r)$ opening into the proximal end of the dark-coloured colon $(\mathrm{col})$, or first portion of the large intestine. The colon has a much greater diameter than the small intestine, and presents a series of sacculations arranged in three rows, separated by flat regions of its wall: it passes insensibly into the second portion of the large intestine or rectum $(r c t)$, which is of about the same diameter as the small intestine, and is recognisable by its rounded swellings containing the pill-like fæces: it passes into the pelvic cavity to open by the anus $(a)$. At the junction of the ileum and colon is a relatively enormous blind-gut or cacum (cac)-a structure not met with in either the dogfish or the frog, and only reaching 
such a relatively large size as in the rabbit in certain other herbivorous Mammals in which the stomach has a simple form: in those which possess a complicated stomach (viz., Ruminants) the cæcum is comparatively small. It is continuous with the proximal end of the colon, which contains an intra-colic valve and into which the round sac at the distal end of the ileum opens by a circular aperture provided with an ileo-colic valve. From this point arises the thin-walled cæcum, which lies coiled on itself amongst the folds of the rest of the intestine : it is about an inch in diameter, and a spiral constriction is seen on the outside marking the attachment, on the inside, of a spiral valve-like that of the dogfish's intestine but narrower-which makes about twenty-four turns and ends at the base of a blind, finger-shaped process, the vermiform appendix, which forms the apex of the cæcum. The whole canal is supported by a mesentery (p. 27), the right and left halves of which are united, and which has a very complicated arrangement in correspondence with the numerous folds of the intestine.

It will be noticed that the intestine is much more differentiated as regards its subdivisions than in the Vertebrates previously examined, and also that it is relatively much longer, being fifteen or sixteen times as long as the body (compare p. 207).

On cutting open the small intestine, its mucous membrane is seen to be raised into minute, finger-shaped elevations or villi, and here and there certain patches present a honeycombed appearance; these portions are known as Peyer's patches, and, like the tonsils, thymus, and spleen, consist of masses of lymphoid follicles composed of a connectivetissue framework in which numerous leucocytes are embedded. Other so-called "lymphatic glands" or adenoids are present in the mesentery and elsewhere. Pcyer's patches also occur in the proximal end of the colon, close to the ileo-colic aperture; and the round sac with which the colon communicates, as well as the vermiform appendix, is lined with similar lymphoid tissue. The mucous 
membrane of the colon has no villi, but, like that of the spiral valve, is raised into small papillæ; while that of the rectum is smooth.

The liver (Figs. I35, lr, and I37) is a large organ, consisting of five lobes. Its anterior surface is convex and is applied to the diaphragm, its posterior concave surface fitting against the stomach. A median vertical fold of the peritoneum attaches it to the diaphragm, and marks the boundary between the right and left central lobes (Fig. I37, r.c, l.c). Externally to the left central lobe, between it and the cardiac end of the stomach, is the left lateral lobe $(l . l)$, and externally to the right central lobe the caudate lobe (cau), applied to the pyloric end of the stomach and hollowed posteriorly, when it fits over the right kidney : a small Spigelian lobe (spg) fits closely against the concave anterior surface of the stomach. The bile-duct is made up of several hepatic ducts (h. d) from the various lobes of the liver, as well as of a cystic $d u c t(c y . d)$ from the pear-shaped gall-bladder (g. b), which is embedded in the right central lobe of the liver: the common bile-duct $(c, b, d)$ thus formed opens into the dorsal side of the duodenum by a prominent aperture a short distance beyond the pylorus.

The pancreas (Figs. I35 and I37, $p n$ ) is a diffuse gland, consisting of a number of small lobules looking not unlike masses of fat, spread all over the mesentery which connects the two limbs of the duodenum. The small ducts from the various lobules run together to form the main pancreatic duct ( $p n$. d) which opens, independently of the bile-duct, into the distal limb of the duodenal loop a couple of inches or so beyond the bend.

The spleen (Fig. 137, spl) is a long, flat body of a dark red colour, attached to the cardiac end of the stomach by a sheet of peritoneum (compare pp. 23 and 447). 


\section{Organs of Respiration and Voice.-Owing to the} presence of a neck, the lungs are situated at some distance from the glottis (Fig. I35), and, instead of a short laryngo-tracheal chamber, as in the frog (p. I4I), there is a windpipe or trachea ( $t r$ ) extending along the neck just ventrally to the gullet, its anterior end forming the larynx or organ of voice (p. 506), and communicating with the pharynx through the glottis. The cartilaginous rings of the trachea are incomplete dorsally, and the cartilages of the larynx are more highly differentiated than in the frog, apart from the presence of an epiglottis (epg, see p. 5I2). The largest and most anterior laryngeal cartilage is the thyroid, which, like the epiglottis, is peculiar to Mammals : it has the form of a broad ring, incomplete dorsally, and is the part of the larynx which can be felt externally. The second cartilage is the cricoid, represented in the frog by a ring-shaped cartilage at the base of the lungs (p. I53): its form is somewhat like that of a signet ring, being broad dorsally -where it lies mainly between the edges of the thyroidand narrow ventrally. A pair of arytenoid cartilages are articulated to the dorsal and inner surface of the cricoid, and each is produced into a projecting process situated between the two edges of the thyroid cartilage. The rocal cords (p. I44) are a pair of elastic folds extending across the cavity of the larynx from the thyroid below to the arytenoids above, each bounded in front by a depression.

In the position of rest, the vocal cords lie at an acute angle to one another, as in the frog; they can be brought into parallelism and regulated by the action of a number of intrinsic and extrinsic muscles, and are set in vibration by the respiratory current of air.

After entering the thorax, the trachea divides into two bronchi (Fig. 135, br), one entering each lung and giving off branches to its different lobes: the bronchi, like the 
trachea, are supported by incomplete cartilaginous rings at their proximal ends, but these gradually disappear after the bronchi have entered the lungs.

The elastic lungs (Figs. I35 and $138, \operatorname{lng}$ ) are not hollow sacs, like those of the frog, but are spongy bodies, of a light pink colour, situated on either side of and above the heart, and filling the greater part of the thoracic cavity, but collapsing as soon as the wall of the thorax is perforated. Each is subdivided into two main lobes, and the right lung has in addition two small accessory lobes, an anterior and a posterior, the latter lying in the median line, behind the heart and being closely applied to the gullet.

Each pulmonary artery (Fig. 135, p.a) crosses the main bronchus anteriorly to the point at which it branches into the various lobes, except in the case of the anterior accessory lobe, the bronchus to which comes off in front of the artery and may even arise from the trachea before its bifurcation. Microscopic examination shows that the bronchi divide and subdivide to form a ramifying system of tubes, each ultimate branch of which opens into a minute chamber or infundibulum, which in structure closely resembles a frog's lung in miniature.

The parietal layer of the pleura (p. 506) lines the cavity of the thorax, and is reflected over each lung at the entrance of the bronchus to form the visceral layer: in the median line it forms a vertical partition, the mediastinum, with which it is continuous on the ventral side of the vertebral column above, and beneath the pericardium below (Fig. I38). Thus each lung (l. lng, r. $\operatorname{lng}$ ) has its own separate pleural cavity (l. $p l, r . p l)$, separated from its fellow by the right and left mediastinum, the space between which is called the mediastinal space. The anterior and dorsal parts of this space are narrow, and enclose the posterior part of the trachea and the bronchi, as well as the gullet (œs) and main blood- 
vessels (aort, $a \approx . v, p t$. cav) ; its middle part is wide, and encloses the heart ( $l$. vent, $r$. vent), the mediastinum here fusing with the visceral layer of the pericardium (vis. per) and thus obliterating the space; below this it again narrows to form the ventral mediastinal space ( $v$. med), in which the thymus (p. 507) is situated.

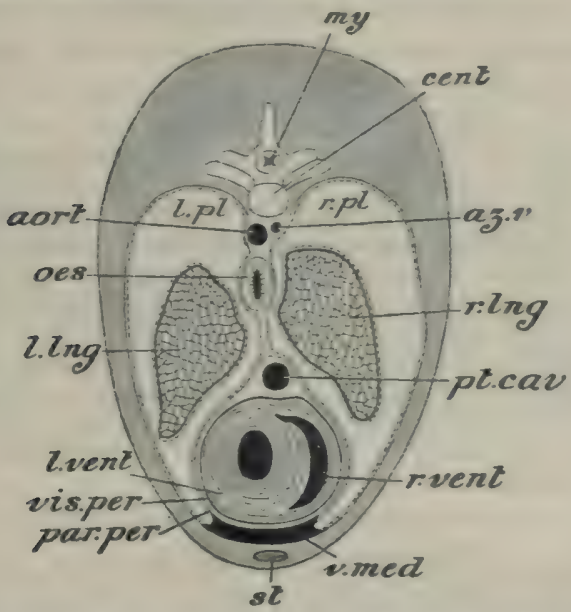

F1G. $\times 3$ 8.-Diagrammatic transverse section of the Rabbit's thorax in the region of the ventricles, to show the relations of the pleura and mediastinum (dotted line), etc. The lungs are contracted. ( $x \neq$. $)$

aont. dorsal aorta: as. $v$. azygos vein; cent. ventrum of thoracic vertebra; 1. lng. left lung $; l$ pl. left pleural navity $; l$. west. lefi ventricle; my. spinal cord : oes. gullet: par. per. parietal laver of pericardium; pt. car'. postcaval, close to its entrance into right auriclp; $r$. Ing. right lung; $r$. bl. risht pleural cavity; r. vent. right ventricle: st. sternum : tis. per. visceral layer of pericardium; v. med. ventral mediastinal space. (From Parker and Haswell's Zoology.)

In the entire animal, the air-tight pleural cavities are completely filled by the lungs, so that the parietal and visceral layers of the pleuræe are practically in contact, there being only a lubricating serous fluid (lymph) between them. The pressure of the air in the bronchial cavities of the lungs is therefore sufficient to keep them distended; but as soon as the pleural cavities are 
perforated, air passes in and equalises the pressure: the elasticity of the lungs then comes into play, causing them to collapse. When the muscles of the diaphragm contract (p. 507), air is drawn into the lungs, and this process is aided by the external intercostal muscles (p. 506) and, in forced respiration, by other muscles of the body-wall also. The mechanism of respiration may therefore be compared with a suction-pump, while that in the frog resembles a force-pump (p. 142).

On either side of the larynx is a soft, vascular, gland-like thyroid body, consisting of two lateral portions connected ventrally by a median bridge. Its function is not thoroughly understood : morphologically it represents a gland developed from the pharynx, but it loses its connection with the latter and thus has no duct. The glandular vesicles of which it is composed give rise to an albuminous substance containing iodine, which is passed into the blood and lymph; if extirpated in the living animal, various functional disturbances result. We are also ignorant of the function of the thymus (p. 507), which is largest in young animals, becoming reduced in size in adults (compare p. 447).

Organs of Circulation.-The heart, as in all Vertebrates, is enclosed in a pericardium consisting of parietal and visceral layers (Fig. I38), between which is a serous pericardial fluid. There is a complete separation between the arterial and venous blood in the heart, for in addition to an auricular septum, as in the frog (p. 88), the ventricular portion is divided into right and left chambers by a partition (Fig. 138), the arterial blood from the lungs entering the left auricle and thence passing into the left ventricle to be pumped into the aorta, and the venous blood entering the right auricle and thence into the right ventricle to pass to the lungs through the pulmonary artery. A distinct conus arteriosus and sinus venosus (pp. 79 and 449) can no longer be recognised, the former having become practi- 
cally absorbed into the ventricular portion of the heart, and the latter into the right auricle; so that the aorta (together with the carotids) and the pulmonary artery now arise directly from the left and right ventricles respectively, and the precavals and postcaval enter the right auricle directly (Figs. 135, I39, and 140).

The line of separation between the two ventricles can be seen externally as an oblique depression extending from the base of the heart backwards and to the right, but not reaching the apex, which is formed by the left ventricle only. The small, irregular cavity of the latter is enclosed by very thick muscular walls, and is partly surrounded by the right ventricle, the cavity of which is crescentic in transverse section (Fig. 138), while its walls are much thinner than those of the left ventricle as it has only to pump the blood to the lungs. The auricles have thin walls: each is produced into a little flap or appendix which envelops the base of the corresponding ventricle, and the walls of which are strengthened by a network of muscular bands.

In the auricular septum is a thin, oval area, the fossa ovalis (Fig. I39.f. ov'), which in the embryo is perforated and so allows the blood from the body to pass directly into the left auricle without going to the lungs, which are not, of course, functional until the animal is born.

The two auriculo-ventricular apertures are guarded by valves--that of the left side, or mitral valve, consisting of two membranous flaps, that of the right, or tricuspid valve (Fig. I39, tri.v), of three flaps: the valves are attached by their bases to the margins of the apertures, their apices extending into the corresponding ventricles. Attached to their edges are tendinous cords arising from conical elevations of the ventricular walls known as papillary muscles, which are much larger in the left ventricle than in the right $(m . p a p):$ these serve to 
prevent the valves from being pushed into the auricles when the ventricles contract.

The right ventricle narrows towards its base, on the ventral side of the heart, to form a conical prolongation from which arises the pulmonary artery (Figs. I35 and I $40, p . a)$, its aperture being guarded by three pocketlike, semilunar valves (Fig. I39, sem. v) : the aperture

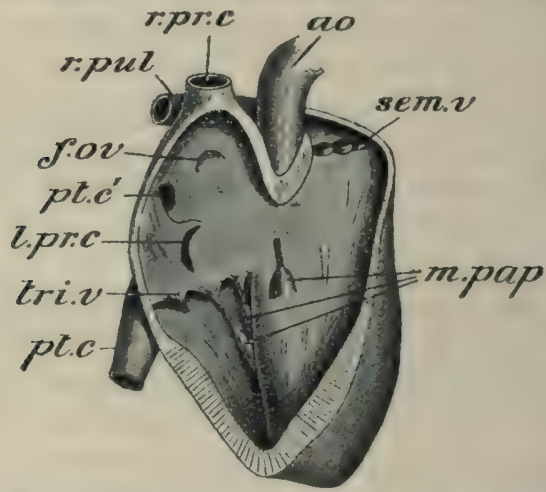

FIG. 139.-Heart of the Rabbit, seen from the right side, the walls of the right auricle and right ventricle partly removed so as to expose the cavities. ( $\times \mathrm{I} 1$. )

ao. aorta; $f$. ov. fossa ovalis; $l$. pr. $c$. opening of left precaval; $m$. pap. papillary muscles; $p t$. c. postcaval; $p t . c^{\prime}$. opening of postcaval, with Eustachian valve below; $r$. pr. c. right precaval; $r$. pul. right pulmonary artery; sem. $v$. semilunar valves at base of pulmonary artery; tri. v. tricuspid valve. (From Parker and Haswell's Zoology.)

of the aorta from the left ventricle is similarly provided with three semilunar valves. The two precavals $(l \cdot p r . c$, $r . p r . c)$ and the postcaval (pt.c) communicate, as we have seen, directly with the right auricle, the right precaval opening into it anteriorly, the left precaval posteriorly, the aperture of the postcaval being just anterior to that of the left precaval. The pulmonary veins from each lung unite and open close together into the left auricle. 
A membranous fold, the Eustachian talie, extends into the right auricle between the apertures of the postcaval and left precaval : in the embryo this helps to conduct the blood through the aperture in the auricular septum (p. 52I). It, like the "ductus arteriosus" (p. 550, and compare Fig. I 20), affords another example of a vestigial structure, representing the remains of the sinu-auricular valves (pp. 89 and 449 ).

You will remember that in the frog (p. So) there are two systematic trunks, representing the second arterial arch of the tadpole and fish (p. $45 I$ and Fig. I20), and uniting above to form the dorsal aorta. In the Mammal, one of these-the right-disappears in the course of development and all the blood from the left ventricle passes into the single left aortic arch (Figs. I35 and Ifo) from the base of which both carotid arteries arise, the aortic arch then curving over the left bronchus to pass into the dorsal aorta $(d$. ao).

Close to the origin of the aortic arch, just bevond its semilunar valves, two small coronary arteries are given off to the walls of the heart; and more anteriorly, at the curve of the arch, arise the vessels which supply the head and fore-limb. There is a certain amount of variation as to the origin of these, which is asymmetrical, and is usually as foilows. Springing from the arch of the aorta towards the right side is an innominate artery (Fig. Ifo, in), which gives off close to its origin the left common carotid (l. c. c), and then, passing forwards, divides into the right common carotid ( $\boldsymbol{r} . c . c$ ) and the right subclavian (s. cl. a), the left subclavian (br) taking its origin independently from the left side of the arch. Each common carotid passes forwards along the neck, close to the trachea, and at about the level of the larynx divides into an internal carotid $(i . c)$, which supplies the brain, and an external carotid $(e . c)$, which goes to the head and face. Each subclavian forms several branches, 
the most important of which are a brachial $(b r)$ to the fore-limb, a vertebral (vr) which passes through the vertebrarterial canal of the cervical vertebræ (p. 498) and supplies the spinal cord and brain, and an anterior epigastric or internal mammary (a. epg) running along the inner side of the ventral wall of the thorax. The aorta gives off, in the thorax, a series of small paired intercostal arteries ( $i . c s)$ to the body-walls, and then passes into the abdomen, between the pillars of the diaphragm.

A short distance behind the diaphragm the coeliac artery (Figs. $\mathrm{I} 37$ and $\mathrm{I} 4 \mathrm{O}, \mathrm{c} \propto$ ) arises, and supplies the liver, stomach, and spleen; and about half or threequarters of an inch further back is the anterior mesenteric artery $(a . m . a)$, the branches of which pass to the small intestine, pancreas, cæcum, and colon. Close behind the anterior mesenteric is the right-and rather further back the left-renal artery (Fig. I40, r), and still more posteriorly, a posterior mesenteric $(p . m)$ to the rectum, and a pair of spermatic or ovarian arteries $(\mathrm{spm})$ to the spermaries or ovaries, as the case may be. A small caudal artery $(m . s c)$, corresponding to the caudal continuation of the aorta, arises from the dorsal surface of the posterior part of the latter just in front of a pair of large common iliac arteries (c. il. a), which appear like a bifurcation of the aorta. These are continued outwards and backwards towards the hind-limbs, each giving off an ilio-lumbar artery (i. $l)$ to the dorsal body-wall and then dividing into an internal iliac (i. il. a) passing along the dorsal side of the pelvic cavity, and an external iliac (e. il. a) which gives off an artery to the bladder (s.vs), and in the female one to the part of the oviduct known as the uterus $(u t)$; and then, passing beneath Poupart's ligament (p. 506) to the hind-limb, becomes the femoral artery ( $f m$. a), from the proximal end of which a posterior cpigastric $(p . e p g)$ runs forwards 
in the ventral abdominal wall. Small lumbar arteries are also given off from the aorta to the walls of the abdomen.

The pulmonary artery $(p, a)$ divides soon after its origin from the right ventricle into two branches, one supplying each lung. Just before its bifurcation it is connected by a short cord, known as the ductus arteriosus, with the aorta : this is the solid vestige of the embryonic connection between the fourth arterial arch and the aorta (compare p. 452 , and Fig. I20).

Each precaval $(l . p r . c, r . p r . c)$ receives-a subclavian (s. cl. v) from the fore-limb; an external jugular (e. juc) from the head, running along the neck just beneath the skin; a small anterior epigastric from the ventral thoracic wall, as well as small vessels from some of the anterior intercostal spaces (i. cs) and the anterior surface of the diaphragm (a.ph); and a small internal jugular $(i . j u)$ from the brain, opening into the corresponding external jugular nearly opposite the subclavian. An azygos vein $(a z . v)$, representing part of the right cardinal of the embryo (compare p. 456 ) and receiving blood from the posterior intercostal spaces, also opens into the base of the right precaval.

There is no renal portal system, as in the dogfish and frog (pp. 454 and 85). A pair of internal iliac veins (i.il.v) in the pelvic cavity unite to join a median vessel (c.il. v), the hinder end of the postcaval, which receives on either side an external iliac (e. il. $v)$ : this is constituted by a femoral vein ( $f m . v)$ from the hind-limb; a posterior epigastric (p.epg), from the ventral walls of the abdomen, entering the femoral just external to Poupart's ligament; and by small veins from the bladder as well as from the uterus in the female. Slightly in front of the external iliacs the postcaval receives a pair of large ilio-lumbar veins (i. l) from the body-walls: the left ilio-lumbar 


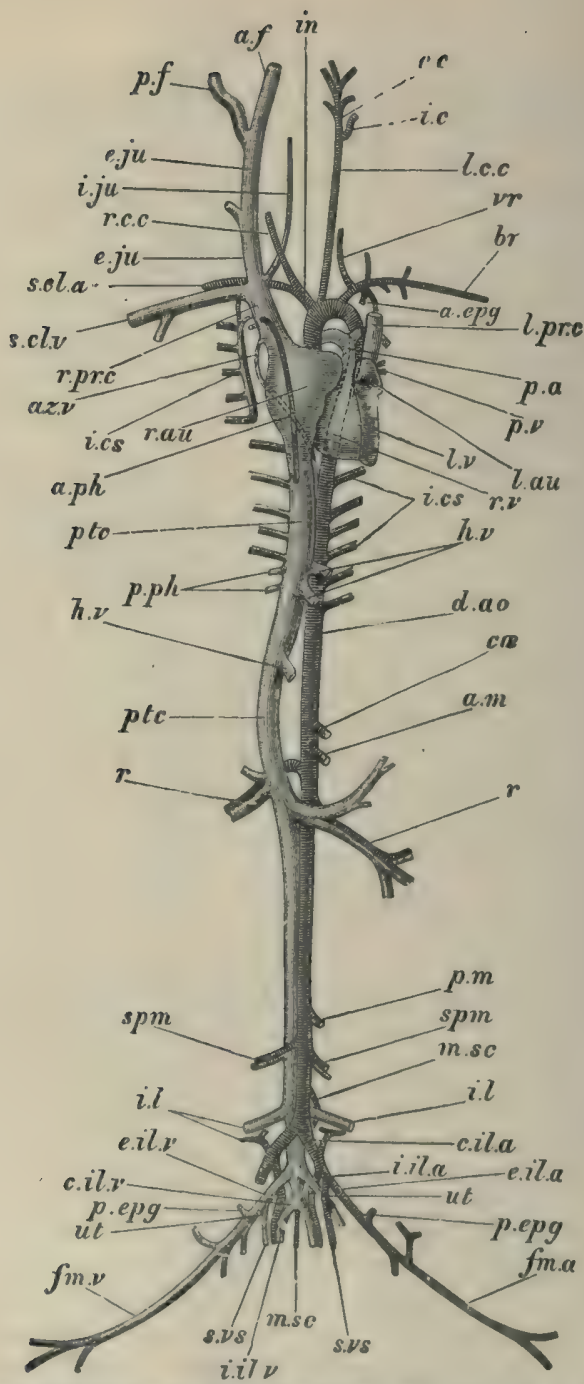

Fig. 140.-The vascular system of the Rabbit from the ventral side. The heart is somewhat displaced towards the left of the subject; the arteries of the right and the veins of the left side are in great measure removed. $(x+1$. 
a. epg. anterior epigastric artery; a. $f$. anterior facial vein; a. $m$. anterior mesenteric artery; $a$. ph. anterior phrenic vein: as. $v$. azygos vein; $b$. right brachial artery ; c. $\boldsymbol{l}$. u. common iliac artery; $c$. il. $v$. hinder end of post-

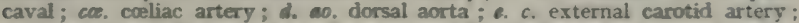
e. ii. a. external uliac artery; c. i. $v$. external iliac vein; $\iota$. ju. external jugular vein : $f m . a$. femoral artery; fm. $v$. femoral vein; $h . v$. hepatic veins; $i: c$. internal

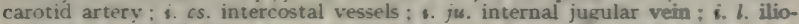
lumbar artery and vein: in. innominate artery : $l$. au. left auricle : $l$. c. c. left common carotid artery ; $l$. pr. $c$. left precaval vein ; $l$. $v$. left ventricle; $m . s c$. caudal artery $; p$. a. pulmonary artery ; $p$. epg. posterior epigastric artery and vein : $p . f$. posterior facial vein: $p . m$. posterior mesenteric artery ; $p . p h$. posterior phrenic veins; ptc. postcaval vein; $p . v$. pulmonary vein; $v$. renal artery and vein; $\%$. au. right auricle; $\boldsymbol{r} . c$. c. right common carotid artery; r. prc. right precaval vein; $7 . v$. right ventricle; scl. a. right subclavian artery ; scl. $v$. right subclavian vein; spm. spermatic artery and vein; s. vs. vesical artery and vein; ut. uterine artery and veis; $v$. vertebral artery. (From Parker's Zootomy.)

sometimes runs forwards to open into the corresponding renal vein. Rather more anteriorly still are a pair of spermatic (spm) or ovarian veins, and a large renal vein $(\boldsymbol{r})$ enters the postcaval from each kidney. As the postcaval passes through the dorsal border of the liver, it receives several large hepatic veins (Figs. I 37 and $\mathrm{I}_{4} \mathrm{O}, h . v$ ) from the lobes of that organ. Other small veins from the bodywalls and from the posterior surface of the diaphragm also open into the postcaval, which then passes through the central tendon of the diaphragm and runs forward in the mediastinal space (Fig. $138, p t$. cav) to open into the right auricle.

The hepatic portal vein (Fig. I 37, p. v) is a large vessel situated in the mesentery, ventral to the postcaval. Anteriorly it passes into and divides up in the liver, sending a branch to each lobe: posteriorly it is constituted by a large anterior mesenteric vein $(m$. v) returning the blood from the small intestine, colon, and cæcum, and by smaller veins from the stomach, spleen, and duodenum, as well as by a posterior mesenteric vein ( $p . m . v)$ from the rectum.

The pulmonary veins have already been described (p. 522). In the freshly-killed animal a number of the delicate, transparent lymphatic i'essels (p. 97) can be made out, those from the intestine (lacteals) running in the mesentery. They 
come into connection with numerous adenoids (p. 5 I 5 ) in the mesentery and elsewhere, and most of them communicate with a main trunk - the thoracic duct-which extends from the abdomen through the thorax on the left and upper side of the aorta. The thoracic duct also receives the lymphatics from the right side of the head and neck and the fore-limb, and opens into the veins at the junction of the left external jugular and subclavian: the lymphatics of the right side of the head and neck and right fore-limb communicate with the corresponding veins of the right side.

Nervous System.-The brain (Figs. I35, I4I, and I42) reaches a much higher development than in the other Vertebrates we have already studied. The prosencephalon is subdivided into two cerebral hemispheres (c.h), of much larger relative size than those of the frog (Fig. 49) and forming about two-thirds of the whole brain. They are closely applied to one another along their flat internal surfaces, are roughly conical in form, narrower in front (frontal lobes), broadening out posteriorly (parietal lobes)-where they overlap the diencephalon and optic lobes and abut against the cerebellum, and produced downwards into the prominent temporal lobes which partly overlap the crura cerebri below. Their external layer or cortex is formed of grey matter, and their surface is convex and smooth, except for the presence of slight lateral grooves between the lobes: in many Mammals the hemispheres are highly convoluted, i.e., raised into numerous winding elevations or gyri, separated by narrow grooves or sulci. A broad transverse band of nerve-fibres forms a commissure connecting the two hemispheres known as the corpus callosum (Figs. I $f$ I and I $42, c p . c l):$ this structure is confined to the Mammalia, and is even wanting in certain of the lower members of the class. The olfactory lobes (olf) are club-shaped, and extend backwards along the ventral surface of the hemispheres in the form of narrow bands as far as the temporal lobes. 
The diencephalon consists of a right and left optic thalamus (o. th) between which is the slit-like third

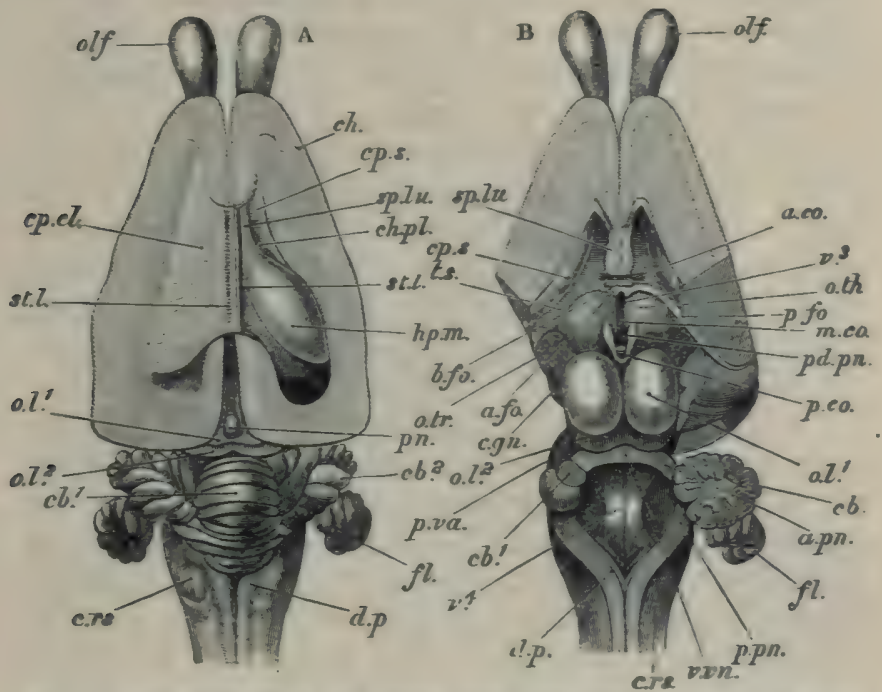

Fig. IfI.- Two dissections of the Rabbit's brain, from above. ( $x$ It.) In A, the left hemisphere is dissected down to the level of the corpus callosum: on the right side the lateral ventricle is exposed. In $\mathrm{B}$, the hemispheres are dissected down to a little below the level of the anterior end of the corpus callosum ; only the frontal lobe of the left hemisphere is retained, of the right a portion of the temporal lobe also remains : the choroul plexus and pineai bodv are removed, as well as the greater part of the body of the fornix and the whole of its left posterior pillar; the certbollum is removed with the exception of a part ot its right lateral lobe with the flocculus.

a. co. anterior commissure : $a$. fo. anterior pillar of fornix; $a$. pn. anterior peduncles of cerebellum; $b$. fo. body of fornix ; $c^{1}$. central lobe of cerebrllum : $c b^{2}$. its lateral lobe: c. gn. elevation on the optic thalamus; $c$. h. cerebral hemisphere; ch. pi. part of choroid plexus : $c p$. $c l$. corpus callosum: $c p$. s. corpus striatum; c. rs. and $d$. $p$. elevitions on the bulb; $f$. fl xeculus; $h p$. $m$. hippocampus; $m$. co. middie commissure : $0 . l l, o . l 2$, optic lobes; olf. oliactory lobe; 0 . th. optic thalamus : 0 . $t r$. optic tract (continuation of chiasma); $p$. co. posterior commissure : $p$. fo. posterior pillar of fornix: $p n$. pineal budy ; $p d$. $p n$. its perturucle: p. pn. ponterior peduncies of certbellum; $p$. va. fibres of pons Varolii formitig middle peduncies of cerebellum; $s p$. in. septum lucidum; st. $l$. line on corpus callosum ; $t$. $s$. band of white matter lying beneath choroid plexus: $v$. vn. valve of Vieussens; $\tau^{3}$. third ventricle; $i^{4}$. fourth ventricle. (From Parker's Zootomy.)

ventricle $\left(v^{3}\right)$ roofed over by a thin membrane continuous with a vascular choroid plexus (Fig. $\mathrm{I} \neq 2, v l . i p$ ), and from 
its hinder part arises a stalk bearing at the end a small, rounded pineal body $(p n)$. The floor of the diencephalon is produced downwards to form the infundibulum (inf), to which the pituitary body (pty) is attached. In front of the infundibulum is the optic chiasma $(o, c h)$, and behind it a small, rounded lobe (c.ma).

Each optic lobe is divided into two by a transverse furrow, so that there are four rounded elevations in this region-an anterior, larger pair $\left(o . l^{1}\right)$, and a posterior, smaller pair $\left(o, l^{2}\right)$. Below the optic lobes are the crura cerebri (c. c) - -two strong, diverging bands passing forwards and outwards from the bulb to the hemispheres.

The bulb or medulla oblongata $(m . o)$ is slightly flattened dorso-ventrally, and passes behind into the spinal cord, the dorsal and ventral fissures of which are continued into it : the fourth ventricle $\left(v^{4}\right)$ which it contains is roofed over by the thin pia mater only (p. I55). Ventrally its anterior border is marked by a stout band of nerve-fibres running transversely, and known as the pons Varolii ( $p$. $v a)$. The large cerebellum is connected with the dorsal surface of the brain by three pairs of peduncles (Fig. I4I, a. $p n, p . v a, p . p n)$, and consists of a median central lobe $\left(c b^{1}\right)$ and of two lateral lobes $\left(c b^{2}\right)$, on the outer side of each of which is a smaller floccular lobe $(f)$. The grey matter is superficial, and the surface is marked by numerous folds which in section present a tree-like pattern (arbor vitce), brought about by the arrangement of the grey and white matter (Fig. I42).

The fourth ventricle is not prolonged into the cerebellum to any extent: it is continued forwards as the iter, from which no optic ventricles are given off (compare pp. I57 and 459) and which passes into the narrow but deep third ventricle in front (Fig. I42): this is bounded anteriorly by a thin wall, the lamina terminalis 
(l. $t)$, and extends into the infundibulum below. At its anterior end are the foramina of. Mouro $(f . m)$, leading into the middle of the lateral ventricles in the hemispheres (Fig. IfI). In this region each lateral ventricle is broad from side to side, but narrow from above downwards ;

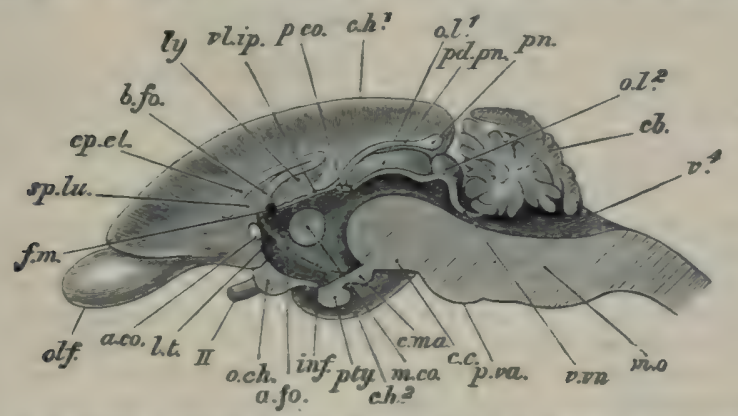

F1G. 142.-Rabbit. Longitudinal vertical section of the rabbit's brain. ( $₹$ I 1 )

Letters as in preceding figure; in addition $-c b$. central lobe of cerebellum, showing arbor vitæ; $c$. c. crus cerebri; c. $h$. pasietal, and $c p . h^{2}$. temporal lobe of cerebral hemisphere; $c$. ma. elevation behind the infundibulum; $f . m$. foramen of Monro; $s u f$. infundibulum ; l. $t$. lamina terminalis ; $l y$. part of hippocampus ; m. o. medull oblongata; $o$. ch. optic chiasma; $p t y$. pituitary body; $v l$. ip. choroid plexus; v. vn. valve of Vieussens; II. optic nerve. (From Parker's Zootomy.)

it extends forwards into the frontal lobe, backwards into the parietal lobe, and downwards into the temporal lobe. The olfactory lobes are solid.

A prominent, convex ridge of white matter-the hippocampus (Fig. I $4 \mathrm{I}, h p . m$ )-projects into the inner side and floor of each lateral ventricle where it descends into the temporal lobe, and closely applied to it is a continuation of the choroid plexus $(\mathrm{ch} . \mathrm{pl}$ ), which passes from the roof of the third ventricle into the lateral ventricle through the foramen of Monro. In front of the hippocampus the outer side and floor of the anterior part of the lateral ventricle is thickened to form an eminence of grey matter, the corpus striatum (cp. s). Just beneath the corpus callosum the internal wall of each lateral ventricle is thin, and is known as the septum lucidum (sp.lu); and below it and above the foramina of Monro is another commissure known as the body of the fornix (Figs. $\mathrm{I}_{4} \mathrm{I}$ and $\mathrm{I}_{42}, b$. fo) which is continuous on either 
side with two bands - one (posterior pillar) lying along the anterior edge of the hippocampus, and the other (anterior pillar) passing backwards in the side walls of the third ventricle. Connecting the two optic thalami are three transverse bands of nerve-fibres, known respectively as the anterior $(a . c o)$, middle $(m$. co), and posterior $(p . c o)$ commissures: the middle commissure, which is much the largest, is not represented in the lower Vertebrata.

The spinal cord (Fig. I35, sp.c) is similar in structure to that already described in other Vertebrates (pp. I55 and 459). It extends through the entire neural canal, ends in a filum terminale, and is swollen opposite the fore- and hind-limbs, where the nerves arise which form the limb-plexuses (pp. I6I and I62).

The dorsal and ventral roots of the spinal nerves lie in the same transverse plane, as in the frog (p. 163), but are relatively shorter than in that animal; after uniting to form the nerve-trunks, they pass directly outwards through the intervertebral foramina. The brachial plexus is formed from the four posterior cervical and the first thoracic nerves, and gives off a number of nerves to the shoulder and fore-limb. The sciatic or lumbosacral plexus is constituted by the two or three hindermost lumbar and the first two or three sacral nerves, and gives off branches to the pelvic region and hindlimb, a femoral and a peroneal going to the extensor muscles, and a large sciatic and an obturator (which passes through the obturator foramen, p. 503) supplying the flexor muscles. Arising from the fourth cervical spinal nerve of either side is a phrenic nerve (Fig. I 35, phr. $n$, which passes backwards, between the heart and lungs, to supply the muscles of the diaphragm; and a large auricular nevve, arising from the third cervical nerve, supplies the external ear.

In addition to the ten cerebral nerves enumerated in the frog (p. I63) and dogfish (p. 46r), two others-the spinal accessory and the hypoglossal (represented in the frog by fibres in connection with the vagus and by the first spinal nerve respectively, p. I60)-emerge from the skull and are therefore counted as the eleventh and twelfth cerebral nerves. The former arises from the side of the spinal cord and bulb by numerous fibres, the 
posterior of which are opposite the fifth spinal nerve, from which point it runs forwards between the dorsal and ventral roots and leaves the skull together with the glossopharyngeal and vagus (p. 496), supplying certain muscles of the neck and shoulder. The hypoglossal arises by a number of fibres from the ventral surface of the bulb, passes out through the condylar foramen, and supplied the muscles of the tongue, as well as certain muscles of the neck.

The origin and distribution of the first ten pairs of cerebral nerves correspond in their main features with those seen in the frog (p. 163). The facial is almost entirely a motor nerve and is chiefly important in supplying the facial muscles, which are very highly developed in Mammals. An anterior and a posterior or vecurrent laryngeal nerve are given off from the vagus.

The relations of the sympathetic nerves (Fig. I35, sy) are also essentially similar to those occurring in the frog (p. I62). Each passes backwards along the neck close to the vagus $(v g)$ and alongside the carotid artery, enlarging to form an anterior and a posterior cervical ganglion. In the thorax it runs just beneath the heads of the ribs, having a ganglion in each intercostal space: it then passes into the abdomen, lying close to the centra of the vertebræ and having ganglia at intervals. From all the sympathetic ganglia branches are given off connecting them with the spinal nerves, others going to the blood-vessels: others again, in the thorax and abdomen, are connected with plexuses from which nerves pass to the heart and abdominal viscera. In the abdomen these plexuses can be seen in the mesentery, a large caliac or solar plexus being present close to the origin of the anterior mesenteric artery.

Sensory Organs.-The sense of touch is situated in microscopic tactile organs in the skin, and groups of cells, 
called taste-buds, are present on the papillæ of the tongue (p. 509) and on the soft palate (compare pp. I79 and I80).

The organs of smell are situated in the olfactory capsules, the form of which has already been described (p. 493). They open externally by the external nostrils, and are produced backwards above the palate into the passage of the internal nostrils, which communicate with the naso-pharynx (Fig. I35, $i . n, n$. ph, and see p. 512). The olfactory epithelium, supplied by the olfactory nerves, is situated on the ethmo-turbinal $(e, t b)$ : the mucous membrane of the maxillo-turbinal ( $m . t b)$ probably serves merely to warm the inspired air.

On the ventral side of the nasal septum is a pair of small, tubular structures known as the vomero-nasal or Jacobson's organs (Fig. I35, $j$ ), lined by epithelium and encloser in cartilages situated just to the inner side of the palatine processes of the premaxillie (p. 494). Each of them opens anteriorly into the corresponding naso-palatine canal (p.507), and receives a special branch of the olfactory nerve. The function of these organs is not understood.

The structure of the eye is similar to that already described in other Vertebrates (pp. $18 \mathrm{I}$ and 465 ), except that the sclerotic is not cartilaginous, but is composed of dense fibrous tissue, and the lens is relatively smaller than in the dogfish and frog and is markedly biconvex in form, the outer surface being rather flatter than the inner : it is capable of adjustment by means of the ciliary muscles contained in the radiating ciliary processes into which the choroid is thrown just externally to the iris (compare p. I84).

The eyelids have already been described (p. 486). The four recti muscles ensheath the optic nerve, as in the frog (p. 186, compare Fig. I 26), but the superior oblique, instead of arising-like the inferior oblique - in the anterior part of the orbit, takes its origin further back, near the recti, passes forwards through a fibro-cartilaginous pulley at the anterior angle of the orbit, and then backwards and outwards to its insertion on the eyeball. 
Between the wall of the orbit and the eveball are two glands, the secretion of which, passing through ducts perforating the conjunctiva lining the eyelids, serves to keep the outer surface of the eye moist, and is then conducted into the nasal chambers by means of the naso-lacrymal duct (pp. I86 and 494). These two glands correspond to special differentiations of a primarily continuous structure : one, the Harderian gland-already met with in the frog-is situated in the antero-ventral region of the orbit: the other, or lacrymal gland proper, in its postero-dorsal region. Besides these, a series of small Meibomian glands is present on the inner side of the edges of the eyelids, and produces a fatty secretion.

The essential part of the auditory organ consists, as in other Vertebrates, of the membranous labyrinth with its three semicircular canals (pp. I $\$ 6$ and 465 ) enclosed in the auditory capsule (periotic bone, p. 492), and constituting the internal ear. The small outgrowth of the sacculus seen in the dogfish and frog, and known as the cochlea (Fig. 59, cc), is represented by a relatively larger structure, coiled on itself in a spiral manner, and specially important as regards the sense of hearing. The part of the periotic bone which directly surrounds the cavity in which the membranous labyrinth lies is especially hard, and when the outer portion of the bone is cut away, is seen to form a sort of cast of the enclosed organ, the form of which it repeats: this is known as the bony labyrinth (Fig. I43). Internally it is separated from the membranous labyrinth by a narrow space all round, containing the perilymph (p. I89) and only shut off from the tympanic cavity at the fenestra ovalis and fenestra rotunda (p. 193) by the membrane which closes each of them.

The membranous cochlea does not run up the middle of the spiral of the bony cochlea, but is attached between its 
outer wall and a spiral shelf arising from its inner wall. Thus the entire cochlea shows three cavities in transverse section - a middle, the membranous cochlea or scala media, and a scala vestibuli and a scala tympani on either side of it respectively, which communicate with one another at the apex of the cochlea and with the perilymphatic cavity surrounding the rest of the membranous labyrinth at its base, where the scala tympani abuts against the membrane of the fenestra rotunda, and the scala vestibuli against that of the fenestra ovalis. On the wall of the scala media which separates it from the scala tympani is a specially modified series of auditory cells forming what:is known as the organ of Corti, which receives nerve-fibres from a branch of the auditory nerve extending along the spiral shelf of the cochlea.

The middle ear (p. 466) is constituted by the tympanic cavity in the tympanic bulla (p. 492), and communicates with the pharynx by the Eustachian tube (Fig. I43, E). The tympanic membrane $(\mathrm{M})$, situated obliquely at the boundary of the bulbous and the tubular portions of the tympanic bone, separates the middle ear from the external ear, consisting of the auditory passage (Ex) and the pinna (p. 486).

The fenestra ovalis is plugged by a small stirrupshaped bone, the stapes (Fig. I43, $\mathrm{O}_{1}$ ), one of the three auditory ossicles (p. 492) connecting the internal ear with the tympanic membrane, and probably corresponding morphologically to part or the whole of the frog's columella (Fig. Io) : with it is connected a small stapedius muscle, serving to keep the membrane of the fenestra ovalis on the stretch. The middle bone of the chain is the incus (Fig. I43, $\mathrm{O}_{2}$ ), a short process of which is articulated to the stapes by the intermediation of a small bony nodule, while its body articulates with the outer bone of the series, the malleus $\left(\mathrm{O}_{3}\right)$. Arising from the body of the malleus is a handle-like process or manubrium, which is attached to the tympanic membrane (M) : this has the form of the roof of a tent, and is kept on the stretch by a small muscle, the tensor tympani. 
arising from the wall of the tympanic cavity and inserted in to the manubrium of the malleus.

The study of development indicates that the malleus corresponds to the ossified articular part of the mandibular cartilage of lower Vertebrates, and the incus to the quadrate

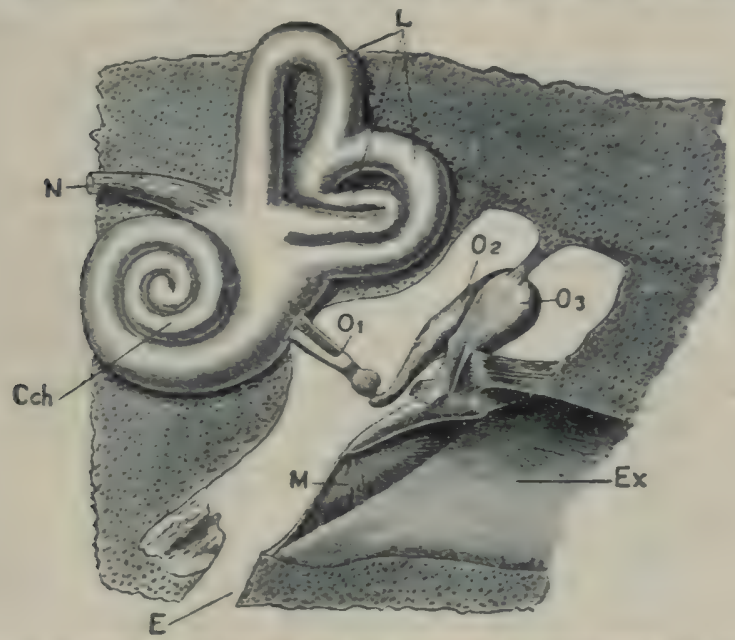

FIG. 143.-Diagram of the mammalian body labyrinth, tympanic cavity, and external auditory passage.

Cch. bony cochlea : $E$. Eustachian tube : Ex. external auditory passage; $L$. bony semicircular canals:.$M$. tympanic membrane; $N$. auditory nerve; $O_{1}$ stapes; $\mathrm{O}_{2}$ incus; $\mathrm{O}_{3}$ malleus. (Áfter Headley.)

(pp. 44 and 438): the articulation of the bony mandible with the squamosal in Mammals has rendered these parts unnecessary for their original purpose; and they have undergone a change of function (p. 449), forming an accessory part of the auditory apparatus.

Urinogenital Organs.-The kidneys (Fig. I35, k) are of a somewhat compressed, oval shape, with a notch or hilus on the inner side. They are in close contact with the dorsal wall of the abdominal cavity, the right being somewhat in advance of the left. Towards the hilus, 
the tubules of which the kidney is composed (p. I46) converge to open into a wide chamber or pelvis, which forms the dilated commencement of the ureter. When the kidney is cut across, its substance is seen to be divided into a central mass or medulla and a peripheral portion or cortex. The former appears radially striated, owing to the tubules in this region being straight and converging to open on the surface of a conical process or pyramid, which projects into the pelvis : the cortex contains the coiled portions of the tubules and the Malpighian bodies, and thus has a dotted appearance. The ureter (Figs. I35 and $I 44, u r)$ runs backwards along the dorsal wall of the abdomen to open into the urinary bladder $(u . b l, b l)$, a pyriform sac with elastic walls which vary in thickness according as the organ is dilated or contracted. Near the front end of each kidney towards its inner side is a small yellowish adrenal body (Fig. I35, $a d r$, compare p. 447).

In the male rabbit the spermaries are oval bodies which in the young animal are situated close to the kidneys on the dorsal wall of the abdomen, but which pass backwards and downwards as the animal approaches maturity until they come to lie each in a scrotal sac (p.487), situated at the side of the urinogenital opening. The cavity of each scrotal sac is in free communication with the cavity of the abdomen by an opening-the inguinal canal. A convoluted epididymis (p. 466), closely adherent to the spermary and connected with the distal end of the scrotal sac, forms the proximal part of the spermiduct or vas deferens (Fig. $\mathbf{I}_{4} \mathrm{~A}$, v. d), which, together with the blood-vessels and nerves of the spermary, passes out through the inguinal canal: it then loops round the corresponding ureter, and extends back between the neck of the bladder and a median sac on the dorsal side of the latter known as the uterus masculinus $(u, m)$. The neck 
of the bladder is continued backwards, through the pelvic carity, as the urinogenital canal or urethra, on the dorsal side of which, just in front of a rounded
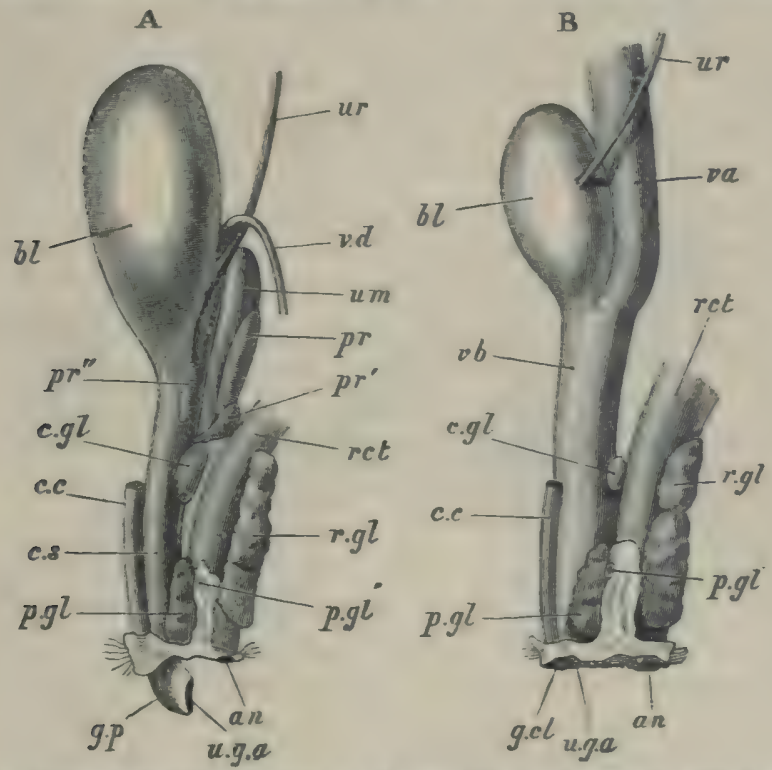

Fig. 144.-The urinogenital organs of the Rabbit. - $A$, of male : B, of temale : from the left side (about nat. size). The kidney and proximal end of the ureter, in A the spermary, and in B the svary, uterine tube and uterus are not shown. an. anus ; $b$. urinarv bladder ; $c$. c. corpus cavernosum : c. s. corpus spongiosum; c. gl. Cowper's glend; g. $c l$. apex of elitoris : $g$. p. apex of penis : $p$. gi. perineal sland, and $p . g l$. aperture of its duct on the perineal space : $p r$. anterior: $p p^{\prime}$. posterior, and $\mathrm{pr}^{\prime}$. lateral lobes of prostate : rot. rectum : $r$. gl. rectal gland;

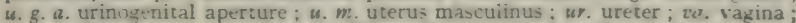
vb. vestibule $; \tau$. d. vas deferens. (From Parker's Zootomy.)

elevation, is an aperture by means of which the uterus masculinus and vasa deferentia open into it.

A prostate gland ( $p r . g l$ ), consisting of several lobes, is embedded in the walls of the uterus masculinus and opens by smal! ducts on either side of the elevation just referred to ; and a pair of smaller, ovoid Cowper's glands communicate with the urinogenital canal further back. 
The terminal part of the urethra traverses the copulatory organ or penis; that part of its wall which in the retracted condition is dorsal is constituted by a soft vascular portion, the corpus spongiosum $(c . s)$, while the opposite surface is strengthened by two harder bodies, the corpora cavernosa (c.c), which are closely applied together through the greater portion of their length, but diverge proximally and are attached to the ischia.

In both sexes a pair of perineal glands $(p . g l)$ open on the perineal spaces (p. 486) at the sides of the penis, and two larger rectal glands $(r, \mathrm{gl})$ lie at the side of the rectum.

In the female the ovaries (Figs. I35 and I45, ov) are small ovoid bodies attached by peritoneum to the dorsal wall of the abdomen behind the kidneys, the ovarian follicles or ovisacs (p. I95), which are hollow, forming, when ripe, very small, rounded projections on their outer surface. ${ }^{1}$

The oviducts, instead of remaining separate along their whole length, are fused proximally to form a wide, median portion, the vagina (Figs. I44, B, and I45, va), opening into the urinogenital canal or vestibule $(v b)$, with which the bladder communicates and which opens externally at the vulva (Fig. I44, B, u.g.a). Into the other or distal end of the vagina, the paired, thick-walled uteri (Fig. I45, r. ut, l. ut), or middle portions of the oviducts, open by separate thick-walled apertures. The eggs undergo development in the uteri, which vary in size according to whether or not they contain embryos, and according to the stage of development of these. Each uterus is continued forwards as a narrow, slightly coiled tube-the anterior section of the oviduct, or uterine tube $(f t . t)$, which communicates with the colome by a small aperture $\left(f l \cdot t^{\prime}\right)$ surrounded by a wide, membranous

1 After the ova are discharged, a proliferation of cells into the follicles takes place, and gives rise to the so-cailed corpora lutea. 
funnel with thin walls and folded margins, which is applied to the outer surface of the corresponding ovary. On the ventral wall of the hinder or proximal end of the urinogenital canal is a small, hard, rod-like body, the clitoris (Fig. I35, cl), corresponding to the penis of the male, and strengthened by two small corpora cavernosa (Fig. If , B, c. c) attached at their proximal ends to the ischia.

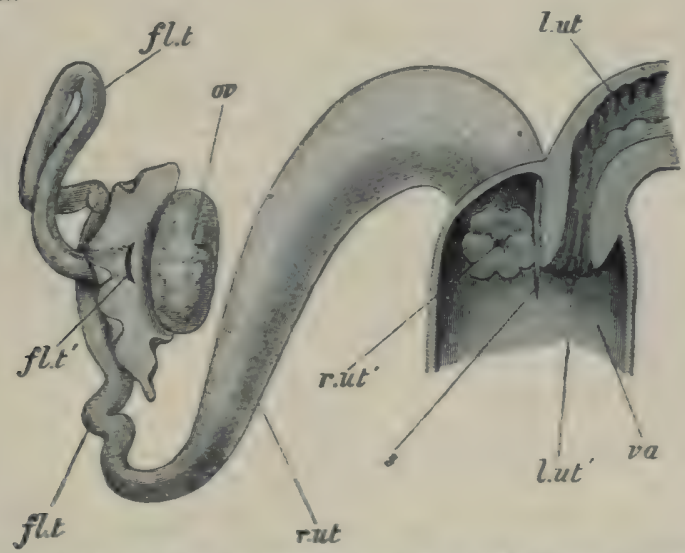

Fic. If5.-The anterior end of the vagina, with the right uterus, Fallopian tube, and ovary of the Rabbit ( about 2). Part of the ventral wall of the vazina is removed, and the proximal end of the left uterus is shown in longitudinal section.

f. $t$. uterine tube ; $f . t^{\prime}$. its colomic aperture : $l$. wt. left uterus ; $l$. w'. aperture of same (os uteri) into vagina; ot. right ovary; $r$. ut. right uterus; $r$. ut'. aperture of right uterus into vagina; s. vaginal septum; 2 . vagina. (From Parker's Zootomy.)

The rabbit is viviparous. The minute ova are discharged into the uterine tubes, where they become fertilised, and then pass into the uterus, in which each develops into a foetus, as the intra-uterine embryo is termed, and is nourished by means of an organ known as the placenta, which will be described in Chapter XIII. The young animal escapes from the uterus in a condition in which all the parts have become fully formed, except 
that it is practically hairless; the eyelids are at first coherent. As many as eight or ten young are produced at a birth, and the period of gestation, i.e., the time elapsing between the fertilisation of the ovum and the birth of the young animal, is thirty days. Fresh broods may be born once a month throughout a considerable part of the year, and, as the young rabbit may begin breeding at the age of three months, the rate of increase is very rapid.

The class Mammalia is divided into a number of orders, that to which the rabbit belongs being called the Rodentia and also including rats and mice, squirrels, beavers, porcupines, and many others. All these are vegetable feeders and are mostly of small size. They possess no canine teeth, and their incisors, which are adapted for gnawing, are never more than two in number in the lower jaw, and in most of them there are only two in the upper jaw also.

\section{PRACTICAL DIRECTIONS}

The specimen used should be over three months old. Place it in a sufficiently large jar or box with a close-fitting lid together with a piece of cotton-wool well soaked in chloroform, and leave it until a short time after all movements have ceased.

A pair of bone-forceps (p. 12) will be requirerl.

A. External characters (see pp. $483-487$ ).

Microscopic preparations of the skin of a Mammal should be examined, and the hairs, hair-sacs, and sebaceous glands (Fig. I3I) noted. (For mode of preparation, see p. I36.)

$B$. Skeleton. If more convenient, the detailed examination of the skeleton may be postponed until after the soft parts have been dissected.

The skeleton of a young rabbit, about six weeks old, as well as that of an adult, should be obtained for examination : the former is the more important of the two for making out the individual bones, which should all be separated from one 
another by prolonged maceration in water, or by boiling for a short time. In the adult skeleton the bones are best kept in their natural cannection. An additional skull should be prepared, and a longitudinal vertical section made of it with a fine saw; but as it is difficult to do this very accurately and perfectly in the rabbit, owing to the delicacy of some of the bones, the skull of a young dog or cat may be used for the purpose.

Work through the account of the skeleton given on pp. $48 ;-505$, as well as of the teeth on pp. 509-511, comparing the form, number, and arrangement of the teeth in the herbivorous rabbit and the carnivorous dog or cat, noting the presence of canines in the two last mentioned; sections of the teeth (see p. 5IO) should be examined. Sketch. typical parts of the skeleton.

\section{Superfleial dissection, and injection of the arteries.}

[For the blood-corpuscles, examine a drop of blood obtained from a freshly-killed rabbit, or by pricking the tip of your finger (compare p. I2I). Note the circular and slightly biconcave form of the red corpuscles, their relatively smail size (about 500 in. or $008 \mathrm{~mm}$. in diameter), and the absence of a nucleus.]

I. Fix the animal on its back on the dissecting-board by inserting large pins or nails through the limbs. If you wish to inject the arteries, cut through the skin on the inner side of one of the thighs, reflect it, and with the seeker expose the femoral artery (p. 524$)$; or if your specimen is a small one, expose one of the carotid arteries (p. 523) instead, by carefully cutting through the skin along the middle ventral line of the neck. Pass a piece of thread round the artery thus laid bare, and make a small slit in it with the fine scissors distally to the thread. Insert and tie in a cannula, directed towards the body, and first inject a little strong formaline (I part formaline and 2 parts water), following this with the coloured starch injection-mass (p. 99), which will force the formaline into the capillaries and help to preserve the specimen : this method is of special advantage for the subsequent examination of the enteric canal, especially if it is not possible to get to the end of $\S$ VI, p. 547 , on the first day: the veins need not be specially injected, as thəy will be naturally injected with blood.

Make a median longitudinal incision through the skin in the sternal region, and continue the cut backwards to the pelvic symphysis and forwards to the mandibular sym- 
physis; then dissect away the skin from the underlying muscles over the whole ventral surface, being careful not to injure any of the larger blood-vessels $(e . g *$ the jugular veins in the neck). Note :-

I. The thin cutaneous muscle, part of which you have very likely removed together with the skin: and in the adult female, the mammary glands (p. 506)-trace the main ducts of some of these to their apertures on the teats.

2. In the neck:- $a$, the trachea and larynx (p. 506), to see which and the following structures clearly the cervical portion of the cutaneous muscle should be dissected away and the underlying parts carefully separated with a seeker; $b$, the hyoid bone, situated in a mass of muscle just anterior to the larynx; $c$, the submaxillary glands (p. 5I2); and $d$, the large external jugular veins (p. 525).

3. In the thorax:- $a$, the sternum and xiphisternum; $b$, the small clavicles (p. 50I) ; $c$, the pectoral muscles; $d$, the vertebral and sternal portions of the ribs and the external intercostal muscles; and $e$, the blood-vessels and nerves going to the fore-limbs.

4. In the abdomen :- $a$, the muscles of the abdominal wall, separated in the middle ventral line by a whitish fibrous band ; b, Poupart's ligament (p. 506) ; $c$, the bloodvessels and nerves passing beneath Poupart's ligament to the hind-limbs.

II. On the same day on which the animal is killed, remove the skin from the dorsal surface of the head and anterior part of the neck, and also the muscles covering the anterior cervical vertebra. A membrane between the skull and first vertebra will then be exposed : cut through this, and part of the spinal cord will be seen. With the bone-forceps cut away the arches of the first three or four vertebra (noting the roots of the spinal nerves) and the roof of the skulltaking particular care in the auditory region-so as to expose the brain: note the olfactory lobes, cerebral hemispheres, cerebellum, and medulla oblongata, and also the dura mater and pia mater. Then cut through the spinal cord about a quarter of an inch beyond its junction with the brain, lever up the latter with the handle of a scalpel, and carefully cut through all the nerves arising from it and separate it from the cranial walls, working from behind forwards. Dissect away the olfactory lobes from their attachments, remove the entire brain, and place it in 3 per cent. formaline or in strong spirit.

The animal may be preserved from day to day by wrap- 
ping a cloth round it soaked in 3 per cent. formaline or strong methylated spirit and placing it in a closed vessel.

\section{$D$. Dissection of the abdomen.}

I. Once more pin the animal down, ventral side uppermost, and make an incision along the middle line of the abdominal muscles from the xiphisternum to the pelvic symphysis, so as to open up the abdominal cavity. From the anterior extremity of the incision make transverse cuts, and turn back the flaps of muscle. ${ }^{1}$ Note :--

I. $a$, The peritoneum; $b$, the diaphragm, with its muscles and central tendon; $c$, the pink lungs, seen through the latter: make a small aperture in the diaphragm on one side of the median line and note the collapse of the corresponding lung.

2. $a$, The liver, stomach, small intestine, cacum, colon, and

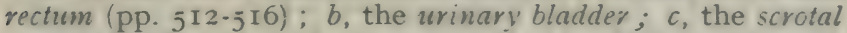
sacs in the male; and-by turning aside the intestines- $d$, the kidney's; in the female $e$, the oraries and oiducts (varying much in size according to age).

3. $a$, The characters and relations of the lobes of the liver (compare Fig. I 37), and the folds of peritoneum which attach the liver to the diaphragm; $b$, the gall-bladder; $e$, the gullet, and its entrance into- $f$, the stomach (note its cardiac and pyloric portions); $g$, the spleen.

4. $a$. The parietal and visceral lavers of the peritoneum and the various subdivisions of the mesentery; $b$, the $\mathrm{L}^{\top}$-shaped duodenum, passing into the ileum, and the connection of the latter with the proximal end of $-c$, the cacum, at the distal end of which is the vermiform appendix, while proximally it passes into $d$, the colon, continuous with-e, the rectum, which enters the pelvic cavity to open by the anus.

II. Turn over the stomach to the animal's right side and make out-

I. $a$, The postcaval vein, passing from the pelvis forwards, near the ventral surface of the backbone, through a notch in the liver to the diaphragm; $b$, the dorsal aorta, running partly above, partly alongside, the postcaval ; $c$, the caliac artery, given off from the aorta about an inch posteriorly to the diaphragm-trace its main branches; $d$, the antevior

1 You will very probably notice a number of transparent, rounded vesicles, about $\frac{1}{4}$ inch in diameter, in the abdomen. These are tape-worms (phylum Platyhe'minthes, p. 112 ) in the encysted stage, which develop into the adult form when swallowed by a dog. 
mesenteric artery, arising about half an inch further back than the cocliac-trace its main branches (Fig. 137). Note the coeliac or solar plexus (p. 533).

2. $a$, The left kidney, with its artery, vein, and ureter; $b$, the yellowish left advenal, anterior to the origin of the renal artery and vein ; $c$, the left ovary, uterine tube, and uterus in the female.

3. $a$, The small posterior mesenteric artery, leaving the aorta a short distance posteriorly to the left kidney and supplying the rectum; $b$, the posterior mesenteric vein, in the mesentery of the rectum.

III. Turn the intestines over to the animal's left side and spread out the duodenum, putting its mesentery slightly on the stretch but taking care not to rupture it, so as to make out-

I. $a$, The large anterior mesenteric vein, into which the posterior mesenteric vein opens; $b$, the pancreas and its duct, opening into the distal limb of the duodenal loop an inch or so beyond the bend.

2. $a$, The right kidney, partly overlapped by the caudate lobe of the liver, and its ureter; $b$, the right adrenal.

IV. Replace the intestines in their natural position; cut through the gullet close to the diaphragm, draw the stomach backwards, turn forwards the lobes of the liver, and dissect out the following structures (Fig. I37) :-

I. The common bile-duct-made up of cystic and hepatic ducts, and its entrance into the duodenum.

2. a, The large hepatic portal vein, running in the mesentery ventrally to the postcaval, made up by the union of the mesenteric, gastric, and splenic veins, and entering the liver, sending a branch to each lobe ; $b$, the transparent lymphatic vessels (lacteals) in the mesentery.

V. Tie two pieces of thread, about half-an-inch apart, round the portal vein just before it enters the liver (the hepatic artery and bile-duct may be included in the ligature); cut through the rectum just anteriorly to the pelvic cavity, and through the portal vein between the ligatures, as well as through the mesenteric attachments of the stomach and intestine, and remove these from the body entire. Unravel the intestine by cutting or tearing the mesenteryexcept that part of it in which the pancreas lies-and spread it out on the dissecting-board or in a large dissecting-dish, arranging it so that the various subdivisions may easily be distinguished from one another, and blowing it up with 
a blow-pipe. In addition to the relations of these parts, note:-

I. The sacculus rotundus (Fig. 135, s. $r$ ), and the characters of the cæcum, which proximally passes insensibly into the colon.

2. The length of the intestine as a whole (about fifteen or sixteen times that of the animal), and the relative length of its five divisions.

Sketch the entire canal.

VI. Remove and cut open the stomach and parts of the small intestine, colon, and rectum: wash thoroughly, and examine under water. Remove the cacum together with a small portion of the ileum and colon, wash it out by directing a stream of water through it, distend with water (it is better to harden it first with formaline) and examine in a dish of water; with the scissors cut away a piece of the wall here and there, between the constrictions and in the appendix. Note :-

I. The mucous membrane, muscular coat, and peritoneum.

2. The longitudinal ridges or mge in the stomach, and the pyloric value.

3. The aperture of the bile-duct, and the villi and Peyer's patches (p. $5^{\mathrm{r}} 5$ ) in the small intestine.

4. $a$, The Peyer's patches and intra-colic ralve in the proximal part of the colon; $b$, the thick lymphoid tissue in the walls of the sacculus rotundus; $c$, the ileo-colic aperture and value.

5. The spira? valie of the crecum, and the !'mphoid tissue of the vermiform appendix.

6. The characters of the mucous membrane of the cæcum and large intestine.

(Microscopic sections of the small intestine-injected and uninjected, should also be examined, compared with Fig. 39, and the villi and intestinal glands noted.)

VII. I.igature the postcaval at the points where it enters and leaves the liver: remove the entire liver, noting as you do so the aorta. gullet, postcaial, hepatic ieins, and phrenic veins. Sketch the liver from the posterior surface. Then return to the examination of the abdomen, and make out (Fig. 140)-

I. The renal and spermatic (or ovarian) arteries and veins.

2. The caudal artery, arising from the dorsal side of the aorta. 
3 The common iliac arteries, each of which gives off an ilio-lumbar branch and divides into an external and an internal iliac, the former becoming continuous, beyond Poupart's ligament, with the femoral. (The course of these arteries and of the corresponding veins can be more easily traced after the urinogenital organs have been removedsee \& IX.)

4. The ilio-lumbar veins, and the trifurcation of the postcaval into the external iliacs and a median vein formed by the fusion of the two internal iliacs.

Sketch all these vessels later, after the removal of the urinogenital organs.

VIII. Dissect away the peritoneum and fat from the kidneys, ureters, and generative organs, and trace each ureter from the hilus of the kidney backwards to the bladder, which, if contracted, may be inflated from the urinogenital aperture. Then make out-

A. In the male (Fig. I44, A) :-

I. The penis, with the prepuce, and the aperture of the urinogenital canal or urethra, which latter is lined by the soft corpus spongiosum, and strengthened by the corpora cavernosa, which diverge proximally and are attached to the ischia.

2. The scrotal sacs, each communicating with the abdominal cavity by the inguinal canal, through which the corresponding spermiduct and the spermatic artery and vein pass to the spermary, to see which and the epididymis the scrotal sac should be slit open along its ventral wall. (Sperms should be examined from a fresh spermary-compare p. 210.)

3. The course of the spermiducts.

B. In the female (Figs. I44, B, and I45) :-

I. The vulva; and the clitoris, with its corpora cavernosa, on the ventral wall of the urinogenital canal.

2. The vagina, uteri, and uterine tubes with their funnels.

3. The ovaries, studded with ovisacs.

IX. Dissect away the kidneys, ureters-and in the male, the scrotal sacs, in the female, the ovaries-from the surrounding parts: cut through the pelvic symphysis with a knife, sever the attachment of the corpora cavernosa to the ischia, and cut through the skin around the urinogenital aperture and anus. Remove the whole of the urinogenital organs, together with the posterior end of the rectum, from the body and, after noting $\$$ VII, 3 and 4 , pin them out in a dissecting-dish, with the ventral side uppermost, taking 
care to preserve the natural relations of the parts. Dissect away all the superfluous connective-tissue and fat, separate the rectum at its cut end from the urinogenital canal, and turn it on one side, noting the rectal and perineal g! ands. Make out, in addition to the parts referred to in \$ VIII :-

A. In the male. The uterus masculinus and prostate, and the spermiducts passing between the former and the bladder.

Sketch the entire dissection. Then slit open the uterus masculinus so as to see the openings of the spermirlucts, remove the rectum and rectal glands, and make out the relations of the corpus spongiosum. Make a median incision along the whole length of the penis, beginning at the apex and cutting through the fibrous septum between the two corpora cavernosa : continue the incision forwards, so as to open the bladder along its ventral wall, and note and indicate on your sketch the openings of the ureters into the bladder, and the crescentic aperture of the uterus masculinus into the urinogenital canal, just at the anterior edge of a cushion-like fold.

B. In the female. The wide urinogenital cana! with its vascular walls.

\section{Sketch the entire dissection.}

Make a median longitudinal incision through the urinogenital canal, and continue it forwards until the cavity of the bladder is exposed: slit open the vagina and one of the uteri and uterine tubes in the same way. Note and indicate on your sketch the openings of the ureters into the bladder, the connection of the neck of the bladder with the urinogenital canal, the large aperture in the latter leading into the vagina, and the thick-lipped aperture (os uteri) communicating between the vagina and thick-walled uterus, between which and its fellow is a vestigial septum, indicating the primarily paired character of the vagina. Insert a seeker in the ccolomic aperture of the uterine tube. If your specimen is in an early stage of pregnancy, young embryo (blastocysts, p. 586) may be present in the uterine tubes, and should be carefully removed and fixed in corrosive sublimate (p. 136) : if the uteri contains later embryos they present a series of swellings : cut one of the swellings open from the ventral side, and note the fotus, enclosed by the ammion (p. 606), and attached to a discoid placenta (p. 6ro); cut out the other swellings, together with their contents, and preserve them in formaline or alcohol. Microscopic sections of the ovary should be examined, and the hollow oxisacs noted, each consisting of follicular cells enclosing a minute ounm. 
With a scalpel or razor cut across one of the kidneys through the hilus, parallel to the dorsal and ventral faces of the organ, and note the pelvis, and its prolongations-the calices, the urinary pyramid, and the cortical and medullary portions. Sketch. Examine also microscopic preparations of injected and uninjected kidney (compare pp. I46 and 537).

$\mathrm{X}$. Dissect out the lumbo-sacral plexus in the pelvic cavity (p. 532), and then cut through the body just behind the diaphragm; the abdomen will be no longer required, but you should retain one of the hind-limbs if you wish to examine any of its muscles and joints (compare p. 64).

\section{$E$. Dissection of the thorax and neck.}

I. Dissect away the pectoral muscles, cut through all the vertebral ribs of the left side except the last five, at about a quarter of an inch from their junction with the sternal ribs : from the posterior end of the incision thus made, cut downwards (i.e., towards the sternum) for about an inch, and then forwards through the sternal ribs. Turn forward the flap thus separated and carefully dissect it away from the underlying tissues at the anterior end, so as to detach it altogether without injuring the jugular and brachial veins. Note in the thoracic cavity thus laid open :-

I. The pericardium and heart, and the thymus (Fig. I35).

2. The collapsed lungs: make a small aperture in the trachea and inflate.

3. The parietal and visceral layers of the pleura and the mediastinum (Fig. 138). To make out the relations of these and of the mediastinal space more accurately, cut away a flap from the right wall of the thorax, as you have already done on the left side, leaving the sternum intact.

4. The relations of the pericardium, to see which cut through the posterior end of the sternum: separate the mediastinum from the dorsal surface of the bone, turn the latter forwards and remove it: then cut the pericardium open longitudinally, noting the pericardial fluid.

II. Dissect away the pericardium, the thymus, and any fat about the base of the heart which may obscure the vessels arising from it. Follow out these vessels to the head by clearing away the connective-tissue, fat, \&c., by which they are surrounded, but taking care not to injure any of the nerves of the neck. Make out:-

I. $a$, The left and right ventricles and auricles, and the ramifications of the coronary artery and vein; $b$, the two bronchi. 
2. $a$, The pulmonary artery and its division into left and right trunks $; b$, the pilmonary z'eins (the course of which will be better seen at a later stage) ; $c$, the two precaial veins, each formed by the union of a subclavian, an external jugular, and a smaller internal jugular; $d$, the thoracic portion of the postcaval vein.

3. $a$, The gillet (cervical and thoracic portions); $b$, the phrenic nevies (p. 532) and the thoracic portions of the vagi (Fig. I35); $c$, the arch of the aoria, continuous with the dorsal aorta, and the short ligament (vestige of the foetal ductus arteriosus) connecting the former with the pulmonary artery: $d$, the thoracic portions of the sympathetic neries and their ganglia, lying on the heads of the ribs; $e$, the azygos vein (p. 525), best seen by turning the heart and lungs over to the left side of the animal. (The thoracic duct (p. 528) cannot easily be made out at this stage.)

4. $a$, The innominate artery, giving off the left and right common carotids and the right subclarian; $b$, the left subclavian artery; $c$, the division of each common carotid, at about the level of the anterior and of the larynx, into an external and an internal carotid.

Sketch the heart and origins of the main vessels.

III. Now dissect out certain of the nerves and other structures in the neck, as follows:-

I. The vagus (Fig. I35), running to the outer side of each common carotid : with the seeker carefully separate it from the carotid, and trace it forwards to the angle between the head and neck-at which point it enlarges to form a gang!ion -and backwards to the thorax. Branches are given off to the larynx (anterior and posterior or recurrent laryngeal nerves) and to the heart (depressor) nerves, but the dissection of these may be omitted by the beginner.

2. The sympathetic (Fig. 135), also running alongside the carotid artery. It is most easily distinguished by seizing the carotid with the small forceps just at the junction of the head and neck and putting it slightly on the stretch: the vagus ganglion will then be seen on the outer side of the artery, and the more elongated anterior cervical ganglion of the sympathetic on its inner side: trace the sympathetic nerve from this ganglion backwards to the thorax, at the anterior end of which (close to the subclavian artery) it enlarges to form the posterior cervical ganglion, and then becomes continuous with the thoracic portion of the cord.

3. The hypoglossal nerie will be seen at about the level of the vagus ganglion, passing forwards to the tongue. 
4. Note- $a$, the thyroid gland; $b$, the thyroid and cricoid cartilages of the larynx; $c$, the brachial and vertebral branches of the subclavian artery; and $d$, the brachial plexus (p. 532).

IV. Remove the heart and lungs from the body, together with the posterior end of the trachea and recognisable portions of the aorta, precaval, and postcaval. Fasten out the organs under water with their ventral surface uppermost, and after making out the course of the pulmonary arteries and veins, cut through them close to the lungs and separate the latter from the heart. Note :-

I. The two main lobes of each lung and the two accessory lobes on the right side.

2. The cartilages of the trachea and bronchi; trace the bronchi for a short distance into the lungs. Sketch.

$V$. Cut away the outer walls of both auricles so as to expose their cavities, taking care not to injure the veins which enter them. Wash out the contained clots of blood, and note-

I. $a$, The appendix of each auricle, and the network of muscular bands in its walls; $b$, the septum auricularum and fossa ovalis (Fig. I39)-best seen from the left side by holding the heart between your eyes and the light.

2. $a$, The auriculo-ventricular apertures; $b$, the apertures of the precaval and postcaval veins; $c$, the Eustachian valve; $d$, the aperture of the coronary vein within the tunnel-like opening of the left precaval; $e$, the apertures of the pulmonary veins.

Then cut away both aurirles so as to expose the bases of the ventricles, and remove all but about an eighth of an inch of the aorta and pulmonary artery; pour water into the ventricles through the auriculo-ventricular apertures and squeeze the ventricles, noting-

3. $a$, The bicuspid and tricuspid valves, and the semilunar valves at the origins of the aorta and pulmonary artery respectively; $b$, the apertures of the coronary arteries just distally to the aortic valves.

Sketch.

Now remove the outer walls of both ventricles by making first a transverse incision along the base of each, and then, from its extremities, converging incisions nearly to the apex of the heart. Make out:-

4. The relative thickness of the walls of the right and left ventricles, the form of the septum ventriculorum, the cavities of the two ventricles, and the muscular ridges in their walls. 
5. The flaps of the tricuspid and bicuspid valies and their tendinous cords and papillary muscles (Fig. I 39).

6. The apertures of the aorta and pulmonary artery into the left and right ventricle respectively.

Sketch.

\section{F. Dissection of the head.}

I. Carefully dissect the skin away from one side of the head ; either side will do, but if you are using the head from which you have already removed the brain, choose the side on which the auditory region is least damaged : notice the Meibomian glands (p. 535). Cut away, with bone-forceps, the supraorbital process of the frontal, being careful not to injure any of the contents of the orbit. Note-

I. $a$, i large mass of muscle (masseter) covering the posterior half of the mandible, on which branches of the facial nerie will be seen; $b$, the thin irregular parotid gland, at the base of the pinna, and the large infraorbital gland lying mainly within the orbit below (p. 5 I 2).

2. The four recti and the two oblique muscles of the eye. Note the course of the superior oblique through its tendinous loop (p. 534).

3. The Iacrymal and Harderian glands, situated in the postero-superior and antero-inferior regions of the orbit respectively.

II. Remove the eveball by cutting through the muscles and optic nerve, noting the retractor bulbi muscle around the latter. (For the dissection of the eye, see p. 555). Cut off the pinna, and clear away the muscles, \&c., covering the tympanic bone: remove the entire tympanic and periotic bones ( $\mathrm{p} .492)$ in one piece, lay open the external auditory passage, and very carefully and gradually cut away the outer wall of both tubular and bulbous portions of the tympanic with the bone-forceps. Note:-

I. The tympanic membrane and the handle of the malleus; and after cutting away the former so as not to injure the latter, and removing rather more of the bulla, make out (Fig. I 43) -

2. The tympanic cai ity; the malleus, incus, and stapes; the tensor tympani and stapedius muscles; and the aperture of the Eustachian tube. Remove the auditory ossicles, noting as you do so the fenestra oralis and fenestra rotunda. and examine them under the low power of the microscope : by cutting through the periotic with the bone-forceps, the position of the membranous labyrinth, including the cochlea, may be made out. 
III. Dissect off the muscles covering the mandible on the side you are working; detach its ascending portion from the muscles inserted on its inner surface, remove it entire, cutting through the symphysis, and clear away the underlying muscles. Pass a probe from the cut end of the gullet forwards into the mouth : lay open the gullet along this, and pull the tongue downwards, so as to get a view into the interior of the mouth (Fig. 135). Note :-

I. $a$, The palate and relum palati; $b$, the tongue and its papilla (p. 507); $c$, the pharynx; $d$, the glottis and epiglottis; $e$, the continuation of the pharynx above the velum palati (nasopharyn $x$ ), with which the internal nostrils communicate; $f$, the positions of the teeth (incisors and grinders); $g$, the apertures of the naso-palatine canals.

With a fine saw, make a longitudina! vertical section of the entire head, cutting very slightly to that side of the median line on which you are working, so as not to injure the cartilaginous nasal septum. Pin down and finish dissecting under water, noting again the parts just referred to $(\S \mathrm{I})$ and also-.

2. The cranial cavity, the roof of which will have been cut away if the brain has been removed.

3. The aperture of the Eustachian tube : pass a probe from it into the tympanic cavity.

4. The position of Jacobson's organ, on the ventral side of the nasal septum; and after cutting away the latter, the olfactory nerve, ethmo-turbinal, maxillo-turbinal and nasoturbinal of the other side (pp. 493 and 534, Fig. 135).

Sketch the entire longitudinal section.

IV. Remove the larynx with the anterior part of the trachea. Make a longitudinal vertical section of it, keep one half entire, and from the other dissect away the muscles and mucous membrane so as to see the cartilages clearly. Examine first the cartilages and then the soft parts, noting the relations of the thyroid, cricoid, and arytenoid cartilages, the epiglottis, and the vocal cords (p. 5I7). Sketch.

\section{G. The Brain.}

I. External characters. Note (Fig. If I) :-

I. The medulla oblongata, with its dorsal and ventral fissures and the pons Varolii; the convoluted cerebellum, consisting of a central lobe and two lateral lobes and flocculi.

2. The optic lobes, seen on slightly separating the hemispheres and cerebellum; the crura cerebri.

3. The olfactory lobes; the cerebral homispheres (frontal, 
parietal, and temporal lobes)-by gently separating them, the corpus callosum will be seen, and just behind then the pineal bodv; the diencephalon is covered above by the hemispheres, below it is visible and is continued into the infundibulum; the pituitary body is nearly always left behind in the brain-case when the brain is removed, and a small aperture is seen in the centre of the infundibulum where it was attached; the optic chiasma.

4. The origins of the nerves from the brain; these cannot, of course, be plainly made out unless the nerves have been carefully cut through when removing the brain. Compare pp. I63-166, and note in addition the spinal accessory and hypoglossal.

Sketch the brain from above and from below.

II. Carefully remove successive slices from one of the hemispheres nearly para!lel to its upper surface, down to the level of the corpus callosum, noting the fibres of the latter and the relations of the grey and white matter in the hemispheres. Cut through the corpus callosum longitudinally, near the middle line, and remove sufficient of the hemisphere already dissected to expose the lateral ventricle and its extensions into the frontal, parietal, and temporal lobes. Note :-

I. The hippocampus, the corpus striatum, the choroid plexus, and the septum lucidum (p. 53r). Then cut away the rest of the same hemisphere so as to expose-

2. $a$, The diencephalon and its two lateral halves (optic thalami) between which the third rentricle is enclosed; from its roof the stalk of the pineal body arises $; b$, the two pairs of optic lobes. Cut away the lateral half of the cerebellum of the same side, and note-

3. The rali'e of Vieussens, the fourth rentricle, and the tree-like appearance (arbor vitce) of the cerebellum in section.

III. Now reduce the whole brain to a longitudinal vertical section (Fig. 142), cutting it through with a sharp knife very slightly to that side of the median line which you have already dissected, so as to expose the median ventricles in section. Make out, on the uninjured half, the relations of the third ventricle, iter, fourth ventricle, foramen of Monro, infundibulum, optic chiasma, corpus callosum and fornix, lamina terminalis, anterior, middle, and posterior commissure, crura cerebri, valie of Vieussens, pons Varolii, Ec. Sketch.

IV. A series of thick transverse sections should be made through another thoroughly hardened brain, and the relations of the parts already seen by dissection examined. 


\section{H. The Eye.}

On account of its larger size, it is better to substitute the eye of an ox or sheep-which can be obtained fresh from a butcher-for that of the rabbit. Two specimens should be obtained, and the fat, muscles, and any portions of the eyelids remaining attached cleared away. Dissect under water.

I. After noting the conjunctiva, cornea, sclexotic, optic nevve, iris, and pupil, insert the scissors into the margin of the cornea, and cut round and remove it so as to expose the aqueous chamber, noting the aqueous humour, as well as the iris, lens, and pupil (compare Fig. 57). Sketch.

Insert the handle of a scalpel between the iris and sclerotic and separate off the latter from the choroid around the outer half of the eyeball, first making four radial cuts in the sclerotic, beginning at its margin, at equal distances from one another. Turn back the four flaps thus made, and insert pins through them so as to fix the eye firmly down under water with the iris uppermost : note the ciliary muscle around the outer margin of the iris, the choroid, and the ciliary vessels and nevves. Then make two radial cuts, a short distance from one another, through the iris, and turn back the portion between the cuts, noting the radially arranged ciliary processes. Sketch.

Cut through the choroid and the parts enclosed by it, horizontally, around the line up to which it has been already exposed, so as to separate the eyeball into an inner and outer hemisphere: in doing so, the gelatinous vitreous humour must be cut with the scissors. Examine and sketch both sections, noting in the outer hemisphere-the retina, stopping short at the outer margin of the ciliary processes, and the lens with its capsule; and in the inner hemisphere-the retina with its blood-vessels, and the blind-spot (or point of entrance of the optic nerve). Then remove the retina and observe the choroid with its iridescent tapetum.

2. Cut your second specimen into vertical halves with the scissors, making the cut pass through the cornea, pupil, and optic nerve. As it is difficult to cut through the lens without disturbing its relations, it should be carefully separated from one half, and left entire on the other half. Examine the relations of the parts once more, compare Fig. 57, and sketch.

\section{Dissection of typical flexor and extensor museles and joints of the fore-limb.}

I. Expose the biceps (chief flexor of the fore-arm). Its 
origin is from the anterior edge of the glenoid cavity on the pectoral arch : it arises by a single long tendon, working in the bicipital froove of the humerus. It is spindle-shaped, consisting of a single belly, and is inserted on the proximal end of the radius.

2. Expose the triceps (chief extensor of the fore-arm). It arises by three main heads from the pectoral arch and humerus, and is inserted on the olecranon-process of the ulna.

3. Remove the muscles from the shoulder-joint, and note the capsular ligament (p. 55). Cut through this so as to open the synorial capsule, and note the synorial membrane and fuid and the cartilaginous articular surfaces of the glenoid cavity and head of the humerus respectively : or, prepare as directed on p. 64 in the case of the hip-joint of the frog.

4. As an example of a muscle with a multiple insertion, dissect out the extensor communis digitorum (dorsal). It arises from the distal end of the humerus, and at the distal end of the fore-arm divides into four tendons, which pass through the annular ligament to digits $2-5$ and are inserted on the middle and distal phalanges. Note the sesamoid bones on the palmar side of the joints of the digits.

\section{$J$. Side Dissection.}

Obtain another rabbit, and make a dissection from the side (compare Fig. 135) as directed in the case of the dogfish and frog (p. 480). Nake out the structure and relations of all the principal organs once more, and sketch your dissection.

\section{$K$. Transverse sections.}

The examination of transverse sections is most easily and satisfactorily done by preparing microscopical sections of a fotus not more than a couple of inches in length, as directed in the case of the young dogfish (p. +80$)$ : a fotal rat or mouse will answer the purpose equally well.

With a sharp knife, cut transverse sections, about th inch thick, from the following regions: $a$, snout; $b$, cranial region; $c$, neck; $d$, thorax; and $e$, abdomen. Stain and embed, and then cut and mount one or two sections of each, examining them with a low power and making sketches.

Then put on the high power, and make out as much as possible of the histology of the various organs. 


\section{CHAPTER XII}

THE MINUTE STRUCTURE OF CELLS : CELL-DIVISION : STRUCTURE OF THE OVUM : GAMETOGENESIS (SPERMATOGENESIS AND OOGENESIS) : MATURATION AND FERTILISATION OF THE OVUM: DIFFERENT TYPES OF OVA AND OF SEGMENTATION-EFFECT OF FOOD-YOLK ON DEVELOPMENT

Structure of the Cell.-We have learnt in previous chapters that all organisms are formed essentially of one or more cells (compare e.g. pp. IIo and II 8 ) ; that the cell consists of protoplasm, and contains a nucleus, enclosing chromatin and usually one or more nucleoli (p. 129) ; and that cells multiply by a process of binary fission (pp. ro6, 236). It will now be necessary to study the structure of a typical animal-cell and of its mode of division in somewhat greater detail.

There seems to be a good deal of variation in the precise structure of various animal- and plant-cells, but the more recent researches show that in the cell-body or cytoplasm two constituents may be distinguished-a clear semi-fluid substance, traversed by a delicate spongework. Now under the microscope the whole cell is not seen at once, but only an optical section of it-that is, all the parts which are in focus at one time (see e.g. 
Fig. 7I, B, and p. 323): by altering the focus we view the object at successive depths, each view being practically a slice parallel to the surface of the lenses. This being the case, protoplasm presents the microscopic appearance of a clear or slightly granular matrix traversed by a delicate network (Fig. It6). In the epithelial cells of animals the protoplasm is bounded externally by a very delicate cell-membrane: in many amœboid cells the ectoplasm, or transparent, non-granular portion of the

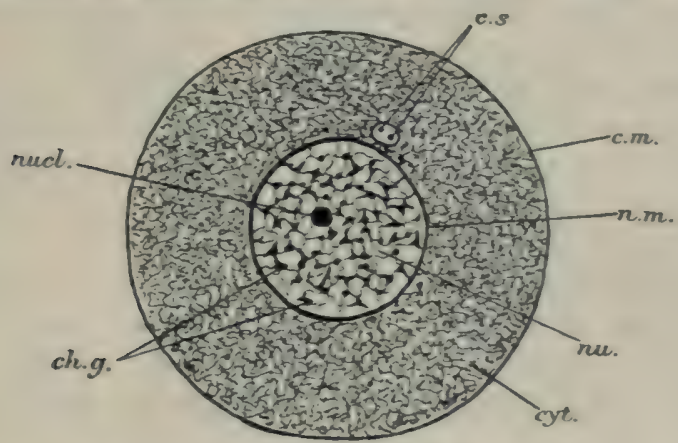

FIc. I\$6-Diagram of a typical cell.

ck. g. chromatin granules; $c$. $m$. cell-membrane; $c$. s. centrosomes lving in centrosphere: cyt. cytoplasm : n. m. nuclear membrane; nu. nucleus ; nucl. nucleolus. (From Dendy's Outlue's of Evolutionary Biology.)

cell, consists of clear protoplasm only, the granular endoplasm alone possessing the sponge-work.

It is quite possible that the reticular character of the cell may be merely the optical expression of an extensive but minute vacuolation, or may be due to the presence of innumerable minute granules developed in the protoplasm as products of metabolism.

The nucleus is spherical or oval in form, and is enclosed in a delicate nuclear membrane (Figs. I 46 and I 47 . A). Its contents (nucleoplasm) consist of a homogeneous semi-fluid substance throughout which an extremely 
fine network of threads extends : this is called the linin network, and in it are embedded granules of chromatin (ch. $g, c h r)$, which are distinguished by their strong affinity for aniline and other dyes (compare e.g. Part I, Chaps. VII and VIII). Frequently, as we have seen, one or more minute globular structures, the nucleoli (nucl, $k k$ ), occur in the nucleus and are usually looked upon as transient structures and not as actual living parts of the cell : they also have a strong affinity for dyes although often differing considerably from the chromatin in their micro-chemical reactions.

Close to the nucleus a small globular body, called the centrosphere $(c s p h)$, enclosing a minute particle or centrosome, is present in the cell ; it is inconspicuous and often difficult to recognise, but probably occurs in all animalcells which are capable of division.

Cell-division.-The precise changes which take place during the fission of a cell are, like the structure of the cell itself, subject to considerable variation. We will consider what may probably be taken as a typical case (Fig. I47).

First of all, the chromatin granules (chr) come together so as to form a loose coil or skein, the spireme (B). The centrosome and then the centrosphere (csph) divide, and from the latter fine cytoplasmic filaments are seen to radiate ; the products of its division gradually separate from one another (C), ultimately passing to opposite poles of the nucleus (D), delicate protoplasmic threads extending from one to the other in the form of a spindle $(k s p)$. At the same time, the nuclear membrane and the nucleoli usually disappear, and the chromatin skein divides into a number of separate pieces of equal length called chromosomes (C, chrs), the form of which varies in different cases : they may be spherical, rod-shaped, or $\mathrm{V}$-shaped, and, in general, their number is constant in 


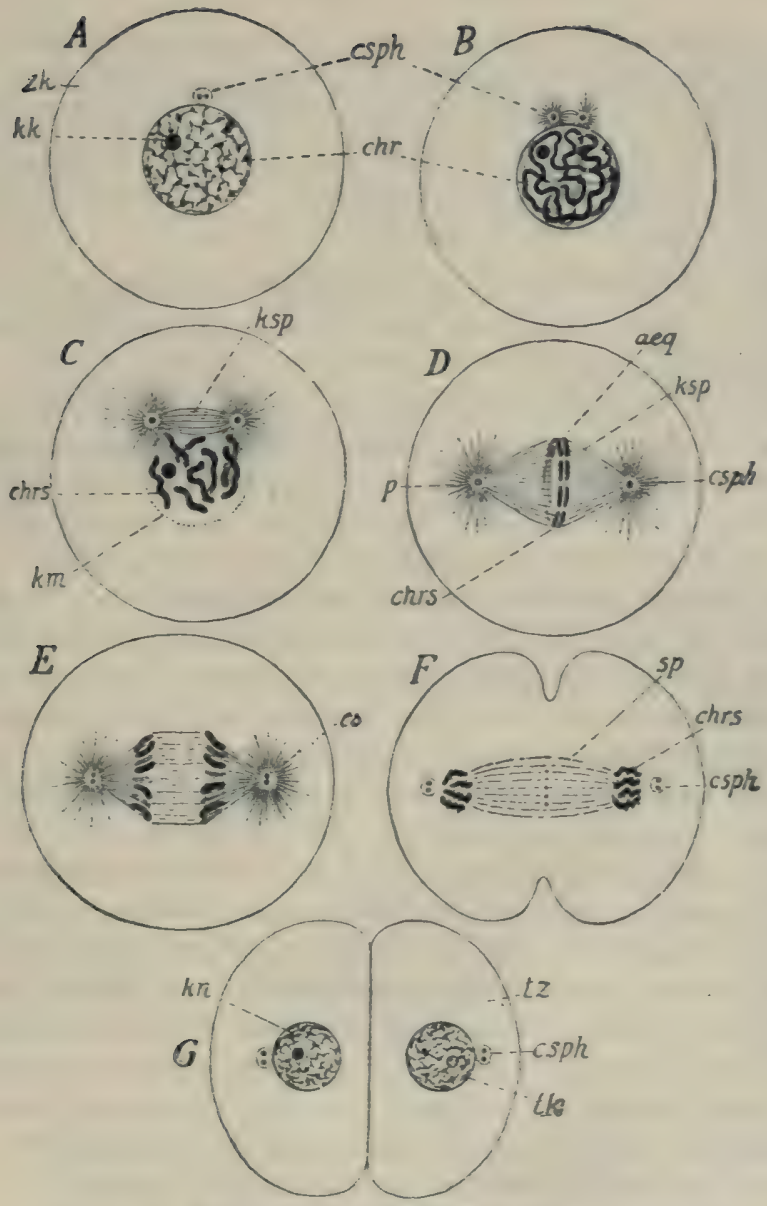

Fic. 147.-Diagram of nuclear division.

$A$, resting-cel, with cytoplasm $(s k)$, centrosphere $(i s p h)$ which contains two centrosomes, nucleolus $(k k)$, and chromatin $(c h r)$, the last distributed in the linin network; the whole nucleus is surrounded by a nuclear membrane. $B$, the chromatin united in a coiled thread; the centrosphere divided in to two and giving off rays which unite the two. $C$. the nuclear spindle (ksp) formed, the ravs more strongly developed, the nuclear membrane (km) in process of dissolution, the chromatin thread divided into eight similar chromosomes (chrs), the rays 
becoming attached to the chromosomes. $D$, perfected nuclear spindle with the two centrospheres at the poles $(c s p h)$ and the eight chromosomes (chrs) in the equator of the spindle, all now longitudinally split. $E$, daughter chromosomes diverging from one another, but still united by filaments ; the centrosomes $(c s)$ are already doubled for the next division. $F$, daughter chromosomes, quite separated from one another, are already beginning to give off processes, only the four of one aspect of the spindle being indicated ; the cell-body is beginning to be constricted. $G$, end of the process of division; two daughter-cells $(t z)$ with similar nuclear network $(t k)$ and centrospheres $(c s p h)$ as in $A$. (From Weismann's Evolution Theory, adapted from E. B. Wilson.)

any given species of animal or plant, although it may vary in different species from two to a hundred and eighty-six or more.

The chromosomes become arranged in the equatorial plane of the spindle and each of them splits along its whole length, so as to form two parallel rods or loops in close contact with one another (D), and arranged in a radiating manner so as to present a star-like figure or aster when the cell is viewed in the direction of the long axis of the spindle: in this way the number of chromosomes is doubled, each one being represented by a pair. Everything is now ready for division, to which all the foregoing processes are preparatory.

The two chromosomes of each pair now gradually pass to opposite poles of the spindle (E), two distinct groups being thus produced and each chromosome of each group being the twin of one in the other group. The mechanism by means of which this is effected is not definitely known: possibly the fibres of the spindle are the active agents in the process, the chromosomes being dragged in opposite directions by their contraction: on the other hand, it is possible that the centrosomes may possess an attractive force, or that the movement is due to the contractility of the chromosomes themselves.

After reaching the poles of the spindle (F) the chromosomes of each group unite with one another to form a network around which a nuclear membrane finally makes its appearance $(G)$. In this way two nuclei are produced within a single cell, the chromosomes of the daughter- 
nuclei, as well as their attendant centrosomes, being formed by the binary fission of those of the mother-nucleus.

But pari passu with the process of nuclear division, fission of the cell-body is also going on. This takes place by a simple process of constriction ( $F, G)$ - in much the same way as a lump of clay or dough would divide if a loop of string were tied round its middle and then tightened.

In comparatively few cases the dividing nucleus instead of going through the complicated processes just described divides by simple constriction, but this seems to occur only in the case of certain highly differentiated cells or of wornout cells. We have therefore to distinguish between direct and indirect nuclear division. 1

From what we have now learnt in this chapter as well as in previous ones about the nucleus (compare e.g. p. 237) and its complicated mode of division, we conclude that it is of prime importance in regulating the life and determining the characters of the cell, and that the chromatin is probably the substance on which these characters depend.

In this connection the reader will not fail to note the extreme complexity of structure revealed in cells and their nuclei by the highest powers of the microscope. When the constituent cells of the higher animals and plants were discovered, during the early years of last century, by Schleiden and Schwann, they were looked upon as the ultima Thuie of microscopic analysis. Now the demonstration of the cells themselves is an easy matter, the problem is to make out their ultimate constitution. What would be the result if we could get microscopes as superior to those of to-day as those of to-day are to the primitive instruments of a hundred years ago, it is impossible even to conjecture.

1 To the latter very elaborate method the name mitosis or karyokinesis is applied: direct division is then distinguished as amitosis. 
Strueture of the Ovum.-The striking general resemblance between the cells of the higher animals and entire unicellular organisms has been commented on as a very remarkable fact : there is another equally significant circumstance to which we must give our attention.

All the higher animals begin life as an egg, which is either passed out of the body of the parent, as such, as in earthworms, crayfishes, frogs, birds, \&c. (oviparous forms), or undergoes development in the body of the parent, as in some dogfishes (p. 470) and nearly all mammals (viviparous forms).

The structure of an egg is, in essential respects, the same in all animals from the highest

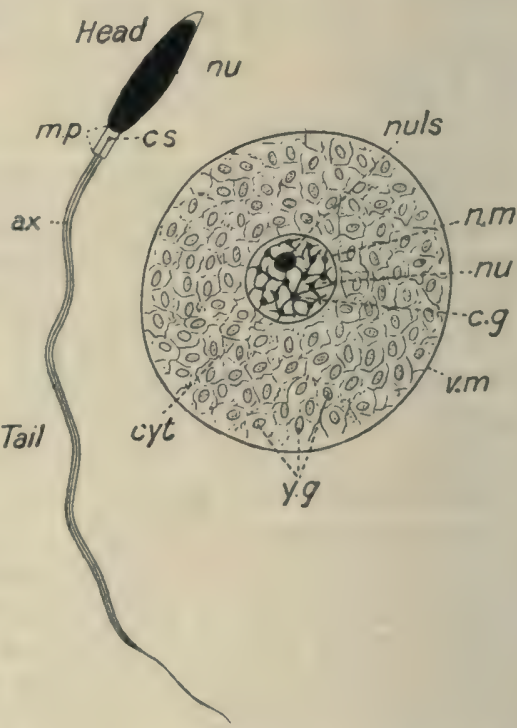

F1G. 148.-Diagram of typical sperm and ovum, the former much more magnified than the latter. ax. axial filament; c.g. chromatin granules; cs. centrosome ; $c y t$. cytoplasm; $m . p$. middle piece ; n.m. nuclear membrane; nucleolus; $v . m$. vitelline membrane; $y . g$. yolkgranules. (From Dendy's Evolutionary Biology.)

to the lowest (compare p. I95). It consists (Fig. I48) of a more or less globular mass of protoplasm $(c y t)$, spoken of as the vitellus, in which are deposited proteinaceous particles known as yolk-granules $(y \cdot g)$. Within the protoplasm is a large nucleus containing chromatin as well as one or more nucleoli $(n u, n u l s)$ - which are often known 
as germinal spots, the entire nucleus of the ovum being sometimes called the germinal vesicle. In other words the egg, as we have already seen, is a cell. An investing vitelline membrane $(v . m)$ is usually present, though often only formed after fertilisation.

The young or immature ova of all animals present this structure, but in many cases certain modifications are undergone before the egg is fully formed. For

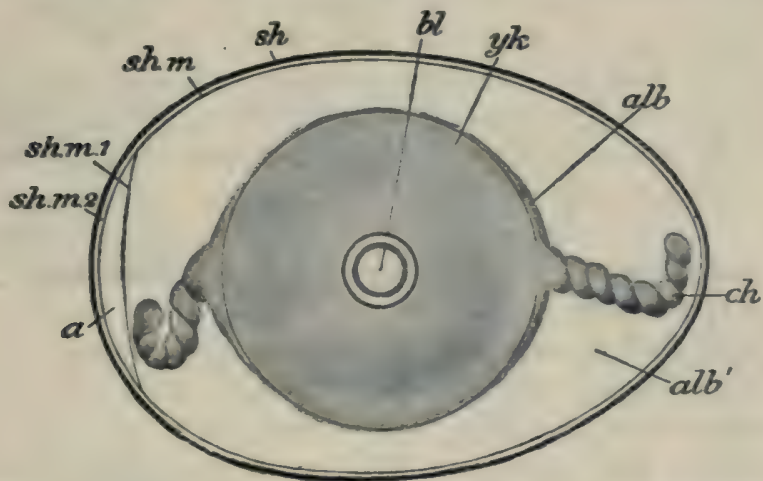

FIG. 149.-Semi-diagrammatic view of the egg of the Fowl at the beginning of incubation. (Nat. size.)

a. air-space; alb. dense layer of albumen; $a l b b^{\prime}$ more luid albumen ; $b l$. blastoderm ; ch. chalaza, a twisted cord of the dense layer of albumen at either end of the egg, formed as the latter rotates down the oviduct: $s h$, shell: $s h . m$. shellmembrane; sh. m. $1, s h . m$. 2, its two layers separated and enclosing air-cavity. (From Parker and Haswell's Zoology, after Marshall, slightly altered.)

instance, the protoplasm may throw out pseudopods, the egg becoming amceboid (p. 307); or, as mentioned above, an investing membrane may be secreted by the egg, or by the follicular cells : this is often of considerable thickness, and may be perforated at one pole by an aperture, the micropyle (p. 4I5 and Fig. I5I, B, C). The most extraordinary modification takes place in some Vertebrata, such as dogfishes (p. 470) and birds. In a hen's egg, for instance (Fig. I49), the yolk-granules 
increase immensely, swelling out the microscopic ovum until it becomes what we know as the "yolk" "of the egg : around this layers of albumen or "white" are deposited by the glands of the oviduct, and finally the double shell-membrane and the shell. Hence we have to distinguish carefully in eggs of this character between the entire "egg" in the ordinary acceptation of the term, and the ovum or egg-cell. But complexities of this sort do not alter the fundamental fact that all the higher animals begin life as a single cell; or in other words, that multicellular animals, however large and complex they may be in their adult condition, originate as unicellular bodies of microscopic size; and the same is the case with plants.

\section{Gametogenesis (Spermatogenesis and Oogenesis).- - In} the preceding chapters it has more than once been stated that sperms arise from undifferentiated epithelial cells in the spermary, and that ova are produced by the enlargement of similar cells in the ovary. Fertilisation has also been described (p. I97) as the conjugation of two different kinds of gametes, the ovum and sperm (compare p. 573). We have now to consider in greater detail what is known as to the precise mode of development of sperms (spermatogenesis) and of ova (oogenesis), as well as the exact steps of the process by which an oosperm or unicellular embryo is formed by the union of the two sexual elements.

Both ovary and spermary are at first composed of cells of the ordinary kind, the primitive germ- or sex-cells ; and it is only by the further development of these that the sex is determined.

In the spermary the sex-cells (Fig. I50, A) undergo repeated fission, forming what may be called the spermmother-cells (B) or male gametocytes (pp. 287 and 348). 
In these, as well as in the egg-mother-cells (p. 569), the nucleus, instead of dividing in the ordinary way, as described on p. 560 , undergoes a special kind of division.

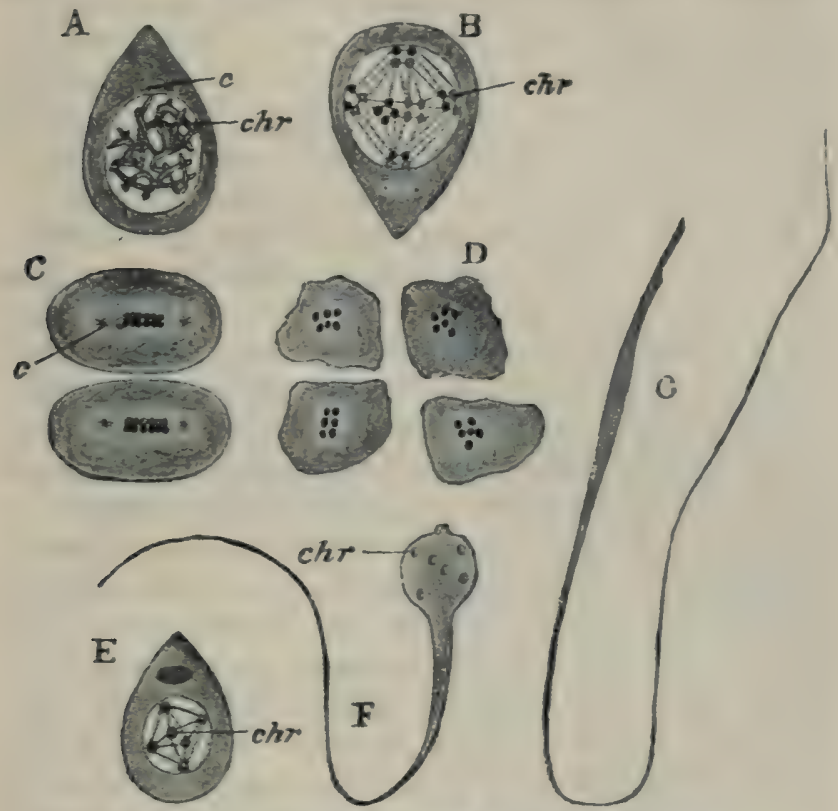

F1G. I50.-Spermatogenesis in the Mole-cricket (Gryllotalpa).

A, primitive sex-cell, just preparatory to division, showing twelve chromosomes arranged in tetrads $(c h r) ; c$. the centrosnme. B, sperm-mother-cell. formed by the division of $\mathrm{A}$, and containing twent $\mathrm{y}$-four chromosomes arranged in tetrads; the centrosome has divided into two. C, the sperm-mother-cell has divided into two, each daughter-cell containing twelve chromosomes. D, each daughtercell has divided asain, a group of four sperm-cells being produced, each with only six chromosomes. E, a single sperm-cell about to elongate to form a sperm. F, immature sperm; the six chromosomes are still visible in the head. G, fully-formed sperm. (From Parker's Biology, after vom Ratb.)

We saw that the number of chromosomes is in general constant in any given animal, though varying greatly in different species. Now in the nucleus of the spermmother-cells the chromosomes become applied close 
together in pairs, and the two members of each pair undergo longitudinal division, so that a group of four (tetrad) is formed from each pair. Thus, supposing the number of chromosomes in the ordinary cells of the body, including the primitive sex-cells, to be twelve (Fig. I50, A), there will be six groups, each containing four chromosomes, i.e., twenty-four in all, or double the number characteristic of the species (B). As the spindle is formed, these tetrads become arranged on its equator, two chromosomes from each group then passing to one pole and two to the other. The cell now divides, and the nucleus of each daughter-cell thus contains twelve chromosomes (C). The process of division, without a return to the spireme stage (p. 56o), is then repeated in each; but the chromosomes this time do not split longitudinally, six of them passing entire to each pole of the spindle respectively. Cell-division follows, and it will be seen that each of the four cells derived from the original mother-cells contains only six chromosomes (D), i.e., half the normal number, and the process is therefore known as a reducing division. The four cells thus produced are the immature sperms $(\mathrm{E})$ : in the majority of cases the protoplasm of each undergoes a great elongation, being converted into a long vibratile thread, the tail of the sperm (F, G), while the nucleus constitutes its more or less spindle-shaped head and the centrosome is included in a small middle piece at the junction of head and tail (Fig. I48).

Thus the sperm or male gamete is a true cell specially modified in most cases for active movement. This actively motile, tailed form is, however, by no means essential: in some animals (c.g. crayfish, p. 382) the sperms are non-motile and of peculiar form.

The ova arise from primitive sex-cells, precisely resembling those which give rise to sperms. They 
divide and give rise to the egg-mother-cells, or female gametocytes, which do not immediately undergo division, but remain passive and increase in size by the absorption of nutriment from surrounding parts : in this way each egg-mother-cell gives rise to an orum. Sometimes this nutriment is simply taken in by diffusion or osmosis. or by means of protoplasmic connection between the orum and the follicle-cells; in other cases the growing ovum actually ingests neighbouring cells after the manner of an Amœeba. Thus in the developing egg the processes of constructive are vastly in excess of those of destructive metabolism.

We have seen (p. 249) that the products of destructive metabolism may take the form either of waste products which are got rid of, or of plastic products which are stored up as an integral part of the organism. In the developing egg, in addition to the increase in the bulk of the protoplasm itself, a formation of plastic products usually goes on to an immense extent. In plants the stored-up materials may take the form of starch, of oil, or of proteid substance: in animals it consists, as mentioned above, of rounded or angular grains of proteid material, known as yolk-granules. These being deposited, like plums in a pudding. in the protoplasm, have the effect of rendering the fully-formed egg opaque, so that its structure can often be made out only in sections.

Before, however, the ovum is ripe for conjugation with a sperm or able to undergo the first stages of segmentation it has to go through a process known as maturation, which consists essentially in a twice repeated process of cell-division. and thus resembles the process just described in the case of the sperms.

Let us take a case,.as before, in which the number of chromosomes characteristic of the species is twelve. 
These, in the egg-mother-cells, become arranged in pairs, and each splits longitudinally, so that six tetrads are formed. The nucleus, which thus contains double the normal number of chromosomes, loses its membrane, travels to the surface of the egg, and a spindle is formed. Next the protoplasm grows out into a small projection or bud, into which one end of the spindle projects (Fig. I5I, A). Nuclear division then takes place, without a splitting of the chromosomes, one of the daughter nuclei remaining in the bud ( $p o l)$, the other in the ovum itself, each of these nuclei containing twelve chromosomes. Then follows, as usual, a division of the protoplasm, and the bud becomes separated as a small cell distinguished as the first polar cell.

In some cases development from an unfertilised female gamete takes place, the process-which occurs in various invertebrate groups and is not uncommon during a greater part of the year among insects (e.g. the common little green plant-louse or Aphis) and crustaceans (e.g. water-fleas)being distinguished as parthenogenesis. It has been shown that in many such cases the egg begins to develop after the formation of the first polar cell, when the ovum contains the number of chromosomes normal to the species.

As a general rule, development takes place only after fertilisation, and a second polar cell $(\mathrm{B}, \mathrm{pol})$ is formed from the ovum, a reducing division occurring just as in the case of the sperm-cells (p. 568), so that the ovum and the second polar cell each contain only six chromosomes. Thus the ovum has now lost a portion of its protoplasm together with three-fourths of its chromatin, half having passed into the first polar cell and half of what remained into the second: the remaining onefourth of the chromatin becomes enclosed in a nucleus which is distingnished as the female pronucleus ( $\mathrm{B}$, $q$ pron). The first polar body has in many cases been 

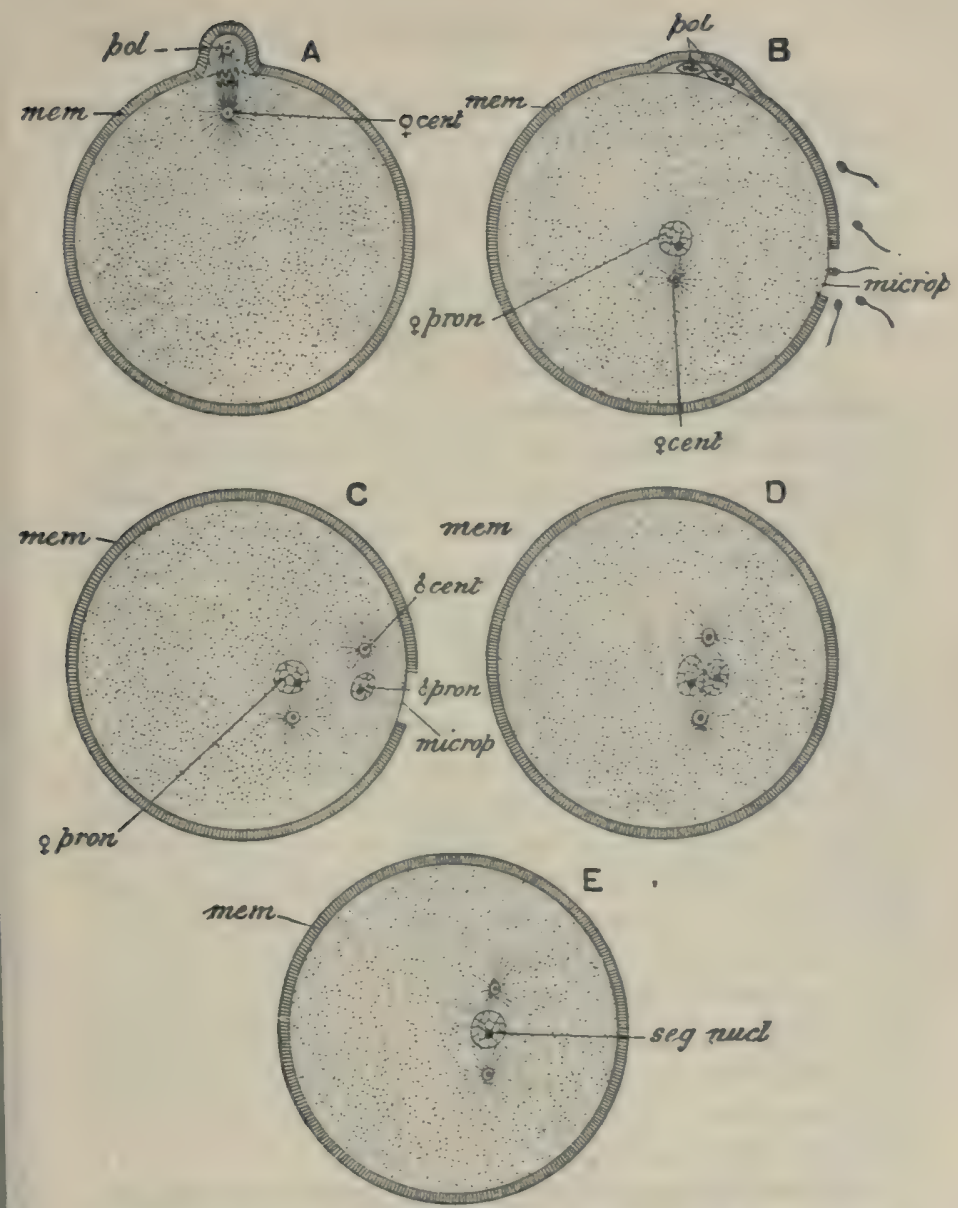

Fig. I51.- The maturation and impregnation of the animal ovum (diagrammatic). $A$, the ovum, surrounded by the vitelline membrane (mem), in the act of forming the first polar cell (pol) ; o cent. centrosome. B, both polar cells (poi) are formed, the female pronucleus ( 2 prom lies near the centre of the orum, and one of the several sperms is shown making its way into the ovum at the micropvle (microp). $\mathrm{C}$, the hear of the sperm has become the male pronucleus ( 3 pron), its middle piece the male centrosome ( 3 cent) ; other structures as before. D, the male and female pronuclei are in the act of conjugation. E, conjugation is complete, and the segmentation nucleus (seg. nucl) formed. (From Parker and Haswell's Zoology.) 
observed to divide after separating from the egg, so that the egg-mother-cell or immature ovum gives rise to a group of four cells-the mature ovum, and three abortive ova or polar cells which develop no further, ${ }^{1}$ just as the sperm-mother-cell gives rise to a group of four cells, all of which, however, become sperms (Fig. I5o).

Fertilisation of the Ovum.- Shortly after maturation, the ovum is fertilised by the conjugation with it of a single sperm. As we have found repeatedly, sperms are produced in vastly greater numbers than ova, and it often happens that a single egg is seen quite surrounded with sperms, all apparently about to conjugate with it (Fig. I5I, B). It has, however, been found to be a general rule that only one of these actually conjugates: the others, like the drones in a hive, perish without fulfilling the one function they are fitted to perform.

The successful sperm (B) takes up a position at right angles to the surface of the egg and gradually works its way into the ovum, passing through the micropyle (microp) when present, until its head lies within the egg-protoplasm. The tail is then lost, and the head, accompanied by the centrosome (sce p. 568 and Fig. I 8 ), penetrating deeper into the protoplasm, takes the form of a rounded body, the male pronucleus ( $\mathrm{C}$, of pron). The centrosome of the ovum disappears, that of the sperm dividing subsequently.

The two pronuclei approach one another (D) and finally unite to form what is called the conjugationor segmentation-nucleus ( $\mathrm{E}, \mathrm{seg}$. nucl), the single nucleus of what is not now the ovum but the oosperm--the

1 It will be remembered that there is an analogous casting off of nuclear material in the process of conjugation in Paramocium (p. 268). 
impregnated egg or unicellular embryo (compare pp. 197 and 198 ).

The fertilising process is thus seen to consist essentially in the intermingling in equal proportions of chromatinmaterial derived from two conjugating cells or gametes, and the term is often restricted to those cases of conjugation in which the gametes differ from one another, so that one can distinguish between a male gamete and a female gamete, known in the Ifctazoa as a sperm and an ovum respectively. It follows from what has been said above that the essential nuclear matter or chromatin of the oosperm-often spoken of as the germ-plasm and probably constituting the material basis of heredity-is derived in equal proportions from each of the two parents. Moreover, as both male and female pronuclei contain only half the number of chromosomes found in the ordinary cells of the species, the union of the pronuclei results in the restoration of the normal number to the oosperm; and as the reducing divisions and conjugation of the germ-cells thus apparently render possible very varied permutations and combinations of the hereditary qualities of both sexes, these processes seem to have a great importance in connection with the phenomenon of individual variation (p. 216).

Fertilisation being thus effected, the process of segmentation or division of the oosperm takes place as described in previous chapters.

Different Types of Ova and of Segmentation.-Before passing on to consider further details in the process of development of the oosperm, we must briefly refer to some differences already noted in the ova of different animals.

We have seen that in all cases the immature egg is a simple, minute cell, but that, owing to the deposition of 
yolk-granules in its protoplasm, it may reach a comparatively large size (e.g. crayfish, dogfish, bird). The presence of a greater or less amount of yolk in the ovum results, as we know, in very considerable differences as regards its mode of segmentation, as well as in its subsequent development. The minute eggs of the lancelet and rabbit, for instance, which are each only $\frac{\mathrm{l}}{10} \mathrm{~mm}$. (about $\frac{1}{2} \frac{1}{50}$ inch) in diameter, contain so comparatively small an amount of food-yolk as not to interfere materially with the process of segmentation: such ova are called microlecithal. When the quantity of food-yolk is relatively greater, the eggs may be described as megalecithal: in these the yolk may become accumulated towards the centre of the egg, eventually leaving a layer of protoplasm comparatively free from yolk round the periphery (centrolecithal ova, e.g. crayfish, Fig. 98) ; or, as in the case of telolecithal ova (Figs. 64, I28, and I49), the yolk-granules may become aggregated more at the lower than at the upper pole (frog), until in the most extreme cases there is only a layer of yolkless protoplasm - the germinal disc (dogfish, bird)-lying at the upper pole of the egg.

As yolk is an inert substance, the more of it an egg contains in proportion to the amount of protoplasm, the less actively can the latter divide, and the quantity may be relatively so great in some parts as to prevent segmentation in these parts altogether. We can therefore distinguish between holoblastic oosperms, which undergo entire segmentation (e.g., hydroids, earthworm, mussel, lancelet, frog, rabbit), and meroblastic oosperms, in which segmentation is limited to that part of the egg in which the protoplasm is comparatively free form yolk (e.g., crayfish, dogfish, bird), this portion, after segmentation, being known as the blastoderm. In the centrolecithal ovum it is evident that the segmentation 
must be superficial or peripheral (p. 383), and in the meroblastic telolecithal ovum discoid, or restricted to the small germinal disc at its upper pole (Figs. I28 and I 49 ). In the case of holoblastic ova the segmenting cells or blastomeres may be equal, or nearly equal, in size (e.g., lancelet, rabbit); or if the yolk is present in greater quantities towards the lower pole, unequal (e.g., earthworm, frog).

The influence of the food-yolk in modifying the early processes of development is thus evidently very great, and in order to understand these processes in their simplest form it is necessary to select for our study a microlecithal holoblastic egg, such as that of the lancelet.

Before reading the next chapter, however, you should once more glance at the account of the development of the tadpole given on pp. 198-210, in order to refresh your memory of some of the more important facts and of the terms used.

\section{PRACTICAL DIRECTIONS}

A. Special methods are required to follow out the details of the structure and division of nuclei, but if you have not the opportunity of examining preparations illustrating this, a good deal may be made out as regards the chromatin and the changes which the chromosomes pass through in the process of mitosis by the following simple method.

Obtain a young gilled-larva or tad pole of the common newt (p. 2 I8) and place it in corrosive sublimate for about half an hour, wash thoroughly in water and transfer first into weak and then into strong alcohol (p. 137). Stain entire, either with a solution of hematorylin or of alum-carmine (p. r36).

Strip off small pieces of the skin of the stained preparation, and after putting them through weak and strong alcohol, transfer to absolute alcohol, then to turpentine, xylol, or oil of cloves, and mount in Canada balsam (p. I 39). Examine, comparing Figs. $\mathrm{I}+6$ and $\mathrm{I}+7$ and pp. 558-563, and sketch as many different stages as possible. 
B. For the structure of ova, examine again your sections of the frog's and rabbit's ovary (see pp. 2 I I and 549). Also obtain an unincubated fowl's egg, place it in a small dish or tin about $2 \frac{1}{2}$ inches deep : mix some plaster of Paris in water and pour it round the egg (first greasing the latter to prevent it adhering to the plaster) so as to cover the lower half, and then let it set. Fill the dish with normal salt-solution, and with the forceps tap the portion of the shell lying uppermost so as to fracture it, when it can be removed bit by bit. Note: $a$, the accessory parts of the egg developed from the oviduct, viz., the porous shell, the double shell-membrane-the layers of which are closely applied together except at the thicker end of the egg (see Fig. I.49), and the white or albumen, with the twisted chalaze at either end; and, $b$, the essential part (the yolk) with its vitelline membrane and circular blastoderm, which always floats uppermost.

Living sperms have already been examined (see, e.g., pp. 210 and 548 , and compare Fig. I48); the head, middle piece, and tail should be noted.

C. Observation of the details concerned in the process of maturation and fertilisation of the ovum is too difficult for the beginner ${ }^{1}$ but polar bodies may be seen in the living, freshly-laid eggs of one of the common pond-snails (e.g., Limncea stagnulis), in which also some of the stages of segmentation can easily be observed. Keep some of these snails in a glass vessel with water-weeds, and notice that the eggs, when laid, are enclosed, a number together, in a common gelatinous envelope. Separate the eggs from one another with needles, and examine in water under the microscope. Note that the small egg is surrounded by fluid enclosed in a relatively large egg-case, and observe the minute polar bodies at the periphery of the ovum.

1 The swarming of sperms round the ova previously to fertilisation can easily be observed in the common olive-brown seaweeds (Fucus), by getting fresh material and examining it in sea-water. 


\section{CHAPTER XIII}

DEVELOPMENT OF THE LANCELET : EARLY DEVELOPMENT OF OTHER TYPES, INCLUDING THE CHICK AND RABBIT : FORMATION OF THE CHIEF ORGANS OF VERTEBRATES, AND of the AMNion, ALLANTOIS, AND PLACENTA.

The oosperm of Amphioxus (Fig. 152, A) undergoes binary fission (B), each of the two resulting cells dividing again into two (C). This process is continued until a globular mass of cells or blastomeres is produced by the repeated division of the one cell which forms the starting point of the series. Owing to there being rather more yolk at the lower than at the upper pole, the lower cells are slightly larger than the upper, so that the segmentation is not quite equal (D, E). The embryo has now arrived at the polyplast or morula stage, and sections show that it is hollow, the blastomeres being arranged in a single layer around a central segmentation-cavity or blastocoele (compare p. 200): such a hollow morula is known as a blastula (F, G). The lower side of the blastula then becomes tucked in, or invaginated, the result being that the single-layered sphere is converted into a double-layered cup $(\mathrm{H}, \mathrm{I})$. This process can be sufficiently well imitated by pushing in one pole of a hollow india-rubber ball with the finger. The resulting embryonic stage is known as the gastrula: 

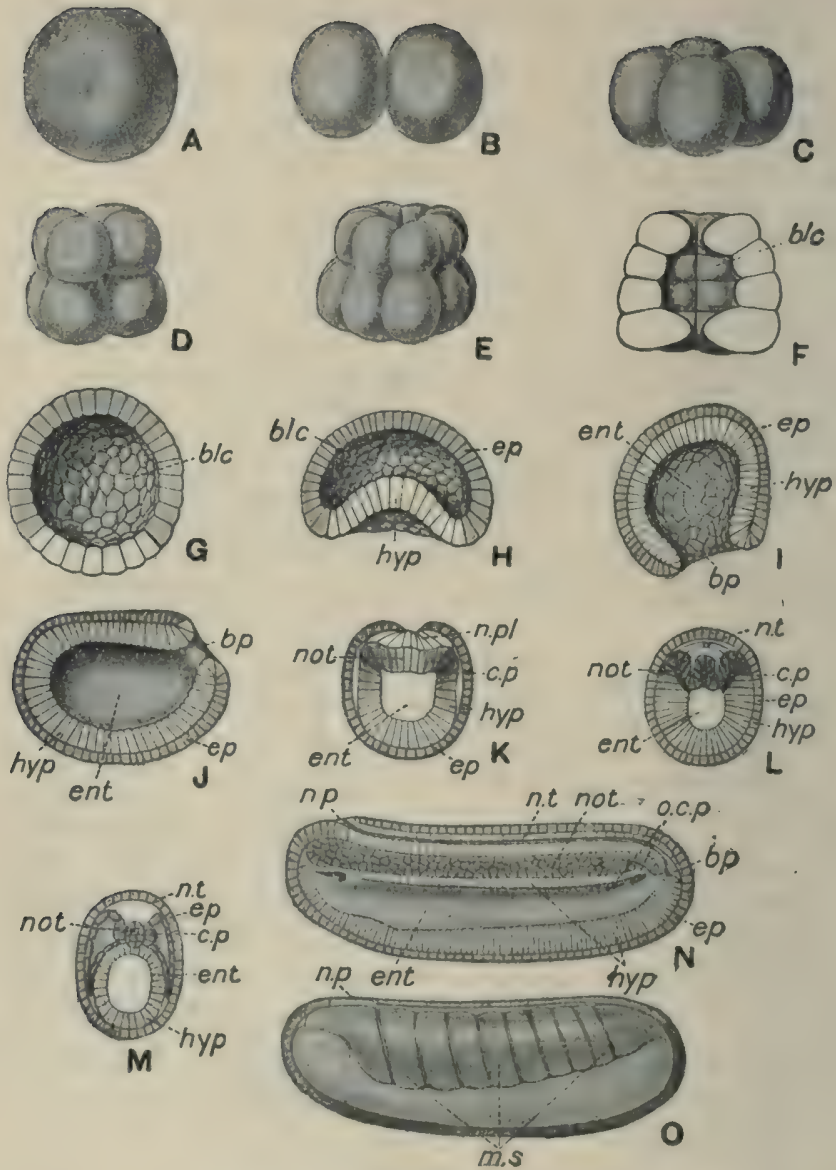

Frg. I52.-Early development of Amphioxus.

$A$, the oosperm ; $B$, stage with two blastomeres; $C$, stage with four blastomeres : $D$, stage with eight blastomeres; $E$, stage with sixteen blastotneres; $F$, stage with thirty-two blastomeres. cut in half vertically; $G$, blastula stage, cut in half ; $H$, early stage of gastrulation, cut in half; $I$, young gastrula in longitudinal section; $J$, older gastrula in longitudinal section; $K, L, M$, transverse sections of older ernbryos, showing the formation of the cielomic pouches or enterocueles, notochord, and medullary tube ; $\mathrm{N}$, longitudinal section of embryo of about the same age as $L ; O$, side view of embryo of same age as $N$, with the ectoderm stripped off from one side to show the mesodermic segments formed from the coelomic pouches. 
bp. blastopore ; blc. segmentation-cavity ; $c p$. coelomic pouch (enterocole) ; ent. enteron; $e p$. ectoderm; hyp. endoderm; m.s. mesodermic segments; not. notochord; n.p. neuropore, or anterior opening of medullary tube ; n.pl. medullary plate; n.t. medullary tube; o.c.p. openings of coelomic pouches into enteron. (From Dendy, adapted from Ziegler's models, after Hatschek.)

its cavity is the primitive enteron or archenteron (ent), and is bounded by the invaginated cells which now constitute the endoderm (hyp), the remaining cells forming the outer wall of the gastrula being the ectoderm (ep) (compare Pp. 201 and 202). The two layers are continuous at the aperture of the cup, the gastrula-mouth or blastopore $(b p)$. Between the ectoderm and endoderm is at first a space, the greatly diminished segmentationcavity, which soon becomes entirely obliterated, so that the ectoderm and endoderm are in contact. The general resemblance of the gastrula to a simplified Hydra, ${ }^{1}$ devoid of tentacles, will be at once apparent, and the stage in the development of the frog's egg represented in Fig. 64 , I, though much modified by the quantity of food-yolk (compare p. 574), will be seen to correspond to the gastrula-stage. As in the frog, the blastopore soon closes over, the mouth and anus being subsequently formed from the stomodæum and the proctodæum respectively (p. 204).

The gastrula becomes elongated, flattened on one side and convex on the other $(\mathrm{J})$. The flattened side corresponds to the dorsal surface of the adult, and the blastopore now comes to be situated, as in the frogembryo (Fig. 64, H, K), at the posterior end of the dorsal surface. A medullary or neural plate and groove (K, n.pl) are then formed, the central nervous system being developed in a manner essentially similar to that already described in the case of the tadpole (p. 202), except that

1 It must, however, be remembered (pp. 308 and 321) that the ectoderm and endoderm of Hydroids are differentiated before the mouth is formed, so that the mouth does not correspond to the blastopore of the gastrula. 
the central canal of the medullary or neural cord $(\mathrm{L}, n . t)$ is formed after the plate is separated from the outer ectoderm. In the mid-dorsal line a thickening of the endoderm $(\mathbf{K}-\mathrm{N}$, not) soon becomes constricted off to form the notochord (pp. 203, 4I9, and 44r), and on either side of this a series of hollow endodermic pouches arise from the archenteron, arranged metamerically (K-O, c.p, o.c.p, m.s). The cavities of these, which subsequently give rise to the body-cavity, are thus at first in free communication with the definitive enteron and are known as enterocoles; from their walls the mesoderm ${ }^{1}$ is derived. Subsequently the communications between the enteric and enterocolic cavities become closed, and the paired pouches gradually extend between the ectoderm and endoderm, so as eventually to meet one another both dorsally and ventrally (M), their outer walls (parietal or somatic layer of the mesoderm) being in contact with the ectoderm and forming with it the somatopleure or body-wall, and their inner walls (visceral or splanchnic layer of the mesoderm) in contact, below the notochord, with the endoderm and with it forming the splanchnopleure or wall of the enteric canal (compare p. 203). Thus the body-wall and the enteric canal are separated by a cavity, the colome, which, much as in the adult earthworm, is divided into a series of metamerically arranged portions: later on, however, the adjacent walls of these colomic sacs disappear, and the colome becomes a continuous cavity.

The embryo lancelet is hatched soon after reaching the gastrula-stage, when it moves about by means of cilia developed on the ectoderm cells, and has to get its own living, having by this time used up its small

1 The terms epiblast, hypoblast, and mesoblast are often used to designate the embryonic ectoderm, endoderm, and mesoderm respectively. 
reserve of yolk. It then passes through a complicated series of larval stages, gradually leading up to the adult form.

Early Development of other Types.-The presence of a greater amount of food-material in the egg renders it possible for the embryo to go on developing further than the gastrula-stage before being hatched, and, as a general rule, the greater the relative quantity of yolk present in the ovum of an animal, the less clearly can a gastrula-stage be recognised.

In the earthworm and mussel, it will be remembered (pp. 349 and $f(10)$, the segmentation is entire, but unequal, and the larger lower cells become invaginated to form the endoderm and enteron while the smaller upper cells give rise to the ectoderm. In the earthworm the blastopore does not become closed, but gives rise to the mouth.

In the frog (p. 20I) the enteron arises by a split appearing amongst the yolk-cells, beginning at the edge of the blastopore and gradually extending inwards: the process is supplemented by an invagination of the ectoderm. The enteron is at first a very narrow cleft, but soon widens considerably (Fig. 64, I, ent): for some time it does not actually communicate with the exterior, the blastopore $(b l p)$ being filled up by a yolkplug $(y k$. $p l)$. As the enteron extends forwards, and the relatively small segmentation-cavity (bl. coel) gradually disappears, the edges of the lower margin of the blastopore approach one another and, uniting in the median plane, give rise to a vertical, grooved streakthe primitive streak and groove, as it is called.

In the centrolecithal egg of the crayfish (Fig. (, ) a gastrula-stage is formed by invagination, but as the centre of the oosperm is filled with solid rolk in the place of a segmentation-cavity containing fluid, the invagination only extends a short distance inwards, the enteron being rela- 
tively very small and the ectoderm separated from the endoderm by the yolk.

The gastrula-stage is much less clearly distinguishable in the segmenting telolecithal eggs of the dogfish and bird (pp. 470 and 583), in which the relatively enormous mass of unsegmented yolk is, as in the crayfish, sufficient to nourish the embryo until it has reached a stage closely resembling the adult in almost every essential respect except size. A blastopore can sometimes be recognised in such cases, but in the embryo of the common fowl it is to some extent represented by a primitive streak and groove (see p. $58 \mathrm{I}$ and Fig. I53, E, $p r . s t)$. The blastoderm becomes differentiated into an outer ectoderm and an inner, lower layer of cells (compare Fig. I28), between which and the yolk the enteric cavity is formed : a segmentation-cavity is recognisable in early stages. As the embryo develops, it becomes folded off from the yolk, which is gradually reduced in amount and in later stages is contained in an extraembryonic part of the embryo-the yolk-sac (Figs. I29 and 159 ).

A more detailed account of the early stages of the chick is given below, as the fowl's egg is an easily procurable type for the practical study of development.

The fowl's egg has already been described in detail (p. 565. Fig. 149). Its essential part, the yolk, corresponds, as we have seen, to a single cell enormously enlarged by the mass of yolk-granules it contains, the only part in which they are absent being a small area which, owing to its lesser specific gravity, is always found at the upper pole; this area, in which the nucleus lies, is known as the germinal disc. Fertilisation takes place in the anterior end of the oviduct, and then the nucleus divides and initiates the process of segmentation which is completed by the time the egg is laid, when the germinal disc has thus become converted into a multicellular blastoderm (p. 574). 
The first stage of segmentation is indicated by the formation of a vertical furrow across the centre of the germinal disc (Fig. I 53, A), and this is followed by another furrow at right angles to it $(\mathrm{B})$. Further radial and concentric furrows then arise (C) until the blastoderm is seen to consist of a number of irregular cells (D). Horizontal furrows are in the meantime formed, so that the originally single lavered blastoderm, beneath which a narrow space representing the segmentation-carity can be recognised, comes to consist of several layers of cells, the outer of which corresponds to the ectoderm; the inner, or lower layer cells, extend rapidly through the segmentation-cavity between the ectoderm and the yolk and give rise to the endoderm, the space remaining beneath this laver and the rolk forming the rudiment of the enteron (F). New cells are gradually formed round the edge of the blastoderm, which, when the egg is laid, forms a circular patch, about $3.5 \mathrm{~mm}$. in diameter, on the surface of the yolk (Fig. If9): its central part, or area pellucida, owing to the presence of fluid in the underlying space, is more transparent than the rim, or area opaca.

When the egg is incubated, the blastoderm grows rapidly at its periphery (area opaca). and eventually, as we shall see, encloses the whole yolk (Fig. I59). The area pellucida extends less rapidly and becomes pear-shaped in outline (Fig. I53, E), its broader end corresponding to what will become the anterior end of the embryo : in this region most of the lower layer cells are arranged so as to form a definite layer of endoderm, which can be recognised somewhat later in the area opaca also. The ectoderm and lower laver cells are at first continuous round the edge of the blastoderm, but later on this is only the case at the posterior border (F), and results in the appearance of a narrow band, the primitive streak ( $\mathrm{E}, \mathrm{pr} . \mathrm{st}$ ), along which a median primitice grooce is formed. This, as already mentioned, represents the fused lips of the blastopore of the frog and other forms; it extends from the centre to the posterior border of the area pellucida, and rapidly grows backwards. The primitive streak is due to the multiplication of ectoderm cells, which grow inwards and spread out right and left under the covering ectoderm, between it and the endoderm. so as to form a horizontal wing of cells on either side $(\mathrm{G})$. The mesoderm is formed from these wing-like ectodermic sheets, with which the endoderm becomes united, so that the three lavers are for a time indistinguishable in this region. The mesoderm eventually forms a sheet of loosely arranged cells which spreads rapidly in all directions except anteriorly (E), in which region, known as the pro-amnion, the blastoderm consists of ectoderm and 

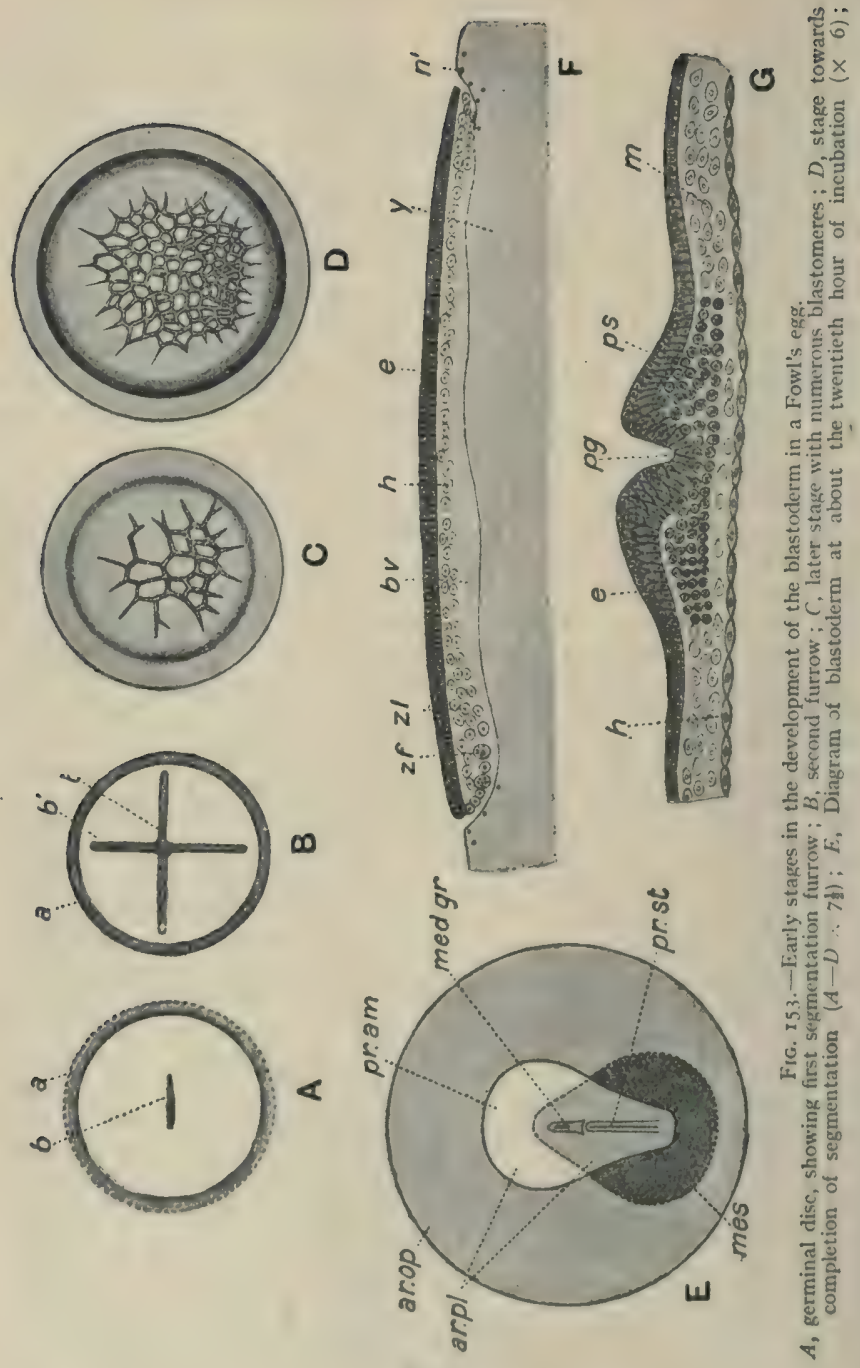

象ํ. 
$F$, vertical section of blastorlerm and adjacent part of the egg at the time of laving, before incubation: the anterior edge of the blastmerm is to the right, the postorior edge to the left of the figure $(.25):(;$, transverse section of blastoderm at about the twentieth hour of incubation, the section passing throush the primitive streak and groove at about the midile of its length (. 200).

a. periphery of germinal disc: ar. op. area opaca: ar. $p l$ area pellucida $b$. first, and $b^{\prime}$. second furrow: $b c$. rilliment of enteron; $e$. ectoderm: $h$. endoderm; $n^{\prime}$. nucleus in yolk around which a cell will be formerl later; $m$. mes. mesinlerm; med. gr. medullary grnove: pg. primitive groove; pr. am. proamaion : pr. st. primitive streak: y. yolk; $=f$. formative cell : $z l$. lower layer cells. ( $A$ and $B$, from Foster and Baifour; $C-G$, from Marshall, after Duval and Coste.)

endoderm only until the end of the second day of incubation, and by the third day the whole blastoderm has become three-layered.

Before tracing the development of the various organs derived from the three germinal layers respectively, it must be remembered that the actual chick embryo is formed from the area pellucida, and gradually becomes folded off from the yolk, while the area opaca extends over the yolk until the latter is entirely covered by it ( 1 ; th day of incubation) : thus we can distinguish between an embrionic and an extra-embryonic portion of the blastoderm. In the mesoderm of the latter portion blood-vessels are formed, so that it is then known as the area vasculosa (see Fig. I 59).

The minute egg of the rabbit (compare p. 574) and of most other mammals, although microlecithal and undergoing a holoblastic segmentation, has presumably been derived from a meroblastic type with abundant yolk, like that of the bird, and some lower mammals (Ornithorhynchus and Echidna) living in Australia at the present day still possess eggs of this type. In the higher Mammalia the yolk has disappeared, as it is no longer needed, the embryo, as we have seen ( $p .483$ ), being nourished by means of a placcnta, which will be described presently. The early processes of development are therefore somewhat peculiar, and though the segmentation is holoblastic, the subsequent development is for some time essentially similar to that of the bird: it is not until later stages that the more special characteristics of the mammal on the one hand and the bird on the other become apparent. 
Fertilisation and segmentation take place in the uterine tubes (p. 540), and each oosperm is surrounded by a membrane, the zona radiata, apparently formed from the follicle cells, and a layer of albumen secreted from the oviduct. The oosperm divides into two blastomeres (Fig. I 54, A), and then further divisions follow and result in the formation of a mulberry-like mass (B), which, however, is not hollow, as in Amphioxus (Fig. I 52), but consists of an outer layer enclosing a solid mass of larger and more granular cells (C), and at this stage it passes into the uterus. The outer layer of cells then grows rapidly, so that a space appears and gradually increases in size, between it and the inner mass except at one point where the latter is attached to the former $(\mathrm{D}, \mathrm{E})$. This hollow ball is known as the blastocyst or blastodermic vesicle, and may be compared to the embryo and yolk-sac of a bird, except that the latter contains a fluid instead of yolk. The outer layer is called the trophoblastic ectoderm or trophoblast; it probably corresponds to the extra-embryonic ectoderm of the chick (p. 585) and takes no direct part in the formation of the embryo, which arises from the inner mass (embryonic area), in much the same way as the chick is formed from the blastoderm in the case of the fowl's egg. Thus later on an embryonic portion with a primitive streak, and an extra-embryonic portion gradually growing round beneath the trophoblast, can be recognised in the embryonic area, the body of the embryo being graciually folded off from the blastocyst, which is then often known as the umbilicalvesicle and has similar relations to the embryo that the yolk-sac has to the body of the chick, to which it corresponds (see p. 582 and Figs. I 59 and I66).

In the lancelet alone amongst the triploblastic animals described in this book does the mesoderm arise as a series of enterocœelic pouches: as we have seen, it is usually at first solid, and may be budded off from the ectoderm and endoderm, or from the ectoderm only, at the margin of the blastopore or primitive groove (chick, p. 583) ; or both endoderm and mesoderm may be differentiated at the same time from the lower-layer cells or yolk-cells (frog, p. 202) ; or finally, it may arise in all these ways. The colome is formed secondarily by a split taking place in the mesoderm on either side (Figs. 65 and 155 ), the split gradually extending with the 

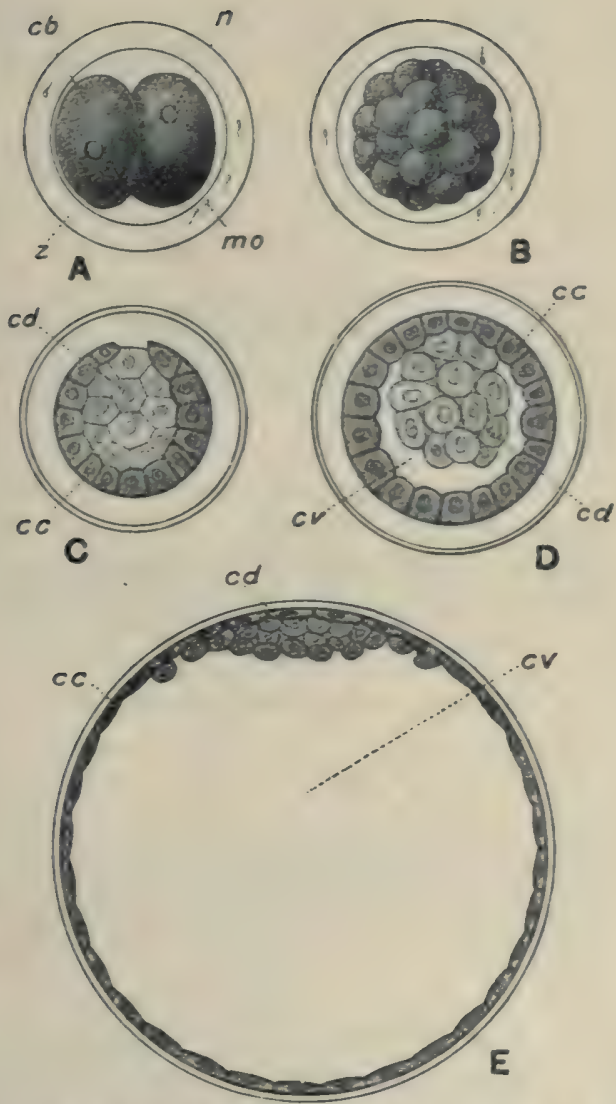

Fic. 154.--Early stages in the development of the oosperm of the Rabbit.

$A$, two-celled stage, from middle: part of the uterine tube, abcut twenty-two hours after copulation; $B$, polyplast or morula stage, from lower end of uterine tube, about the mididle of the third day : $C$. clost of sezmentation, from lower end of uterine tube just before entering uterus, seventy hours aiter copslation ; $D$. first stage in formation of blastocyst, from uterus, seventy-tive hours after copulation; $E$, section of blastocyst at the end of fourth day. (is about 160.)

$c b$, blastomere ; $c c$, trophoblastic ectoderm; $c d$, inner mass of cells (embryonic area); $c v$, cavity of blastixyst; mo, sperms imbedded in the zona radiata $(z)$ : $n$, nucleus. (From Marshall ; $A, B$, after Bischoff ; $C-E$, after Van Beneden.) 
extension of the mesoderm between the ectoderm and endoderm. Thus the colome is formed not as an enterocœle, but as a schizocoele.

In Vertebrates each mesoderm-band becomes differentiated into a dorsal portion abutting against the medullary cord and notochord known as the vertebral plate - which soon loses its colomic space, and a ventral portion, the lateral plate, which is divided into parietal and visceral layers by the colome (Figs. 65, I52, and I55). The vertebral plate undergoes metameric segmentation, becoming divided into a row of squarish masses, the mesodermal segments or "protovertebra" (pr.v, m. s, ms. s), from the outer parts of which (muscleplates, Fig. I6. $m . p$ ) the muscular segments or myomeres are formed (p. 203), and from the inner parts the vertebral column, the segmentation of which alternates with that of the myomeres (compare p. 44I).

\section{Outline of the Development of the Chief Organs in the} Craniata (compare pp. 20I-2IO).- The nervous system, as well as the essential parts of the sensory organs, are, as we have seen, in all cases formed from the ectoderm. The development of the central nervous system takes place in essentially the same manner as in the lancelet (p. 579) and frog (p. 202).

In the chick the ectoderm in front of the primitive streak early becomes thickened along the median line to form the medullary plate (Fig. $153, \mathrm{E}$ ); this gradually increases in length at the expense of the primitive streak, from the cells of which the greater part of the embryo is eventually developerl. A medullary groore, bounded by medullary folds (Fig. $155, \mathrm{~A}$ and $\mathrm{B}$ ), is formed along the median line of the medullary plate and passes into the primitive groove posteriorly; while near their anterior ends the folds meet and unite, so as to constrict off a medullary tube from the outer ectoderm (Figs. I 5.5 and I $58, A$ ). This closure of the tube then gradually extends both forwards and backwards, the groove remaining open longest at its posterior end. By 
the formation of a head-fold in the region of the pro-amnion (p. 583 , Figs. $\mathrm{I}_{53}, \mathrm{E}$, and $\mathrm{I}_{5} 8, \mathrm{~A}$ ) the anterior end of the embryo (head-process) becomes marked off from the blastoderm.

In craniate Vertebrates the anterior end of the hollow medullary tube becomes dilated, forming three bulb-like
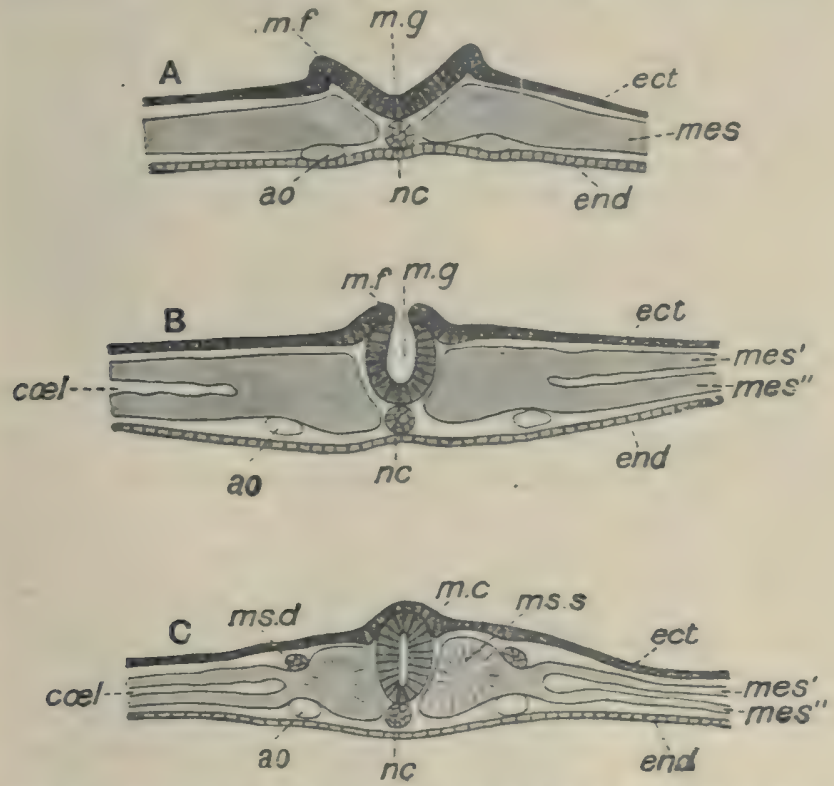

Frg. I55.- Transverse sections across the body of a chick embryo at the twentyfourth to thirtieth hour of incubation, to show stages in the formation of the medullary cord and coelome. ( $x$ roo.)

ao. aorta ; mes. mesodierm; mes' periutal, and mes" visceral laver of méoderm; m. $f$. medullary fold; $m . g$. medulhary groove; ms. d. mesonephric duct; ms. $s$. mesodermal segment; ne. notochord.

swellings - the fore-brain (Fig. I56, A, f. b), mid-brain $(m . b)$, and hind-brain $(h . b)$. Soon another hollow swelling grows forwards from the first vesicle (B, prs. en ), and the third gives off a similar hollow outgrowth (cblm) 
from its dorsal surface. The brain now consists of five divisions : the prosencephalon (prs. en) and diencephalon (dien) derived from the fore-brain, with the pineal structures $(p n . b, p n . e)$ and the infundibulum, to which the pituitary body becomes attached (inf, pty); the mid-brain or mesencephalon $(m . b)$, which gives rise to the optic lobes and crura cerebri ; and the epencephalon or cerebellum $(\mathrm{cblm})$ and metencephalon or medulla oblongata (med. obl) derived from the hind-brain. The original cavity of the brain becomes correspondingly divided into a series of chambers or ventricles (compare Figs. I56 and 50), all communicating with one another, and called respectively the fore-ventricle or prosoccele, third ventricle or diacoele, mid-ventricle or mesocale (iter, and optic ventricles or optocales), cerebellar ventricle or epicoele, and fourth ventricle or metacale.

In some fishes (e.g. dogfish, Fig. I24) the brain consists throughout life of these five divisions, but in most cases (Figs. 49 and I4I) the prosencephalon grows out into paired lobes, the right and left cerebral hemispheres or parencephala (Figs. I56, I-L, c. h), each containing a cavity, the lateral ventricle or paraccele (pa. co) which communicates with the diacœle $(d i . c œ)$ by a narrow passage, the foramen of Monro $(f . m)$. From the prosencephalon or the hemispheres are given off a pair of anterior prolongations, the olfactory lobes or rhinencephala ${ }^{1}$ (olf. l), each containing an olfactory ventricle or rhinocoele (rh. $c \propto)$.

In the preceding description the brain has been described as if its parts were in one horizontal plane ; but, as a matter of fact, at a very early period of development the anterior part becomes bent down over the end of the notochord so that the whole organ assumes a retort-shape, the axis of the

1 The prosencephalon together with the olfactory lobes is often described as the telencephalon. 


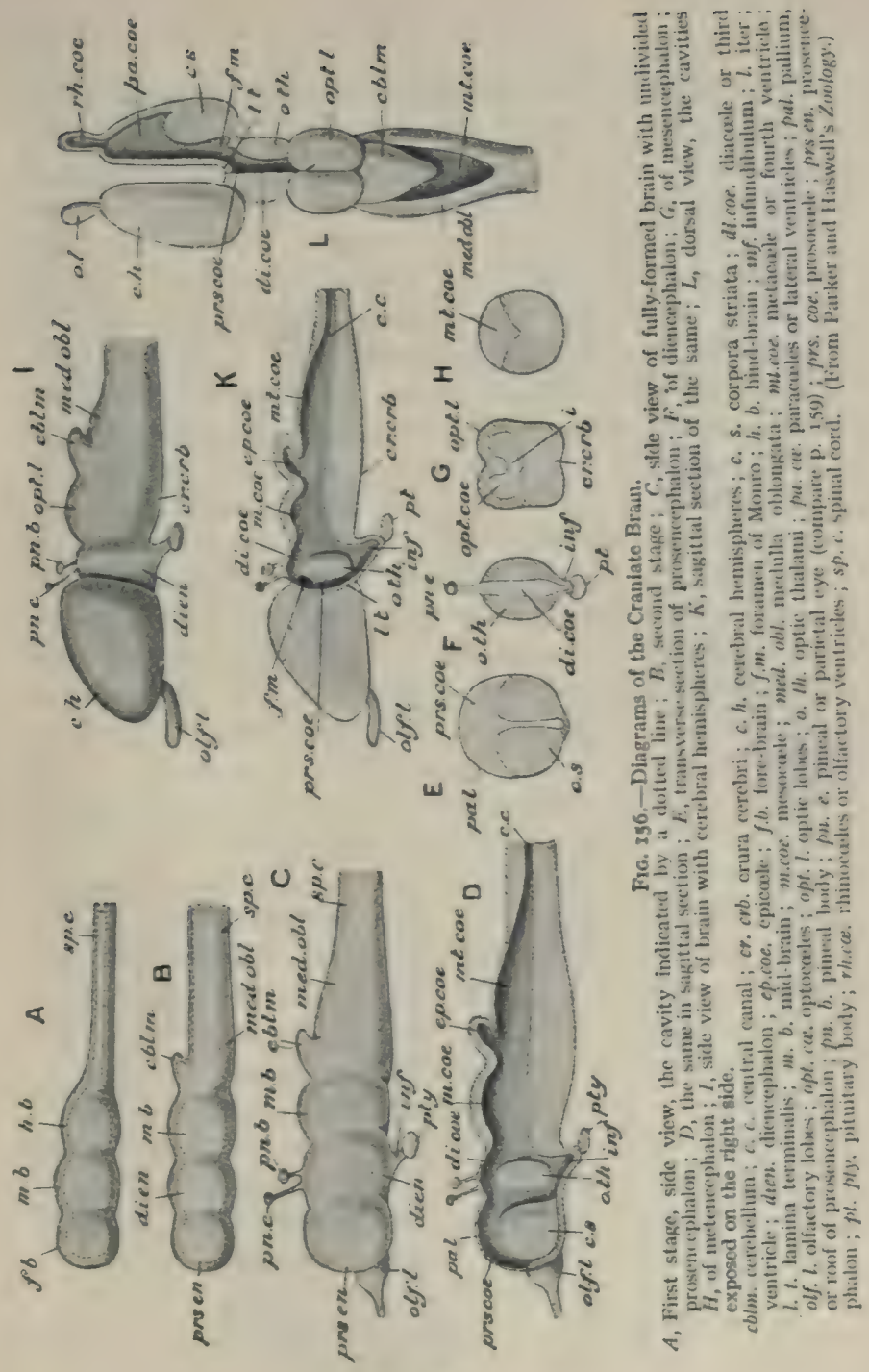


fore-brain being strongly inclined to that of the hind-brain. The bend is known as the cerebral flexure (Figs. I 58 and 159): it is really permanent, but as the hemispheres grow forwards parallel to the hind-brain, and the floor of the mid-brain and hind-brain thickens, it becomes obscure and is not noticeable in the adult.

The central cavity of the embryonic spinal cord has at first the form of a narrow vertical slit (Fig. I55, c), the walls of which eventually fuse dorsally, while ventrally part of the slit forms the central canal of the adult spinal cord, in which dorsal and ventral fissures are developed.

The ganglia of the dorsal roots of the spinal nerves are developed from a paired neural crest (Figs. 65 B, I6r, and I64) arising close to the junction of the medullary plate and outer ectoderm : this becomes segmented to form the ganglia, from the cells of which the sensory fibres arise and extend into the spinal cord. The ventral roots arise as direct outgrowths from cells in the ventral region of the medullary cord. Certain of the cerebral nerves are developed in an essentially similar manner to the dorsal roots of the spinal nerves, while others arise as direct ventral outgrowths from the brain, like the ventral roots. The fibres of all the typical nerves grow peripherally until they reach the parts which they ultimately supply.

The olfactory organs arise as sac-like invaginations of the ectoderm, one on either side of the snout (Fig. I58), and become enclosed by the cartilaginous olfactory capsules, developed, with the rest of the skeleton, from the mesoderm. The aperture of invagination gives rise to the external nostril, the internal nostrils (in air-breathing forms) being developed subsequently.

The mode of development of the paired eye of vertebrates is peculiar and characteristic. 
At an early stage of development a hollow outgrowth - the optic vesicle (Fig. I57, A, op. v) -is given off from either side of the fore-brain and extends towards the side of the head, where it meets with an in-pushing of the ectoderm ( $I)$, which becomes thickened, and finally, separating from the ectoderm, forms a closed, spherical $\operatorname{sac}(B, l)$ with a very small cavity and thick walls (compare Figs. 64, L, e, and I58). This bodv is the rudiment of the lens: as it enlarges it pushes against the optic vesicle which becomes invaginated, the singlelayered optic vesicle thus being converted into a twolayered optic cup (Fig. I57, B, oc', oc ), its cavity, originally continuous with the diacole, becoming obliterated. Between the edge of the cup and the lens, on the ventral side, is a small space which gradually extends towards the stalk of the cup, and thus gives rise to a slit in the wall of the latter: this choroid fissure (C, aus), as it is called, soon becomes closed by the union of its edges. The outer layer of the optic cup gives rise to the pigment-layer of the retina (p. I83): from its inner layer the rest of the retina-including the rods and cones-is formed. The stalk of the optic cup occupies, in the embryonic eye, the place of the optic nerve, but the actual fibres of the nerve are formed from the nerve-cells of the retina and grow inwards to the brain.

During the formation of the lens, mesoderm extends in between the ingrowth from which it arises and the external ectoderm; from this the main substance of the cornea and its inner or posterior epithelium are formed, the adjacent ectoderm becoming the external epithelium, i.e., that of the conjunctiva (p. I82). Mesoderm also makes its way into the optic cup through the choroid fissure, and gives rise to the vitreous humour. Lastly, the mesoderm immediately surrounding the optic cup 

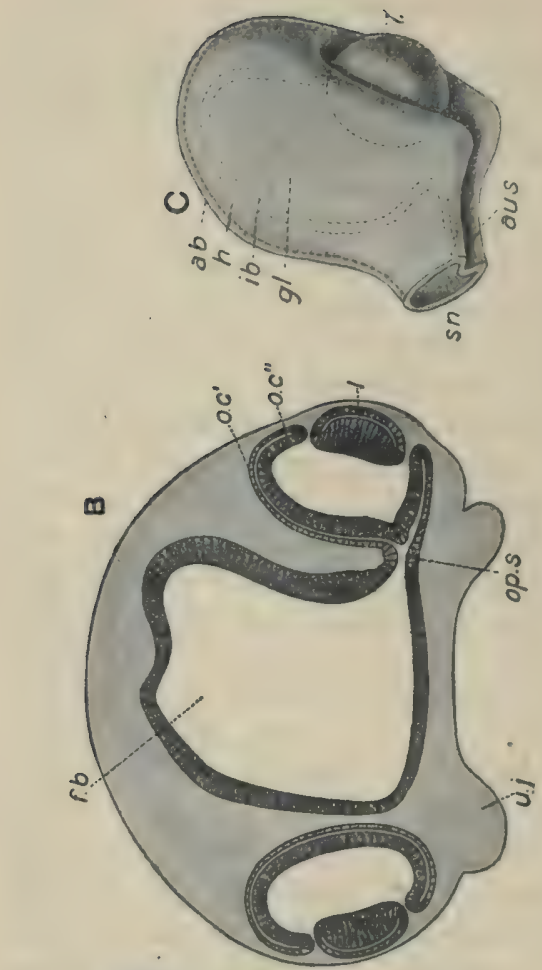

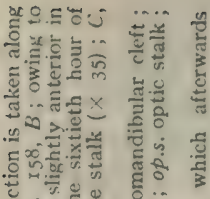

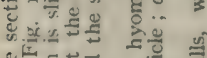

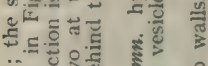

ह5

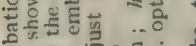

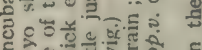

造造

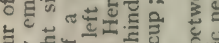
के

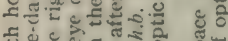

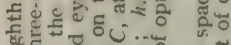
远起.. خ้.

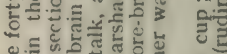
อ.

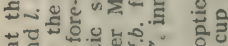
त्टिए

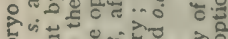

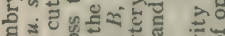
है है ०ण टण

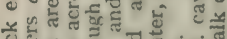

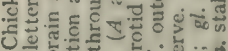
- 5 空

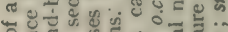

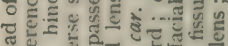

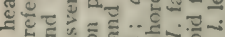

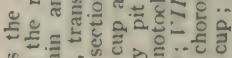

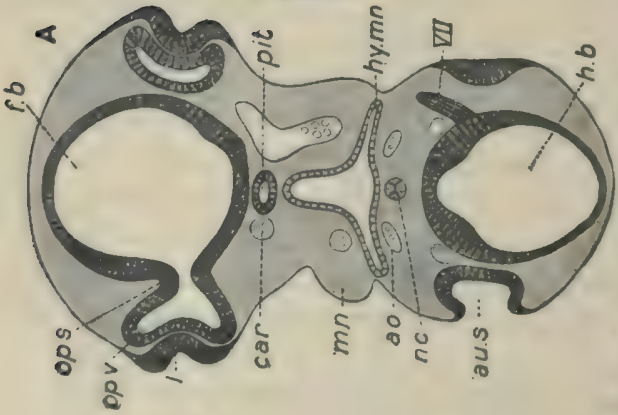

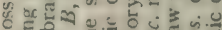

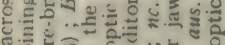
ํํํำ

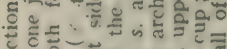

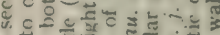
प. $s^{-\frac{\pi}{v}}$ ह

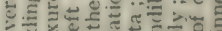
u.

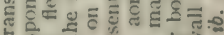
$\Rightarrow$ F ₹ 1.0 c $\therefore \therefore$ 을 $\therefore$ 들

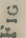


is differentiated to form the choroid, the iris, and the sclerotic.

Thus the eye of Vertebrates has a threefold origin: the sclerotic, choroid, iris, vitreous humour, and the greater part of the cornea are mesodermal ; the lens and external epithelium of the cornea are derived from the ectoderm of the head: the retina and optic nerve are developed from a hollow pouch of the brain, and are therefore in their ultimate origin ectodermal. The sensory cells of the retina-the rods and cones - although not directly formed from the external ectoderm, as in Invertebrates, are ultimately traceable into the superficial layer of ectoderm. since they are developed from the inner layer of the optic cup, which is a prolongation of the inner layer of the brain, the latter being continuous, before the closure of the medullary groove, with the ectoderm covering the general surface of the body (compare Figs. I55 and 157).

The organ of hearing, like that of smell, arises in the embryo as a paired invagination of the ectoderm in the region of the hind-brain, a shallow depression being formed (Figs. 64, L, I57, A, and I58, au. s) which deepens and becomes flask-shaped; and finally, as a rule (compare p. 465), loses its connection with the external ectoderm, giving rise to a closed sac surrounded by mesoderm in which the cartilaginous auditory capsule is subsequently developed. At first simple, it soon becomes divided by a constriction into dorsal and ventral compartments, from the former of which arise the utriculus and semicircular canals, and from the latter the sacculus and cochlea.

The early development of the enteric canal has already been dealt with in the case of the frog (pp. 204 and 2I0), and it will be remembered that the greater part of it (mesenteron) is lined by endoderm, its cavity being at first bounded by the yolk below, but gradually becoming closed in by endoderm cells. When a yolk-sac is formed (dogfish, bird, mammal, Figs. I29, I59, I66), it 


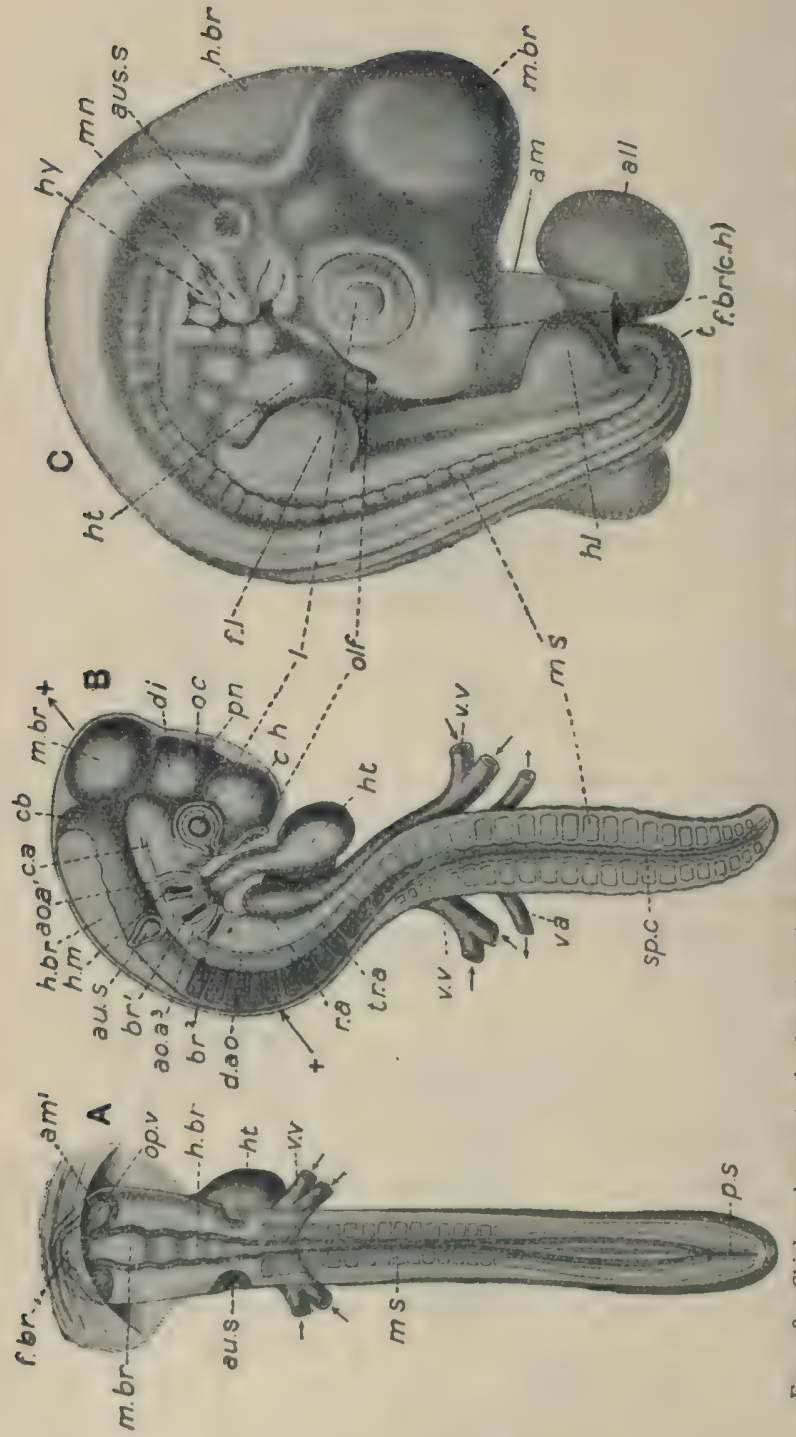

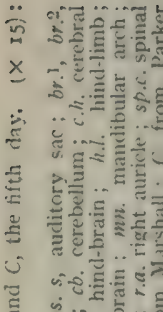

"

i. w

त क

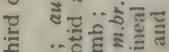

घ

ङ ह

ธै

ஏ

을 है है

¿.ल...

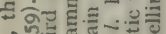

$\infty \rightarrow 0$.

...

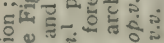

है औौ है

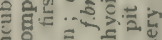

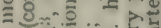

\% \&

응.

- ..

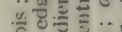

s.

를. है

흘.

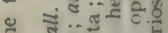

\& वैँ

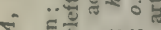

ष ธี …

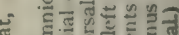

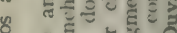

는

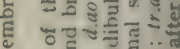

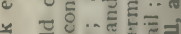

늘 岁

$\div=$ ㄴ.

है है है. हू

- $\rightarrow$ है इ

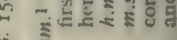

i 
of course communicates with the mesenteron; but as the embryo is gradually folded off from the yolk-sac, the mesenteron becomes tubular along its whole length, and eventually the stalk of the yolk-sac becomes solid, at hatching (or birth) its point of communication with the body being marked by the navel (umbilicus). The ectodermic stomodaum (p. 204), which gives rise to the mouth-cavity and also to the greater part of the pituitary body, comes to communicate with the mesenteron anteriorly, and the ectodermic proctodaum opens into it posteriorly. The muscular and peritoneal layers of the canal are formed, it will be remembered, from the mesoderm.

The first traces of the liver and pancreas are seen as simple endodermic offshoots of the mesenteron, which gradually become branched in a complicated manner, the numerous lobules being more or less closely connected together by mesoderm. The gill-pouches arise as paired outgrowths of the endoderm lining the pharynx, which come into contact with the ectoderm, the latter becoming perforated to form the external branchial apertures. Four gill-clefts appear in the embyro of reptiles, birds, and mammals - animals in which gills are never developed (Figs. I58 and I60); but they early disappear with the exception of the first cleft, corresponding with the spiracle of the dogfish, which gives rise in all Vertebrates above fishes to the tympanoeustachian passage (p. 449): the branchial skeleton, as we have seen, undergoes a corresponding reduction or modification (pp. 438 and 496). In air-breathing Vertebrates the lungs arise as a ventral outgrowth of the pharynx, lined by endoderm and covered by mesocierm.

The circulatory organs are developed from the mesoderm, the heart arising in the visceral layer on the 


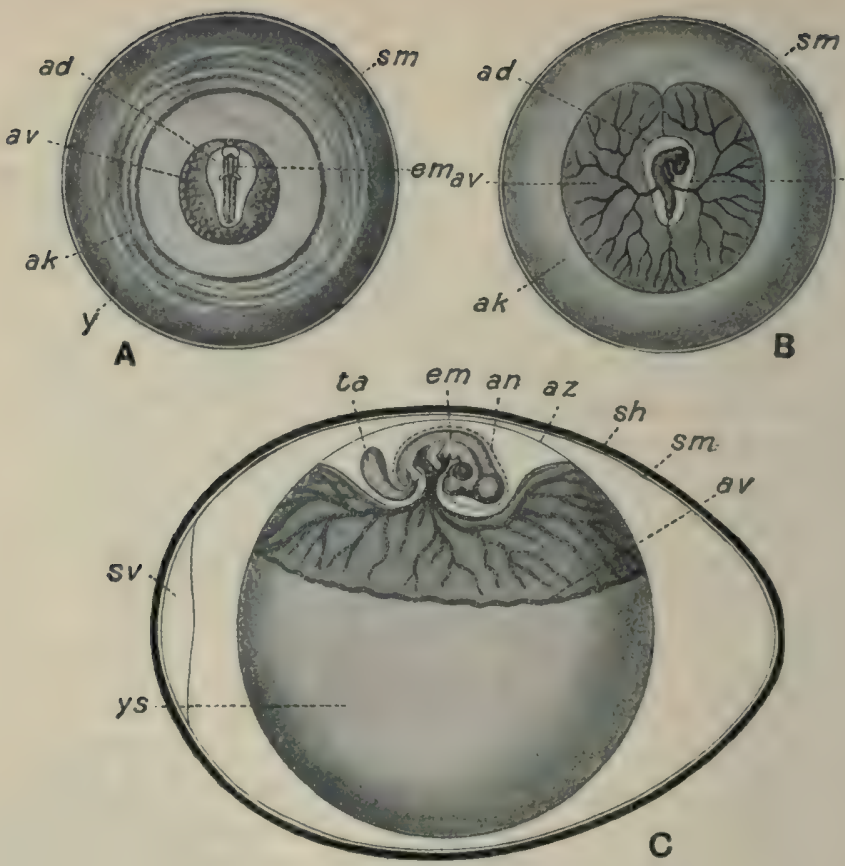

$\theta m$

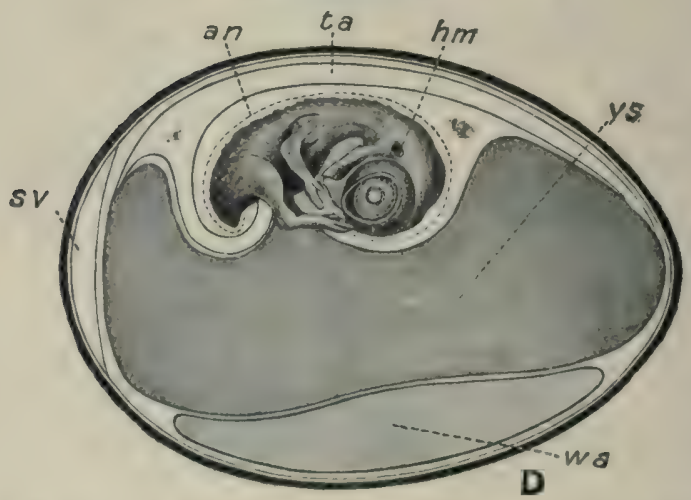

FiG. I59.- The yolk of a Hen's egg at, $A$, the thirty-sixth hour from the beginning of incubation ; $B$, the third day; $C$, the end of the fitth day; and $D$, the end of the ninth day (about nat. size). (Compare Fig. $15^{8}$.)

ad. area pellucida ; an. inner or "true " amnion ; $u k$. area opaca ; av, area vasculosa (in C its nuter markin); az. cuter or "false " amnion (serous membrane), together with the vitelline membrane: em. embryo: hm, hyomandibular eleft; sh. egrg-shell : sm. vitelline memibrane (in $($, shell membrane) : st. anr-chamber; ta. allantois ; $" a$. white or albumen; $y$. ys, yolk-sat. (Frow Marshall.) 
ventral side of the pharynx. It has at first the form of a straight tube, developed in the chick and rabbit by the fusion of two longitudinal vessels (vitelline veins, see below, and Figs. I6I and I6 4 ); it soon becomes twisted into an S-shape, and transverse constrictions are formed dividing it into the different chambers. The auricular and ventricular portions are each at first single, but from the Amphibia onwards the former subsequently becomes divided into two by a septum, and in birds and mammals the ventricle is similarly subdivided. The modification of the arterial arches in the examples studied has already been described (pp. 452 and 523).

In the dogfish, bird, and rabbit the dorsal aorta, in addition to its other branches, gives rise to paired vitelline arteries: these branch over the extra-embryonic part of the blastoderm ( $p$. 585) which spreads over the yolksac, and in the two former types the capillary network takes an important share in the absorption of the

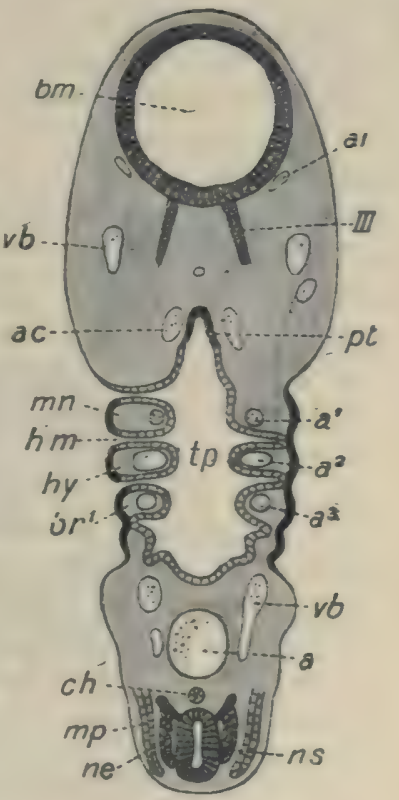

Fic. 160.-A section through a Chick embryo at the end of the third day of incubation, the section being taken along a plane indicated by the two arrows and crosses in Fig. $158, B$; the right side of the section is at a level slightly dorsal to that of the left side. ( $x$ 30.) (After Marshall.)

a. dorsal aorta ; 1 . first aortic arch, in the mandibular arch; $a^{2}$. second aortic arch, in the hyoid anch; 3 . third aortic arch, in the first branchial arch ; $e c$. carotid artery ; ai. internal carotid artery; bm. cavity of midbrain; brl. first branchial arch; ch. notochord; hm. hyomandibular cleft ; hy. hyoid arch ; wh. mandibular arch; mp. muscle-plate; me. ganglion of spinal nerve; ns. spinal cord; $\mu$. pituitary pouch; $\phi$. pharynx; ob. jugular vein. 
yolk by the embryo. From this area vasculosa (Fig. I59) the blood is returned by splanchnopleuric (p. 580) vitelline veins, which join with a subinlestinal vein (compare Amphioxus, Fig. IIO) and open into vessels which eventually give rise to the hepatic portal veins. The chief somatopleuric veins in all embryonic Craniates are, as in the dogfish, the jugulars and the cardinals (Figs. I60 and I64). In all Vertebrates above

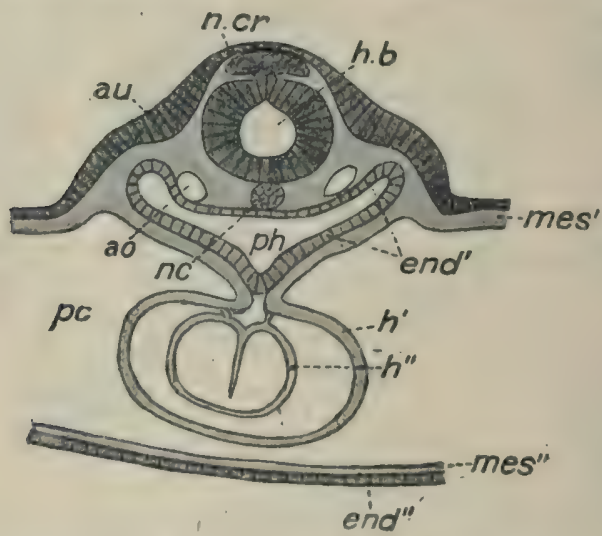

Fic. $16 \mathrm{r}$.- Transverse section across the head of a Chick embiyo at about the fortieth hour of incubation, in the region of the hind-brain and heart. ( $x$ about go.)

ao. aorta; au. beginning of auditory pit; $\epsilon$ nd. endoderm of pharynx ; cnd". endoderm of yolk-sac; $h^{\prime}$. muscular wall of heart $; h^{\prime \prime}$. epithelial lining of heart (the septum, which subsequently disappears, indicates the paired origin of the heart) ; h.b. hind-brain ; mes'. parietal, and mes". visceral layer of niesoderm; nc. notochord; $n$. cr. neural crest; $p c$. pericardial coxlome; ph. pharynx.

the fishes, the cardinals become subsequently more or less entirely replaced functionally by the postcaval (compare p. 456) : the anterior part of one or both cardinals may, however, persist as the azygos vein or veins (e.g. rabbit, p. 525).

Urinogenital Organs.-The excretory organs, speaking of craniate Vertebrates as a whole, arise as a series of tubules having the general character of nepliridia 
(pp. I46 and 340) situated along the dorsal region of the colome ; but owing to the fact that they do not all arise at the same time and that their development is modified by neighbouring structures, there is much variation as regards these tubules and their relations. They may in general be classified into three groups, constituting what are known respectively as the fore-kidney or pronephros (Fig. I62, A, p. nph), the mid-kidney or mesoncphros (ms. $n p h$ ), and the hind-kidney or metanephros (mt. nph).

The pronephros appears at an early stage of development, and very rarely remains functional throughout life, though in some cases (e.g. tadpole) it serves as an excretory organ in the larva : in many cases it is merely represented by vestiges (e.g. dogfish, bird, rabbit). The pronephric tubules open at one end into the colome by ciliated nephrostomes, and at the other into a longitudinal pronephric duct (sg. d), which communicates with the cloaca posterioriy. They are generally from two to four in number, are not strictly segmental, and are confined to the anterior end of the cœlome: opposite their nephrostomes a single large glomerulus (see p. If6) is present on either side of the body.

The mesonephros appears rather later than the pronephros, and extends along the greater part of the cœlome. Its tubules are segmentally arranged, and thus furnish another example of metamerism in the Vertebrate body; each tubule is provided with a separate glomerulus enclosed within a Malpighian capsule $(m c)$. The nephrostomes of the mesonephros may lose their connection with the tubules and come to open into certain veins (e.g. frog, Fig. 47 and p. 98), or may disappear at a later stage to a greater or less extent (dogfish) or entirely (e.g. bird, rabbit): at their other ends the tubules communicate with the pronephric duct, which may now be spoken of as the mesonephric duct (Fig. 

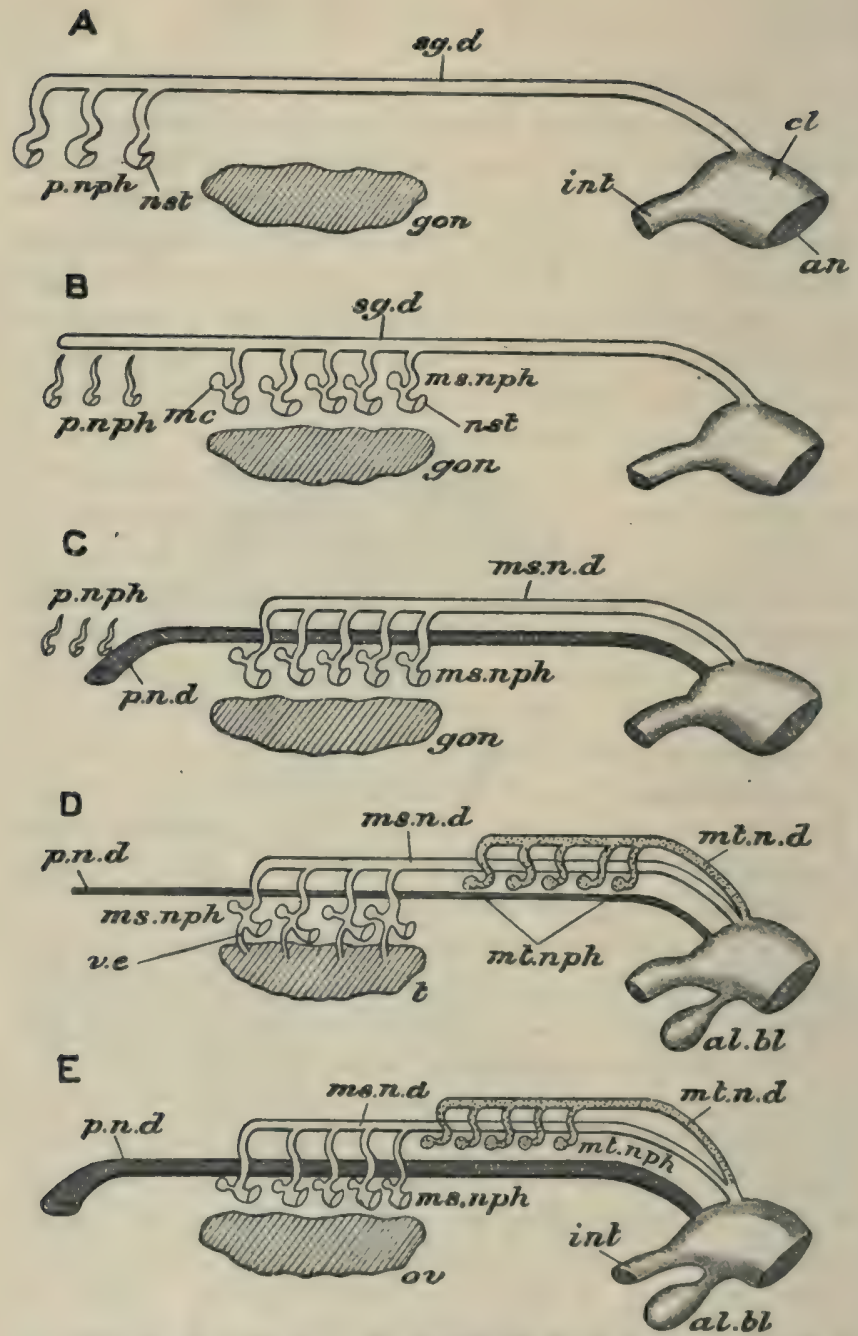

Frg. r62.-Diagrams illustrating the development of the urinogenital organs of Craniata.

$A$, pronephros and its duct; $B$, atrophy of pronephros, development of mesonephrus; $C$, differentiation of Wolffian and Müllerian ducts; $D$, development of metanephros, male type; $\boldsymbol{E}$, female type. 
al. bl. allantoic bladder; an. anus; cl. cloaca : gon. gonad; int intestine; mc. Maluishian cap-ule; msn.d. mesonephric (Wolfian) duct : ms.nph. mesonephros; mt.n. d. metanephric duct; mt.nph. metanephros; nst. nephrostomes; ov. ovary; p. n. d. Multerian duct; p.nph. pronephros; sg. d. prone phric duct

t. spermary; v.e. efferent ducts. (From Parker and Haswell's Zoology.)

I64, $m s . d, m s . t)$. In Fishes and Amphibians the mesonephros remains as the functional kidney throughout life, though the posterior part alone may retain the renal function and develop special ducts (dogfish, Fig. I27), thus foreshadowing the metanephros of higher Vertebrates.

The metanephros is characteristic of Reptiles, Birds, and Mammals. Its tubules arise later than those of the mesonephros and open into a special ureter or metanephric duct, which arises as an outgrowth from the posterior end of the mesonephric duct : they resemble the mesonephric tubules except that they possess no nephrostomes and usually show no signs of a metametric arrangement.

In the majority of Vertebrates the generative organs take on a close connection with certain of the urinary tubules and their ducts (pp. 193 and 466 ), which may thus undergo a change of function. In the dogfish the pronephric duct early undergoes longitudinal subdivision into two, one of which is retained as a mesonephric duct (now often spoken of as the Wolffian duct); while the other (Miillerian duct), retaining its connection anteriorly with the cœlome by a persistent pronephric nephrostome, assumes the function of an oviduct in the female, and atrophies in the male, though traces of it may persist in the adult (Fig. I27, m.d). Owing to the connection which is formed between the spermary and certain of the mesonephric tubules by means of efferent ducts, the Wolffian duct may now serve as a urinogenital duct (frog, p. 193); or, when an epididymis is formed from the mesonephros, and special ureters arise 
in connection with the functional renal tubules, the Wolffian duct serves as a spermiduct mainly if not entirely (dogfish, p. $4^{t} 6$; mammal, p. 538).

In most Vertebrates the oviducts arise quite independently of the mesonephric ducts, each being formed as a groove of the cœlomic epithelium which becomes closed in and grows from before backwards to the cloaca, its anterior opening into the cœlome representing a nephrostome of the pronephros.

In the higher Vertebrates, in which the kidney of the adult is a metanephros (p. 537), the only parts of the mesonephros and its duct which persist as more than a vestige are the epididymis and spermiduct of the male.

The gonads arise as ridges covered by cœlomic epithelium on the dorsal wall of the body-cavity close to the inner side of the developing kidneys. Their epithelium is known as germinal epithelium, and from it either ova or sperms are eventually developed (pp. I94I96, and 566).

The majority of the muscles are developed, as we have seen (pp. 203 and 588), from the mesodermal segments, others arising from the parietal and visceral layers of the mesoderm.

The first part of the endoskeleton to be formed is the notochord (pp. 203 and 580), developed primarily from the endoderm, but in the chick and rabbit arising from the central part of the mass of cells in the primitive streak (Fig. I53, G), in which the layers become indistinguishable from one another (p. 583). In the mesoderm surrounding the notochord cartilages appear and give rise to the vertebra, the notochord becoming constricted by the ingrowing cartilage and eventually disappearing more or less completely (p. 44I) : it at first 
extends into the head as far as the pituitary body (Fig. I63). The cranial cartilages do not become segmented, but give rise to a pair of horizontal bars, the parachordals $(p a . c h)$ : these are continued forwards, diverging around the pituitary body, as the trabecula cranii (tr). The two parachordals and trabeculæ then unite respectively with one another, and so form a firm floor for the future brain-case: this is gradually developed by the cartilage growing up on either side and eventually meeting to a greater or less extent above the brain : there is never, however, a complete cartilaginous roof to the cranium, parts of which are only membranous and form the fontanelles (pp. 43 and 436). In the meantime the cartilaginous sensecapsules are developed, the olfactory

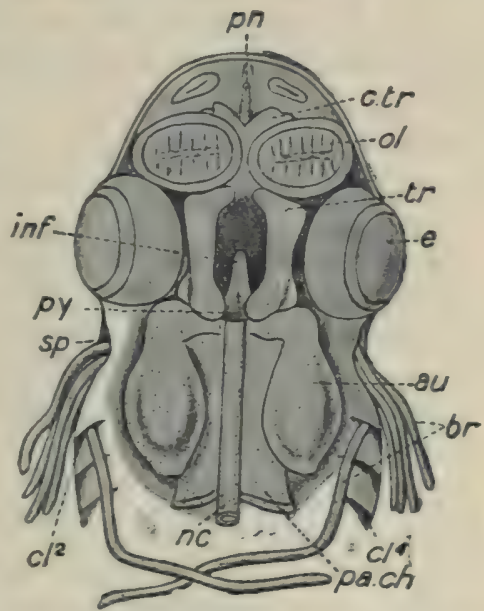

Fig. I63.-Dissection of the head of an embryo Dogfish (Scyllium canicula) from the dorsal side, to show the developing chondrocranium (dotted). ( $\times$ 8.)

ai. auditory capsule; br. external gills ; c.tr. cornu of trabecula; $c l^{2}, c l^{4}$. gill-clefts; inf. infundibulum of brain (left in situ); nc. nntochord; ol. olfactory sac; pa. ch. parachordal cartilage ; $\boldsymbol{p}$. prenasal cartilage ; py. pituitary borly (left in situ) ; $s p$. spiracular cleft; $t r$. trabecula. (After W. K. Parker.)

and auditory capsules uniting with the brain-case in front and behind respectively.

The visceral skeleton is formed as a series of cartilaginous bars within the visceral arches, the first of which forms the mandibular arch, the second the hyoid, and the others the branchial arches. 
The limbs appear as small buds (Fig. I58, C) composed of ectoderm with a core of mesoderm, in which latter their skeleton arises by the formation of cartilage extending inwards to form the arches, and outwards to form the skeleton of the free portions of the limbs.

As we have seen, the endoskeleton may remain practically entirely cartilaginous in the adult (e.g. dogfish), but in higher forms extensive processes of ossification set in, certain bones replacing the cartilage to a greater or less extent, and others being formed in the surrounding connective-tissue (compare p. 43).

\section{Development of the Amnion, Allantois, and Placenta. -}

We must now consider some important and characteristic structures which are developed in the embryos of Reptiles, Birds, and Mammals, and known as embryonic membranes. Taking the chick as a convenient example, these are formed as follows.

The blastoderm, as we have seen (p. 585 and Fig. I59), gradually extends peripherally so as to cover the yolk, and thereby becomes divisible into an embryonic portion, from which the embryo is formed, and an extraembryonic portion, which invests the yolk-sac and takes no direct share in the formation of the embryo. The extension of the ectoderm and endoderm takes place regularly and symmetrically; but the mesoderm, while extending equally in the lateral and posterior regions, grows forwards in the form of paired prolongations which afterwards unite, so that for a time there is an area of the blastoderm in front of the head of the embryo formed of ectoderm and endoderm only and called the pro-amnion (p. 583 and Fig. I53, pr. am).

Before the embryo has begun to be folded off from the yolk the rudiment of one of the two embryonic membranes, the amnion, has appeared. A crescentic 
amniotic fold (Fig. I58, A, and $165, \mathrm{~A}$, am. $f^{\prime}$ ) arises in front of the head end of the embryo from the region of the pro-amnion : it consists at first of ectoderm only, the mesoderm not having yet spread into the pro-amnion. The fold is soon continued backwards along the sides of the body (Fig. I65, B) and round the tail (A), but in these regions $(a m . f)$ it consists from the first of ectoderm plus the parietal layer of mesoderm, i.e., it is a fold of

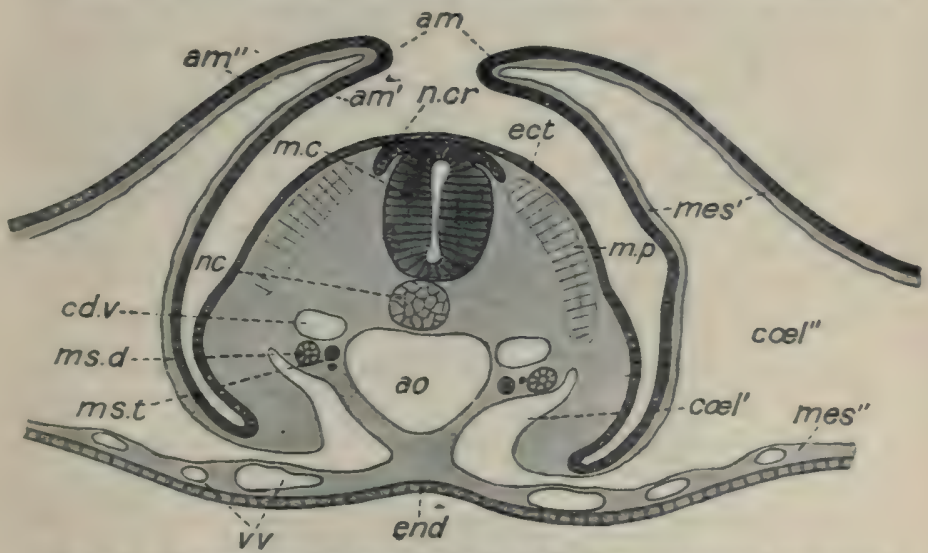

Fic. 164.- Transwerse section across the body of a Chick embryo at about the sixtieth hour of incubation. ( $x$ about go.)

ao. aorta ; am. amniotic folds; $a m^{\prime}$. true amnion, and $a m^{\prime \prime}$. serous membrane; $c d . v$. cardinal vein ; cel'. coelome in body of embryo ; $c c l^{\prime}$. extra-embrvonic coelome ; et. ectoderm; end. endnlerm; m. c. medullary cord ; mes'. parietal. and mes". visceral laver of mesoderm; $m$. $p$. muscle-plate : $m s$. $d$. mesonephric duet; ms. $t$. mesonephric tubule: $m$. notochord; $n$. $v$. neural crest $: v$. $v$. vitelline veins.

what may be called the embryonic body-wall or somatopleure (p. 580, Fig. I64, am). Its cavity is a prolongation of the space between the parietal and visceral layers of mesoderm-i.e., is an extension of the extraembryonic coelome.

The entire amniotic fold gradually closes in above (Fig. I65, C) forming a double-layered dome over the embryo. Its inner layer, formed of ectoderm internally and meso- 


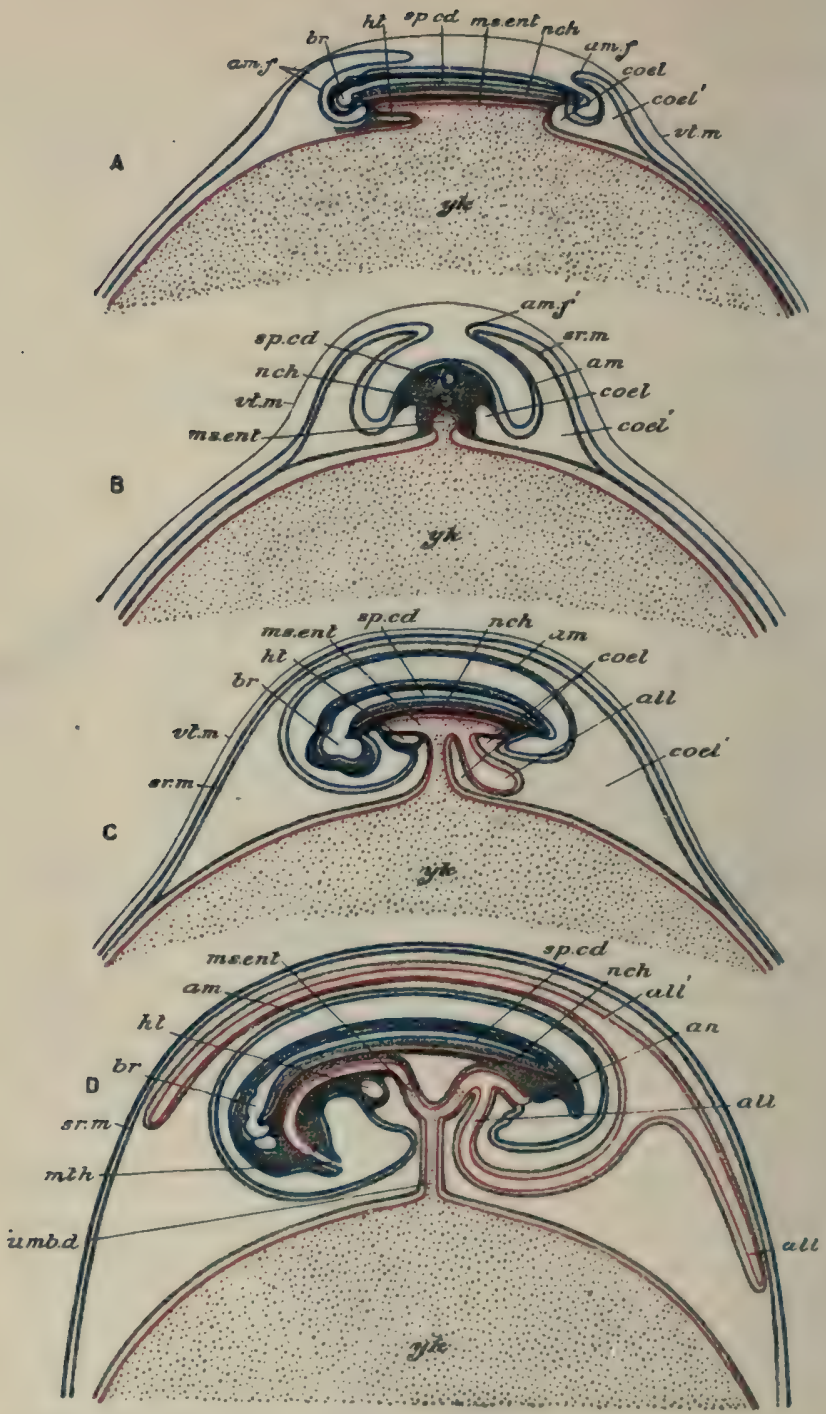

Fig. I65.-Diagrams illustrating the development of the foetal membranes of a Bird. $A$, early stage in the formation of the amnion longitudinal vertical section; $B$, 608 
slightly later stage, transverse section; $C$, stage with completed amnion and commencing allantois; D, stage in which the allantois has begun to envelop the embryo and yolk-sac. The ectoderm is represented by a blue, the endoderm by a red line ; the mesoderm is grey.

all. allantois; all' the same growing round the embryo and yolk-sac; aw. amnion ; um.f. amniotic fold; an. anus; br. brain ; cal. coelome; coxl'. extra-embryonic cuelome; $h t$. heart; ms. ent. mesenteron; mth. mouth; nch. notochord; $s p . c d$. spinal cord; $s \%$. $m$. serous membrane; $u m b$. $d$. umbilical duct; $v t$. $m$. vitelline membrane; $y k$. yolk-sac. (Reduced from Parker and Haswell's Zoology.)

derm externally, is the amnion (am, and Fig. I64, $a m^{\prime}$ ), the cavity enclosed by which becomes filled with a watery amniotic fluid, serving as a protective water-cushion to the contained embryo. Its outer layer, formed of ectoderm externally and mesoderm internally, is the serous membrane, sometimes spoken of as the false amnion (sr. $\left.m, a^{\prime \prime}\right)$ : this comes to lie just beneath the vitelline membrane (p. 565), with which it subsequently fuses.

The second of the embryonic membranes, the allantois, is developed as an outpushing of the ventral wall of the mesenteron near its posterior end (Figs. I65, C, all, and I66), and consists, therefore, of a layer of visceral mesoderm lined by endoderm (splanchnopleure, p. 580). It has at first the form of a small, ovoid sac having the precise anatomical relations of the urinary bladder of the frog. Increasing rapidly in size, it makes its way, backwards and to the right, into the extra-embryonic coelome, between the amnion and the serous membrane (C, D). Allantoic arteries pass to it from the dorsal aorta, and its veins, joining with the vitelline veins from the yolk-sac, take the blood through the liver to the heart (p. 600). Next, the distal end of the sac spreads itself out and extends all round the embryo and yolk-sac (D, all'), fusing, as it does so, with the serous and vitelline membranes, and thus coming to lie immediately beneath the shellmembrane. It finally encloses the whole embryo and yolk-sac, together with the remains of the albumen, which has, by this time, been largely absorbed (Fig. I59, D). The allantois serves as the embryonic respira- 
tory organ, gaseous exchange readily taking place through the porous shell; its cavity is an embryonic urinary bladder, excretory products being discharged into it from the kidneys.

At the end of incubation the embryo breaks the shell by means of a little horny elevation or caruncle at the end of the beak. By this time the remainder of the yolk-sac has been drawn into the colome, and the ventral body-walls have closed round it. On the shell being broken the allantois gradually shrivels up, respiratory movements begin, the aperture in the shell is enlarged, and the young bird is hatched and begins a free life.

In the higher Mammalia the allantois takes on a further important function. The relations of the amnion and allantois in the rabbit are essentially similar to those described above in the case of the bird. But the later history of the allantois is widely different, owing to the modifications which it undergoes in order to take part in the formation of the placenta, the structure by means of which the foetus receives its nourishment from the walls of the uterus, with which the blastocyst (p. 586) first becomes adherent over the future placental area by the proliferation of the cells of its outer layer (trophoblastic ectoderm) in this region, which forms irregular processes extending into the thickened mucous membrane on the dorsal side of the uterus (Fig. I66, $\left.e^{\prime}\right)$. As the embryo develops, it sinks down and causes the vascular " yolk-sac" or umbilical vesicle ( 1 's) to be doubled in and take on a flattened form (Figs. I67, $d s, I 66$ and $I 68, y s):$ this early becomes attached to the mucous membrane of the uterus, and represents a vitelline or yolk-sac placenta such as occurs in some viviparous dogfishes; but its nutritive function in the mammal is of only minor importance.

The fatal part of the placenta is formed from the outer layer of the amnion (serous membrane) in a 
limited disc-shaped area covered by the thickened part of the trophoblastic ectoderm where the distal portion of the allantois coalesces with it (Fig. r66). The membrane thus formed (chorion) develops vascular processes or septa-the chorionic, villi, which are

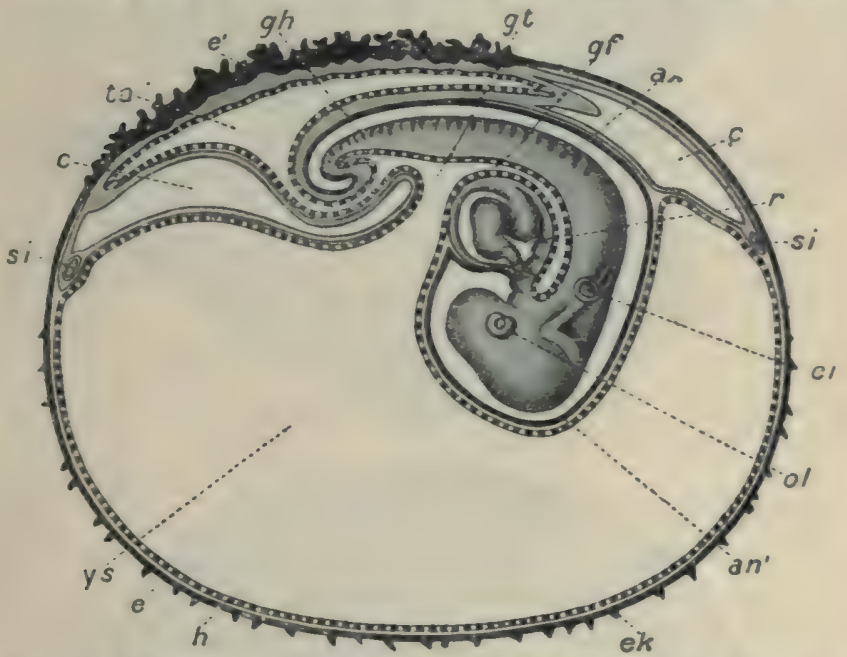

Fic. It5. A Rabbit embryo and blastodermic vesicle (" yolk-sac ") at the ent of the tenth day. The embryo is represented in surface view from the right side, the courie of the enteric canal being indicated by the broad dotted line: the blastodermic vesicle is shown in median longitudinal section. The greater part of the tail has been removed. ( $x>7$.)

an'. pro-amnion; a.x. cavity of amnion, between the inner or true amnion and the embryo; $c$. extra-embryonic purtion of cuelome : $e$. trophoblastic ectoderm: $c$. thickened part of $c$ by which the blastolermic vesicle is attached to the uteru- and from which the development of the fotal part of the placenta

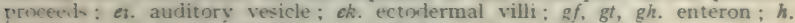
intorlerm: ol. eye : $r$. heart : si. circular marginal vensel of area vasculosa ; ta. cavity of allantois: ys. cavity of "yolk-sac." (From Marshall, in part after Van Beneden and Julin.)

received into depressions - the uterine crypts - in the thickened placental folds of mucous membrane on the dorsal wall of the uterus which constitute the maternal portion of the placenta (Figs. I67 and I6S). The foetal portion of the placenta with its villi is supplied with 

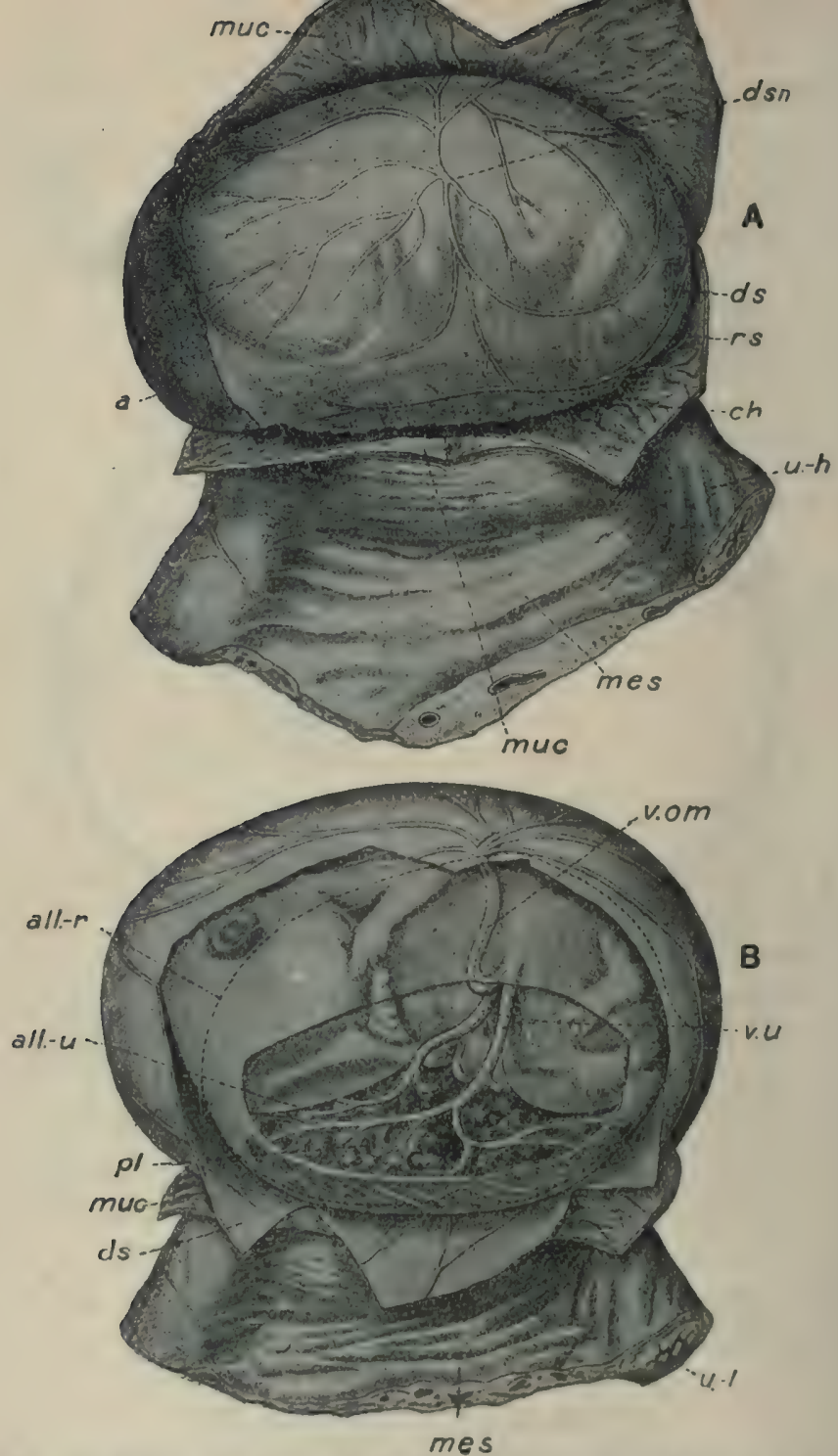

FiG. 167 .- Portion of the uterus of a Rabbit containing an advancer foetus. $(\times>1)$ In $\mathrm{A}$, the uterus has been opened and its wall reflected, and part of the "yolk$6 r 2$ 
sac" (umbilical vesicle) cut away (left of the figure) to show the underlying amnion. In $\mathrm{B}$, part of the uterine wall has been removed and part of the yolksac and allantois cut out so as to expose the foetus and placenta.

a. amnion; all. $-\boldsymbol{r}$. dotted line indicating margin of allantois (compare Figs. $\mathbf{1} 66$ and $168, t a)$; all. $-u$. line along which the allantois is reflected over the placental region; $c h$. chorion : $d s$. yolk-sac; $d s n$ point at which the umbilical vessels branch out from the umbilical stalk; mes. mesentery of uterus; muc. mucous membrane of uterus; $p l$. foetal surface of placenta covered by the distal wall of the allantois; rs. circular marginal vessel of area vasculosa of yolk-sac $; u-h$. undilated narrow portion of uterus between two foetus-containing swellings; $u-l$. lumen of uterus; $v$. om. vitelline vessels; $v$. $u$. allantoic vessels. (From Grosser's Verol. Anat. is. Endurick. geschichte der Eihüute und der Placenta.)

blood by the allantoic vessels, and the blood-supply of the uterus is at the same time greatly increased: the fœtal and dilated maternal capillaries and sinuses are thus brought into intimate relation with one another in the placenta, being only separated by thin layers of epithelium: diffusion can thus take place between them, nutrient matter and oxygen diffusing from the blood of the mother into that of the fœtus, while excretory substances pass from the blood of the fœtus into that of the mother.

The disc-shaped or discoidal placenta of the Rabbit is of the type termed deciduate, its villi being so intimately connected with the uterine mucous membrane that a part of the latter comes away with it at birth in the decidua, or afterbirth, which is attached to the newly-born young by the umbilical cord, consisting of the stalks of the allantois and flattened yolk-sac twisted together (Figs. I67 and I68).

The blood-vessels at the plane of separation of the placenta and uterus are very small, and the uterus contracts so rapidly when the young are expelled at parturition that very little bleeding takes place, and the mucous membrane rapidly undergoes regeneration, ready for the next conception. The cord is gnawed through by the parent rabbit, the blood-vessels being compressed in the process; and it soon shrivels up and comes away at the navel or umbilicus (see p. 597) 
which represents the point of connection between the fœtus and the placenta. The intra-abdominal portion

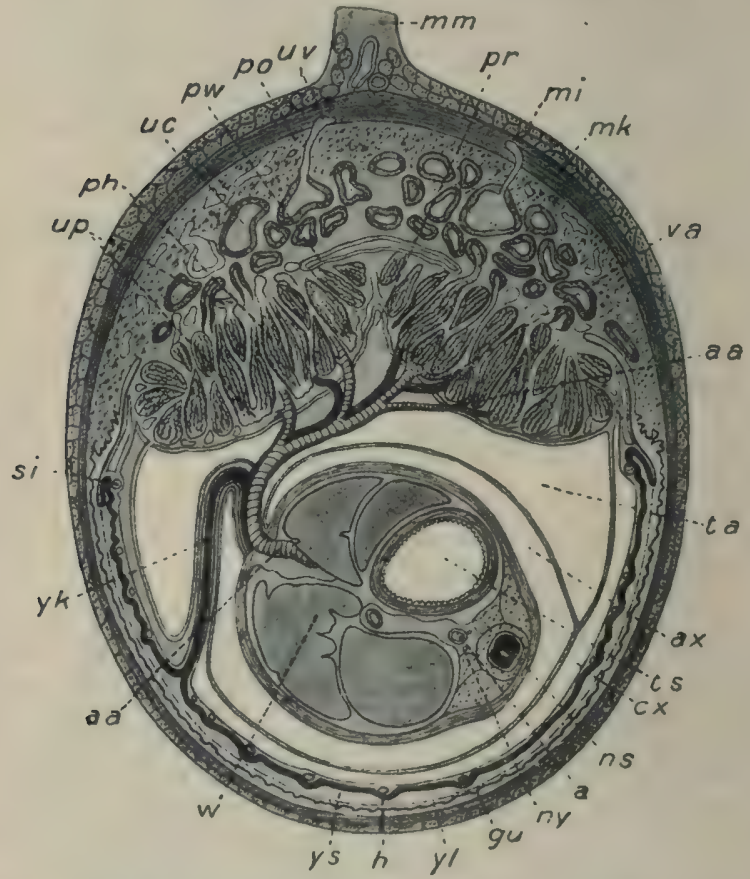

FIG. 168.-A transverse section across the uterus and the contained embryo of a Rabbit at the end of the nineteenth day. The embryo is cut transversely about the middle of the body, the section passing through the "yolk-stalk" and allantoic stalk. ( $x$ 2\%. $)$

A, clorsal aorta; aa. allantoic artery; a.x. cavity of amnion, between the inner or true amnion and the embryo; $c x$. space between the juner and outer layers of the amnion; $g u$. uterine glauds; $h$. endoderm of upper or vascular wall of "yolk-sac" (umbilical vesicle); $m i, m k$. outer or longitudinal, and inner or circular muscles of wall of uterus; $m m$. mesentery of uterus ; $n s$. spinal cord; $n y$. sympathetic nerve-cord; ph. lobule of placenta; po. region along which the separation of the placenta occurs at birth; $p r$. inter-placental groove : pre. sub-placental cavity; si. circular marginal vessel of yolk-sac; la. cavity of the allantois (see Fig. I66)； ts. stomach; wc. dilated uterine capillary, with thick perivascular wall; up. uterine or maternal sinuses of placenta ; uv. blood. vessels of uterus; $v a$. allantuic vein ; $w$. liver ; $y k$. stalk of yolk-sac; $y l$. dotted line representing the lower or non-vascular wall of the yolk-sac, now completely alssorberl; $y$ s. cavity of yolk-sac, continuous with the uterine cavity owing to absorption of the lower wall of the yolk-sac. (From Marshall.) 
of the allantois is represented by a cord or ligament, the urachus, which connects the navel with the apex of the bladder, so that only a small portion of the allantoic outgrowth and not the whole of it, as in the frog, persists in the adult.

\section{PRACTICAL DIRECTIONS}

A. A series of models of the development of Amphioxus, to be found in most Zoological Museums, should be examined. Note-

$a$, the various stages of segmentation and the formation of the segmentation-cavity (Fig. I52, A-G) ; $b$, the obliteration of the segmentation-cavity during the formation of the gasirula, with its ectoderm, endoderm, and blastopore (H, I) ; $c$, the elongation of the gastrula, the flattening of the surface which will become dorsal, and the position of the blastopore at the posterior end $(\mathrm{J}) ; d$, the formation of the medullary plate and cord, the mesodermal segments and enterocceles, and the separation of the latter from the enteron, the walls of these cavities forming the parietal and visceral layers of the mesoderm ; and the formation of the notochord and the neurenteric canal $(\mathrm{K}-\mathrm{O})$.

B. In order to follow out the development of the chief organs in the Vertebrata, it is necessary to make a number of serial sections of various embryonic stages. For this purpose chick-embryos (see below) are, on the whole, the most convenient and satisfactory; but if you have not already followed out the instructions given on pp. 212-2I 4 , you should not only examine externally the stages in the development of the tadpole up to hatching, but also attempt to make sections of some of the early stages so as to show $a$, the segmentation-carity (Fig. 64, E); $b$, the enteron, blastopore, and yolk-plug (I): and c, the medullary cord, notochord, mesoderm, and coelome (Figs. $64 \mathrm{~K}$, and 65, A and B). Compare with the corresponding stages in Amphioxus.

C. A number of fresh, impregnated fowls' eggs should be obtained and placed in an incubator at a temperature of about $39.5^{\circ} \mathrm{C}$. $\left(103^{\circ} \mathrm{F}\right.$.), or under a "broody " hen, first marking each with the date. One or two should be examined each day or oftener for the first four or five days of incubation. Expose the embryo as directed on p. 576 , using warm normal ( $0 \cdot 75$ per cent.) salt solution (temperature as above), in order that after the first day the beating of the heart and the circulation of the blood may not be 
stopped. Remove sufficient of the shell and shell-membrane to expose the entire blastoderm, as well as the embryo proper when developed. Examine with a hand-lens before removing, and then, after carefully cutting through the vitelline membrane with fine scissors around the margin of the blastoderm, the latter should be floated off in a watchglass, and the covering vitelline membrane removed by the aid of a needle-an operation which requires considerable care in the early stages. Fix with corrosive sublimate, and after about half an hour wash in water and preserve in increasing strengths of alcohol up to go per cent. (see p. I36).

Two specimens of each stage should be preserved, one for examining entire, and the other for sectioning ${ }^{1}$; they should subsequently be stained entire with borax-carmine or hrematoxylin, the one mounted in Canada balsam on a slide after treating with absolute alcohol and xylol or oil of cloves, and the other imbedded in paraffin (see p. 137). Serial sections of each embryo should be mounted in order, after smearing the slide with collodion and oil of cloves or glycerine and albumen (p. I39).

Sketch typical preparations of the various stages, both entive embryos and sections.

I. External characters.

I. Unincubated egg. Segmented blastoderm (Figs. I49, 153, D).

2. First day of incubation (18-20 hours). Note the area pellucida, area opaca, primitive streak, and medullary plate (Fig. I53, E).

3. End of first day (about 24 hours). Note the area pellucida, area opaca, pro-amnion, head-process and medullary groove, primitive streak and groove, and the small number of mesodermal segments, which increase in number from before backwards (compare Figs. I 53, E, and I $58, A$ ).

4. Second day. Note- $a$, that the blastoderm has further increased in size, that blood-vessels are apparent in the area opaca, and that the head end of the embryo has become raised above the yolk and is beginning to be covered by the head-fold of the amnion; $b$, the medullary folds, which meet in the middle line except at the posterior end, in front of the primitive streak, while in front the medullary cord thus formed has become swollen to form the vesicles of the brain, and the optic vesicles are seen standing out right and left

1 The early stages are difficult to prepare, and the most important of those referred to below are from the end of the first to the third day of incubation. As the medullary groove only closes gradually from before backwards in the body-region, sections showing different stages in the development of the central nervous system may be obtained from the same embryo at these stages. 
from the fore-brain; $c$, the auditory pits, one on either side of the hind-brain region; $d$, the increase in number of the mesodermal segments; $e$, the heart and main vitelline veins (Figs. 518, A, and I 59, A).

5. Third day. Note- $a$, the further extension of the area vasculosa and its blood-vessels and the folding off of the embryo from the rest of the blastoderm investing the yolk ; $b$, the amnion ; $c$, the head, which is proportionately very large, has become twisted over-so that its right side is now uppermost, and has undergone flexure, the prominent midbrain being now at the anterior end; observe the cerebral hemispheres and the cerebellum in addition to the other parts of the brain; $d$, the visceral arches and clefts; $e$, the olfactory pits, optic cups and lens, and closed auditory vesicles; $f$, the mesodermal segments; $g$, the S-shaped heart, arterial arches, dorsal aorta, and vitelline veins (Figs. $\mathbf{1}_{5} 8, \mathrm{~B}$, and $159, \mathrm{~B})$.

6. Fourth to sixth days. Note- $a$, the advances in the parts mentioned above, including the further extension of the area vasculosa over the yolk, the narrowing of the yolkstalk, the flexure of the whole embryo, and the amnion ; $b$, the budding fore- and hind-limbs; $c$, the allantois, at first a small sac extending from the ventral side of the body and gradually increasing in size (Figs. I $58, C$, and $1_{59}, C$ ). By the sixth day, the embryo can be recognised as that of a bird from the characters of the fore-limbs (wings), hindlimbs, head, etc. : feather-rudiments cannot be recognised until the ninth day.

7. A few further stages should be examined (compare Fig. I 59, D), especially that just before hatching, at the end of the third week of incubation, when it will be found that the albumen has become absorbed, and the very vascular allantois will be seen all round the egg directly under the shell, its stalk entering the body at the umbilicus; the remains of the yolk-sac have been withdrawn into the body at the same point. Note also the neb or caruncle on the beak, used for bieaking the egg-shell at hatching.

II. Transuerse sections.

I. Unincubated egg. ${ }^{1}$ Note the ectoderm and the lower layer cells (Fig. I53, F).

2. First day of incubation. Less difficulty will be experienced if you select embryos towards the end of the first day (20-24 hours). Distinguish between the ectoderm, endoderm, and mesoderm. Note- $a$, the formation of the medullary groove and folds; $b$, the primitive streak and groove; $c$, the notochord (Figs. I 53, G, and I 55).

1 See note about early stages on p. 6r6. 
2. Second day. Note- $a$, the parts mentioned under first day, tracing the series of sections through the whole embryo ; $b$, the gradual meeting of the medullary folds to form the hollow medullary cord; $c$, that anteriorly the head has become folded off from the yolk, so that the enteron in this region forms a closed tube, lined all round by endcderm; $d$, the cerebral vesicles, and the optic vesicles arising from the fore-brain, the lens and the auditory pits from the ectoderm ; $e$, the mesoderm, consisting on either side of dorsal mesodermal segments and of a ventral portion, split into parietal and visceral layers, with the calome between them-the former layer, with the ectoderm, constituting the body-wall or somatopleure, and the latter, with the endoderm, the wall of the enteron, or splanchnopleure; $f$, the heart, vitelline vessels, paired aorta, and cardinal veins (Figs. I 55 and I $57, \mathrm{~A}$ ).

3. Third day. Note- $a$, the parts mentioned above under the first day, and their further development, including the flexure of the brain, formation of the optic cup or secondary optic vesicle, choroid fissure, and the closure of the auditory pits; $b$, the mesonephric (Wolffian) ducts on the dorsal side of the cœlome ; $c$, in the pharyngeal region, the relations of the visceral arches and clefts and of the arterial arches ; $d$, the somatopleuric amniotic folds, and the way in which they meet to form the serous membrane ("false amnion") and the true amnion and amniotic cavity. "The allantois arises from the hinder part of the splanchnopleure on the fourth or fifth day).

(Figs. I57, B, C, I60, I6I, and I64.)

D. I. If you have been fortunate enough to obtain some blastocysts of the rabbit (see p. 549), examine them carefully with a lens, and note the outer layer of cells (trophoblast) and the smaller mass (embryonic area) attached to the former on the inner side of the vesicle at one pole (Fig. I 54, E).

2. Obtain, either fresh or preserved, a gravid uterus of the rabbit or rat containing advanced embryos. Slit open each swelling of the uterus carefully on the ventral side, and note- $a$, the flattened umbilical vesicle (" yolk-sac ") ; $b$, the foetus, enclosed by the thin, transparent amnion, containing a fluid; $c$, the vascular discoid placenta, closely attached to the dorsal side of the uterus, and connected with the navel or umbilicus of the foetus by a vascular umbilical stalk, consisting of the stalk of the allantois and that of the umbilical vesicle twisted around one another (Figs. I67 and I68).

3. Sections should be made through the uterine wall and placenta in order to make out the relations between the maternal and foctal portions of the placenta (Fig. I68). 


\section{INDEX}

(The numbers in italics refer to practical directions.)

A

Abdomen, abdominal cavity, 20, $36 \mathrm{r}, 434,483,506$

Abdominal pore, 432

Abiogenesis, 288

Acanthias, 431, 469

Acetabulum, 50, 503

Aciculum, 352

Acoustic spots, 188

Acrania, 42I

Acromion, 500

Adaptation, 225

Adenoids (lymphatic glands), 507,515

Adrenal bodies, $145,447,53^{8}$

Agamobium, $32 \mathrm{I}$

Albumen, of bird's egg, 566

Alimentary canal, see Enteric canal

Allantois, 609, 6ro

Allolobophora, see Earthworm

Alternation of generations, $32 \mathrm{I}$ Alveoli of jaws, 510

Amnion, 606, 610

Амбви: occurrence and general characters, 229; movements, 232 ; resting condition, 232; nutrition, 233; growth, 234 ; excretion, 235 ; respiration, 235 ; metabolism, 236 ; reproduction, 236 ; immortality, 236 ; conjugation, 237 ; death, 237 ; animal or plant? 255 ; practical directions, 238

Amœboid movements, 106, 23r Amphibia, 219, 418, 482

Amphicoelous, 439
Amphioxus, see Lancelet

Ampulla, of semicircular canals, I 88

Ampulla, of sensory canals of the integument, 464

Anal segment, 327,352

Analogous, analogy, 217

Anatomy, 217

Angular process of mandible, 496

Animals and Plants: comparison of typical forms, 255 ; discussion of doubtful forms, 255, 257; boundaries artificial, 258

Ankle, see Tarsus

Annulata, 220, 349

Anodonta, see Mussel

Anopheles, 287

Antenna, 366

Antennary gland, 375

Antennule, 366

Anura, 219

Anus, 6, 267, 272, 327, 352, 371, $40 \mathrm{I}, 4 \mathrm{I} 8,426,432,486,5 \mathrm{I} 4$

Aorta, aortic arches, see Arteries

Aperture, or apertures (see also Foramen, and under Nephridium, Kidney, Pores, Reproductive organs, \&c.), auditory: Crayfish, 381; Rabbit, 486, 492, 536; cloacal, see anus ; exhalant and inhalant, 397

Aphis, $57^{\circ}$

Appendages, lateral, and their skeleton: Frog, 5, 29, 36, 48, 5I ; Crayfish, 360,363 ; Dogfish, 433, 44I ; Rabbit, 50I, 503; development of, in Vetebrates, 606 
Aqueous chamber and humour, 183

Arachnoid fluid, 155

Archenteron, 579

Archicerebrum, 380

Area opaca, $5^{83}$

Area pellucida, 583

Area vasculosa. $5^{85}$

Arm, see Fore-limb

Arterial arches, 80, 452, 523, 599

Arteries: Frog, 27, 80 ; Crayfish, 376; Mussel, 407; Dogfish, 449 ; Rabbit, 522 ; Vertebrate embryo, 599, 609

Arthobranch, 375

Arthropoda, 220, 360, 386, 4 I I

Articular membrane, 361, 368

Articular processes, see Zygapophyses

Artificial selection, 227

Arytenoid cartilage, I 44, 5I 7

Asexual generation, see Agamobium

Asexual reproduction, see Fis. sion, Budding, Spore

Assimilation, 149, 2.34, 305

Astacus, see Crayfish

Aster, 562

Astragalus, 5I, 504

Atlas vertebra, 498

Atrial pore, 420

Atrium, 420, 42I

Atrophy, see Vestige

Auditory capsule, 39, 4r, 436, 492

Auditory organ (incl. statocyst): Frog., 45, 186; Crayfish, 381 ; Mussel, 409; Dogfish, 465; Rabbit, 535; development of, in Vertebrates, 595

Auditory ossicles, 492, 536

Auricle, see Heart

Automatism, see Movements

Aves, 219,418

Axial fibre: of Vorticella, 272 : of Carchesium, 277

Axial parts, 4, 29

Axis fibre, see Neuraxis

Axis vertebra, 499

Axon, 167

Backbone, see Vertebral column BACTERIA, $\mathrm{r}_{52}$; structure, 257 ; nutrition, 257 ; animals or plants? 257 ; rapid multiplication, 289; practical directions, 260

Basal cartilages of fins, or basalia, $442-443$

Bell of medusæ, see Umbrella

Bilateral symmetry, 296

Bile, 68,148

Bile-duct, $68,447,5$ I 6

Bile-passages, I 34

Binomial nomenclature, 2 I5

Biogenesis, 288

Biology, I

Bird, development of, 582 et seq.

Birds, see Aves

Bladder, see Gall-bladder and Urinary bladder

Blastocole, see Segmentationcavity

Blastocyst, see Blastodermic vesicle

Blastoderm, 384, 470, 574, 582 , embryonic and extraembryonic portions, $58.5,606$

Blastodermic vesicle, 586,6 ro

Blastomere, 577

Blastopore, 201, 349, 384, 579, $58 \mathrm{r}, 582$

Blastostyle, $3 \mathrm{Ir}, 3 \mathrm{I}_{3}, 3 \mathrm{r}_{4}$

Blastula, 577

Blind spot, I83, 556

Blood : Frog, 20, 78, 85, 105 ; Earthworm, 336, 339; Crayfish, 379; Mussel, 408 ; Lancelet, 424 ; Dogfish, 458; Rabbit, 483,543

Blood-corpuscles : colourless, see Leucocytes ; red, 105, 458, 483,543

Blood-sinus, see Sinus

Blood-vessels : Frog, 80 ; Earthworm, 337; Crayfish, 376; Mussel, 407; Lancelet, 424； Dogfish, 449; Rabbit, 520 
Body-cavity, see Coelome

Body of vertebra, see Vertebra Bodỳ-segments, see Metamere Bojanus, organ of, 406

Bone: replacing and investing, 43,489 ; nature of, 52 ; $\mathrm{mi}$ croscopical structure of, II6

Bones, see Endoskeleton, Skull, Vertebra, Ribs, Sternum, and under individual bones of limbs

Botany, I

Brachial plexus, see Nerve-plexus Brain: Frog, 28, 156, 202 ; Earthworm, 342 ; Crayfish, 379: Mussel, 408; Lancelet, 420, 424; Dogfish, 459 ; Rabbit, 528 ; development of, in Vertebrates, 588

Brain-case, see Skull

\section{Branchia, see Gill}

Branchial apertures, arches, clefts, and septa: Tadpole, 204, 206; Lancelet, 420, 422 ; Dogfish, 432, 438, 448 ; development of, 597

Branchial rays, $438,44^{8}$

Branchial vessels: "Crayfish, 377; Mussel, 408; Lancelet, 424; Dogfish, 449; Tadpole, $45^{\mathrm{I}}$

Breast-bone, see Sternum

Bronchus, 517

Brood-pouch, 4 io

Buccal carity, $16,335,445,507$

Buccal groove, 262

Bud, budding, 306, 309

Bufo, Bufonidæ, 218

Bulb, see Medulla oblongata

Bulbus aortæ, 89

Bulla, tympanic, 492

Byssus, 4I I

\section{C}

Cæcum, 5r 4

Cxnosarc, 3Ir

Calcaneum, 5r, 504

Calcar, 52

PRACT. ZOOL.
CaÍciferous glands, 335

Canal : central, of spinal cord, I 56, 424, 459; naso-palatine, 507 ; neural, see Vertebral column; neurenteric, 203; radial and circular of Medusa, 315 ; semicircular, of ear, see Auditory organ and Membranous labyrinth : sensory, of Dogfish, 432, 4646 ; sternal, of Crayfish, 363 ; vertebrarterial, 498

Canaliculi, see Bone

Canine teeth, 509

Capillaries, 95, 377, 451, 454

Capitular facet and capitulum, 498,500

Carapace, 36r

Carbohydrates, 72

Carbon dioxide, 66

Carchesium, 277

Cardiac division, see Stomach

Carotid arch, see Arteries of Frog, Dogfish, and Rabbit

Carpus, 50, 502

Cartilage, 20, 35, II 5, 43I, 435 . 487 ; calcified, $46,48,435$

Caruncle, 610

Castings of earthworm, 326

Cell, IIO, 23I, 558; and see under various types

Cell-colony, see Colony

Cell-differentiation, see Differentiation, and under development of various types

Cell-division, 106, 560, 575

Cell-membrane or wall, 232,244 , $254,255,275,285,559$

Cellulose, $244,254,42$ I

Cement, 445, 5 Io

Centrale, 502, 505

Centrolecithal ova, 574

Centrosome, 560, 568, $57^{2}$

Centrosphere, 560

Centrum, see Vertebra

Cephalothorax, 36r

Cerebellum: Frog, I 57 ; Dogfish, 459 ; Rabbit, 530 ; development of, 590

Cerebral flexure, 592 
Cerebral ganglion, see Brain

Cerebral hemispheres: Frog, 160; Rabbit, 528; development of, 590

Cerebral nerves, see Nerves

Cerebral vesicles, 202, 589

Cerebro-pleural ganglion, 408

Cervical groove, $36 \mathrm{I}$

Chretopoda, $35^{\circ}$

Chalaza, 576 (Fig. 149)

Change of function, 449, 537

Chela, 364, 366

Cheliped, 364

Chiasma, optic, $164,459,530$

Chick, see Bird

Chitin, 232, 328, 36x

Chlorophyll, 242, 251, 304

Chorion, 6I I

Choroid, 183,556

Choroid fissure, 593

Choroid plexus, 157,529

Chromatin, 129, 23I, 560, 573

Chromatophores, 243, 25I

Chromosomes, $560,569,587$

Cilia, and ciliary movement, Io9, 242, 250, 265, 27I, 280

Cilia, absence of, in Crayfish, 386

Ciliary folds, muscles, nerves, and vessels, 184,534

Ciliata, Ciliate Infusoria, 26r, 292

Circulation of blood, $86,90,337$. $375,407,425,449,520$

Circulatory organs, see Bloodvessels and Lymphatic system

Cirri, $352,42 \mathrm{r}$

Clasper, 433, 442, 469

Class, 219

Classification, 2I 7, 220, 223, 4 I I

Clavicle, $47,50 \mathrm{r}$

Clitellum, $327,34^{8}$

Clitoris, $54 \mathrm{I}$

Cloaca, 23, 401, 445

Cloacal aperture, see Anus

Cnemial crest, 504

Cnidoblast, 302

Cnidocil, 302

Coagulation of blood, 78, ro 7

Cochlea, 187,535

Cocoon, 328, 349
Colenterata, 322

Coelomata, 322

Colome, 20, 203, 322, 328, 40r, $422,434,506$; development of, $203,580,586$; extraembryonic, 607

Colomic epithelium, 330; and see Ephithelium and Germinal Epithelium

Coelomic fluid, 336

Collaterals, I $7 \mathbf{I}$

Colon, 514

Colony, Colonial organisms, 277

Columella, 46, I89

Commissures, see Blood-vessels and Nervous system; of brain, I 58, 53 I

Conchiolin, 399

Concrescence, $36 \mathbf{I}$

Condyle, occipital, see Skull

Cones of retina, 185

Conjugation, 197, 237, 251, 268, $274,283,286,287,308,566$, 572 ; and see under development of various types

Conjunctiva, 182

Connectives, $342,379,408$

Connective-tissue, I8, II 3

Contractility, nature of, 112

Conus arteriosus, 79, 88, 449, 520

Coracoid, 47, 443

Coracoid process, 500

Cordylophora, 325

Cornea, 182, 354, 380, 555

Coronoid process, 496

Corpus adiposum, see Fat-body : callosum, 528; cavernosum and spongiosum, 540; striatum, 53I

Corpuscles, see Blood-corpuscles and Leucocytes

Cortex of brain, 160,528

Cortical layer, 264, 269, 280, 286

Craniata, 421

Cranium, see Skull

CRAYFISH: general characters, 360,386 ; limited number and concrescence of metameres, 360 ; appendages, 363,393 ; exoskeleton, $36 \mathrm{r}, 363,387$, 
394 ; muscular system, 369 , 390,392 ; enteric canal, 370 , 390 ; gills, 374,388 ; kidney, 375,393 ; blood-system, 375 , 389; nervous system, 379, 392 ; sense-organs, 380,393 ; reproductive organs, 382,390 ; development, $383,58 \mathrm{I}$

Creation, 22I

Cribriform plate, $49 \mathrm{I}$

Cricoid cartilage, $5^{1} 7$

Crop, 335

Cross-fertilisation, $34^{8}$

Crura cerebri, $157,459,530,590$

Crustacea, 297, 386

Crystalline lens, see Lens of Eye Crystalline style, 4 or

Ctenidia, 398

Cutaneous glands: Frog, I29: Rabbit, $4^{8} 4$

Cuticle: in unicellular animals, $251,264,269,286$; in multicellular animals, 313, 328, 369

Cyst, see Cell-wall and Encystation

Cystic duct, see Bile-duct

Cytoplasm, $55^{8}$

D

Daughter-cells and nuclei, 250, 254,562

Death, II, I 52, 236

Decalcifying, directions for, 137

Decidua, 61 3

Decomposition, II, I52 ; and see Putrefaction.

Degeneration, 42I ; and see Vestige

Dehydrating, directions for, 137

Dendron, 167

Dental formula, 5II

Dental lamina and papilla, 445

Denticles (Nereis), 352

Dentine, 445, 5 Io

Deric epithelium, see Epiderm

Derm, 1 28, 369, 433, 485

Dermal teeth, 434
Descent, doctrine of, see Evolution

Development, meaning of the term, 9, 276, 284. For development of the various multicellular types, see under their names. Practical work $212-214,615$

Dialyser, 73,77

Diaphragm, 483,506

Diastema, 507

Diastole of heart, 90 ; of contractile vacuole, 265

Diencephalon, $157,459,529,590$

Differentiation, 204, 206, 237. 26I

Diffusion, 73, 305, 336, 6r 3

Digestion, 68,233 ; intra- and extra-cellular, 305, 336

Digestive glands, $373,40 \mathrm{r}$, and see Digestion, Enteric canal, Liver, Glands, Pancreas

Digestive system, see Enteric canal

Digits, 50, 502, 505

Dimorphic, Dimorphism, 250

Diøecious, 320,382

Diploblastic 298, 3 I I

Directions for dissecting, 14 ; for drawing, 14; for killing, 31, $239,259,278,293,324,354$, $386,412,542$; for preparing skeletons, $53,394,472,542$; for injecting blood-vessels, 99 . $389,414,474.543$; for microscopic work, 119; for histological and embryological work, $135,575,615$

Disc of Vorticella, $27 \mathrm{I}$

Discoid segmentation, $\mathbf{5 7 5}$

Dispersal, $274,283,287$

Dissecting instruments, etc., 12

Distal, 6

Distribution of food-materials, $14^{8}$

Divergence of character, 223

Division of physiological labour, 206,238

Dogfish: General characters, 431 ; exoskeleton, 433, 472; 
endoskeleton, 435,472 ; enteric canal, 444,473 ; gills, 448, 478; blood-system, 449, 474,477 ; nervous system, 458,478 ; kidneys, 466,476 ; reproductive organs, 466,475 ; sense-organs, 464,479 ; development, 469,582

Dorsal, 6

Drum-membrane, see Tympanic membrane

Ducts, see under names of individual ducts and glands,

Ductus arteriosus, 550

Duodenum, 22; and see Enteric canal

Dura mater, I 55

\section{E}

Ear, see Auditory organ: internal, middle, and external, $466,492,535,595$

EARTHWORM : general characters, 326 : metameric segmentation, 327; coelome and enteric canal, $328,333,335,355$, 358 ; cell-layers, 329,358 ; bloodsystem, $337,355,357,358$; nephridia, $340,355,357,358$ : nervous system, $342,357,358$; differentiation of organs and tissues, 344 ; reproduction and reproductive organs, 345,355 , 356 ; development, 349,581

Ecdysis, 369

Echinodermata, 412

Ectoderm, 202, 209, 298, 3II, $314,317,349,384,470,583$, 586

Ectoplasm, 23I

Efferent duct of spermary, r93. $347,466,603$

Egestion, 233

Egg, 8

Egg of fowl, 565

Egg-cell, see Ovum

Egg-sac, 346

Elasmobranchii, 43i
Embryo, 9, 200; and see under various types

Embryology, 2I7

Embryonic area, 586

Embryonic membranes, 606

Emulsification of fats, 75

Enamel, 445, 5 Io

Enamel-organ, 445

Encystation, 232, 244, 254, 275 . 283,285

Endoderm, 202, 210, 298, 303, $3 \mathrm{II}, 3 \mathrm{I}_{4}, 349,384,47 \mathrm{O}, 583$, 586

Endoderm-lamella, 315, 3I7

Endolymph, I 88

Endolymphatic duct, 436,465

Endoparasite, see Parasite

Endoplasm, 23I

Endophragmal system, 363

Endopodite, 364

Endoskeleton: Frog, I6, 35, 205; Lancelet, 422; Dogfish, 435; Rabbit, 487 ; development of, in Vertebrates, 604

Endostyle, 424

Energy, conversion of potential into kinetic, 235 ; source of, in chlorophyll-containing organisms, 248

Enteric canal: Frog, 22, 204 ; Earthworm, 328, 335; Crayfish, 370 ; Mussel, 401 ; Lancelet, 424 ; Dogfish, 444 ; Rabbit, 507-516; development of, in Vertebrates, 204-210, 595

Enteric epithelium, see Epithelium

Enteroccele, 580

Enteron or enteric cavity, 296, 298,3 II, 3I 4, 330, 373, 580, 595

Epencephalon, 590

Epiblast, see Ectoderm and p. 580

Epiderm: Frog, 128; Earthworm, 328, 329 ; Crayfish, 369 ; Mussel, 400 ; Lancelet, 4I9 ; Dogfish, 433: Rabbit, 484

Epididymis, 466, 538,603

Epiglottis, 512, 51 7 
Epipharyngeal groove, $42 \%$

Epiphysis, 497,503

Epipodite, 366

Epistoma, 363

Epistylis, 277

Epithelial cells : columnar, 107: ciliated, ro9; glandular, I 30 et seq. ; squamous, IIo; stratified, 128

Epithelium, 109, 319 ; colomic, 330 (and see Peritoneum): deric-see Epiderm; enteric, 330 (and see Endoderm)

Equilibration, organ of, see Auditory organ

Equivocal generation, see Abiogenesis

EUGLENA : occurrence and general characters, 25I; movements, 251 ; structure, $25 \mathrm{I}$; nutrition, 254; resting stagc, 254 ; reproduction, 254; animal or plant? 255; practical directions, 259

Eustachian tube or recess, 17. I 89, 512, 536

Eustachian valve, 523

Evolution: organic, 293 : of animals and plants, 293; of animals and plants, $25^{8}$

Excretion, I48, I 50, 235

Execretory organs, see Kidney and Nephridium, and compare Contractile vacuole

Exopodite, 364

Exoskeleton: cuticular, $3 \mathbf{I}_{3}$, 369,398 ; dermal, 434, 444: epidermal, $482,4^{84}$

Expiration, I43

Extra-cellular digestion, 234. 305

Eye: Frog, 5, r81; Nereis, 35r, 353; Crayfish, 380 ; Lancelet, 424; Dogfish, 465; Rabbit 486, 534; development in Vertebrates, 593

Eye : compound, 380

Eyelids, 5, 432, 486

Eye-muscles, see Muscles of eye
Eye-spot: Euglena, 254; Lancelet, 424

Eye-stalks, 363, 380
Fabellæe, 504

Facet, 38

Fæces, 8

Family, $2 \times 8$

Fascia, I 8, 59

Fat-body, 23

Fats, 72

Femur, 5I, 504

Fenestra ovalis, $46,189,492,536$

Fenestra rotunda, 493, 536

Ferment, fermentation : amylolytic, 74 ; peptonising or proteolytic, 74 ; putrefactive, 256,257

Fertilisation, r97, 566, 572 ; and see also Conjugation, and under development of various types

Fibrin, 107

Fibula, 5I, 504

Filum terminale, I55, 532

Fingers, see Digits

Fin-rays, $422,430,44 \mathrm{I}-443$; dermal, $44^{2}$

Fins: Tadpole, 207; Lancelet, 42I; Dogfish, 433, 44I

Fishes, see Pisces

Fission, 106, 198, 236, 250, 268, 273,283 ; multiple, 237,254 , 283,286

Fissures of spinal cord, 155

Fixing, directions for, 136

Flagellata, Flagellate Infusoria, $26 \pi, 292$

Flagellum, 242, 25I, 256, 303

Flocculus, 493, 530

Foetus, 54 I

Follicle, ovarian, see Ovisac

Fontanelle, 44, 436

Foods, 67, 72

Foot, of mussel, 397; and sec Pes Foramen : lacrymal, 494 ; intervertebral, $38,440,498$ : mag- 
num, 40, 436, 487; obturator, 503 ; of Monro, 160,531 Foramina for cerebral nerves, see Skull

Fore-brain, 202, 589

Fore-gut, $37 \mathrm{I}$

Fore-limb or fin, see Appendages

Fornix, 53I

Fossa ovalis, $52 \mathrm{I}$

Fossils, 223

Frog: Preliminary account, 4 ; mouth-cavity, r6; skin and muscles, 17,31 ; abdomen and its contents, 20, 32; neural cavity and its contents, 27. 33; structure of limbs, 28 , 34 ; skeleton, 35,53 ; joints, 55, 64; muscles, 57,64 ; enteric canal and digestion, 67,76 ; vascular system, 78,98 ; circulation of blood, 89,103 ; lymphatic system, $97, .98$; simple tissues, 104, 121; compound tissues and glands, 126, 139 ; lungs and larynx, I4I, 152; kidneys, 145,153 ; structure and functions of nervous system, I54, 175 ; sense-organs, $x 79,191$; reproductive organs, 193,210 ; fertilisation of eggs, 9, 197 ; development, 9, 198, 212, 214, 575. 58I ; metamorphosis, II, 206, 212; classification, 215 ; side dissection, 481 ; summary of characters, 482

Function, see Physiology

\section{G}

Gall-bladder, 20, 68, 447, 516

Gallus, see Bird

Gamete, I97,250, 268, 275, 286, $287,566,573$

Gametocyte, 286, 287, 348, 356, 566

Gametogenesis, 566
Gamobium, 32 I

Ganglion, 163,167 ; and see Nerve-ganglia

Gastric glands, see Gland

Gastric juice, 71, 74, I32

Gastric mill, 372

Gastrocnemius, 59

Gastrolith, 373

Gastrula, 577,582

Gemmation, see Budding

Generation, alternation of, see Alternation of generations

Generation, asexual, see Agamobium

Generation, sexual, see Gamobium

Generative organs, see Reproductive organs

Genus, 215,218

Germ-cells, see Sex-cells

Germ-plasm, 573

Germinal disc, 575, 582

Germinal epithelium, I94, I96, $345,383,566$

Germinal vesicle and spots, 565

Gestation, 542

Giant-fibres, 343

Gill-arches and clefts, see Branchial apertures, arches, ciefts and septa

Gill-cover, 36r, 374

Gill-rays, see Branchial rays

Gills: Tadpole, 9, 204, 207; Crayfish, 374; Mussel, 398, 401; Dogfish, 448, 470

Gizzard : Earthworm, 335 ; Crayfish, $37^{\circ}$

Gland-cells: Hydra, 303, 305 ; Earthworm, 335; and see Glands and Goblet-cells

Glands : Cowper's, 539; cutaneous, I29, 484; digestive, see Enteric canal; ductless, 447: gastric, I3 1 , 447, 514; green, 375 ; Harderian, 186 , 535 ; lacrymal, 535 : mammary, 483, 486, 506; Meibomian, 535; cesophageal, 335 ; perineal, $486,54^{\circ}$; prostate, 539; racemose, 133 ; 
rectal, 540; salivary, $5 \mathrm{II}$; sebaceous, 484 ; and see kidney, liver, pancreas, etc.

Glands, ductless, 447, 507

Glenoid cavity, 47, 500

Glochidium, 4 I I

Glomerulus, 146,601

Glottis, I7, 512

Glycogen, 135

Goblet-cells, I09, 130, I3I

Gonad, 193; and see Reproductive organs

Gonaduct, see Reproductive organs

Gonotheca, 312

Gregarines, $28+$

Grey matter of spinal cord and brain, $156,160,167$

Growth, 236

Gryllotalpa, 567

Gullet, 22, 253, 265, 27I ; and see Enteric canal

\section{$\mathbf{H}$}

Hæmal arch and spine, $44 \mathrm{I}$

Hæmatochrome, 243

Hæmatococcus, see SPHerRELA

Hæmocœle, 374

Hremocyanin, 379

Haemoglobin, 107, 339

Hairs, 482,484

Hallux, 505

Hand, see Manus

Hardening, directions for, 136

Harderian gland, 186,535

Head, 4, 350, 36 $\mathrm{r}, 43 \mathrm{I}, 484$, 486

Head-fold and process, 589

Heart, $20,79,87,378,407,449$, 520: development of, 458 , 597 ; pulsation of, 90

Heat, evolution of, I 52

Hemibranch, $44^{8}$

Hepatic cacum, 424

Hepatic ducts, see Bile-duct

Hepatic portal system, see Portal system
Heredity, 225

Hermaphrodite, see Monoecious

Heterocercal tail, 433

Heterogenesis, 29I

Hibernation, 8

Higher (triploblastic) animals, uniformity in general structure, 332

Hind-brain, 202, 589

Hind-gut, $37 \mathrm{I}$

Hind-limb or Fin, see Appendages

Hinge of lamellibranchiate shell, 396,398

.Hip-girdle, see Pelvic arch

Hippocampus, 531

Histological methods, 120, 135 , 575

Histology, ro4

Holoblastic segmentation, 574

Holobranch, $44^{8}$

Holophytic nutrition, 247, 255

Holozoic nutrition, 247, 255

Homogenesis, 290

Homology and homologous, 217 . 318

Homology, serial, 39, 52, 327

Host, 282, 284, 287

Humerus, 48 , 5 or

Hybrids, 216

HYDRA : occurrence and general characters, 294 ; species, 296 ; movements, 296; mode of feeding, 297; microscopic structure, 297; digestion, 304: asexual, artificial, and sexual reproduction, 306 ; development, 308 ; - practical directions, 322

Hydranth, 309

Hydroid polypes, 308

Hydrotheca, 312

Hydrozoa, 322

Hyoid, $40,44,438,496$

Hyomandibular, 438

Hypoblast, see Endoderm and P. 580

Hypobranchial groove, see Endostyle

Hypostome, 295, 309, 317 


\section{I}

Ileum, 22; and see Enteric canal

Ilium, 50, 503

Imbedding, directions for, 138

Immortality, 236

Impregnation, see Fertilisation

Incisors, 509

Income and expenditure, $I_{4} 8$, 236,245

Incubation, 615

Incus, see Auditory ossicles

Individual, 220,277

Individuation, 277, 306

Infundibulum, of brain, I59, $459,530,590$; of lung, 518

Infusoria, 261, 292

Ingesta and egesta, balance of, 236

Ingestion, 8, 233

Inguinal canal, 538

Injection of blood-vessels, 99, $389,414,474,477,543$

Innominate bone, 5I, 503

Insertion of muscle, 60

Inspiration, I43

Integument, structure of, 127 ; and see under various types

Integumentary sense-organs, 430,464

Intercellular substance, I I 5, x I 8

Interneural plate, $44^{\circ}$

Interrenals, 447

Interstitial cells, 298

Intervertebral discs, 497

Intervertebral foramina, see Foramen

Intervertebral substance, 440, 44 I

Intestine, 22; see its various subdivisions and Enteric canal

Intracellular digestion, 234, 305

Invagination, 384,577

Invertebrata, 412

Iris, 5, I82, 555

Irritability, 60, I69, 232, 272, 319

Ischium, 5I, 503
Jacobson's organ, 534

Jaws, $17,353,364,367,436$, 489,496

Joints, 55, 362, 368

\section{K}

Karyokinesis, 563

Keber's organ, see Pericardial gland

Kidney: Frog, 25, I45; Crayfish, 375; Mussel, 406; Dogfish, 466; Rabbit, 537; development of, in Vertebrates, 600

\section{L}

Labial palp, see Palp

Labrum, 370

Lacrymal glands, I86, 535

Lacunæ, see Bone and cartilage

Lamellæ and laminæ of gills (Mussel), 40r

Lamellibranchiata, 4 II

Lamina terminalis, 530

LANCELET : general characters, 4I9; fins, 4I9; skeleton, 422 ; gill-slits and bars, 420,422 ; enteric canal, 424: blondvessels, 424 ; nephridia, 424 ; nervous system, 424 ; gonads, $42 \mathrm{~T}, 425$; development, 425 . 577 ; practical directions, 615 Larva, 9, 354, 384, 4II, 425, 580 Laryngo-tracheal chamber, I $4 \mathrm{I}$ Larynx, I 44, 506, 517

Lateral line, 432, 464

Laverania, see Malaria organism Legs, see Appendages

Lens of eye, $183,353,534,593$ LEPUS, see Rabbit

Lencocyte, I05, 336, 379, $45^{8}$ Life, origin of, 288 ; and see Biogenesis

Life-history, 8, 206; and see under various types 
Iigaments, 55, 57

Limbs, see Appendages

Limnæa, 576

Linin, 560

Lips, 486, 507

Lithite, 315, 409

Lithocyst, 3 I 5

Liver, 20, I33, I 47, 204, 424, $447,516,597$

Liver-fluke, 412

Lobster, 360

Lumbricidæ, $35^{\circ}$

LUMBRICUS, see Earthworm

Lungs, 10, 22, 141, 204, 208, 518, 597

Lymph, 18

Lymph-hearts, 97

Lymphatic glands, see Adenoids Lymphatic system, I 8, $97,45^{8}$, 527

\section{M}

Malaria organism, 287

Malleus, see Auditory ossicles Malpighian capsule, 146, 538, 601

Mammalia, 219, 418,482, 585, 610

Mammary glands, see Glands

Mandible, 16, 44, 366. 438, 496, 537

Mantle, 396

Mantle-cavity, 400

Manubrium: of Medusa, 3T4; of malleus, 536 ; of sternum, 500

Manus, 5, 50, 484, 502

Marginal sense-organs, see Lithocyst

Marrow-cavity, 48

Matrix, see Inter-cellular substance

Maturation of ovum, 569

Maxilla: Crayfish, 366

Maxilliped, 364

Meckel's cartilage, 44,438

Mediastinum, $5 \times 8$

Medulla oblongata, I57, 459, 530,590
Medullary cord, folds, groove, and plate, 202, 588 ; sheath, I67; substance of Protozoa, $264,269,280,286$

Medusa, medusa-buds, 3 II, 3 I 3 , $3 I_{4}, 3$ I 5

Megagamete, 275, 287

Megalecithal ova, 574 .

Meganucleus, 265, 271

Megazooid, 250, 275

Meibomian glands, 535

Membranous labyrinth, 186,465 , 535,595

Meroblastic segmentation, 574 Mesencephalon, see Mid-brain Mesenteron, 371, 595; and see Enteron

Mesentery, 23, 27,447, 51 5

Mesoblast, see Mesoderm and p. 580

Mesoderm, 202, 203, 210, 349 , $384,470,580,586$; vertebral and lateral plates of, 588

Mesodermal segments, 580

Mesogloea, 298, 3II, 3I4, 3I5, 317

Mesonephros and mesonephric duct, $60 \mathrm{I}$

Metabolism, 149, 236

Metacarpus, 50,502

Metacromion, 500

Metagenesis, $32 \mathrm{I}$

Metamere, Metameric segmentation, $327,360,368$

Metamorphosis, II, 209, 276; and see Larva

Metanephros and metanephric duct, 603

Metapleural folds, $42 \mathrm{I}$

Metatarsus, 5I, 505

Metazoa, 293

Metencephalon, see Medulla oblongata

Microgamete, 275, 287

Microlecithal ova, 574

Micrometer, 121

Micromillimetre $(\mu)=10^{1} \mathrm{o}$ of a millimetre, or grbo of an inch

Micronucleus, 265, 27 I

Micropyle, 4I5, 572 
Microtome, 139

Microzooid, 250, 275

Mid-brain, 202, 589

Mid-gut, 37 I

Milk-glands, see Glands

Milk-teeth, 5 ro

Milt, see Spermatic substance

Mitosis, 563

Molars, 509

Mollusca, 220, 4 II

MoNADS : occurrence ana general characters, 256 ; movements, 256 ; nutrition, 256 ; animals or plants? 258 ; rapid multiplication, 289 ; practical directions, 260

Monocystis : occurrence, 284 ; structure, 286 ; parasitic nutrition, 286 ; conjugation and reproduction, 286,287 ; encystation and spore-formation, 285,286 ; means of dispersal, 287; practical directions, 293

Monœecious, 307

Morphology, 2I 7

Morula, see Polyplast

Mosquito, 288

Mother of pearl, see Nacre

Mounting sections, directions for, 139

Mouth, 4, I6, 253, 265, 27x, 295, 3II, 3I 4, 37O, 40I, 42I, 43I, 486

Mouth-cavity, see Buccal cavity Movement, spontaneous, voluntary, and involuntary, $7, \mathbf{I r}$, II2, I72, 232, 319, 327, 433 : and see under various types

Mucous membrane, I7

Mule, see Hybrid, 2x6

Müllerian duct, 603

Multinucleate, II 3, 28I

Muscle-fibres : striped, I12 ; unstriped, III, I3I, $\mathbf{r}_{32}$; and see under various types

Muscle-plate, 580

Muscle-processes, 298, 304, 3 II 318

Muscles : Frog, 18, 57, 59, 63 ,
205; Obelia, 3II ; Earthworm, 329; Cravfish, 369; Mussel, 399, 400 ; Lancelet, 419: Dogfish, 434; Rabbit, 505 ; and see Myomere

Muscles : of eye, $186,465,534$; of middle ear, 536

Muscles, papillary, $52 \mathrm{I}$

Muscular contraction, 60 , III, 273

Muscular impressions on shell, 399

Muscular layers of enteric canal, $7 \mathrm{O}, 75$, I $3 \mathrm{I}, \mathrm{I} 32$

Muscular system, see under various types; development of, in Vertebrates, 203, 588, 604

Muscularis mucosæ, I33

Mussel: general characters, 396 ; 412 ; mantle, shell, and foot, $396,397,412$; foodcurrent, 404, 412 ; gills, 397. 401,414 ; muscles, 399, 400, 413 ; enteric canal, 401,416 ; nephridia,-406, 415; bloodsystem, 407, 414; nervous system, 408, 415; senseorgans, 409; 416; gonads, 409, 417; development and metamorphosis, 410, 415, 58I

Mustelus, 43I, 469

Myocomma, 434

Myomere, 203, 369, 419, 423, 434,588

\section{$\mathrm{N}$}

Nacre: nacreous layer, 399

Nasal organ, see Olfactory organ

Naso-lacrymal duct, $\mathbf{I} 86,535$

Naso-palatine canals, see Canals

Naso-pharynx, 5I 2

Natural History, 2

Natural selection, 227

Nauplius, 384

Navel, 597, 613

Navicular bone, 505

Neck, 484 
Nemathelminthes, $4 \mathrm{r} 2$

Nematocyst, $300,3 I I, 3 I 7$

Nephridiopore, 34I

Nephridium, 146, 340, 406, 421, 424,600

Nephrostome, 98, 145 (Fig. 46), I 46 (Fig. 47), 340, 424, 60I

Nereis: occurrence and general characters, 350 ; parapods and setae, 350-352; senseorgans, 353 ; trochosphere larva, 354 ; practical directions, 359

Nerve-cells, $167,303,318,343$

Nerve-collar, 342

Nerve-cord, ventral, 342,379

Nerve-fibres, I 67

Nerve-forzmina, 495; and see Skull and Vertebral column

Nerve-ganglia, I62, I63, I64, $167,342,379,408,592$

Nerve-plexus: brachial, I6r, 532 ; coeliac, 533 ; sciatic or lumbosacral, I62, 532

Nerve-roots, I63, 425, 46r, 532

Nerves: afferent and efferent, I66, I75 ; cerebral, I63, 46r, 532,$592 ;$ of lateral line, 463 ; motor and sensory, I62, I69: phrenic, 532 ; sciatic, 62, I62, 532 : spinal, I $60,425,46 \mathrm{r}$, 532 ; sympathetic, 162,461 , 533 ; vaso-motor, 174 ; visceral, 380

Nervous impulse, 62, 154

Nervous system: Frog, 155; Hydra, 303; Obelia, 318; Earthworm, 342 ; Crayfish, 379; Mussel, 408; Lancelet, 424; Dogfish, 459; Rabbit, 528 ; development of, in Vertebrates, 588

Neural arch, spine, and canal, see Vertebra

Neural cord and plate, see Medullarv cord

Neural crest, 592

Neural plate and process, $44^{\circ}$

Neuraxis, 167

Neurenteric canal, see Canal
Neuroccele, 459 ; and see Canal, central of spinal cord, and Ventricles of brain

Neuroglia, 169

Neurolemma, 167

Neurone, 167

Neuropod, 35I

Newt, 218

Nictitating membrane, 5, 486

Nodes of nerve-fibre, 167

Nose, see Olfactory organ

Nostrils, $4,17,180,432,464,4^{87}$. 534

Notochord, 203, 419, 441, 580, 604

Notopod, 35I

Nuclear division, 560; indirect and direct, 563

Nuclear membrane, 559 ; spindle, 560

Nucleolus, ro9; and see under Nucleus

Nucleoplasm, 599

Nucleus, ro6 ot seg., r29, 23I, $243,254,265,269,280,286$, $559,567,569$

Nucleus, conjugation-, 268 ; segmentation-, $57^{2}$

Nutrition, 67; and see under various types

O

ObeLIA : occurrence and general characters, 309; microscopic structure, $3 I I$; structure of medusa, 314 ; nervous system, 318 ; lithocysts, 315 ; reproduction and development, 320 ; alternation of generations, 321 ; practical directions, 325

Occipital condyle, see Skull

Ocellus, see Eye-spot

Odontoblasts, 445

Odontoid process, 499

Esophageal glands, see Calciferous glands

Ossophagus, see Gullet

Olecranon, 49, 502 
Olfactory capsule, $39,42,436$, 493

Olfactory lobe, I6o, 460,528

Olfactory organs: Frog, r8o ; Crayfish, 38r ; Mussel, 409; Dogfish, 432, 464; Rabbit, 534; development of, in Vertebrates, 592

Olfactory pit, 424

Oligochreta, $35^{\circ}$

Ommatideum, 380

Omosternum, 48

Ontogeny, 224

Oogenesis, 196, 568

Oosperm, 198, 572; and see under various types

Oosperm, holoblastic and meroblastic, 573, 574

OPALINA : occurrence and general characters, 280 ; nuclei, 280 ; parasitic nutrition, 282 ; reproduction, 282 ; means of dispersal, 283; development, 283 ; practical directions, 293

Optic chiasma, see Chiasma; cup, 593 ; lobe, I57, 459, 530, 590 ; thalamus, I59, 529 ; vesicle, 593

Optical section, $323,55^{8}$

Oral cavity, see Buccal cavity

Oral hood, 42 I

Orbit, see Skull

Order, 218

Organ, 30, 3I, I 5I, 238, 344

Organism, 23 I

Origin: of muscle, 60 ; of species, 225

Osmosis, 73

Osphradium, 409

Ossicles, auditory, see Auditory ossicles

Ossification, 44

Osteoblasts, II 7

Ostia : of heart, 376 ; of gills, 404

Otocyst, 315 (see also under Statocyst)

Otoliths, 188, 382

Ovary: Frog, 23, 193, 195; and see Reproductive organs
Oviduct, 25, r96, 346, 382, 467 $54^{\circ}, 603$

Ovisac, I95, 540

Ovum, 195, 564, 568; and se under various types

Ovum : microlecithal, megaleci thal, centrolecithal, and telo lecithal, 574

Oxidation of protoplasm, I 49 $24^{8}$

\section{P}

Palæontology, 224

Palate, 494, 507

Palatoquadrate, 44,436

Pallial line, 399

Pallium, see Mantle

Palp: Nereis, 35I ; Crayfish 367 ; Mussel, 4 or

Pancreas, 22, 70, 133, 204, 447 516, 597

Pancreatic juice, 70, 74

Papillæe of tongue, I80, 509

Parachordal cartilages, 605

Paragnatha, 370

Paramylum, 25I

PARAMCECIUM : structure. 262 mode of feeding, 266; repro duction, 268; conjugation 268 ; practical directions, $2 \%$

Parapod, $35^{\circ}$

Parasite, parasitism, 33, 153 $280,282,284,287$

Parietal layer of peritoneum, 26 331 ; see also Mesoderm

Parthenogenesis, 570

Patella, 504

Pectoral arch, 20, 36, 46, $44^{2}$ 500,606

Pectoral fin or limb, see Appen dages

Pedal ganglion, 408

Pelecypoda, 4II

Pelvic arch, 20, 36, 50, 442, 503 606

Pelvic fin or limb, see Appendages

Pelvis of kidney, 538

Penis, 487,540 
Pepsin, peptone, 74

Pericardial gland, 406

Pericardial sinus, 376

Pericardium, 20, 40I, 434, 520

Perichondrium, 55

Perilymph, I89

Perinæum, 486,540

Periosteum, 55

Periostracum, 399

Perisarc, 312

Peristaltic movements, 75

Peristome, 27I

Peristomium, 327, 35I

Peritoneum, 22, 26, 330, 434, 506

Pes, 5, 5I, 484, 505

Peyer's patches, 5I5

Phalanges, 50, 5I, 502, 505

Pharynx, I $7,335,445,448,597$

Photosynthesis, 248

Phylogeny, 224

Phylum, 219

Physiology, I, 217

Pia mater, 155

Pigment cells, 128

Pigment-layer of retina, I 85

Pigment-spot, -254, 424

Pineal body, I 59, 459, 530

Pineal eye, 159.591 .

Pinna, 483,486

Pisces, 219; general characters of, $4 \mathrm{I} 8$

Pisiform bone, 502

Pituitary body, I 59, 459, 530

Placenta, $483,54 \mathrm{I}, 610$

Placoid scales, 434

Planula, 32 I

Plasma, $\mathrm{IO}_{4}$

Plastic products, 249

Platyhelminthes, 412,545

Pleopod, 364

Pleura, pleural membrane, 506, 518

Pleurobranch, 375

Pleuron, Cray fish, 36r

Podobranch, 375

Podomere, 363

Polar cells, 570

Pollex, 502

Polychæta, 350

Polymorphic, polymorphism, 3r 8
Polype, 294, 309

Polyplast, 200, 577; and see under various types

Polystomum, 33

Pons Varolii, 530

Pores, dorsal, 333

Portal system : hepatic, 85,456 , 527 ; renal, 85,454

Post-axial and pre-axial borders of $\operatorname{limb}, 501,503$

Poupart's ligament, 506

Premolars, 509

Prepuce, 487

Preservative fluids, 13

Primitive streak and groove, $5^{81}, 5^{83}$

Prismatic layer, 399

Proamnion, 583, 606

Proboscis, 353

Processes of skull, $489 \mathrm{et}$ seq.

Procoelous, 36

Proctodaeum, 204, 384, 447,'597

Pronation, 50I

Pronephric duct, 6oI

Pronephros, 6or

Pronucleus, male and female, $570,57^{2}$

Prosencephalon, 460, 590

Prostate, see Gland

Prostomium, 327, 35 I

Proteids, 72

Protista, 258

Protococcus, see SPHArella

Protoplasm, 106, 558; and see Cell

Protopodite, 364

Protovertebra, 588

Protozoa, 220, 292

Proximal, 6

Pseudobranch, 449

Pseudopod, I06, 231, 250, 303

Pterygiophores, $44 \mathrm{I}$

Ptyalin, 511

Pubis, 5I, 503

Pulmonary artery and vein, see Arteries and Veins

Pulse, 86

Pupil, 5, 182, 353

Putrefaction, 256, 257; and see Decomposition 
Pyloric division and.valve, of stomach, see Stomach

Pyramid of kidney, 538

Pyrenoid, 243, 249

\section{$\mathbf{R}$}

RABBIT: general characters, 482,542 ; skeleton, 487,542 ; muscles and body-wall, 505 . $543,544,549,552,556$; coelome, $506,545,550$; di. gestive organs, $507,545-547$; respiratory and vocal organs, $517,544,551,554$; circulatory organs, $520,544,545$ $547,550-554$; nervous system, $528,544,549,554$; senseorgans, $533,552-556$; urinogenital organs, $537,545,548$; development, $54 \mathrm{I}, 549,585$

Racemose gland, I 33

Radial canals, see Canal

Radial symmetry, 296

Radio-ulna, 49

Radius, 50, $50 \mathrm{r}$

Rana esculenta, 216

Rana temporaria, see Frog

Ranidæ, 218

Reagents, hardening, preserving, mounting, and staining, 135

Recapitulation, theory of, 224

Rectal gland, 447,540

Rectum, 23; and see Enteric canal

Reducing division, 568,570

Reflex action, I69, 319

Regeneration, 268, 307, 345

Renal portal system, see Portal system

Replacement of organs, 209

Reproduction, 8 ; and see under various types, and alsoAsexual reproduction

Reproductive organs, 23, 23 , I93, 307, 320, 345, 382, 409, $421,425,467,538,603$

Reptilia, 219, 418
Reservoir of contractile vacuole, 254

Respiration, I4I, I 43, 235, 339, $374,448,517$

Respiratory movements, $7, \mathrm{I}_{42}$, 375,520

Retina, $\mathbf{1 8 3}, \mathbf{1 8 4}, 353,556$

Retinula, 380

Rhabdome, 380

Rhabdonema, 153

Rhinencephalon, 590

Rhizopoda, 292

Ribs, $486,498,500$

Rocks, sedimentary and stratified, 223

Rodentia, 542

Rods and cones, 185

Rostrum, 363. 436

Rudiment, often used for Vestige, $(q . v$.

S

Sacculus, 187

Sacculus rotundus, 515.547

Sacrum, 38, 499

Salamander, 218

Salivary glands, see Glands

Saprophytic nutrition, 256, 257

Sarcolemma, II 3

Scales, 430, 433

Scapula, 47, 443, 500

Schizocoele, 588

Sclerite, 390

Sclerotic, 182, 534,555

Scrotal sac, $487,538,548$

Scyllium, see Dogfish

Sebaceous glands, see Glands

Section-cutting, directions for, I $26,1_{3} 8$

Secretion, 130

Segment, see Metamere, Podomere

Segmentation-cavity, 200, 577 . $581,5^{83}$

Segmentation of oosperm, 198, $200,212,308,320,349,383$, $410,470,574,577,581,582$, $5^{8} 5$ 
Segmentation, equal and unequal, 575 ; discoid, 575

superficial, 383 , 575 metameric, see Metamere

-nucleus, 572

Selection, natural and artificial, 226,227

Self-fertilisation, $34^{8}$

Seminal funnel, 347

vesicle, $194,347,467$

Sense-organs and cells, 179, 31 5 . $344,351,380,409,424,432$, $464,533,592$

Septa, of earthworm, 333

Septum: lucidum, 53I; nasal, 42,493 ; interorbital, $4^{87}$

Serous membrane of embryo, 609,610

Sesamoid bones, 502, 505

Seta, $328,350,351,362$

Sex-cells, primitive, 566,568

Sexual characters, external, 7 , $382,433,4^{86}$

Sexual generation, see Gamobium

Sexual organs, see Reproductive organs

Sexual reproduction, see under various types

Shank, 5

Shell, 396 ; larval, 4 ro

Shell of egg, 470,566

Shell-gland of mussel-embryo, 410; of dogfish, 469

Shoulder-girdle, see Pectoral arch

Sinus: blood-, 376, 377, 408, 457 ; lymph-, I 8,27 ; urinary and urinogenital, 466 , 467 ; venosus, $80,89,449$, 520

Siphon, inhalant and exhalant, 397

Skeleton, see Endo- and Exoskeleton

Skin, see Integument

Skull: Frog. 16, 35, 39 ; Dogfish,
436; Rabbit, 487; development of, 604

Smell, organ of, see Olfactory organ

Snout, 4

Somatic layer of mesoderm, 580

Somatopleure, $5^{80}$

Spawn, 9

Species, 215 et seg. ; origin of, 222,225

Sperm, or Spermatozooid, I94, 382,568 ; and see under various types

Spermary, 25, r93, r94; and see Reproductive organs

Spermatic substance, 9

Spermatogenesis, 194, 566

Spermatophore, 382

Spermiduct, 193, 347, 382, 466, 538,603

Spermotheca, 347

Sperm-reservoir, 347

Sperm-sac, 347,467

SPHARELLA : general characters, 240 ; rate of progression, 240 ; ciliary movements, 242,250 ; colouring matter, 242 ; motile and stationary phases, 244 : nutrition, $245 ;$ source of energy, 248; dimorphism, 250 ; reproduction, 250 ; conjugation, 25I ; animal or plant? 255; practical directions, 259

Sphincter, $7 \mathbf{I}$

Spinal cord, 27, 155, 424, 459. 532,592

Spindle, nuclear, 560

Spiracle, 432, 449

Spiral valve, 445,5 I 5

Spireme, 560

Splanchnic layer of mesoderm, $5^{80}$

Splanchnopleure, 580

Spleen, 23, 98, 447, 51 6

Spontaneous generation, see Abiogenesis

Spores, $254,275,286$

Sporozoa, 284, 292

Sporozoite, 287 
Staining, directions for, $\mathbf{I} 37$

Stalk of Vorticella, 269, 272

Stapes, 46, I89, 492, 536

Starch, 72, 243, 252

Starfishes, 4I 4

Statocyst, 31 5, 381, 409

Sterilised infusions, 289

Sternal canal, 363

Sternebræ, 500

Sternum, I6, 47, 361, 486, 500

Stigma, 254

Stimulus, various kinds of, 62

Stock, see Colony

Stomach, 22, 70, 401, 445, 5I 2

Stomodaum, 203, 384, 445, 447 , 597

Struggle for existence, 226

Submucosa, 1 30

Substitution of organs, 209

Sucker, 203

Superficial or peripheral segmentation, 575

Supination, 5 Or

Supporting lamella, see Mesoglœea

Suprarenals, 447; and see Adrenals

Suspensorium, 40, $43^{8}$

Sutures, 50, $4^{87}$

Swimmeret, see Pleopod

Symbiosis, 304

Sympathetic, see Nerves

Symphysis, 496, 503

Syn-cerebrum, 380

Synovial capsule, 56

Systęmic arch, 80, 452, 523

Systole : of heart, 90 ; of contractile vacuole, 266

\section{$\mathrm{T}$}

Tactile organs, $179,353,382,533$

Tadpole, 9,203 , et seq.

Tail, 9, 209, 432, 483, 484

Tape-worms, 412, 545

Tapetum, 556

Tarsus, 5I, 504

Taste-organs, $180,509,534$
Teasing, directions for, 123

Teats, 486,506

Teeth, I7, 434, 444, 509

Telencephaion, 590

Teleostomi, 43I

Telolecithal ova, 574

Telson, 36r

Tendon, 59

Tentacles, 295, 309, 31 4, 31 5, $35^{\mathrm{I}}$

Tergum, 36r

Testis, see Spermary

Tetrad, 568

Thalamencephalon, see Diencephalon

Thigh, 5

Thoracic duct, 528

Thorax, 36r, $483.486,506$

Thread-cell, see Nematocyst

Thymus, 447, 507, 519

Thyroid, 447,520

Thyroid, cartilage, $5 \mathrm{I} 7$

Tibia, 5I, 504

Tibio-fibula, 5 I

Tissues, enumeration of, $3 \mathrm{I}$

Toad, see Bufo

Toes, see Digits

Tongue, 8, 17, 445, 509

Tonsil, 507

Trabeculæ cranii, 605

Trachea, 506, 5I 7

Transverse process, see Vertebra

Trichocyst, 267

Trimorphic, 3 I 8

Triploblastic, 31 I, 322, 332

Trochanter, 504

Trochlea, 501

Trochosphere, 354

Trophoblast, 586

Trophozoite, 286

Trunk, 4, 432, 484

Trypanosoma, 284

Trypsin, 74

Tubercle, and Tubercular facet, 498,500

Tuberosity, 50r, 503

Tunicata, 42 I

Turbinals, 493, 534

Tympanic cavity, membrane, and ring, 5, 45, 189, 536

Typhlosole, 335,401 
U

Ulna, 50, 502

[mbilical cord and umbilicus, $597,6 \mathrm{I}_{3}, 6 \mathrm{I}_{5}$

Umbilical vesicle, 586

Umbo, 398

Umbrella of Medusa, 314

Unicellular, $23 \mathbf{I}$

Unio, see Mussel

Trachus, 6I 5

Urea, 66, I47

Ureter, 25: and see under various types

Urethra, see Urinogenital canal

Uric acid, 66

Urinary bladder, 23, I47, 375, $406,538,6$ i 5

Urinary tubules, see Nephridium

Urine, 8, 66, I 47

Urinogenital aperture, 486 canal, $539,54^{\circ}$ duct, I94, 603 organs, I93, 466, 537 organs, develop-

Urodeles, 219 ment of, 600

Uropod, 364

Urostyle, 35,39

Uterine crypts, 6 I I

Uterine tube, 540

Uterus, 540 .. masculinus, $53^{8}$

Utriculus, 187

\section{V}

Vacuole: contractile, 232,243 . $254,265,269$; food $-, 233,266$, 272

Vagina, 5 to

Valve: of Vieussens, 529, 531 (Figs.); spiral, 445, 515; ileocolic, 515

Valves: of heart, $88,376,52 \mathrm{I}$, 523 ; of shell, 396 ; of veins, 89

Variation, 216, 225, 292, 483
Variety, 225

Vascular system, see Bloodvessels, Arteries, Veins

Vas deferens, see Spermiduct, Wolffian duct

Vasa efferentia, see efferent ducts

Veins: Frog, 19, 82; Crayfish,

377; Mussel, 408; Dogfish.

454; Rabbit, 525; embryo

Vertebrate, 599,600

Veliger, $4^{\text {I I }}$

Velum: of Medusa, 315; of Lancelet, 424

Velum palati, 507

Vena cava, see Veins

Vent, see Anus

Ventral, 6

Ventricle, see Heart

Ventricles of brain, I57, I 59, 424 $459,529,531,590$

Vermiform appendix, 515

Vertebra and vertebral column, I $6,35,36,439,497,604$

Vertebrarterial canal, see Canal

Vertebrata, 220 ; general characters of, 418

Vessels, see Blood-vessels

Vestibule, see Urinogenital canal

Vestige, vestigial, I 59, 364, 521, 550

Vibrissae, 486

Villi: of intestine, 515; of chorion, $6 \mathrm{II}$

Viscera, abdominal, 20, 444, 512

Visceral arches and clefts, 438 , $44^{8}$; and see Branchial apertures

Visceral ganglion, 408

Visceral layer of peritoneum, 27, 331

Visceral mass, 399

Vitelline membrane, 565, 609

Vitelline vessels, 599

Vitreous body of compound eye, 380

Vitreous chamber and humour, 183

Vocal cords, I44, 5I 7

Vocal sacs, 218

Vomero-nasal organ, 534 
VORTICELLA : occurrence and general characters, 269 ; structure, 269; reproduction, 273 ; conjugation, 274 ; means of dispersal, 274; encystation, 275 ; spore-formation, 276 ; metamorphosis, 276 ; practical directions, $27 \gamma$

Vulva, $4^{87}, 540$

W

Waste-products, $8,66,249$

White matter of brain and spinal cord, I 56, I 60,167

Wolffian body, see Epididymis duct, 603

Work and waste, $66, x_{4} 8,234$

Worms, 349

Wrist, see Carpus

\section{$\mathrm{X}$}

Xiphisternum, 48, 500

\section{Y}

Yellow cells of Earthworm, 33x, 335,34 I

Yolk, yolk-granules or spheres, r95, 564, 569; and see under various types

Yolk-cells, 200, 202

Yolk-plug, 20I, 581

Yolk-sac, $470,582,5^{86}$
Zona radiata, 586

Zoochlorella, 304

Zooid, 277, 309; and see Megazooid and Microzooid

Zoology, I

Zoophytes, see Hydroid polypes Zygapophysis, 36,498

Zygoma, zygomatic arch, 494. 495

Zygote, I97, 25I, 275, 284 
By Prof. T. JEFFERY PARKER and Prof. WILLIAM A. HASWELL.

\section{A TEXT-BOOK OF ZOOLOGY. In Two}

Vols. With Illustrations. Medium 8vo, $36 s$. net.

VATLRAL SCIEVCE. - "Parker and Haswell' has been looked forward to with expectation for cone time. We welcome it gladly $n$ ow that it appears; it hould be in the hand, of all -tudents, ant even teachers will find it of value. . . It is so we.l illustrated, so c early pri ted, and generally good, that it will be fourd a useful addition to the student's and teacher's shelves."

\section{A MANUAL OF ZOOLOGY. Illustrated. Crown 8 vo, 10s. $6 d$.}

BY Prot. T. JEFFERY PARKER.

\section{LESSONS IN ELEMENTARY BIOLOGY.}

Illustrated. Crown 8vo, i2s. $6 d$.

N.ATURE. - "Prof. Jeffery Parker is to be congratulated on having produced an extremely weil-written, well-considered, and original class-book."

\section{WILLIAM KITCHEN PARKER, F.R.S.}

A Short Memoir. Crown 8vo, 4s. net.

ATHEN.EC:M._- Prof. Jeffery Parker is to be warmly cingratulated on thicharming sketch of his beloved fatner's life. . . As a sketch it is, we think, perfect."

By Prof. T. JEFFERY PARKER and Prof. W. NEWTON PARKER.

\section{ELEMENTARY COURSE OF PRAC- TICAL ZOOLOGY. Illustrated. Crown 8vo, I2s. $6 t$.}

NATURE.- "Will be welcomed by many as a handy and inexpensive Manual of Zoology adapied to the need; of elementary, and especially mecical, stucients, which is at the sime time free from the futts and vices of the harmful, unnecessary cram. book. .... Mary be confidently recommended."

MACMILLAN AND CO., LTD., LONDON. 


\section{THE GAMBRIDGE NATURAL HISTORY}

Edited by

S. F. HARMer, Sc.D., F.R.S, and A. E. Shipley, M.A., F.I In Ten Volumes. Fully Illus!rated. Medium 8vo, 2is. net each Vol. I. PROTOZOA, by Marcus Hartog, M.A., D.S PORIFERA (SPONGES), by IGERna B. J. SOLlas, B.SC.; COELENTER. AND CTF.NOPHORA, by S. J. HICKSON, M.A., F.R.S. ; ECHINODERMA by E. W. MACBRIDE, M.A., F.R.S.

Vol. II. FLATWORMS AND MESOZOA, by F. Gamble, D.Sc.; NEMERTINES, by Miss L. Sheldon; THREAD-WO AND SAGITTA, by A. E. SHIPLEY, M.A., F.R.S. ; ROTIFERS, by MAI HARTOG, M.A., D.Sc. ; POLYCHAET WORMS, by W. BLAXland BeNI D.SC., M.A.; EARTHWORMS AND I.EECHES, bY F. E. BEDDARD, A F, R.S. ; GEPHYREA AND -PHORONIS, by A. E. SHIPLEY, M.A., F.F POLYZOA, by S. F. HARMER, Sc.D., F.R.S.

Vol. III. MOLLUSCS, by the Rev. A. H. Cooke, M. BRACHIOPODS (Recent), by A. E. SHIPLEY, M.A., F.R.S. ; BRACHIOPC (Fossil), by F. R. C. REED, M.A.

Vol. IV. CRUSTACEA, by Geoffrey Smith, M and the late W. F. R. WELDON, M.A. ; TRILOBITES, by HENRY Wo M.A. ; Introduction to ARACHNIDA AND KING-CRABS, by A. E. SHIP M.A., F.R.S. : EURYPTIRIDA, by HENRY WOONS, M.A.; SCORPIC SPIDERS, MITES, TICKS, \&c.. by CECIL WARBURTON, M.A. : TARIIICR. (WATER-13ears), by A. i. ShIPley, M.A., F.R.S.; PENTAST()MI by A. E. ShIPLEY, M.A., F.R.S.; PYCNOGONIDA, by D'ARCY W. Thoup C.B., M.A.

Vol. V. PERIPATUS, by AdAM SEdgwick, M.A., F.R. MYRIAPODS, by F. G. Sinclair, M.A. ; INSECTS, Part I., Introduct Aptera, Orthoptera, Neuroptera, and a portion of Hymenoptera (Sessiliver and Parasitica), by David Sharp, M.A., M.B., F.R.S.

Vol. VI. HYMENOPTERA, continued (Tubulifera Aculeata), Coleoptera, Strepsiptera, Lepidoptera, Diptera, Aphaniptera, sanoptera, Hemiptera, Anoplura, by DAvir SHarp, M.A., M.B., F.R.S.

Vol. VII. HEMICHORDATA, by S. F. HARMer, Sc. F.R.S. ; ASCIDIANS AND AMPHIOXUS, by W. A. HERUMAN, D F.R.S.; FISHES (Exclusive of the Syitematic Account of Teleostei), by T BRIDGE, Sc.D., F.R.S.; FISHES (Systematic Acrount of Teleostei), G. A. BOULENGER, F.R.S.

Vol. VIII. A.IIPHIBIA AND REI'TILES, by $\mathrm{H}$ GADOW, M.A., F.R.S.

Vol. IX. BIRIS, by A. H. Evans, II A.

Vol. X. MAMMalia, by Frank Evers Bedda M.A., F.R.S.

MACMILLAN ANI) (O., LTD., LUNI)ON. 



\section{.}

$$
1
$$




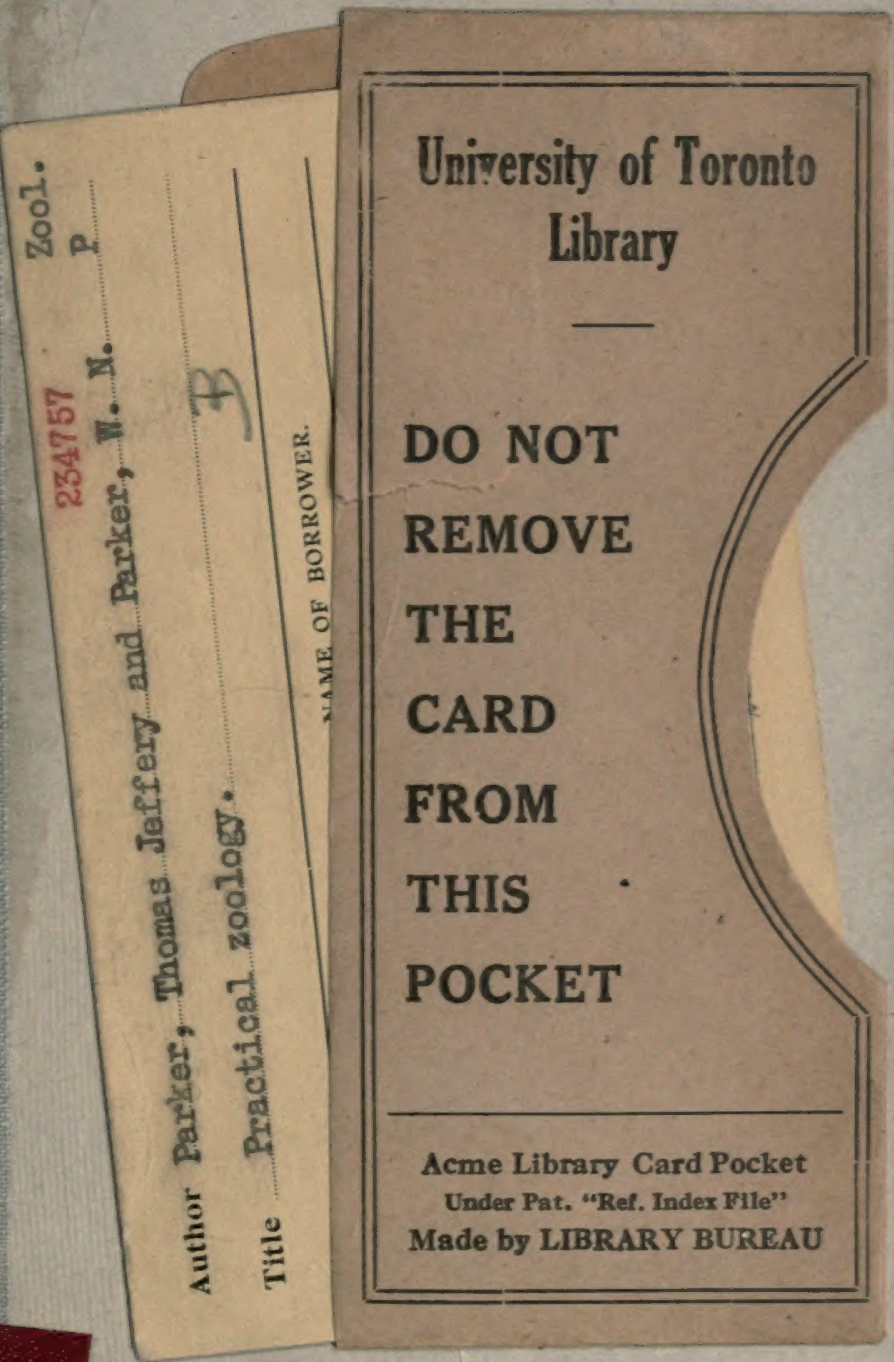




\section{$7 \mathrm{x}$}

(1)

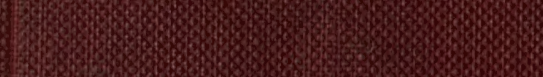

